DISSERTATION

\title{
DECONTAMINATION OF BIOAEROSOLS WITHIN ENGINEERING TOLERANCES OF AIRCRAFT MATERIALS
}

\author{
Submitted by \\ John S. Frazey \\ Department of Environmental and Radiological Health Sciences
}

\author{
In partial fulfillment of the requirements \\ For the Degree of Doctor of Philosophy \\ Colorado State University \\ Fort Collins, Colorado
}

Fall 2012

Doctoral Committee:

\author{
Advisor: Stephen Reynolds \\ John Volckens \\ Thomas J. Keefe \\ Robert P. Ellis \\ Chad J. Roy
}


Copyright by John S. Frazey 2012

All Rights Reserved 


\section{ABSTRACT \\ DECONTAMINATION OF BIOAEROSOLS WITHIN ENGINEERING TOLERANCES OF AIRCRAFT MATERIALS}

Bacillus anthracis spores are generally considered the most difficult biological agents to decontaminate or inactivate. Inactivation of these spores is further complicated on aircraft because engineering specifications do not allow for chemical disinfectants to be used. Aircraft, however, must meet strict engineering specifications, requiring extended storage at temperatures greater than $185^{\circ} \mathrm{F}$ at $100 \%$ relative humidity $(\mathrm{RH})$. Heat and humidity near these levels have been tested to determine if they can inactivate spores; however, these studies have only evaluated spores in high concentrations $\left(10^{6}\right.$ spores $)$ on aluminum coupons. This dissertation research was designed to evaluate the effectiveness of high heat and humidity on Bacillus atrophaeus subsp globigii (BG) spores, a simulant commonly used for Bacillus anthracis, when delivered via three different methods onto two different materials.

In Chapter 2, an innovative bioaerosol deposition chamber design and testing is described. The test chamber was designed to deposit Bacillus atrophaeus subsp globigii (BG) spores onto coupons modeling real aircraft components. Deposition equations were derived to model the spore deposition. Initial deposition tests with fluorescent particles were inconclusive because the limit of quantification could not be reached; therefore, the BG spores were used to test deposition. Initial tests demonstrated the parameters that could be manipulated throughout the experiments to control the spore deposition. After these were evaluated, four final tests were completed to perform more in-depth statistical analysis. The coefficients of variation for these tests were within acceptable ranges (all were $25.5 \%$ or less). Ryan-Joiner tests were performed 
on the data and showed that 2 of the 4 tests displayed a lognormal distribution, while the other 2 tests were inconclusive. All data was therefore treated as a lognormal distribution. Contour plots were then constructed to determine if a discernible pattern was present. While these contour plots showed a somewhat even dispersion, there were no discernible patterns. Additionally, the plots showed a wide range of spore deposition throughout the four tests. Finally, the equations derived for spore deposition were validated. The data showed that $8.67 \%$ up to $31.0 \%$ (average of $20.25 \%$ ) of the spores modeled could actually deposit and be recovered through culture methods. These losses could have occurred during the nebulization through inactivation or clumping after the spores were aerosolized. Regardless, this showed that the equations could be used after accounting for these losses. The study demonstrated that the test chamber can be used for spore depositions with the caveat that future studies include an appropriate control coupon next to each sample.

In Chapter 3, decontamination of aluminum coupons was evaluated using the BG spores inoculated in three different methods - high direct inoculation $\left(10^{6}\right.$ spores per coupon), low direction inoculation $\left(10^{4}\right.$ spores per coupon), and an aerosol deposition using the test chamber from Chapter 2 (deposition goal of $10^{4}$ spores per coupon). Initial tests found the optimal method to remove the spores from coupons was sonication followed by vortexing, which was nearly five times more effective at removing the spores than shaking. Equations, derived to model spore depositions in the aerosol test chamber, were tested and showed that $10 \%$ of the spores could be effectively recovered. Five different test conditions of temperature and humidity (ranging from an upper limit combination of $180^{\circ} \mathrm{F}$ and $90 \%$ relative humidity $[\mathrm{RH}]$ to a lower limit of $160^{\circ} \mathrm{F}$ and $70 \% \mathrm{RH}$ ) were evaluated over 24 hour increments with an upper time limit of 120 hours. Decontamination tests showed that the high concentrations of spores were all 
inactivated within 24 hours at $180^{\circ} \mathrm{F}$ with $90 \% \mathrm{RH}$ and partially inactivated at $170^{\circ} \mathrm{F}$ with $80 \%$ RH. Tests using low direct inoculations showed complete kills at 48 hours when treatment was $180^{\circ} \mathrm{F}$ with $90 \% \mathrm{RH}$ and at 96 hours when treatment was $170^{\circ} \mathrm{F}$ with $80 \% \mathrm{RH}$. All spores deposited by aerosols were inactivated within the 120 hour time period. A stepwise regression was performed to determine which variables are significant to predict the inactivation rates $(\alpha=$ 0.05 was used to keep or discard terms). For this regression, there were three variables required to be in each model — time, temperature, and humidity. The data for the stepwise regression retained more variables for high direct inoculation (10 predictors) than low (8 predictors) or aerosol deposition (5). The only variable retained by all three models, besides the mandatory variables, was Temp ${ }^{2} * \mathrm{Time}^{2}$. For both of the direct inoculation methods, several of the same variables were retained, which included Temp*Humidity, Temp*Time, Humidity ${ }^{2}$, and $\mathrm{Temp}^{2} * \mathrm{Time}^{2}$. These variables were then used to complete a final regression model to determine inactivation rates. The final regression models had $\mathrm{R}^{2}$ values for high and low inoculation methods of $76.4 \%$ and $71.5 \%$, respectively. The $\mathrm{R}^{2}$ for the aerosol deposition model was not as strong, being only $38.5 \%$. The ideal humidity and temperature range is clearly the highest levels that can be delivered, reasonably maintained, and within proper engineering specifications. If $90 \%$ humidity cannot be easily generated or maintained throughout the body of an aircraft, the results show that $80 \%$ at the proper temperature $\left(170^{\circ} \mathrm{F}\right.$ or higher) can be effective as well.

The study in Chapter 4 evaluated decontamination rates on plastic coupons, using the same inoculation methods as Chapter 3. Decontamination tests showed that the high concentrations of spores were inactivated within 48 hours at $180^{\circ} \mathrm{F}$ and $90 \% \mathrm{RH}$. No other treatment temperatures or humidity ranges inactivated all spores within the time allotted of 120 
hours. Tests using low direct inoculations showed complete kills at 48 hours with a treatment of $180^{\circ} \mathrm{F}$ with $90 \% \mathrm{RH}$ and $170^{\circ} \mathrm{F}$ with $80 \% \mathrm{RH}$. Additionally, all spores were inactivated at 120 hours $160{ }^{\circ} \mathrm{F}$ with $90 \%$ RH. Aerosol deposited spores were inactivated within 48 hours for all five test conditions, except for treatment with $160^{\circ} \mathrm{F}$ with $70 \% \mathrm{RH}$, which still had active spores at the 120 hour point. A stepwise regression was performed to determine which variables are significant to predict the inactivation rates ( $\alpha=0.05$ was used to keep or discard terms). For this regression, there were three variables required to be in each model—-time, temperature, and humidity. The stepwise regression resulted in approximately the same number of terms being retained in the models with high, low, and aerosol deposition have 7, 6, and 8 terms, respectively. Besides the mandatory variables (time, temperature, and humidity), there were no variables retained in all three models. The statistical analysis does indicate humidity is a critical factor, as nearly all variables retained in these models contain humidity — each model only has one variable that does not contain humidity. The $\mathrm{R}^{2}$ values are reasonable for these models, with the values being $76.6 \%, 68.8 \%$, and $77.8 \%$, for high and low direct inoculation and aerosol deposition, respectively. Thus most of the variability for the spore inactivation is explained by the models.

Data from Chapters 3 and 4 were compared to determine if there were significant differences in the inactivation rates between aluminum and plastic. The slopes for inactivation plots were compared for plastic and aluminum coupons for each test condition. For high direction inoculation, there was a significant difference for test condition $5\left(170^{\circ} \mathrm{F}\right.$ with $80 \%$ $\mathrm{RH})$ and test condition $7\left(160^{\circ} \mathrm{F}\right.$ with $\left.90 \% \mathrm{RH}\right)$. For low direct inoculation there was only one test condition that was significantly different - test condition $7\left(160^{\circ} \mathrm{F}\right.$ with $\left.90 \% \mathrm{RH}\right)$. A tobit analysis showed the plastic coupon inactivation rates were significantly different, indication a 
faster inactivation for plastic coupons at test condition $3\left(180^{\circ} \mathrm{F}\right.$ with $\left.70 \% \mathrm{RH}\right), 5\left(170^{\circ} \mathrm{F}\right.$ with $80 \% \mathrm{RH})$, and $7\left(160^{\circ} \mathrm{F}\right.$ with $\left.90 \% \mathrm{RH}\right)$. This shows that only 6 of the 15 test conditions were significantly different for plastic versus aluminum coupons. All of the 6 tests that were significantly different showed the plastic coupons were inactivated with less time, thus demonstrating that if the spores are inactivated on aluminum coupons, they will likely be inactivated on plastic coupons as well

Taken together, these three studies demonstrate that high heat and humidity can safely and effectively decontaminate aircraft materials at the proper time intervals. Furthermore, it appears that plastic coupons can be decontaminated at a faster rate for some of the temperature and humidity ranges. Finally, the spores that were deposited by aerosol were decontaminated more effectively than the spores inoculated directly. 


\section{ACKNOWLEDGEMENTS}

First and foremost a very sincere thank you to Kirsten, Justis, and Britin. You have been the inspiration throughout this project. I cannot thank you three enough for the patience you've shown over the last three years during the long hours it took to complete this project. Also, thank you Mom and Dad for teaching me the value of hard work and education.

Thank you to my committee for the guidance and assistance throughout these last three

years. A special thanks to Dr. Reynolds for all the help, especially on those days when it was not going so well. Also, thank you to Dr. Volckens for the guidance in the aerosol portion-without that help, this project would not have been completed. I also want to thank Dr. Keefe for the help with the statistics, especially towards the end of the project. Also, thank you Dr. Ellis for help with the biosafety aspects and also Dr. Roy for the bioaresol portion.

This project would not have started without the funding provided by Dr. Reynolds through the grant from the Mountain and Plains Research Center (MAP ERC). Additional funding was provided through a grant from AFMSA/SGRS, with considerable assistance from Neredya Sevilla. The Air Force funds were committed with assistance of William Kilpatrick, Gregory Sudberry, Robert Arrington, and Ashley Grant - a considerable amount of work was required to obligate and track these funds. Again, this research would not have been possible without these funds.

Several different organizations donated supplies. Aluminum coupons were provided by William Culhane (USAF Coating Technology Integration Office) and plastic coupons were provided by Dr. Ken Heater and Daniel E. Badowski (METSS Corporation). Spores were provided by Joe Dalmasso, Yakibou, Inc. and Jeremy Beard, Dugway Proving Grounds. Thank you to all these organizations who provided these supplies 
I have many people to thank for their help with this project. Thank you to Kirsten Koehler for all the assistance with the aerosol deposition chamber; Tim Provens for background knowledge and future needs of this decontamination method; Joe Dalmasso for the microbiological advice for the spores; James zumBrunnen for assistance with statistical analysis; Stefan Tonazzi for computer assistance; John Mehaffy for help in the lab and ordering supplies;, Jaclyn Adkins for assistance with the scanning electron microscope, and finally, to Josh Schaeffer for research collaboration throughout the entire project and also all the coursework.

I have had several exceptional mentors who have helped guide me throughout my career - Chad Cunningham, Joe Costantino, David Hammiel, Dave Byer, and Mike Chulick. Without this guidance, I could not have been accepted and completed this program. 


\section{DEDICATION}

To Kirsten, Justis, and Britin. 


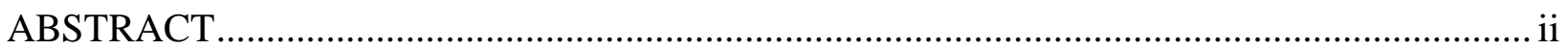

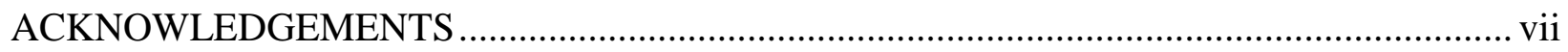

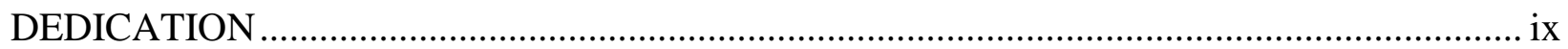

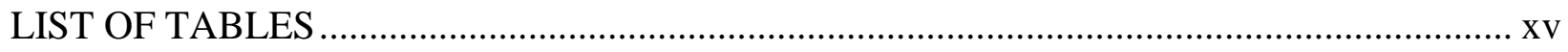

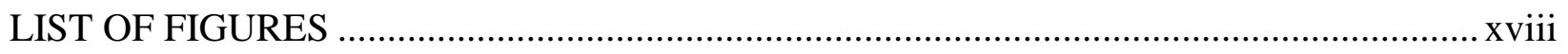

CHAPTER 1 -- INTRODUCTION, GOALS, AND BACKGROUND .......................................... 1

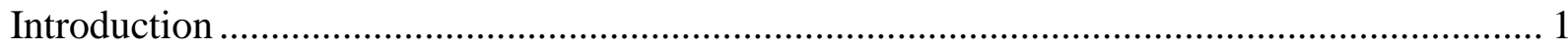

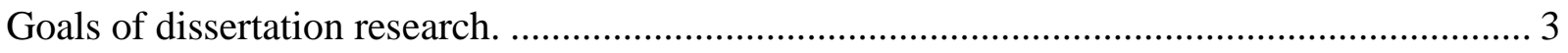

Specific aims and hypotheses ……………………........................................................ 3

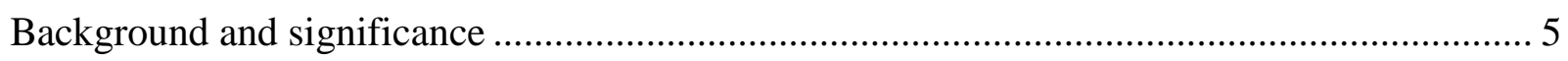

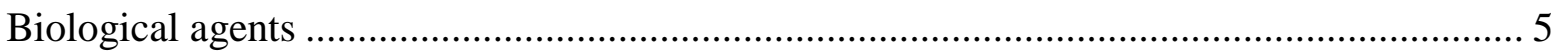

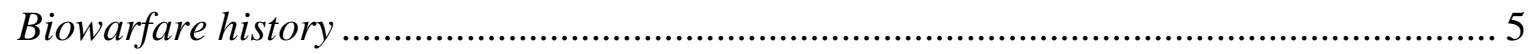

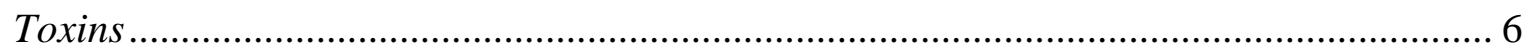

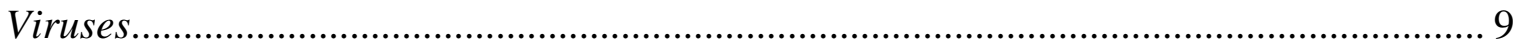

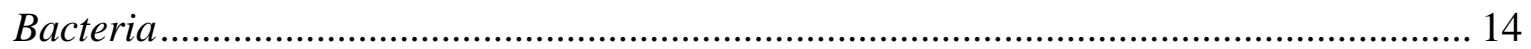

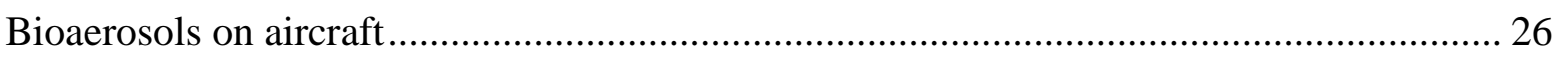

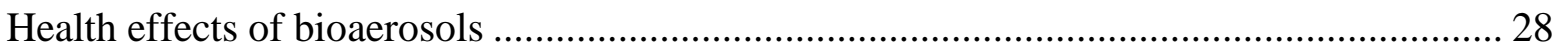

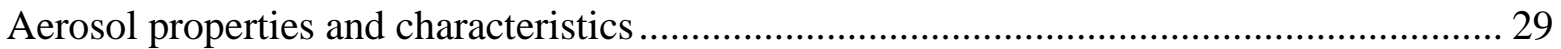

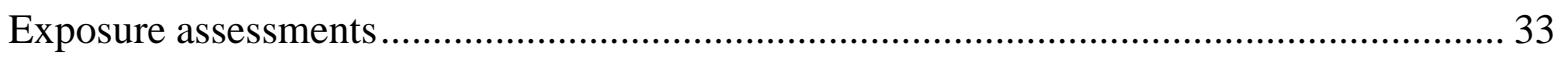

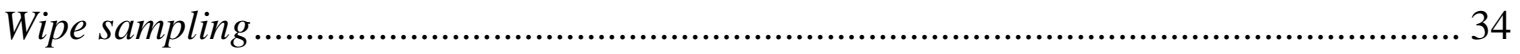

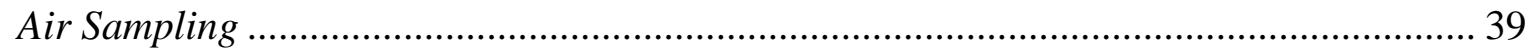

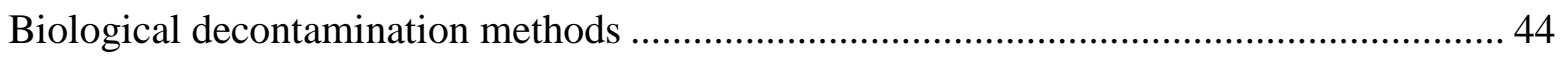

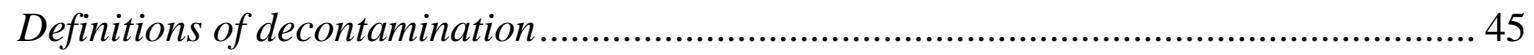

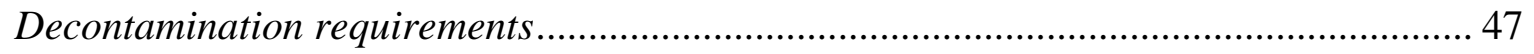

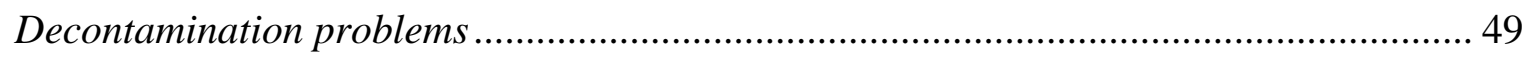

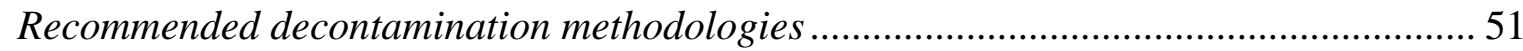

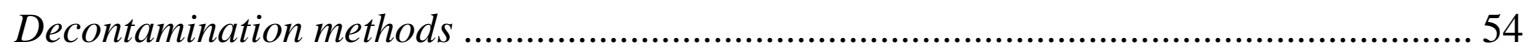

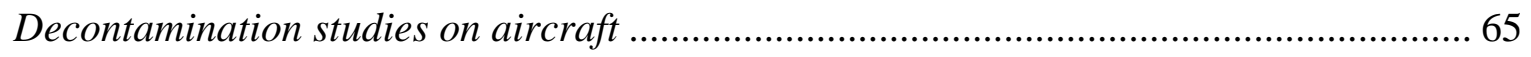




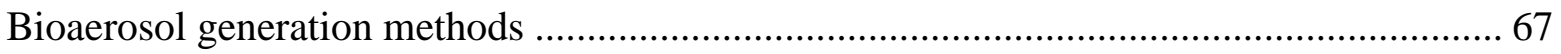

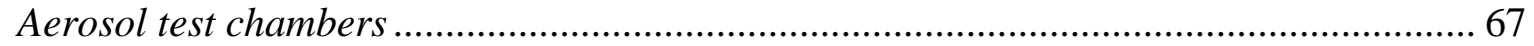

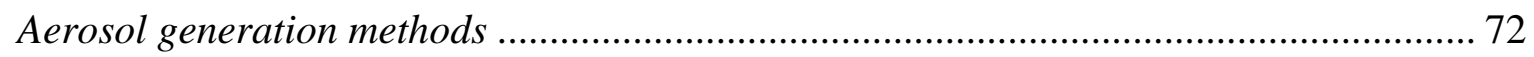

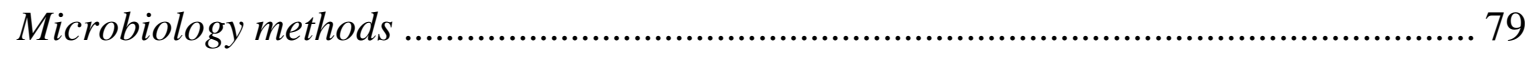

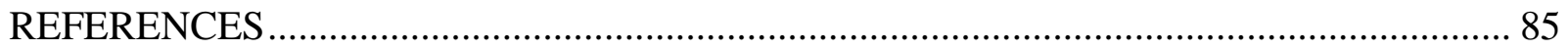

CHAPTER 2 -- DESIGN AND EVALUATION OF A BIOAEROSOL DEPOSITION CHAMBER FOR TESTING DECONTAMINATION OF AEROSPACE MATERIALS ........ 106

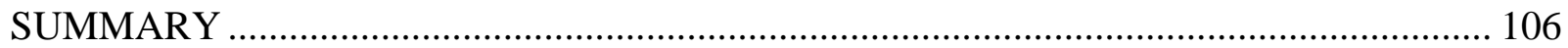

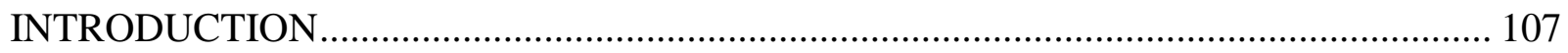

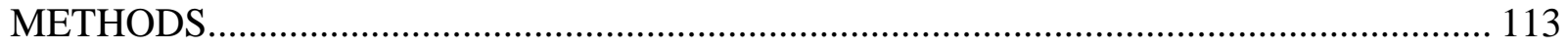

Bioaerosol Test Chamber Design ....................................................................... 113

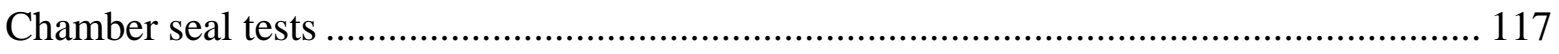

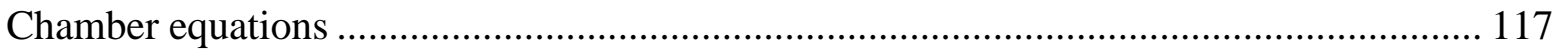

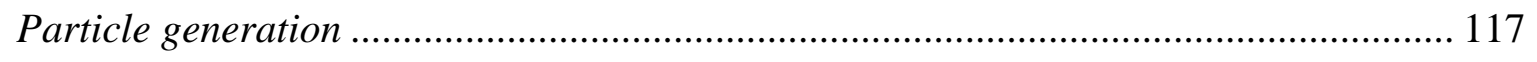

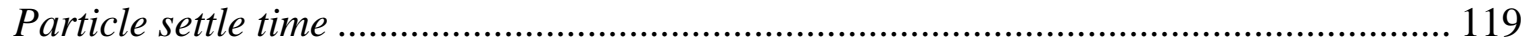

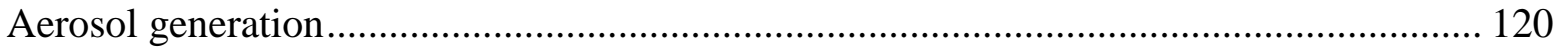

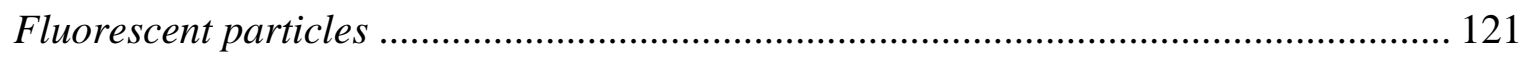

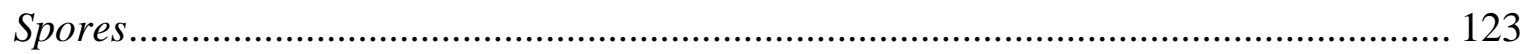

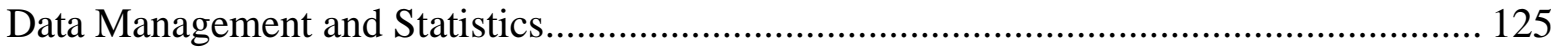

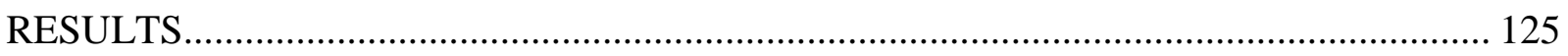

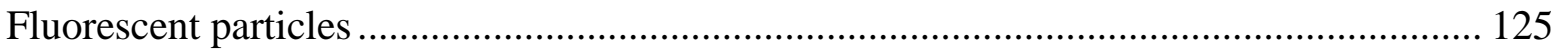

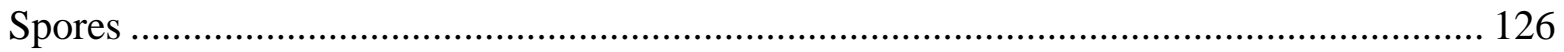

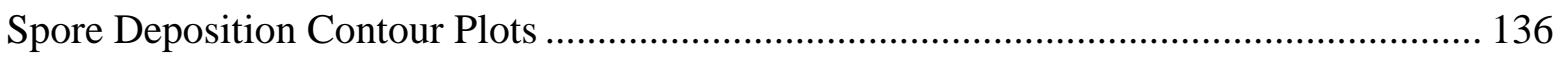

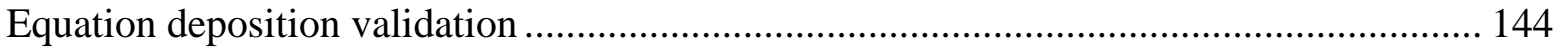

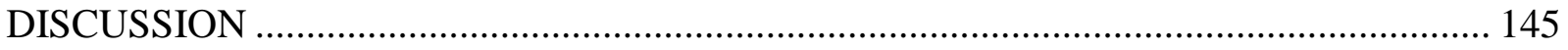

ACKNOWLEDGEMENTS .................................................................................. 146

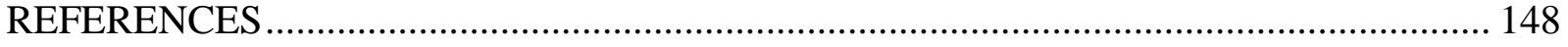

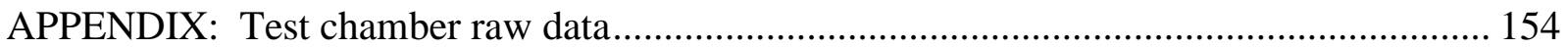

CHAPTER 3 -- DECONTAMINATION OF A BACILLUS ANTHRACIS SPORE SIMULANT ON AIRCRAFT ALUMINUM COUPONS USING HIGH HEAT AND HUMIDITY WITHIN AIRCRAFT ENGINEERING TOLERANCES ............................................................ 156

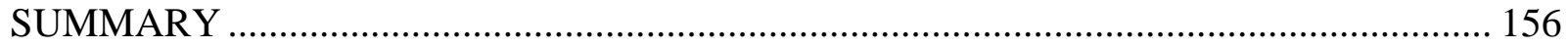




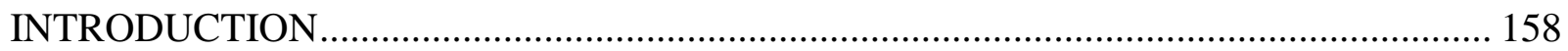

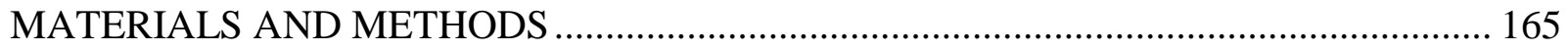

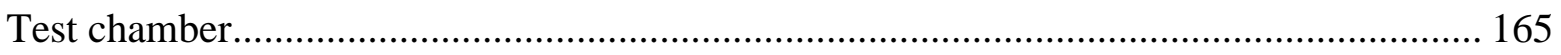

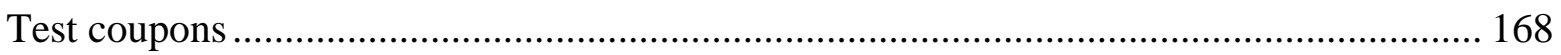

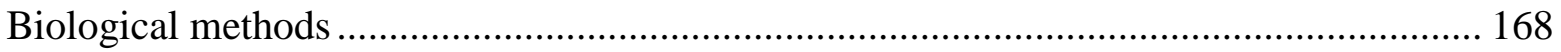

Spores

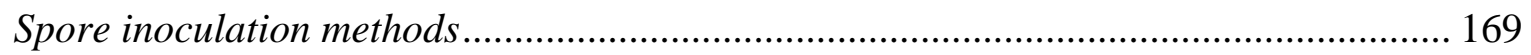

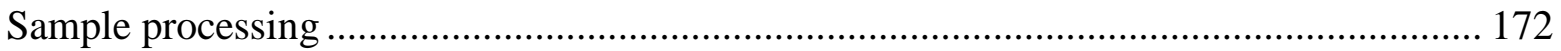

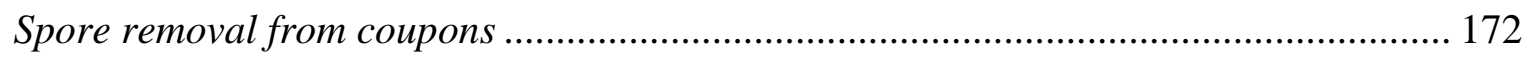

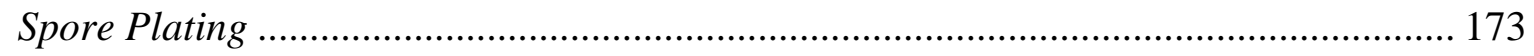

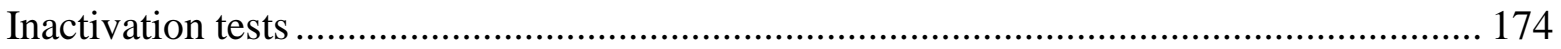

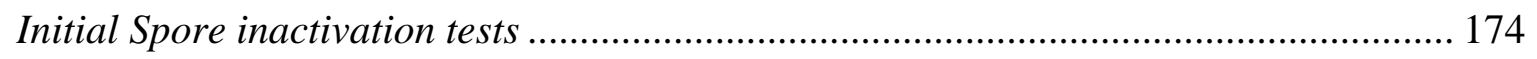

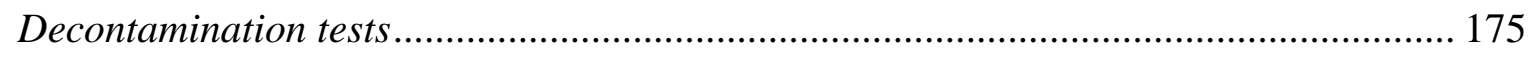

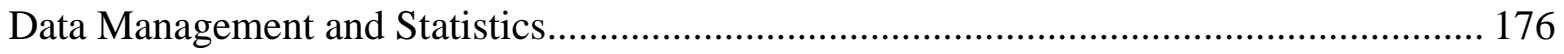

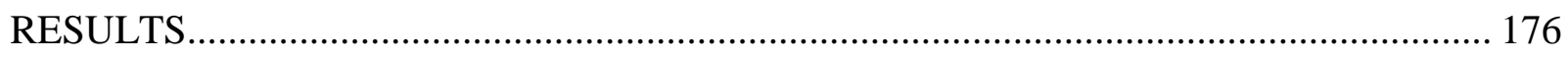

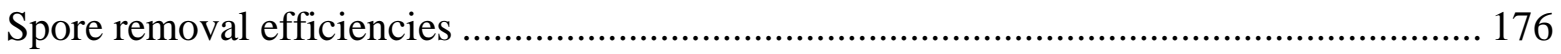

Aerosol deposition removals ...................................................................................... 177

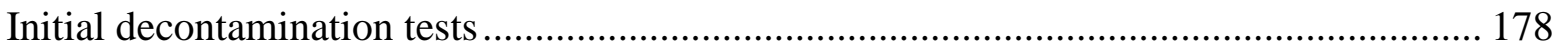

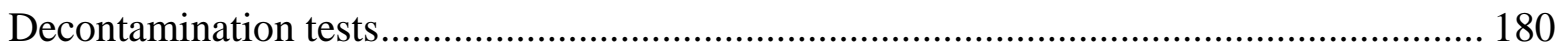

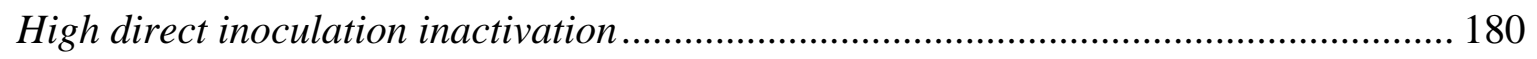

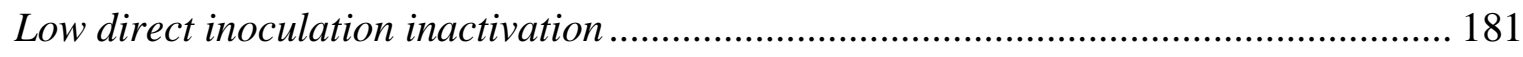

Aerosol deposition inoculation inactivation ………………………………………..... 182

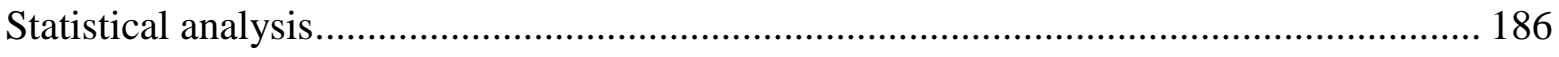

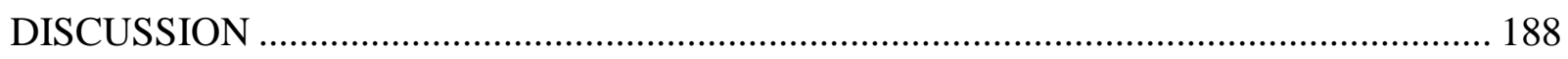

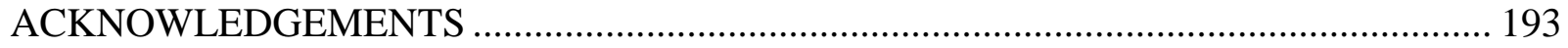

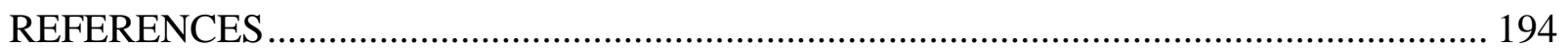

APPENDIX 1: High direct inoculation test plots............................................................... 201

APPENDIX 2: Low direct inoculation test plots ............................................................... 204

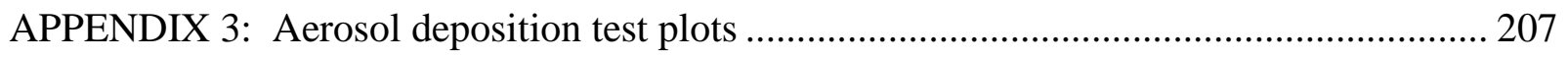

APPENDIX 4: High direct inoculation data .................................................................. 210

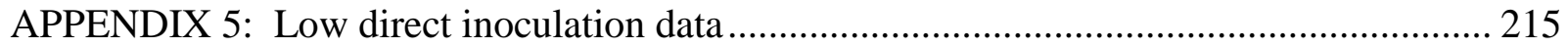


APPENDIX 6: Aerosol deposition.

CHAPTER 4 -- DECONTAMINATION OF A BACILLUS ANTHRACIS SPORE SIMULANT ON AIRCRAFT PLASTIC COUPONS USING HIGH HEAT AND HUMIDITY WITHIN AIRCRAFT ENGINEERING TOLERANCES.

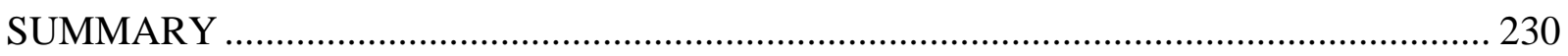

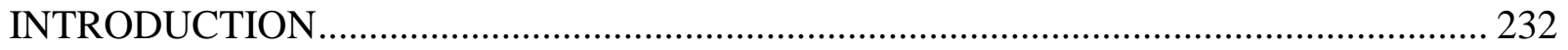

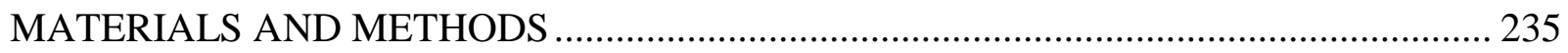

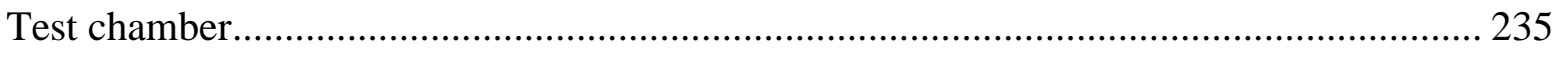

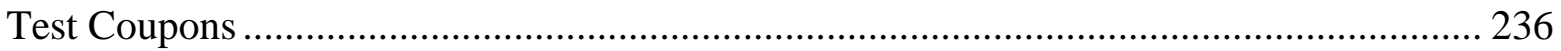

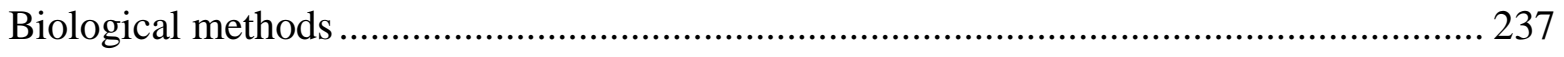

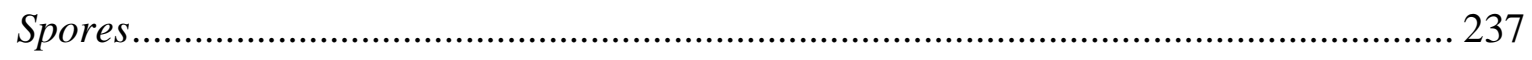

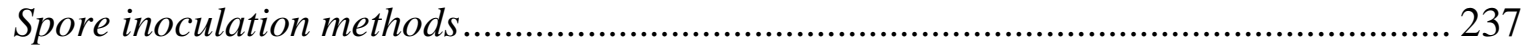

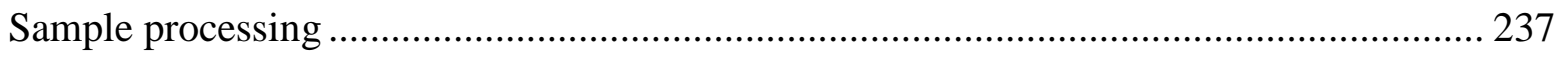

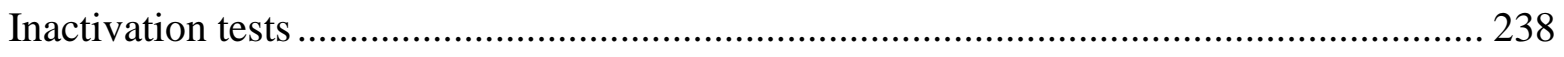

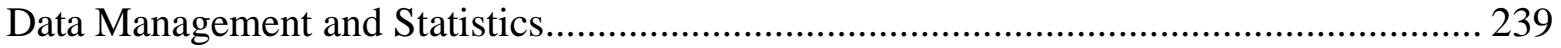

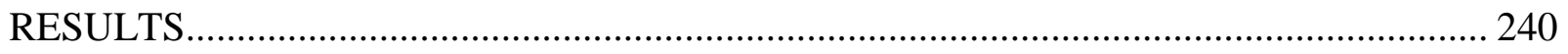

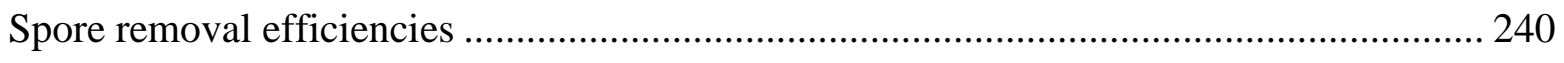

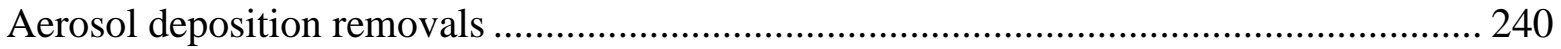

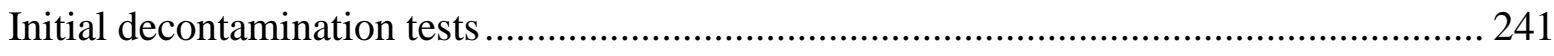

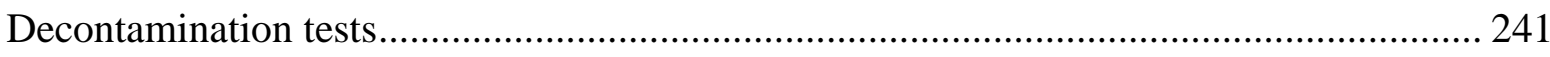

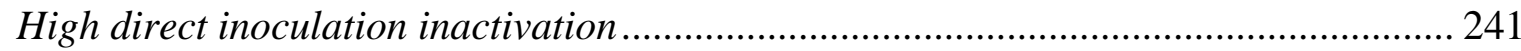

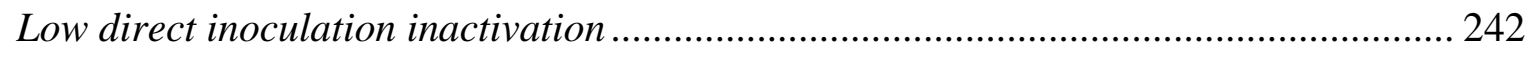

Aerosol deposition inoculation inactivation .......................................................... 243

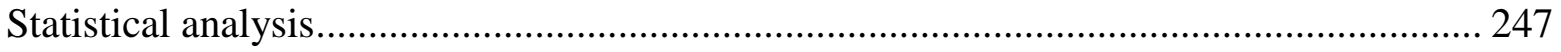

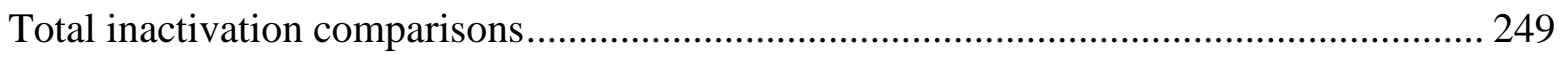

Direct inoculation inactivation rate comparisons ................................................... 250

Aerosol deposition inactivation rate comparisons ................................................... 251

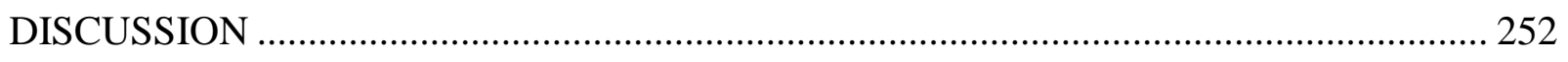

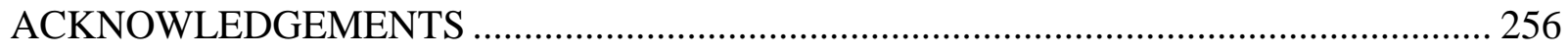

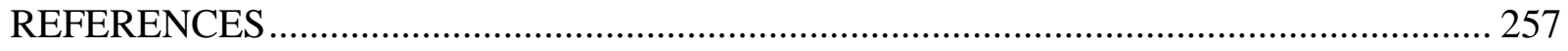

APPENDIX 1: High direct inoculation test plots........................................................... 264 


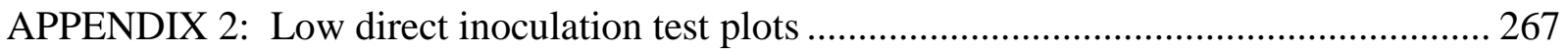

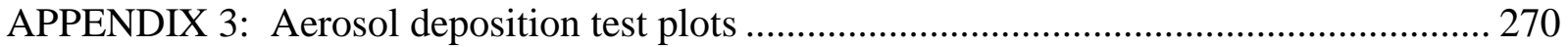

APPENDIX 4: High direct inoculation data .................................................................. 273

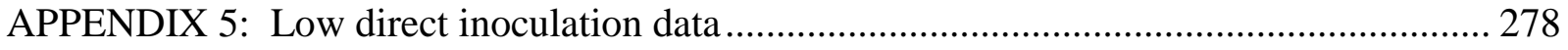

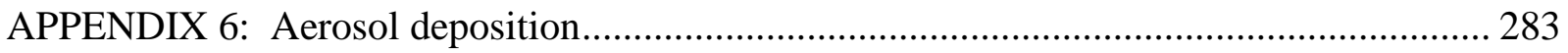

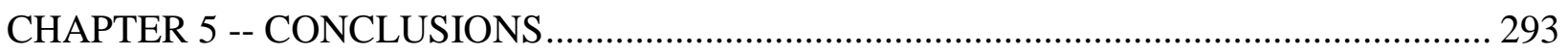

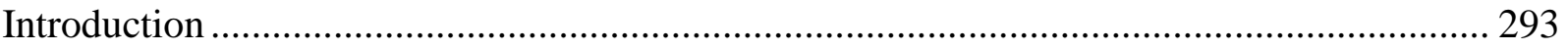

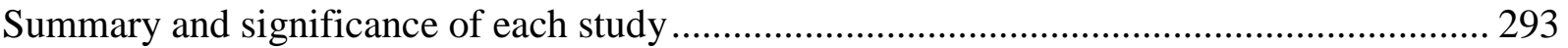

A bioaerosol test chamber can be designed and built to model biological agent deposition on aircraft materials...................................................................................................... 293

Aluminum coupons, indicative of aircraft materials, can be effectively decontaminated using high heat and humidity. ....................................................................................................... 294

Plastic coupons, similar to materials used on aircraft, can be effectively decontaminated using high heat and humidity....

There were six tests that showed a significant difference between the two different materials.

Conclusion 297

Future Research 297

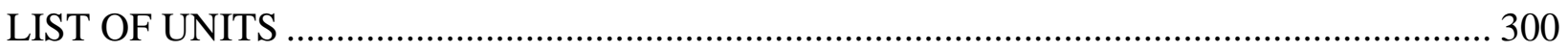

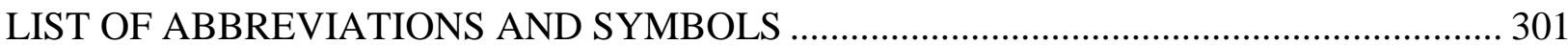




\section{LIST OF TABLES}

Chapter 1

TABLE 1 - 1 - VIRUSES THAT COULD BE USED AS BIOLOGICAL WARFARE AGENTS

TABLE 1 - 2 - BACTERIA THAT COULD BE USED AS BIOLOGICAL WARFARE AGENTS 15

TABLE 1 - 3 - SUMMARY OF CHEMICAL DISINFECTANTS ........................................ 55

TABLE 1 - 4 - DRY BIOAEROSOL DISSEMINATION METHODS .................................. 74

Chapter 2

TABLE 2 - 1 - FINAL TEST CHAMBER OPERATING PARAMETERS .......................... 126

TABLE 2 - 2 - SPORE DEPOSITION TESTS SUMMARY ............................................ 127

TABLE 2 - 3 - RYAN-JOINER ANALYSES, SPORE DEPOSITION TESTS ..................... 136

TABLE 2 - 4 - TEST 72 SPORE DEPOSITION STATISTICAL SUMMARY ..................... 140

TABLE 2 - 5 - TEST 76 SPORE DEPOSITION STATISTICAL SUMMARY ..................... 140

TABLE 2 - 6 - TEST 80 SPORE DEPOSITION STATISTICAL SUMMARY ...................... 141

TABLE 2 - 7 - TEST 81 SPORE DEPOSITION STATISTICAL SUMMARY ..................... 141

TABLE 2 - 8 - SPORE DEPOSITION SUMMARIES .................................................... 144

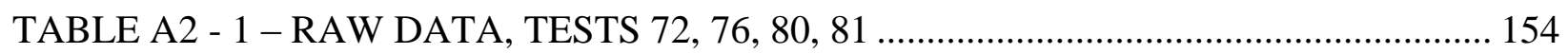

Chapter 3

TABLE 3 - 1 - SPORE RECOVERY PERCENTAGES FROM INOCULATED COUPONS 177

TABLE 3 - 2 - SPORE RECOVERY PERCENTAGES FROM SPIKED TUBES ................. 177

TABLE 3 - 3 - SPORE RECOVERY FROM AEROSOL DEPOSITION, ALUMINUM

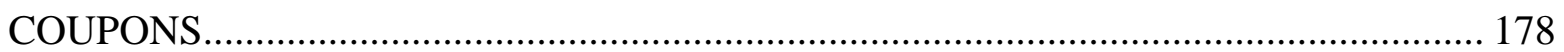

TABLE 3 - 4 - SAMPLE SIZE REQUIRED BASED ON INITIAL DECONTAMINATION TESTS

TABLE 3 - 5 - STEPWISE REGRESSION TERMS RETAINED IN MODELS................... 187

TABLE 3 - 6 - HIGH DIRECT INOCULATION, REGRESSION MODEL ......................... 187

TABLE 3 - 7 - LOW DIRECT INOCULATION, REGRESSION MODEL .......................... 188

TABLE 3 - 8 - AEROSOL DEPOSITION, REGRESSION MODEL ................................... 188

TABLE A3 - 1 - TEST CONDITION 1: 180 DEG F, 90\% RH...............................210

TABLE A3 - 2 - TEST CONDITION 3: 180 DEG F, 70\% RH ........................................ 211

TABLE A3 - 3 - TEST CONDITION 5: 170 DEG F, 80\% RH ......................................... 212

TABLE A3 - 4 - TEST CONDITION 7: 160 DEG F, 90\% RH .......................................... 213

TABLE A3 - 5 - TEST CONDITION 9: 160 DEG F, 70\% RH …........................................ 214

TABLE A3 - 6 - TEST CONDITION 1: 180 DEG F, 90\% RH .......................................... 215

TABLE A3 - 7 - TEST CONDITION 3: 180 DEG F, 70\% RH .......................................... 216 


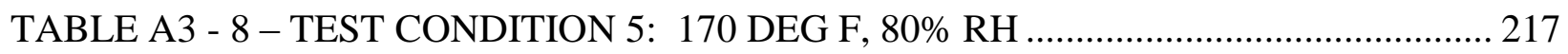

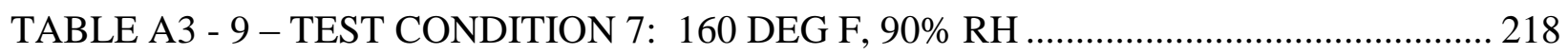

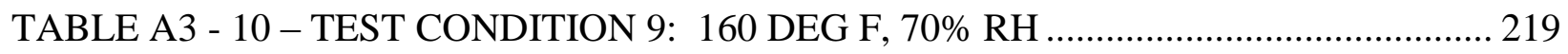

TABLE A3 - 11 - TEST CONDITION 1: 180 DEG F, 90\% RH, SAMPLES …………........... 220

TABLE A3 - 12 - TEST CONDITION 1: 180 DEG F, 90\% RH, CONTROLS ....................... 221

TABLE A3 - 13 - TEST CONDITION 3: 180 DEG F, 70\% RH, SAMPLES ………............... 222

TABLE A3 - 14 - TEST CONDITION 3: 180 DEG F, 70\% RH, CONTROLS ....................... 223

TABLE A3 - 15 - TEST CONDITION 5: 170 DEG F, 80\% RH, SAMPLES ……................... 224

TABLE A3 - 16 - TEST CONDITION 5: 170 DEG F, 80\% RH, CONTROLS ....................... 225

TABLE A3 - 17 - TEST CONDITION 7: 160 DEG F, 90\%, SAMPLES …………………...... 226

TABLE A3 - 18 - TEST CONDITION 7: 160 DEG F, 90\% RH, CONTROLS ………............ 227

TABLE A3 - 19 - TEST CONDITION 9: 160 DEG F, 70\% RH, SAMPLES …………........... 228

TABLE A3 - 20 - TEST CONDITION 9: 160 DEG F, 70\% RH, CONTROLS ....................... 229

Chapter 4

TABLE 4 - 1 - SPORE RECOVERY FROM AEROSOL DEPOSITION, PLASTIC AND

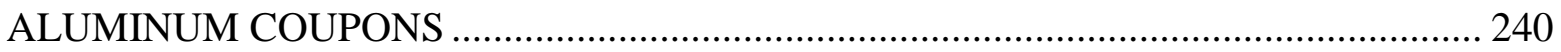

TABLE 4 - 2 - STEPWISE REGRESSION TERMS RETAINED IN MODELS...................... 248

TABLE 4 - 3 - HIGH DIRECT INOCULATION, REGRESSION MODEL …......................... 248

TABLE 4 - 4 - LOW DIRECT INOCULATION, REGRESSION MODEL …......................... 248

TABLE 4 - 5 - AEROSOL DEPOSITION, REGRESSION MODEL ….................................... 249

TABLE 4 - 6 - HIGH DIRECT INOCULATION TEST SUMMARIES, PLASTIC AND

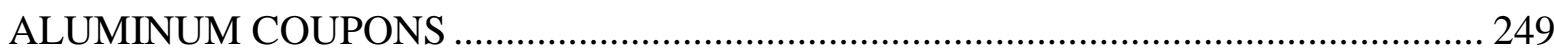

TABLE 4 - 7 - LOW DIRECT INOCULATION TEST SUMMARIES, PLASTIC AND

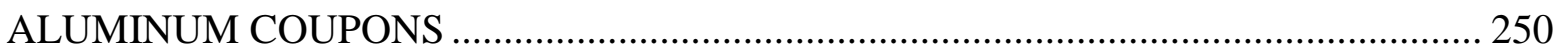

TABLE 4 - 8 - AEROSOL DEPOSISTION TEST SUMMARIES, PLASTIC AND

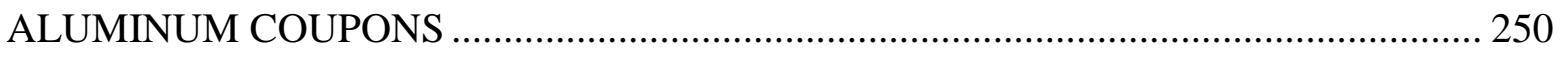

TABLE 4 - 9 - SLOPE COMPARISON, HIGH DIRECT INOCULATIONS............................ 251

TABLE 4 - 10 - SLOPE COMPARISON, LOW DIRECT INOCULATIONS........................... 251

TABLE 4 - 11 - D-VALUE ANALYSIS, PLASTIC VERSUS ALUMINUM COUPONS ..... 252

TABLE A4 - 1 - TEST CONDITION 1: 180 DEG F, 80\% RH................................273

TABLE A4 - 2 - TEST CONDITION 3: 180 DEG F, 70\% RH ……………………….......... 274

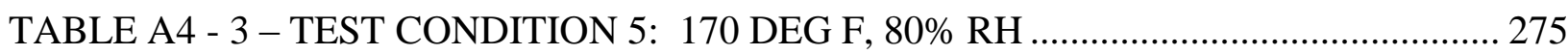

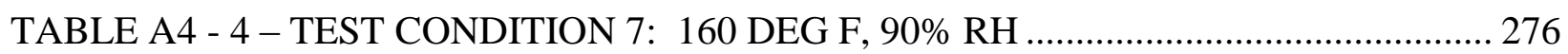

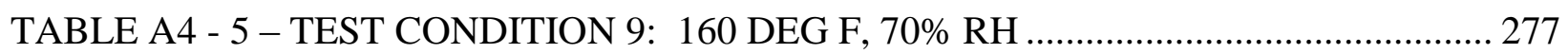

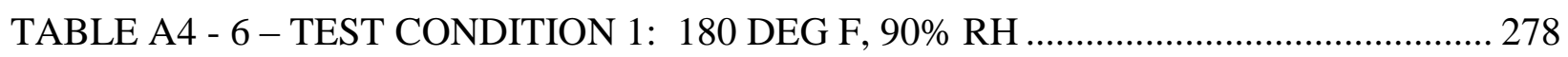

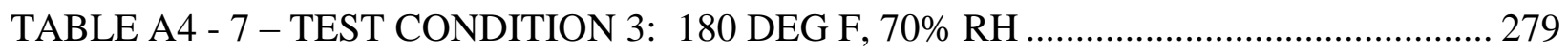

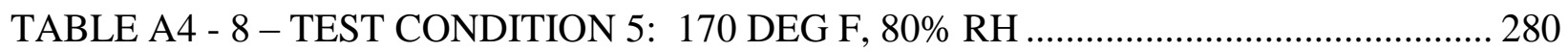

TABLE A4 - 9 - TEST CONDITION 7: 160 DEG F, 90\% RH …………………………..... 281 


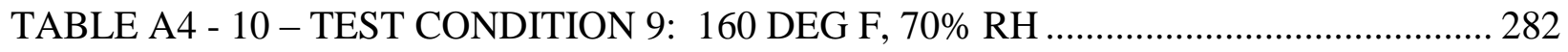

TABLE A4 - 11 - TEST CONDITION 1: 180 DEG F, 90\% RH, SAMPLES .......................... 283

TABLE A4 - 12 - TEST CONDITION 1: 180 DEG F, 90\% RH, CONTROLS ....................... 284

TABLE A4 - 13 - TEST CONDITION 3: 180 DEG F, 70\% RH, SAMPLES ........................... 285

TABLE A4 - 14 - TEST CONDITION 3: 180 DEG F, 70\% RH, CONTROLS ………............ 286

TABLE A4 - 15 - TEST CONDITION 5: 170 DEG F, 80\% RH, SAMPLES ………............... 287

TABLE A4 - 16 - TEST CONDITION 5: 170 DEG F, 80\% RH, CONTROLS ....................... 288

TABLE A4 - 17 - TEST CONDITION 7: 160 DEG F, 90\% RH, CONTROLS ........................ 289

TABLE A4 - 18 - TEST CONDITION 7: 160 DEG F, 90\% RH, CONTROLS ....................... 290

TABLE A4 - 19 - TEST CONDITION 9: 160 DEG F, 70\% RH, SAMPLES ……………....... 291

TABLE A4 - 20 - TEST CONDITION 9: 160 DEG F, 70\% RH, CONTROLS ....................... 292 


\section{LIST OF FIGURES}

\section{Chapter 2}

FIGURE 2- 1 - TEST CHAMBER, WORKING SIDE …................................................ 115

FIGURE 2- 2 - TEST CHAMBER, GLOVE PORT SIDE ................................................ 115

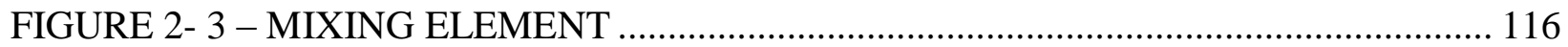

FIGURE 2- 4 - TEST CHAMBER, WORKING SIDE ..................................................... 116

FIGURE 2- 5 - TEST CHAMBER, GLOVE PORT SIDE............................................... 116

FIGURE 2- 6 - TEST CHAMBER, DEPOSITION MODEL ............................................... 118

FIGURE 2- 7 - FLUORESCENT PARTICLE TEST LOCATIONS ON CHAMBER FLOOR

FIGURE 2- 8 - SPORE DEPOSITION TEST LOCATIONS ON CHAMBER FLOOR .......... 125

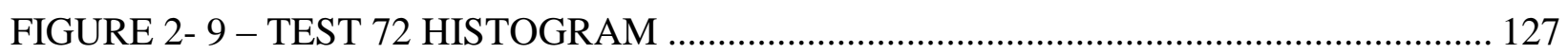

FIGURE 2- 10 - TEST 72 HISTOGRAM, LOG VALUES ............................................ 128

FIGURE 2- 11 - TEST 76 HISTOGRAM ....................................................................... 128

FIGURE 2- 12 - TEST 76 HISTOGRAM, LOG VALUES ................................................ 129

FIGURE 2- 13 - TEST 80 HISTOGRAM ….................................................................. 129

FIGURE 2- 14 - TEST 80 HISTOGRAM, LOG VALUES .............................................. 130

FIGURE 2- 15 - TEST 81 HISTOGRAM …....................................................................... 130

FIGURE 2- 16 - TEST 81 HISTOGRAM, LOG VALUES ............................................... 131

FIGURE 2- 17 - TEST 72 PROBABILITY PLOT, NON-TRANSFORMED DATA .............. 132

FIGURE 2- 18 - TEST 72 PROBABILITY PLOT, LOG TRANSFORMED DATA............... 132

FIGURE 2- 19 - TEST 76 PROBABILITY PLOT, NON-TRANSFORMED DATA .............. 133

FIGURE 2- 20 - TEST 76 PROBABILITY PLOT, LOG TRANSFORMED DATA............... 133

FIGURE 2- 21 - TEST 80 PROBABILITY PLOT, NON-TRANSFORMED DATA .............. 134

FIGURE 2- 22 - TEST 80 PROBABILITY PLOT, LOG TRANSFORMED DATA............... 134

FIGURE 2- 23 - TEST 81 PROBABILITY PLOT, NON-TRANSFORMED DATA .............. 135

FIGURE 2- 24 - TEST 81 PROBABALITY PLOT, LOG TRANSFORMED DATA ............. 135

FIGURE 2- 25 - TEST 72 SPORE DEPOSITION CONTOUR PLOT ................................... 137

FIGURE 2- 26 - TEST 76 SPORE DEPOSITION CONTOUR PLOT ................................... 138

FIGURE 2- 27 - TEST 80 SPORE DEPOSITION CONTOUR PLOT .................................. 138

FIGURE 2- 28 - TEST 81 SPORE DEPOSITION CONTOUR PLOT .................................. 139

FIGURE 2- 29 - TEST 72 RESIDUAL PROBABILITY PLOT .......................................... 142

FIGURE 2- 30 - TEST 76 RESIDUAL PROBABILITY PLOT ............................................ 142

FIGURE 2- 31 - TEST 80 RESIDUAL PROBABILITY PLOT ........................................... 143

FIGURE 2- 32 - TEST 81 RESIDUAL PROBABILITY PLOT ............................................. 143

FIGURE 2- 33 - SPORE DEPOSITION TEST LOCATIONS ON CHAMBER FLOOR ........ 155 
Chapter 3

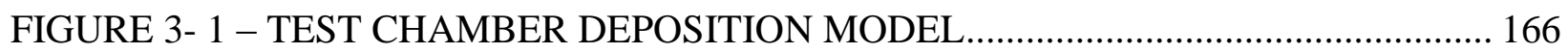

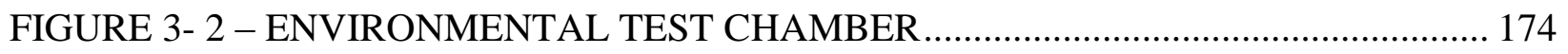

FIGURE 3- 3 - DECONTAMINATION TEST CONDITION MATRIX …………................. 175

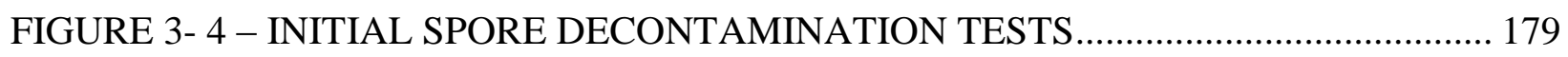

FIGURE 3- 5 - DECONTAMINATION TESTS, HIGH DIRECT INOCULATION .................. 181

FIGURE 3- 6 - DECONTAMINATION TESTS, LOW DIRECT INOCULATION.................. 182

FIGURE 3- 7 - DECONTAMINATION TESTS, AEROSOL DEPOSITION, TEST

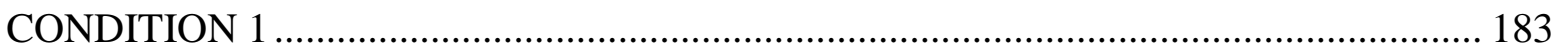

FIGURE 3- 8 - DECONTAMINATION TESTS, AEROSOL DEPOSITION, TEST

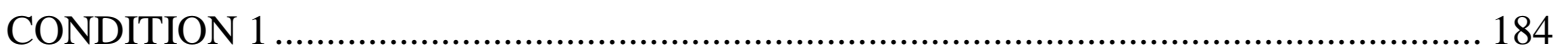

FIGURE 3- 9 - DECONTAMINATION TESTS, SEROSOL DEPOSITION, TEST

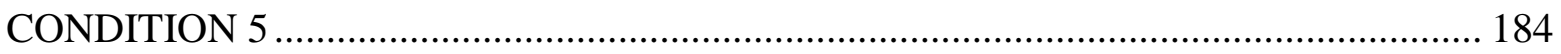

FIGURE 3- 10 - DECONTAMINATION TESTS, AEROSOL DEPOSITION, TEST

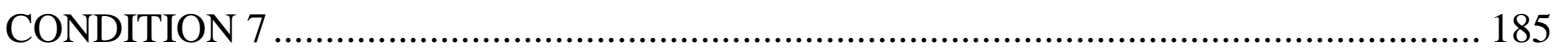

FIGURE 3- 11 - DECONTAMINATION TESTS, AEROSOL DEPOSITION, TEST

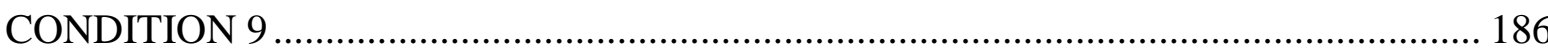

FIGURE 3- 12 -- HIGH DIRECT INOCULATION SPORE SURFACE PLOT-SPORE LOG

VERSUS TEMPERATURE AND HUMIDITY ............................................................ 201

FIGURE 3- 13 - HIGH DIRECT INOCULATION SPORE SURFACE PLOT-SPORE LOG VERSUS HUMIDITY AND TREATMENT TIME ...................................................... 201

FIGURE 3- 14 - HIGH DIRECT INOCULATION SPORE SURFACE PLOT-SPORE LOG VERSUS TEMPERATURE AND TREATMENT TIME ............................................. 202

FIGURE 3- 15 - HIGH DIRECT INOCULATION CONTOUR PLOT—SPORE LOG VERSUS

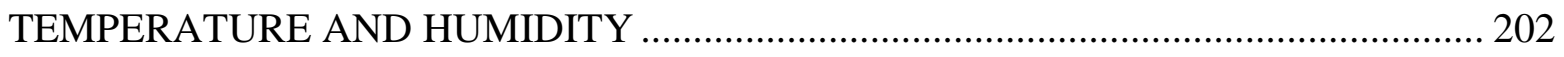

FIGURE 3- 16 - HIGH DIRECT INOCULATION CONTOUR PLOT-SPORE LOG VERSUS

HUMIDITY AND TREATMENT TIME ................................................................ 203

FIGURE 3- 17 - HIGH DIRECT INOCULATION CONTOUR PLOT—SPORE LOG VERSUS

TEMPERATURE AND TREATMENT TIME ………................................................ 203

FIGURE 3- 18 - LOW DIRECT INOCULATION SPORE SURFACE PLOT-SPORE LOG

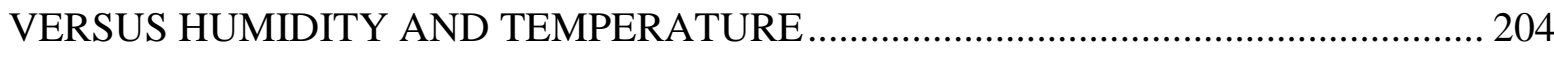

FIGURE 3- 19 - LOW DIRECT INOCULATION SPORE SURFACE PLOT-SPORE LOG

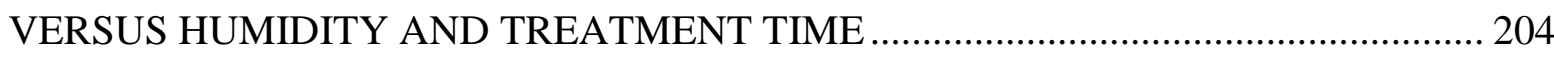

FIGURE 3- 20 - LOW DIRECT INOCULATION SPORE SURFACE PLOT-SPORE LOG

VERSUS TEMPERATURE AND TREATMENT TIME ………................................... 205

FIGURE 3- 21 - LOW DIRECT INOCULATION CONTOUR PLOT-SPORE LOG VERSUS

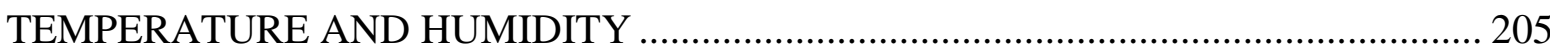

FIGURE 3- 22 - LOW DIRECT INOCULATION CONTOUR PLOT-SPORE LOG VERSUS

HUMIDITY AND TREATMENT TIME

206 
FIGURE 3- 23 - LOW DIRECT INOCULATION CONTOUR PLOT-SPORE LOG VERSUS

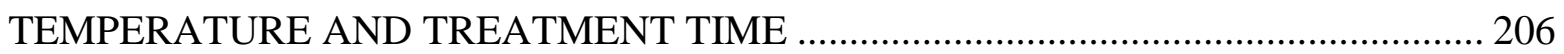
FIGURE 3- 24 - AEROSOL DEPOSITION SURFACE PLOT-SPORE LOG VERSUS

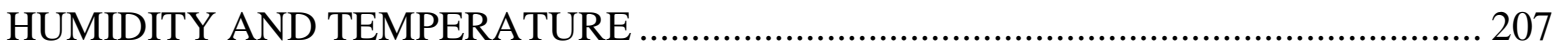

FIGURE 3- 25 - AEROSOL DEPOSITION SURFACE PLOT—SPORE LOG VERSUS

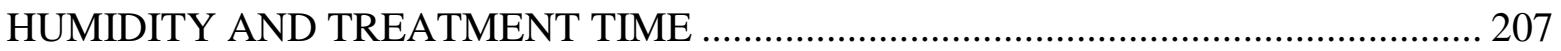

FIGURE 3- 26 - AEROSOL DEPOSITION SURFACE PLOT-SPORE LOG VERSUS

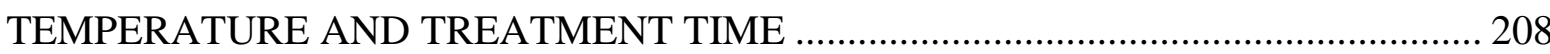

FIGURE 3- 27 - AEROSOL DEPOSITION CONTOUR PLOT-SPORE LOG VERSUS

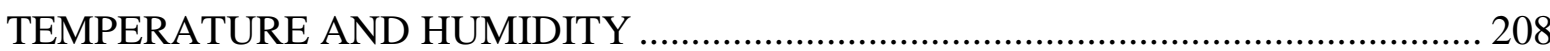

FIGURE 3- 28 - AEROSOL DEPOSITION CONTOUR PLOT—SPORE LOG VERUSUS

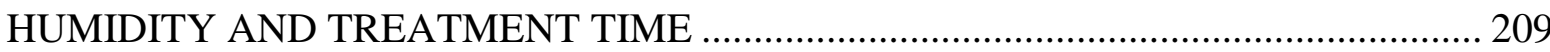
FIGURE 3- 29 - AEROSOL DEPOSITION CONTOUR PLOT - SPORE LOG VERSUS TEMPERATURE AND TREATMENT TIME 209

Chapter 4

FIGURE 4- 1 - DECONTAMINATION TEST MATRIX …………………………….......... 238 FIGURE 4- 2 - DECONTAMINATION TESTS, HIGH DIRECT INOCULATION ................. 242 FIGURE 4- 3 - DECONTAMINATION TESTS, LOW DIRECT INOCULATION .................. 243 FIGURE 4- 4 - DECONTAMINATION TESTS, AEROSOL DEPOSITION, TEST

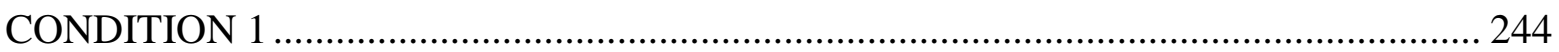
FIGURE 4- 5 - DECONTAMINATION TESTS, AEROSOL DEPOSITION, TEST CONDITION 3 245

FIGURE 4- 6 - DECONTAMINATION TESTS, AEROSOL DEPOSITION, TEST CONDITION 5 245

FIGURE 4- 7 - DECONTAMINATION TESTS, AEROSOL DEPOSITION, TEST CONDITION 7 246

FIGURE 4- 8 - DECONTAMINATION TESTS, AEROSOL DEPOSITION, TEST CONDITION 9

FIGURE 4- 9 - HIGH DIRECT INOCULATION SPORE SURFACE PLOT-SPORE LOG VERSUS TEMPERATURE AND HUMIDITY .............................................................. 264

FIGURE 4- 10 - HIGH DIRECT INOCULATION SPORE SURFACE PLOT-SPORE LOG

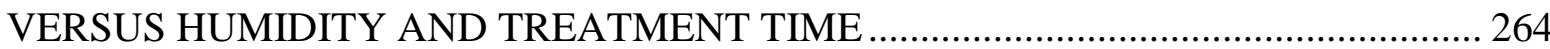

FIGURE 4- 11 - HIGH DIRECT INOCULATION SPORE SURFACE PLOT-SPORE LOG VERSUS TEMPERATURE AND TREATMENT TIME ……........................................... 265 FIGURE 4- 12 - HIGH DIRECT INOCULATION CONTOUR PLOT-SPORE LOG VERSUS TEMPERATURE AND HUMIDITY 265 FIGURE 4- 13 - HIGH DIRECT INOCULATION CONTOUR PLOT-SPORE LOG VERSUS HUMIDITY AND TREATMENT TIME 266 
FIGURE 4- 14 - HIGH DIRECT INOCULATION CONTOUR PLOT—SPORE LOG VERSUS

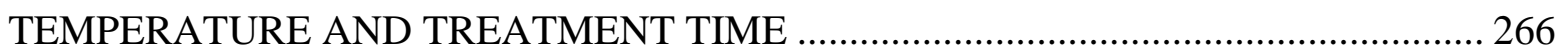
FIGURE 4- 15 - LOW DIRECT INOCULATION SPORE SURFACE PLOT-SPORE LOG

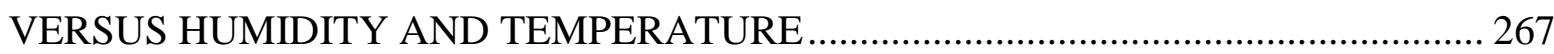

FIGURE 4- 16 - LOW DIRECT INOCULATION SPORE SURFACE PLOT-SPORE LOG

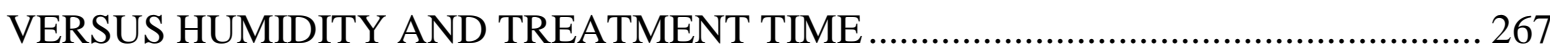

FIGURE 4- 17 - LOW DIRECT INOCULATION SPORE SURFACE PLOT-SPORE LOG VERSUS TEMPERATURE AND TREATMENT TIME …............................................... 268 FIGURE 4- 18 - LOW DIRECT INOCULATION CONTOUR PLOT-SPORE LOG VERSUS TEMPERATURE AND HUMIDITY 268 FIGURE 4- 19 - LOW DIRECT INOCULATION CONTOUR PLOT-SPORE LOG VERSUS HUMIDITY AND TREATMENT TIME 269 FIGURE 4- 20 - LOW DIRECT INOCULATION CONTOUR PLOT-SPORE LOG VERSUS TEMPERATURE AND TREATMENT TIME 269

FIGURE 4- 21 - AEROSOL DEPOSITION SPORE SURFACE PLOT—SPORE LOG VERSUS HUMIDITY AND TEMPERATURE 270

FIGURE 4- 22 - AEROSOL DEPOSITION SURFACE PLOT-SPORE LOG VERSUS HUMIDITY AND TREATMENT TIME 270

FIGURE 4- 23 - AEROSOL DEPOSITION SURFACE PLOT-SPORE LOG VERSUS TEMPERATURE AND TREATMENT TIME

FIGURE 4- 24 - AEROSOL DEPOSITION CONTOUR PLOT-SPORE LOG VERSUS TEMPERATURE AND HUMIDITY

FIGURE 4- 25 - AEROSOL DEPOSITION CONTOUR PLOT-SPORE LOG VERSUS HUMIDITY AND TREATMENT TIME 272

FIGURE 4- 26 - AEROSOL DEPOSITION CONTOUR PLOT-SPORE LOG VERSUS TEMPERATURE AND TREATMENT TIME 272 


\section{CHAPTER 1 -- INTRODUCTION, GOALS, AND BACKGROUND}

\section{Introduction}

Bioterrorism can be defined as a use or threatened use of biological agents against individuals to obtain an advantage for specific purpose such as intimidation, ideological principles, or disruption of everyday activities (Brachman, 2002). In any act of biological terrorism or warfare, diagnosis of the agent can be difficult (Estill et al., 2009), which may hamper decontamination efforts. To minimize illnesses, decontaminating materials to acceptable levels in a very short time is critical (Uhm et al., 2007). Once decontamination is conducted, another difficulty is detecting the agents to ensure they have been adequately inactivated (Uhm et al., 2007). Any of these complications can impact military missions.

DoDI 3150.09 “The Chemical, Biological, Radiological, and Nuclear (CBRN) Survivability Policy" requires all DoD assets to be compatible with conducting operations in the presence of biological warfare agents for extended periods of time. This capability must include conducting operations without the need to undertake maintenance or repairs specific to operating in environments with biological agents present (DoD, 2009). There is currently a gap within these requirements and the fielded capabilities. According to the Air Force Research Laboratory (AFRL), all existing biological decontamination solvents (liquids or vapors) shown to inactivate biological agents are at least somewhat hazardous to the environment and aircraft materials (AFRL, 2008). For these reasons, there are currently no methods approved to decontaminate Air Force aircraft (AFRL, 2008).

Any type of aircraft decontamination must meet strict engineering specifications, which do not currently allow for chemical disinfection. All U.S. Air Force inventory aircraft, however, must withstand storage at temperatures greater than $185^{\circ} \mathrm{F}$ at $100 \%$ relative humidity (AFRL, 
2008). These ranges have been tested on aircraft and no damage was seen after 10 continuous days. These levels give a potential method to decontaminate an aircraft if the agent can be inactivated within these ranges (AFRL, 2008).

The biological agent to be decontaminated is an important consideration as well. While not considered a communicable disease, anthrax would make a good biowarfare agent because the spores are easy to produce, highly resistant to decontamination efforts, and readily dispersed (USAMRIID, 2005). Infection can occur with a low number of Bacillus anthracis spores, the causative agent. Estimates of infectious doses vary; however, current research shows the dose for inhalation anthrax to be between 8,000 to 15,000 spores (USAMRIID, 2008). Other studies have shown that the $\mathrm{LD}_{50}$ in primates (for inhalation anthrax) can be in the range of 2,500-55,000 spores (Inglesby et al., 2002) up to over 100,000 spores (Bartrand et al., 2008). The concentration for the cutaneous version of anthrax can be 10 spores or fewer (Watson and Keir, 1994). This shows that even a very low concentration can present a significant health risk.

Bacillus anthracis spores also present a unique problem because of their persistence, remaining viable for over 60 years in dried soil at room temperature (Perkins, 1983). Because of this long term viability, these spores can present a significant health risk if not inactivated properly. The long term activity of the spore was seen was seen after the 2001 anthrax attacks in the United States, when re-aerosolization occurred, causing several cases when personnel had no direct exposure to the spore release zone (CDC, 2001; Jernigan et al., 2001). For these reasons, the Bacillus anthracis spore is considered the ultimate decontamination requirement and military decontamination efforts have been tested on some type of anthrax simulant.

One final complication for decontamination efforts is that there is disagreement on the level of inactivation required. Some have said a 6-log reduction is usually considered adequate; 
however, Gale et al. (2009) state that some in the bio-defense community have suggested a 12$\log$ reduction. Most field trials have targeted a 6-log reduction.

\section{Goals of dissertation research.}

Previous studies have been completed analyzing the effects of high heat and humidity on inactivation rates for Bacillus anthracis spore simulants at high concentrations. In those studies spores were directly deposited onto aluminum coupons and focusing only on the upper limits of the heat and humidity engineering limits of the aircraft. A better understanding of the inactivation rates of these spores on other aircraft materials, lower heat and humidity ranges, and lower inoculation rates is critical for understanding the best method for safely decontaminating aircraft. The overall goal of this dissertation research was to design an aerosol test chamber to deposit these spores to model a more realistic contamination event. Additionally, two other methods of delivery were provided, including high and low direct inoculation. These three methods were used to inoculate spores onto aluminum and plastics — both aircraft material components. Once the spores were delivered, the inactivation rates were tested using five different combinations of heat and humidity.

\section{Specific aims and hypotheses}

1. Develop and validate an aerosol dispersion method to model and analyze real-world bioaerosols contamination events. All the decontamination tests completed to date have been completed using direct inoculation methods. This novel test method provided a more realistic method for spore deposition on the testing materials, which were typical of aircraft components. This study tested one main hypothesis: differing levels of spore 
concentrations can be produced to test different concentrations of spore dispersal within the test chamber. Changing the concentration can model a direct contamination event (high spore loading) and a re-aerosolization event (low spore loading).

2. Test viability of decontamination technology using a Bacillus anthracis spore simulant while determining the effectiveness of different temperature, humidity, and time combinations on aluminum coupons. This was tested using five different temperature and humidity settings over a minimum of five different times. Three different delivery methods were used-high direct inoculation, low direct inoculation, and aerosol deposition. This study tested three hypotheses: 1) the time required for aerosol deposition will follow a similar relationship as those found in direct inoculation; 2) a higher direct inoculation will require more time to decontaminate than a low direct inoculation; and 3) higher temperate and humidity levels will have a much quicker decontamination on all methods of delivery.

3. Test viability of decontamination technology using a Bacillus anthracis spore simulant while determining the effectiveness of different temperature, humidity, and time combinations on plastic coupons. This was tested using five different temperature and humidity settings over a minimum of five different times. Three different delivery methods were used-high direct inoculation, low direct inoculation, and aerosol deposition. This study tested three hypotheses: 1) the time required for aerosol deposition will follow a similar relationship as those found in direct inoculation; 2) a higher direct inoculation will require more time to decontaminate than a low direct inoculation; and 3) higher temperate and humidity levels will have a much quicker 
decontamination on all method of delivery. An additional aim was to determine the difference in inactivation rates of the plastic versus aluminum coupons.

\section{Background and significance}

\section{Biological agents}

Biowarfare history

The use of biological agents in warfare dates back to the $6^{\text {th }}$ century BC when Assyrians poisoned wells with rye ergot (a fungus). In the $4^{\text {th }}$ century BC, Scythian archers tipped arrows with blood, manure, and tissues from decomposing bodies. In $1350 \mathrm{AD}$, attackers catapulted dead horses and other animals over castles in Hainault, France. In the $15^{\text {th }}$ century, Francisco Pizarro reportedly gave clothing contaminated with the smallpox virus to South American natives. In a letter dated 16 July 1763, General Jeffry Amherst approved a plan to spread smallpox to Delaware Indians using blankets. This same tactic was used in the Civil War by Dr. Luke Blackburn. A Japanese unit numbered 731, conducted biological warfare research until 1945, and completed several attacks, including poisoning Soviet water sources with intestinal typhoid bacteria. They also released plague infected fleas over several villages in China and Mongolia in 1941. Around the same time, several thousand German and Soviet troops acquired tularemia, of which $70 \%$ was the respiratory form of the disease. Just the year before, the Soviets had developed tularemia as a biological weapon (Ryan and Glarum, 2008).

The United States had a biological weapon research program as well. One of the experiments the United States completed to show biological warfare feasibility was to release Bacillus globigii (BG) spores secretly in the New York Subway in 1966 using a broken light 
bulb. This was kept secret from the general public until 1977. Spores were also released in St. Louis, Minneapolis, and San Francisco in the 1950s and 1960s (Ryan and Glarum, 2008).

The Soviet Union had a much larger biological weapons research project. They examined more than 50 biological agents, while employing a peak of 60,000 people at 8 production facilities, 4 major testing grounds, and 7 mobilization facilities (which were to be activated in case of war). Their program included plague, anthrax, tularemia, glanders, smallpox, Marburg, Ebola, and Q fever. During their program, the Soviets produced more than 100 tons of anthrax, 20 tons of plague, and 20 tons of smallpox. This research resulted in a release of anthrax spores in 1979 near Sverdlovsk in Russia with 66 fatal human cases. Sheep and cattle were affected as far as $50 \mathrm{~km}$ downwind (Alibek, 2005).

Biological weapons can clearly be used and maintained by countries; however, these weapons can also be developed by non-state groups because they are inexpensive to produce. Some estimate costs as low as $\$ 7,320$ to outfit a laboratory with used equipment capable of producing either concentrated live virus or finely milled anthrax in quantities in the range of kilograms per week. The weekly costs for this production would be only a few hundred dollars, not including personnel costs (Alibek, 2005). These low costs and ease of production shows that even small non-state groups could conceivably develop some type of biological agent.

\section{Toxins}

Biological agents can be classified into different categories, including toxins, viruses, and bacteria. Biological toxins, produced by a variety of living organisms (bacteria, plants, and animals), are some of the most toxic materials known (Seto, 2011). Biological toxins are polar, high molecular weight compounds that have large ranges of sizes. For instance, sarin gas (a 
chemical weapon) has a molecular weight of 140, while ricin and botulinum toxins have molecular weights of 66,000 and 150,000, respectively (Ryan and Glarum, 2008). Biological toxins are not volatile, which is an important consideration because toxins are thus less likely to produce either secondary or person-to-person exposures or a persistent environment threat (Seto, 2011).

The CDC groups biological agents into different categories based on the corresponding health risk. Category A agents are the highest priority agents and are defined as agents which “can be easily disseminated or transmitted, cause high mortality, severely affect Public Health (PH), might cause public panic and social disruption, and require special action for $\mathrm{PH}$ preparedness." Botulinum toxin from Clostridium botulinum fits into this category (Ryan and Glarum, 2008). Category B agents are the second highest agents and defined as "moderately easy to disseminate, result in moderate morbidity rates and low mortality rates, and require specific enhancements for diagnostic capacity and disease surveillance”. The toxins in Group B include Clostridium perfringens epsilon toxin, Staphylococcal enterotoxin B (SEB), and ricin toxin (from Ricinus communis) (Ryan and Glarum, 2008). The WHO and CDC state that Saxotoxin (STX), ricin, botulinum toxin, and Staphylococcal enterotoxin B all could be used as toxins in bioterrorism (CDC, 2000; WHO, 2004). Several of these agents are discussed in-depth below.

\section{Toxins: Ricin}

Ricin, another important toxin, is one of the most lethal and easily produced plant toxins (Ryan and Glarum, 2008), with a lethality 30 times greater than VX gas by weight (Alibek, 2005). The $\mathrm{LD}_{50}$ for humans via oral ingestion is $1,000 \mu \mathrm{g} / \mathrm{kg}$, with a fatality rate of $6 \%$ 
(Thomas et al., 2008). Ricin is found in seeds of Ricinus communis (the castor bean plan) (Seto, 2011) and can be made into powder, mist, or pellet. Ricin is water soluble, therefore is can be dissolved in water or weak acid. Ricin is very stable and not affected by extremes in temperatures (Seto, 2011). There are three routes of exposure-inhalation, ingestion, and injection. Only $500 \mathrm{mg}$ would be enough to kill an average adult through injection; however, a greater amount would be required for inhalation or ingestion exposures. Ricin is not contagious or infectious, so person-to-person transmission is not an issue (Ryan and Glarum, 2008). There is no treatment or vaccine currently available for ricin poisoning (Seto, 2011).

Ricin has been used several times as biological agent. In 1978, the Bulgarian government (using Soviet Union technology) assassinated Georgi Markov, a Bulgarian defector. Small metal pellets containing ricin crystals were injected into his calf muscles by using an umbrella. Markov died three days after the incident. There were also several small-scale incidents in 2003 in the US involving ricin (Seto, 2011).

\section{Toxins: Botulinum toxin}

Another type of toxin is the botulinum toxins, which include several different types produced by an obligate anaerobic spore-forming bacillus bacteria Clostridium botulinum (Seto, 2011; Ryan and Glarum, 2008). These toxins are highly lethal, easily produced, and easily released. Botulinum toxin is the most toxic substance known - up to 15,000 times more toxic than VX nerve agent (Ryan and Glarum, 2008). The $\mathrm{LD}_{50}$ for humans via oral ingestion is 0.01 $\mu \mathrm{g} / \mathrm{kg}$, with a fatality rate of 50-60\% (Thomas et al., 2008). If evenly dispersed, one gram of pure botulinum toxin could theoretically kill one million people (Ryan and Glarum, 2008). 
Clostridium botulinum bacteria are ubiquitous in soil and, because they are very resistant to heat, light, drying and radiation, the spores may survive boiling for several hours at $100^{\circ} \mathrm{C}$ $\left(212^{\circ} \mathrm{F}\right)$. The spores are inactivated at $120^{\circ} \mathrm{C}\left(248^{\circ} \mathrm{F}\right)$ within 30 minutes. The neurotoxins are released after germination (Ryan and Glarum, 2008). A point source aerosol release of botulinum toxin could incapacitate or kill $10 \%$ of persons within half a kilometer downwind of the release; however, the CDC maintains a well-established surveillance system for reporting human botulism cases that would promptly detect such an event (Seto, 2011). The toxin is normally spread through ingestion of the spores; however, the inhalation route is possible which would most likely be the route used in bioterrorism attack. The toxin could be effective in smallscale poisonings or aerosols in enclosed facilities (such as theaters) (Seto, 2011).

The Japanese cult Aum Shinrikyo used botulinum toxin aerosols at several sites between 1990 and 1995; however, the attempts failed (Seto, 2011).

\section{Viruses}

Viruses are multiple nucleic acids surrounded by an envelope of proteins or lipids called a nucleocapside. Viruses are much smaller than bacteria, with sizes between 20 to $300 \mathrm{~nm}$ in diameter (Seto, 2011), which is 2 to 60 times smaller than bacteria (Ryan and Glarum, 2008).

Again, the CDC groups these in the same categories as listed above for the toxins. The category A viruses include several viruses from the Arenaviridae, Filoviridae, Bunyaviridae, and Flavividae families, which cause hemorrhagic fevers. The category B viruses include several arboviruses, which cause viral encephalitis. Several viruses also fall into Category C. These include Nipah virus, Hantavirus, West Nile fever, and SARS. The Category C list changes as world disease outbreak situations change. For instance, H5N1 has been received attention 
because there have only been 300 confirmed cases in a 10 year period (Ryan and Glarum, 2008).

Viruses that could be used as biological warfare agents are included in Table 1-1 (Cordesman, 2005).

Table $1-1-$ Viruses that could be used as biological warfare agents

\begin{tabular}{|c|c|c|}
\hline Lethal & Lethal/incapacitating & Incapacitating \\
\hline $\begin{array}{ll}\text { - } & \text { Bolivian hemorrhagic } \\
& \text { fever } \\
\text { - } & \text { Ebola infection } \\
\text { - } & \text { Lassa infection } \\
\text { - } & \text { Marburg infection } \\
\text { - } & \text { Smallpox } \\
\text { - } & \text { Yellow fever }\end{array}$ & $\begin{array}{ll}\text { - } & \text { Congo-Crimean } \\
& \text { hemorrhagic fever } \\
\text { - } & \text { Japanese encephalitis } \\
\text { - } & \text { Monkeypox infection } \\
\text { - } & \text { Omsk hemorrhagic fever } \\
\text { - } & \text { Russian S/S encephalitis }\end{array}$ & $\begin{array}{ll}\text { - } & \text { VEE, EEE, WEE } \\
\text { - } & \text { Dengue fever } \\
\text { - } & \text { West Nile encephalitis } \\
\text { - } & \text { Epidemic typhus } \\
\text { - } & \text { Murine typhus } \\
\text { - } & \text { Rift Valley fever } \\
\text { - } & \text { Influenza A }\end{array}$ \\
\hline
\end{tabular}

An important consideration with viruses is their survivability. The influenza virus can usually survive on hands for only a few minutes (Weber and Stilianakis, 2008); however, the virus has been detected in air samples up to 24 hours after aerosolization at low levels of humidity (Branskston et al., 2007).

Viruses: Pandemic Influenza

Another potential biowarfare agent is the influenza A virus, a highly transmissible virus that has caused pandemics with high mortality rates when a new strain was introduced. Such new strains occurred in 1918, 1957, and 1968, causing extremely high morbidity and mortality. New technology is available which can be used to alter genetic composition and can change genes, enabling genic shifts (Mahy, 2003). Terrorists, however, would likely not have the knowledge, facilities, or ingenuity to carry out these recombinant DNA experiments. One important consideration for the influenza virus is that vaccinations are effective; however, these vaccinations take six months to produce so they would be of limited value if not completed before an outbreak starts (Krug, 2003). 
Viruses: Smallpox

Smallpox, caused by the variola major or minor double stranded DNA virus, is another potential biological weapon which could be very dangerous because the virus is easily transmitted from person-to-person, no effective treatment exists, and few people carry immunity (Ryan and Glarum, 2008). Because smallpox is unique to humans, the virus must be transmitted person-to-person (Whitley, 2003). The virus is strongly infective by air transmission, splash, or contact (Seto, 2011; Whitley, 2003) and is spread through respiratory droplets, typically within 2 meters or less (Ryan and Glarum, 2008). Transmission in the past usually required direct and fairly prolonged face-to-face contact; however, on rare occasions, the virus was carried in enclosed settings such as buildings, buses, and trains (Ryan and Glarum, 2008). Smallpox can also be transmitted through contaminated bedding as well (Whitley, 2003).

The fatality estimates for smallpox range from 50-90\% (Seto, 2011) to 20-60\% (Ryan and Glarum, 2008) but decreases to 3\% in vaccinated populations (Ryan and Glarum, 2008). A critical point to consider is the small infective dose required-only 10 to 100 virions (Alibek, 2005). Also, one case can lead to a 10 to 20 -fold increase in cases based on the high infectivity of the virus (Alibek, 2005). Estimates have shown that a successful bioterrorist infecting 1,000 people initially could be capable of spreading to the whole population within 180 days if no intervention is completed (Meltzer et al., 2001). All of these reasons make smallpox a threat to be used as a biological warfare agent (Whitley, 2003).

Small quantities of the virus exist in two secure facilities in the United States (Atlanta) and Russia (Novosibirsk, Siberia); however, there are likely unrecognized stores of smallpox virus exist elsewhere (Mahy, 2003; Ryan and Glarum, 2008). Unclassified reports have stated Iran, Libya, North Korea, and Syria could have retained cultures, but these may only be 
speculation (Hedgpeth, 2000). There are reports that the Soviet Union conducted bioweapon research on smallpox from 1980 to 1990 (Mahy, 2003).

Smallpox is first believed to have appeared around 10,000 BC during the first agricultural settlements in northeastern Africa (Ryan and Glarum, 2008; Whitley, 2003) and was deployed as a biological weapon during the French and Indian Wars (1754-1767) by British forces through contaminated blankets (Whitley, 2003). There were two European outbreaks in the 1970s which demonstrated the infectivity of the virus. One outbreak occurred in Germany in 1970 when an unintentional aerosol deployment led to a widespread outbreak even with low dosages released (Whitley, 2003). The second outbreak occurred in Yugoslavia in 1972 when a single case led to an exponential increase in the number of transmissions from person-to-person. Both of these outbreaks showed that a limited number of individuals could result in expansion factor of 10- to 20-fold (Whitley, 2003). The last natural case was seen in Somalia in October of 1977 (Mahy, 2003; Whitley, 2003); however, there was one case in 1980 when a laboratory photographer obtained smallpox because of transmission of the virus through the building HVAC system (Alibek, 2005).

The smallpox virus becomes inactive after a period of 48 hours (Whitley, 2003). Vaccination is effective, providing a level of immunity up to $95 \%$ for 3 to 5 years, after which the effectiveness decreases (Ryan and Glarum, 2008)

\section{Viruses: Filoviruses}

Another potential viral bioweapon are the Filoviruses, which are enveloped singlestranded negative-sense RNA viruses with an unusual filamentous morphology (Bray, 2003). This group, which includes Ebola and Marburg, exist in unknown reservoirs but cause occasional 
outbreaks with fatality rates up to $80 \%$ (Mahy, 2003). These diseases would create great public concern and panic, but could be controlled once recognized (Mahy, 2003).

Since Marburg was discovered, there have only been 10 outbreaks involving 30 or more victims and a total victim count of less than 2,000. These outbreaks burned out fairly rapidly. Transmission of the Marburg virus is through direct contact with body fluids or contaminated items that contain the virus. Transmission is usually from hands to eyes or mouth; therefore, the greatest risk is to family members caring for those that are ill. Fatality rates are usually 23-33\% (Bray, 2003).

Marburg viruses were not tested as a biological weapon in the United States because the testing program ended before the virus was discovered; however, the Soviet Union did have a strong interest because the virus was highly suitable for biological warfare use. The virus was maintained after the first outbreaks in the 1960s and 1970s so there is availability. Additionally, the Marburg virus could be obtained through an outbreak in Africa (Bray, 2003).

Ebola, also caused by a Filovirus, has four recognized subtypes (Bray, 2003). The virus was first reported in Zaire and Sudan in 1976, with two distinct subtypes being isolated. Both of these caused mortality rates greater than 50\%. A third type was later found in macaques imported from the Philippines into the US in 1989. A fourth type was found in 1994. Scattered outbreaks have occurred since. The primary reservoir is still unknown, even after 3,000 species have been tested (Ryan and Glarum, 2008). The virus could be obtained because of on-going outbreaks in Africa (Mahy, 2003).

Transmission of the Ebola virus is typically intimate person-to-person contact. Nosocomial transmission has presented major problems in outbreaks in Africa because of needle re-use. Additional exposure to infected tissues, fluids, and hospital materials has transmitted the 
virus. Aerosol transmission is possible in primates, but is not a major transmission method for humans. Ebola is the most severe hemorrhagic fever in humans, with fatality rates from 53-58\%. The virus causes abrupt onset of fever, chills, malaise, and myalgia with death or recovery coming in 7-11 days. The health impacts last for weeks following the initial fever (Ryan and Glarum, 2008).

These viruses degrade in hours in UV light, but they may survive at room temperature in liquid or dried material for a number of days. Steam sterilization is the most effective decontamination method, but bleach (1:100) is effective as well (Bray, 2003).

\section{Bacteria}

Bacteria are single celled organisms which vary in shape and size and have no distinct nucleus (Ryan and Glarum, 2008). Bacteria have rigid cell walls and are divided into positive and negative types by Gram staining, which identifies the cell wall composition (Seto, 2011). Some produce toxins and spores. The spores are made in a dormant form, making them more resistant to environmental factors. The interior of the cells contain DNA, cytoplasm, and cell membrane. Finally, some can only grow inside cell hosts (Rickettsia, Coxiella, Chlamydia) (Ryan and Glarum, 2008).

The CDC groups these in the same categories as described above. Category A agents are Bacillus anthracis (anthrax), Yersinia pestis (plague), and Francisella tularensis (tularemia). The Category B agents are Brucella species (brucellosis), Burkholderia mallei (glanders), Burkholderia pseudomallei (melioidosis), and Chlamydophila psittaci (psittacosis). Additionally, there are several food and water safety threats caused by different bacteria. These include Salmonella species; Shigella dysenteriae Type 1; Escherichia coli O157:H7; and Vibrio 
cholera. Finally, the rickettsia Coxiella burnetii which causes Q fever is considered a Class B agent (Ryan and Glarum, 2008). Table 1-2 includes several different bacteria that could be used as biological weapons in both state and non-state use (Cordesman, 2005).

Table $1-2$ - Bacteria that could be used as biological warfare agents

\begin{tabular}{|c|c|c|}
\hline Lethal & Lethal/incapacitating & Incapacitating \\
\hline Anthrax & - Brucellosis & \\
\hline - Glanders & - Diphtheria & - Legionellosis \\
\hline - Meliodosis & - Psittacosis & - Murine typhus \\
\hline - Plague & - Tularemia & \\
\hline
\end{tabular}

\section{Bacteria: Yersinia pestis}

Yersinia pestis, the causative agent of plague, could be used as a biological warfare agent (Seto, 2011; Ryan and Glarum, 2008). The bacterium is facultative anaerobic, gram negative, and rod-shaped (Seto, 2011). Because of high production and ability to aerosolize easily, Yersinia pestis could be used as a biological weapon. The bacterium can also be transmitted from person-to-person in some forms and is also widely distributed in research laboratories around the world (Ryan and Glarum, 2008).

Plague is acquired by humans most often through a flea bite (Ryan and Glarum, 2008; Seto, 2011); however, transmission is also possible through respiratory droplets, direct contact with infected patients with pneumonic plague, direct skin or mucous membrane contact with tissues, and fluids of infected animals less common (Ryan and Glarum, 2008). Inhalation of infective aerosols is rare with naturally occurring plague in the United States; however, inhalation would be the most likely route of transmission in a bioterrorist event. Infection acquired naturally through respiratory routes requires direct and close contact with an ill person - this has not occurred in the US for decades (Ryan and Glarum, 2008). 
Naturally occurring plague has been documented in the US since 1900. There are 1,500 to 3,000 cases worldwide every year, 5 to 15 of which are in the US, mostly in Arizona, Colorado, and New Mexico. The incident rate in the US is $2 \%$ pneumonic, $83 \%$ bubonic, and 15\% septicemic. The last person-to-person case in the US was an epidemic in 1925 (Ryan and Glarum, 2008).

The infective dose for plague is low, down to 100 cells (Seto, 2011). Bubonic plague is the most common, accounting for approximately $80 \%$ of the cases. The incubation time is 2 to 6 days and symptoms include vomiting, nausea, and petechia. Infection is through a flea bite or exposure to infected material through a break in the skin. Plague cannot be transmitted from person-to-person. If untreated, this disease can spread through the bloodstream and infect the lungs, causing secondary infection pneumonic or septicemic plague, resulting in fatality rates up to $60 \%$. Septicemic plague is another form and occurs when bacteria enter the bloodstream and disperse throughout the body. Symptoms include prostration, circulatory collapse, septic shock, organ failure, hemorrhage, disseminated intravascular coagulation, and necrosis of extremities often seen in fingertips, tip of nose, and toes-the result of small blood clots blocking capillaries and circulation of to these areas. These conditions are $100 \%$ fatal without treatment. The last form is pneumonic plague which occurs when bacteria are inhaled and gain direct access to the lungs. Pneumonic plague is the least common form but also the most fatal and can be transmitted person-to-person through respiratory droplets with direct close contact. If definitive treatment is not given, plague is considered universally fatal due to respiratory failure and shock (Ryan and Glarum, 2008)

Plague requires prompt antibiotic treatment and supportive therapy. Without quick treatment, most forms are $100 \%$ fatal. About $14 \%$ of cases in the US are fatal, but these are 
linked to delay in seeking medical care. Prophylactic antibiotics should be given to those who had a close exposure ( 2 meters or less) to persons suspected of having pneumonic plague (Ryan and Glarum, 2008).

Japan is the only country to have ever tried to use plague as a biowarfare agent (Alibek, 2005). As discussed previously, this was done by Unit 731 which dropped plague-infected fleas over China on several occasions and caused some cases, although the scale was not known (Ingelsby et al., 2000). The Soviets conducted massive weaponization efforts during the Cold War, which included 10 institutes and thousands of scientists (Alibek, 2005).

The bacterium is easily destroyed by sunlight and drying, but can survive briefly in soil and longer in frozen or soft tissues. Survival for can be up to 1 hour (depending on conditions) when released into air (Ryan and Glarum, 2008).

\section{Bacteria: Francisella tularensis}

Another potential biological agent is Francisella tularensis, the causative agent for tularemia. This bacterium is a gram-negative, non-spore forming, intracellular bacterium with a very low infectivity dose, requiring only 10 to 50 cells (Seto, 2011) making it one of the most infectious agents known (Ryan and Glarum, 2008). This level of infectivity and its ability to be aerosolized make Francisella tularensis a potential biological weapon. There are about 100 cases of tularemia per year in the United States, with occurrences in every state except Hawaii, but more than half the cases are found in Arkansas, Missouri, South Dakota, and Oklahoma. The source of these infections is usually ticks and rabbits (Ryan and Glarum, 2008). The bacterium is relatively resistant in the environment, with survival times of 3 to 4 months in mud, water, or dead animals. Survival can be for weeks in low temperatures, but disinfectants (hypochlorite, 
ethanol, and formaldehyde) and moist heat $\left(121^{\circ} \mathrm{C}\right.$ for at least 15 minutes) and dry heat (160$170^{\circ} \mathrm{C}$ for at least one hour) are effective at decontamination (Ryan and Glarum, 2008).

\section{Bacteria: Bacillus anthracis}

The causative agent of anthrax is Bacillus anthracis, an encapsulated, aerobic, grampositive, spore-forming, rod shaped (bacillus) bacterium (CDC, 2002; USAMRIID, 2005). Anthrax is a zoonotic disease of herbivores (cattle, sheep, goats, and horses) (USAMRIID, 2005) and is not considered a communicable disease because it is acquired through environmental exposures. Most mammals (Chosewood and Wilson, 2009) and all humans (USAMRIID, 2005) are susceptible.

Bacillus anthracis endospores are metabolically inactive and are highly resistant to many physical stresses such as wet and dry heat, chemical agents, UV and gamma radiation, oxidizing agents, vacuums and ultra-high hydrostatic pressures (Nicholson et al., 2002). The spores are stable for years in soil and water and can resist sunlight for varying periods (Chosewood and Wilson, 2009). Because of this, the spores create a serious and lasting health risk (Nicholson et al., 2002). The endospore, while in the dormant state, may not be hazardous but germination of the endospore and proliferation of vegetative cells cause human health implications (Atrih and Foster, 2002).

Bacillus anthracis would make a good biowarfare agent for several reasons. First, the bacterium is easy to cultivate and spore formation is readily induced. Second, the spores are highly resistant to sunlight, heat, and disinfectants are not as effective in inactivating the spores. Additionally, the spores can be produced in wet or dry form, can be stabilized for weaponization, and can be delivered as an aerosol cloud either from line source (aircraft) or as point source 
(spray) (USAMRIID, 2005). Bacillus anthracis is the most stable Category A agent in the environment (Sinclair et al., 2008).

There are three different routes of infection for anthrax — cutaneous (via broken skin), gastrointestinal (via ingestion), and inhalation. The cutaneous version is the most common and also the most treatable form, while the other versions are rare (CDC, 2002). The inhalation version is known as "Woolsorter disease" because of its prevalence in textile mill workers that handle wool and other contaminated animal products. Anthrax still occurs frequently in parts of central Asia and Africa and only sporadically in animals throughout the West, Midwest, and Southwest portions of the United States (Chosewood and Wilson, 2009). Natural incidence is extremely low, and the outbreaks that have occurred have been in California, Louisiana, Mississippi, Nebraska, North Dakota, Oklahoma, South Dakota, and Texas (Ryan and Glarum, 2008). Only 18 cases of inhalation anthrax were recorded in the US from 1900 to 1978, two of which were from lab experiments. A significant outbreak occurred in Zimbabwe from 1979 to 1985, with 10,000 people dying (Alibek, 2005). Anthrax is considered an occupational infection because infection is possible when in contact with contaminated animals, animal products, or even pure cultures of Bacillus anthracis (Chosewood and Wilson, 2009). As mentioned above, 66 people died in 1979 because of an accidental release in Sverdlovsk, Russia (Alibek, 2005).

Determining the magnitude of inhalational risks from aerosolized Bacillus anthracis spores is uncertain for several reasons. Data is lacking for human infective doses. There are also several different characteristics that influence the exposure and response, including individual susceptibility, virulence of the strain, and spore physical characteristics (Druett et al., 1953; Fitch, 2008; Watson, 1994). The inhalation infective doses for humans have been primarily extrapolated from inhalation challenges for nonhuman primates or studies conducted in 
contaminated mills (Chosewood and Wilson, 2009). The research that has been completed has very wide estimates (Cohen and Whalen, 2007; Fennelly et al., 2004). For instance, the ranges for the $\mathrm{LD}_{50}$ are from 5,000 to 20,000 (Fitch, 2008); 8,000 to 15,000 (USAMRIID, 2005), and 2,500 to 55,000 spores (Inglesby et al., 2002; Keim and Kauffman, 1999). These estimates go as high as 100,000 for analysis completed using guinea pigs and rhesus monkeys exposed to spores when inhaling 1-um particles (Bartrand et al., 2008). Some primate studies have shown inhalational infectivity of Bacillus anthracis following minimal exposures (Brachman, 1980) and have been as low as a few spores (Peters and Hartley, 2002) with some risk predictions have shown that infective doses may be as low and 1 to 3 spores (Patrick, 1999). The infective dose is believed to be very few spores (10 or less) for cutaneous anthrax (Watson and Keir, 1994).

Each different form of anthrax needs to be considered. Inhalation anthrax is extremely rare and transmitted by the inhalation of aerosolized Bacillus anthracis spores (CDC, 2002). Because inhalation anthrax is so rare, a single case should be presumed to be an intentional exposure until proved otherwise (USAMRIID, 2005). The incubation period is usually 1 to 6 days; however, people were still getting ill up to six weeks after an aerosol release in the Soviet Union (USAMRIID, 2005). The incubation time can be up to two months (CDC, 2002) and some primate studies have shown times up to 100 days (USAMRIID, 2005). The symptoms are dependent on dose and strain, but generally the initial symptoms are non-specific and include fever, malaise, headache, fatigue, and drenching sweats. Sometimes these include nausea, vomiting, confusion, non-productive cough, and mild chest discomfort. These non-specific symptoms make anthrax difficult to diagnose (CDC, 2002; USAMRIID, 2005). These are seen for 2 to 5 days, followed by a short period of improvement which can be on the scale of hours up to 3 days. After this short time of improvement, there is an abrupt development of severe 
respiratory distress with dyspnea, diaphoresis, stridor, and cyanosis. Shock and death occurs with 24-36 hours. There is evidence of mediastinal widening or pleural effusions on chest x-ray or CAT scan (CDC, 2002; USAMRIID, 2005). These symptoms can be complicated by hemorrhagic meningitis in up to $50 \%$ of cases and GI hemorrhagic in $80 \%$ of cases (USAMRIID, 2005). Early antibiotic treatment is critical for patient survival. The treatment for anthrax includes high doses of intravenous antibiotic treatments including ciprofloxacin or doxycycline combined with one or two additional antibiotics. Such treatment is required for 60 days, then switching to oral antibiotics. A vaccination is available, which includes a $0.5 \mathrm{ml}$ dose given subcutaneously at $0,2,4$ weeks, then $6,12,18$ months, followed by an annual booster. Historically the mortality rates have been greater than $85 \%$; however, the mortality rates from the 2001 attacks were $45 \%$ because of intensive care medicine and aggressive treatment (USAMRIID, 2005):

The cutaneous version of anthrax, the most common, has an incubation time of 1 to 12 days (USAMRIID, 2005). Responses can be immediate in some cases (CDC, 2002). Cutaneous anthrax is caused by direct contact with the spore from infected animals or animal products, which is usually the hands or forearms of people working with animals; however, other routes have resulted in the disease include fly bites and exposure to mail (USAMRIID, 2005). The signs and symptoms include localized itching followed by a painless papule lesion that turns vesicular with subsequent development of black eschar in 7-10 days. The eschar falls off in 1 to 2 weeks. If cutaneous anthrax is left untreated, a local infection may disseminate into a fatal systematic infection, which occurs in 10 to $20 \%$ of the cases. When treated, the mortality is less than $1 \%$ (USAMRIID, 2005). 
The gastrointestinal version of anthrax is rare in humans and is acquired after eating insufficiently cooked meat or dairy products from infected animals (CDC, 2002; USAMRIID, 2005). There is no person-to-person transmission of GI anthrax. After the incubation time of 17 days, edema can start which can lead to airway compromise and the disease can progress to sepsis, with case mortality rates of 10 to $50 \%$. If untreated, overall mortality can be greater than 50\% (USAMRIID, 2005). The initial symptoms are nausea, anorexia, vomiting, and fever. A subsequent phase starts 2-4 days after the initial onset. Shock and death can occur within 2-5 days of onset (CDC, 2002)

Iodine can be used to inactivate the spore in medical situations; however, disinfectant strength must be used (anti-septic strength iodophors are not usually sporicidal). Chlorine (either sodium or calcium hypochlorite) can be used, but caution should be used because the activity of these is greatly reduced in the presence of organic material (USAMRIID, 2005).

Anthrax has been used as a biological weapon throughout history. Anthrax outbreaks are thought to have started as early as $1250 \mathrm{BC}$. The Soviet Union researched anthrax as a biological weapon. This was proven during the accidental release of Bacillus anthracis spores in 1979 in Sverdlovsk, USSR (now Yekaterinaburg, Russia) (Claude, 1997; Meselson et al., 1994). The United States conducted research in anthrax in the 1950s and 1960s (USAMRIID, 2005). In 1993 the Aum Shinrikyo cult tried to complete a biological attack using anthrax near Tokyo, Japan; however, the attack did not work because they used the wrong strain (Fitch, 2008). The cult inadvertently used a vaccine strain of Bacillus anthracis, so no casualties were caused (Keim et al., 2001; Olson, 1999). The most recent use of anthrax as a biological weapon was in the fall of 2001 in the US, when there were 22 cases of confirmed or suspected anthrax related to letters. These 2001 letters resulted in 22 persons being infected (11 inhalation anthrax cases and 11 
cutaneous anthrax cases). Five of the inhalation cases ultimately died (Jernigan et al., 2001; Shieh et al., 2003).

Testing revealed where the contamination originated. The letters and sorting equipment that did come into contact with the letter were found to be contaminated with spores as high as 8 X $10^{6}$ CFU/100 cm² (Beecher, 2006; Sanderson et al., 2004; Sanderson et al., 2002). There was no official quantification of the material present in the letter sent to Senator Daschle's office. The estimates were that the letters contained 2 grams of "weapons-grade" Bacillus anthracis and the concentration was somewhere between $10^{11}$ and $10^{12}$ CFU per gram (Inglesby et al., 2002). Scanning electron microscopy showed the spores were ranged in sizes from individual particles to aggregates of $100 \mu \mathrm{m}$ or more (Weis et al., 2002).

The exposures during this incident provided new data on the spread of the anthrax and bioaerosols in general. Before the incident, studies showed that Bacillus subtilis spores could reaerosolize with varying activities in outdoor environments (Davids and Lejeune, 1981; Resnick et al., 1990). There were limited studies available on primary aerosolization during these types of incidents (Brachman et al., 1966; Druett et al., 1953; Meselson et al., 1994; Watson and Keir, 1994) and reaerosolization research was limited (Weis et al., 2002). In fact, before these attacks, most scientists (and likely also the perpetrators) did not expect the spores to disperse through envelopes and buildings at the extent they did (Carrera et al., 2005).

The re-aerosolization question was answered with the sampling completed. Nasal swabs were collected for more than 7,000 building occupants. Twenty of the 38 workers in the office suite tested positive - 13 of those workers were in the vicinity of the mail area, 7 workers on interconnected lower flow, 2 from adjacent office suites, and 6 emergency workers (Weis et al., 2002). There were cases of anthrax among workers which did not open the letters but were 
within the same room (Kournikakis et al., 2011)—only 8 cases actually handled the mail (Weis et al., 2002). The spores were found to be uniform in size and appearance and the aggregates which were larger had a propensity to pulverize; therefore, the particles dispersed into smaller particles (Parker, 2001). Also, some of the material was believed to be in a form that had silica added, or a fluidized form (Baron et al., 2008), which was a powdered suspension easily dispersed into the air (Parker, 2001). Overall, this showed that some individuals were exposed to aerosols generated from residual spore material on contaminated surfaces and demonstrated that spores can be reaerosolized from surfaces during office activities (such as paper handling, foot traffic, moving containers, etc) even after a period of no entry and no ventilation for several days (Weis et al., 2002).

During the anthrax responses, agencies collected over 10,000 samples. The CDC responded to anthrax releases in Florida, New Jersey, New York, Connecticut, and Washington, D.C. In all but two cases, CDC identified the sources of the anthrax release. The CDC never found the source of anthrax affecting a health care working in New York City or an elderly woman in Connecticut. In order to identify the sources, CDC followed a consistent sampling strategy. For anthrax delivered through the mail, they sampled the mail-sorting machines and electrostatic collection points (e.g., computer monitors). At Capitol Hill, CDC collected samples from elevators, furniture, floors, ventilation systems, vehicles, and clothing. The CDC personnel collected primarily bulk samples or surface samples and rarely collected air samples. At the time no method to validate spore sampling results existed (Martinez, 2005).

The recovery operations from these attacks were complicated because there no sampling or decontamination standards existed (Edmonds et al., 2009). The CDC did provide guidelines for office personnel who might encounter a letter containing a suspicious powder. These 
guidelines, developed during the crisis, did not include experimental data from laboratory or field investigations but were based on expert opinion applying the best available information (Kournikakis et al., 2011). Overall, the response showed the sampling methods were not adequately validated, especially at low surface readings (Estill, 2010). The lack of guidelines at the time led to much more research on wipe sampling and air sampling.

The anthrax responses caused several studies to be conducted immediately, some to verify the how the spores spread. The Defence R\&D Canada completed a statistically validated model system that was developed to assess objectively the aerosol exposure risk in an office environment from letters containing Bacillus anthracis spores. The spread of the aerosols through the building was assessed as well as the effectiveness of several potential mitigation procedures. This study showed that the letter opener effectively became a walking disseminator of spores (Kornikakis et al., 2009). Kournikakis et al. used $\mathrm{SF}_{6}$ tracer gas and smoke tubes to visualize airflow, culturable aerosol sampling, and aerosol spectrometry in order to characterize airflow and unmitigated spore aerosol dissemination within the office test site during letter opening. The study was designed to evaluate the risks and benefits of having the letter opener remain in place for five minutes and then closing the doors and HVAC system. The study found that the sharpest peak of spores (above 50 culturable particles/L) occurred from 40-52 seconds after the start of opening the letter. The study also found that spore aerosol concentrations reached equilibrium in approximately 10 minutes, which was 30 minutes faster than the $\mathrm{SF}_{6}$ concentrations reached equilibrium, likely because of the gravitational deposition of the spores (Kournikakis et al., 2011).

An additional study was designed to evaluate exposures and contamination levels in both semiquiescent and active offices. Active offices included paper handling, foot traffic, mail 
sorting, moving trash, and patting chairs. There was little contamination found on the vertical surfaces. The study showed 5 of 17 open plates were positive in the semiquiescent period. Personal air samples were collected on the personnel completing the study, all of which were positive, but with significantly more spores collected during the active period. Results also showed breathing zone samples had an increase in spores compared to the floor level samples. They concluded that the activities simulated caused higher airborne concentrations of spores and even minimal movements may result in the re-suspension of spores, with secondary aerosolization as high 15,000 CFU/hour (Weis et al., 2002).

\section{Bioaerosols on aircraft}

There have been historical concerns about the air quality on aircraft, with questions raised about whether poor air can cause illness. There has only been one documented instance of influenza linked to a specific flight; however, these illnesses followed a four-hour mechanical delay during which the passengers were kept onboard and the ventilation system was off and cabin doors were closed (Moser et al., 1979).

Studies have shown that the concentration of microorganisms in airline cabin air is much lower than in ordinary city locations and the small number found in US airliner cabin environments does not contribute to the risk of disease transmission among passengers. The study selected 36 domestic flights by one of the largest US airlines, including sampling a variety of different aircraft (narrow and wide-body) four intercontinental flights, two international flights (all in western hemisphere), and three short commuter flights (turbopropellor flights). The study showed there was little difference in the levels between seat level and higher locations; however, the highest concentrations were about 1 foot above the floor level near the 
outflow vents. The results showed an order of magnitude less than the levels found city buses and streets, thus the overall conclusion was the risk of disease transmission is low (Wick and Irvine, 1995).

Osman et al. (2008) conducted a study to extend sampling methods to identify significant differences in the total microbial burden and composition among individual aircraft $(747,757$, and 777) and different flights. The results showed that viable microbial burden within these cabin air parcels constituted only $1-10 \%$ of the total microbial population and ranged from below detection limits to $1.2 \times 10^{4}$ cells per meter ${ }^{3}$. Cultivable bacterial diversity was almost entirely limited to Gram-positive bacteria such as Staphylococcus and Bacillus. Isolations of staphylococci and micrococci were expected because these genera are found in association with human skin cells. Bacillus species were not widely distributed across all flights and were limited almost entirely to international flights, even though the artificially dry conditions aboard flights would promote their prevalence. Overall the study showed there is no significant difference between domestic and international flights, which is most likely due to constant HEPA filtration. Additionally, the controls are adequate for exposures from microbes which may cause disease. Any health symptoms are likely to be caused from the lower humidity, which the passengers constantly encounter (Osman et al., 2008).

During the SARS outbreak, there were only 4 flights in which SARS was transmitted among passengers (Freedman, 2003). The Aerospace Medical Association Task Force (AMATF) was established to evaluate the impact of SARS on passengers. After evaluating the data, they felt the transmission of SARS would occur due to person-to-person contact and not due to dispersion through aircraft ventilation system, which was also supported by the CDC. Additionally, they recommended that the aircraft be decontaminated through appropriately 
sanctioned procedures and also thoroughly ventilated after any ill individuals traveled (AMATF, 2004).

The design of aircraft HVAC systems is such that microbial growth would be limited. According to the FAA, first generation airliners used 100\% fresh air; however, later generations use different methods to maintain cabin pressure (which is usually maintained at 8,000 feet). The air on newer aircraft used for this is provided by bleeding air from the main jet engine compression stages, which is at $250^{\circ} \mathrm{C}$ or greater. The return air is then cooled to $112^{\circ} \mathrm{C}$ and passed through heat exchangers back to the cabin, meaning the air is virtually upon reentry into the cabin. The system also maintains low humidity, which does not favor microbiological growth (FAA, 1991). Up to $50 \%$ of air is recirculated on some aircraft; however, the air that is recirculated is passed through HEPA filters before going back to the cabin (AMATF, 2004). The normal air exchange rate for US airliners is between 15 and 20 per hour (FAA, 1991). Offices in the US are typically 10 per hour (Kodama and McGee, 1986) and can be as low as 5 per hour for some homes (Macher et al., 1991).

\section{Health effects of bioaerosols}

Bioaerosols can present a significant health threat, even aerosols which are not biological warfare agents. Past studies have shown a reduction in lung functions with airborne mold concentrations (Dahlqvist et al., 1992). Several studies have demonstrated that the onset of Sick Building Syndrome (SBS) could at least be partially due to the exposure to the biological agents (Bholah and Subratty, 2002; Cooley et al., 1998; Teeuw et al., 1994; Walinder et al., 2001). Exposures to airborne biological results in a wide range of respiratory and health disorders

(Douwes et al., 2003). These are so prevalent that some estimates have been made that up to 250 
million episodes of respiratory infections could be attributed to bioaerosol exposures every year in the United States (Cox and Wathes, 1995).

\section{Aerosol properties and characteristics}

An important aspect of bioaerosols is that both viable and non-viable bioaerosol particles are capable of causing adverse health effects (Adhikari et al., 2009; Gorny et al., 2002; Robbins et al., 2000). The response depends on the exposure and the host; however, both of these are related (Fitch, 2008). Another critical factor is the particle size.

Particle size plays a critical role in the infectivity of the bioaerosol in several ways. Particle size determines the survival in the aerosols (Lighthart and Shaffer, 1997; Tong and Lighthart, 1998) as well as the time the aerosolized microbe remains suspended in the atmosphere (Knight, 1980). Particle size also determines the deposition location (Harper and Morton, 1953; Heyder et al., 1986; Lippmann and Albert, 1969; Raabe et al., 1988). Particles 10 $\mu \mathrm{m}$ or smaller can penetrate beyond the head and thoracic regions during mouth breathing. Particles $4.0 \mu \mathrm{m}$ or smaller are considered respirable aerosols which can penetrate into lower airways during nasal breathing (Fitch, 2008). Bacteria cells are normally $1 \mu \mathrm{m}$ in size, meaning they can penetrate into lower airways and deposit in the pulmonary region (Fitch, 2008).

Particles smaller or approximately the same size of a bacterium cell (between 1 to $5 \mu \mathrm{m}$ ) deposit in the alveoli (respirable particles), while larger particles (greater than $10 \mu \mathrm{m}$ ) deposit in the upper respiratory tract (thoracic particles) (Menache et al., 1995; Raabe et al., 1988; Schlesinger, 1985). Past research has shown that for atmospheric particles containing bacteria, approximately $40 \%$ are greater than $7 \mu \mathrm{m}$ due to adherence to debris (Lighthart, 1997). 
The relationship of particulate number to particle size is important because the size impacts the number of particles required to be inhaled to initiate infection (i.e., the infective dose) or the quantity of material which can be deposited on surfaces. Because $12 \mu \mathrm{m}$ particles contain greater numbers of bacteria or spores than do $1 \mu \mathrm{m}$ particles, fewer large particles need to be inhaled to reach the lethal dose and initiate infection (Thomas et al., 2008). This has been seen in the laboratory when induction animals with these larger particles have different clinical features. Larger particle sizes usually result in lower mortality rates and longer incubation periods. For example, particles in the range of 1 to $5 \mu \mathrm{m}$ with Bacillus anthracis spores, caused hemorrhagic mediastinitis in rhesus macaques (similar to humans); but $12 \mu \mathrm{m}$ resulted in massive edema of soft tissues of head and neck, which was likely the result of initial spread of infection to cervical lymph nodes (Druett et al., 1953). Also, ricin particles transmitted via smaller aerosols caused death more frequently, while material in the form of $12 \mu \mathrm{m}$ particles did not cause death (Roy et al., 2003).

Aerosols with a high proportion of particles consisting of individual microbes could have higher infectious efficacy when compared to the same number of microbes but in larger aggregated particles. The aggregated particles may have a better survival rate though (Carrera et al., 2005). The severity of diseases increases with the average number of microbes or mass of toxin in each particle. When aerosols contain a variety of particle sizes, those particles in the range of $5 \mu \mathrm{m}$ will always pose the greatest threat, even if they represent only a minor fraction of inhaled material (Hatch, 1961). Additionally, research has shown that Brucellosis can be caused from particles sized from a single organism up to particles as large as $12 \mu \mathrm{m}$; however, the smallest were 600 times more likely to cause the disease (Druett et al., 1956). Additionally, monkeys exposed to $F$. tularensis via a mean particle size of 2.1 to $7.5 \mu \mathrm{m}$ became ill and died 
more quickly than those exposed to same agent in 12 or $24 \mu \mathrm{m}$ particles (Day and Berend, 1972). Particle size and deposition was studied using the murine inhalation models. Within the respiratory tract, the 1 - to $3 \mu \mathrm{m}$ particles containing $E$. coli preferentially deposited in the lungs as opposed to the nasal passages. The same organisms, delivered as $12 \mu \mathrm{m}$ particles, preferentially deposited in the nasal passages as opposed to the lungs (Thomas et al., 2008). These studies have shown that the particle size is a critical component of exposures.

Another factor that affects deposition is airway morphology and breathing physiology (Fitch, 2008). When a particle is inhaled, particles can deposit inside the respiratory system due to the impaction, settling, or diffusion mechanism (Yah and Mainelis, 2007). Larger particles are removed through inertial impaction and smaller particles are removed through diffusion. Other forces, such as electrostatic effects, may enhance or modify deposition. Additionally, condensation of water in humid environments may cause particles to increase in size and also impact their viability (Thomas et al., 2008). The humidity in the respiratory tract may do this as well (Fitch, 2008). Large respirable particles result in the generation of smaller particles comprising the dried components after evaporation of water surrounding the particle (Nicas et al., 2005). Additionally, mouth breathing will increase deposition in lungs for agent-containing particles of all sizes, which is especially true during heavy workloads (Fitch, 2008).

Transmission of disease is an important consideration as well. The chance of illness is largely due to the properties and characteristics of the aerosol (Fitch, 2008). The settling velocity is an important characteristic, but the chance of illness is not entirely dependent on this. Bacterial cell or spore survival to UV irradiation, desiccation, atmospheric gases, decontamination, and other damaging effects also impacts the transmission of the disease (Carrera et al., 2005). 
The idea of infectivity and the number of active biological units has caused scientists to recommend how to change the method in which bioaerosols are measured. The current method is a simple agent-containing particles per liter of air (ACPLA). This is a simple and clearly quantifiable method, but this method does not necessarily provide useful information because no differential diagnosis is made between active and inactive organisms, virulent and innocuous strains, single organisms and hundreds or thousands. For these reasons, the method fails to capture the one relevant characteristic which is the capacity to interact with the body and cause harm. Another proposed method is to use a new method which will account for the biological activity of the aerosol. The new method would be called the BAULA Dae - Biologically Active Units/Liter of Air and the health hazard would be a function of the physical characteristics and the biological characteristics. The physical characteristics would be the particles per liter of air, how much agent per particle, particle size distribution. The biological characteristics would include the agent present, how much of the agent is active, and what the $\mathrm{LD}_{50}$ of the agent is. The $\mathrm{LD}_{50}$ would be different for each agent. For these reasons, the new method would require assessment of not only the physical properties of the aerosol, but also an assessment of the biological activity (Fitch, 2008).

An additional consideration for health effects is the use of protective equipment. Personnel efficiency and effectiveness is decreased due to restrictive protective equipment, either individual or collective protective equipment. This will also have a larger logistical requirement, making the military mission more difficult to accomplish. In military specific environments, the decision maker must weigh perceived risks from exposure to the biological agents versus impacts of the intervention (Fitch, 2008). 


\section{Exposure assessments}

There are numerous ways to sample for biological warfare agents. Herzog et al. completed a study on the detection limits for Bacillus anthracis spores in several different types of media (air, water, and soil samples). They found the most sensitive detection method for anthrax is real-time PCR, which has a median instrument LOD of 440 cells $/ \mathrm{ml}$. The most sensitive method for environmental samples is PCR-enzyme-linked immunosorbent assay (ELISA), which has a detection limit of $0.1 \mathrm{CFU} /$ gram. The most sensitive method for air samples is ELISA-biochip system, which can detect down to $17 \mathrm{CFU} /$ liter for air. Finally, cultivation methods can detect down to $1 \mathrm{CFU} /$ liter for water and $1 \mathrm{CFU} / \mathrm{cm}^{2}$ after removal from a stainless steel surface. The median limit of detection for spores in soil samples was $1.2 \times 10^{4}$ CFU per 100 grams of soil; however, this type of sampling is highly dependent on sample pretreatment so there was a range of nine orders of magnitude because of the different approaches (Herzog et al., 2009). Finally, the BiSKit method was found to have the highest recovery for swipe samples, with recovery efficiencies for the fomite studies ranged from 10 to $50 \%$ and the extraction efficiencies ranging from 75 to $99 \%$. These recovery efficiencies are greatly by the fomite survival studies, which are greatly impacted by surface characteristics, relative humidity, and temperature (Sinclair et al., 2008). They concluded, however, that a low concentration release would be more likely detected by symptoms than using current sampling technology (Herzog et al., 2009). This was just one study showing the different methods to collect and analyze samples for Bacillus anthracis spores. Since the 2001 anthrax attacks, there have been numerous other studies evaluating the best methods for collecting and analyzing these samples. Recent research on wipe and air sampling is discussed more in-depth below. 


\section{Wipe sampling}

Past research showed low precision in swab and wipe sampling were due to errors inherent in the sampling mechanism itself such as wipe material composition, surface composition, and mechanical removal action but are also subject to collection and processing errors (Angelotti et al., 1958). Another factor is operator technique (such as angle and pressure or sampling), variations in extraction method, and processing errors (Angelotti et al., 1958; Rose et al., 2004). Additional errors include non-homogeneous surface deposition of spore material; incomplete removal of spores from reference coupon (Brown et al., 2007b). This historical data showed that the sampling methods underestimated the number of spores present on surfaces, and because of this, empirical studies were needed (GAO, 2005; National Research Committee, 2005). Newer methods are being researched to help with these errors, especially after the anthrax attacks.

Brown et al. (2007b) completed several studies using polyester-rayon blend wipes to test recovery of powdered Bacillus atrophaeus spores from stainless steel and painted wallboard surfaces, both considered non-porous surface. The wipes used were sterile polyester-rayon blend gauze wipe (10 by $10 \mathrm{~cm}$, catalog no. 9728; Alliance Medical, Russellville, MO). The extraction method was sonication and included a mean efficiency of 93\%. Studies showed that the wipe method was better than a swab method and the removal efficiency was significantly lower from the painted wallboard. The spores used contained non-spore material and no attempt was made to evaluate the method efficiency in presence of dust, bacterial vegetative cells, cells, fungal spores, or other native background material which might interact with removal, extraction, or plating efficiency. The mean efficiency for recovery with wipe sampling was $35 \%$ for stainless steel (standard deviation of \pm 0.12 ) and painted wallboard was $29 \%$ (standard deviation of \pm 0.15 ). 
Brown et al. (2007a) also conducted a study for recovery efficiencies on porous and nonporous surfaces using a vacuum filter sock. The surfaces evaluated were non-porous surfaces (stainless steel and painted wallboard) and porous surfaces (two different types of carpets) with stainless steel used as a reference coupon. The recovery efficiency was a measure of overall transfer effectiveness from surface to culture, which was calculated as a number of CFU from filter sock to those on the sample. Bacillus atropheaus spores were again used for the tests. The recovery fractions from stainless steel were 0.062 to $0.551($ mean $=0.289, \mathrm{SD}=0.138, \mathrm{n}=36$ ) and painted wall board was 0.035 to 0.577 (mean $=0.248, \mathrm{SD}=0.145, \mathrm{n}=36$ ).

Edmonds et al. (2009) conducted a study to evaluate dry deposition of spores on four representative sampling surfaces. They completed the tests using a liquid spore deposition and aerosolized spores which were allowed to deposit on the coupons. The tests were completed on four different swabs, including cotton-tipped, Dacron-tipped, rayon-tipped, and a polyurethane macrofoam-tipped swab. The coupons tested were all cut $1 / 8$ inch think and $2 \mathrm{~cm}$ by $5 \mathrm{~cm}$ and included were glass, chemical agent-resistant coating (CARC)-painted steel, polycarbonate, and vinyl tile. They found that recovery of liquid-deposited spores differs significantly than from dry aerosol-deposited spores in most instances. The variation in recovery efficiency across all surface materials with aerosol-deposited spores is significantly smaller than with liquiddeposited spores. They found no single swab outperformed other ones. They concluded an optimal sampling methodology requires accurately recreating the contamination events in the laboratory. Previous studies have shown that using the same method of surface contamination, the percentage of recovery of liquid-deposited Bacillus atrophaeus spores on glass coupons decreased from 92.7 to 42.1 as the concentration of spores deposited on surfaces dropped from $10^{7}$ to $10^{4} \mathrm{CFU}$; therefore, concentration plays a role as well. 
Another study was completed by Baron et al. in which they designed a test chamber to aerosolize Bacillus anthracis Sterne spores in order to achieve very low surface loadings—as low as 3, 30, and $200 \mathrm{CFU}$ per $100 \mathrm{~cm}^{2}$. Previous wipe sample studies have primarily investigated sampling techniques by direct inoculation using a high-concentration suspension of bacteria or spores (Hodges et al., 2006; Rose et al., 2004) or by evaluating environmental samples (Krischner and Puleo 1979; Sanderson et al., 2004). These earlier tests have not directly simulated the dry deposition of bacteria or spores from the aerosol state or may not have sufficient precision or accuracy to adequately differentiate between techniques. The tests completed by Baron's group used steel and carpet coupons and sampled with swabs, wipes, or vacuums, with agar settle plates used as a reference. The results showed wipe methods at these lower surface concentrations could detect as low as $15 \mathrm{CFU} / 100 \mathrm{~cm}^{2}$ and vacuum samples could detect as low as $44 \mathrm{CFU} / 100 \mathrm{~cm}^{2}$. Some of these positive results observed at low or the very low target concentrations could be based on sample contamination instead of recovery efficiency. Even with the caution they used, they required a sample to have had 3 or more CFU to be considered a positive when they estimated the LOD for wiping and vacuuming on steel. They found with the low number of chamber runs and the high variability, the confidence intervals were very wide. Additionally, re-aerosolization of spores during these samples was an issue, especially at the lower levels (Baron et al., 2008).

Lewandowski et al. (2010) evaluated Bacillus atrophaeus to determine the recovery efficiencies from glass and stainless steel surfaces using polyester swab and macrofoam sponge wipe. They found that swabbing with a macrofoam sponge wipe was more efficient in recovering spores from surfaces contaminated with high bioaerosol concentrations than swabbing with a polyester swab. Sampling materials tested were pre-moistened with PBS and 
placed in a sterile $50-\mathrm{mL}$ vial. The macrofoam sponge wipe and foam spatula were hand mixed for $1 \mathrm{~min}$, and then they were squeezed with sterile forceps and removed. The polyester swabs were sonicated for 12 minutes at a frequency of $40 \mathrm{kHz}$ or vortexed for 1 minute. The suspensions were serially diluted prior to inoculation onto agar. The plates were incubated at $35^{\circ} \mathrm{C}$ for 24 hours. They found the median recovery efficiency from the surfaces using foam spatulas was equal to $9.9 \%$ for Bacillus atrophaeus spores when the recovery was calculated relative to the theoretical surface spore load.

NASA has a swab protocol which has not changed for decades. Probst et al. (2010) completed a study comparing a novel nylon-flocked swab, evaluated for recovery of different Bacillus atrophaeus spore concentrations on stainless steel and other surfaces and compared this new method to the NASA standard. The new protocol recovered 3 to 4 fold more ( $45.4 \%$ and 49.0\% recovery efficiency) Bacillus atrophaeus spores than the NASA standard method (13.2\%) The recovery efficiencies were different for different surfaces -5.9 to $62 \%$ for rough surfaces and $80 \%$ for direct inoculation. Worker variability was a factor, with inexperienced experimenters achieving a removal efficiency of $39.3 \%$ versus $45.4 \%$ (Probst et al., 2010). Past spacecraft were found to be susceptible to heat sterilization protocols - the Viking Lander Capsule could be exposed to temperatures of $111.7^{\circ} \mathrm{C} \pm 1.7^{\circ} \mathrm{C}$ for 23 to 30 hours; however, current NASA craft cannot withstand temperatures this high (Puleo et al., 1977). Because of this, NASA has been looking into different methods to decontaminate their spacecraft (Schuerger et al., 2008). The Mars Exploration Rover mission craft is an example of the difficulty in completing sampling and decontamination — the spacecraft contains many different types of materials - aluminum, aluminum honeycomb structures, titanium and graphite composite (carbon fiber-reinforced plastic [CFRP]). There are also vectran and polyester/nylon fabrics. 
These materials are challenging for sampling tools and no studies have been completed for many of these materials (Probst et al., 2010).

Martin and Moore (2001) completed a study using Bacillus globigii to contaminate surfaces by both aerosol methods and by application directly onto material. The recovery efficiencies for aerosol and droplet contamination were similar, but aerosol contamination had a higher variability. Studies completed by Hodges et al. (2006) and Rose et al. (2004) used direct application of droplets to inoculate steel surfaces to determine recovery efficiencies. The directly inoculated surfaces resulted in greater recovery efficiencies. Both studies concluded that the lower efficiencies could have been a result of inoculation methods or surface loadings being 100 times lower in this study.

Cotton swabs have a high DNA content, which makes them a poor choice for molecular technologies. A better choice is the rayon or macrofoam (Probst et al., 2010). Different studies have shown different removal efficiencies for the spores: vacuum filter sock sampler-28.2\% (Brown et al., 2007c); nylon-flocked swabs-41.6\% (Brown et al. 2007c), and BiSKit have had efficiencies up to $47.3 \%$ (Buttner et al., 2004). Despite the increase in studies, there is still an overall lack of consensus for spore removal in the literature (Probst et al., 2010). The removal efficiencies are different when the spores are applied to porous and non-porous surfaces (Buttner et al., 2004). The inoculation method also plays an important role in the removal rates. Direct inoculation recovery efficiencies for Bacillus atrophaeus spores can range from anywhere from $75.6 \%$ up to $96.6 \%$ (Brown et al., 2007b; Rose et al., 2004). Additionally, there are errors for poor precision which includes inconsistent spore release from the testing swabs because of variations in vortexing, sonication, pipetting, and colony counting errors (Rose, 2004). 


\section{Air Sampling}

Air sampling devices are also a critical component for exposure assessments. There are several different types of samplers. For example, bioaerosol concentrators are used to either increase the number of particles available for analysis or to augment the statistical significance of detection or identification of a hazardous bioaerosol. Concentrators use either continuous flow (a virtual impactor) or batch type (impinger). A virtual impactor concentrates aerosol particles from a larger volume of air into a smaller volume of air. Virtual impactors use the same principles as the traditional impactors, where an aerosol stream is first accelerated and then caused to change direction. The direction change imposes a centrifugal force on the particle which causes the particles to move perpendicular to the direction of the turning air stream. A traditional impactor collects the particles on a real surface (such as culture media, filters, or plates) placed traverse to the initial air flow direction, which is different than a virtual air impactor. A virtual impactor uses a port system where the larger particles are driven into a port. Approximately $10 \%$ of the air stream is drawn into the port to transport the larger particles away from the fractionation zone, while the remaining $90 \%$ of the air (the portion which does not have large particles now) is exhausted away from the port. These airstreams are referred to the minor (concentrated aerosol) and major flows. These virtual impactors can be operated in series, where the minor flow from a first stage then becomes the inflow to a second stage which could then provide much higher concentrations than a single stage alone. Impingers use the same basic principle as a real impactor, with the major difference being that the collection surface is a liquid or solid (such as glass) immersed in liquid. Particles that are larger than approximately $1 \mu \mathrm{m}$ are captured by the inertial mechanisms in the liquid. Particles are collected in the same liquid volume over time, therefore the collection is on a batch basis and the particles are concentrated 
in the liquid. Another method is a fan arrangement, which collects particles by direct impaction onto the moving fan blades. As the fan moves, large particles will strike the blade and remain on the blades because of inertia. If operated for a long time, the particles can be concentrated on the blade surfaces (Kesavan et al., 2008a).

Numerous studies have been completed to evaluate these different air sampling devices. Park et al. (2009) evaluated the performance of six different aerosol samplers. These included Anderson samplers, total suspended particulate (TSP), RespiCon, PM10, DustTracks, and SidePaks. They found that the Anderson samplers underestimated total suspended PM, while overestimating thoracic and respirable particulate matter. This was largely due to particle bounce and carryover between stages. The TSP samplers analyzed provided total suspended particulate matter as reference samples and quantified by a coulter counter multisizer provide no information below an equivalent spherical diameter of $2 \mu \mathrm{m}$; therefore, they underestimate respirable PM. They found the RespiCon samplers were free from particle bounce as inhalable samplers but underestimated total suspended PM. The PM10 samplers overestimated thoracic PM. Finally, the DustTrak and SidePak samplers profile relative PM concentrations instead of absolute PM concentration.

Another study analyzed the impact of time on the overall performance of seven portable impactors. The impactors tested were the SMA MicroPortable, BioCulture, Microflow, MAS100, Millipore Air Tester, SAS Super 180, and RCS High Flow, evaluated by collecting airborne bacteria and fungi from 2 to 30 minutes indoors and outdoors. The stationary BioStage impactor was used as a reference, with a collection time of 2 minutes. For outdoor sampling, the average concentration ratio of all test samplers relative to the reference sampler was 0.64 , but decreased to 0.04 when bacteria were collected for 2 min first and subsequently exposed to particle-free air 
for $28 \mathrm{~min}$ (30 min total sampling time). The study showed that, when impactors are used for the collection of airborne bacteria and fungi, sampling times should be as short as reasonably possible to minimize the under-representation of airborne microorganism concentration which could be a factor of 10 or higher for prolonged sampling times. This study evaluated the effects of desiccation damage to the already collected microorganisms as well as the effect of agar desiccation prior to collecting the microbial particles. The results indicated that the recovery of airborne bacteria and fungi by microbial impactors decreases as the sampling time increases, even after the media is exposed to particle free air. The bacteria collected decreased by a factor close to 20 when the media was exposed to particle free air for 28 minutes prior to collection. The likely reason for such a dramatic decrease in microorganism recovery is the desiccation and hardening of agar under the impaction jet which results in particle bounce and reduced collection efficiency. Visual observation of dented media was seen (Mainelis and Tabayoyong, 2010). A remedy for this could be to increase the jet-to-plate distance; however this results in decreased collection efficiency (Yao and Mainelis, 2007). Another solution is to keep the sampling time as short as possible (Mainelis and Tabayoyong, 2010). The detrimental impacts of long sampling times were seen previously (Hensel and Petzoldt, 1995). This longer sampling time can also lead to increased particle bounce (Juozaitis et al., 1994). Sample overload can take as little as a few seconds (Chang 1995; Rinsoz et al., 2008), and some studies have found that sampling less than 40 minutes did not significantly influence bacterial recoveries (Li and Lin, 1999).

Portable microbial samplers are being increasingly used to determine the presence of microbial agents in the air. Because of this, other researchers have studied the collection efficiencies of MAS-100, Microflow, SMA MicroPortable, Millipore Air Tester, SAS Super 180, BioCulture, and RCS High Flow portable microbial samplers by sampling six bacterial and 
fungal species ranging from 0.61 to $3.14 \mu \mathrm{m}$ in aerodynamic diameter. This study did not take into account biological performance of the samplers, but rather focused on the physical aspects of the aerosol collection. The researchers compared the sampler collection efficiency curves with particle inhalation and deposition conventions for the human lung, focusing on the physical performance for the collection efficiency curve and cutoff size, or $\mathrm{d}_{50}$. These sampling devices were tested using the airborne concentrations up and down stream, where both were measured isokinetically by a Grimm optical particle counter (OPC) (model 1.108, Grimm Technologies, Inc., Douglasville, GA, USA). The study noted that, when graphed, there were small peaks to the left of the main peak, as in the case of the Bacillus subtilis, which is likely the culture medium. The results showed the collection efficiency increased with increasing microorganism aerodynamic size (Yah and Mainelis, 2007).

Additional studies have been completed to analyze new technologies for collecting aerosols. One newer collection method is the Electrostatic Precipitator with Superhydrophobic Surface (EPSS) use to collect bioaerosols for analysis with the whole-cell QPCR. The EPSS is novel sampler using a combination of electrostatic collection mechanism with superhydrophobic collection surface. The combination of these devices allows for efficient particle collection, removal, and concentration in water droplets which can be as small as $5 \mu \mathrm{L}$. This mechanism was tested using Pseudomonas fluorescens and Bacillus subtilis, with collection efficiency determined using the traditional method of microscopic counting and whole-cell quantitative real-time polymerase chain reaction assay (QPCR). Research has shown samplers can achieve collection efficiencies as high as $72 \%$. The researchers also found the collection efficiency for both bacteria obtained by the two different methods was not statistically different, which indicates the sampler's compatibility with the PCR-based sample analysis techniques. The 
airborne concentrations were further evaluated using an APS. They found Bacillus subtilis had a collection efficiency ranging from $59 \%$ to $72 \%$ for the same sampling flow rates. The study showed that the sampling device could detect a low microorganism concentration with low power requirements due to the absence of pressure drop inside the EPSS (Han et al., 2010).

Farnsworth et al. (2006) conducted a study to evaluate the effectiveness of building air handling units and determining whether they can serve as high volume samplers for airborne bacteria and viruses. To test this, they nebulized and injected aerosols into a test facility upstream of a MERV 14 filter. They used a biosampler similar to an AGI-39 with a sampling rate of $12.5 \mathrm{~L} / \mathrm{min}$ and a duration of 10 minutes. The collection solution used was $20 \mathrm{~mL}$ of phosphate buffered saline. The researchers found the overall collection efficiency to be between $97.6 \pm 0.2 \%$ and $105 \pm 19 \%$. The level that corresponded to greater than $100 \%$ is because the level removed from the HVAC sampler is more efficient than the media sampler. Additionally, they found Bacillus subtilis can be removed from HVAC media with little loss of culturability. Viruses had a much lower recoverability. Finally, they found that relative humidity can impact the recovery efficiencies of these organisms.

Numerous studies have been completed using inert particles to model bioaerosols. One study, completed by Li et al., evaluated the collection efficiency of six samplers in the inhalable particle size range. They used fluorescein particles to determine how well these samplers matched the inhalable convention. This study showed that the sampling efficiency can depend on the "stickiness" of the particles (Li et al., 2000). John and Kreisberg (1999) characterized samplers with dry polystyrene beads generated with a fluidized bed and analyzed with an Aerodynamic Particle Sizer (APS). Maynard et al. developed a system to rapidly measure sampler performance using polydisperse glass microspheres and an APS analysis method 
(Maynard et al., 1999). McFarland et al. (1991) used liquid fluorescent oleic acid with fluorometer analysis in their sampler characterization tests. Gao et al. (1997) used a fluidized bed aerosol generator to generate ceramic and polystyrene beads for characterizing samplers. Aizenberg et al. (2000a) and Witschger et al. (1998) used aluminum oxide particles with gravimetric analysis to characterize sampler performance. Finally, Willeke et al. (1998) conducted tests with PSL aerosols generated with a Collison nebulizer and analyzed the results with an Aerosizer (Willeke et al., 1998).

Another important consideration is relative humidity, which can decrease impactor performance. One study evaluating sensitive bacteria Pseudomonas fluorescens found total recovery of the bacteria at relative humidity of $30 \%$ decreased from $1.5 \%$ to $0.3 \%$ when sampling was extended from 10 to 30 minutes. When sampling was repeated at relative humidity of $90 \%$, the total recovery had higher variability (compared to lower humidity levels) and the influence of sampling time was not substantial (Thompson et al., 1994).

\section{Biological decontamination methods}

Decontamination of biological agents can be a very complicated task, but acceptable decontamination in short time is critical to protect health (Uhm et al., 2007). There are several complicating issues, including the fact that detecting the agents before and after is difficult, there are no set standards for decontamination levels, and several different methods which could be used for decontamination (Uhm et al., 2007). Each of these is discussed more in-depth below. 


\section{Definitions of decontamination}

Decontamination can entail several different levels of inactivation for biological agents. Probably the most basic definition is "a process or method whereby an object or material...freed of the contamination agent(s) and rendered safe for human handling without further recourse to individual protective measures" (Perkins, 1983). In effect, the decontamination process is the equivalent of sterilization which all infective agents must be destroyed or irreversibly inactivated. Inactivation is rendering the biological particle inert. While this seems straightforward, there is not always agreement concerning the thermal death requirements of microbial life in part because the mechanisms responsible for microbial death due to heat are not clearly understood (Perkins, 1983).

A key term in biological discussions is sterilization, which can be defined as a procedure that kills all microorganisms, including high numbers of bacterial endospores. This can be accomplished in a number of ways — heat, ethylene oxide and hydrogen peroxide gases, plasma, ozone, radiation, etc. To be considered sterile, the item has to be completely free of all living microorganisms and viruses, which is a categorical and absolute definition; that is, an item is either sterile or not (Chosewood and Wilson, 2009).

A process that is generally less lethal than sterilization is disinfection. Disinfection eliminates nearly all recognized pathogenic microorganisms; however, the method does not necessarily eliminate all microbial forms on inanimate objects. Disinfection does not ensure an "overkill" and lacks the margin of safety achieved by sterilization. The distinguishing difference between sterilization and disinfection is that disinfection does not inactivate spores. This may be over-simplified because some chemical germicides used as disinfectants do kill large numbers of spores even though high concentrations and several hours of exposure may be required. Non- 
sporicidal disinfectants may differ in their capacity to accomplish disinfection or decontamination. Some germicides rapidly kill only the ordinary vegetative forms of bacteria (such as Staphylococci and Streptococci), some forms of fungi, and lipid-containing viruses, whereas others are effective against such relatively resistant organisms as Mycobacterium tuberculosis var. bovis, non-lipid viruses, and most forms of fungi (Chosewood and Wilson, 2009). The effectiveness of these procedures is controlled significantly by a number of factors, each one of which may have a significant impact on the end result. Some of these factors include the nature and number of microorganisms (especially the presence of bacterial spores); the amount of organic matter present (e.g., soil, feces, and blood); the type and condition of the object to be disinfected, and the temperature, and also the time and contact of the agent with the organism (Chosewood and Wilson, 2009).

There are several different levels of disinfection. For example, high-level disinfection kills vegetative microorganisms and inactivates viruses, but not necessarily high numbers of bacterial spores. Some of these disinfectants are capable of sterilization when the contact time is relatively long (e.g., 6 to 10 hours). These high-level disinfectants are used for relatively short periods of time, usually 10 to 30 minutes. These chemical germicides can be potent sporicides and, in the United States, are classified by the FDA as sterilant/disinfectants. These are formulated for use on medical devices, but not on environmental surfaces such as laboratory benches or floors (Chosewood and Wilson, 2009). The next level is intermediate-level disinfection, which kills vegetative microorganisms, including Mycobacterium tuberculosis, all fungi, and inactivates most viruses. Chemical germicides used in this procedure often correspond to Environmental Protection Agency (EPA)-approved "hospital disinfectants", which requires them to be "tuberculocidal." These types are used commonly in laboratories for 
disinfection of laboratory benches and as part of detergent germicides used for housekeeping purposes. The last level is low-level disinfection, which kills most vegetative bacteria except Mycobacterium tuberculosis, some fungi, and some viruses. The EPA approves these as "hospital disinfectants" or "sanitizers" (Chosewood and Wilson, 2009).

There are several military definitions which need to be considered as well. The first one is "Chemical, Biological, and Radiological (CBR) decontamination", which is defined as a "process making material safe by absorbing, destroying, neutralizing, rendering harmless, or removing chemical or biological agents and radiological contamination". Additionally, CBR decontaminability is defined as the "ability of a system to be rapidly and effectively decontaminated to reduce the hazard to personnel operating, maintaining, and re-supplying it" (DoD, 2009). Finally, military equipment must be able to survive in a CBRN environment. This means the system must have a capability to "avoid, withstand, or operate during and/or after exposure to a CBR environment (and relevant decontamination) or a nuclear environment, without losing the ability to accomplish the assigned mission." This also requires the system to withstand chemical, biological, or radiological contaminated environments, decontaminants, and decontamination processes, without losing the ability to accomplish the assigned mission. There are three elements to CBR contamination survivability - CBR hardness (ability to continue to operate in contamination), CBR compatibility, and CBR decontaminability (DoD, 2009). Therefore, the decontamination method is a critical piece for military equipment and readiness.

\section{Decontamination requirements}

There are no set limits for decontamination requirements and even the recommendations made are very wide ranging. For example, Herzog et al. (2009) stated that any detectable 
Bacillus anthracis spore would constitute an unacceptable risk. Detection would be very difficult at these low concentrations. In fact, detection at these low concentrations would more likely be seen in a human infection than current sampling methods, demonstrating that a significant risk posed by undetectable concentrations of these spores (Sinclair et al., 2008). There is a general disagreement on the level of spore inactivation required. Some in the biodefense community have suggested a 12-log inactivation, but most of the general consensus is that a 6-log reduction would be sufficient (Gale et al., 2008). This 6-log reduction has been used in most field studies. Several EPA guidance for decontaminating surfaces requires $98-99.999 \%$ reduction, but these do not address the original quantity of the organisms, which is a relevant consideration (Raber et al., 2001)

Another complicating factor is that background levels differ widely because some are indigenous in certain areas. This is true for Bacillus anthracis as explained previously. Additionally, outdoor air may contain between 0.5 to 5 endotoxin units per cubic meter of air. While these are not necessarily viable, this level demonstrates there are certain levels of biological contamination in the air (Cox and Wathes, 1995).

Finally, guidelines on the dose required for infection is limited because established threshold limit values are not available for many biological warfare agents. Those limits which are available are associated with production and battlefield use and not intended for general public use, thus they cannot be used for public exposures, such as children, immunocompromised persons, etc. Additional data is limited as well, including incomplete regulatory standards, definitions of terms and cleanup are problematic, sampling and analysis strategies are not adequately defined for decontamination protocol, and the site- and populationspecific nature of health risks and procedures are not defined. Because of these limitations, the 
potential residual health effects for a biological agents in a situation involving infrastructure decontamination, some type of scenario-based health risk assessment would be necessary (Raber et al., 2001)

Regardless of the level of reduction required, decontamination protocols and methods need to be tested against spores. The U.S. Army Edgewood Chemical and Biological Center (ECBC) requires that the decontamination methods be effective against spores and not just viruses. This is not only to facilitate the inactivation, but also because a first responder will not necessarily know the differences among viruses, biological agents, and spores (Brickhouse, 2005).

\section{Decontamination problems}

The decontamination or inactivation of spores can be complicated because of the nature of the spore itself. Spores from the Bacillus species are metabolically inactive and highly resistant to many physical stresses such as wet and dry heat, chemical agents, ultraviolet and gamma radiation, oxidizing agents, vacuums and ultra-high hydrostatic pressures and they can remain viable for many years creating a serious and lasting health risk (Nicholson et al., 2002). Additionally, there are many factors which have an impact on decontamination efforts, including the number of organisms and resistance; the state of the organisms (i.e., spore or vegetative) protection afforded to the organisms by extraneous matter (such as oils, greases, protein, soil, etc.), and the exponential death rate of the organism (which is proportional to the beginning concentration of organisms) (Perkins, 1983). This death rate is referred to as the "D-value", defined as the time (in hours) required to inactivate $90 \%$ of the organisms (Prescott et al., 2002). Decontamination for spores is much more complicated than for normal vegetative cells. Cell 
death due to thermal decontamination is generally due to oxidation or a low combustion process within the cell. During hot air exposure, vegetative bacteria are dehydrated greatly before the temperature rises sufficiently to cause death by coagulation. Cell death by coagulation requires complete dehydration which is ultimately a burning process. However, water is not in a free state in spores but is rather bound and thus less reactive. Some past studies have shown bound water content is 60 to $70 \%$ in spores, compared to 3 to $21 \%$ in vegetative cells (Henry and Friedman, 1937). These levels are comparable to dehydrated proteins (Powell and Strange, 1953; Ross and Billing, 1957). Thus, the very nature of the spore makes decontamination much more difficult.

Another problem is the general information on the agent will likely be incomplete. This data that will be lacking will include incomplete regulatory standards, problematic definitions of terms and cleanup criteria, inadequately defined sampling and analysis strategies in the context of a broader decontamination protocol, and the site- and population-specific nature of health risks and procedures. Additionally, the area of contamination can be a concern - urban areas will present more challenges, including collateral damage and recertification. For urban areas, time may be less of a factor and public perception will become more important (Raber et al., 2001).

An additional problem is that when spores are involved, they are usually attached to aerosol particles or other organic matter. The presence of this organic matter requires longer contact time with a decontamination method if the item or area is not pre-cleaned (Chosewood and Wilson, 2009). This organic matter is such an issue that some studies have used soot to show elimination of the particle the agent is attached to (Uhm et al., 2007). 
Finally, current decontamination methods focus on killing the agent and not on returning the item to use. Any type of decontamination agent needs to be environmentally benign to people and environment, and many of the current decontamination methods are not benign (Uhm et al., 2007).

\section{Recommended decontamination methodologies}

While there are no definite standards for decontamination, there are recommendations which can be followed. The DoD has had methods for decontamination for quite some time (Raber et al., 2001). Other possibilities include the Biosafety Reference Manual, American Industrial Hygiene Association (1995), Biosafety in Microbiological and Biomedical Laboratories, Centers for Disease Control (1999b), and the NASA Standards for Clean Rooms and Work Stations for Microbially Controlled Environments, National Aeronautics and Space Administration (1967). There have also been publications based on the anthrax attacks in 2001, as discussed previously.

Before a course of action can be determined, the actual location and delivery of the contamination must be evaluated. For instance, there are three broad categories of such an attack scenario - open air (such as a stadium), semi-enclosed (a subway), and enclosed (building or airplane). Two of these involve HVAC systems, which must be considered for decontamination (Carlsen, 2005).

Raber et al. provided general recommendations for decontamination. The overall objective of the effort should be to determine an effective level of cleanup, meeting all health and environmental regulations while ensuring stakeholders considerations are met. Clearly, health and personal property should be protected. Secondary objectives are the time and cost for 
the process to be completed, including if the area needs to be re-occupied quickly for some reason (such as some mission critical area which cannot be easily relocated). The overall method should be a risk-based approach, with the clean-up activities based on a defined, acceptable level of risk to the health of those exposed (Raber et al., 2001).

The scenario-based health-risk assessment approach would start with a multimedia, multi-pathway dose assessment. For example, the delivery and re-suspension, subsequent multimedia transport, and fate of a substance or microorganism must be determined. Thus the ability of the contaminant to move into and off of contaminated materials must be determined or assumed. The mobility of the contaminant must be considered as well, which will include the typical movements as well as unusual movements such as fires, water-off from on-site work, and even building paint. The toxicology of the agent, human morbidity, mortality, and latency of effects must be evaluated. Integrating multimedia transport and fate with multi-pathway exposure (e.g., inhalation, secondary ingestion, or dermal absorption) and physiologically based pharmacokinetics (if available). Because of the different exposure routes, each must be considered (including inhalation, ingestion, dermal absorption) (Raber et al., 2001).

The clean-up criteria will be defined more after the release is quantified. The standard used should ensure public health and property is protected; however, the decontamination efforts must still be realistic. The decontamination criteria should be population specific, meaning the standard used must consider who will occupy the area. For instance, a higher standard may be needed if the area will be occupied by children, elderly, immunocompromised, etc. The cleanup criteria should be site specific as well. For example, there should be stricter criteria for indoor, long-term reuse than for outdoor scenarios where natural attenuation may be effective for the 
decontamination or destruction of biological agents, biological toxins, or chemical warfare agents (Raber et al., 2001).

When developing a decontamination plan, education of the public is a critical component. The public generally accepts hospital disinfectant methods but those do not guarantee zero risk. Another example is swimming pools which must meet defined risks, but there are still risks. Children have died from exposure to and ingestion of E. coli from pools; however, the general public accepts these standards (Raber et al., 2001).

Perceptions of the cleanup requirements and methods are also critical drivers for decontamination efforts. The actual cleanup goals will be strongly driven by stakeholder concerns, which include both acceptance and perception. Many stakeholders may demand zero living organisms for some areas (Raber et al., 2001)

When selecting a decontamination method, consideration should be given to building security, interagency relationships, incident command structure, preparation and review of technical documents, contractor selection, and crisis exemption applications and approvals. Project schedules should also be considered. The last three above will have the most effect on the project schedule (Martin, 2005). Additional items to consider are sampling during the decontamination efforts to quantify the exposures to those doing the work. Also, the controls for the workers should be evaluated, including possible prophylactic drugs or vaccines should be considered for those which have been exposed and all responders (Raber et al., 2001).

The final item to consider is verification after the work is completed. A sound and defensible sampling strategy to verify the decontamination efforts must be completed. Such sampling may require some type of "hot-spot" environmental sampling type strategy. The sampling after decontamination must include a cost analysis (Raber et al., 2001). 
An alternative and commonly accepted approach is to study contaminated versus uncontaminated environments. Elevated concentrations of an agent can then be used as an index for evaluating relative contamination levels and to determine whether decontamination treatment should be repeated. For example, to evaluate anthrax contamination in a building located in a farming community, where anthrax is indigenous, one would measure the relative anthrax concentration inside versus outside the building to ascertain whether decontamination should be implemented or repeated. Acceptable levels should be somewhere between background and the lowest dose for any kind of infection (Raber et al., 2001).

\section{Decontamination methods}

There are many different types of germicides which can be ranked differently based on activity levels. For instance, chlorine can be in liquid or solid form (sodium or calcium hypochlorite), can be in different concentrations, and thus can be used for many disinfecting applications. Chlorine compounds are highly corrosive though, so they cannot be used on certain materials. These are just a few examples of the advantages and disadvantages of one disinfectant agent. Several others are described below. Several different disinfectants are listed in Table 1-3 (Chosewood and Wilson, 2009). 
Table $1-3$ - Summary of chemical disinfectants

\begin{tabular}{|c|c|c|}
\hline \multicolumn{3}{|c|}{ Disinfection } \\
\hline Procedure / Product & Aqueous Concentration & Activity Level \\
\hline Glutaraldehyde & Variable & High to intermediate \\
\hline Ortho-phthalaldehyde & $0.5 \%$ & High \\
\hline Hydrogen peroxide & $3-6 \%$ & High to intermediate \\
\hline Formaldehyde & $1-8 \%$ & High to low \\
\hline Chlorine dioxide & Variable & High \\
\hline Peracetic acid & Variable & High \\
\hline Chlorine compounds & $\begin{array}{l}500 \text { to } 5000 \mathrm{ml} / \mathrm{L} \\
\text { free/available chlorine }\end{array}$ & Intermediate \\
\hline Alcohols (ethyl, isopropyl) & $70 \%$ & Intermediate \\
\hline Phenolic compounds & 0.5 to $3 \%$ & Intermediate to low \\
\hline Iodophor compounds & $\begin{array}{l}30-50 \mathrm{mg} / \mathrm{L} \text { free iodine up } \\
\text { to } 10,000 \mathrm{mg} / \mathrm{L} \text { available } \\
\text { iodine } 0.1-0.2 \%\end{array}$ & Intermediate to low \\
\hline
\end{tabular}

Clearly the type of decontamination method must be effective against the agent. For example, Bacillus atrophaeus spores are typically diluted in up to $40 \%$ ethanol, which is harmless to the spores (Uhm et al., 2007).

In the United States, decontamination agents are regulated by the Federal Insecticide, Fungicide, and Rodenticide Act (FIFRA). When the anthrax attacks occurred in 2001, there were no chemicals that had been registered for decontaminating Bacillus anthracis. For this reason, the government created a crisis exemption process to allow agent approval. During the responses, the EPA received 63 requests, approving 28. Each request included remediation action plans, sampling and analysis plans, and ambient air monitoring plans. The EPA granted crisis exemptions for 4 liquid anthrax sporicides to use on hard, nonporous surfaces only (aqueous chlorine dioxide, hydrogen peroxide/peracetic acid, sodium hypochlorite, and hydrogen peroxide/quarternary ammonium foam. Five gases were approved: gaseous chlorine dioxide and vaporized hydrogen peroxide (both for buildings), paraformaldehyde (for use on equipment in tented enclosures), methyl bromide (for field and laboratory studies), and ethylene oxide (for specialized off-site treatment of specific items). Additionally, four liquid sporicides were 
approved, which included aqueous chlorine dioxide, hydrogen peroxide/peracetic acid, sodium hypochlorite, and hydrogen peroxide/quarternary ammonium foam. These liquids were approved for use on hard, nonporous surfaces only (Kempter, 2005). Several of these different methods are covered more in-depth below.

Decontamination methods: Natural attenuation

One approach that could be used in some areas is natural attenuation. This could be used more for chemical agents, such as phosgene and lewisite agents because they rapidly decompose at a relative humidity levels above 70\%. Many biological toxins are also water soluble and hydrolyze under neutral to basic conditions. Weather patterns will impact this as well. Inversion weather conditions cause some agents (mainly chemical agents) to remain near the ground, reducing the rate of dispersion. Warmer ground-surface temperatures tend to increase the dissipation of liquid chemicals through the evaporation process. Various matrices (soils, concrete, and gasket materials) were contaminated outdoors with GD, VX, and HD, then sampled and analyzed as a function of time. Results showed that the chemical warfare agents GD and VX degrade or hydrolyze in 3 to 5 days to nonhazardous chemicals. Biological agents require specific environmental conditions to survive, thus environmental factors, such as available sunlight, temperature, relative humidity or rain, and the presence of atmospheric pollutants, should be considered. For example, Yersinia pestis can survive near freezing temperatures for months to years, but this agent is killed by several hours of exposure to sunlight. Ultraviolet light will naturally inactivate most biological agents. High-molecular-weight toxins are usually more sensitive to ultraviolet light, heat, and oxidation than are low-molecular-weight toxins (Department of the Army, 1990). However, Bacillus anthracis spores are so resistant to 
environmental conditions and can survive in their dormant state for years; therefore, this method is not practical in most situations.

Decontamination methods: VHP

Another relatively new method of large-scale decontamination is using hydrogen peroxide. There are two different delivery methods: vaporized hydrogen peroxide (VHP) when the compound remains in the vapor phase and hydrogen peroxide vapor (HPV) when a very small amount of condensation is induced deliberately (Gale et al., 2009). VHP is an option for decontamination because of hydrogen peroxide has a high efficacy and a low environmental impact; however, the very nature of hydrogen peroxide mandates extreme care-hydrogen peroxides a fairly strong oxidizing agent, with a pH of about 3 (Gale et al., 2009). Hydrogen peroxide decomposes to water and oxygen so residual contamination is not a concern (Herd, 2005; McVey, 2005). Because of the high decomposition rate, VHP requires repeated injections, preferably at different locations in the building (McVey, 2005). By nature, VHP is residue-free because the degradation products are only oxygen and water. A treated area can be reoccupied when the concentration there reaches a time-weighted average of 1 ppm (Herd, 2005).

Several past studies and real world decontamination work have evaluated Bacillus species decontamination using hydrogen peroxide. Oh et al. (2005) tested the efficacy of aerosolized peroxyacetic acid $\left(\mathrm{C}_{2} \mathrm{H}_{4} \mathrm{O}_{3}\right)$ and hydrogen peroxide $\left(\mathrm{H}_{2} \mathrm{O}_{2}\right)$ as disinfectants and found a 3.09-log reduction of Bacillus cereus cells. Andersen et al. (2006) applied a 5\% hydrogen peroxide dry fume disinfectant to Bacillus subtilis endospores for 30, 60, and 120 minute intervals. Their study found an $87 \%$ reduction in endospores after 120 minutes, while treatments at 30 and 60 minutes offered no reduction in endospore concentration. Other tests 
have evaluated the effect of the material on the decontaminating agent. Tests with VHP in a medium-scale HVAC system indicated that galvanized steel reduced the hydrogen peroxide concentration, whereas PVC had less of an effect. In another test, using 90 feet of galvanized steel ductwork with sensors located throughout, the hydrogen peroxide concentration decreased as a function of distance traveled along the ductwork, and VHP decreased with increasing temperature and decreasing flow rate (Carlsen, 2005). Also, Verce et al. (2008) found similar results in tests in a clean, room-scale galvanized steel (GS) and polyvinyl-coated steel air ducts to determine decontamination of larger systems. They found that VHP decreases along the length of the duct in the GS, thus reducing the concentration of available hydrogen peroxide. This suggested that the VHP decomposes faster in the GS, undergoing a surface-catalyzed heterogeneous decomposition. They did find oxidative damage was minimal after 100 experiments over one year, but there was a patina on the surfaces area where the VHP was introduced. VHP contact time of $100 \mathrm{mg} / \mathrm{L} \mathrm{H}_{2} \mathrm{O}_{2}$ (g) per minute was required for a 6-log reduction of $2.5 \times 10^{6}$ Geobacillus stearothermophilus spores used as indicators. The decomposition was reduced at lower temperatures and high flow rates, which led them to conclude a better process is to decontaminate GS separately as opposed to the entire building. The decreasing concentration of VHP along the length of the GS-duct is a strong indication that the peroxide undergoes a surface-catalyzed, heterogeneous decomposition as the VHP passes through GS ducting. The decomposition can be minimized by increasing temperature, flow rate, and initial concentration; however, lower decomposition will result in a slower killing of the biological (Verce et al., 2008). Additionally, studies have found that the reduction of organisms using hydrogen peroxide gas is impacted by the porous and nonporous nature of the surfaces (Rogers et al., 2005). VHP use has been around for some time, but on smaller scale uses such as 
in pharmaceutical companies and clean rooms (McVey, 2005). STERIS modified their technologies following the 9/11 and anthrax attacks so the VHP could be used to decontaminate larger buildings. Two buildings were fumigated following the anthrax attacks: the GSA Building 410, a 1.4 million- $\mathrm{ft}^{3}$ building used for office supply storage area and a mail-sorting facility for the White House. The contents were fumigated in place, with the building being separated into $200,000 \mathrm{ft}^{3}$ fumigation zones because no data for fumigation of a whole building were available. Each HVAC system was treated as separate zones, taking a total of three weeks. The second building was building SA-32, which was decontaminated using a simplified system based on information from the GSA building 410. This building was 1.5 million- $\mathrm{ft}^{3}$ and was also separated into 200,000- $\mathrm{ft}^{3}$ zones. All contents were removed from the building, and the total decontamination time was two weeks (McVey, 2005). These efforts have shown the real-world application of VHP technology.

Hydrogen peroxide has been tested on a C-141 cargo aircraft. The unit, made by STERIS, included a modified vaporous hydrogen peroxide unit, with ammonia as an activator. The system set-up took 2 days and the aircraft materials were exposed to hydrogen peroxide for 100 hours. The tests showed VHP did affect structural components, but there were no ill effects on the avionics. This has shown greater than 99.9\% kill rates for bare metal coupons (McVey, 2005).

Gale, et al. (2009) showed the material impacts of VHP on aluminum alloys and stainless steel. The testing involved exposing the specimens to vapor phase using VHP 1000ED decontamination unit (STERIS). The chamber concentration was $450 \mathrm{ppm}$ for 4 to 8 hours. The study found that overall the micro structural effects were relatively small and confined to a region near the exposed surface. The metals had a small but measurable weight loss when 
placed in liquid hydrogen. A single exposure cycle to VHP had negligible effects, but after 25 cycles of the vaporous hydrogen peroxide, the materials had weight gains indicating oxidation. Overall, the decontamination procedures had little effect on tensile properties and corrosion resistance. Exposure of VHP to the 2024 and 7075 aluminum alloys showed no measureable effect on subsequent corrosion behavior. Surface softening was slight and confined to the immediate vicinity of the surface; however, the conclusion made by the research is further work is needed on metal fatigue (Gale et al., 2009).

While VHP offers some advantages, the decontaminant must be used under conditions controlled very tightly and still may have detrimental material impacts. While the methods listed above meet the requirements for decontamination, they cannot be used on aircraft until further materials research is developed. Because of this, current AF research has focused on heat coupled with humidity for decontamination.

\section{Decontamination methods: Chlorine}

Another chemical used for decontamination is chlorine, which can be used in several different types-including chlorine dioxide $\left(\mathrm{ClO}_{2}\right)$ and sodium hypochlorite $(\mathrm{NaOCl})$. Several studies have been completed on the effectiveness of chlorine. Perez et al. (2005) found that liquid disinfectants on hard surfaces were effective to reduce organism load (including Bacillus subtilis) although chlorine dioxide did take longer. Wagner et al. (2008) conducted a study evaluating the inactivation rates of deposited Bacillus subtilis on untreated gypsum board, similar to what would be found in interior spaces. The treatments used included aerosolized solutions of distilled water, $0.05 \%$ chlorine dioxide $\left(\mathrm{ClO}_{2}\right)$, and $0.6 \%$ sodium hypochlorite $(\mathrm{NaOCl})$. The endospores were inoculated onto commercially available gypsum board with 
paper facing. Commercial bleach (sodium hypochlorite, $\mathrm{NaOCl}$ ) and aqueous chlorine dioxide were chosen as the chemical challenges. Sodium hypochlorite (Clorox Co.) was diluted to $10 \%$ using media grade water resulting in a total sodium hypochlorite concentration of $0.6 \%$. The chlorine dioxide used was a commercially available product available at $0.05 \%$ chlorine dioxide (500 ppm) aqueous solution (Biocide, Inc., Stamford, CT). The distilled water had a negative kill rate and the chlorine dioxide had no effective kill rate (thus there was no antimicrobial effect). This could have been from the reaction with the gypsum board, thus reducing the oxidizing potential of the chlorine dioxide. Finally, the sodium hypochlorite solution had 1.55 to $1.92 \log$ kill rates, which was the highest antimicrobial properties (Wagner et al., 2008).

Chlorine dioxide gas was used in the cleanup of building interiors contaminated with Bacillus anthracis spores in 2001 (Barth et al., 2003; Rastogi et al., 2009). Aqueous $\mathrm{ClO}_{2}$ was also used on used on nonporous surfaces in two mail sorting machines (Canter et al., 2005). The Trenton mail facility, contaminated in 2001, did not have fumigation completed until October 2003 and restoration activities began in February 2004. The HVAC system continued to run even after the building was closed; however, some of the components failed so the building temperatures reached $100^{\circ} \mathrm{F}$. The recommendations were to keep the environmental controls working by sending workers in the scene with PPE if needed. Recommendations have been made to use bleach sparingly because it is highly damaging to many materials (Orlusky, 2005).

Decontamination methods: UV

Ultraviolet radiation, especially in the maximum germicidal $254 \mathrm{~nm}$ range, has been recommended to inactivate a variety of infectious organisms (CDC, 1994). Studies have been completed to determine the inactivation rates by UV radiation on aerosolized Serratia 
marcescens, Escherichia coli, Mycobacterium bovis and Mycobacterium parafortuitum, spores of Bacillus subtilis, and the fungus Penicillium citrinum. These studies found that the UVC required to inactivate these bioaerosols is related to sufficient dose of radiation over time, the ability of different microbial species to recover UV radiation-induced damage, and the levels of relative humidity (Ko et al., 2002; Lai et al., 2004; Lin and Li, 2002; Riley and Kaufman, 1972). Previous research suggests these bioaerosols survive better, with or without UV exposure, at relative humidity levels above 50\% (Cox, 1971; Cox and Goldberg, 1972; Lin and Li, 2002; Marthi et al., 1990; Riley and Kaufman, 1972; Peccia and Hernandez, 2004); however, other studies did not identify an effect of RH on the survival of airborne bacteria (Ko et al., 2002) so the data is contradictory for $\mathrm{RH}$.

The data for the effect of UV on aerosolized bacteria are available for only a limited number of species and is less for human pathogens, especially for potential biological agents. Though there is some data for surface decontamination of aerosols, there is little data which can be applied to decontamination of aerosol deposition using UV. One potential problem with UV is that the light waves must make contact with the actual organism, so any organic matter present provides protection (King et al., 2011).

Decontamination methods: Methyl bromide

Another disinfectant is methyl bromide, used for over 60 years for termite control and fumigating ships importing produce to kill bacteria which may be present. Because methyl bromide diffuses rapidly and is very stable, clearing any building is required. Methyl bromide is relatively cheap (approximately $\$ 150$ per $1,000 \mathrm{ft}^{3}$ ), has a rapid turnover to completion time (approximately 200 hours), and treats all porous materials, voids, and HVAC system. The 
chemical can be applied at any humidity and there is no damage to other materials. One major disadvantage is that methyl bromide depletes stratospheric ozone (Scheffrahn, 2005).

Tests using methyl bromide to fumigate Bacillus anthracis were completed in a 30,000

$\mathrm{ft}^{3}$ home which was tented as commonly done for termite treatments. Gaseous methyl bromide was generated by passing the liquid through a heat exchanger. Because there is better inactivation efficiency at higher temperatures, fans and heaters were used to maintain a target temperature of $35^{\circ} \mathrm{C}$ within the house. The test showed that after 48 hours at $37^{\circ} \mathrm{C}$, complete kill was observed for Bacillus anthracis and Geobacillus stearothermophilus; however, Bacillus atrophaeus and Bacillus thuringiensis experienced only partial kills. After fumigation for two days, essentially all 50 spore strips placed throughout the house indicated no growth at 48 of 50 spore strip locations, no growth was observed. The two positive locations were reported to be in a refrigerator and at an improperly mounted spore strip location. No damage to electronic equipment was observed (Scheffrahn, 2005).

Decontamination methods: Others

Yet another type being used is ozone and acidic electrolyzed water or electrolyzed ozone water. When used, this inactivates most of the organisms within three minutes. This fast inactivation is because of the synergistic effects of the ozone and acidic water, providing a kill rate up to $99.98 \%$ on viruses (except the CoxB3 virus). The method also kills Bacillus atrophaeus spores within three minutes because the outer surface of the spore appears to be vulnerable to oxygen radicals from the electrolyzed ozone water. Following the action, the only byproducts are water and oxygen with no trace of harmful materials. The electrolyzed ozone can 
be formulated promptly and in large amounts while being applied in a mist or fog. It is also environmental friendly because it decomposes into water and oxygen (Uhm et al., 2007).

One other chemical used for decontamination is paraformaldehyde. This was used to decontaminate mail sorting machines. Paraformaldehyde has been used in the past to decontaminate biosafety cabinets (Canter et al., 2005).

Decontamination methods: High heat and humidity

Another method used for decontamination for many years is heat and humidity. It has long been known that most spores have a greater resistance to dry heat than moist heat (Perkins, 1983); however, the effect of relative humidity on decontamination is not fully understood, even after four decades of study (Peccia et al., 2001). Perkins states that dry heat (60 minutes at $\left.320^{\circ} \mathrm{F}\right)$ has the same effect as moist heat $\left(15 \mathrm{~min}\right.$ at $250^{\circ} \mathrm{F}$ in moist heat) for sterilization purposes (Perkins, 1983). However, high relative humidity is generally accepted to be optimal for the stability and survival of aerosols generated from liquid bacterial suspensions (Peccia et al., 2001). Still, other studies did not identify an effect of relative humidity on the survival of airborne bacteria (Ko et al., 2002). Clearly further research is required to determine the optimal levels of heat and humidity.

AFRL has helped develop vaporous hydrogen peroxide; however, because VHP must be used under tightly controlled conditions and may have undesirable material effects, AFRL has also evaluated additional decontamination methods including heat and humidity. AFRL has conducted laboratory and field tests on a Large Frame Aircraft (LFA) to determine the feasibility of using high temperatures and relative humidity to inactivate known biological organism threats. Heated air for decontamination offers the advantages of being benign as long as all 
components are compatible with $180^{\circ} \mathrm{F}\left(82.2^{\circ} \mathrm{C}\right)$ storage and the technology exists for this method, therefore efforts to field such a unit would be minimal (AFRL, 2008).

Past studies showed injecting hot air acts as accelerated weathering. A key component is that the heat must be applied evenly. Additional items to consider include the material compatibility, treatment volume, and air distribution (Brickhouse, 2005).

High heat was tested against ricin after this was found in a US Senator's office on February 2, 2004. During the course of the incident, the EPA collected 670 samples from three affected rooms and identified 19 positive results, all of which were from one room. All items were removed from the room which could be removed. The items which could not be removed were decontaminated with sodium hypochlorite solution, which was found to be effective as well. Clothing and office materials were heat treated. The EPA tested heat treatment using both crude and pure ricin, with the temperature of 82 to $88^{\circ} \mathrm{C}$. The pure ricin was inactivated up to $100 \%$ and the crude ricin was found to be inactivated from 94.4 to $99.7 \%$, until the treatment was increased in time. After 4 days, 99.8 to $99.99 \%$ of the ricin was inactivated and all the crude ricin was inactivated up to $99.99 \%$ after three weeks. The purified ricin was $100 \%$ inactivated after only 4 days, but was 99.92 to $99.99 \%$ after three weeks. The EPA never determined why this was the case, but believed these differences could have been due to protein refolding. If the heat and humidity method did not work, the EPA was prepared to use ethylene oxide (Kelly, 2005).

\section{Decontamination studies on aircraft}

Several studies have been conducted on grounded aircraft to determine if this technology is feasible in this environment. These studies conducted by AFRL have used the simulant 
Bacillus thuringiensis var kurstaki (BtK or Bt). Phase I of these studies was completed in a laboratory to determine the feasibility of high temperature and high humidity decontamination. Test temperatures were limited to $5^{\circ} \mathrm{F}$ below the maximum high temperature storage limitations for USAF aircraft (which is approximately $185^{\circ} \mathrm{F}$ ). A 5 to 6 - $\log$ reduction was achieved for initial spore contaminations of $10^{6}$ per test coupon for test temperatures of $180^{\circ} \mathrm{F}, 170^{\circ} \mathrm{F}, 160^{\circ} \mathrm{F}$ and relative humidity of 75 to $90 \%$, indicating a near kill or neutralization of all contamination. Phase II of the study was completed on an intact DC-9, owned by AeroClave, LLC, of Orlando, FL. Test results using BtK confirmed the Phase I results with a 5- to 6-log reduction for each of the three trials on aluminum coupons placed in three areas of the aircraft, including the passenger compartment, flight deck, and near the environmental control system (ECS). If viable, this process could use off-the-shelf equipment to control both temperature and humidity over long periods of time. The AFRL study recommends that the next steps include testing the bio-thermal decontamination of other materials within the aircraft, including cloth and plastics. Thus far, all testing has been on aluminum coupons with a dry preparation of the biological agent (AFRL, 2008).

Tests have been completed on other aircraft, in attempts to up-scale the methods used on the DC-9 study. One test was done using VHP alone and VHP coupled with thermal decontamination on the main cargo bay of a wide-body aircraft (Boeing 747). These were found to be sporicidal at several locations in the cabin; however, there were several areas where a 6-log of Geobacillus stearothermophilus reduction could not be achieved, such as locations the VHP could not reach. Additionally, there were "hot-spots" of peroxide, but no way to identify exactly where. The required concentration for spore inactivation was found to be 250 parts per million (ppm) of hydrogen peroxide for 2 hours. The study concluded there was a good kill of the spores 
in specific areas; however, the aircraft did not have absorbent surfaces which are typically found on aircraft and this could have decreased the inactivation rate. The researchers were able to maintain the VHP between 125 to 200 ppm for 2 hours and there was not condensation in the main cabin when the levels were maintained at $175 \mathrm{ppm}$. There was some condensation in the return air systems of the aircraft. They also found they could heat the aircraft to these temperatures, but maintaining the temperature and humidity levels at these levels for 2 hours on a wide-body aircraft was difficult. Steam generators were required to maintain the humidity levels, but even with these, humidity was still only maintained at $30 \%$ (Gale et al., 2008).

There are several methods which can be used for biological decontamination. Of all the methods described, only high heat and humidity meet the Air Force aircraft engineering specifications. Additionally, to date, these studies have not been completed on aerosolized spores, but rather spores placed on coupons with the liquid allowed to dry. As previously

described, the real-world spores will likely be attached to an aerosol particle. Spores with fumed silica will more accurately depict the real-world spore requirements. This will not only affect their dispersal, but likely also their survival. Perkins states that when spores are suspended in oily materials, their heat resistance markedly increases (Perkins, 1983). Thus material surrounding the spore can help protect or insulate it and should be part of future studies.

\section{Bioaerosol generation methods}

\section{Aerosol test chambers}

The literature shows many different designs for test chambers used to aerosolize particles. These test chambers have been constructed from different materials, including Plexiglas $^{\mathrm{TM}}$, stainless steel, and aluminum. Several chambers have been constructed from 
aluminum, which has a significant advantage in that it decreases static electricity on the particles being generated.

One chamber used to deposit bioaerosols was designed and build by the Centers for Disease Control (CDC) and Dugway Proving Ground and designed to uniformly deposit spore particles on surfaces (Baron et al., 2008). Used for several different experiments (Baron et al., 2007; Baron et al., 2008; Estill et al,. 2009), this chamber was constructed from Plexiglas ${ }^{\mathrm{TM}}$ with dimensions of 1.22 meters by 1.22 meters by 2.44 meters. The chamber was used to target Bacillus anthracis spore (mixed with fumed silica) concentrations from 3 to 200 colony forming units (CFU) per $100 \mathrm{~cm}^{2}$ for steel and carpet coupon sampling. Once constructed, the chamber seal was tested with smoke particles. Prior to aerosol generation, the chamber was evacuated for at least 20 minutes using a vacuum pump. Spores were introduced into the chamber through an ion air cannon static eliminator using ultra high purity nitrogen at a flow rate of 150 liters per minute for about 5 minutes, with neutralization occurring before the aerosol entered the chamber. Brushless electronic fans were used to stir the aerosol in 1 minute consecutive intervals, with each fan activated for as short of time as possible. These were provided to mix the aerosols while keeping the air velocity at the sampling surface at minimum to reduce impaction and resuspension of the spores. Operation of the fans was kept to a minimum to ensure settling was the primary deposition mechanism. Once injected, calculations showed $99 \%$ of the particles had settled within 10 hours. The team used agar plates to measure the amount of spores settling. They found with using these agar plates to help determine deposition rates and correlated this to (Baron et al., 2008).

Kesavan et al. (2008) constructed a 64 cubic meter chamber, including temperature and humidity control via computer, used to compare different bioaerosol concentrators. Aerosol 
generation was completed a 24 -jet Collison nebulizer and a 10 milliCurie $\mathrm{Kr}-85$ source used for neutralization. The study found that a uniform aerosol concentration was produced after approximately 45 seconds. The tested deposition with aluminum oxide particles, monodisperse polystyrene spheres, and oleic acid droplets; however, they ultimately concluded that biological particle tests are needed due to the additional complications they add to the tests.

Farnsworth et al. (2006) developed a closed-loop wind tunnel as a test chamber to determine recovery efficiencies from HVAC systems. The system was constructed from stainless steel, $61 \mathrm{~cm}$ by $61 \mathrm{~cm}$. Aerosol injection made of $\mathrm{KCl}$ salt particles was completed using a Collison nebulizer. Buttner and Stetzenbach (1993) designed a room-sized chamber (4 meters by 4 meters by 2.2 meters) in which dry deposition of Penicillium chrysogenum spores were completed through an acoustically fluidized bed through air supply registers. They used an Aerodynamic Particle Sizer (TSI, Inc., Shoreview, MN) to measure the spores in concentrations of the size ranges of $1.8 \mu \mathrm{m}$ to $3.5 \mu \mathrm{m}$ in airborne concentrations of about $1,000 \mathrm{~m}^{3}$. The same chamber was used later to settle Bacillus atrophaeus subsp. globigii (BG) spores onto flooring materials (Buttner et al., 2004).

Brown et al., aerosolized BG spores into a chamber and produced surface concentrations in the range of $10^{2}$ to $10^{5}$ colony forming units per square centimeter for the purposes of swipe sampling (Brown et al., 2007a, 2007b, 2007c). The chamber included a cylinder mixing chamber, constructed from carbon steel with enamel-coated surface, and a diameter of $45 \mathrm{~cm}$, a height of $30 \mathrm{~cm}$, and a total volume of $0.048 \mathrm{~m}^{3}$. The aerosol was fed from the mixing chamber to the deposition chamber through valved feed through port at the top of the chamber. The aerosol was then fed into a cubic deposition chamber with dimensions of $90 \mathrm{~cm}$ on each side, with an interior volume of $0.73 \mathrm{~m}^{3}$ (Brown et al., 2007b). Feather and Chen (2003) constructed a 
compact and low cost chamber, developed for evaluating personal samples in a calm-air environment. The chamber, made from aluminum in a 28 centimeter diameter and 68 centimeter long tube, used fluorescein particles to test the dispersal. They found the chamber worked well for particles less than $6 \mu \mathrm{m}$ adequately, thus resembling larger test chambers up to $1 \mathrm{~m}^{3}$.

Edmonds et al. (2009) developed a circular deposition chamber, with three separate zones. The chamber used ionizing fans to decrease static charges and to continually mix the air during spore aerosolization. The chamber included a rotating base platform to ensure no single point in the chamber was exposed for an extended period of time. The analysis of the chamber showed that spore concentrations were even throughout the zones and more closely resembles real-world encounters.

Byrne et al. (1995) developed a test chamber to measure deposition on different surface types. Constructed as an aluminum cube with sides 2 meters each, it was used to determine deposition velocities for 3 rough vertical surfaces (wallpaper, short-pile caret, and Astroturf) using $4.5 \mu \mathrm{m}$ particles. They found there was greater deposition velocities measured for the rougher surfaces (wallpaper, carpet, Astroturf) than for smoother aluminum surfaces. Lai et al. (2002) developed a test chamber facility with a volume of $8 \mathrm{~m}^{3}$ to study particle deposition under well-stirred conditions using monodisperse particles in the size ranges of 0.7 to $5.4 \mathrm{um}$. The chamber was aluminum with 2 meter sides. The walls of the chamber were insulated with 2.5 $\mathrm{cm}$ think expanded polystyrene foam sheets. The particle generation took five minutes and the aerosol was neutralized with a $0.4 \mathrm{MBq}$ Americium-241 radiation source. The chamber was used to investigate aerosol deposition on smooth surfaces and regular arrays of 3D roughness elements, using three different airflow speeds. Four rough and five smooth samples were used. 
The research found that under the lowest airflow condition and smallest particle size, deposition onto rough samples was less than the smooth surfaces.

King et al. (2011) developed two test chambers to study UV exposures on bioaerosols. The chambers, made of $1 / 4$ " Plexiglas ${ }^{\circledR}$, had an internal volume of $0.137 \mathrm{~m}^{3}$ and measured 0.91 $\mathrm{m}$ long, $0.43 \mathrm{~m}$ tall, and $0.35 \mathrm{~m}$ deep. The vegetative cells were aerosolized into an exposure chamber and then exposed for various lengths of time to a $254 \mathrm{~nm} \mathrm{UV} \mathrm{light} \mathrm{source.} \mathrm{The} \mathrm{aerosols}$ were collected onto gelatin filters, which were dissolved, diluted, plated, and incubated to enumerate colony formation. Ten-fold dilutions of the bacterial suspensions were completed with $100 \mu \mathrm{L}$ of suspension containing the specific species of bacteria under study was spread onto nutrient agar in plastic Petri dishes. These dishes were placed on chamber floor below the UV lamp housing, which faced downward and was centered $118 \mathrm{~cm}$ over the dishes. Four trials were conducted with bacteria, with the required amount of UV energy required for inactivation being calculated.

There have been several tests completed using rotating bioaerosol test chambers which allowed aerosol evaluation longer. Goldberg et al. (1958) used a $0.61 \mathrm{~m}$ long by $1.83 \mathrm{~m}$ diameter (volume of $1.6 \mathrm{~m}^{3}$ ) rotating reactor to maintain constant airborne pathogen concentrations for animal exposure test. Several additional reactors like this were constructed and then later called the "Goldberg Drum", which were used in several other studies to study airborne microorganisms, microbial survival based on environmental effects, or to maintain bioaerosol concentrations for animal exposure studies (Krumins et al., 2008). Krumins et al. (2008) designed a rotating chamber to create an ambient air active ecosystem where the bacteria retain their activity and the air is not just a medium for transport. This allowed the researchers to keep the particles suspended for prolonged periods of time so their activity could be measured. 
The challenge is the keep the bioaerosol suspended so it will not settle under the influence of gravity. The reactors were sufficiently sealed to limit ethane leakage to less than $5 \%$ per day. They set the drum to rotate at $1.3 \mathrm{rpm}$, resulting in a $1.0 \%$ loss of $1 \mu \mathrm{m}$ particles per day, for a half-life of 54 days.

Another consideration for these test chambers is the decontamination, which is especially important if high threat organisms are used. The test chamber designed by Baron et al. was decontaminated using VHP at a concentration greater than 1,000 ppm for 90 minutes (model VHP1000; Steris Corp., Mentor, OH). This was required because Bacillus anthracis strain Sterne spores (a BSL 3, virulent form of anthrax) were used. They found that VHP could be released for an extended period of time because it absorbed onto the Plexiglas $®$ surfaces in the sample holders they used and then out gassed, for up to 23 hours. The concentration remained at 3 ppm for several days later, which was high enough to inactivate the spores during subsequent tests. To alleviate this, they used space heaters in the chamber to facilitate high temperature degassing (temperature was approximately $43^{\circ} \mathrm{C}$ ) for 3 days (Baron, et al., 2007b; Estill and Deye, 2010). This limited the test runs that could be done to just one per week. Because of this phenomenon, the researchers recommended a material other than Plexiglas ${ }^{\mathrm{TM}}$ be used for these test chambers (Baron et al., 2007).

\section{Aerosol generation methods}

Aerosols can be generated using either wet or dry techniques. Wet techniques work by converting a liquid which contains the biological test material to small droplets. This is referred to as aerosolization, nebulization, atomization, or spraying. Air blast nebulization is a wet technique that uses compressed air to draw liquid from a reservoir, with the high velocity of the 
air breaking the liquids into droplets which are suspended as part of the aerosol (Fitch, 2008). This method produces a volume median diameter of 1-10 $\mu \mathrm{m}$ and geometric standard deviation of 1.4 to 2.5 (Cheng and Chen, 2001). Examples of these are the Collison (BGI, Waltham, MA) and Hudson nebulizers (CAN Medical, Rockwell, TX), which generate particles in the size ranges from 0.1 to $5 \mu \mathrm{m}$. This depends on the amount of test material in the aerosol suspension as well as operational conditions. Neither of these nebulizers can produce large droplets or large amounts of aerosols. Agricultural sprayers (Micro Spray Ltd., Bromyar, UK) have been used successfully to produce biological simulants in tests required high dissemination rates. Some can be vehicle mounted. These larger nebulizers use a rotary atomization technology for droplet generation. Finally, ultrasonic nebulizers use mechanical energy to atomize liquid into droplets using a vibrating piezoelectric crystal driven by a variable-frequency electrical oscillator. These nebulizers do not a pressurized air source. Ultrasonic nebulizers do not have issues with evaporating effects and different particle sizes can be made by adjusting the concentration of the suspended material in the liquid and also the nozzle size, frequency of the driving crystal, and the liquid feed rate (Fitch. 2008).

The other major delivery technique is the dry technique. This method uses a mechanism to store, transport, and deliver a test material in powder form. This method may use a gravity feed (hopper), conveyor belt, screw feed, rotating disks, brushes, or compressed cylindrical packs. The delivery rate is adjustable and is key factor in the aerosol generation. Two major factors influence the stability of dry powder dissemination - feed mechanism and flow property of the powder. The resulting aerosol size distributions depend critically on the material properties of the power itself as well as the generation method. Several different dry dissemination methods are listed in the Table 1-4 (Fitch et al., 2008). 
Table 1 - 4 - Dry bioaerosol dissemination methods

\begin{tabular}{|l|l|c|c|}
\hline \multicolumn{1}{|c|}{ Product } & \multicolumn{1}{c|}{$\begin{array}{c}\text { Feeding } \\
\text { Mechanism }\end{array}$} & $\begin{array}{c}\text { Particle size } \\
\text { (um) }\end{array}$ & $\begin{array}{c}\text { Generation rate } \\
\text { (mg/min) }\end{array}$ \\
\hline Wright Dust Feed & $\begin{array}{l}\text { Scraping the packed } \\
\text { plug }\end{array}$ & $1-100$ & $0.1-1000$ \\
\hline Fluidized Bed & Chain conveyor & $1-100$ & $0.5-10$ \\
\hline $\begin{array}{l}\text { Small Scale } \\
\text { Powder Disperser }\end{array}$ & Rotating disk & $0.5-50$ & $0.05-2.0$ \\
\hline $\begin{array}{l}\text { Jet-O- } \\
\text { Mizer/Screw Feed }\end{array}$ & Screw feed & $2-50$ & $0.1-3$ \\
\hline RBG 1000 & Rotating brush & $0.1-100$ & $0.5-9000$ \\
\hline GRIMM 7840 & Belt conveyor & $<200$ & $15-9000$ \\
\hline $\begin{array}{l}\text { Vilnius Aerosol } \\
\text { Generator }\end{array}$ & $\begin{array}{l}\text { Rotating/Vibrating } \\
\text { Turbine }\end{array}$ & $1-50$ & --- \\
\hline
\end{tabular}

Collison nebulizers are widely used for aerosolization of liquid supplies. These nebulizers, first described in 1973 by May, are used generate small-particle aerosols with mass median aerodynamic diameter (MMAD) of 1 to $3 \mu \mathrm{m}$. They work by generating a fine mist through the compression of air (May, 1973). The literature has many examples of how Collison nebulizers were used to deliver a bioaerosol, especially Bacillus subtilis spores. For instance, Wagner et al. (2008) used a 6-jet Collison nebulizer (BGI, Inc, Waltham, MA) to aerosolize Bacillus subtilis spores to test the effectiveness of decontamination of gypsum boards using $0.05 \%$ chlorine dioxide $\left(\mathrm{ClO}_{2}\right), 0.6 \%$ sodium hypochlorite $(\mathrm{NaOCl})$, and distilled water. They reported a solution delivery of $10 \mathrm{~mL}$ per hour and a mass median diameter of $2 \mu \mathrm{m}$. Another study, conducted by Han et al. used a 6-jet Collison nebulizer to aerosolize the vegetative form of Bacillus subtilis. The nebulizer operated at a flow rate of $4 \mathrm{~L} / \mathrm{min}$ with HEPA-filtered dilution air delivered at $36 \mathrm{~L} / \mathrm{min}$ (Han et al., 2010). Krumins et al. (2008) used a Collison nebulizer to aerosolize a test aerosol with $5 \mathrm{~L} / \mathrm{min}$ of filtered nitrogen. The outflow from the nebulizer was mixed with $45 \mathrm{~L} / \mathrm{min}$ of ambient air which was filter-sterilized through a $45 \mu \mathrm{m}$ filter to dry any liquid water that could have resulted in particle agglomeration. Another study involved generating several different types of bioaeroaols using a 24-jet Collison nebulizer. The 
bioaerosols generated were $50 \mathrm{ml}$ of Bacillus atrophaeus cells in PBS $\left(10^{9}\right.$ cells $\left./ \mathrm{ml}\right)$. The agents were introduced through a $1 \frac{1 / 4}{4}$ diameter port through the outlet of the nebulizer. The nebulization was completed by with 20 psi air through the nebulizer for 10 minutes. The bioaerosols were used to test different air sampling techniques (Kesavan et al., 2008). Yah and Mainelis used a Collison nebulizer to aerosolize microorganisms to test portable microbial samplers. The microorganisms were suspended in water and then aerosolized from the nebulizer which was operated at a flow rate from 2 to $10 \mathrm{~L}$ per minute. These aerosols were then neutralized using a Po-120 bipolar charger (Mainelis, 2007). It should be noted that a Collison nebulizer can have deleterious impacts on sensitive microorganisms. This is because the fluids are recirculated every 6 seconds (May, 1973), subjecting them to shear stress. This increases the amount of injury and thus their viability decreases (Mainelis and Tabayoyong, 2010) and has been demonstrated in several studies with the sensitive bacteria, in the vegetative form (Mainelis et al., 2005; Reponen et al., 1997). There are other methods to generate bioaerosols. Baron et al. used a Small Scale Powder Disperser (SSPD, Model 3433, TSI, Inc.), a generation device which uses a venturi aspirator. The researchers placed an impactor between the aspirator and the mixing chamber to remove particles larger than approximately $5 \mu \mathrm{m}$ aerodynamic diameter (Model 266, Sierra Instruments). The aerosol used had a flow-enhancing powder, which appeared to affect the spore's ability to grow on agar medium. They found that spore agglomerates present were re-suspended by the sampling activities; this increased the variability of deposited particles (Baron et al., 2008). Brown et al. used a TSI 3400A fluidized bed aerosol generator (TSI Inc., Minneapolis, MN) to generate a bioaerosol. The aerosols were injected and mixed with the circulating fans for 15 minutes followed by a settling time of 24 hours. The surface loading goals were in the ranges of 100 to 1,000 and 10,000 to $100,000 \mathrm{CFU} / \mathrm{cm}^{2}$. 
(Brown et al., 2007b ; Brown et al., 2007a). Lewandowski et al. completed a study to evaluate Bacillus atrophaeus on vertical, horizontal top, and horizontal bottom surfaces and the how those could be recovered from glass and stainless steel with polyester swab and macrofoam sponge wipe. The researchers aerosolized Bacillus atrophaeus spores and Pantoea agglomerans to test foam spatulas and polyester swabs to remove the spores. The bioaerosols were aerosolized with a compressed air nebulizer Monsun 2 MP2 equipped with a RF6 head (Medbryt, Warsaw, Poland), which was $65 \mathrm{~cm}$ above the test surface. The study generated $10 \mathrm{~mL}$ samples of Bacillus atrophaeus spore suspension (concentration of $1 \times 10^{8}$ to $2 \times 10^{8} \mathrm{CFU} / \mathrm{ml}$ ) in SDW or a Pantoea agglomerans suspension in 0.01 M phosphate-buffered saline (PBS), pH 7.4, were aerosolized at $46.4 \mathrm{lb} / \mathrm{in} 2$ pressure with an airflow rate of 15.5 liters/min, and resulted in a liquid generation rate of $0.48 \mathrm{ml} / \mathrm{min}$. There was an overall low recovery for vegetative cell capture which may in part be due to stress of desiccation or damage by aerosolization (Lewandowski et al., 2010). Carrera et al. generated Bacillus globigii spores by using small, pressurized metereddose inhalers generators. These metered dose inhalers were filled with $5 \mathrm{ml}$ spores of Bacillus globigii 0.05\% using Dymel 134a as propellant (DuPont, Wilmington, DE 19898) (Carrera et al., 2005). Feather and Chen (2003) used two different aerosol generators to produce homogenously distributed monodisperse aerosols. The first method was a Venturi feeder (In-Tox Products Albuquerque, NM), which can generate powders $6 \mu \mathrm{m}$ and larger. The generation was controlled through a computer solenoid valve, creating a pulsating flow in airstream. The second method used a medical nebulizer (Hospitak Inc, Cat. No. 952, Farmingdale, NY), which can generate particles smaller than $6 \mu \mathrm{m}$. This method requires a diffusion dryer with desiccant installed to remove water droplets in the aerosol. Edmonds et al. (2009) also used an Aeroneb Go 7070 micropump nebulizer (Active Forever, Scottsdale, AZ) to generate $1 \mathrm{ml}$ of $10^{10}$ stock solution 
(Edmonds et al., 2009). Kesavan et al. (2008) used an ink-jet aerosol generator (TSI, Inc) to aerosolize Bacillus atropheus spores to test several different bioaerosol samplers. The Ink Jet Aerosol Generator (IJAG; TSI Inc., Shoreview) was filled with a suspension of Bacillus atropheus in water. During operation, droplets generated from the cartridge are passed through a heated drying tube, where the water was evaporated leaving only a cluster of Bacillus atropheus spores. The IJAG has a maximum aerosol output rate of approximately 1000 particles per second, which is lower than other devices.

Another aerosol generator is the flow-focusing aerosol generator (FFAG), which is used to generate particles larger than $10 \mu \mathrm{m}$. This instrument relies on the formation of a stable microjet which disintegrates at a defined distance from a critical orifice. Thomas et al. (2008) used the FFAG, operated at a pressure of 16 psi and dilution air at $50 \mathrm{l} / \mathrm{min}$, to characterize 12 $\mu \mathrm{m}$ particles. These larger particles were compared to smaller-particles generated by a 3-jet Collison nebulizer operated at a pressure of $26 \mathrm{psi}$. The aerosol generated by the FFAG demonstrated an MMAD of 12.63 um, with a particle distribution ranging from 8 to $20 \mu \mathrm{m}$, while the Collison nebulizer produces smaller aerosols, ranging from 1 to $3 \mu \mathrm{m}$. The FFAG generated fewer 1 to $3 \mu \mathrm{m}$ particles than Collison nebulizer. The researchers found there were more entrapped particulates (15.9- to 19.2-fold) incorporated into 9- to 17 - $\mu \mathrm{m}$ particles generated by the FFAG than by the Collison nebulizer. They tested culturability of both generation methods and found the $E$. coli cells aerosolized using the FFAG survived better those made by Collison nebulizer (Thomas et al., 2008).

Yet another method to generate particles larger than $10 \mu \mathrm{m}$ is with a spinning-top aerosol generator (STAG) connected to a drying column (Druett et al., 1953; Druett et al., 1956a; Druett et al., 1956b; Druett and May, 1952; Harper et al., 1953; Roy et al., 2003). This device has been 
used to deposit large particles containing ricin into murine upper respiratory tracts. The distribution for this was bimodal, with peaks at 5 and $12 \mu \mathrm{m}$ (Roy et al., 2003).

To alleviate the particle charges accrued during nebulization, some type of neutralization is usually required. There are different methods to neutralize an aerosol. The aerosol can be passed through a 2-mCi Po-210 neutralizer, as was done by Han et al. (2010) for vegetative Bacillus subtilis and Bacillus subtilis spores by Yah and Mainelis (2007). Another commercially available neutralizer is a Kr-85 bipolar ion source (TSI, Inc.). This was used by Feather and Chen (2003) to attempt to neutralize an aerosol. They found some problems with this because of re-aerosolization of particles which were deposited within the $\mathrm{Kr}-85$ source. Several attempts to free these particles were unsuccessful, which eventually led to generation of undesirable sizes and thus error. This was pronounced with particles greater than $6 \mu \mathrm{m}$. The source was then removed, the chamber was grounded, and conductive samplers were used to eliminate electrostatic fields in the chamber; therefore, the aerosol was introduced without neutralization. Kesavan et al. used a $10 \mathrm{mCi} \mathrm{Kr}-85$ source (TSI, Inc.) to neutralize particles generated through a 24-jet Collison (Kesavan et al., 2008). The system designed by Baron et al. had several different methods to neutralize the aerosols. These included an Ion Cannon static eliminator (Exair, Inc., Cincinnati, $\mathrm{OH}$ ) which was used to neutralize the dilution air. A second static eliminator (Exair, Inc.) was situated on the settling chamber wall near the HEPA filter. This provided additional neutralization of the aerosol during the pump-down and settling phases (Baron et al., 2008).

An important consideration is the survival of the organism being aerosolized. The survival of bacteria during the initial process of aerosolization in the apparatus will influence survival during extended aerosolization and thus infection upon deposition in the respiratory tract. Initially, mechanical and shear stresses are imparted by the actual act of aerosol generation 
within the device (Thomas et al., 2008). After this, bacteria are rapidly inactivated due to several environmental stresses including desiccation and UV radiation (Lighthart, 1997; Tong and Lighthart, 1997; Tong and Lighthart, 1998). The survival of bacteria has been related to particle size, with survival better in $7 \mu \mathrm{m}$ particles than within $1.1 \mu \mathrm{m}$ particles This phenomenon is likely due to larger particles containing higher numbers of bacteria in aggregates, enabling a fraction to survive deleterious stresses encountered during aerosolization because the outer layer of bacteria effectively is sacrificial, enabling the bacteria within the core of the aggregate to survive. This was seen in the study completed by Thomas, et al (2002).

\section{Microbiology methods}

Bioaerosol simulants are often used as safe alternatives for research. The following organisms are used to simulant different types of microorganisms: Bacillus subtilis var niger (also known as Bacillus globigii, or BG) (simulant for Bacillus anthracis); Erwinia herbicola (simulant for vegetative bacteria); MS2 bacteriophage (simulant for viruses); and Ovalbumin (simulant for toxins) (Fitch, 2008). The stimulants listed are considered Biosafety Level 1 organisms by the $\mathrm{CDC}$ and do not require engineering and safety protocols as rigorous as higher levels. To be classified a level 1 organism, the agent has to be well-characterized and not known to cause disease in normal, healthy humans, thus they present minimal hazards to laboratory personnel and the environment (Chosewood and Wilson, 2009). Additionally, the NIH classifies infectious microorganisms by risk groups. Risk Group 1 are "agents not associated with disease in healthy adult humans" (NIH, 2011) or "unlikely to cause human or animal disease" presenting no individual and community risk" (WHO, 2004). Laboratories using BSL-1 do not have to be separated from the general traffic patterns in buildings and work can be 
conducted on open bench tops using standard microbiological practices. Special containment equipment or facility design is not required, but may be used as determined by appropriate risk assessment (NIH, 2011).

One of the most used surrogates is Bacillus subtilis, used in place of Bacillus anthracis. Bacillus subtilis is a gram-positive, endospore producing, motile, rod shaped bacteria that produces highly resistant endospores (Hill et al., 1999; Mainelis et al., 2002). These spores are widely used in research due to their ubiquity, hardiness, availability, and ability to simulate safely Bacillus anthracis spores (Burton et al., 2005; Farnsworth et al., 2006; Foarde et al., 1999; Maus et al., 2001; Li and Lin, 2001). These spores react similarly to disinfectants as Bacillus anthracis (Sagripanti et al., 2007) and are non-pathogenic for humans and animals (Priest, 1993; Sonenshein et al., 1993).

Bacillus subtilis is one of nature's best-studied organisms, second among prokaryotes only to E. coli in the level of detail understood (Sonenshein et al., 2002). The completion of the gene sequence was completed on in 1997 (Moszer, 2002). It is universally distributed in oceans and soils (Aizawa et al., 2002). Because soil is a reservoir this bacterium is transmitted to plants, plant materials, foods, animals, and marine/fresh-water inhabitants (Priest, 1993) and has at times contaminated various foodstuffs. This is largely ignored though because they are nonpathogenic (Priest, 1993). It has been used is used to control fungal disease for fruits, vegetables, field crops, and flowers (Hall and Davis, 1990).

The diameter of a Bacillus subtilis spore ranges from 0.9 to $1.3 \mu \mathrm{m}$ (Farnsworth et al., 2006). The size of the spores (particle diameter, $D_{p}$ ) does change slightly with relative humidity, with the following equation showing the relationship: $\mathrm{D}_{\mathrm{p}}(\mu \mathrm{m})=0.94+0.003 \mathrm{RH}(\%)$ (Farnsworth et al., 2006; Johnson et al., 1999). 
Bacillus subtilis spores have been used extensively in research projects. Burton et al. used Bacillus subtilis var Niger in dried powder form as a simulant for Bacillus anthracis to determine filter efficiencies for sample collection (Burton et al., 2005). Other studies have focused on the differences on spores of Bacillus anthracis compared to other Bacillus species (Carrera et al., 2005; Sagripanti et al., 2007). Wagner et al. (2008) measured inactivation rates of Bacillus subtilis spores on gypsum board through using different chemical disinfectants aerosolized into the room. Other studies have included research on the sensitivity of the spores in the UV regions of light used for decontamination purposes (Coohill and Sagripanti 2008). Farnsworth et al. (2006) found Bacillus subtilis could be removed from HVAC filters because of their hardiness, while live viruses were inactivated. Burton et al. (2005) used these spores to determine the filter material and extraction methods for environmental sampling of Bacillus anthracis. These studies have not been limited to just the spores. Edmonds et al. (2009) seeded test coupons with five- $20 \mu \mathrm{L}$ drops of $10^{7}$ stock of BG. These coupons were allowed to air-dry a minimum of 3 hours or until all the liquid was completely evaporated. Yah and Mainelis used the vegetative state of Bacillus subtilis to estimate inhalation exposures to the vegetative form of the species (Yah and Mainelis, 2007). Baron et al. (2008) used both BG and Bacillus anthracis strain Sterne (BaS) spores in wipe sampling experiments. The BaS spores were BSL 3, posing severe risk to the researchers if not controlled properly. Because of this, they used the BG spores first to evaluate the test chamber (Baron et al., 2008). Additionally, it has been used to evaluate bioaerosol samplers (Jensen, 1992). Aizenberg et al. (2008b) compared inert particles to $B$. atropheus, finding there were no significant differences between the collection efficiency of PSL microspheres and microorganisms. These are just a few examples of how Bacillus subtilis has been used as a simulant for Bacillus anthracis. 
The bioaerosols must be diluted in some solution and then properly removed for analysis. There have been several different methods used to accomplish this dilution. For example, Brown et al. (2007b) used simple sterile, deionized water to moisten their wipe samples. After the samples were completed, they were placed in a 50-mL screw top container with $30 \mathrm{~mL}$ of sterile Butterfield Buffer with Tween 80 (BBT). Estill et al. (2009) used two different solutions for suspending the spores. The first one was BBT $(0.01 \%$, pH 7.2; Becton Dickson Microbiology, Franklin Lakes, NJ) and the second one was phosphate-buffered saline (PBS, Sigma Aldrich, St. Louis, MO).

Another critical piece of the bacteriological analysis methods is the removal of the spores from the medium, whether that be a wipe or filter sampler. Several studies completed have evaluated the best methods for this. Lewandowski et al. (2010) completed both sonication and vortexing methods and found no different in the recovery efficiencies between the two methods. They did note there was no method to estimate the actual number, which settle on a coupon 100 $\mathrm{cm}^{2}$ during experiment and the recovery was based on the theoretical number of the spores depositing. Other research has been completed on vortexing. Wagner et al. (2008) collected swab surface samples and vortexed each sample for 10 seconds, followed by a serial dilution and plating to TSA. The plates were incubated for 48 hours at $37^{\circ} \mathrm{C}$ and plate counts were conducted after 48 hours. Kesevan et al. (2008) also used vortexing to suspend spores after a test filter was disintegrated. Following vortexing, they diluted the samples and plated $100 \mu \mathrm{L}$ onto tryptose agar petri dishes. If the counts were greater than 300 colonies, the samples were diluted again and re-plated. Brown et al. (2007b) found the optimal method for removal was to sonicate in BBT for 15 minutes. They tested this by using 24 reference coupons seeded to approximately $200,000 \mathrm{CFU} / \mathrm{cm}^{2}$. Colonies forming on the reference surface with distinct margins were 
counted by eye. Similar methods have been used for the vegetative forms of Bacillus subtilis cells after collection onto filters. Wang et al. (2001) used a procedure in which they soaked the filter for 10 minutes in sterile deionized water, followed by vortexing for 2 min and sonicating for $15 \mathrm{~min}$. The number of bacteria in the resulting suspension was determined using epifluorescence microscopy. This value was then compared to the APS reading and it was found they agreed within $8 \%$ (Wang et al., 2001). This number compares very well to the standard deviation of microscopy counting, which has a standard deviation of approximately 20\% (Han et al., 2010).

Burton et al. conducted studies on the most efficient filter materials and extraction methods when sampling B. subtilis. They used MCE (pore size of 3 um), polytetrafluoroethlyene (PTFE) (pore sizes of 1 and $3 \mathrm{um}$ ), and gelatin (pore size of $3 \mathrm{um}$ ). The test used Bacillus subtilis var niger (BG), using a SKC Button aerosol sampler (SKC, Inc, Eighty Four, PA). The results showed MCE and $1 \mu \mathrm{m}$ PTFE had the best results. In order to complete this, they tried different extraction methods to determine the culturability. They found vortexing with shaker agitation showed significantly higher physical extraction efficiency for MCE and 1 $\mu \mathrm{m}$ PTFE filters than the vortex with ultrasonic agitation extraction method. Sampling times up to 4 hours did not affect relative culturability and extraction of $B$. subtilis off the sampling filters (Burton et al., 2005).

The BG spores analysis is straightforward when the culture method is used. Estill et al. (2009) used plates with trypticase soy agar (TSA) with 5\% sheep blood (TSAII, Becton Dickenson Microbiology, Franklin Lakes, NJ). These plates were incubated at $35^{\circ} \mathrm{C}$ to $37^{\circ} \mathrm{C}$ for 16 to 18 hours before colonies were enumerated. Hill et al. also used culturing method to analyze Bacillus subtilis spores by plating them onto tryptic soy agar (Becton Dickinson 
Microbiology Systems, Cockeysville, MD, USA) filled petri dishes which were kept in the incubator for $18 \mathrm{~h}$ at $30^{\circ} \mathrm{C}$ (Hill et al., 1999). Baron et al. (2008) evaluated several different methods to plate the spores. They eventually determined that spreading the spores onto an agar plate using a hockey-stick shaped glass rod was the most reproducible method (Baron et al., 2008).

Caution must be used when culturing because this method depends on stresses of the test organisms. Dead organisms can be quantified by PCR or other molecular techniques, but they will clearly not germinate. Because of this, sensitivity and repeatability may not be particularly good when using culture methods (Kesavan, 2008). Additionally, dehydration effects can kill sensitive bacteria. This was shown by Li et al. when they conducted tests with E. coli and Bacillus atropheus - the E. coli was more affected than the B. atropheus spores (Li et al., 1999). This can especially be a problem for cells during aerosolization when they are injured and remain "viable" but not "culturable" (Heidelber et al., 1997; Rule et al., 2007; Terzieva et al., 1996).

In conclusion, the literature shows limited studies on decontamination using high heat and humidity have been conducted. This is a research focus area because it is the only method which can be used on aircraft. These studies have been very limited, focusing on only a high direct inoculation method on aluminum coupons and at very high temperatures and humidity levels. The literature also shows that several different aerosol deposition chambers have been constructed and tested, but not to test decontamination efforts. A Bacillus anthracis simulant could be used to conduct tests safely, potentially closing research gaps on different inoculation levels and methods, as well as different aircraft materials. 


\section{REFERENCES}

ACGIH: Particle size-selective sampling for particulate air contaminants. Cincinnati, OH: ACGIH, 1999.

Adhikari, A., J. Jung, T. Reponen, J.S. Lewis, E.C. Degrasse, L.F. Grimsley, G.L. Chew, and S.A. Grinshpun: Aerosolization of fungi, $(1 \rightarrow 3)-\beta$-d glucan, and endotoxin from floodaffected materials collected in New Orleans homes. Environmental Research 109(3): 215-224 (2009).

Aerospace Medical Association Task Force: Emerging infectious diseases including severe acute respiratory syndrome (SARS): guidelines for commercial air travel and air medical transport. Aviation, Space, and Environmental Medicine 75: 85-86 (2004).

Air Force Instruction (AFI): Air Force Chemical, Biological, Radiological, and Nuclear (CBRN) Survivability, Pub No. AFI 10-2607, 25 Feb 2010.

Air Force Research Laboratory (AFRL): Bio-thermal decontamination tests for large frame aircraft application laboratory and field test report, AFRL Document HEPC OSMSWP8Z, 5 Dec 2008.

Aizenberg, V., S.A. Grinshpun, K. Willeke, J. Smith, and P.A. Baron: Performance characteristics of the button personal inhalable aerosol sampler. American Industrial Hygiene Association Journal 61: 398-404 (2000a).

Aizenberg, V., T. Reponen, S.A. Grinshpun, and K. Willeke: Performance of air-o-cell, burkard, and button samplers for total enumeration of airborne spores. American Industrial Hygiene Association Journal 61: 855-864 (2000b).

Alibek, K.: (2005, April 22-23). Biological Weapons/Bioterrorism Threat and Defense-Past, Present, and Future. Paper presented at ETH international conference, Meeting the Challenges of Bioterrorism: Assessing the Threat and Designing Biodefense Strategies, Furigen, Switzerland.

American Industrial Hygiene Association: Biosafety Reference Manual. 2nd ed, Pub. No. 204-RC-95. Falls Church, VA, 1995.

Andersen, B.M., M. Rasch, K. Hochlin, F.H. Jensen, P. Wismar, and J.E. Fredriksen: Decontamination of rooms, medical equipment and ambulances using an aerosol of hydrogen peroxide disinfectant. Journal of Hospital Infection Hosp Infection 62: 149-155 (2006).

Angelotti, R., M.J. Foter, K.A. Busch, and K.H. Lewis: A comparative evaluation of methods for determining the bacterial contamination of surfaces. Food Research International 23: 175185 (1958). 
Atrih, A., and S.J. Foster: Bacterial endospore the ultimate survivors. International Dairy Journal. 12: 217-223 (2002).

Baldwin, P.E.J., and A.D. Maynard: A survey of wind speeds in indoor workplaces. Annals of Occupational Hygiene 20: 303-313 (1998).

Baron, P.A., C.F. Estill, G.J. Deye, M.J. Heim, J.K. Beard, L.D. Larsen, and G.E. Dahlstrom: Development of an aerosol system for uniformly depositing Bacillus anthracis spore particles on surface. Aerosol Science and Technology 42: 159-172 (2008).

Baron, P.A., C.F. Estill, J.K. Beard, M.J. Hein, and L. Larsen: Bacterial endospore inactivation caused by outgassing of vapourous hydrogen peroxide from polymethyl methacrylate (Plexiglas $\left.{ }^{\circledR}\right)$. Letters in Applied Microbiology 45: 485-490 (2007).

Barth, E., R. Rupert, F. Stroud, E. Rice, and B. Potoka: Environmental response to intentional dissemination of Bacillus anthracis spores in the United States 2001. Risk Management Research 13: 99-111 (2003).

Bartrand, T.A., M.H. Weir, and C.N. Haas: Dose-response models for inhalation of Bacillus anthracis spores: interspecies comparisons. Risk Analysis 28: 1115-1124 (2008).

Beecher, D. J.: Forensic application of microbiological culture analysis to identify mail intentionally contaminated with Bacillus anthracis spores. Applied Environmental Microbiology 72: 5304-5310 (2006).

Berry R.D., and Froude S: An investigation of wind conditions in the workplace to assess their effect on the quantity of dust inhaled, Internal Report—Pub No IR/L/ DS/89/3. Health and Safety Executive, London (1998).

Betty, R.: DF-200 Decontamination of CBW Agents, Other Biological Pathogens, and Toxic Industrial Chemicals. In Report on Workshop on Decontamination, Cleanup and Associated Issues for Sites Contaminated with Chemical, Biological, or Radiological Materials, S. Dun, and J. Wood, US EPA: October 2005.

BGI, Inc: Collison Nebulizer Droplet Number Output (Online) Available at http://bgiusa.com/agc/droplet_number_output.htm (accessed March 2011).

Bholah R., and A.H. Subratty: Indoor biological contaminants and symptoms of sick building syndrome in office buildings in Mauritius. Int J Environ Health Res 12: 93-98 (2002).

Brachman, P.S.: Bioterrorism: an update with a focus on anthrax. American Journal of Epidemiology 155: 981-987 (2002).

Brachman P.S.: Inhalation anthrax. Annals of the New York Academy of Sciences 353: 83-93 (1980). 
Brachman P.S., A.F. Kaufmann, and Dalldorf F.: Industrial inhalation anthrax. Bacteriology Review 30: 646-659 (1966).

Brankston, G., L. Gitterman, Z. Hirji, C. Lemieux, and M. Gardam: Transmission of influenza A in human beings. The Lancet Infectious Diseases 7(4): 257-265 (2007).

Bray, M.: Defense against filoviruses used as biological weapons, Antiviral Research 57:53-60. In Handbook of Viral Bioterrorism and Biodefense, E. De Clercq, and E.R. Kern (eds.). New York: Elsevier, 2003.

Brickhouse, M.: Innovative and Emerging Decontamination Technologies. In Report on Workshop on Decontamination, Cleanup and Associated Issues for Sites Contaminated with Chemical, Biological, or Radiological Materials, S. Dun, and J. Wood, US EPA: October 2005.

Brooks, L.: DHS S\&T Biological and Chemical Restoration Programs. In Report on Workshop on Decontamination, Cleanup and Associated Issues for Sites Contaminated with Chemical, Biological, or Radiological Materials, S. Dun, and J. Wood, US EPA: October 2005.

Brown, G.S.; R.G. Betty, J.E. Brockman, D.A. Lucero, C.A. Souza, K.S. Walsh, R.M. Boucher, M.S. Tezak, and M.C. Wilson: Evaluation of vacuum filter sock surface sample collection method for Bacillus spores from porous and non-porous surfaces. Journal of Environmental Monitoring 9(7): 666-671 (2007a).

Brown, G.S., R.G. Betty, J.E. Brockman, D.A. Lucero, C.A. Souza, K.S. Walsh, R.M. Boucher, M. Tezak, M.C. Wilson, and T. Rudolph: Evaluation of a wipe surface sample method for collection of Bacillus spores from nonporous surfaces. Applied and Environmental Microbiology 73(3): 706-710 (2007b).

Brown, G.S., R.G. Betty, J.E. Brockmann, D.A. Lucero, C.A. Souza, K.S. Walsh, R.M. Boucher, M.S. Tezak, M.C. Wilson, T. Rudolph, H.D. Lindquist, and K.F. Martinez: Evaluation of rayon swab surface sample collection method for Bacillus spores from nonporous surfaces. Journal of Applied Microbiology 103(4): 1074-1080 (2007c).

Burgess, W.A., M.J. Ellenbecker, and R.D. Treitman: Ventilation for Control of the Work Environment. Hoboken, NJ: John Wiley \& Sons, 2004.

Burke, S.A., J.D. Wright, M.K. Robinson, B.V. Bronk, and R.L. Warren: Detection of molecular diversity in Bacillus atrophaeus by amplified fragment length polymorphism analysis. Applied and Environmental Microbiology 79(5): 2786-2790 (2004).

Burton, N.C., A. Adhikari, S.A. Grinshpun, R. Hornung, and T. Reponen: The effect of filter material on bioaerosol collection of Bacillus subtilis spores used as a Bacillus anthracis stimulant. Journal of Environmental Monitoring 7: 475-480 (2005). 
Buttner, M.P., P. Cruz-Perez, and L.D. Stetzenbach: Enhanced detection of surfaceassociated bacteria in indoor environments by quantitative PCR. Applied Environmental Microbiology. 67: 2564-2570 (2001).

Buttner, M. P., P. Cruz, L.D. Stetzenbach, A.K. Klima-Comba, V.L. Stevens, and P.A. Emanuel: Evaluation of the biological sampling kit (BiSKit) for large-area surface sampling. Applied Environmental Microbiology 70: 7040-7045 (2004).

Buttner, M.P., and L.D. Stetzenbach: Monitoring fungal spores in an experimental indoor environment to evaluate sampling methods and the effects of human activity on air sampling. Applied Environmental Microbiology 59:219-226 (1993).

Byrne, MA; A.J.H. Goddard, C. Lange, and J. Roed: Stable tracer aerosol deposition measurements in a test chamber. Journal of Aerosol Science 26(4): 645-653 (1995).

Canter, D.A., D. Gunning, P. Rodgers, L. O'Connor, C. Traunero, and C.J. Kempter: Remediation of Bacillus anthracis contamination in the U.S. Department of Justice mail facility. Biosecurity Bioterror 3(2): 119-127 (2005).

Carlsen, T.: Use of HVAC Systems in Building Decontamination. In Report on Workshop on Decontamination, Cleanup and Associated Issues for Sites Contaminated with Chemical, Biological, or Radiological Materials, S. Dun, and J. Wood, US EPA: October 2005.

Carrera, M., J. Kesavan, R. Zandomeni, and J.-L. Sagripanti: Method to determine the number of bacterial spores within aerosol particles. Aerosol Science and Technology 39: 960-965 (2005).

Caudle, L.C., III: The biological warfare threat, p. 451-466. In Medical Aspects of chemical and Biological Warfare, F.R. Sidell, E.T. Takafuji, and D.R. Franz (eds.),. Office of the Surgeon General, Borden Institute, Walter Reed Army Medical Center, Washington, DC. Available at http://www.bordeninstitute.army.mil/published_volumes/chemBio/Ch21.pdf. (accessed July 2011).

Centers for Disease Control: Biosafety in Microbiology and Biomedical Laboratories, 4th Ed. DHHS Pub. No. 93-8395. Atlanta, GA, 1999b.

Centers for Disease Control: Bioterrorism overview (Online) Available at http://www.bt.cdc.gov/bioterrorism/overview.asp (accessed November 2011).

Centers for Disease Control: Exposure of passengers and flight crew to Mycobacterium tuberculosis on commercial aircraft. Morbidity and Mortality Weekly Report, 44: 137-140 (1995).

Centers for Disease Control: Fact Sheet: Anthrax Information for Health Care Providers. (Online). Available at www.bt.cdc.gov/agent/anthrax/anthrax-hcp-factsheet.asp, 2002 
Centers for Disease Control: Guidelines for preventing the transmission of Mycobacterium tuberculosis in health-care facilities. Morb Mort Wkly Rep, 43: 1-132 (1994).

Centers for Disease Control: Investigation of bioterrorism-related anthrax-Connecticut. Morb Mortal Wkly Rep, 50: 1077-1079 (2001).

Chang, C.-W., S.A. Grinshpun, K. Willeke, J.M. Macher, J. Donnelly, S. Clark, and A. Juozaitis: Factors affecting microbiological colony count accuracy for bioaerosol sampling and analysis. American Industrial Hygiene Association Journal 56: 979-986 (1995).

Chen, B.T., M.D. Hoover, G.J. Newton, S.J. Montano, and D.S. Gregory: Performance evaluation of the sampling head and annular kinetic impactor in the Savannah River site alpha continuous air monitor. Aerosol Science and Technology 31: 24-38 (1999).

Cheng, Y.S. and B.T. Chen: Aerosol sampler calibration. In Air Sampling Instruments. Cincinnati: ACGIH, 2001.

Chosewood, L.C., and D.E. Wilson, eds.: Biosafety in Microbiological and Biomedical Laboratories, $5^{\text {th }}$ Ed., Pub. No. 21-1112. Atlanta, GA: US Department of Health and Human Services, Centers for Disease Control, NIH, 1992.

Cohen, M.L., and T. Whalen: Implications of low level human exposure to respirable Bacillus anthracis. Appl. Biosafety 12: 109-115 (2007).

Cordesman, A.H.: The Challenge of Biological Terrorism. Washington, DC: The CSIS Press (Center for Strategic and International Studies), 2005.

Cole, E.C., and C.E. Cook: Characterization of infectious aerosols in strategies. American Journal of Infection Control 26: 453-464 (1998).

Cooley J.D., W.C. Wong, C.A. Jumper, and D.C. Straus: Correlation between the prevalence of certain fungi and sick building syndrome. Journal of Occupational and Environmental Medicine 55: 579-584 (1998).

Corsi, R.: Building Disinfection Byproducts: Experimental Evaluation and Decision Tool. In Report on Workshop on Decontamination, Cleanup and Associated Issues for Sites Contaminated with Chemical, Biological, or Radiological Materials, S. Dun, and J. Wood, US EPA: October 2005.

Cox C.S.: Aerosol survival of Pasteurella tularensis disseminated from the wet and dry states. Applied Microbiology 21: 482-486 (1971).

Cox C.S., and L.J. Goldberg: Aerosol survival of Pasteurella tularensis and the influence of relative humidity. Applied Microbiology 23(1): 1-3 (1972). 
Cox, C.S., and C.M. Wathes, eds: Bioaerosols Handbook. New York: Lewis Publishers; 1995.

Dahlqvist, M., and U. Johard: Lung function and precipitating antibodies in low exposed wood trimmers in Sweden. American Journal of Industrial Medicine 21(4): 549- 559 (1992).

Davids D.E., and A.R. Lejeune: Secondary Aerosol Hazard in the Field. Report No. 321, No 18: Defense Research Establishment Suffield: Ralston, Alberta (1981).

Department of Defense: The Chemical, Biological, Radiological, and Nuclear (CBRN) Survivability Policy, Pub No. DoDI 3150.09 (August 17, 2009).

Department of the Army: Occupational and Environmental Health, Swimming Pools and BathingFacilities. Washington, DC: US Government Printing Office, Technical Bulletin No. Med 575 (1982).

Druett, H.A., and K.R. May: A wind tunnel for the study of airborne infections. Journal of Hygiene 50: 69-81 (1952).

Druett, H.A., D.W. Henderson, L. Packman, and S. Peacock: Studies on respiratory infection I. The influence of particle size on respiratory infection with anthrax spores. Journal of Hygiene 51(3): 359-71 (1953).

Druett, H.A., J.M. Robinson, D.W. Henderson, L. Packman, and S. Peacock: Studies on respiratory infection II. The influence of aerosol particle size on infection of the guinea-pig with Pasteurella pestis. Journal of Hygiene 54: 37-48 (1956a).

Druett, H.A., D.W. Henderson, and S. Peacock: Studies on respiratory infection III. Experiments with Brucella suis. Journal of Hygiene 54: 49-57 (1956b).

Donaldson, A.I., and S. Alexandersen: Predicting the spread of foot and mouth disease by airborne virus. Rev. Sci. Tech 21: 569-575 (2002).

Douwes, J., P. Thorne, N. Pearce, and D. Heederik: Bioaerosol health effects and exposure assessment: progress and prospects. Annals of Occupational Hygiene 47(3): 187-200 (2003).

Edmonds, J.M., P.J. Collett, E.R. Valdes, E.W. Skowronski, G.J. Pellar, and P.A. Emanuel: Surface sampling of spores in dry-deposition aerosols. Applied and Environmental Microbiology 75(1): 39-44 (2009).

Estill, C.F., and G.J. Deye: Personal communication, January 7, 2010.

Estill, C.F., P.A. Baron, J.K. Beard, M.J. Hein, L.D. Larsen, L. Rose, F.W. Schaefer III, J. Noble-Wang, L. Hodges, H.D.A. Lindquist, G.J. Deye, and M.J. Arduino: Recovery efficiency and limit of detection of aerosolized Bacillus anthracis sterne from environmental surface samples. Applied and Environmental Microbiology 75(13): 4297-4306 (2009). 
Farnsworth, J.E., S.M. Goyal, S.W. Kim, T.H. Kuehn, P.C. Raynor, M.A. Ramakrishnan, S. Anantharaman, and W. Tang: Development of method for bacteria and virus recovery from heating, ventilation, and air conditioning (HVAC) filters. Journal of Environmental Monitoring 8: 1006-1013 (2006).

Feather, G.A., and B.T. Chen: Design and use of a settling chamber for sampler evaluation under calm-air conditions. Aerosol Science and Technology 37: 261-270 (2003).

Federal Aviation Administration: FAR Part 25. Title 14 CFR, Washington, D.C., 1991.

Fennelly, K.P., A.L. Davidow, S.L. Miller, N. Connell, and J.J. Ellner: Airborne infection with Bacillus anthracis - from mills to mail. Emerging Infectious Disease 10: 996-1001 (2004).

Fennelly, K.P., J.W. Martyny, K.E. Fulton, I.M. Orme, D.M. Cave, and L.B. Heifets:

Cough-generated aerosols of Mycobacterium tuberculosis. a new method to study infectiousness. American Journal of Respiratory and Critical Care Medicine 169: 604-609 (2004).

Fitch, J.P.: A Framework for Assessing the Health Hazard Posed by Bioaerosols, Washingtong, DC: The National Research Council, National Academis Press, 2008.

Flight Safety Foundation: Guidelines enable health authorities to assess risk of tuberculosis transmission aboard aircraft. In Cabin Crew Safety, Flight Safety Foundation. pp 1-24 (1998).

Foarde, K.K., J.T. Janley, D.S. Ensor, and P. Roessler: Development of method for measuring single-pass bioaerosol removal efficiencies of a room air cleaner, Aerosol Science Technology 30: 223-234 (1999).

Gale, W.F., S. Hyacinth, and J. Watson: Field Evaluation of Whole Airliner Decontamination Technologies - Wide Body Aircraft with Dual-Use Application for Railcars, Federal Aviation Administration, Pub No. DOT/FAA/AM-08/4, (2008).

Gale, W.F., N.I. Sofyan, H.S. Gale, S.-F. Chou, J.W. Fergus, and C.G. Shannon: Effect of vapour phase hydrogen peroxide, as a decontaminant for civil aviation applications, on microstructure, tensile properties and corrosion resistance of 2024 and 7075 age hardenable aluminum alloys and 304 austenitic stainless steel. Materials Science and Technology 25(1): 7684 (2009).

Gao, P., H.K. Dillon, and W.E. Farthing: Development and evaluation of an inhalable bioaerosol manifold sampler. American Hygiene Association Journal 58: 196-206 (1997).

Goldberg, L.J., H.M.S. Watkins, E.E. Boerke, and M.A. Chatigny: The use of a rotating drum for the study of aerosols over extended periods of time. American Journal of Hygiene 68: 85 (1958). 
Gorny, R.L., T. Reponen, K. Willeke, D. Schmechel, E. Robine, M. Boissier, and S.A. Grinshpun: Fungal fragments as indoor air biocontaminants. Applied Environmental Microbiology 68: 3522-3531 (2002).

Government Accountability Office (GAO): Agencies Need to Validate Sampling Activities in Order to Increase Confidence in Negative Results, Government Accountability Office Report to the Chairman Subcommittee on National Security, Emerging Threats, and International Relations, House Committee on Government Reform, House of Representatives GAO-05-251, Washington, DC, 2005, p. 76.

Hall, T.J., and W.E.E. Davis: Survival of bacillus subtilis in silver and sugar maple seedlings over a two-year period. Plant Disease 74: 608-609 (1990).

Han, Taewon; H.R. An, G. Mainelis: Performance of an electrostatic precipitator with superhydrophobic surface when collecting airborne bacteria. Aerosol Science and Technology 44(5): 339-348 (2010).

Harper, G.J., and J.D. Morton: The respiratory retention of bacterial aerosols: experiments with radioactive spores. J. Hyg 51: 372-385 (1953).

Hatch, T.F.: Distribution and deposition of inhaled particles in respiratory tract. Bacteriology Review 25: 237-40 (1961).

Hedgpeth, D.: (2000, August 24). BioReliance vs Bioterrorism: Maryland Firm Prepares Smallpox Vaccine for Military. Washington Post, pp E-1.

Heidelberg, J.F., M. Shahamat, M. Levin, I. Rahman, G. Stelma, C. Grim, and R.R. Colwell: Effect of aerosolization on culturability and viability of gram-negative bacteria. Applied Environmental Microbiology 63: 3585-3588 (1997).

Henry, B.S. and C.A. Friedman: The water content of bacterial spores. J. Bact, 33, 323-329, (1937). In Principles and Methods of Sterilization in Health Sciences, $2^{\text {nd }}$ ed, J.J. Perkins. Springfield, Illinois: Charles C. Thomas Publishers, 1983.

Hensel, A., and K. Petzoldt, K.: Biological and biochemical analysis of bacteria and viruses. In Bioaerosols Handbook, C. S. Cox and C.M.Wathes (eds). New York: Lewis Publishers, 1995.

Herd, M.: Hydrogen Peroxide Vapor for Room/Building Decontamination Following a Chemical or Biological Agent Attack: Overview of Efficacy and Practical Issues. In Report on Workshop on Decontamination, Cleanup and Associated Issues for Sites Contaminated with Chemical, Biological, or Radiological Materials, S. Dun, and J. Wood, US EPA: October 2005.

Herzog, A.B., S.D. McLennan, A.K. Pandey, C.P. Gerba, C.N. Hass, J.B. Rose, and S.A. Hashsham: Implications of limits of detection of various methods for Bacillus anthracis in 
computing risks to human health. Applied and Environmental Microbiology 75(19): 6331-6339 (2009).

Heyder, J., J. Gebhart, G. Rudolf, C.F. Schiller, and W. Stahlhofen: Deposition of particles in the human respiratory tract in the size range. Journal of Aerosol Science 17: 811-825 (1986).

Hill S.C., R.G. Pinnick, S. Niles, Y.L. Pan, S. Holler, R.K. Chang, J. Bottiger, B.T. Chen, C.S. Orr, G. Feather, and A.P. Snyder: Real-time measurement of fluorescence spectra from single airborne biological particles. Field Analysis and Chemical Technology 3: 221-239 (1999).

Hinds, W.C.: Aerosol Technology: Properties, Behavior, and Measurement of Airborne Particles. New York: John Wiley \& Sons, Inc., 2004.

Hodges, L.R., L.J. Rose, A. Peterson, J. Noble-Wang, and M.J. Arduino: Evaluation of a macrofoam swab protocol for the recovery of Bacillus anthracis spores from a steel surface. Applied Environmental Microbiology 72: 4429- 4430 (2006).

Ijaz, M.K., A.H. Brunner, S.A. Satta, R.C. Nair, and C.M. Johnson-Lussenburg: Journal of General Virology 66: 2743-2748 (1985).

Inglesby, T.V., D.A. Henderson, J.G. Bartlett et al.: Anthrax as a biological weapon. Journal of American Medical Association 281(18): 1735-45 (1999).

Inglesgy, T.V., et al: Plague as a biological weapon: medical and public health management. Journal of American Medical Association 283 (18),2281-2290 (2000).

Inglesby, T.V., T. O’'Toole, D.A. Henderson, J.G. Bartlett, M.S. Ascher, E. Eitzen, A.M. Friedlander, J. Gerberding, J. Hauer, J. Hughes, J. McDade, M.T. Osterholm, G. Parker, T.M. Perl, P.K. Russell, and K. Tonat. Anthrax as a biological weapon, 2002: updated recommendations for management. Journal of American Medical Association 287: 2236-2252 (2002).

Jensen, P.A., W.F. Todd, G.N. Davis, and P.V. Scarpino: Evaluation of eight bioaerosol samplers challenged with aerosols of free bacteria. American Industrial Hygiene Association Journal 53: 660-667 (1992).

Jernigan, D.B., P.L. Raghunathan, B.P. Bell et al.: Investigation of bioterrorism-related anthrax, United States, 2001: epidemiologic findings. Emerging Infectious Diseases 8: 10191028 (2002).

Jernigan, J.A., D.S. Stephens, D.A. Ashford, C. Omenaca, M.S. Topeil, M. Galbraigh, M. Tapper, T.L. Fish, S. Saki, T. Popovic, R.F. Meyer, C.P. Quinn, S.A. Harper, S.K. Fridkin, J.J. Sejvar, C.W. Shepard, M. McConnell, J. Guarner, W. Sheih, J.M. Malecki, J.L. Gerberding, J.M. Hughes, B.A. Perkins, and members of the Anthrax Bioterrorism 
Investigation Team: Bioterrorism-related inhalational anthrax: the first 10 cases reported in the United States. Emerging Infectious Diseases 7: 933-944 (2001).

John, W., and N.M. Kreisberg: Calibration and testing of samplers with dry, polydisperse latex. Aerosol Science Technology 31: 221-225 (1999).

Johnson, D.L., T.A. Pearce, and N.A. Esmen: The effect of phosphate buffer on aerosol size distribution of nebulized Bacillus subtilis and Psuedomonas fluorescens bacteria. Aerosol Science Technology 30: 202-210 (1999).

Juozaitis, A., K. Willeke, S.A. Grinshpun, and Donnelly, J.: Impaction onto a glass slide or agar versus impingement into a liquid for the collection and recovery of airborne microorganisms. Applied Environmental Microbiology 60(3): 861-870 (1994).

Keim, P., K.L. Smith, C. Keys, H. Takahashi, T. Kurata, and A. Kaufmann: Molecular investigation of the Aum Shinrikyo anthrax release in Kameido, Japan. J. Clin. Microbiol 39: 4566-4567 (2001).

Keim, M., and A.F. Kauffman: Principles for emergency response to bioterrorism. Annals of Emergency Medicine 34: 177-182 (1999).

Keith, L.H., W. Crummett, J. Deegan, R.A. Libby, J.K. Taylor, and G. Wentler: Principals of environmental analysis. Annals of Chemistry 55: 2210-2218 (1983).

Kelly, J.: Capitol Hill Ricin Incident: Decontamination Dilemmas. In Report on Workshop on Decontamination, Cleanup and Associated Issues for Sites Contaminated with Chemical, Biological, or Radiological Materials, S. Dun, and J. Wood, US EPA: October 2005.

Kempter, J.: Crisis Exemptions for Products Intended to Inactivate Bacillus anthracis, In Report on Workshop on Decontamination, Cleanup and Associated Issues for Sites Contaminated with Chemical, Biological, or Radiological Materials, S. Dun, and J. Wood, US EPA: October 2005.

Kenny, L.C., R.J. Aitken, P.E.J. Baldwin, G.C. Beaumont, and A.D. Maynard: The sampling efficiency of personal inhalable aerosol samplers in low air movement environments. Journal of Aerosol Science 30(5): 627-638 (1999).

Kesavan, J., J.R. Bottiger, and A.R. McFarland: Bioaerosol concentrator performance: comparative tests with viable and with solid and liquid nonviable particles. Journal of Applied Microbiology 104: 285-295 (2008b).

Kirschner, L.E., and J.R. Puleo: Wipe-rinse technique for quantitating microbial contamination on large surfaces. Applied Microbiology 38(3): 466-470 (1979). 
King, B., J. Kesavan, and J.-L. Sagripanti: Germicidal UV sensitivity of bacteria in aerosols and on contaminated surfaces. Aerosol Science and Technology 45(5): 645-653 (2011).

Knight, V.: Viruses as agents of airborne contagion. Annals of the New York Academy of Sciences 353: 147-156 (1980).

Ko, G., M.W. First, and H.A. Burge: The characterization of upper-room ultraviolet germicidal irradiation in inactivating airborne microorganisms. Environ Health Perspectives 110(1): 95-101 (2002).

Koch, W., W. Dunkhort, and H. Lodding: Design and performance of a new personal aerosol monitor. Aerosol Science (1999).

Kodama, A.M., and R.I. McGee: Airborne microbial contaminants in indoor environments: naturally ventilated and air-conditioned homes. Archives of Environmental Health 41: 306-11 (1986).

Kournikakis, B., K.F. Martinez, R.E. McCleery, S.V. Shadomy, and G. Ramos: Anthrax letters in an open office environment: effects of selected CDC response guidelines on personal exposure and building contamination. Journal of Occupational and Environmental Hygiene 8: 113-122 (2011).

Kournikakis, B., M. Walker, J. Ho et al.: Statistical analysis of bacterial spore aerosols created by opening a spore containing anthrax letter in an office. Journal of Aerosol Science 40: 514-522 (2009).

Krug, R.M.: The potential use of influenza virus as an agent for bioterrorism, Antiviral Research 57: 47-150. In Handbook of Viral Bioterrorism and Biodefense, E. De Clercq, and E.R. Kern (eds.). New York: Elsevier, 2003.

Krumins, V., E.-K. Son, G. Mainelis, and D.E. Fennell: Retention of inactivated bioaerosols and ethene in a rotating bioreactor constructed for bioaerosol activity studies. Clean 36(7): 593600 (2008).

La Duc, M., T. Stucker, and K. Venkateswaran: Molecular bacterial diversity and bioburden of commercial airliner cabin air. Canadian Journal of Microbiology 53: 1259-1271 (2007b).

Lai, A.C.K.; M.A. Byrne, A.J.H. Goddard: Experimental studies of the effect of rough surfaces and air speed on aerosol deposition in a test chamber. Aerosol Science and Technology 36: 973-982 (2002).

Lai K.M., H.A. Burge, and M.W. First: Size and UV germicidal irradiation susceptibility of Serratia marcescens when aerosolized from different suspending media. Applied Environmental Microbiology 70(4): 2021-2027 (2004). 
Lester, E.D., G. Bearman, and A. Ponce: A second-generation anthrax "smoke detector." IEEE Eng. Med. Biol. Mag 23: 130-135 (2004).

Lewandowski, R., K. Kozlowska, M. Szpakowska, M. Stepin'ska, and E.A. Trafny: Use of a foam spatula for sampling surfaces after bioaerosol deposition. Applied and Environmental Microbiology 76(3): 688-694 (2010).

Lighthart, B.: The ecology of bacteria in the alfresco atmosphere. FEMS Microbiology Ecology 23: 263-274 (1997).

Lighthart, B., and B.T. Shaffer: Increased airborne bacterial survival as a function of particle content and size. Aerosol Science Technology 27: 439-446 (1997).

Li, C.S., and Y.C. Lin: Storage effects on bacterial concentration: determination of impinge and filter samples. Science of the Total Environment 278: 231-237 (2001).

Li, C.S., M.L. Hao, W.H. Lin, C.W. Chang, and C.S. Wang: Evaluation of microbial samplers for bacterial microorganisms. Aerosol Science Technology 30: 100-108 (1999).

Li, C.-S., and Y.-C. Lin: Sampling performance of impactors for bacterial bioaerosols. Aerosol Science Technology 30(3): 280-287 (1999).

Li, S.N., D.A. Lundgren, and D. Rovell-Rixx: Evaluation of six inhalable aerosol samplers. American Industrial Hygiene Association Journal 61: 506-516 (2000).

Lin, C.Y., and C.S. Li: Control effectiveness of ultraviolet germicidal irradiation on bioaerosols Aerosol Science Technology 36: 474-478 (2002).

Lippmann, M., and R.E. Albert: The effect of particle size on the regional deposition of inhaled aerosols in the human respiratory tract. American Industrial Hygiene Association Journal 30: 257-275 (1969).

Load, J.M., L.M. Roberts, and J.D. Robertus: Ricin: structure, mode of action, and some current applications. Journal of the Federation of American Societies for Experimental Biology 8: 201-208 (1994).

Macher J.M., F.Y. Huang, and M. Flores: A two-year study of microbiological indoor air quality in a new apartment. Archives of Environmental Health 46: 25-29 (1991).

Mahy, B.W.: An overview on the use of a viral pathogen as a bioterrorism agent: why smallpox?, Antiviral Research 57:1-5. In Handbook of Viral Bioterrorism and Biodefense, E. De Clercq, and E.R. Kern (eds.). New York: Elsevier, 2003.

Mainelis, G., and M. Tabayoyong: The effect of sampling time on the overall performance of portable microbial impactors. Aerosol Science and Technology 44: 75-82 (2010). 
Mainelis, G., A. Adhikari, K. Willeke, S. Lee, T. Reponen, and S.A. Grinshpun: Collection of airborne microorganisms by a new electrostatic precipitator. Aerosol Science 33: 1417-1432 (2002).

Mainelis, G., D. Berry, H.R. An, M.S. Yao, K. DeVoe, D.E. Fennell, and R. Jaeger: Design and performance of a single-pass bubbling bioaerosol generator. Atmospheric Environment 39(19): 3521-3533 (2005).

Marple, V.A., and K.L. Rubow: An aerosol chamber for instrument evaluation and calibration. American Industrial Hygiene Association Journal 44: 361-367 (1983).

Marthi, B., V.P. Fieland, M. Walter, and R.J. Seidler: Survival of bacteria during aerosolization. Applied Environmental Microbiology 56(11):3463-3467 (1990).

Martin, B.: Opening Remarks, In Report on Workshop on Decontamination, Cleanup and Associated Issues for Sites Contaminated with Chemical, Biological, or Radiological Materials, S. Dun, and J. Wood, US EPA: October 2005.

Martin, D. D., and L. M. Moore: Technical report for the comparison of swab-sampling techniques for recovery of bacterial spores from three surfaces. West Desert Test Center document no. WDTC-TR-00-018, U.S. Army Dugway Proving Ground, Utah, (2001).

Martinez, K.: CDC/NIOSH and Health Response to Biothreat Agents: Environmental Monitoring, In Report on Workshop on Decontamination, Cleanup and Associated Issues for Sites Contaminated with Chemical, Biological, or Radiological Materials, S. Dun, and J. Wood, US EPA: October 2005.

Maus, R., A. Goppelsroder, and H. Umhauer: Atmospheric Environment 35: 105-113 (2001).

May, K. R.: The collison nebulizer: description, performance and application. Journal of Aerosol Science 4: 235-243 (1973).

Maynard, A.D.: Measurement of aerosol penetration through six personal thoracic samplers under calm air conditions. Journal of Aerosol Science 30: 1227-1242 (1999).

Maynard, A.D., L.C. Kenny, and P.E.J. Baldwin: Development of a system to rapidly measure sampler penetration up to $20 \mathrm{~lm}$ aerodynamic diameters in calm air, using the aerodynamic particle sizer. Journal of Aerosol Science 30: 1215-1226 (1999).

McCleery, R. E., K.F. Martinez, G.A. Burr, and D.A. Mattorano: Health hazard evaluation: Trenton processing and distribution center, Pub No. 2002-0109-2927, Washington, DC: Department of Health and Human Services, (2004). 
McFarland, A.R., E.L. Bethel, C.A. Ortiz, and J.G. Stanke: A CAM sampler for collection and assessing a-emitting aerosol particles. Health Physics Journal 61: 97-103 (1991).

McVey, I.: STERIS Chem-Bio Decontamination, In Report on Workshop on Decontamination, Cleanup and Associated Issues for Sites Contaminated with Chemical, Biological, or Radiological Materials, S. Dun, and J. Wood, US EPA: October 2005.

Meselson, M., J. Guillemin, M. Hugh-Jones, A. Langmuir, I. Popova, A. Shelokov, and O. Yampolskaya: The Sverdlovsk anthrax outbreak of 1979. Science 266: 1202-1208 (1994).

Menache, M., F. Miller, and O. Raabe: Particle inhalability curves for humans and small laboratory animals. Annals of Occupational Hygiene 39: 317-328 (1995).

Meltzer, M.I., I. Damon, J.W. LeDuc, J.D. Miller: Modeling potential responses to smallpox as a bioterrorist weapon. Emerging Infectious Diseases 7: 959-969 (2001).

Moser, M.R., T.R. Bender, H.S. Margolis, G.R. Noble, A.P. Kendal, and D.G. Ritter: An outbreak of influenza aboard a commercial airliner. American Journal of Epidemiology 110: 1-5 (1979).

Moszer, I.: The Bacillus subtilis Genome, Genes, and Function, 7-12. In Bacillus subtilis and Its Closest Relatives: From Genes to Cells. A.L. Sonenshein, J.A. Hoch, and R. Losick (eds). Washington, DC: ASM Press, 2002.

National Aeronautics and Space Administration: NASA Standards for Clean Rooms and Work Stations for the Microbially Controlled Environments, Pub No. NHB 5340.2. Washington, DC: US Government Printing Office, 1967.

National Institutes of Health: NIH Guidelines for Research Involving Recombinant DNA Molecules (Online) Available at http://oba.od.nih.gov/oba/rac/Guidelines/ NIH_Guidelines_prn.pdf., October 2011.

National Research Committee: How Clean is Safe? In Reopening Public Facilities After a Biological Attack: A Decision Making Framework. National Research Council Committee on Standards and Policies for Decontaminating Public Facilities Affected by Exposure to Harmful Biological Agents, Washington, DC: The National Research Council, The National Academies Press, 2005.

Nicas, M., W.W. Nazaroff, and A. Hubbard: Toward understanding the risk of secondary airborne infection: emission of respirable pathogens. Journal of Occupational Environmental Hygiene 2: 143-154 (2005).

Nicholson, W.L., P. Fajardo-Cavazos, R. Rebeil, T.A. Slieman, P.J. Riesenman, J.F. Law, and Y. Xue: Bacterial endospores and their significance in stress resistance. Antonie Leeuwenhoek 81: 27-32 (2002). 
Office of the Surgeon General: Medical Aspects of Chemical and Biological Warfare, Part I. Textbook of Military Medicine. Department of the Army, 1997.

Oh, S.W., P.M. Gray, R.H. Dougherty, and D.H. Kang: Aerosolization as novel sanitizer delivery system to reduce food-borne pathogens. Lett Appl Microbiol 41: 56-60 (2005).

Olson, K.B.: Aum Shinrikyo: once and future threat? Emerging Infectious Diseeases 5: 513-516 (1999).

Orlusky, R.: Restoration From Decontamination: USPS Experience. In Report on Workshop on Decontamination, Cleanup and Associated Issues for Sites Contaminated with Chemical, Biological, or Radiological Materials, S. Dun, and J. Wood, US EPA: October 2005.

Osman, S., M.T. La Duc, A. Dekas, D. Newcombe, and K.Venkateswaran: Microbial burden and diversity of commercial airline cabin air during short and long durations of travel. International Society for Microbial Ecology 2: 482-497 (2008).

Pace, N.R.: A molecular view of microbial diversity and the biosphere. Science 276: 734-740 (1997).

Pace, N.R., D.A. Stahl, D.J. Lane and G.J. Olsen: The analysis of natural microbial communities by ribosomal RNA sequences. Microb Ecol 9: 1-56 (1985).

Park, J.M., J.C. Rock, L. Wang, Y.C. Seo, A. Bhatnagar, and S. Kim: Performance evaluation of six different aerosol samplers in a particulate matter generation chamber. Atmospheric Environment 43: 280-289 (2009).

Park, J.M., J.C. Rock, L. Wang, S. Yong-Chil, A. Bhatnagar, S. Kim: Performance evaluation of six different aerosol samplers in a particulate matter generation chamber. Atmospheric Environment 43: 280-289 (2009).

Parker, J. S.: Terrorism through the mail: protecting the postal workers and the public. Report submitted to the Committee on Governmental Affairs and the Subcommittee on International Security, Proliferation and Federal Service, October 31, 2001, Available at http://hsgac.senate.gov/103101parker.htm.

Patrick W.C.: Risk Assessment of Biological Warfare Primary and Secondary Aerosols and Their Requirements for Decontamination. Science Applications International Corp, Vienna, Va (1999).

Peccia J., and M. Hernandez: UV-induced inactivation rates for airborne Mycobacterium bovis BCG. Journal of Occupational Environmental Hygiene 1: 430-435 (2004).

Peccia J., H.M. Werth, S. Miller, \& M. Hernandez: Effects of relative humidity on the ultraviolet induced inactivation of airborne bacteria. Aerosol Science Technology 35: 728-740 (2001). 
Perez, J., S. Springthorpe, and S.A. Sattar: Activity of selected oxidizing microbicides against the spores of Clostridium difficile: relevance to environmental control. American Journal of Infection Control 33: 320-325 (2005).

Perkins, J.J.: Principles and Methods of Sterilization in Health Sciences,. $2^{\text {nd }}$ ed.. Springfield, IL, Charles C. Thomas Publishers, 1983.

Peters, C.J., and D.M. Hartley: Anthrax inhalation and lethal human infection. Lancet. 359: 710-711 (2002).

Prescott, L.M., J.P. Harley, and D.A. Klein: Microbiology, $5^{\text {th }}$ ed. New York: McGraw-Hill Higher Education, 2002.

Priest, F.G.: Systematics and Ecology of Bacillus, 3-16. In Bacillus subtilis and Other GramPositive Bacteria: Biochemistry, Physiology, and Molecular Genetics. A.L. Sonenshein, J.A. Hoch, and R. Losick (eds.). Washington, DC: ASM Press, 1993.

Probst, A., R. Facius, R. Wirth, and C. Moissl-Eichinger: Validation of a nylon-flocked-swab protocol for efficient recovery of bacterial spores from smooth and rough surfaces. Applied and Environmental Microbiology 76(15): 5148-5158 (2010).

Puleo, J.R., N.D. Fields, S.L. Bergstrom, G.S. Oxborrow, P.D. Stabekis, and R. Koukol: Microbiological profiles of the Viking spacecraft. Applied Environmental Microbiology 33: 379384 (1977).

Raabe, O.G., M.A. Al-Bayati, S.V. Teague, and A. Raslot: Regional deposition of inhaled monodisperse coarse and fine aerosol particles in small laboratory animals. Annals of Occupational Hygiene 32: 53-63 (1988).

Raber, E., A. Jin, K. Noonan, R. McGuire, and R.D. Kirvel: Decontamination issues for chemical and biological warfare agents: how clean is clean enough? International Journal of Environmental Health Research 11: 128-148 (2001).

Rastogi, V.: (No date). Presentation: JBADS Surrogate Efficacy Testing, DTRA/Joint Science and Technology Office (Army).

Rastogi, V.K., L. Wallace, L.S. Smith, S.P. Ryan, and M. Blair: Quantitative method to oetermine sporicidal decontamination of building surfaces by gaseous fumigants, and issues related to laboratory-scale studies. Applied and Environmental Microbiology 75(11): 3688-3694 (2009).

Reponen, T., K. Willeke, V. Ulevicius, S.A. Grinshpun, and J. Donnelly: Techniques for dispersion of microorganisms into air. Aerosol Science Technology 27: 405-421 (1997).

Resnick I.G., D.D. Martin, and L.D Larsen: Evaluation of Need for Detection of Surface Biological Agent Contamination. Dugway Proving Ground, Utah: US Dept of the Army; 1990. 
Riley, R.L., and J.E. Kaufman: Effect of relative humidity on the inactivation of airborne Serratia marcescens by ultraviolet radiation. Applied Microbiology 23(6): 1113-1120 (1972).

Riley, R.L., S. Permutt, and J.E. Kaufman: Room air disinfection by ultraviolet irradiation of upper air. Archives of Environmental Health 23: 35-39 (1971).

Rinsoz, T., P. Duquenne, G. Greff-Mirguet, and A. Oppliger: Application of real-time PCR for total airborne bacterial assessment: comparison with epifluorescence microscopy and culturedependent methods. Atmospheric Environment 42(28): 6767-6774 (2008).

Robbins, C.A., L.J. Swenson, M.L. Nealley, and R.E. Gots,: Health effects of mycotoxins in indoor air: a critical review. Applied Occupational and Environmental Hygiene 15: 773-784 (2000).

Rogers, J.V., C.L.K. Sabourin, Y.W. Choi, W.R. Richter, D.C. Rudnicki, K.B. Riggs, M.L. Taylor, and J. Chang: Decontamination assessment of Bacillus anthracis, Bacillus subtilis, and Geobacillus stearothermophilus spores on indoor surfaces using a hydrogen peroxide gas generator. Journal of Applied Microbiology 99: 739-748 (2005).

Rose, L.J., B. Jensen, A. Petersen, S.N. Banerjee, and M.J. Arduino,: Swab materials and Bacillus anthracis spore recovery from nonporous surfaces, Emerging Infectious Disease 10(6): 1023-1029 (2004).

Roy, C.H., M. Hale, J.M. Hartings, L. Pitt, and S. Duniho: Impact of inhalation exposure modality and particle size on the repiratory desposition of ricin in BALB/c mice. Inhalation Toxicology 15(6): 619-38 (2003).

Rule, A.M., J. Kesavan, K.J. Schwab, and T.J. Buckley: Application of flow cytometry for the assessment of preservation and recovery efficiency of bioaerosol samplers spiked with Pantoea agglomerans. Environmental Science Technology 41: 2467-2472 (2007).

Ryan, J.R., and J.F. Glarum: Biosecurity \& Bioterrorism: Containing and Preventing Biological Threats. Elsevier: New York, 2008.

Sanderson, W.T., M.J. Hein, L. Taylor, B.D. Curwin, G.M. Kinnes, T.A. Seitz, T. Popovic, H.T. Holmes, M.E. Kellum, S.K. McAllister, D.N. Whaley, E.A. Tupin, T. Walker, J.A. Freed, D.S. Small, B. Klusaritz, and J.H. Bridges: Surface sampling methods for Bacillus anthracis spore contamination. Emerging Infectious Diseases 8: 1145-1151 (2002).

Sanderson, W.T., R.R. Stoddard, A.S. Echt, C.A. Piacitelli, D. Kim, J. Horan, M.M. Davies, R.E. McCleery, P. Muller, T.M. Schnorr, E.M. Ward, and T.R. Hales: Bacillus anthracis contamination and inhalational anthrax in a mail processing and distribution center. Journal of Applied Microbiology 94: 1048-1056 (2004). 
Sagripanti, J.L., M. Carrera, J. Insalaco, M. Ziemski, J. Rogers, \& R. Zandomeni: Virulent spores of Bacillus anthracis and other bacillus species deposited on solid surfaces have similar sensitivity to chemical decontaminants. Journal of Applied Microbiology 102: 11-21 (2007).

Scheffrahn, R.: Whole-Structure Decontamination of Bacterial Spores by Methyl Bromide Fumigation. In Report on Workshop on Decontamination, Cleanup and Associated Issues for Sites Contaminated with Chemical, Biological, or Radiological Materials, S. Dun, and J. Wood, US EPA: October 2005.

Schlesinger, R.B.: Comparative deposition of inhaled aerosols in experimental animals and humans: a review. Journal of Toxicology and Environmental Health 15: 197-214 (1985).

Schuerger, A.C., S. Trigwell, and C.I. Calle: Use of non-thermal atmospheric plasmas to reduce the viability of Bacillus subtilis on spacecraft surfaces. International Journal of Astrobiology 7: 47-57 (2008).

Seto, Y.: Research and development of on-site decontamination system for biological and chemical warfare agents. Journal of Health Science 57(4): 311-333 (2011).

Shieh, W., J. Guraner, C. Paddock, P. Greer, K. Tatti, M. Fischer, M. Layton, M. Philips, E. Bresnitz, C.P. Quinn, T. Popovic, B.A. Perkins, S.R. Zaki, and the Anthrax Bioterrorism Investigation Team: The critical role of pathology in the investigation of bioterrorism-related cutaneous anthrax. American Journal of Pathology 163: 1901-1910 (2003).

Shin-Ichi, A., I.B. Zhulin, L. Marquez-Magana, and G.W. Ordal: Chemotaxis and Motility. In Bacillus subtilis and Its Closest Relatives: From Genes to Cells. A.L. Sonenshein, J.A. Hoch, and R. Losick (eds.), pp 437-452. Washington, DC: ASM Press, 2002.

Sinclair, R., S.A. Boone, D. Greenberg, P. Keim, and C.P. Gerba: Persistence of category A select agents in the environment. Applied Environmental Microbiology 74: 555-563 (2008).

Sonenshein, A.L., J.A. Hoch, and R. Losick: Bacillus subtilis and Its Closest Relatives. In From Genes to Cells, xv-xvi, Washington, DC: ASM Press, 2002.

Sonenshein, A.L., J.A. Hoch, and R. Losick: Bacillus subtilis and Other Gram-Positive Bacteria: Biochemistry, Physiology, and Molecular Genetics, xi-xiii, Washington, DC: ASM Press, 1993.

Stratis-Cullum, D.N., G.D. Griffin, J. Mobley, A.A. Vass, and T. Vo-Dinh: A miniature biochip system for detection of aerosolized Bacillus globigii spores. Analytical Chemistry 75: 275-280 (2003).

Tang, J.W., Y. Li, I. Fames, P.K. Chan, and G.L. Ridgway: Factors involved in the aerosol transmission of infection and control of ventilation in healthcare premises. Journal of Hospital Infection 64: 100-114 (2006). 
Teeuw, K.B., C.M. Vandenbroucke-Grauls, and J. Verhoef: Airborne gramnegative bacteria and endotoxin in sick building syndrome. A study in Dutch governmental office buildings. Archives of Internal Medicine 154: 2339-2345 (1994).

Tellier, R.: Review of aerosol transmission of influenza A virus. Emerging Infectious Diseases 12: 1657-1662 (2006).

Terzieva, S., J. Donnelly, V. Ulevicius, S.A. Grinshpun, K. Willeke, G.N. Stelma, and K.P. Brenner: Comparison of methods for detection and enumeration of airborne microorganisms collected by liquid impingement. Applied Environmental Microbiology 62: 2264-2272 (1996).

Thatcher, T.L., and W.W. Nazaroff: Effects of small-scale obstructions and surface textures on particle deposition from natural convection flow. Aerosol Science Technology 27: 709-725 (1997).

Thomas, R.J., D. Webber, W. Sellors, A. Collinge, A. Frost, A.J. Stagg, S.C. Bailey, P.N. Jayasekera, R.R. Taylor, S. Eley, R.W. Titball: Characterization and deposition of respirable large- and small-particle bioaerosols. Applied and Environmental Microbiology 74(20): 64376443 (2008).

Thompson, M.W., J. Donnelly, S.A. Grinshpun, A. Juozaitis, and K. Willeke: Method and test system for evaluation of bioaerosol samplers. Journal of Aerosol Science 25(8): 1579-1593 (1994).

Tong, Y., and B. Lighthart: Effect of simulated solar radiation on mixed outdoor atmospheric bacterial populations. FEMS Microbiology Ecology 26: 311-316 (1998).

Tong, Y., and B. Lighthart: Solar radiation has a lethal effect on natural populations of culturable outdoor atmospheric bacteria. Atmospheric Environment 31: 897-900 (1997).

Uhm, H.S., H.Y. Lee, Y.C. Hong, D.H. Shin, Y.H. Park, Y.F. Hong: A decontamination study of simulated chemical and biological agents. Journal of Applied Physics 102 (2007).

United States Army Medical Research Institute for Infectious Diseases (USAMRIID): Medical Management of Biological Casualties Handbook, $6^{\text {th }}$ Ed. J.B. Woods (Ed) Fort Detrick, Maryland, 2005.

United States Environmental Protection Agency: Federal on-scene coordinator's report for the Capitol Hill site, EPA Pub No. GS-10F-0076K, Washington DC, 2002.

Verce, M.F., B. Jayaraman, T.D. Ford, S.E. Fisher, A.J. Gadgil, and T.M. Carlsen: Minimizing decomposition of vaporized hydrogen peroxide for biological decontamination of galvanized steel ducting. Environ Sci Technol 42: 5765-5771 (2008). 
Wagner, A., C.F. Green, V. Pedregon, E. Barth, S.G. Gibbs, and P.V. Scarpino: Inactivation of Bacillus subtilis on gypsum board using aerosolized chemical agents. Journal of Environmental Engineering Science 7: 159-164 (2008).

Walinder, R., D. Norback, B. Wessen, and P. Venge: Nasal lavage biomarkers: effects of water damage and microbial growth in an office building. Archives of Environmental Health 56: 30-36 (2001).

Wang, Z., T. Reponen, S.A. Grinshpun, R.L. Gorny, and K. Willeke: Effect of sampling time and air humidity on the bioefficiency of filter samplers for bioaerosol collection. Journal of Aerosol Science 32(5): 661-674 (2001).

Watson, A., and D. Keir: Information on which to base assessments of risk from environments contaminated with anthrax spores. Epidemiology and Infection 113: 479-90 (1994).

Weber, T.P., and N.I. Stilianakis: Inactivation of influenza A viruses in the environment and modes of transmission: a critical review. Journal of Infection 57: 361-373 (2008).

Weis, C.P., A.J. Intrepido, A.K. Miller, P.G. Cowin, M.A. Dumo, J.S. Gebhardt, and R. Bull: Secondary aerosolization of viable Bacillus anthracis spores in a contaminated US Senate office. Journal of the American Medical Association 288(22): 2853-2858 (2002).

Whitley, R.J.: Smallpox: a potential agent of bioterrorism, Antiviral Research 57: 7-12. In Handbook of Viral Bioterrorism and Biodefense, E. De Clercq, and E.R. Kern (eds.). New York: Elsevier, 2003.

Wick, R.L., and L.A. Irvine: The microbiological composition of airliner cabin air. Aviation, Space, and Environmental Medicine 66: 220-224 (1995).

Willeke, K., X. Lin, and S.A. Grinshpun: Improved aerosol collection by combined impaction and centrifugal motion. Aerosol Science Technology 28: 439-456 (1998).

Witschger, O., K. Willeke, S.A. Grinshpun, V. Aizenberg, J. Smith, and P.A. Baron: Simplified method for testing personal inhalable aerosol samplers. Journal of Aerosol Science 29: 855-874 (1998).

World Health Organization: Public health response to biological and chemical weapons: WHO guidance, Geneva, Switzerland, 2004, Available at http://www.who.int/csr/delipepidemics/biochemguide/en

World Health Organization: Global Tuberculosis Program-Tuberculosis Fact Sheet. Geneva, Switzerland; 1998, Available at http://wwwwho.int/gtb/publications/ factsheet/index.htm. 
World Health Organization: Laboratory biosafety manual, $3^{\text {rd }}$ ed. Geneva, Switzerland; 2004, Available at http://www.who.int/csr/resources/publications/biosafety/en/Biosafety7.pdf.

Yah, M., and G. Mainelis: Use of portable microbial samples for estimating inhalation exposure to viable biological agents. Journal of Exposure Science and Environmental Epidemiology 17: 31-38 (2007). 


\section{CHAPTER 2 -- DESIGN AND EVALUATION OF A BIOAEROSOL DEPOSITION CHAMBER FOR TESTING DECONTAMINATION OF AEROSPACE MATERIALS}

\section{SUMMARY}

The aim of this study was to design, build, and test an aerosol deposition chamber that could be used to model real-world contamination using a Bacillus anthracis simulant. The test chamber, constructed from aluminum, offered a unique approach to deposit spores onto coupons to test decontamination rates using high temperature and humidity. Initial testing was completed using fluorescent particles; however, the limit of quantification could not be reached with these particles so Bacillus atrophaeus subsp globigii (BG) spores were used to model deposition. Initial tests demonstrated the parameters that could be controlled through the experiments. After these were evaluated, four final tests were completed to perform more in-depth statistical analysis. The coefficients of variation for these tests were within acceptable ranges (all were $25.5 \%$ or less). Ryan-Joiner tests were performed on the data and showed that 2 of the 4 tests displayed a lognormal distribution, while the other 2 tests were inconclusive. All data was therefore treated as a lognormal distribution. Contour plots were then constructed to determine if a discernible pattern was present. While these contour plots showed a somewhat even dispersion, there were no discernible patterns. Additionally, the plots showed a wide range of spore deposition throughout the four tests. Finally, the equations derived for spore deposition were validated. The data showed that $8.67 \%$ up to $31.0 \%$ (average of $20.25 \%$ ) of the spores modeled could actually deposit and be recovered through culture methods. These losses could have occurred during the nebulization through inactivation or clumping after the spores were aerosolized. Regardless, this showed that the equations could be used after accounting for these 
losses. The study demonstrated that the test chamber can be used for spore depositions with the caveat that future studies include an appropriate control coupon next to each sample.

\section{INTRODUCTION}

Bioterrorism is defined as a use or threatened use of biological agents against individuals to obtain advantage for specific purpose such as intimidation, ideological principles, or disruption of everyday activities (Brachman 2002). In an act of biological terrorism or warfare, diagnosis of the agent in a short time can be difficult (Estill et al., 2009), which may hamper decontamination efforts. To minimize illnesses, decontamination of materials to an acceptable level in a short time is critical. Once decontamination is conducted, another difficulty is detecting the agents post-decontamination to ensure they have been adequately removed and/or inactivated (Uhm et al., 2007). Any of these complications can impact military missions, both here and in deployed locations. DoDI 3150.09 "The Chemical, Biological, Radiological, and Nuclear (CBRN) Survivability Policy" requires all DoD assets to be able to continue operations even in the presence of biological agents, including the capability to be decontaminated properly (DoD, 2009).

All existing biological decontamination solvents shown to inactivate biological agent threats are at least somewhat hazardous to aircraft materials, therefore, there are currently no methods approved to decontaminate Air Force aircraft. However, all aircraft must meet strict engineering specifications. While these specifications do not allow for chemical disinfection, all aircraft must withstand high temperature storage greater than $185^{\circ} \mathrm{F}$ at $100 \%$ relative humidity. These ranges give a potential decontamination method if an agent can be inactivated within ranges (AFRL, 2008). 
Any decontamination methods must be able to inactivate Bacillus anthracis. The US Army Edgewood Chemical and Biological Center (ECBC) requires that the decontamination methods be effective against spores. These spores are required not only to facilitate the inactivation, but also because a first responder will not necessarily know the type of microorganism involved (Brickhouse, 2005). The Bacillus anthracis spore is the target because they are considered the most difficult biological warfare agent to decontaminate. Bacillus anthracis endospores are metabolically inactive and are highly resistant to many physical stresses such as wet and dry heat, chemical agents, UV and gamma radiation, oxidizing agents, vacuums and ultra-high hydrostatic pressures (Nicholson et al., 2002). The spores are very stable for up to 60 years in soil and water and can resist sunlight for varying periods (Chosewood and Wilson, 2009; Perkins, 1983). For these reasons, the spores can remain viable for years creating a serious and lasting health risk (Nicholson et al., 2002).

Additionally, these spores can become re-aerosolized after an initial exposure, causing illnesses when personnel had no direct exposure to the spore release zone (CDC 2001; Jernigan et al., 2001). The anthrax letter attacks in 2001 showed that re-aerosolization was a problem in that there were cases of anthrax among persons that did not handle the contaminated materials but were in the same room. Further studies have shown that the person who opened the letter effectively became a walking disseminator of spores (Kornikakis et al., 2009).

Bacillus anthracis spores are a target for decontamination because anthrax is considered a good biowarfare agent for several reasons. First, the species is easy to cultivate and spore formation is readily induced. Second, the spores are highly resistant to sunlight and heat and disinfectants are not as effective in inactivating the spores. Additionally, the spores can be produced in wet or dry form and can be stabilized for weaponization, and the spores can be 
delivered as an aerosol cloud either from line source (aircraft flying upwind) or as point source (spray) (USAMRIID, 2005). Of the Category A agents, which are the highest risk agents identified by the CDC, Bacillus anthracis spores are the most stable in the environment (Cordesman, 2005; Ryan and Glarum, 2008; Sinclair et al., 2008). Also, diagnosis for anthrax is difficult (Estill et al., 2010). Finally, the dose for anthrax infectivity is small. For instance, the number of spores required for inhalation exposures is in the range of 8,000 to 15,000 spores (USAMRIID, 2008), down to 2,500-55,000 spores (Fitch, 2008; Inglesby et al., 2002).

Cutaneous anthrax infection can be caused by 10 spores or fewer (Peters and Hartley, 2002; Watson and Keir 1994) while some risk predictions having shown that infective doses may be as low as 1 to 3 spores (Patrick, 1999). Anthrax could be available for non-state actors as well: the Soviet Union reportedly had a large biowarfare program, producing up to 100 tons of anthrax. The Soviet research resulted in a release of anthrax spores in 1979 near Sverdlovsk in Russia. There were 66 human fatal cases. Sheep and cattle were affected as far as $50 \mathrm{~km}$ downwind (Alibek, 2005).

The causative agent for anthrax is Bacillus anthracis, which is an encapsulated, aerobic, gram-positive, spore-forming, rod shaped (Bacillus) bacterium (CDC, 2002; USAMRIID, 2005). Because of the health risk of the spores, simulants are often used as safe alternatives for research, with Bacillus subtilis var niger (also known as Bacillus globigii, or BG). These spores are used because they are considered a human risk group 1 organism by the CDC meaning they are not known to cause disease in normal, healthy humans (Chosewood and Wilson, 2009) and may be manipulated at BSL-1 levels. These spores are also very similar to Bacillus anthracis, especially the endospore (Hill et al., 1999; Mainelis et al., 2002; Sagripanti et al., 2007). These spores, like most of the Bacillus species, are non-pathogenic to humans (Priest, 1993; Sonenshein et al., 
1993). These spores are widely used in research due to their ubiquity, hardiness, availability, and relation to known pathogens (Aizenberg et al., 2000; Burton et al., 2005; Carrera et al., 2005; Coohill and Sagripanti 2008; Farnsworth et al., 2006; Foarde et al., 1999; Jensen, 1992; Maus et al., 2001; Li and Lin, 2001; Sagripanti et al., 2007; Wagner et al., 2008; Yah and Mainelis, 2007).

The literature also refers to Bacillus atrophaeus, which is a strain that is indistinguishable from Bacillus subtilis except that it creates a pigment on certain media. Some of the Bacillus subtilis species were actually renamed a new strain, Bacillus atrophaeus subsp globigii (Burke et al., 2004). Again, there are several tests and studies which have used Bacillus atropheaus (Brown et al., 2008a; Carrera et al., 2005; Kesavan, 2008; Lewandowski et al., 2010; Martin and Moore, 2001; Thomas et al., 2008;).

There are several methods for depositing a bioaerosol onto a testing medium. Direct inoculation is frequently used; however, the direct inoculation is not the most realistic method. For this reason, bioaerosol deposition test chambers are frequently used. There are many different examples of bioaerosol test chambers used for a variety of purposes, and these have been constructed from a number of materials, including Plexiglas ${ }^{\mathrm{TM}}$, stainless steel, and aluminum. One such chamber was constructed from Plexiglas ${ }^{\mathrm{TM}}$ with dimensions of 1.22 meters by 1.22 meters by 2.44 meters. Used to deposit actual Bacillus anthracis spores at low concentrations, it had to be air-tight, which was checked with several smoke particle tests. The chamber had an aerosol mixing element as well, where spores were introduced into the chamber through an ion air cannon static eliminator. Before generation, the chamber was evacuated for at least 20 minutes. Electronic fans were used to stir the aerosol in 1 minute consecutive intervals, with each fan activated for as short a time as possible to ensure settling was the primary 
deposition mechanism (Baron, et al., 2008). The chamber was used for several experiments, testing spore removal through wipe sampling (Baron et al., 2007; Baron et al., 2008; Estill et al., 2009). Another chamber was 64 cubic meters and included temperature and humidity control via computer. The size of the chamber required that a 24 -jet Collison nebulizer be used followed by a 10 milliCurie $\mathrm{Kr}-85$ source used for neutralization. Initial tests used inert particles; however, research has shown that biological particle tests are ultimately required (Kesavan, 2008). Another chamber, used to deposit Bacillus atrophaeus subsp. globigii (BG) spores onto flooring materials, had a volume over 35 cubic meters (Buttner et al., 2004). Farnsworth et al. (2006) developed a closed-loop wind tunnel test chamber to determine recovery efficiencies from HVAC systems, which was constructed from stainless steel (Farnsworth et al., 2006). Brown et al. (2007a, 2007b, 2007c) aerosolized BG spores into a chamber and produced surface concentrations in the range of $10^{2}$ to $10^{5}$ colony forming units per square centimeter for the purposes of swipe sampling. Their chamber had a cylinder mixing chamber, constructed from carbon steel with enamel-coated surface, and a diameter of $45 \mathrm{~cm}$, a height of $30 \mathrm{~cm}$, and a total volume of $0.048 \mathrm{~m}^{3}$. Edmonds et al. (2009) developed a circular deposition chamber, with three separate zones, including a rotating base platform to ensure no single point in the chamber was exposed for an extended period of time (Edmonds et al., 2009). King et al. (2010) developed two test chambers study UV exposures on bioaerosols. The chambers, made of $1 / 4$ " Plexiglas ${ }^{\circledR}$, had an internal volume of $0.137 \mathrm{~m}^{3}$ and measured $0.91 \mathrm{~m}$ long, $0.43 \mathrm{~m}$ tall, and $0.35 \mathrm{~m}$ deep. Other chambers have been constructed from aluminum to study different aerosol depositions (Byrne et al., 1995; Feather and Chen, 2003; Lai et al., 2002). Lewandowski et al. (2010) constructed a test chamber made clear cast acrylic with an interior volume of $0.5 \mathrm{~m}^{3}$ and bottom surface area was $89 \mathrm{~cm}$ wide by $74 \mathrm{~cm}$ wide. Several other researchers have constructed 
chambers over $1 \mathrm{~m}^{3}$ in volume (Chen et al., 1999; Marple and Rubow, 1983; Kenny et al., 1999; Koch et al., 1999). Park et al. (2009) completed a cubical test chamber of $2.5 \mathrm{~m}$ to study fly ash. Thatcher and Nazaroff (1997) constructed a 1.8 cubic meter aluminum chamber to measure deposition velocity under natural convective conditions on rough surfaces.

Aerosolization of the bioaerosol is another key consideration. One of the most widely used methods is the Collison nebulizer, which uses an air blast nebulization technique utilizing compressed air to draw liquid from a reservoir. The high velocity of the air breaks the liquids into droplets which are suspended as part of the aerosol (Fitch, 2008). First described in 1973 by May, these nebulizers have been used to generate small-particle aerosols with mass median aerodynamic diameter (MMAD) of 1 to $3 \mu \mathrm{m}$ (May, 1973). The literature has many examples these nebulizers are used to deliver a bioaerosol, especially spores. For instance, Wagner et al. (2008) used a 6-jet Collison nebulizer (BGI, Inc, Waltham, MA) to aerosolize Bacillus subtilis spores to test the effectiveness of decontamination of gypsum boards using $0.05 \%$ chlorine dioxide $\left(\mathrm{ClO}_{2}\right), 0.6 \%$ sodium hypochlorite $(\mathrm{NaOCl})$, and distilled water. Han et al. (2010) used a 6-jet Collison nebulizer to aerosolize the vegetative form of Bacillus subtilis to test the collection methodology of a new system using electrostatic forces. Krumins et al. (2008) used a Collison nebulizer to aerosolize a test aerosol with $5 \mathrm{~L} / \mathrm{min}$ of filtered nitrogen, diluted with $45 \mathrm{~L} / \mathrm{min}$ of ambient air that was filter-sterilized through a $45 \mu \mathrm{m}$ filter to dry any liquid water that could have resulted in particle agglomeration. A Collison nebulizer was used to generate $\mathrm{KCl}$ salt particles at duct flow rates of $240 \mathrm{~L} / \mathrm{s}$ and $940 \mathrm{~L} / \mathrm{s}$ (Farnsworth et al., 2006). Another study involved generating several different types of bioaeroaols using a 24-jet Collison nebulizer to generate Bacillus atrophaeus cells in PBS (Kesavan, 2008). 
This research project designed and tested an aerosol test chamber and dispersion method to model real-world bioaerosol contamination methods. All previous decontamination tests have utilized direct inoculation methods. The test chamber was designed to provide a more realistic method in which the spores will deposit on the testing materials, which were typical aircraft components. The chamber was eventually used to disperse Bacillus anthracis spore simulants onto aircraft materials to test different inactivation rates using high heat and humidity. This was the first chamber designed for this purpose.

\section{METHODS}

\section{Bioaerosol Test Chamber Design}

A bioaerosol test chamber was designed to allow biological particles to deposit uniformly onto test coupons. The initial design was created using TurboCAD ${ }^{\circledR}$ Deluxe 2D/3D (C) 2010 Open Design Alliance, IMSI/Design, Novato, CA). The overall interior dimensions of the test chamber were 1.49 meter (4.9 feet) in length, 1.22 meter ( 4 feet) in height, and 0.86 meter (2.82 feet) in width. The original design called for a width of 0.9 meters; however, this was reduced later because a slightly smaller width resulted in reduced construction costs. The total volume of the chamber was 1.4 cubic meters (49 cubic feet) and the surface area was 1.18 square meters (12.7 square feet).

To decrease static electricity impacts on aerosol generation and deposition, the chamber was constructed from metal. The chamber construction was priced for construction from 16gauge steel powder, 16-gauge stainless steel, and 16-gauge aluminum. Aluminum was chosen based on price and weight considerations.

A mixing element was designed to deliver contaminant free air into a Collison nebulizer for delivery into the test chamber. The mixing element was made from aluminum like the rest of 
the test chamber and was constructed by Design Metal Manufacturing (DMM) of Fort Collins, Colorado. The dimensions of the mixing element were 0.5 meter in length, 0.25 meter in height, and 0.25 meter in width. The mixing element had a working opening of 0.28 meter by 0.11 meter, sealed with Plexiglas ${ }^{\circledR}$. These dimensions were large enough to include space for both a Collison nebulizer and neutralizer.

The test chamber was designed with two glove port openings. These glove ports were purchased from TerraUniversal $^{\circledR}$ (Glove port, plastic, 10” diameter; Glove, 13 inch, unlined, nitrile; straight sleeve, nitrile, 10 " port, 22" long). The openings required for each glove port was circular with a diameter of 0.25 meters. These gloves and ports were self-sealing once installed. Additional openings were closed with Plexiglas $®$, cut and drilled by DMM. There was a permanent viewing window directly above the glove ports. The viewing window had dimensions of 0.75 meter by 0.15 meter. A working area was placed on the opposite side of the glove ports. The working area opening, with dimensions of 0.85 meter by 0.5 meter, was used to insert and remove samples. An additional working and viewing chamber was installed on the top of the chamber with dimensions of 0.8 meter by 0.5 meter. Each of these openings was sealed with Plexiglas ${ }^{\circledR}$ cut to overlap the openings by $0.05 \mathrm{~m}$ to ensure an adequate seal. A silicone rubber gasket, 1/16" (0.062”) thick (DieCutTech, Denver, CO) was used to seal each opening. The gasket material was received in a roll and then cut to conform to each opening. Additionally, silicone coating material (Loctite ${ }^{\circledR}$, Silicone Lubricant) was placed on each gasket to ensure an adequate seal. Fans were also installed in each corner of the test chamber.

A HEPA filter (Air Handler ${ }^{\circledR}$, Dayton Electric Manufacturing Company, SN 665729, 12 inch by 12 inch by 11.5 inch) was installed to ensure that bioaerosols were not released from the chamber. The HEPA filter was purchased prior to the construction and given to DMM for 
fabrication to ensure a proper seal. Numerous additional openings were made in the chamber to include sampling ports and power for mixing fans. Additionally, a Dwyer Magnehelic ${ }^{\circledR}$ differential pressure gauge, Model 2301 (range—inches of water -0.5 to 0.5 ), was installed. These were each bored and sealed with sealant $\left(\mathrm{DAP}^{\circledR}, \mathrm{Kwik} \mathrm{Seal}^{\circledR}\right)$.

The test chamber and mixing element are depicted in Figures 2-1-2-3. Photographs of the completed test chamber are presented in Figures 2-4 and 2-5.

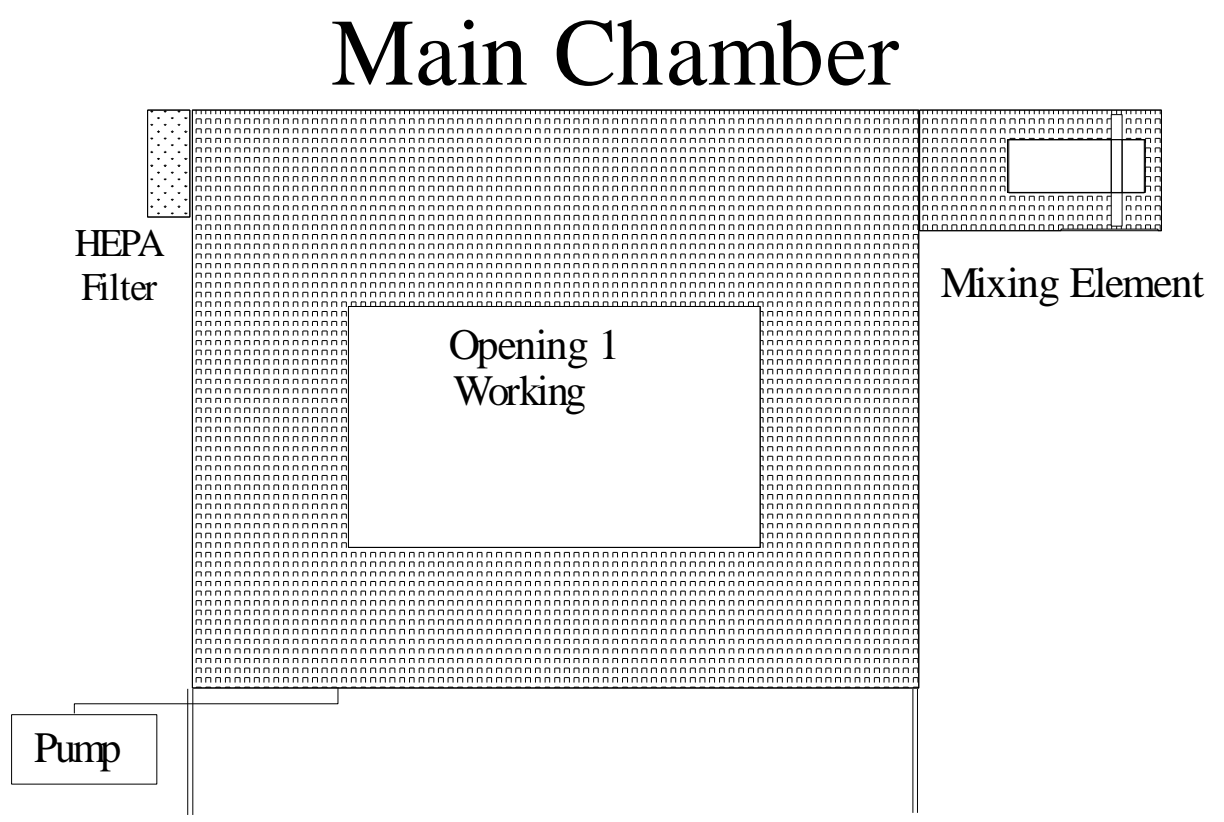

Figure 2- 1 - Test chamber, working side

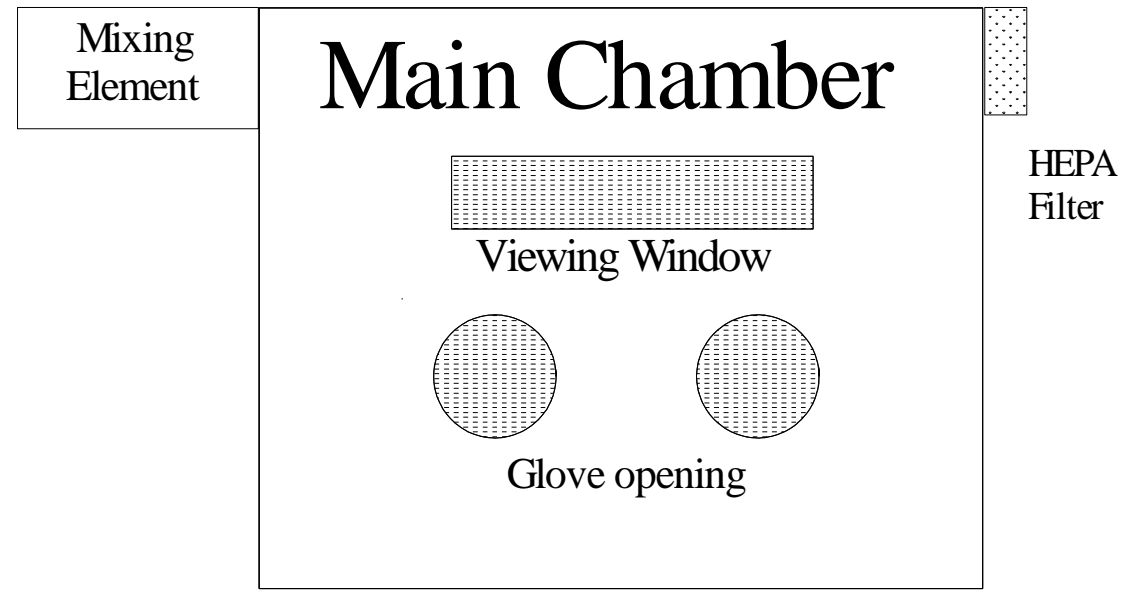

Figure 2- 2- Test chamber, glove port side 
Contaminant Free Air and Aerosol Inlet

Neutralizer Nebulizer

Figure 2- 3 - Mixing element

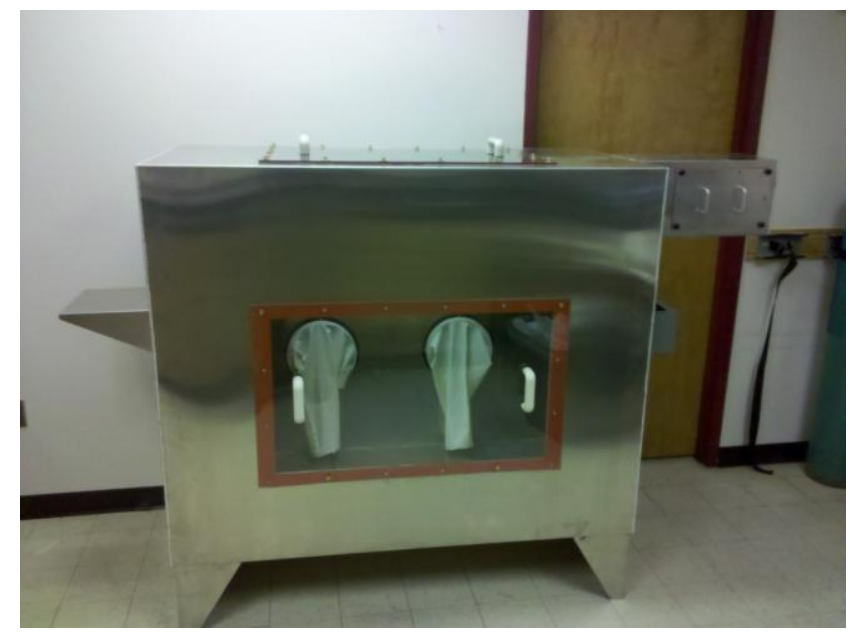

Figure 2- 4- Test chamber, working side

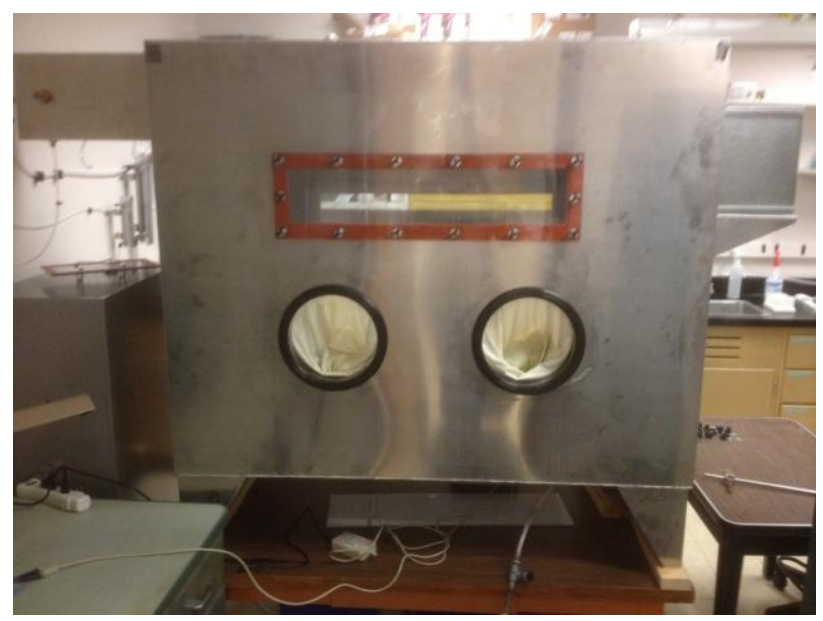

Figure 2- 5 - Test chamber, glove port side 


\section{Chamber seal tests}

After the chamber was constructed and sealed, leak tests were conducted to verify that the chamber was air tight. The first set of tests included using smoke tests (incense and smoke bombs) to observe any smoke leaking from the chamber. These smoke tests showed that there were no visible leaks. The second set of tests included using a leak detector (Swagelok ${ }^{\circledR}$ Snoop $^{\circledR}$ Leak detector) designed to form bubbles if there were a leak. The leak detector was placed on each opening, and then a positive and negative pressure was created in the test chamber using a Maxima C D4B pump (Fisher Scientific, Waltham, MA). Each positive and negative pressure check was completed twice. Finally, the air pump was used to create a vacuum in the chamber, the chamber was sealed, and the pump turned off. This action forced the gloves to raise perpendicular to the chamber floor. The gloves held this position for over 15 minutes, with the pump off and chamber sealed. This test was repeated three additional times.

\section{Chamber equations}

\section{Particle generation}

Equations for aerosol generation and deposition were derived to model the test chamber. This derivation started with the general ventilation equation (equation 1 below), modeled as shown in Figure 2-6 below.

$$
\begin{aligned}
& \text { (1) } \mathrm{C}_{\max }=\frac{\mathrm{G}}{\mathrm{Q}_{\mathrm{in}}} \quad \text { (Burgess, Ellenbecker, and Treitman, 2004) } \\
& \begin{aligned}
\mathrm{C}_{\max } & =\text { Maximum concentration }\left(\frac{\mathrm{CFU}}{\mathrm{m}^{3}}\right) \\
\text { Where: } \quad \mathrm{G} & =\text { Generation rate }\left(\frac{\mathrm{CFU}}{\text { minutes }}\right) \\
\mathrm{Q}_{\text {in }} & =\text { Air generation rate into chamber }\left(\frac{\mathrm{m}^{3}}{\text { minute }}\right)
\end{aligned} \\
& \text { (Controllable throughout experiment) }
\end{aligned}
$$




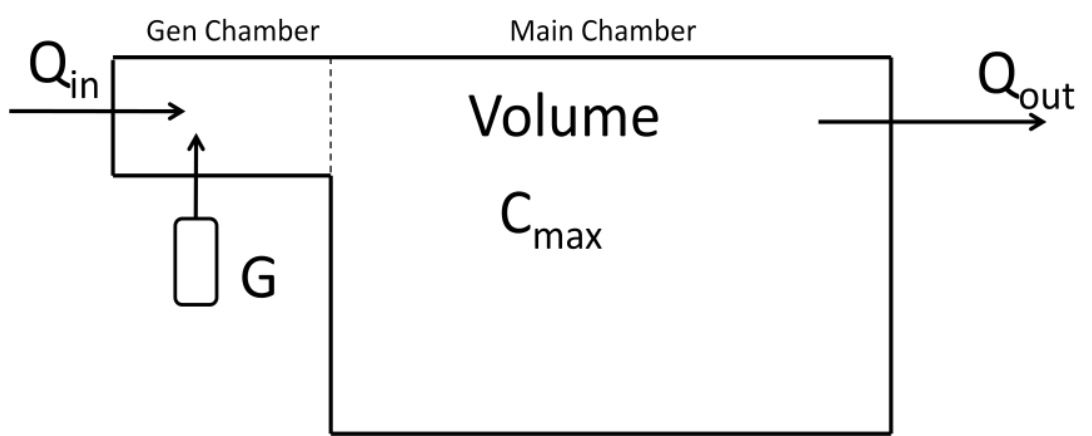

Figure 2- 6- Test chamber, deposition model

$\begin{aligned} \mathrm{C}_{\max } & =\text { Maximum concentration }\left(\frac{C F U}{m^{3}}\right) \\ \text { Where: } \quad \mathrm{G} & =\text { Generation rate }\left(\frac{C F U}{\text { minutes }}\right) \\ \mathrm{Q}_{\text {in }} & =\text { Air generation rate into chamber }\left(\frac{\mathrm{m}^{3}}{\text { minute }}\right)\end{aligned}$

(Controllable throughout experiment)

The generation rate, $\mathrm{G}$, was derived with equation 2 below. The $\mathrm{Q}_{\text {liq }}$ below is the amount of liquid that is generated from a Collison nebulizer and based on the pressure and number of jets of the nebulizer. Each jet requires approximately $2 \mathrm{Lpm}$ of air, producing droplets with a mass median aerodynamic diameter (MMAD) of $2.5 \mu \mathrm{m}$ with a geometric standard deviation (GSD) of 1.8. The amount of liquid used by the nebulizer depends on the back pressure that is applied to the nebulizer and is defined by equation 2 below.

(2) $\mathrm{G}=\mathrm{Q}_{\text {liq }} * \mathrm{C}_{\text {neb }}$

$$
\begin{aligned}
\mathrm{G} & =\text { Generation rate for spores }\left(\frac{\mathrm{CFU}}{\text { minute }}\right) \\
\mathrm{C}_{\mathrm{neb}} & =\text { Spore concentration in nebulizer }\left(\frac{\mathrm{CFU}}{\mathrm{mL}}\right) \\
& =\frac{\text { Total total particles }}{\text { Deionized water }(\mathrm{mL})} \\
\mathrm{Q}_{\text {liq }} & =\text { Liquid use rate for nebulizer }\left(\frac{\mathrm{mL}}{\mathrm{minute}}\right) \\
& =1.5 \frac{\mathrm{mL}}{\mathrm{hr}}=0.0253 \frac{\mathrm{mL}}{\mathrm{min}}(\text { for } 1-\text { jet nebulizer, BGI) } \quad(\mathrm{BGI}, 2008) \\
& =\left[-0.84859+0.2636 * \ln (\mathrm{psig})^{2}\right] \times \text { [number of jets] }
\end{aligned}
$$


Once the value for $\mathrm{C}_{\max }$ was solved, the desirable spore concentration on each coupon was determined.

(3) $\mathrm{Sv}=\mathrm{C}_{\max } * \mathrm{H}$

$$
\begin{array}{ll} 
& \mathrm{S}_{\mathrm{v}}=\text { Viable surface concentration }\left(\frac{\mathrm{CFU}}{\mathrm{m}^{2}}\right) \\
\text { Where: } & \mathrm{C}_{\max }=\text { Total number concentration }\left(\frac{\mathrm{CFU}}{\mathrm{m}^{3}}\right) \\
& \mathrm{H}=\text { Chamber height }(1.22 \mathrm{~m})
\end{array}
$$

This was further evaluated to determine the concentration on a coupon surface, assuming a one square inch area. This settling was based on assuming still air within the test chamber.

(4) $\mathrm{S}_{\mathrm{c}}=\mathrm{S}_{\mathrm{v}} * \mathrm{SA}_{\mathrm{C}}$

$$
\begin{array}{ll} 
& \mathrm{S}_{\mathrm{V}}=\text { Viable surface concentration }\left(\frac{\mathrm{CFU}}{\mathrm{m}^{2}}\right) \\
\text { Where: } & \mathrm{S}_{\mathrm{c}}=\text { Surface concentration per coupon }(\mathrm{CFU}) \\
& \mathrm{SA}_{\mathrm{c}}=\text { Coupon surface area }\left(1 \mathrm{in}^{2}=6.45 \times 10^{-4} \mathrm{~m}^{2}\right)
\end{array}
$$

The final model used for the test chamber was reduced to the equation below, which is the equation used to determine the variables that could be manipulated to change the concentration depositing on the coupons.

$$
\begin{aligned}
& \mathrm{S}_{\mathrm{c}}=\left(\left(\frac{\mathrm{G}}{\mathrm{Q}_{\text {in }}}\right) * \mathrm{H}\right) * \mathrm{SA}_{\mathrm{C}} \\
& \mathrm{S}_{\mathrm{c}}=\text { Surface concentration per coupon (CFU) } \\
& \mathrm{G}=\text { Generation rate for spores }\left(\frac{\mathrm{CFU}}{\text { minute }}\right) \\
& \text { Where: } \quad \mathrm{Q}_{\text {in }}=\text { Air generation rate into chamber }\left(\frac{\mathrm{m}^{3}}{\text { minute }}\right) \\
& \mathrm{H} \quad=\text { Chamber height }(1.22 \mathrm{~m}) \\
& \mathrm{SA}_{\mathrm{c}}=\text { Coupon surface area }\left(1 \mathrm{in}^{2}=6.45 \times 10^{-4} \mathrm{~m}^{2}\right)
\end{aligned}
$$

\section{Particle settle time}

Another critical aspect is the time required for the particle to settle. Settle time is based on the settling velocity (defined in equation 6) and the height of the chamber. 
(6) $\mathrm{V}_{\mathrm{TS}}=\frac{\left(\rho_{\mathrm{p}} * \mathrm{~d}^{2} * \mathrm{~g} * \mathrm{C}_{\mathrm{C}}\right)}{(18 * \eta)}$

(Hinds, 1999)

$$
\begin{aligned}
\mathrm{V}_{\mathrm{TS}} & =\text { Settling velocity }\left(\frac{\text { meter }}{\text { second }}\right) \\
\rho_{\mathrm{p}} & =\text { density of particle }\left(\mathrm{kg} / \mathrm{m}^{3}\right) \\
\mathrm{d} & =\text { particle diameter }(\mathrm{m}) \\
\mathrm{g} & =\text { acceleration of gravity }\left(\frac{\mathrm{meter}}{\mathrm{s}^{2}}\right) \\
\eta & =\text { viscosity of gas }(\text { air }),\left(\frac{\mathrm{Pa}}{\text { second }}\right) \text { or }\left(\frac{\mathrm{kg}}{\mathrm{m} * \mathrm{~s}}\right) \\
\mathrm{Cc} & =\text { Cunningham correction factor } \\
& =1.15 \text { (for } 1.0 \mu \text { m particle) }
\end{aligned}
$$

The required time was then calculated using the height of the test chamber.

(7) Time $_{\text {Set }}=\frac{H}{V_{\mathrm{TS}}}$

$$
\begin{array}{ll} 
& \text { Time }_{\mathrm{Set}}=\text { Time to setting }(\text { seconds }) \\
\text { Where: } & \begin{array}{l}
\mathrm{H} \\
\end{array} \\
\mathrm{V}_{\mathrm{TS}} \quad=\text { Height of chamber }(1.22 \text { meters })
\end{array}
$$

\section{Aerosol generation}

The supply air used for aerosol generation was filtered and dried to ensure it was contaminant free. It was then delivered to a Collison nebulizer (BGI Incorporated, Waltham, MA). Depending on the concentration, a 1-, 3-, or 6-jet nebulizer was used. The flow into the nebulizer was measured using a Dwyer ${ }^{\circledR}$ Rate-Master ${ }^{\circledR}$ Flowmeter, RMB-52 (5-50 SCFH Air) and controlled through an air control valve. An additional stream of air (henceforth called dilution air) was used to force the aerosol into the main testing chamber. This second stream of air was measured using a Dwyer ${ }^{\circledR}$ Rate-Master ${ }^{\circledR}$ Flowmeter, RMC-103 (20-200 SCFH Air) and controlled through an additional air control valve. Both the aerosol and the dilution air were neutralized using a TSI Kr-85 neutralizer (Shoreview, MN). Air pressure provided to the nebulizer was measured using a 2.5 inch pressure gauge, 0 to $30 \mathrm{psi}$, Ashcroft ${ }^{\circledR}$ Instruments (Stratford, CT). 
Polyethylene sheeting (Grainger, Lake Forest, IL) was used to cover the floor of the chamber during tests. All required items (sample coupons, bleach for decontamination, etc.) were placed in the chamber, which was then sealed. Once sealed, it was pumped down for approximately 20 minutes using a Maxima C D4B pump (Fisher Scientific, Waltham, MA) to minimize particles that could interfere with the tests. Following the evacuation, the only air that entered the chamber was the contaminant free air, and all air that exited was through the HEPA filter at a flow rate equal to the dilution air.

\section{Fluorescent particles}

Initial particle deposition tests were completed by aerosolizing Thermo Scientific, Fluoro-Max ${ }^{\mathrm{TM}}$ Green Fluorescent Polymer Microspheres (or polystyrene latex spheres-PSL) of diameter $1.0 \mu \mathrm{m}$ onto 3 -inch by 3-inch square polyethylene sheets. A standardized calibration curve for volume of PSL was constructed using an initial concentration of 1.81 x $10^{10}$ particles per $\mathrm{mL}$. Three replicate trials using seven 1:10 serial dilutions were completed, analyzed, and plotted.

The PSL was dissolved and diluted in a solution containing $25 \mathrm{~mL} 29 \%$ ammonium hydroxide, $200 \mathrm{~mL}$ deionized water, $2 \mathrm{~mL} \mathrm{1 \%} \mathrm{ethyl} \mathrm{acetate,} \mathrm{and} 2 \mathrm{~mL} \mathrm{1 \%}$ pyridine. The ethyl acetate was added to break apart the PSL particles so the dye within the particles could be measured. The fluorescent dye in the extraction solutions was measured using a FLx800 Microplate Fluorescence Reader (BIO-TEK Instruments, Inc.) fluorescence spectrophotometer to determine particle concentration. This was then used to establish a limit of detection (LOD) and limit of quantification (LOQ) based on volume. The LOQ is the concentration at which the quantitative results may be obtained with a certain degree of confidence. According to Keith, et 
al. (1983), the LOQ is the amount of analyte that will rise to a signal that is 10 times the standard deviation of the signal from a series of blanks. Based on this, the limit of quantification (LOQ) was calculated by using the following equation:

Limit of Quantification $(\mathrm{LOQ})=(10 \times$ Standard Deviation $)+$ mean (Keith et al., 1983) After the calibration curve was constructed, tests using aerosols were conducted to verify this calibration curve. The calibration curve was constructed by adding the fluorescent particles and deionized water to a 1 -jet Collison nebulizer generating particles for 60 minutes. The particles were settled and collected on 3-inch by 3-inch square polyethylene sheets. Settling time was at least 10 hours. Six samples were collected for each test, the locations in the test chamber evenly dispersed on the chamber floor as depicted in Figure 2-4. After the deposition time, the sample sheets were placed in $\mathrm{BD}$ Falcon ${ }^{\mathrm{TM}} 15 \mathrm{~mL}$ polystyrene conical tubes with the solution described above. Each tube was then shaken vigorously for 2 minutes and vortexed for 10 seconds. The solution was then added to a 96 well plate and analyzed with a fluorescence detector described above. Thirteen tests were completed, with sample locations depicted in Figure 2-7. 


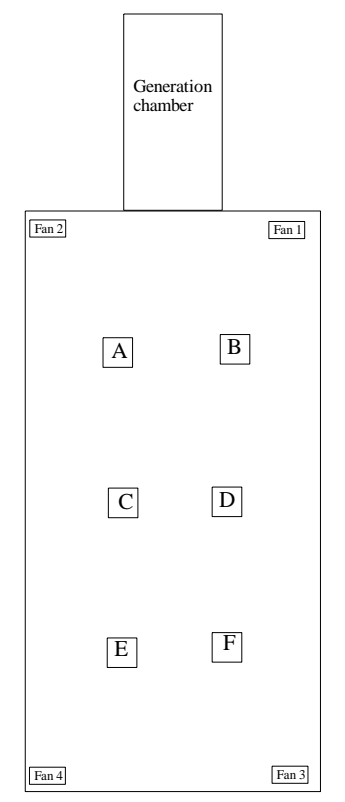

Figure 2- 7 - Fluorescent particle test locations on chamber floor

Spores

Following the fluorescent particle tests, depositions were tested using Bacillus atrophaeus subsp globigii (BG) spores, which were purchased from Yakibou, Inc (Apex, NC). The spores were acquired in two concentrations-3.1 × $10^{8}$ spores $/ \mathrm{mL}$ and $3.1 \times 10^{9}$ spores $/ \mathrm{mL}$. For nebulization, the spores were diluted in sterile Phosphate Buffered Saline with 0.05\% Tween 20 (Fisher Scientific, Waltham, MA).

A deposition goal of 300 spores per petri dish was used as this level was a quantifiable number that could be differentiated. The petri dishes used were $100 \mathrm{~mm}$ x $15 \mathrm{~mm}$ style, Becton, Dickinson and Company (Sparks, MD), filled with 40 mL of BBL ${ }^{\mathrm{TM}}$ TSA II Trypticase ${ }^{\mathrm{TM}}$ Soy Agar, Modified (Becton, Dickinson, and CO, Sparks, MD). Based on this goal, the equations presented previously were used to determine the variables for the experiments. The petri dishes used have a surface area of $6.082 \times 10^{-3} \mathrm{~m}^{2}$, which was used in the models. The equations 
showed that $5.16 \mu \mathrm{L}$ of the $3.1 \times 10^{8}$ spore/mL would provide approximately 300 spores per plate, without consideration for losses or survival of the spores.

Before each test, a new plastic sheet was placed on the chamber floor and a spray bottle with $10 \%$ sodium hypochlorite bleach was placed in the chamber. The chamber was then sealed and evacuated for at least 10 minutes. The covers for the petri dishes were removed and nebulization of the spores was then conducted using a 1-jet Collison nebulizer at 20 psi with a neutralizer installed. The solution in the nebulizer was a volume of $6 \mu \mathrm{L}$ spores $\left(3.10 \times 10^{8}\right.$ spores/mL) and $20 \mathrm{~mL}$ sterile PBS with $0.05 \%$ Tween 20 . The dilution air was set at 50 liter/min. The nebulization continued for 30 minutes. After the 30 minute time period, the air to the nebulizer was shut-off, and the dilution air was continued to run for 5 minutes to disperse the spores into the test chamber. The spores were allowed to settle for at least 10 hours. After the settling time, the petri dishes were recovered and the chamber was sprayed with the $10 \%$ sodium hypochlorite bleach. At least 30 minutes contact time was allowed for proper spore inactivation. Each test run included 18 tests, evenly dispersed throughout the chamber, as depicted in Figure 2-5. The samples included one negative control and one positive control for each sample run. After removal from the chamber, the petri dishes were placed in an incubator at $37^{\circ} \mathrm{C}$ and read at 24, 48, and 72 hours. There were 29 experiments conducted with samples locations depicted in Figure 2-8. 


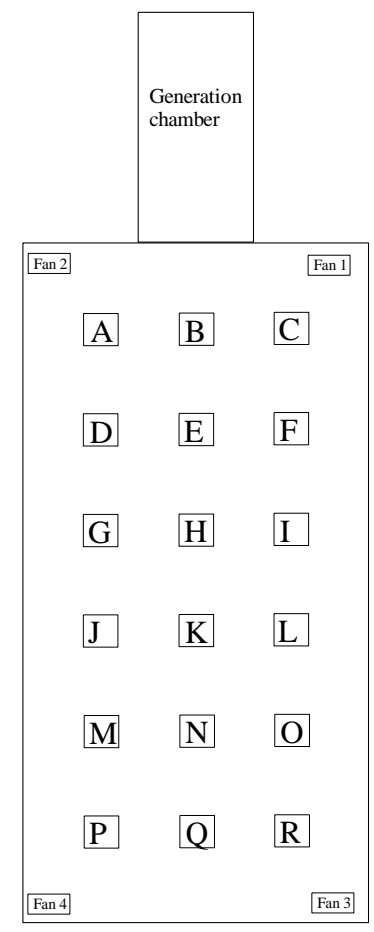

Figure 2- 8 - Spore deposition test locations on chamber floor

\section{Data Management and Statistics}

The distribution of the data (normal versus lognormal) was analyzed using histograms and Ryan-Joiner analyses in Minitab®, v16.1.1 (State College, PA). This analysis was completed using both the non-transformed and log transformed values of the spores that deposited in the chamber. Contour plots were constructed in Minitab® to determine the deposition patterns. Finally, regression models were completed on the deposition data, with residual analysis completed.

\section{RESULTS}

\section{Fluorescent particles}

The limit of detection (LOD) for the fluorescent particles was 11 relative fluorescence units (RFU). After the standardized curves were constructed, the value of $11 \mathrm{RFU}$ correlated to $6.89 \times 10^{8}$ particles. A RFU value of 50 was the limit of quantification (LOQ), which would 
have required a particle count of $3.22 \times 10^{9}$ particles. The RFU of 50 would have required 38 $\mathrm{mL}$ of particle solution for detection. One $60 \mathrm{~mL}$ bottle of these particles costs over $\$ 700$; therefore, the fluorescent particle methods was cost prohibitive to complete. A total of 13 tests were completed, using up to $750 \mu \mathrm{L}$ PSL particles in $20 \mathrm{~mL}$ deionized water. These tests confirmed this method was inconclusive.

\section{Spores}

Twenty-nine total tests were completed using Bacillus atrophaeus subsp globigii (BG) spores to test the deposition. These tests were used to determine the optimal operating parameters of the test chamber. For instance, muffin fans installed were evaluated and shown to increase the coefficient of variation (CV) of the spore deposition to $51.5 \%$. Because of the high CV level, these fans were not used. The final parameters for the deposition tests are included the Table 2-1.

Table 2 - 1 - Final test chamber operating parameters

\begin{tabular}{|l|l|}
\hline Nebulizer & 1 -jet \\
\hline Nebulizer pressure & $20 \mathrm{psi}$ \\
\hline Fans & Off \\
\hline Dilution air & 50 Liters/min \\
\hline Spore volume & $6 \mu \mathrm{L}$ spores \\
\hline Spore concentration & $3.10 \times 10^{8}$ spores $/ \mathrm{mL}$ \\
\hline Neutralizer & $\mathrm{Kr}_{85}$ \\
\hline Dilution fluid & $\begin{array}{l}20 \mathrm{~mL}, \text { sterile PBS } \\
\text { with } 0.05 \% \text { Tween }\end{array}$ \\
\hline Chamber evacuation & 10 minutes \\
\hline Nebulization & 30 minutes \\
\hline $\begin{array}{l}\text { Generation chamber } \\
\text { evacuation }\end{array}$ & 5 minutes \\
\hline Settling time & 9.5 hours \\
\hline
\end{tabular}

These test chamber operating parameters were then used for four additional tests. All tests completed on this project were numerically numbered in the order of completion. Therefore, these four final tests were numbered $72,76,80$, and 81 . These final tests had CV 
values $25.5 \%$ or lower, which was close to the overall goal of a CV of $25 \%$ or less for each deposition test. The spore deposition summaries are included in Table 2-2.

Table 2 - 2 - Spore deposition tests summary

\begin{tabular}{|c|c|c|c|c|}
\hline Spore number & Test 72 & Test 76 & Test 80 & Test 81 \\
\hline High value & 114 CFU/plate & 38 CFU/plate & 68 CFU/plate & 154 CFU/plate \\
\hline Low value & 51 CFU/plate & 18 CFU/plate & 38 CFU/plate & 60 CFU/plate \\
\hline Average & 71 CFU/plate & 26 CFU/plate & 53 CFU/plate & 93 CFU/plate \\
\hline $\begin{array}{c}\text { Standard } \\
\text { Deviation }\end{array}$ & 14.69 & 5.71 & 8.12 & 23.66 \\
\hline $\begin{array}{c}\text { Coefficient of } \\
\text { Variation }\end{array}$ & 20.8 & 22.0 & 15.4 & 25.5 \\
\hline $\begin{array}{c}\text { High value } \\
\text { (log of spores) }\end{array}$ & 2.06 & 1.58 & 1.83 & 2.19 \\
\hline $\begin{array}{c}\text { Low value } \\
\text { (log of spores) }\end{array}$ & 1.71 & 1.26 & 1.58 & 1.78 \\
\hline
\end{tabular}

$\mathrm{n}=18$ for each test

Data from each test $(72,76,80$, and 81$)$ was plotted in two histograms, one with the raw spore count and another with the log values of the spores deposited within the chamber. These plots are below in Figures 2-9-2-16. The purpose of these histograms was to determine if the data was normally or lognormally distributed.

Figures 2-9 and 2-10 are histograms for the non-transformed and log values for test 72 . These figures show the data appears to be a lognormal distribution.

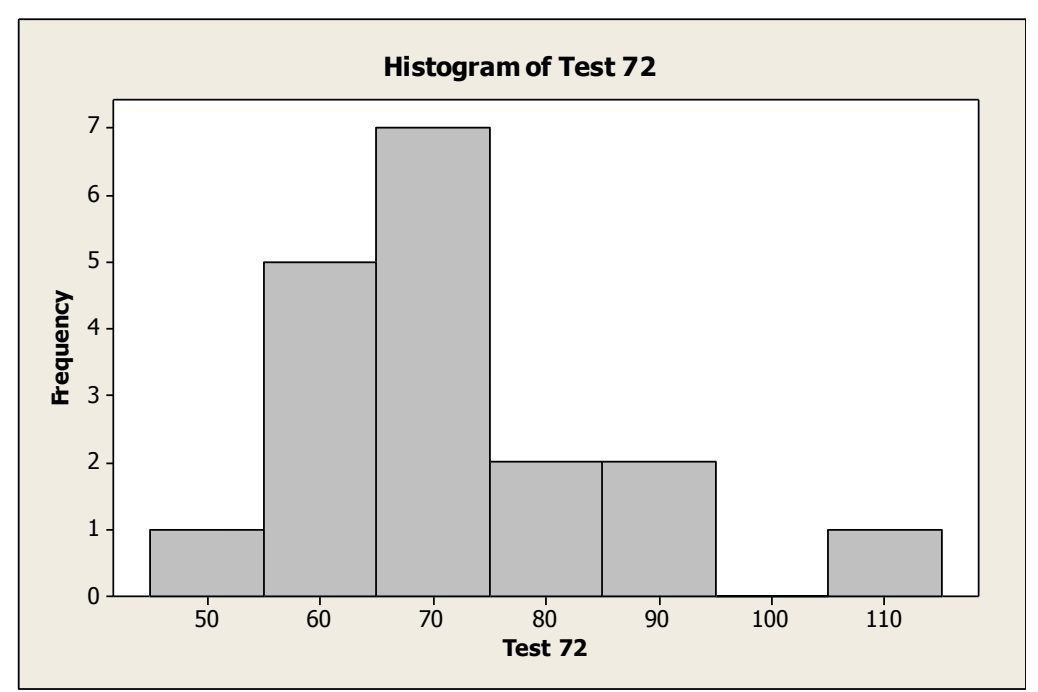

Figure 2- 9 - Test 72 histogram 


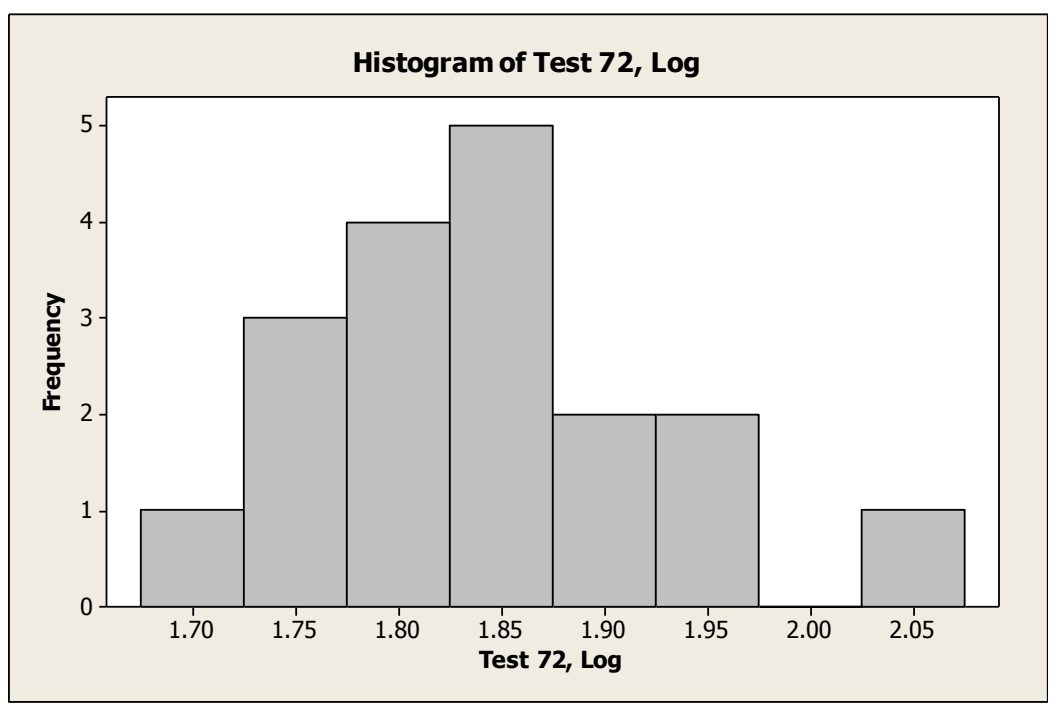

Figure 2- 10 - Test 72 histogram, log values

Figures 2-10 and 2-11 are histograms for the non-transformed and log values for test 76.

These figures show the data appears to be a lognormal distribution.

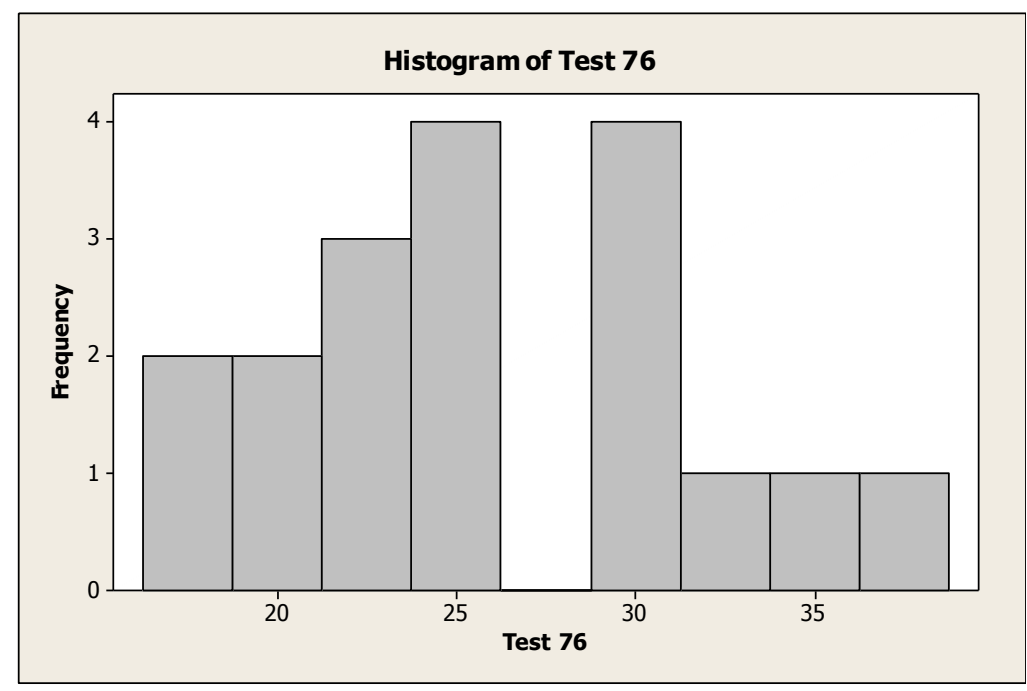

Figure 2- 11 - Test 76 histogram 


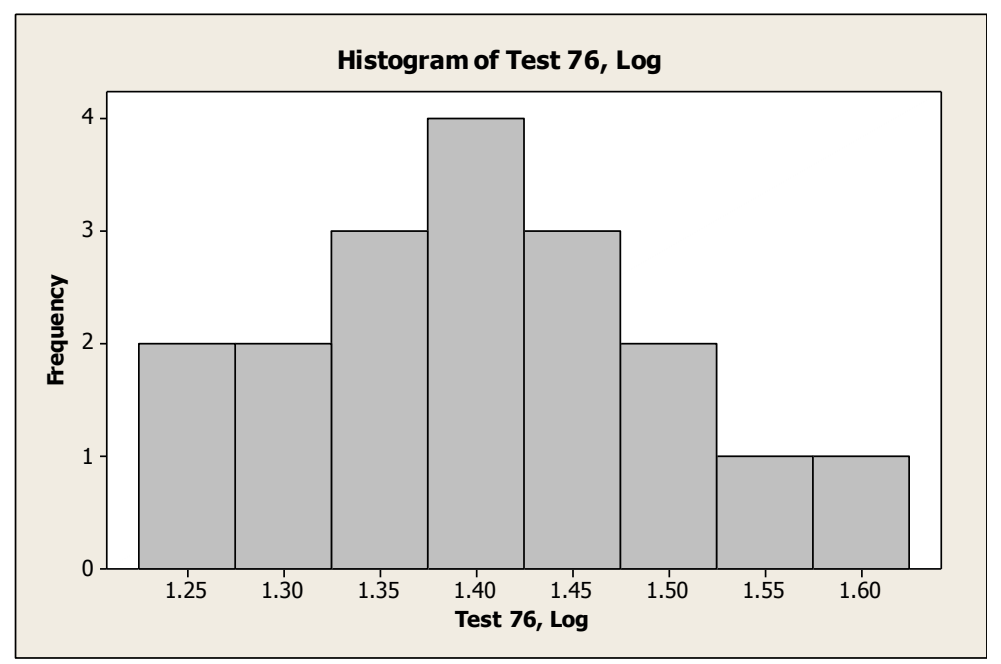

Figure 2- 12 - Test 76 histogram, log values

Figures 2-13 and 2-14 are histograms for the non-transformed and log values for test 80 .

Again, these figures show the data appears to be a lognormal distribution.

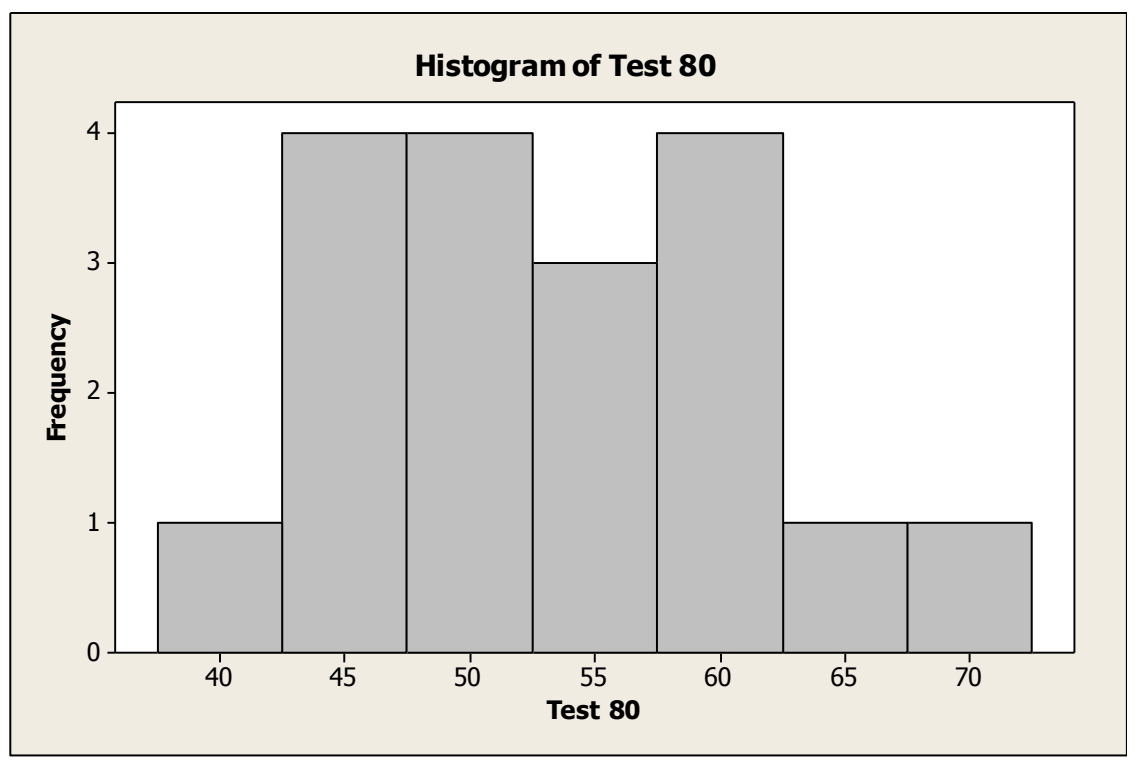

Figure 2- 13 - Test 80 histogram 


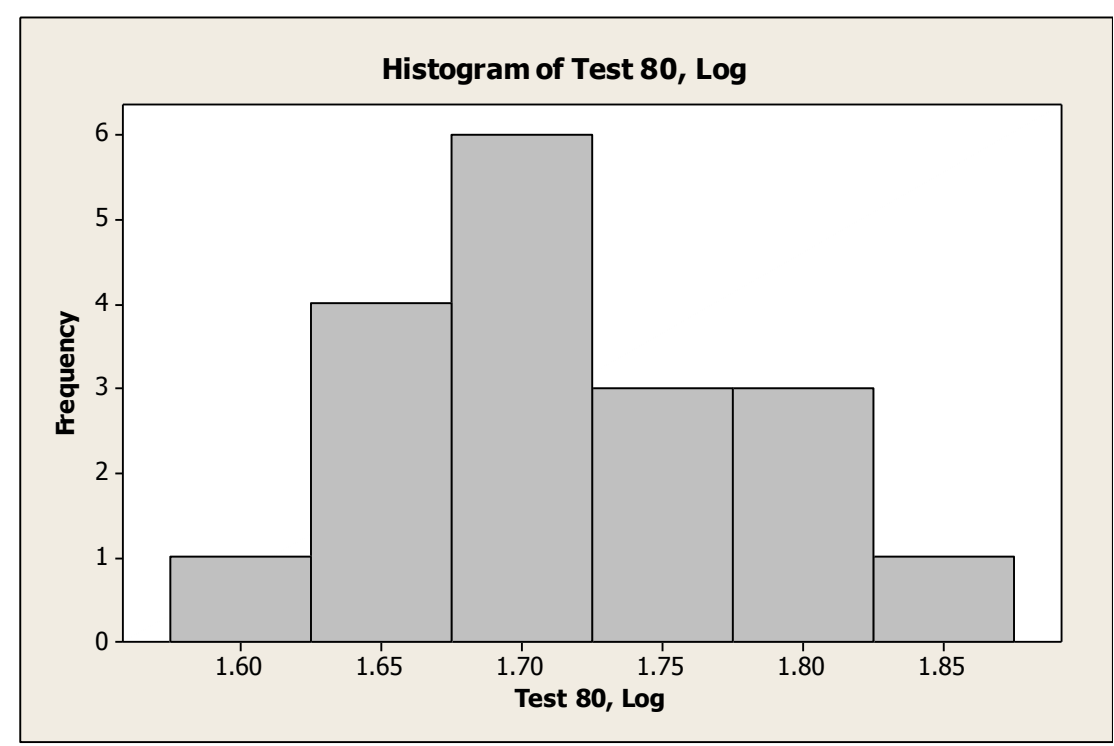

Figure 2- 14 - Test 80 histogram, log values

Finally, Figures 2-15 and 2-16 are histograms for the non-transformed and log values for test 82. Once again, these figures appear to show the data fits best with a lognormal distribution.

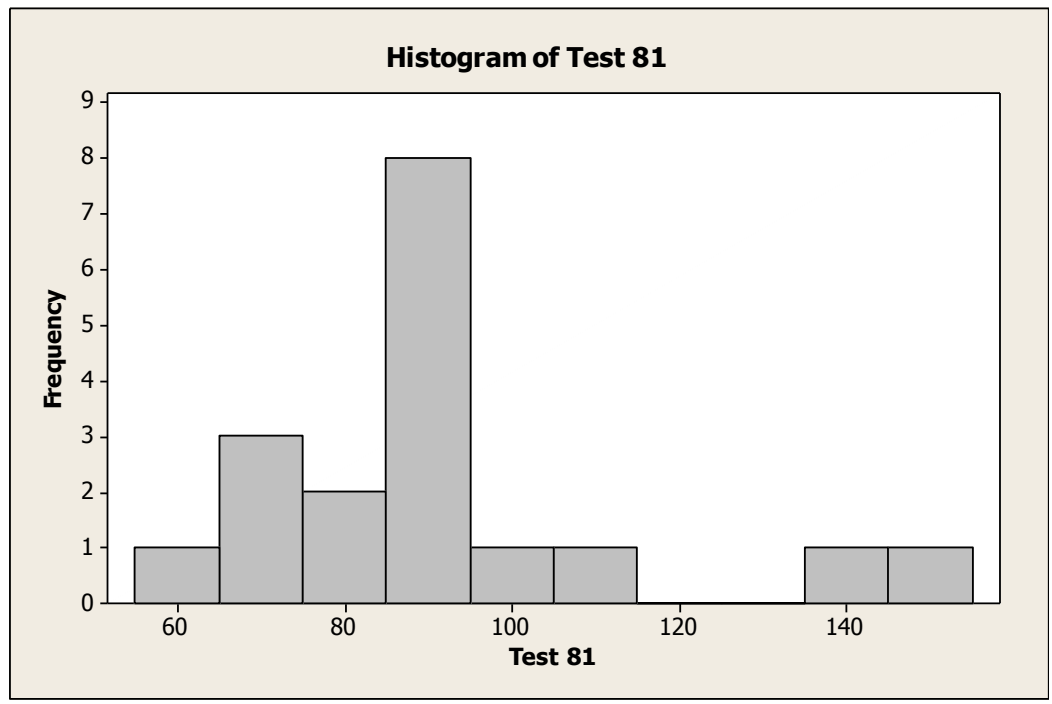

Figure 2- 15 - Test 81 histogram 


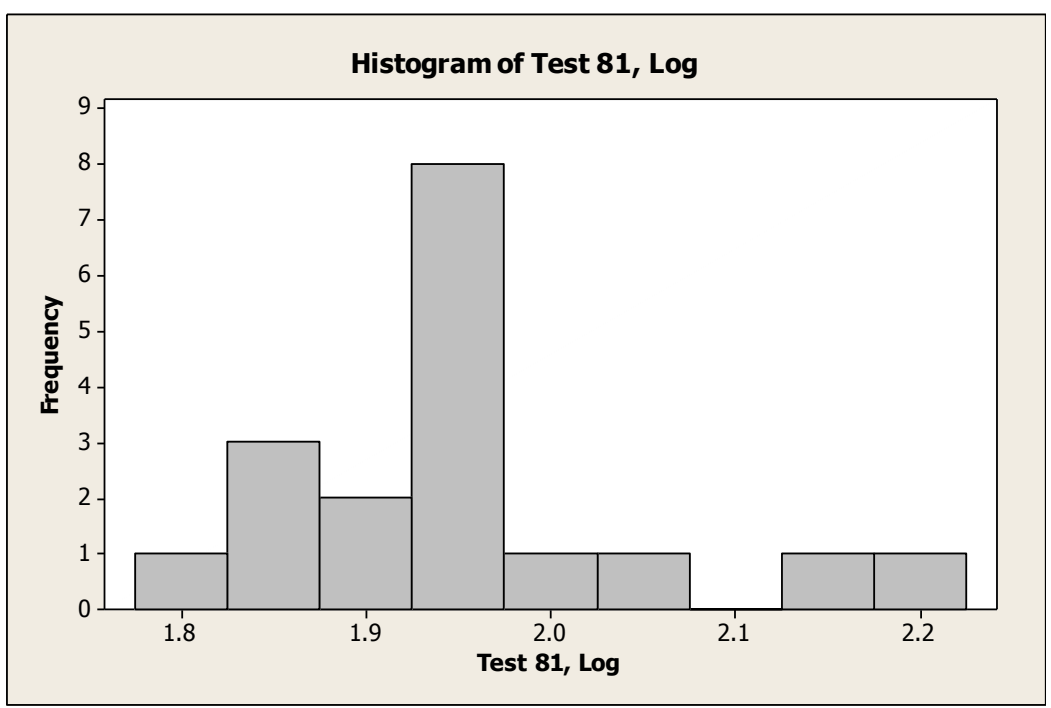

Figure 2- 16 - Test 81 histogram, log values

The histograms are a basic method to visualize the data; however, a statistical test is required to determine if the data is normally or lognormally distributed. The Ryan-Joiner test was completed on both the non-transformed and lognormal data. The null hypothesis $\left(\mathrm{H}_{\mathrm{o}}\right)$ for these tests is that the data is normally distributed, which is the case for both the non-transformed data and the lognormal data tests. Thus, if the p-value is small, then the null is rejected and the data is not normally distributed. If the p-value is large, there is no evidence to reject the null and the data is then considered normally distributed. These tests are included in Figures 2-17 and 218 (Test 72), Figures 2-19 and 2-20 (Test 76), Figures 2-21 and 2-22 (Test 80) and Figures 2-23 and 2-24 (Test 81). 


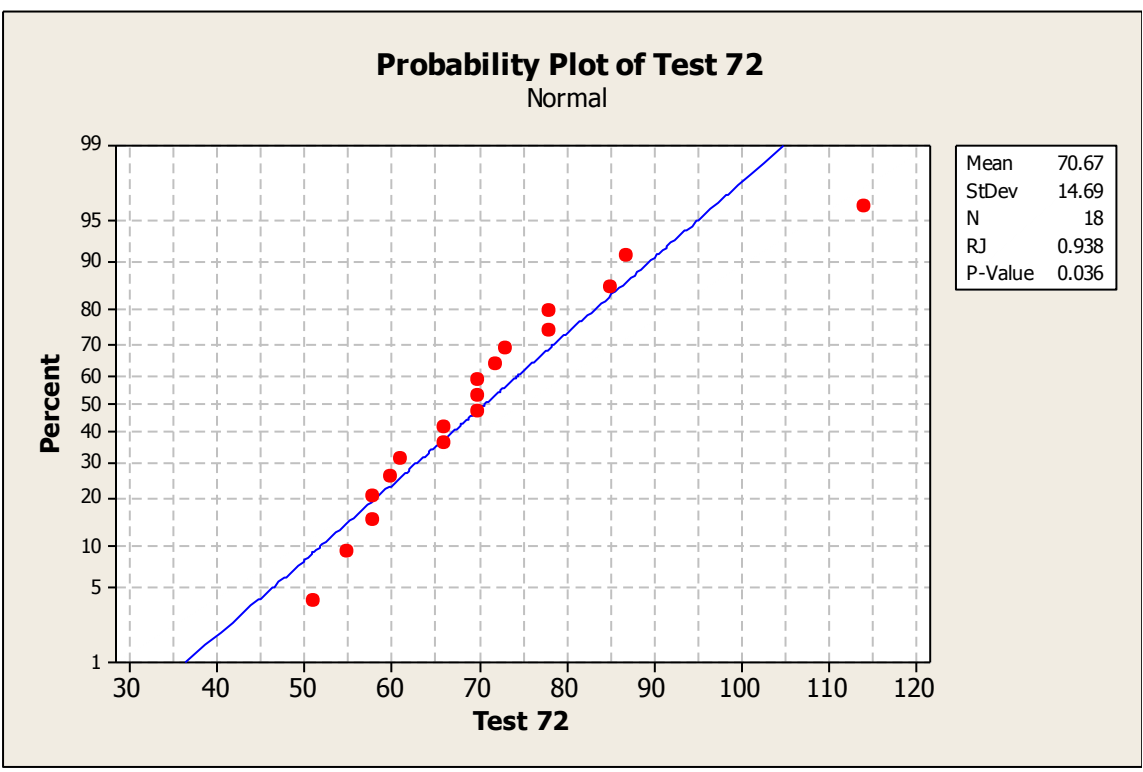

Figure 2- 17 - Test 72 probability plot, non-transformed data

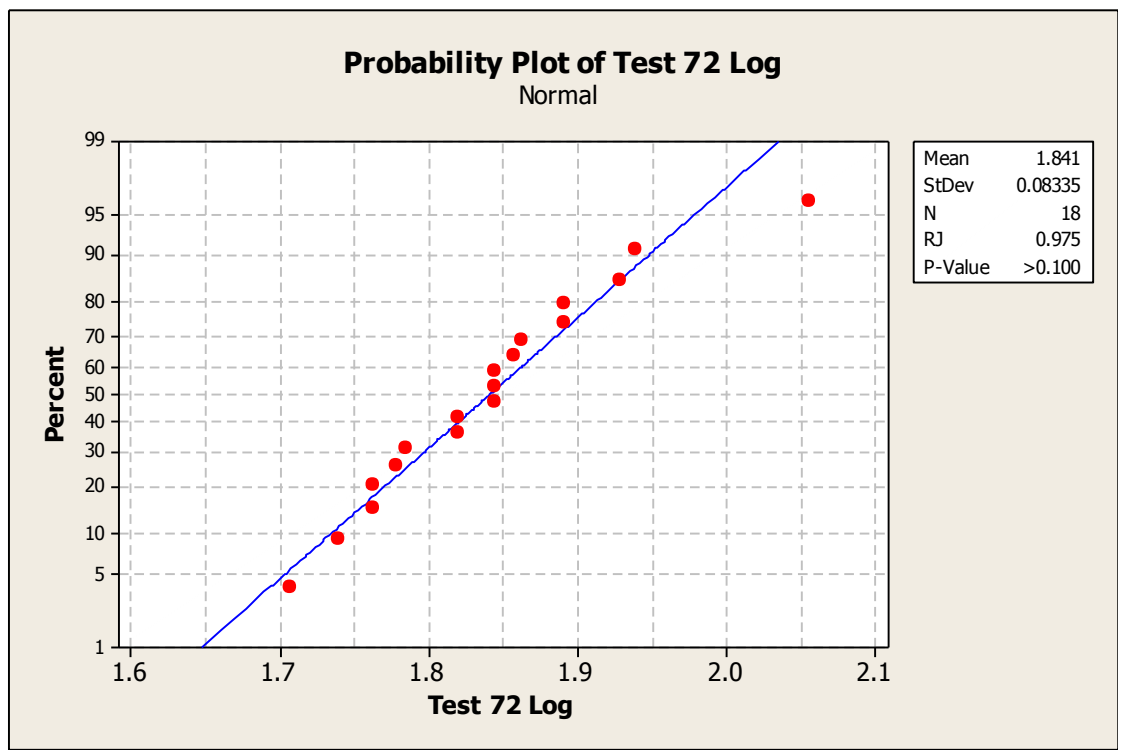

Figure 2-18-Test 72 probability plot, log transformed data 


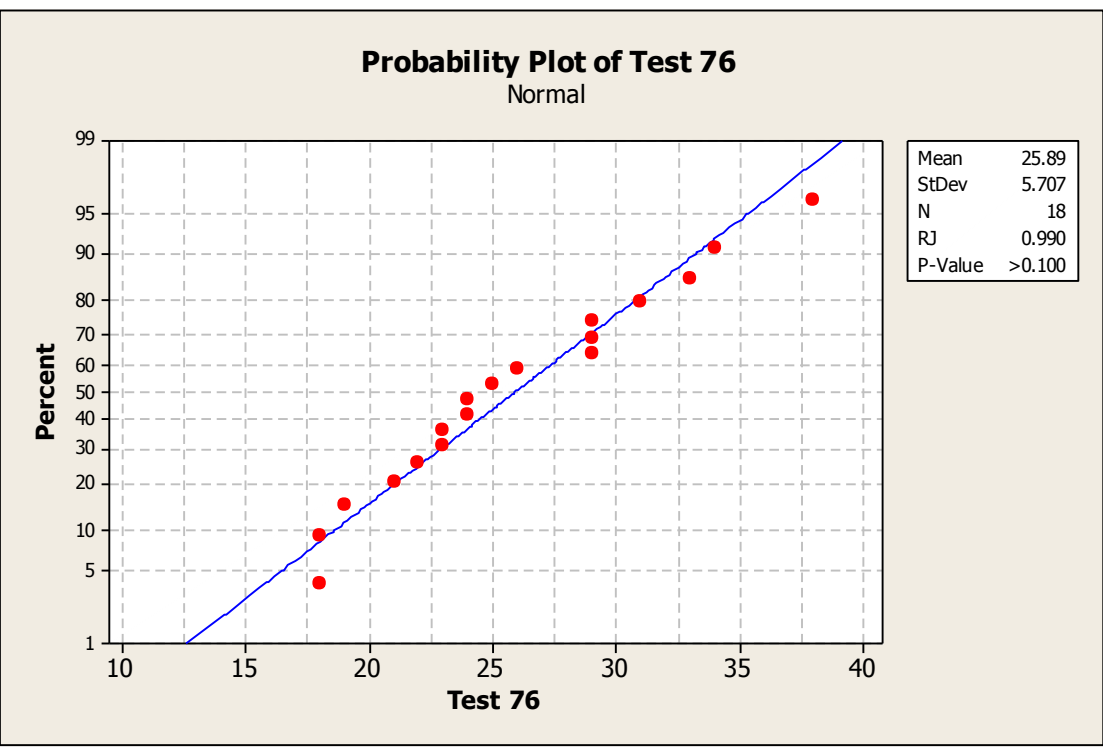

Figure 2- 19 - Test 76 probability plot, non-transformed data

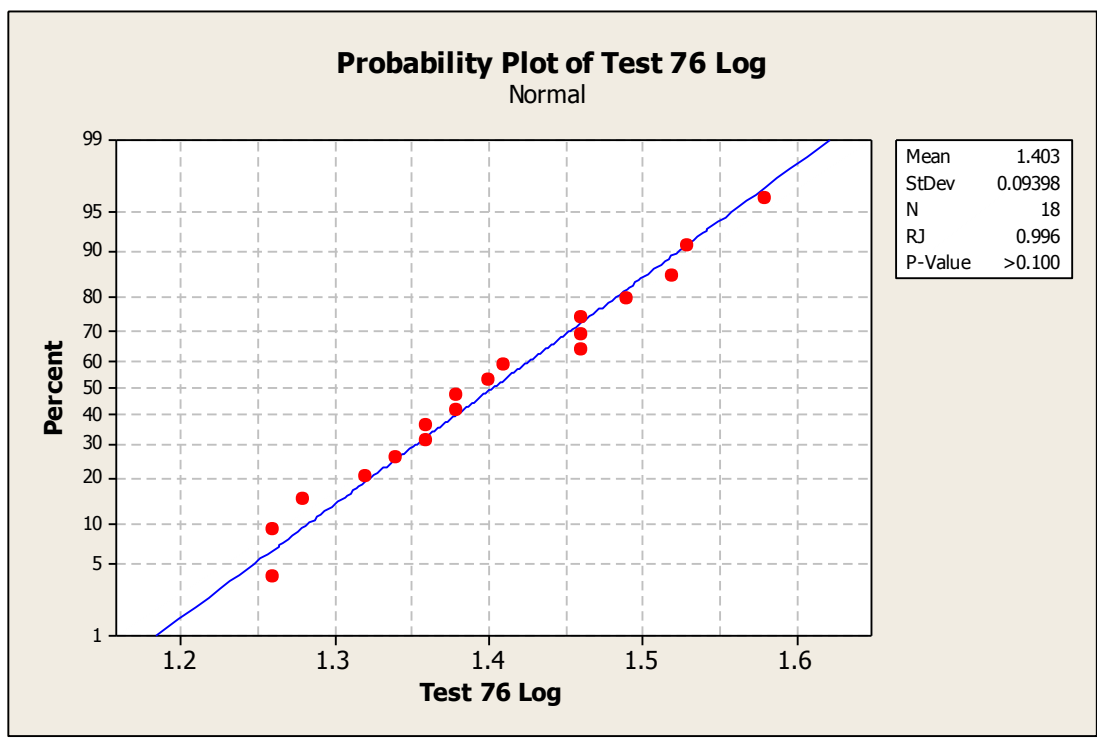

Figure 2- 20 - Test 76 probability plot, log transformed data 


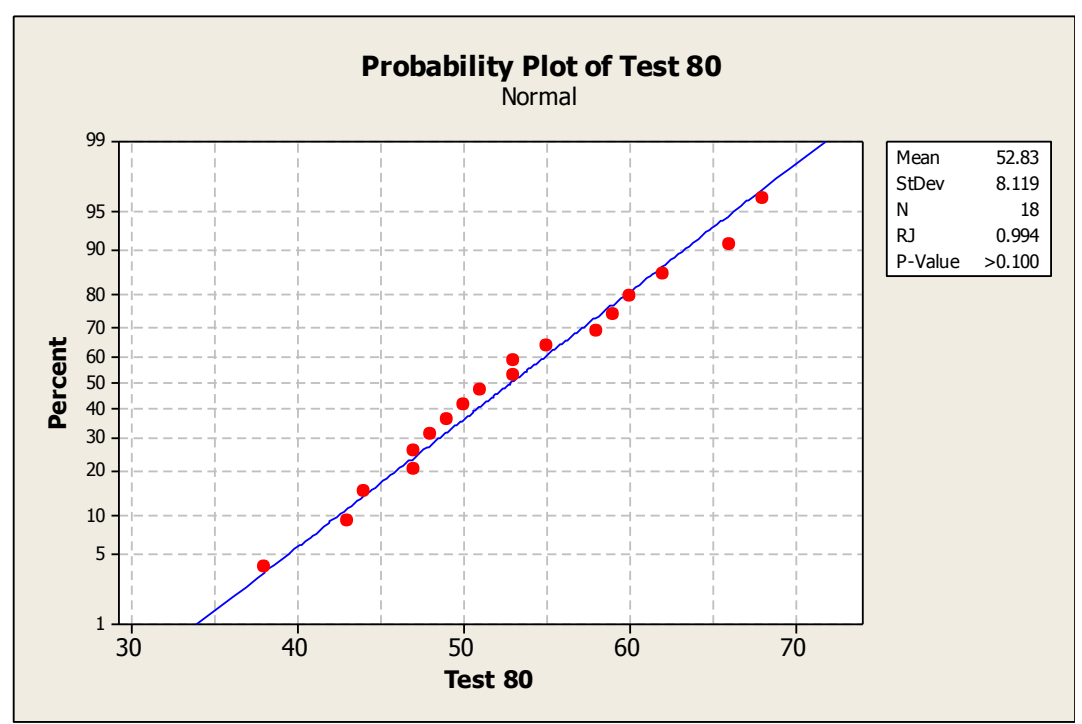

Figure 2- 21 - Test 80 probability plot, non-transformed data

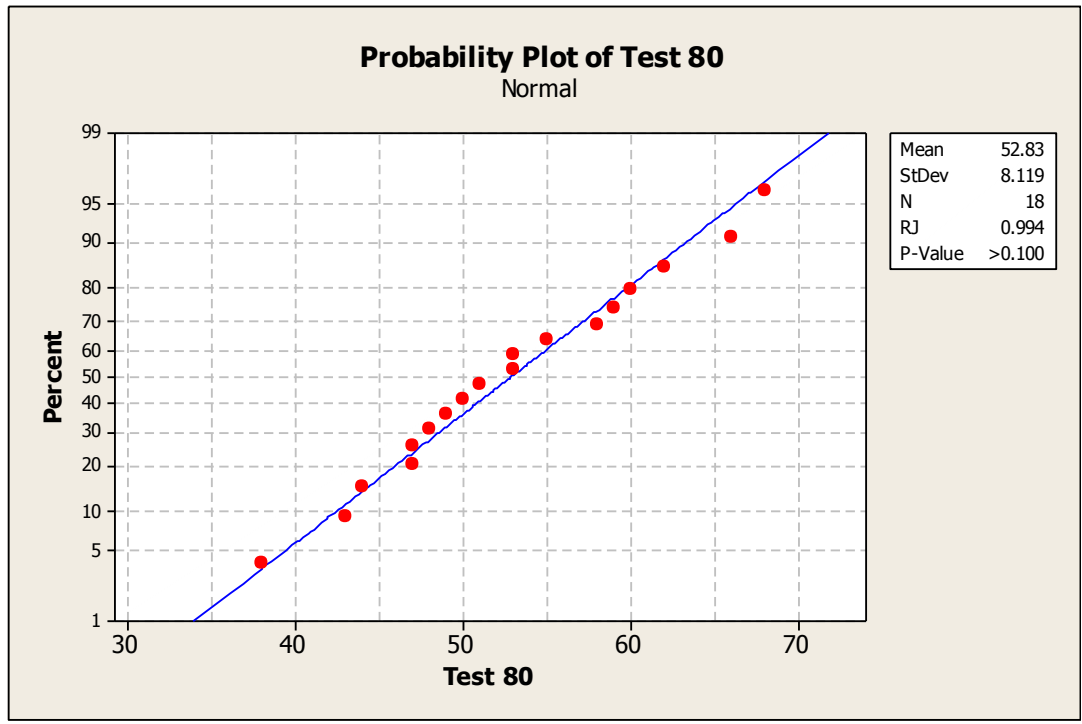

Figure 2- 22 - Test 80 probability plot, log transformed data 


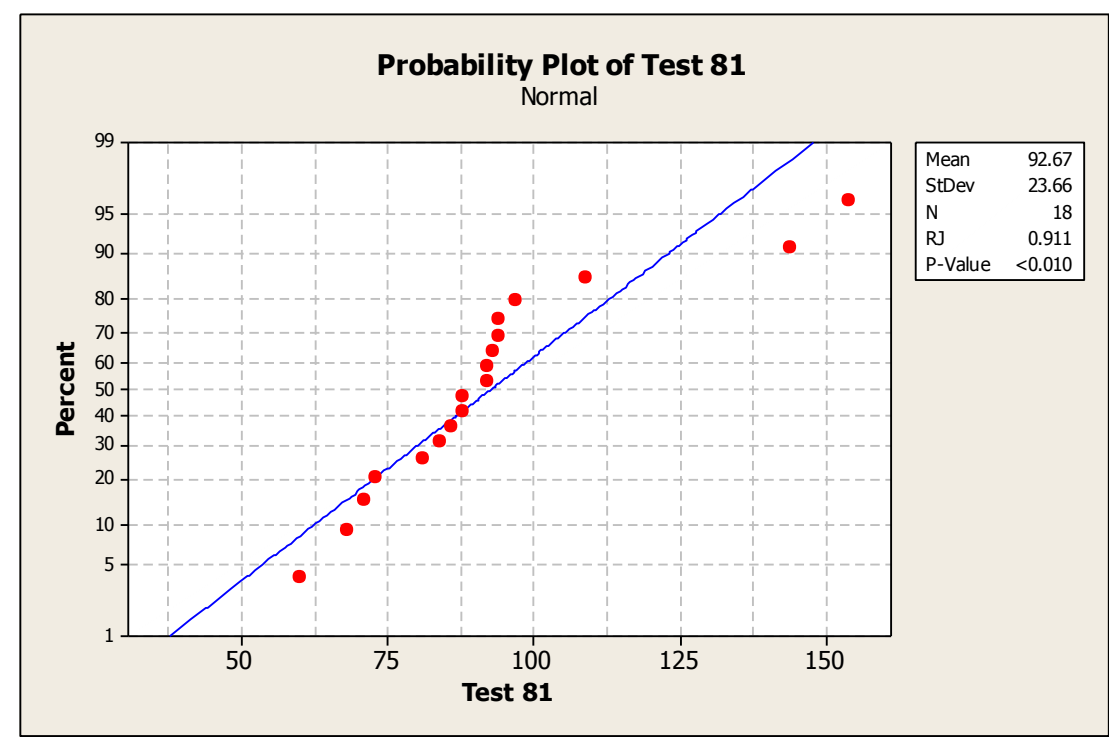

Figure 2- 23 - Test 81 probability plot, non-transformed data

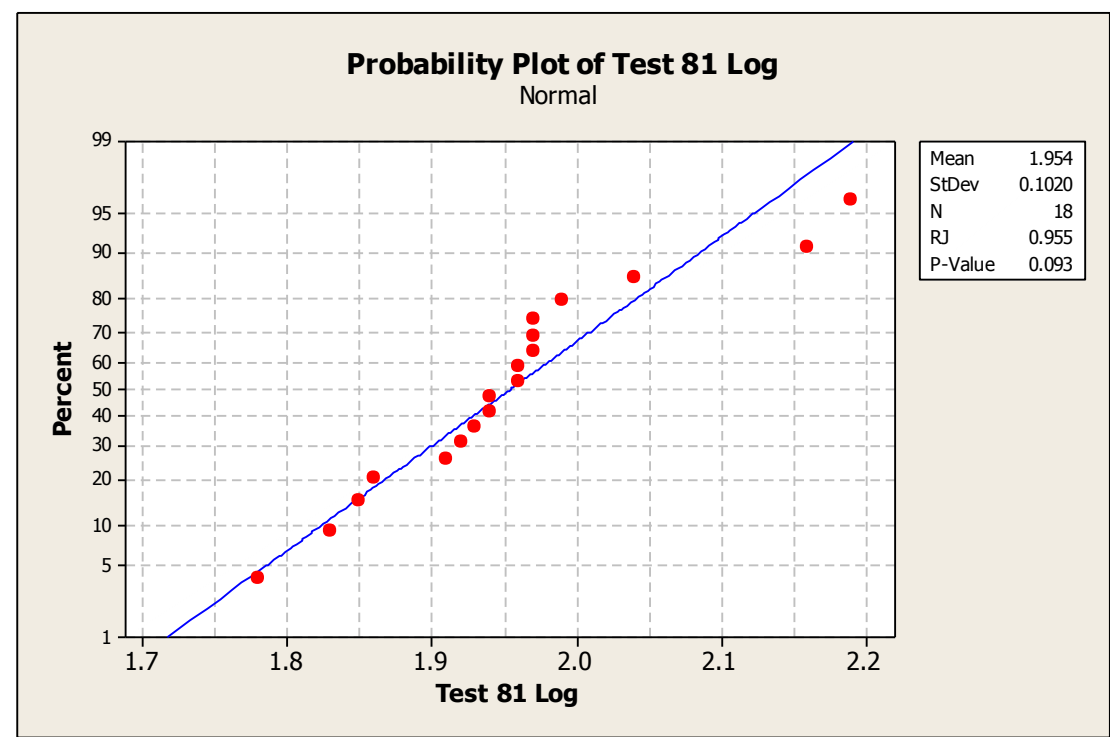

Figure 2- 24 - Test 81 probabality plot, log transformed data

The results of the Ryan-Joiner tests are presented in Table 2-3 below. If the p-value is less than 0.05 , then the normality is rejected. The results show that two of the tests ( 72 and 81 ) are lognormally distributed. The analyses were inconclusive for the other two tests (76 and 80) indicating these tests are neither normal nor lognormal, but rather include characteristics of both of these data distributions. Subsequent analyses on these data were completed on the log- 
transformed values of the data. Such transformations were completed in order to keep all the tests similar.

Table 2 - 3 - Ryan-Joiner analyses, spore deposition tests

\begin{tabular}{|c|c|c|c|c|}
\hline Test & Data & $\begin{array}{c}\text { p- } \\
\text { value }\end{array}$ & p-value Result & Conclusion \\
\hline \multirow{2}{*}{72} & $\begin{array}{l}\text { Non-transformed } \\
\text { data }\end{array}$ & 0.036 & $\begin{array}{l}\text { Reject } \mathrm{H}_{\mathrm{o}} \text { - data is not normally } \\
\text { distributed }\end{array}$ & \multirow{2}{*}{ Data is lognormal } \\
\hline & Lognormal data & $>0.100$ & $\begin{array}{l}\text { No evidence to reject } \mathrm{H}_{\mathrm{o}} \\
\text { (lognormal distribution) }\end{array}$ & \\
\hline \multirow{2}{*}{76} & $\begin{array}{l}\text { Non-transformed } \\
\text { data }\end{array}$ & $>0.100$ & $\begin{array}{l}\text { No evidence to reject } \mathrm{H}_{\mathrm{o}} \text { (normal } \\
\text { distribution) }\end{array}$ & \multirow{2}{*}{$\begin{array}{l}\text { Inconclusive-data has } \\
\text { characteristics of both } \\
\text { normal and lognormal } \\
\text { distributions }\end{array}$} \\
\hline & Lognormal data & $>0.100$ & $\begin{array}{l}\text { No evidence to reject } \mathrm{H}_{\mathrm{o}} \\
\text { (lognormal distribution). }\end{array}$ & \\
\hline \multirow{2}{*}{80} & $\begin{array}{l}\text { Non-transformed } \\
\text { data }\end{array}$ & $>0.100$ & $\begin{array}{l}\text { No evidence to reject } \mathrm{H}_{\mathrm{o}} \text { (normal } \\
\text { distribution) }\end{array}$ & \multirow{2}{*}{$\begin{array}{l}\text { Inconclusive-data has } \\
\text { characteristics of both } \\
\text { normal and lognormal } \\
\text { distributions }\end{array}$} \\
\hline & Lognormal data & $>0.100$ & $\begin{array}{l}\text { No evidence to reject } \mathrm{H}_{\mathrm{o}} \\
\text { (lognormal distribution). }\end{array}$ & \\
\hline \multirow{2}{*}{81} & $\begin{array}{l}\text { Non-transformed } \\
\text { data }\end{array}$ & $<0.010$ & $\begin{array}{l}\text { Reject } \mathrm{H}_{0} \text { - data is not normally } \\
\text { distributed }\end{array}$ & \multirow{2}{*}{ Data is lognormal. } \\
\hline & Lognormal data & 0.093 & $\begin{array}{l}\text { No evidence to reject } \mathrm{H}_{\mathrm{o}} \\
\text { (lognormal distribution) }\end{array}$ & \\
\hline
\end{tabular}

\section{Spore Deposition Contour Plots}

Contour plots using Minitab ${ }^{\circledR}$ version 16.1.1 were constructed to show how the bioaerosols deposited within the test chamber for these test runs. Six different levels were plotted on each contour plot, equally dispersed from the high and low levels of the spore deposition tests completed. Based on this, the contour plots were the log of the spore counts plotted at the levels of $1.2,1.4,1.6,1.8,2.0$, and 2.2 . In the plots below, coordinate $(0,0)$ is the center of the test chamber, with coordinate $(-50,0)$, the point at which the aerosol is introduced into the chamber. All contour plots had $n=18$ evenly dispersed throughout the area. The distribution expected was an even distribution with the highest spore levels at the front of the chamber, or at coordinates $(-50,0)$ with a decrease in concentrations to the end of the chamber These contour plots are presented in Figures 2-25 - 2 -28. 
Figure 2-25, contour plot for spore deposition test 72, shows relative even dispersion, with a lower concentration towards the back of the chamber. Overall, this is the dispersion that would be expected, that is, a high concentration at the front of the chamber followed by a decrease at the back of the chamber.

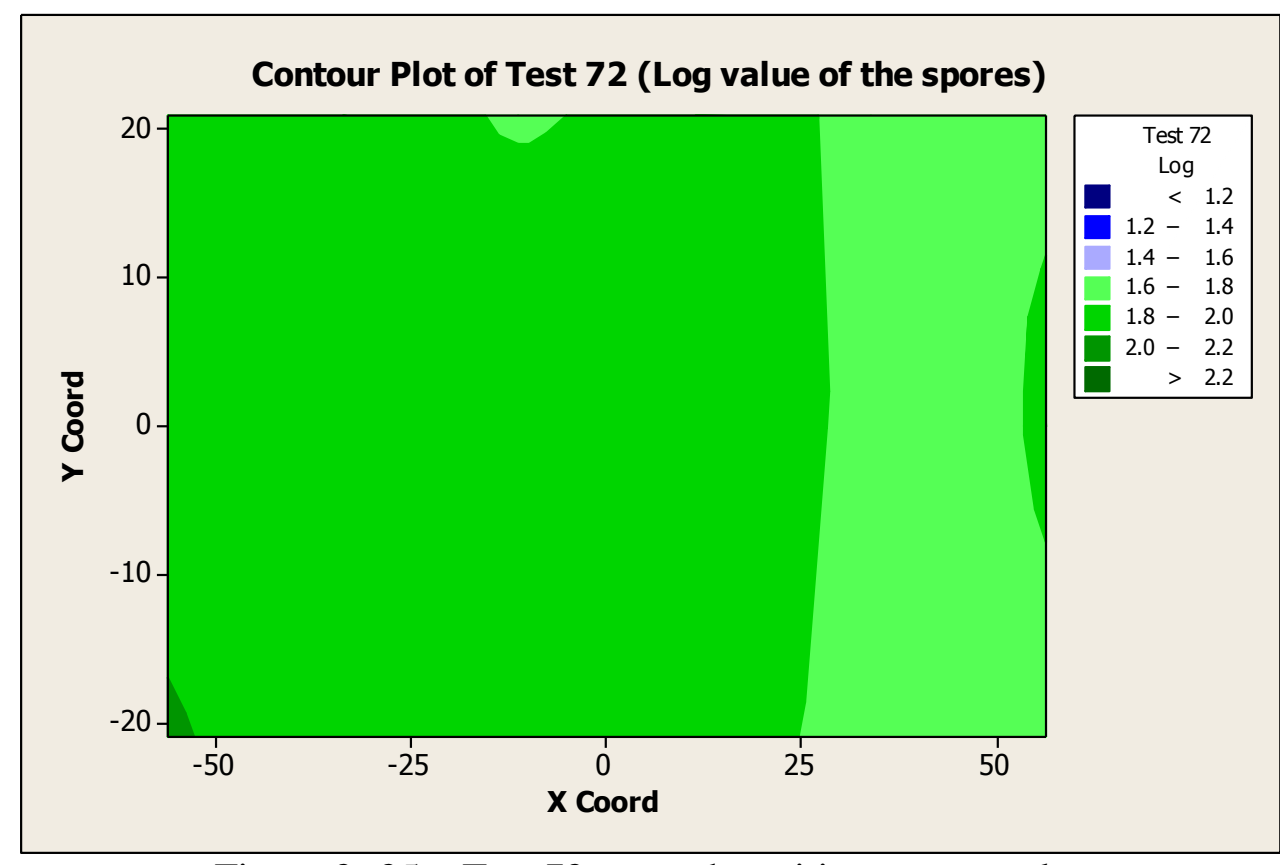

Figure 2- 25 - Test 72 spore deposition contour plot

Figure 2-26, contour plot for spore deposition test 76 shows a pattern with lower distribution of spores. This is an area of higher spore counts towards the bottom of the plot, which corresponds to the area where the glove ports are installed. Also, there is a lower concentration at the front of the chamber. This does not represent the expected deposition pattern. 


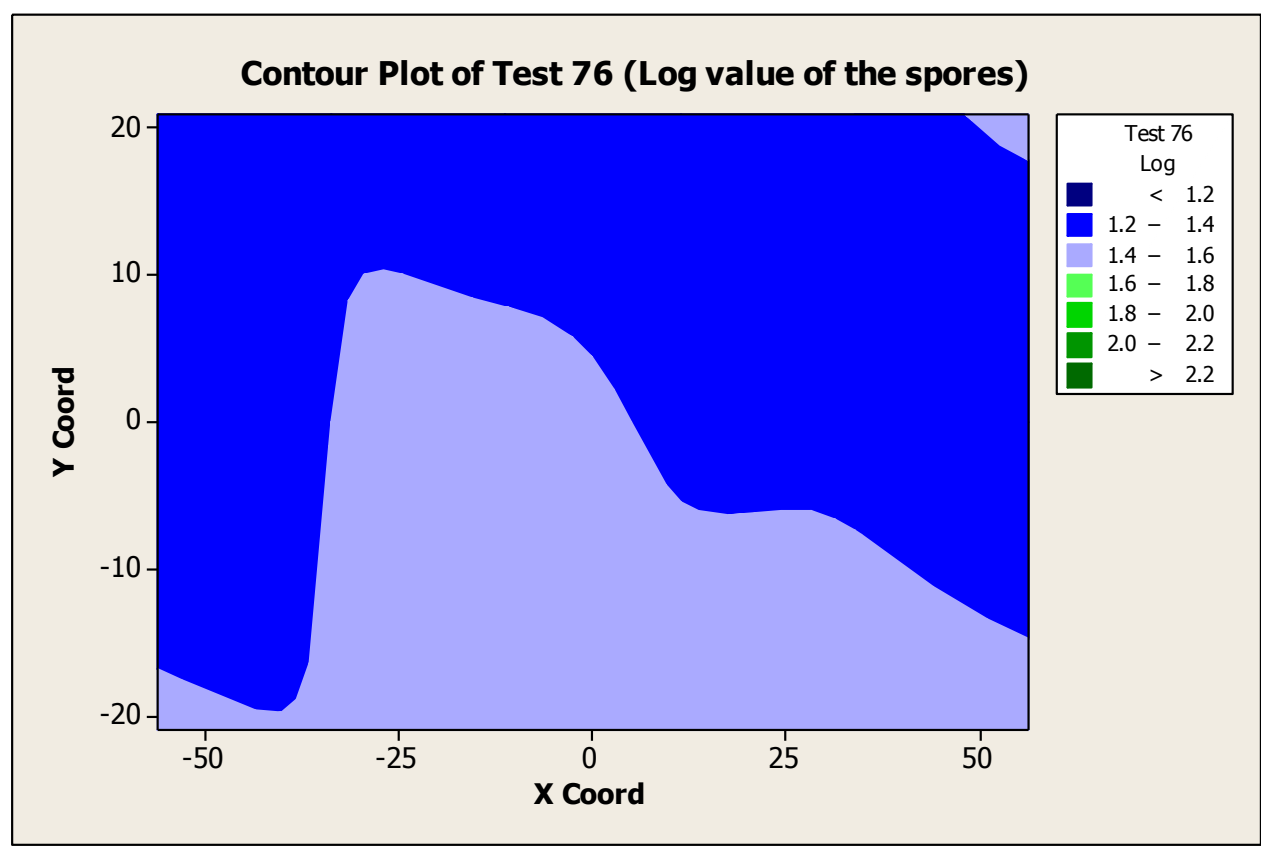

Figure 2- 26 - Test 76 spore deposition contour plot

Figure 2-27, contour plot for deposition test 80 shows a relatively uniform dispersal, with a lower count near the front of the chamber. Again, this is not the dispersion pattern that was expected.

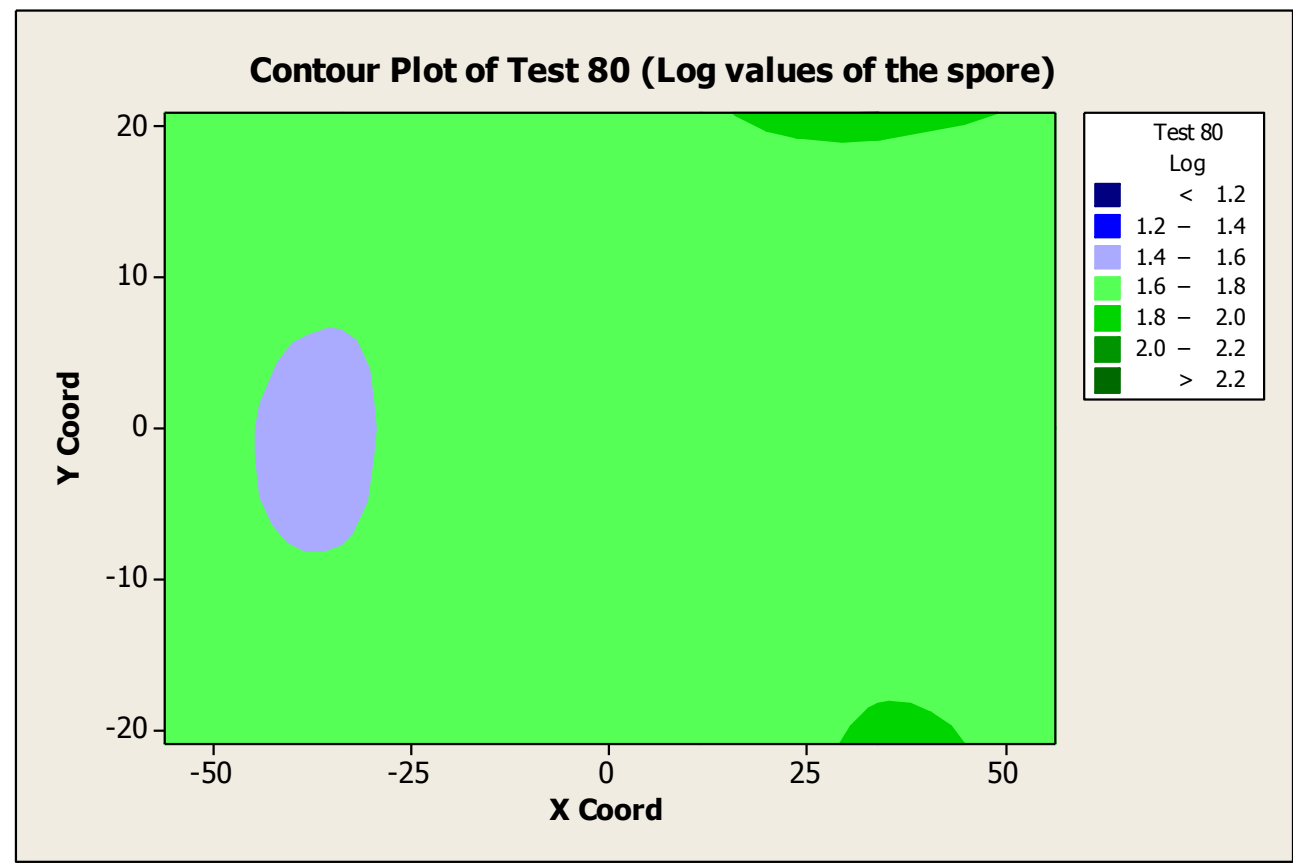

Figure 2- 27 - Test 80 spore deposition contour plot 
Finally, Figure 2-28 shows a higher deposition near the back of the chamber where the HEPA filter was installed. Again, the expected deposition pattern was not seen.

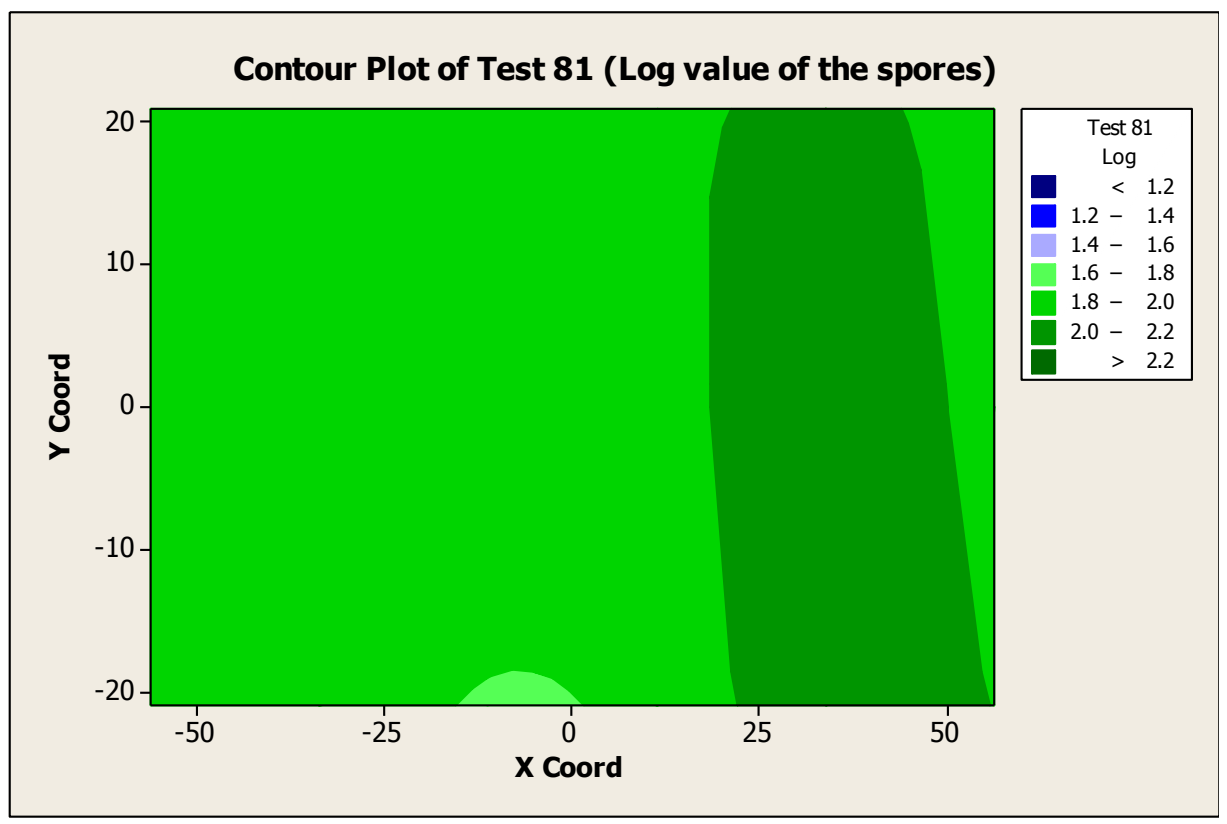

Figure 2- 28 - Test 81 spore deposition contour plot

Overall the contour plots showed somewhat even distribution; however, there was not an overall pattern throughout all the tests.

Finally, multiple forward linear regressions were completed on the tests to determine if spore deposition could be modeled throughout the chamber. This regression analysis included using the $\log$ of the spore counts as the response and the explanatory variables were the coordinates of the floor and the interactions between these variables. Regressions were completed for each of the four tests.

Table 2-4 shows the values for test 72 . All $\mathrm{R}^{2}$ values are greater than $40 \%$, demonstrating that approximately one-half of the dispersion is explained by the model. The best models overall include $\mathrm{X}, \mathrm{X}^{2}, \mathrm{Y}, \mathrm{Y}^{2}$, and $\mathrm{X}$-Y interactions and also the model with just $\mathrm{X}, \mathrm{Y}$, and $\mathrm{X}-\mathrm{Y}$ interactions. Adding the final two variables increases the $\mathrm{R}^{2}$ but decreases the adjusted $\mathrm{R}^{2}$. 
Table 2 - 4- Test 72 spore deposition statistical summary

\begin{tabular}{|l|l|c|c|}
\hline \multicolumn{2}{|c|}{ Explanatory variables } & $\mathbf{R}^{\mathbf{2}}$ & $\mathbf{R}^{\mathbf{2}}$ (adj) \\
\hline $\mathrm{X}:$ & $\mathrm{X}$ coordinate & $42.3 \%$ & $34.6 \%$ \\
$\mathrm{Y}:$ & $\mathrm{Y}$ coordinate & & \\
\hline $\mathrm{X}:$ & $\mathrm{X}$ coordinate & $49.0 \%$ & $38.1 \%$ \\
$\mathrm{Y}:$ & $\mathrm{Y}$ coordinate & & \\
$\mathrm{X}^{*} \mathrm{Y}:$ & $\mathrm{X}, \mathrm{Y}$ coordinate interaction & & \\
\hline $\mathrm{X}:$ & $\mathrm{X}$ coordinate & $44.9 \%$ & $27.9 \%$ \\
$\mathrm{Y}:$ & $\mathrm{Y}$ coordinate & & \\
$\mathrm{X}^{2}:$ & $\mathrm{X}$ coordinate & \\
$\mathrm{Y}^{2}:$ & $\mathrm{Y}$ coordinate & \\
\hline $\mathrm{X}:$ & $\mathrm{X}$ coordinate & & \\
$\mathrm{X}^{2}:$ & $\mathrm{X}$ coordinate & \\
$\mathrm{Y}:$ & $\mathrm{Y}$ coordinate & $51.6 \%$ & $31.4 \%$ \\
$\mathrm{Y}^{2}:$ & $\mathrm{Y}$ coordinate & & \\
$\mathrm{X}^{*} \mathrm{Y}:$ & $\mathrm{X}-\mathrm{Y}$ coordinate interaction & & \\
\hline
\end{tabular}

Table 2-5 shows the values for test 76 . The best model is the third model below, which includes all variables except the X-Y interaction.

Table 2 - 5 - Test 76 spore deposition statistical summary

\begin{tabular}{|c|c|c|c|}
\hline \multicolumn{2}{|r|}{ Explanatory variables } & $\mathbf{R}^{2}$ & $\mathbf{R}^{2}(\mathbf{a d j})$ \\
\hline $\begin{array}{l}X: \\
Y:\end{array}$ & $\begin{array}{l}\text { X coordinate } \\
\text { Y coordinate }\end{array}$ & $42.7 \%$ & $35.1 \%$ \\
\hline $\begin{array}{l}\mathrm{X}: \\
\mathrm{Y}: \\
\mathrm{X}^{*} \mathrm{Y}:\end{array}$ & $\begin{array}{l}\mathrm{X} \text { coordinate } \\
\mathrm{Y} \text { coordinate } \\
\mathrm{X}, \mathrm{Y} \text { coordinate interaction }\end{array}$ & $42.8 \%$ & $30.5 \%$ \\
\hline $\begin{array}{l}\mathrm{X}: \\
\mathrm{Y}: \\
\mathrm{X}^{2}: \\
\mathrm{Y}^{2}:\end{array}$ & $\begin{array}{l}\text { X coordinate } \\
\text { Y coordinate } \\
\text { X coordinate } \\
\text { Y coordinate }\end{array}$ & $61.7 \%$ & $49.9 \%$ \\
\hline $\begin{array}{l}X: \\
X^{2}: \\
Y: \\
Y^{2}: \\
X^{*} Y:\end{array}$ & $\begin{array}{l}X \text { coordinate } \\
X \text { coordinate } \\
\text { Y coordinate } \\
Y \text { coordinate } \\
X-Y \text { coordinate interaction }\end{array}$ & $61.8 \%$ & $45.8 \%$ \\
\hline
\end{tabular}

Table 2-6 shows the values for the regression for test 80 . The $\mathrm{R}^{2}$ values are much lower for this test, with the highest values below 30\%. The best model is the one with all variables except $\mathrm{X}-\mathrm{Y}$ interactions. 
Table 2 - 6 - Test 80 spore deposition statistical summary

\begin{tabular}{|c|c|c|c|}
\hline \multicolumn{2}{|r|}{ Explanatory variables } & $\mathbf{R}^{2}$ & $\mathbf{R}^{2}(\mathbf{a d j})$ \\
\hline $\begin{array}{l}\mathrm{X}: \\
\mathrm{Y}:\end{array}$ & $\begin{array}{l}\text { X coordinate } \\
\text { Y coordinate }\end{array}$ & $10.7 \%$ & $0.0 \%$ \\
\hline $\begin{array}{l}\mathrm{X}: \\
\mathrm{Y}: \\
\mathrm{X}^{*} \mathrm{Y}:\end{array}$ & $\begin{array}{l}\text { X coordinate } \\
\text { Y coordinate } \\
X, Y \text { coordinate interaction }\end{array}$ & $12.0 \%$ & $0.0 \%$ \\
\hline $\begin{array}{l}\mathrm{X}: \\
\mathrm{Y}: \\
\mathrm{X}^{2} \\
\mathrm{Y}^{2}:\end{array}$ & $\begin{array}{l}\text { X coordinate } \\
Y \text { coordinate } \\
\text { X coordinate } \\
\text { Y coordinate } \\
2\end{array}$ & $28.5 \%$ & $6.5 \%$ \\
\hline $\begin{array}{l}\mathrm{X}: \\
\mathrm{X}^{2}: \\
\mathrm{Y}: \\
\mathrm{Y}^{2}: \\
\mathrm{X}^{*} \mathrm{Y}:\end{array}$ & $\begin{array}{l}\text { X coordinate } \\
X \text { coordinate }^{2} \\
\text { Y coordinate } \\
\text { Y coordinate } \\
X-Y \text { coordinate interaction }\end{array}$ & $29.8 \%$ & $0.6 \%$ \\
\hline
\end{tabular}

Finally, Table 2-7 shows the values for test 81 . The $\mathrm{R}^{2}$ values are not over $10 \%$;

however, the best model again is the one that includes all the variables-X, $\mathrm{X}^{2}, \mathrm{Y}, \mathrm{Y}^{2}$, and $\mathrm{X}-\mathrm{Y}$ interaction.

Table 2 - 7 - Test 81 spore deposition statistical summary

\begin{tabular}{|c|c|c|c|}
\hline \multicolumn{2}{|r|}{ Explanatory variables } & $\mathrm{R}^{2}$ & $\mathrm{R}^{2}(\mathrm{adj})$ \\
\hline $\begin{array}{l}\mathrm{X}: \\
\mathrm{Y}:\end{array}$ & $\begin{array}{l}\text { X coordinate } \\
\text { Y coordinate }\end{array}$ & $2.2 \%$ & $0.0 \%$ \\
\hline $\begin{array}{l}\mathrm{X}: \\
Y: \\
X^{*} Y:\end{array}$ & $\begin{array}{l}\text { X coordinate } \\
\text { Y coordinate } \\
\mathrm{X}, \mathrm{Y} \text { coordinate interaction }\end{array}$ & $8.6 \%$ & $0.0 \%$ \\
\hline $\begin{array}{l}\mathrm{X}: \\
\mathrm{Y}: \\
\mathrm{X}^{2}: \\
\mathrm{Y}^{2}:\end{array}$ & $\begin{array}{l}\text { X coordinate } \\
\text { Y coordinate } \\
\text { X coordinate } \\
\text { Y coordinate }\end{array}$ & $3.0 \%$ & $0.0 \%$ \\
\hline $\begin{array}{l}X^{2} \\
X^{2}: \\
Y: \\
Y^{2}: \\
X^{*} Y:\end{array}$ & $\begin{array}{l}X \text { coordinate } \\
X \text { coordinate } \\
\text { Y coordinate } \\
Y \text { coordinate } \\
X-Y \text { coordinate interaction }\end{array}$ & $9.4 \%$ & $0.0 \%$ \\
\hline
\end{tabular}

\section{$\underline{\text { Residual analysis }}$}

The final analysis was completed evaluated the residuals from these models to determine if these residuals are normally distributed. This was completed to determine if the assumption of 
normal distribution was met. The Ryan-Joiner test was completed for the residuals based on the regression model. To keep the analysis consistent, the same regression models were analyzed which was the model with all the variables with the response variable being the log of the spore counts. These plots are included below in Figures 2-29 through 2-33

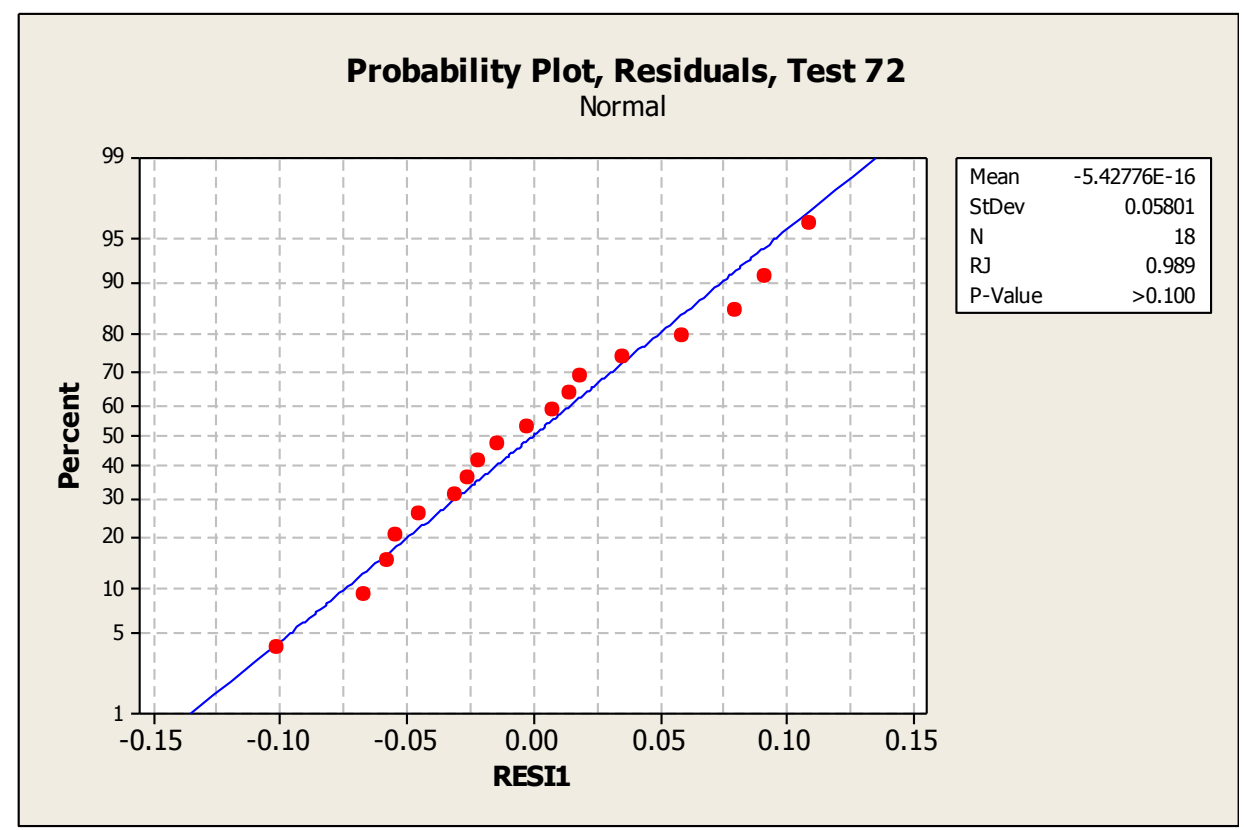

Figure 2- 29- Test 72 residual probability plot

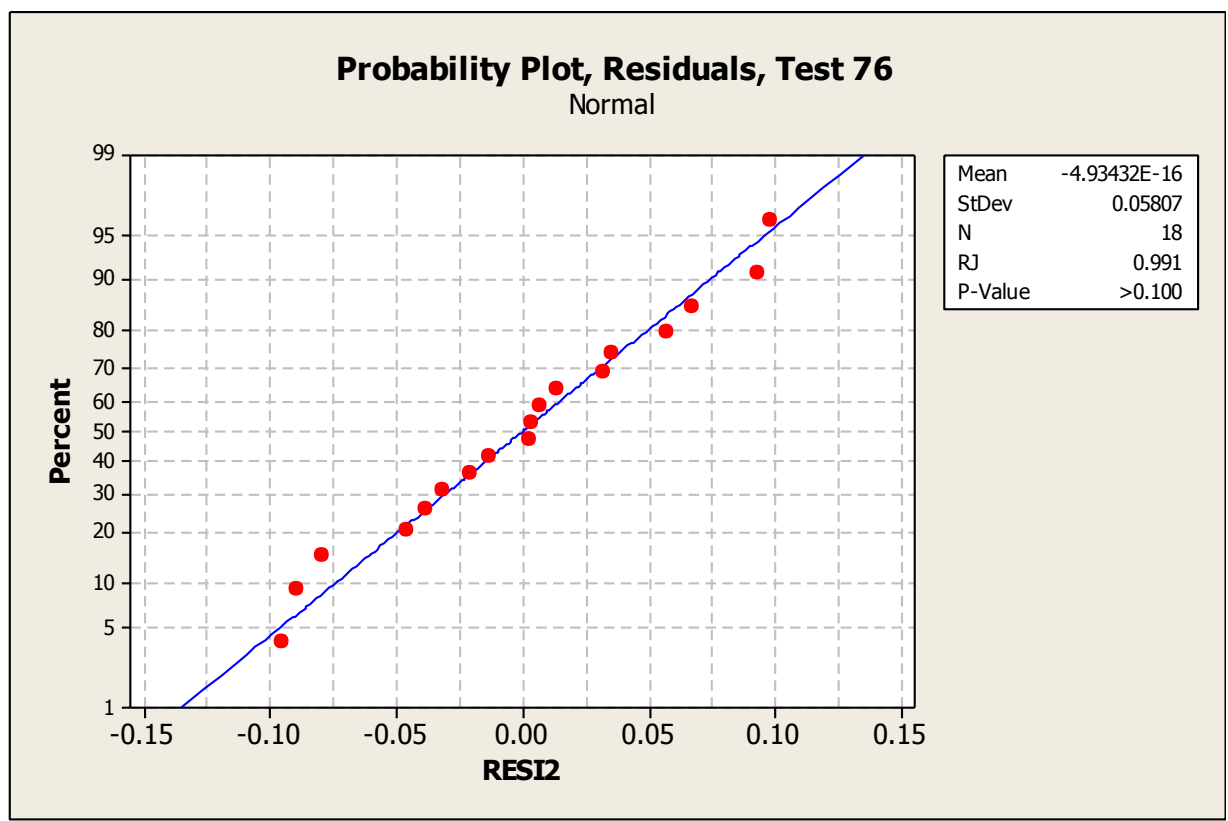

Figure 2-30 - Test 76 residual probability plot 


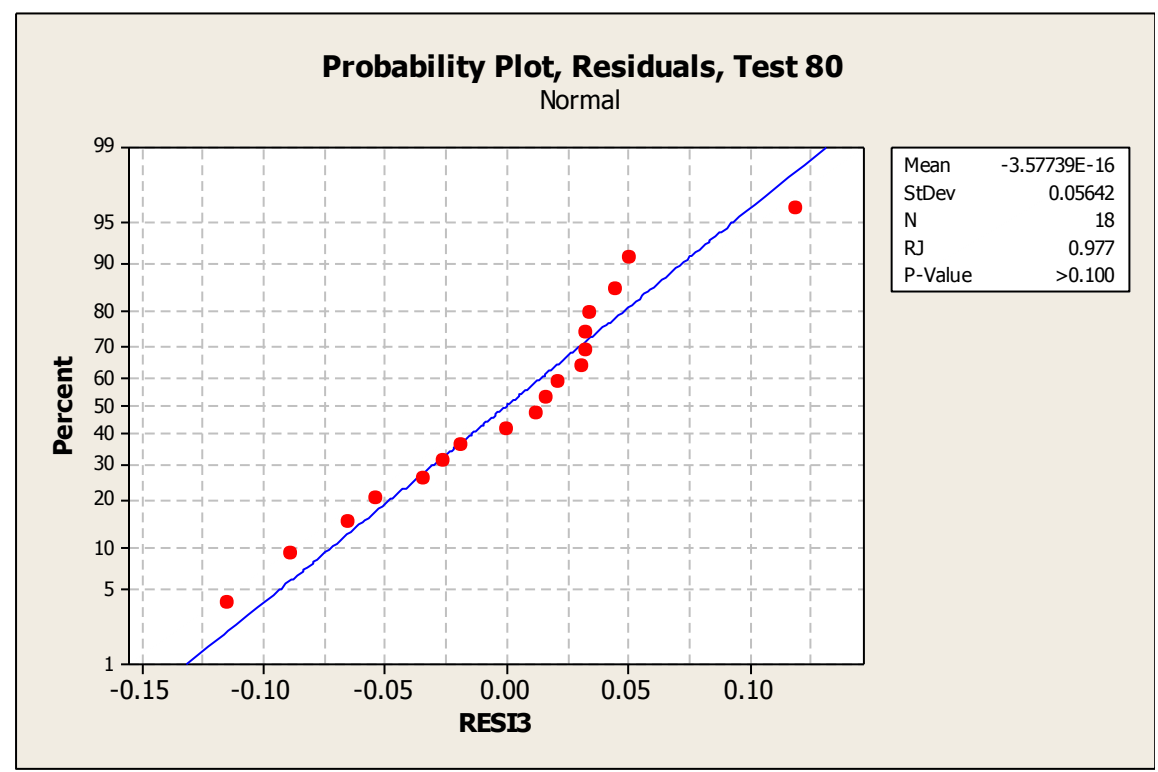

Figure 2- 31 - Test 80 residual probability plot

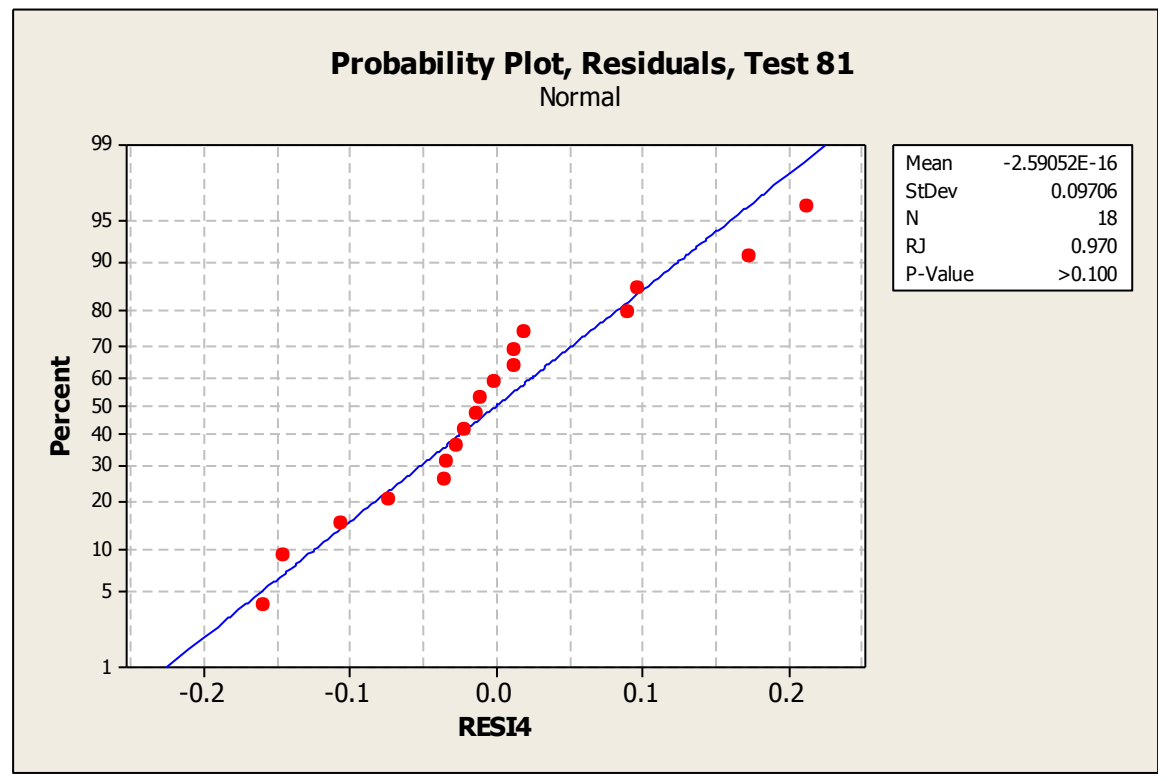

Figure 2- 32 - Test 81 residual probability plot

All p-values for the residual tests were $>0.100$. This shows that there is no evidence to reject $\mathrm{H}_{\mathrm{o}}$, which is the residuals from the regression models are lognormally distributed. This signifies that the models meet the assumption of normal distribution, after the data is transformed to lognormal values. 


\section{Equation deposition validation}

The final evaluation completed was to validate the equations developed for spore depositions. This was equation 5 presented above and also listed below as equation 8 . The surface concentration goal for this deposition was $300 \mathrm{CFU}$ per petri dish or plate.

$$
\begin{aligned}
& \mathrm{S}_{\mathrm{c}}=\left(\left(\frac{\mathrm{G}}{\mathrm{Q}_{\mathrm{in}}}\right) * \mathrm{H}\right) * \mathrm{SA}_{\mathrm{C}} \\
& \mathrm{S}_{\mathrm{c}} \quad=\text { Surface concentration per coupon (CFU) } \\
& \mathrm{G}=\text { Generation rate for spores }\left(\frac{\mathrm{CFU}}{\text { minute }}\right) \\
& \text { Where: } \quad Q_{\text {in }}=\text { Air generation rate into chamber }\left(\frac{\mathrm{m}^{3}}{\text { minute }}\right) \\
& \mathrm{H}=\text { Chamber height }(1.22 \mathrm{~m}) \\
& \mathrm{SA}_{\mathrm{c}}=\text { Petri dish surface area }\left(7.85 \times 10^{-3} \mathrm{~m}^{2}\right)
\end{aligned}
$$

The surface concentration goal for these deposition tests was $300 \mathrm{CFU}$ per petri dish, which was modeled in the equation above. The data for each test is presented in Table 2-8 below. The average recovery efficiencies ranged from 26 to 93 CFU per plate. This corresponded to average recoveries of $8.67 \%$ to $31.0 \%$.

Table 2 - 8 - Spore deposition summaries

\begin{tabular}{|c|c|c|c|c|}
\hline & Test 72 & Test 76 & Test 80 & Test 81 \\
\hline $\begin{array}{c}\text { Modeled } \\
\text { deposition }\end{array}$ & \multicolumn{4}{|c|}{300 CFU/plate } \\
\hline $\begin{array}{c}\text { Average } \\
\text { deposition }\end{array}$ & 71 CFU/plate & 26 CFU/plate & 53 CFU/plate & 93 CFU/plate \\
\hline $\begin{array}{c}\text { Standard } \\
\text { Deviation }\end{array}$ & 14.69 & 5.71 & 8.12 & 23.66 \\
\hline Standard error & 3.46 & 1.35 & 1.91 & 5.58 \\
\hline $\begin{array}{c}\text { Recovery } \\
\text { Efficiency }\end{array}$ & $\begin{array}{c}23.67 \% \pm \\
4.90 \%\end{array}$ & $\begin{array}{c}8.67 \% \pm \\
1.91 \%\end{array}$ & $\begin{array}{c}17.67 \% \pm \\
2.71 \%\end{array}$ & $\begin{array}{c}7.0 \% \pm \\
7.89 \%\end{array}$ \\
\hline $\begin{array}{c}\text { Average } \\
\text { Recovery } \\
\text { Efficiency }\end{array}$ & \multicolumn{4}{|c|}{$20.25 \%$} \\
\hline
\end{tabular}

The sample size was 18 for each test $(n=18)$. 


\section{DISCUSSION}

The aim of this study was to design, build, and test an aerosol deposition chamber that could be used to model real-world contamination using a Bacillus anthracis simulant. The test chamber was constructed from aluminum, with the goal to reduce static electricity which would be detrimental to particle depositions. Equations were derived to model the deposition of particles. Results showed that fluorescent particles could not be used to test deposition because of the high number or particle solution needed. Bacillus atrophaeus subsp globigii (BG) spores were then used to test the deposition.

Twenty-nine deposition tests were completed with these BG spores. These tests were completed to determine the parameters that would result in the most even deposition. Four final tests were completed utilizing these parameters. These tests had relatively low coefficients of variation, ranging from $15.4 \%$ to $25.5 \%$. Ryan-Joiner tests on these data showed that two of the data sets were lognormal distributed and the other two were inconclusive. All subsequent tests were therefore handled as lognormal distributions. Contour plots showed even deposition; however, there were no discernible patterns over all four tests. These plots did demonstrate that the sample should be gathered as closely as possible in the middle of the chamber.

Forward regression models were then completed, using the log of the spores as the response variable and the $\mathrm{X}$ and $\mathrm{Y}$ coordinates of the chamber floor as the explanatory variables. Also considered were these coordinates squared $\left(\mathrm{X}^{2}\right.$ and $\left.\mathrm{Y}^{2}\right)$ and the $\mathrm{X}^{*} \mathrm{Y}$ interaction. There was no one regression model that fitted the best (by analyzing the $\mathrm{R}^{2}$ values). Following the regression models, the residuals were tested to determine if the model variables were normally

distributed. All p-values were $>0.100$; therefore, there was not enough evidence to reject $\mathrm{H}_{\mathrm{o}}$, that is, that the data residuals were lognormal distributed. 
The final analysis was to determine if the equations derived adequately predicted the deposition of the spores. The recovery efficiencies for the spore deposition ranged from $8.67 \%$ to $31.0 \%$, with an average recovery of $20.25 \%$ over the four tests. This shows that almost $80 \%$ of the spores were lost during this process. These losses could have occurred during the nebulization, deposition, or culturing process. One possibility is that the spores clumped during one of these stages. The clumping was controlled as much as possible, which included using a Collision nebulizer generating aerosols with sizes between 1 to $3 \mu \mathrm{m}$ and also a $\mathrm{Kr}-85$ neutralizer. The clumping can occur after the aerosols are generated while the spores are settling. Regardless of where the losses occurred, these tests showed that the recovery efficiency provided a basis to model future experiments which can then account for these losses.

\section{Conclusion}

Overall, this project demonstrated that an aerosol chamber can be designed and built for the purpose of spore deposition onto coupons. The spore deposition can be modeled as long as the losses are accounted for during the processes. The contour plots showed somewhat even deposition for each individual test; however, there was a wide range over the four final tests completed. The wide deposition range occurred even with all the controllable parameters remaining constant. The study demonstrated that the test chamber can be used for spore depositions with the caveat that future studies include an appropriate control next to each sample.

\section{ACKNOWLEDGEMENTS}

This study was funded in part by a grant from the Mountain and Plains Research Center (MAP ERC) through Dr. Steven Reynolds, Deputy Director. Additional funds were provided through a grant from AFMSA/SGRS. Funds were committed with the assistance of William 
Kilpatrick (711 HPQ/RHXS), Gregory Sudberry (711 HPW/RHPC), SSgt Robert Arrington (711 HPW/RHPCB), SrA Ashley Grant (ASC/PKOA), and Tim Provens (711 HPW/RHPC). Aluminum coupons were provided by William Culhane (AFRL/RXSSO), USAF Coating Technology Integration Office. Spores were provided by Joe Dalmasso, Yakibou, Inc. Kirsten Koehler provided assistance with developing and testing the bioaerosol chamber. 


\section{REFERENCES}

Air Force Research Laboratory (AFRL): Bio-thermal decontamination tests for large frame aircraft application laboratory and field test report, AFRL Document HEPC OSMSWP8Z, 5 Dec 2008.

Aizenberg, V., S.A. Grinshpun, K. Willeke, J. Smith, and P.A. Baron: Performance characteristics of the button personal inhalable aerosol sampler. American Industrial Hygiene Association Journal 61: 398-404 (2000a).

Alibek, K.: (2005, April 22-23). Biological Weapons/Bioterrorism Threat and Defense-Past, Present, and Future. Paper presented at ETH international conference, Meeting the Challenges of Bioterrorism: Assessing the Threat and Designing Biodefense Strategies, Furigen, Switzerland.

Baron, P.A., C.F. Estill, J.K. Beard, M.J. Hein, and L. Larsen: Bacterial endospore inactivation caused by outgassing of vapourous hydrogen peroxide from polymethyl methacrylate (Plexiglas $\left.{ }^{\circledR}\right)$. Letters in Applied Microbiology 45: 485-490 (2007).

Baron, P.A., C.F. Estill, G.J. Deye, M.J. Heim, J.K. Beard, L.D. Larsen, and G.E. Dahlstrom: Development of an aerosol system for uniformly depositing Bacillus anthracis spore particles on surface. Aerosol Science and Technology 42: 159-172 (2008).

BGI, Inc: Collison Nebulizer Droplet Number Output (Online) Available at http://bgiusa.com/agc/droplet_number_output.htm (accessed March 2011).

Brachman, P.S.: Bioterrorism: an update with a focus on anthrax. American Journal of Epidemiology 155: 981-987 (2002).

Brickhouse, M.: Innovative and Emerging Decontamination Technologies. In Report on Workshop on Decontamination, Cleanup and Associated Issues for Sites Contaminated with Chemical, Biological, or Radiological Materials, S. Dun, and J. Wood, US EPA: October 2005.

Brown, G.S.; R.G. Betty, J.E. Brockman, D.A. Lucero, C.A. Souza, K.S. Walsh, R.M. Boucher, M.S. Tezak, and M.C. Wilson: Evaluation of vacuum filter sock surface sample collection method for Bacillus spores from porous and non-porous surfaces. Journal of Environmental Monitoring 9(7): 666-671 (2007a).

Brown, G.S., R.G. Betty, J.E. Brockman, D.A. Lucero, C.A. Souza, K.S. Walsh, R.M. Boucher, M. Tezak, M.C. Wilson, and T. Rudolph: Evaluation of a wipe surface sample method for collection of Bacillus spores from nonporous surfaces. Applied and Environmental Microbiology 73(3): 706-710 (2007b).

Brown, G.S., R.G. Betty, J.E. Brockmann, D.A. Lucero, C.A. Souza, K.S. Walsh, R.M. Boucher, M.S. Tezak, M.C. Wilson, T. Rudolph, H.D. Lindquist, and K.F. Martinez: Evaluation of rayon swab surface sample collection method for Bacillus spores from nonporous surfaces. Journal of Applied Microbiology 103(4): 1074-1080 (2007c). 
Burgess, W.A., M.J. Ellenbecker, and R.D. Treitman: Ventilation for Control of the Work Environment. Hoboken, NJ: John Wiley \& Sons, 2004.

Burke, S.A., J.D. Wright, M.K. Robinson, B.V. Bronk, and R.L. Warren: Detection of molecular diversity in Bacillus atrophaeus by amplified fragment length polymorphism analysis. Applied and Environmental Microbiology 79(5): 2786-2790 (2004).

Burton, N.C., A. Adhikari, S.A. Grinshpun, R. Hornung, and T. Reponen: The effect of filter material on bioaerosol collection of Bacillus subtilis spores used as a Bacillus anthracis stimulant. Journal of Environmental Monitoring 7: 475-480 (2005).

Buttner, M. P., P. Cruz, L.D. Stetzenbach, A.K. Klima-Comba, V.L. Stevens, and P.A. Emanuel: Evaluation of the biological sampling kit (BiSKit) for large-area surface sampling. Applied Environmental Microbiology 70: 7040-7045 (2004).

Byrne, MA; A.J.H. Goddard, C. Lange, and J. Roed: Stable tracer aerosol deposition measurements in a test chamber. Journal of Aerosol Science 26(4): 645-653 (1995).

Carrera, M., J. Kesavan, R. Zandomeni, and J.-L. Sagripanti: Method to determine the number of bacterial spores within aerosol particles. Aerosol Science and Technology 39: 960-965 (2005).

Centers for Disease Control: Fact Sheet: Anthrax Information for Health Care Providers. (Online). Available at www.bt.cdc.gov/agent/anthrax/anthrax-hcp-factsheet.asp, 2002

Centers for Disease Control: Investigation of bioterrorism-related anthrax - Connecticut. Morb Mortal Wkly Rep, 50: 1077-1079 (2001).

Chosewood, L.C., and D.E. Wilson, eds.: Biosafety in Microbiological and Biomedical Laboratories, $5^{\text {th }}$ Ed., Pub. No. 21-1112. Atlanta, GA: US Department of Health and Human Services, Centers for Disease Control, NIH, 1992.

Department of Defense: The Chemical, Biological, Radiological, and Nuclear (CBRN) Survivability Policy, Pub No. DoDI 3150.09 (August 17, 2009)

Edmonds, J.M., P.J. Collett, E.R. Valdes, E.W. Skowronski, G.J. Pellar, and P.A. Emanuel: Surface sampling of spores in dry-deposition aerosols. Applied and Environmental Microbiology 75(1): 39-44 (2009).

Estill, C.F., P.A. Baron, J.K. Beard, M.J. Hein, L.D. Larsen, L. Rose, F.W. Schaefer III, J. Noble-Wang, L. Hodges, H.D.A. Lindquist, G.J. Deye, and M.J. Arduino: Recovery efficiency and limit of detection of aerosolized Bacillus anthracis sterne from environmental surface samples. Applied and Environmental Microbiology 75(13): 4297-4306 (2009).

Estill, C.F., and G.J. Deye: Personal communication, January 7, 2010. 
Farnsworth, J.E., S.M. Goyal, S.W. Kim, T.H. Kuehn, P.C. Raynor, M.A. Ramakrishnan, S. Anantharaman, and W. Tang: Development of method for bacteria and virus recovery from heating, ventilation, and air conditioning (HVAC) filters. Journal of Environmental Monitoring 8: 1006-1013 (2006).

Feather, G.A., and B.T. Chen: Design and use of a settling chamber for sampler evaluation under calm-air conditions. Aerosol Science and Technology 37: 261-270 (2003).

Fitch, J.P. (Chair): A Framework for Assessing the Health Hazard Posed by Bioaerosols, Washingtong, DC: The National Research Council, National Academis Press, 2008.

Foarde, K.K., J.T. Janley, D.S. Ensor, and P. Roessler: Development of method for measuring single-pass bioaerosol removal efficiencies of a room air cleaner, Aerosol Science Technology 30: 223-234 (1999).

Han, Taewon; H.R. An, G. Mainelis: Performance of an electrostatic precipitator with superhydrophobic surface when collecting airborne bacteria. Aerosol Science and Technology 44(5): 339-348 (2010).

Hill S.C., R.G. Pinnick, S. Niles, Y.L. Pan, S. Holler, R.K. Chang, J. Bottiger, B.T. Chen, C.S. Orr, G. Feather, and A.P. Snyder: Real-time measurement of fluorescence spectra from single airborne biological particles. Field Analysis and Chemical Technology 3: 221-239 (1999).

Hinds, W.C.: Aerosol Technology: Properties, Behavior, and Measurement of Airborne Particles. New York: John Wiley \& Sons, Inc., 2004.

Inglesby, V., D.A. Henderson, J.G. Bartlett et al.: Anthrax as a biological weapon. Journal of American Medical Association 281(18): 1735-45 (1999).

Jensen, P.A., W.F. Todd, G.N. Davis, and P.V. Scarpino: Evaluation of eight bioaerosol samplers challenged with aerosols of free bacteria. American Industrial Hygiene Association Journal 53: 660-667 (1992).

Jernigan, D.B., P.L. Raghunathan, B.P. Bell et al.: Investigation of bioterrorism-related anthrax, United States, 2001: epidemiologic findings. Emerging Infectious Diseases 8: 10191028 (2002).

Keith, L.H., W. Crummett, J. Deegan, R.A. Libby, J.K. Taylor, and G. Wentler: Principals of environmental analysis. Annals of Chemistry 55: 2210-2218 (1983).

Kenny, L.C., R.J. Aitken, P.E.J. Baldwin, G.C. Beaumont, and A.D. Maynard: The sampling efficiency of personal inhalable aerosol samplers in low air movement environments. Journal of Aerosol Science 30(5): 627-638 (1999). 
Kesavan, J., J.R. Bottiger, and A.R. McFarland: Bioaerosol concentrator performance: comparative tests with viable and with solid and liquid nonviable particles. Journal of Applied Microbiology 104: 285-295 (2008b).

King, B., J. Kesavan, and J.-L. Sagripanti: Germicidal UV sensitivity of bacteria in aerosols and on contaminated surfaces. Aerosol Science and Technology 45(5): 645-653 (2011).

Koch, W., W. Dunkhort, and H. Lodding: Design and performance of a new personal aerosol monitor. Aerosol Science (1999).

Kournikakis, B., M. Walker, J. Ho et al.: Statistical analysis of bacterial spore aerosols created by opening a spore containing anthrax letter in an office. Journal of Aerosol Science 40: 514-522 (2009).

Krumins, V., E.-K. Son, G. Mainelis, and D.E. Fennell: Retention of inactivated bioaerosols and ethene in a rotating bioreactor constructed for bioaerosol activity studies. Clean 36(7): 593600 (2008).

Lai K.M., H.A. Burge, and M.W. First: Size and UV germicidal irradiation susceptibility of Serratia marcescens when aerosolized from different suspending media. Applied Environmental Microbiology 70(4): 2021-2027 (2004).

Lewandowski, R., K. Kozlowska, M. Szpakowska, M. Stepin'ska, and E.A. Trafny: Use of a foam spatula for sampling surfaces after bioaerosol deposition. Applied and Environmental Microbiology 76(3): 688-694 (2010).

Li, C.S., and Y.C. Lin: Storage effects on bacterial concentration: determination of impinge and filter samples. Science of the Total Environment 278: 231-237 (2001).

Mainelis, G., A. Adhikari, K. Willeke, S. Lee, T. Reponen, and S.A. Grinshpun: Collection of airborne microorganisms by a new electrostatic precipitator. Aerosol Science 33: 1417-1432 (2002).

Marple, V.A., and K.L. Rubow: An aerosol chamber for instrument evaluation and calibration. American Industrial Hygiene Association Journal 44: 361-367 (1983).

Martin, D. D., and L. M. Moore: Technical report for the comparison of swab-sampling techniques for recovery of bacterial spores from three surfaces. West Desert Test Center document no. WDTC-TR-00-018, U.S. Army Dugway Proving Ground, Utah, (2001).

Maus, R., A. Goppelsroder, and H. Umhauer: Atmospheric Environment 35: 105-113 (2001).

May, K. R.: The collison nebulizer: description, performance and application. Journal of Aerosol Science 4: 235-243 (1973). 
Nicholson, W.L., P. Fajardo-Cavazos, R. Rebeil, T.A. Slieman, P.J. Riesenman, J.F. Law, and Y. Xue: Bacterial endospores and their significance in stress resistance. Antonie Leeuwenhoek 81: 27-32 (2002).

Patrick W.C.: Risk Assessment of Biological Warfare Primary and Secondary Aerosols and Their Requirements for Decontamination. Science Applications International Corp, Vienna, Va (1999).

Park, J.M., J.C. Rock, L. Wang, Y.C. Seo, A. Bhatnagar, and S. Kim: Performance evaluation of six different aerosol samplers in a particulate matter generation chamber. Atmospheric Environment 43: 280-289 (2009).

Perkins, J.J.: Principles and Methods of Sterilization in Health Sciences,. $2^{\text {nd }}$ ed.. Springfield, IL, Charles C. Thomas Publishers, 1983.

Peters, C.J., and D.M. Hartley: Anthrax inhalation and lethal human infection. Lancet. 359: 710-711 (2002).

Priest, F.G.: Systematics and Ecology of Bacillus, 3-16. In Bacillus subtilis and Other GramPositive Bacteria: Biochemistry, Physiology, and Molecular Genetics. A.L. Sonenshein, J.A. Hoch, and R. Losick (eds.). Washington, DC: ASM Press, 1993.

Ryan, J.R., and J.F. Glarum: Biosecurity \& Bioterrorism: Containing and Preventing Biological Threats. Elsevier: New York, 2008.

Sagripanti, J.L., M. Carrera, J. Insalaco, M. Ziemski, J. Rogers, \& R. Zandomeni: Virulent spores of Bacillus anthracis and other bacillus species deposited on solid surfaces have similar sensitivity to chemical decontaminants. Journal of Applied Microbiology 102: 11-21 (2007).

Sinclair, R., S.A. Boone, D. Greenberg, P. Keim, and C.P. Gerba: Persistence of category A select agents in the environment. Applied Environmental Microbiology 74: 555-563 (2008).

Sonenshein, A.L., J.A. Hoch, and R. Losick: Bacillus subtilis and Other Gram-Positive Bacteria: Biochemistry, Physiology, and Molecular Genetics, xi-xiii, Washington, DC: ASM Press, 1993.

Thatcher, T.L., and W.W. Nazaroff: Effects of small-scale obstructions and surface textures on particle deposition from natural convection flow. Aerosol Science Technology 27: 709-725 (1997).

Thomas, R.J., D. Webber, W. Sellors, A. Collinge, A. Frost, A.J. Stagg, S.C. Bailey, P.N. Jayasekera, R.R. Taylor, S. Eley, R.W. Titball: Characterization and deposition of respirable large- and small-particle bioaerosols. Applied and Environmental Microbiology 74(20): 64376443 (2008). 
Uhm, H.S., H.Y. Lee, Y.C. Hong, D.H. Shin, Y.H. Park, Y.F. Hong: A decontamination study of simulated chemical and biological agents. Journal of Applied Physics 102 (2007).

United States Army Medical Research Institute for Infectious Diseases (USAMRIID): Medical Management of Biological Casualties Handbook, $6^{\text {th }}$ Ed. J.B. Woods (Ed) Fort Detrick, Maryland, 2005.

Wagner, A., C.F. Green, V. Pedregon, E. Barth, S.G. Gibbs, and P.V. Scarpino: Inactivation of Bacillus subtilis on gypsum board using aerosolized chemical agents. Journal of Environmental Engineering Science 7: 159-164 (2008).

Watson, A., and D. Keir: Information on which to base assessments of risk from environments contaminated with anthrax spores. Epidemiology and Infection 113: 479-90 (1994).

Yah, M., and G. Mainelis: Use of portable microbial samples for estimating inhalation exposure to viable biological agents. Journal of Exposure Science and Environmental Epidemiology 17: 31-38 (2007). 


\section{APPENDIX: Test chamber raw data}

Raw data from the chamber tests are presented below in Table II-IX.

Table A2 - 1 - Raw data, tests 72, 76, 80, 81

\begin{tabular}{|c|c|c|c|c|c|c|c|c|c|c|c|c|c|}
\hline \multirow{2}{*}{$\begin{array}{l}\text { Sample } \\
\text { Location }\end{array}$} & \multicolumn{2}{|c|}{ Test 72} & \multicolumn{2}{|c|}{ Test 76} & \multicolumn{2}{|c|}{ Test 80} & \multicolumn{2}{|c|}{ Test 81} & \multicolumn{5}{|c|}{ Coordinates (chamber floor for each test) } \\
\hline & Raw & $\log$ & Raw & $\log$ & Raw & $\log$ & Raw & Log & $x$ & $\mathbf{Y}$ & $x^{\wedge} 2$ & $\mathbf{Y}^{\wedge} \mathbf{2}$ & $\mathrm{X} * \mathrm{Y}$ \\
\hline A & 114 & 2.06 & 29 & 1.46 & 53 & 1.72 & 92 & 1.96 & -56.3 & -20.9 & 3169.7 & 437.6 & $1177 . \varepsilon$ \\
\hline $\mathrm{B}$ & 70 & 1.85 & 18 & 1.26 & 49 & 1.69 & 81 & 1.91 & -56.3 & 0.0 & 3169.7 & 0.0 & 0.0 \\
\hline C & 70 & 1.85 & 21 & 1.32 & 59 & 1.77 & 92 & 1.96 & -56.3 & 20.9 & 3169.7 & 437.6 & $-1177 . \varepsilon$ \\
\hline $\mathrm{D}$ & 66 & 1.82 & 26 & 1.41 & 55 & 1.74 & 84 & 1.92 & -33.8 & -20.9 & 1141.1 & 437.6 & 706.7 \\
\hline $\mathrm{E}$ & 70 & 1.85 & 25 & 1.40 & 38 & 1.58 & 93 & 1.97 & -33.8 & 0.0 & 1141.1 & 0.0 & 0.0 \\
\hline $\mathrm{F}$ & 85 & 1.93 & 24 & 1.38 & 58 & 1.76 & 94 & 1.97 & -33.8 & 20.9 & 1141.1 & 437.6 & -706.7 \\
\hline $\mathrm{G}$ & 78 & 1.89 & 38 & 1.58 & 47 & 1.67 & 60 & 1.78 & -11.3 & -20.9 & 126.8 & 437.6 & 235.6 \\
\hline $\mathrm{H}$ & 78 & 1.89 & 29 & 1.46 & 53 & 1.72 & 86 & 1.93 & -11.3 & 0.0 & 126.8 & 0.0 & 0.0 \\
\hline 1 & 61 & 1.79 & 19 & 1.28 & 43 & 1.63 & 88 & 1.94 & -11.3 & 20.9 & 126.8 & 437.6 & -235.6 \\
\hline $\mathrm{J}$ & 72 & 1.86 & 31 & 1.49 & 44 & 1.64 & 73 & 1.86 & 11.3 & -20.9 & 126.8 & 437.6 & -235.6 \\
\hline $\mathrm{K}$ & 87 & 1.94 & 24 & 1.38 & 51 & 1.71 & 88 & 1.94 & 11.3 & 0.0 & 126.8 & 0.0 & 0.0 \\
\hline $\mathrm{L}$ & 73 & 1.86 & 23 & 1.36 & 62 & 1.79 & 94 & 1.97 & 11.3 & 20.9 & 126.8 & 437.6 & 235.6 \\
\hline $\mathrm{M}$ & 58 & 1.76 & 33 & 1.52 & 68 & 1.83 & 144 & 2.16 & 33.8 & -20.9 & 1141.1 & 437.6 & -706.7 \\
\hline $\mathrm{N}$ & 58 & 1.76 & 23 & 1.36 & 50 & 1.70 & 154 & 2.19 & 33.8 & 0.0 & 1141.1 & 0.0 & 0.0 \\
\hline 0 & 60 & 1.78 & 22 & 1.34 & 66 & 1.82 & 109 & 2.04 & 33.8 & 20.9 & 1141.1 & 437.6 & 706.7 \\
\hline $\mathrm{P}$ & 51 & 1.71 & 34 & 1.53 & 47 & 1.67 & 97 & 1.99 & 56.3 & -20.9 & 3169.7 & 437.6 & -1177.8 \\
\hline Q & 66 & 1.82 & 18 & 1.26 & 48 & 1.68 & 68 & 1.83 & 56.3 & 0.0 & 3169.7 & 0.0 & 0.0 \\
\hline $\mathrm{R}$ & 55 & 1.74 & 29 & 1.46 & 60 & 1.78 & 71 & 1.85 & 56.3 & 20.9 & 3169.7 & 437.6 & 1177.8 \\
\hline Average & 71 & 1.85 & 26 & 1.41 & 53 & 1.72 & 93 & 1.97 & & & & & \\
\hline SD & 14.7 & 1.17 & 5.71 & 0.76 & 8.12 & 0.91 & 23.66 & 1.37 & & & & & \\
\hline $\mathrm{CV}$ & 20.8 & 1.32 & 22 & 1.34 & 15.4 & 1.19 & 25.5 & 1.41 & & & & & \\
\hline Neg control & 0 & & 0 & & 0 & & 0 & & & & & & \\
\hline Pos control & TNTC & & TNTC & & TNTC & & TNTC & & & & & & \\
\hline
\end{tabular}

*Negative controls were petri dishes placed in the chamber to verify no cross-contamination. Positive controls were spiked petri dishes taken to the test chamber during the aerosol generation and deposition to verify the spores would germinate appropriately. 
The locations of the samples are presented in Figure 2-33.

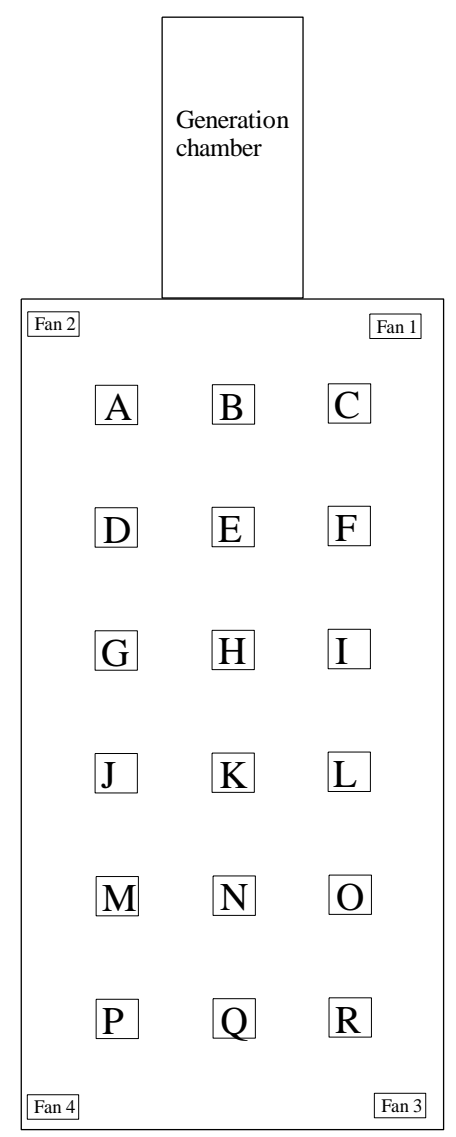

Figure 2- 33 - Spore deposition test locations on chamber floor 
CHAPTER 3 -- DECONTAMINATION OF A BACILLUS ANTHRACIS SPORE SIMULANT ON AIRCRAFT ALUMINUM COUPONS USING HIGH HEAT AND HUMIDITY WITHIN AIRCRAFT ENGINEERING TOLERANCES

\section{SUMMARY}

The goal of this research project was to determine if aluminum coupons, coated like aircraft materials, could be effectively decontaminated from a Bacillus anthracis simulant (Bacillus atrophaeus subsp globigii [BG]) using high heat and humidity within the engineering specifications of aircraft. These spores were deposited using a high direct inoculation $\left(10^{6}\right.$ spores per coupons), low direction inoculation ( $10^{4}$ spores per coupons), and an innovative aerosol deposition method (goal of $10^{4}$ spores per coupons) using a bioaerosol test chamber. Previous studies have evaluated only direct inoculations in the range of $10^{6}$ spores. Initial tests found the optimal method to remove the spores from coupons was sonication followed by vortexing. Sonication was nearly five times more effective at removing the spores than shaking. Equations, derived to model spore depositions in the aerosol test chamber, were tested and showed that $10 \%$ of the spores could be effectively recovered. Five different test conditions of temperature and humidity (ranging from an upper limit combination of $180^{\circ} \mathrm{F}$ and $90 \%$ relative humidity $[\mathrm{RH}]$ to a lower limit of $160^{\circ} \mathrm{F}$ and $70 \% \mathrm{RH}$ ) were evaluated over 24 hour increments with an upper time limit of 120 hours. Decontamination tests showed that the high concentrations of spores were all inactivated within 24 hours at $180^{\circ} \mathrm{F}$ with $90 \% \mathrm{RH}$ and partially inactivated at $170^{\circ} \mathrm{F}$ with $80 \% \mathrm{RH}$. Tests using low direct inoculations showed complete kills at 48 hours when treatment was $180^{\circ} \mathrm{F}$ with $90 \% \mathrm{RH}$ and at 96 hours when treatment was $170^{\circ} \mathrm{F}$ with $80 \% \mathrm{RH}$. All spores deposited by aerosols were inactivated within the 120 hour time period. A stepwise regression was performed to determine which variables were significant to predict the inactivation rates ( $\alpha=0.05$ was used to keep or discard terms). For this 
regression, there were three variables required to be in each model-time, temperature, and humidity. The data for the stepwise regression retained more variables for high direct inoculation (10 predictors) than low (8 predictors) or aerosol deposition (5). The only variable retained by all three models, besides the mandatory variables, was Temp ${ }^{2} * \mathrm{Time}^{2}$. For both of the direct inoculation methods, several of the same variables were retained, which included Temp*Humidity, Temp*Time, Humidity ${ }^{2}$, and Temp ${ }^{2} *$ Time $^{2}$. More of the predictor variables for high inoculation included an interaction with time when compared to the predictors for low inoculation. These variables were then used to complete a final regression model. The final regression models demonstrated $\mathrm{R}^{2}$ values for high and low inoculation methods, $76.4 \%$ and $71.5 \%$, respectively, accounting for a large portion of the variability within the inactivation. The $\mathrm{R}^{2}$ for the aerosol deposition model was not as strong, being only $38.5 \%$, showing that a much smaller portion of the variability is captured by the model. The ideal humidity and temperature range is clearly the highest levels that can be delivered, reasonably maintained, and within proper engineering specifications. If $90 \%$ humidity cannot be easily generated or maintained throughout the body of an aircraft, the results show that $80 \%$ at the proper temperature $\left(170^{\circ} \mathrm{F}\right.$ or higher) can be effective as well. Additionally, the delivery method impacts how long it will take to inactivate the spores, with aerosol delivered spores inactivated more quickly. A critical component that needs to be considered is time required to decontaminate the item. If the item being decontaminated can remain out of service for a longer period of time, lower temperatures and humidity levels could be used. The research demonstrated that these spores, and hence microbiological warfare agents, can be inactivated safely, effectively, and also within aircraft engineering specifications using high heat and humidity. 


\section{INTRODUCTION}

Bioterrorism is defined as a use or threatened use of biological agents against individuals to obtain advantage for a specific purpose such as intimidation, ideological principles, or disruption of everyday activities (Brachman, 2002). In an act of biological terrorism or warfare, diagnosis of the agent in a short time can be difficult, which may hamper decontamination efforts (Estill et al., 2009). To minimize illnesses, decontamination to acceptable levels in a short time is critical (Uhm et al., 2007). Once decontamination is conducted, another difficulty is detecting the agents afterwards to ensure they have been adequately removed and/or inactivated (Uhm et al., 2007).

DoDI 3150.09, "The Chemical, Biological, Radiological, and Nuclear (CBRN) Survivability Policy", requires all DoD assets to be able to continue operations even in the presence of biological agents, including the capability to be decontaminated properly (DoD, 2009). Decontamination can entail several different inactivation levels, with the most basic definition requiring the object to be free of contamination and safe for human handling without further recourse to individual protective measures (Perkins, 1983). Sterilization is a procedure that kills all microorganisms, including high numbers of bacterial endospores, while disinfection is less lethal than sterilization, eliminating nearly all recognized pathogenic microorganisms, but not ensuring "overkill" and lacking the margin of safety achieved by sterilization procedure because spores are not inactivated (Chosewood and Wilson, 2009). The DoD defines decontamination as a "process making material safe by absorbing, destroying, neutralizing, rendering harmless, or removing chemical or biological agents and radiological contamination" (DoD, 2009). These terms are critical because there is disagreement on the level of inactivation required, with some stating a 6-log reduction is usually considered adequate with others 
suggesting that a 12-log reduction is required; however, most field trials have targeted a 6-log reduction (Gale et al., 2009). Others have said that any detectable Bacillus anthracis spore would constitute an unacceptable risk (Herzog et al., 2009).

Regardless of the level of decontamination required, decontamination tests are generally completed on spores. The U.S. Army Edgewood Chemical and Biological Center (ECBC) requires that the decontamination methods be effective against spores. These spores are tested during decontamination to not only to facilitate the inactivation processes, but also because a first responder will not necessarily have the ability to identify the microbiological agent (Brickhouse, 2005). Any decontamination methods must be able to inactivate Bacillus anthracis, which is the target because the spore is considered the most difficult biological warfare agent to decontaminate. The endospores are metabolically inactive and are highly resistant to many physical stresses such as wet and dry heat, chemical agents, UV and gamma radiation, oxidizing agents, vacuums and ultra-high hydrostatic pressures (Nicholson et al., 2002). The spores are stable for up to 60 years in soil and water and can resist sunlight for varying periods (Chosewood and Wilson, 2009; Perkins, 1983). For these reasons, the spores can remain viable for years creating a serious and lasting health risk (Nicholson et al., 2002).

Bacillus anthracis spores are also chosen to test decontamination because they make an ideal biological warfare agent for several reasons - they are easy to produce, easy to disperse, the most stable biological weapon (Cordesman, 2005; Estill, 2010; Ryan and Glarum, 2008), and the number of spores required for infection are low, down to 10 spores or fewer for cutaneous anthrax (Peters and Hartley, 2002; Watson and Keir 1994;). As seen after the 2001 anthrax attacks, the spores can become re-aerosolized, causing illnesses when there was no direct exposure to the spore release zone (CDC 2001; Jernigan et al., 2001; Kornikakis et al., 2009). 
Because of the spore lethality, simulants are used. These simulants have included Bacillus subtilis var niger (also known as Bacillus globigii (BG) or Bacillus subtilis) (Aizenberg et al., 2000; Burton et al., 2005; Carrera et al., 2005; Farnsworth et al., 2006; Foarde et al., 1999; Hill et al., 1999; Jensen, 1992; Mainelis et al., 2002; Maus et al., 2001; Li and Lin, 2001; Sagripanti et al., 2007; Wagner et al., 2008; Yah and Mainelis, 2007). Additionally, Bacillus atrophaeus has been used in the past because the spore is virtually indistinguishable from Bacillus subtilis. Some of the Bacillus subtilis lines used in the past were identified as a new strain, Bacillus atrophaeus susp globigii (Burke et al., 2004). Bacillus atrophaeus spores have been used in several studies as well (Brown et al., 2007a; Carrera et al., 2005; Kesavan, 2008; Lewandowski et al., 2010; Martin and Moore, 2001; Thomas et al., 2008;).

Several test chambers have been designed to aerosolize and then deposit these simulants onto some type of coupon for further testing (Baron et al., 2007; Baron et al., 2008; Brown et al., 2007a, 2007b, 2007c; Buttner et al., 2004; Byrne et al., 1995; Chen et al., 1999; Edmonds et al., 2009; Estill et al., 2009; Farnsworth et al., 2006; Feather and Chen, 2003; Kenny et al., 1999; Kesavan, 2008; Koch et al., 1999; King et al., 2011; Lai et al., 2002; Lewandowski et al., 2010; Marple and Rubow, 1983; Park et al., 2009; Thatcher and Nazaroff, 1997). With few exceptions (King, 2010), these chambers were constructed to evaluate deposition or swipe sampling and have not been used to evaluate the effectiveness of decontamination methods.

The actual decontamination process can be completed in several different ways, some of which were used during the 2001 anthrax attacks. After these attacks, the EPA granted crisis exemptions for four liquid anthrax sporicides - chlorine dioxide, hydrogen peroxide/peroxyacetic acid, sodium hypochlorite, and hydrogen peroxide/quarternary ammonium 
foam. Five gases were approved — chlorine dioxide, vaporized hydrogen peroxide, paraformaldehyde, methyl bromide, and ethylene oxide (Kempter, 2005).

Hydrogen peroxide is one of the disinfectants recently used on spores. It can be used in two methods: vaporized hydrogen peroxide (VHP) when the compound remains in the vapor phase, and hydrogen peroxide vapor (HPV) when a very small amount of condensation is induced deliberately (Gale et al., 2009). VHP has been used for some time on smaller scales, such as in pharmaceutical companies and clean rooms (McVey, 2005); however it was scaled up for use in 2001. Following the 2001 anthrax attacks, the GSA Building 410, a 1.4 million- $\mathrm{ft}^{3}$ building used for office supply storage and area mail-sorting facility, was decontaminated with VHP, effectively inactivating the spores (McVey, 2005). Additional studies have been conducted since 2001 to further validate the efficacy of VHP. One study using aerosolized hydrogen peroxide combined with peroxyacetic acid was found to have a 3.09-log reduction of B. cereus cells (Oh et al., 2005), while VHP alone reduced Bacillus subtilis endospores by $87 \%$ after 120 minutes (Andersen et al., 2006). A VHP system was tested on a non-flying C-141 cargo aircraft, showing an inactivation rate of $99.9 \%$ after 120 hours (McVey, 2005). Another test using VHP with thermal decontamination on a wide-body aircraft demonstrated that heat and VHP were sporicidal at several locations within the cabin; however, several locations did not see 6-log reduction because the VHP could not reach all areas. The spores were killed within two hours if the concentration was 250 parts per million (ppm) of hydrogen peroxide for 2 hours (Gale et al., 2008). A test on a DC-9 found that maintaining proper humidity is critical for lower concentrations of the VHP (Gale et al., 2008). Though very effective with a low environmental impact, hydrogen peroxide is a strong oxidizing agent presenting severe material impacts (Gale et al., 2009). After exposures for 4 to 8 hours at $450 \mathrm{ppm}$, microstructural effects were found on 
aluminum alloys and stainless steel. A single cycle had negligible effects, but after 25 cycles the materials had weight gains indicating oxidation. Surface softening was slight and confined to the immediate vicinity of the surface, necessitating further work (Gale et al., 2009). Studies have shown minimal oxidative damage after 100 experiments over one year, but there was a patina on the surfaces area where the VHP was introduced (Verce et al., 2008).

Another type of decontamination chemical is chlorine in several different forms, including chlorine dioxide $\left(\mathrm{ClO}_{2}\right)$ and sodium hypochlorite $(\mathrm{NaOCl})$. Chlorine dioxide gas was used in the cleanup of building interiors contaminated with spores of Bacillus anthracis in 2001 (Rastogi et al., 2009; Barth et al., 2003). Perez et al. (2005) found that liquid disinfectants on hard surfaces were effective to reduce organism load, including Bacillus subtilis tests. Other studies have shown 1.55 to 1.92 log kill rates on Bacillus subtilis on untreated gypsum wall board for commercially available bleach as well as for chlorine dioxide at 500 ppm (Wagner et al., 2008). Aqueous $\mathrm{ClO}_{2}$ was also used on nonporous surfaces in two mail sorting machines (Canter et al., 2005). Despite its efficacy, cleaning with bleach, as in the mail room facilities, should be done sparingly because it is highly damaging to many materials (Orlusky, 2005).

Yet another chemical is methyl bromide, which has been used for more than 60 years to fumigate ships carrying fruit and vegetables. Cheap and stable, it can treat all porous materials with a rapid turnover time. Methyl bromide was tested on a $30,000 \mathrm{ft}^{3}$ home and after 48 hours at $37^{\circ} \mathrm{C}$, there was a complete kill on Bacillus anthracis and Geobacillus stearothermophilus; however, Bacillus atrophaeus and Bacillus thuringiensis experienced only partial kills in an area where the methyl bromide could easily reach. No damage to electronic equipment was observed (Scheffrahn, 2005). 
All these existing biological decontamination solvents are at least somewhat hazardous to aircraft materials so they cannot currently be used on Air Force aircraft (AFRL, 2008). All aircraft, however, must meet strict engineering specifications that include withstanding high temperature storage greater than $185^{\circ} \mathrm{F}$ at $100 \%$ relative humidity for prolonged periods. Temperatures and humidity levels at these levels may provide a potential method to inactivate biological agents (AFRL, 2008). For this reason, the Air Force has evaluated the use of high heat and humidity for decontaminating aircraft. Most spores have a greater resistance to dry heat than moist heat (Perkins, 1983); however, the effect of relative humidity on decontamination is not fully understood (Peccia et al., 2001). Dry heat $\left(60\right.$ minutes at $\left.320^{\circ} \mathrm{F}\right)$ has the same effect as moist heat $\left(15 \mathrm{~min}\right.$ at $250^{\circ} \mathrm{F}$ in moist heat) for sterilization purposes (Perkins, 1983). AFRL has conducted laboratory and field tests on a Large Frame Aircraft (LFA) to determine the feasibility of using high temperatures and high relative humidity to inactivate known biological organism threats. Heated air for decontamination offers the following advantages - it is benign as long as all components are compatible with $180^{\circ} \mathrm{F}$ storage, the method presents means of "enhanced" weathering, and technology exists for this method; thus, efforts to field such a unit would be minimal (AFRL, 2008). Several studies have been conducted on grounded aircraft to determine if the technology is feasible in this environment. These tests, completed at $180^{\circ} \mathrm{F}$ and relative humidity ranges from 75 to $90 \%$, showed a 5 to $6 \log$ reduction in Bacillus thuringiensis var kurstaki (BtK or Bt) spores. The studies have shown that these ranges are capable of inactivating the spores within the engineering specifications (AFRL, 2008).

Testing decontamination efforts requires the spores, whether active or inactive, to be efficiently removed from the substrate. This is problematic because there is an overall lack of consensus for spore removal in the literature (Probst et al., 2010), with studies noting errors for 
poor precision relating to inconsistent spore removal because of variations in vortexing, sonication, pipetting, and colony counting errors (Rose, 2004). Studies have evaluated both sonication and vortexing methods to remove spores from swabs and found no difference in the recovery efficiencies between the two methods. Some have stated that there was no method to estimate the actual number that settle on a $100 \mathrm{~cm}^{2}$ coupon during experiments and that the recovery had to be based on the theoretical number that might deposit. Using that method, overall recovery efficiencies have been as low as $0.9 \%$ (Lewandowski et al., 2010). Wagner et al. (2008) collected swab surface samples and vortexed each sample for 10 seconds, followed by a serial dilution and plating to TSA. Others have used vortexing alone to remove spores, including sample dilution and replating if the colony counts were greater than 300 colonies (Kesavan, 2008). Some researchers have found that the optimal method for removal was sonication for 15 minutes (Brown et al., 2008) while others have used vortexing for 2 minutes followed by 15 minutes of sonication (Wang et al., 2001). Burton et al. (2005) found that shaking and vortexing lead to a significantly higher physical extraction efficiency for MCE and 1 $\mu \mathrm{m}$ PTFE filters than the vortex with ultrasonic agitation extraction method. The method of deposition also impacts the recovery — an additional study using Bacillus globigii to contaminate surfaces by both aerosol methods and by application directly onto material found that the recovery efficiencies for aerosol and droplet contamination were similar, but that aerosol contamination had a higher variability (Martin and Moore, 2001).

Another aspect of the testing methods is the seeding of the test substrate. Studies have been completed using five-20 $\mu \mathrm{L}$ drops of $10^{7}$ stock of BG spores, allowing the coupons to air dry a minimum of 3 hours or until all the liquid was completely evaporated (Edmonds et al., 2009). Ten-fold dilutions of the bacterial suspensions were completed with $100 \mu \mathrm{L}$ of 
suspension containing the specific species of bacteria under study which was spread onto trypticase $^{\mathrm{TM}}$ soy nutrient agar in plastic Petri dishes (King et al., 2011). Spreading onto the agar plate is another consideration. Baron et al. (2008) tried several different methods including misting and a "hockey stick" method. They eventually determined that spreading the spores onto an agar plate using a "hockey stick" shaped glass rod was the most reproducible method.

Finally, the bioaerosols must be diluted in some solution and then properly removed for analysis. Different solutions have been used, including Butterfield Buffer with Tween 80 (BBT) (Brown et al., 2007b; Estill et al., 2009) and phosphate-buffered saline (Estill et al., 2009).

Previous studies have been completed analyzing high heat and humidity inactivation rates on Bacillus anthracis spore simulants at high concentrations directly deposited onto aluminum coupons. These studies have focused on only the upper limits of the heat and humidity engineering limits of the aircraft. A better understanding of the inactivation rates of these spores on other aircraft materials, lower heat and humidity ranges, and also lower inoculation rates is critical for understanding the best method for safely decontaminating aircraft. This research tested the inactivation rates of a Bacillus anthracis spore simulant using five combinations of high heat and humidity levels, all within the engineering specifications of aircraft. The spores were delivered to aluminum coupons in three different methods - high direct inoculation, low direct inoculation, and an aerosol deposition method using a previously described test chamber.

\section{MATERIALS AND METHODS}

\section{Test chamber}

A bioaerosol test chamber was designed and built to deposit a Bacillus anthracis simulant onto aluminum coupons to test inactivation rates when exposed to high heat and humidity (see 
Chapter 2). This test chamber was 1.49 meter in length, 1.22 meter in height, and 0.86 meter in width, with a total interior volume of 1.4 cubic meters ( 49 cubic feet) and surface area of 1.18 square meters (12.7 square feet). The chamber was constructed from aluminum and included a mixing element where the aerosol was mixed and injected. Several working openings were also installed and sealed with Plexiglass ${ }^{\circledR}$ and rubber sealants; then, the potential for leaks was tested using smoke tests and leak detectors.

Equations were derived and tested to verify the bioaerosol generation requirements using a 6-jet Collison nebulizer (BGI, Waltham, MA). These equations were based on the general ventilation dilution equations and modeled in Figure 3-1.

(1) $\mathrm{C}_{\max }=\frac{\mathrm{G}}{\mathrm{Q}_{\mathrm{in}}} \quad$ (Burgess, Ellenbecker, and Treitman, 2004)

$$
\begin{array}{ll}
\mathrm{C}_{\max } & =\text { Maximum concentration }\left(\frac{\mathrm{CFU}}{\mathrm{m}^{3}}\right) \\
\text { Where: } \quad \mathrm{G} & =\text { Generation rate }\left(\frac{\mathrm{CFU}}{\text { minutes }}\right) \\
\mathrm{Q}_{\text {in }} & =\text { Air generation rate into chamber }\left(\frac{\mathrm{m}^{3}}{\text { minute }}\right)
\end{array}
$$

(Controllable throughout experiment)

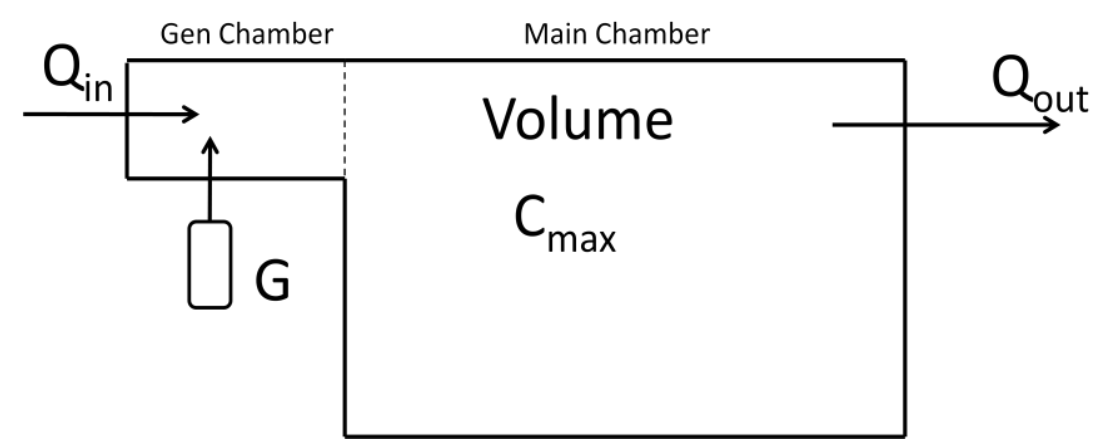

Figure 3- 1- Test chamber deposition model

The generation rate for these equations was derived using the following equations. The variables below are for a Collison 1-jet nebulizer. The $\mathrm{Q}_{\text {liq }}$ below is the amount of liquid that is 
generated from a Collison nebulizer. Each jet requires approximately $2 \mathrm{lpm}$ of air, producing droplets with a mass median aerodynamic diameter (MMAD) of $2.5 \mu \mathrm{m}$ with a geometric standard deviation (GSD) of 1.8. The amount of liquid used by the nebulizer depends on the pressure is applied to the nebulizer and is defined by the equation below.

(2) $\mathrm{G}=\mathrm{Q}_{\text {liq }} * \mathrm{C}_{\mathrm{neb}}$

$$
\begin{aligned}
\mathrm{G} & =\text { Generation rate for spores }\left(\frac{\mathrm{CFU}}{\mathrm{minute}}\right) \\
\mathrm{Q}_{\text {liq }} & =\text { Liquid use rate for nebulizer }\left(\frac{\mathrm{mL}}{\mathrm{minute}}\right) \\
& =1.5 \frac{\mathrm{mL}}{\mathrm{hr}}=0.0253 \frac{\mathrm{mL}}{\mathrm{min}}(\text { for } 1 \text {-jet nebulizer, BGI) } \quad(\mathrm{BGI}, 2008) \\
& =\left[-0.84859+0.2636 * \ln \left(\mathrm{psig}^{2}\right] \times \text { [number of jets }\right] \quad(\mathrm{BGI}, 2008) \\
\mathrm{C}_{\mathrm{neb}} & =\text { Spore concentration in nebulizer }\left(\frac{\mathrm{CFU}}{\mathrm{mL}}\right)
\end{aligned}
$$

(Controllable throughout experiment)

These equations were then manipulated to model the surface concentration defined in equation 3. These equation derivations are explained more thoroughly in Chapter 2.

(3) $\mathrm{S}_{\mathrm{c}}=\left(\left(\frac{\mathrm{G}}{\mathrm{Q}_{\mathrm{in}}}\right) * \mathrm{H}\right) * \mathrm{SA}_{\mathrm{C}}$

$$
\begin{aligned}
& \mathrm{G}=\text { Generation rate for spores }\left(\frac{\mathrm{CFU}}{\text { minute }}\right) \\
& \text { Where: } Q_{\text {in }}=\text { Air generation rate into chamber }\left(\frac{\mathrm{m}^{3}}{\text { minute }}\right) \\
& \text { (Controllable throughout experiment) } \\
& \mathrm{H} \text { = Chamber height }(1.22 \mathrm{~m}) \\
& \mathrm{SA}_{\mathrm{c}}=\text { Coupon surface area }\left(1 \mathrm{in}^{2}=6.45 \times 10^{-4} \mathrm{~m}^{2}\right)
\end{aligned}
$$

Particle free air was used to generate aerosols with the Collison nebulizer (BGI,

Waltham, MA). This air was also used to push the aerosols into the chamber. The flow into the nebulizer was measured using a Dwyer ${ }^{\circledR}$ Rate-Master ${ }^{\circledR}$ Flowmeter (Michigan City, IN) RMB-52 (5-50 SCFH Air) and controlled through an air control valve. An additional stream of air (henceforth called dilution air) was used to force the aerosol into the main testing chamber. This second stream of air was measured using a Dwyer ${ }^{\circledR}$ Rate-Master ${ }^{\circledR}$ Flowmeter, RMC-103 (20- 
200 SCFH Air) and controlled through an additional air control valve. These flowmeters were calibrated using TSI Model 4146 (Shoreview, MN) (0.01 to 20 liters per minute) and TSI Model 4046 ( 2.5 to 300 liters per minute) calibrators. These calibrations were performed before the aerosolization tests were completed. Both the aerosol and the dilution air were neutralized using a TSI Kr-85 neutralizer. A 2.5 inch pressure gauge, 0 to 30 psi, Ashcroft ${ }^{\circledR}$ Instruments (Stratford, CT), was used to measure the air pressure into the Collison nebulizer.

\section{Test coupons}

Aluminum testing coupons were provided by the Coatings Group at the University of Dayton Research Institute (UDRI). The coupons were 0.032" thick 2024-T3 aluminum, pretreated with PreKote, primed with MIL-PRF-23377, and topcoated with Type IV MIL-PRF85285. These coupons were provided in 12 inch by 12 inch sheets and then cut to 1 inch by 1 inch squares by Design Metal Manufacturing (DMM) (Fort Collins, CO). These coupons are indicative of the current materials and coatings on Air Force aircraft.

Before each test, the coupons were rinsed with tap water and then deionized water to remove all biological material and chlorine ion residuals. The coupons were then autoclaved at $121^{\circ} \mathrm{C}$ for 30 minutes. Inoculations were then performed using aseptic techniques explained later.

\section{Biological methods}

Spores

Tests were completed using Bacillus atrophaeus subsp globigii (BG) obtained from Yakibou, Inc (Apex, NC). The spores were provided in two concentrations-3.1 x $10^{8}$ spores $/ \mathrm{mL}$ and $2.2 \times 10^{9}$ spores $/ \mathrm{mL}$. For nebulization, the spores were diluted in Phosphate 
Buffered Saline (PBS) with 0.05\% Tween 20 (Fisher Scientific). The PBS, delivered as a dry powder, was mixed with laboratory grade water from a Barnstead NANOpure Diamond ${ }^{\mathrm{TM}}$ purification system.

\section{Spore inoculation methods}

Direct

All spore inoculations used aseptic techniques, including decontaminating all working surfaces with 1:10 sodium hypochlorite bleach before work began. The direct inoculations were completed in a NUAIRE ${ }^{\mathrm{TM}}$ Class II, A2 Type Biological Safety Cabinet. The spores were inoculated onto the coupons which were placed in $100 \mathrm{~mm}$ x $15 \mathrm{~mm}$ style petri dishes (BD Falcon $^{\mathrm{TM}}$, Becton, Dickinson, and CO, Sparks, MD) and allowed to air dry overnight.

Tests were performed initially to verify that the spores could be inactivated and also to determine future sample number requirements. These tests were conducted with a high concentration of spores on the coupons, with an inoculation of $9.68 \mu \mathrm{L}$ of the spore solution onto aluminum coupons. This amount contained approximately $3 \times 10^{6}$ spores per coupon. The inactivation procedures are covered more in-depth later.

The actual decontamination tests were done at two different direct inoculation levelshigh and low, corresponding to $10^{6}$ and $10^{4}$, respectively. The high inoculation methods were straightforward-3.5 $\mu \mathrm{L}$ of the $3.1 \times 10^{8}$ spores $/ \mathrm{mL}$ was inoculated directly onto the coupons. The low concentration required a dilution to effectively pipette onto a coupon. In order to dilute the spores, $0.05 \mathrm{mLs}$ of the $2.2 \times 10^{9}$ spore suspension was added to a $30 \%$ ethanol solution. The ethanol solution was made with LAL Reagent water (endotoxin content $<0.005 \mathrm{EU} / \mathrm{mL}$ ) (Lonza, Walkersville, MD) and laboratory grade ethanol. This solution corresponded to approximately $2.06 \times 10^{6}$ spores $/ \mathrm{mL}$, with $10 \mu \mathrm{L}$ containing 10,000 spores, which was the inoculation goal. 
Aerosol spore deposition

The spores were also aerosolized within the test chamber and allowed to deposit onto the aluminum coupons. The equations derived were used to model spore deposition. The goal for aerosol deposition was 10,000 spores per coupon which would relate to the low direct inoculation amount. Previous tests, described in Chapter 2, showed that deposition was not even throughout the chamber. The remedy for this was to include a control coupon next to each sample. This was done by placing four total coupons (two for samples and two for controls) in each petri dish. The lids for the petri dishes remained on until nebulization started.

Prior to nebulization, a Maxima C D4B pump (Fisher Scientific, Waltham, MA) was used to evacuate the test chamber for 10 minutes. Then a 6-jet Collison nebulizer was used to aerosolize spores into the test chamber. The volume of spores placed into the nebulizer was 160 $\mu \mathrm{L}$, based on the test chamber models and tests showing the concentrations that could be recovered. These spores were diluted in $20 \mathrm{~mL}$ sterile PBS with Tween. The nebulizer was run at 20 psi until it was empty, taking 50 minutes. During the entire nebulization, dilution air was used to push the aerosol into the chamber at $50 \mathrm{lpm}$. Both the aerosol and dilution were connected to a $\mathrm{Kr}-85$ neutralizer. Once nebulization was completed, the dilution air continued to run for 5 minutes. Once this was completed, all chamber air was shut-down and the particles were allowed to settle for $9 \frac{1 / 2}{2}$ hours.

Each aerosol test run included 1 negative control dish, 1 positive control petri dish, and 2 sample petri dishes — all filled with BL ${ }^{\mathrm{TM}}$ TSA II Typticase ${ }^{\mathrm{TM}}$ Soy Agar, Modified (Becton, Dickinson, and CO, Sparks, MD). These 2 sample petri dishes, located at the front and back of the test chamber, were used to verify that the spores were aerosolized properly during the nebulization. 
The spores were required to be decontaminated in the test chamber before it was opened following each test. A spray bottle with 1:10 bleach was placed in the chamber before the chamber was sealed. Additionally, plastic sheets were placed on the chamber floor before each run. After the spores had settled for the proper time, the petri dishes were covered and the entire chamber was sprayed with the bleach. A contact time of 30 minutes was allowed for spore decontamination. Following this time, the chamber was opened, the petri dishes were removed, and the plastic sheet was discarded.

The petri dishes were removed from the chamber after this 30 minute contact time. The petri dishes were then opened, with the control coupons being placed into a BD Falcon ${ }^{\mathrm{TM}} 50 \mathrm{~mL}$ polypropylene conical tube (Franklin Lakes, NJ) with analysis occurring immediately afterwards. The sample coupons were placed on aluminum shelves, which were placed inside the environmental test chamber. After each treatment time (24, 48, 72, and 96 hours), the aluminum shelves were removed from the test chamber and sterile forceps were used to place the coupons into the $50 \mathrm{~mL}$ tubes. Each sample was analyzed immediately after removal from the environmental chamber.

These procedures were used to test the efficiency of spore recovery and removal after aerosol deposition. The deposition goals were 10,000; 100,000; and 1,000,000 spores per sample, with each sample consisting of two one-inch square aluminum coupons. The deposition equations derived were used to estimate the parameters for spore deposition. All variables were kept constant except the spore concentration placed into the nebulizer. The test conditions used were a 6-jet Collison nebulizer, operated at 20 psi with 50 lpm dilution air. The spores were diluted into $20 \mathrm{~mL}$ sterile PBS with Tween, corresponding to the deposition goals of 10,000, 
100,000, and 1,000,000 spores per sample coupon, respectively. Each of these tests was performed once with 18 different sample coupons.

\section{Sample processing}

Spore removal from coupons

Removal of the spores from the coupons is a critical step for accurate data collection. To evaluate the effectiveness of this process, aluminum coupons were inoculated with $9.68 \mu \mathrm{L}$ spore solution $\left(3.1 \times 10^{8}\right.$ spores $\left./ \mathrm{mL}\right)$. These coupons were allowed to air dry and then placed in $30 \mathrm{~mL}$ Sterilized Phosphate Buffered Solution with $0.01 \%$ Tween, into a $50 \mathrm{~mL}$ Blue Falcon conical tube.

The following removal techniques were tested:

- Test 1: Sonicating and vortexing. Coupons were sonicated in an ultrasonic bath (Fisher Scientific FS110 Ultrasonic cleaner, Pittsburg, PA) for 30 minutes at $40 \mathrm{kHz}$, followed by vortexing (Fisher Scientific Mini-vortexor, Pittsburg, PA) for two minutes.

- Test 2: Shaking and vortexing. Coupons were placed in a New Brunswick Scientific, C24KC Refrigerator, Incubator, Shaker (Edison, NJ) for 60 minutes followed by vortexing for two minutes.

- Test 3: Vortexing. Coupons were vortexed for two minutes.

- Test 4: Negative Control. Coupons without spores were placed in a tube and analyzed with the other tests. This followed the sample procedures as test 1 but was completed to verify that there was not cross contamination in these procedures.

- Test 5: Positive control. The same amount of spore solution was placed directly into a tube. 
After the samples were treated, a 20:1 serial dilution was performed in three additional tubes. Following the dilutions, $100 \mu \mathrm{L}$ of each sample was plated onto TSA agar. This amount was then spread using a "hockey-stick" method with sterilized glass rods. A total of 32 samples was analyzed. All samples were incubated at $37^{\circ} \mathrm{C}$ with counting done at 24,48 , and 72 hours.

The results (covered more in-depth later) showed that the optimal method to remove spores was to use a 30 minute sonication followed by 2 minutes vortexing. Spore removal from the coupons was completed using this procedure for all subsequent samples.

\section{Spore Plating}

Plating was completed by transferring a volume of $100 \mu \mathrm{L}$ from all samples and placing this onto $100 \mathrm{~mm}$ x $15 \mathrm{~mm}$ petri dishes, BD Falcon ${ }^{\mathrm{TM}}$ (Becton, Dickinson, and CO, Sparks, MD) filled with BBL ${ }^{\mathrm{TM}}$ TSA II Typticase ${ }^{\mathrm{TM}}$ Soy Agar, Modified (Becton, Dickinson, and CO, Sparks, MD). A deposition goal of 300 spores per petri dish was used as this was a differentiable value, with serial dilutions completed to meet this level. These serial dilutions were 1:20 dilutions, taken from the $30 \mathrm{~mL}$ initial solution and placed into $9.5 \mathrm{~mL}$. Sterile PBS with Tween was used for all these samples. All samples were spread using the "hockey-stick" method, which included sterilized glass rods bent in the form of a hockey stick. The spore solution was spread using a turn-table through two complete revolutions.

Negative control coupons were performed each day. Additionally, a positive control high inoculation coupon was analyzed daily to verify that there was no cross contamination and to ensure the spores would germinate properly. Once the plates were removed, they were placed in an incubator at $37^{\circ} \mathrm{C}$ and counted at 24,48 , and 72 hours. 


\section{Inactivation tests}

The environmental test chamber used was a reconditioned Blue M, HR-381C Temperature/Humidity chamber purchased from Technical Equipment Sales, Inc, (Severance, $\mathrm{CO})$, capable of maintaining a temperature up to $150^{\circ} \mathrm{C}\left(300^{\circ} \mathrm{F}\right)$ and relative humidity levels from $10 \%$ to $95 \%$. The chamber was updated with an electronic controller operated by Watlow Electric Manufacturing Co, Watview® Runtime Version 2.6.4 software (St. Louis, MO). Figure 3-2 shows a picture of the chamber.

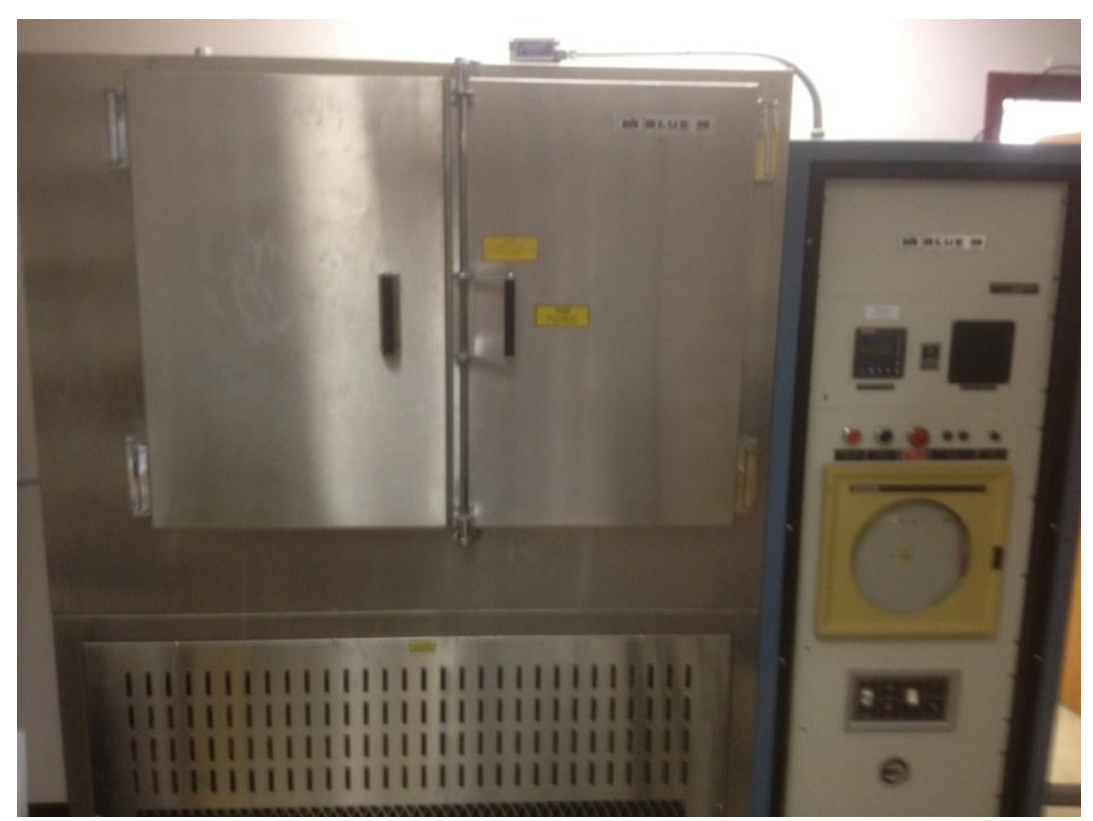

Figure 3- 2 - Environmental test chamber

\section{Initial Spore inactivation tests}

Initial decontamination tests were performed to determine future sample numbers

required. These tests were performed with a $10 \mu \mathrm{L}$ spore inoculation $\left(3.1 \times 10^{8}\right.$ spores $\left./ \mathrm{mL}\right)$ onto both plastic and aluminum coupons. After inoculation, the coupons were air dried overnight in a Class II, A2 Type Biosafety Cabinet. Following this, they were treated in the environmental heat and humidity chamber at $80^{\circ} \mathrm{C}\left(176^{\circ} \mathrm{F}\right)$ and $87 \% \mathrm{RH}$ with treatment times of 24,48 , and 72 
hours. After the treatment, the coupons were analyzed as described above. This testing allowed initial data to be used to determine sample numbers required in subsequent testing.

\section{Decontamination tests}

The decontamination tests were performed at temperatures ranging from $160^{\circ} \mathrm{F}$ to $180^{\circ} \mathrm{F}$ and relative humidity ranges from 70 to $90 \%$, temperature and humidity ranges within Air Force engineering tolerances. A matrix representing tests completed is presented in Figure 3-3. Each different temperature and humidity setting has a corresponding test condition number, which is the number referred to in the rest of this chapter.

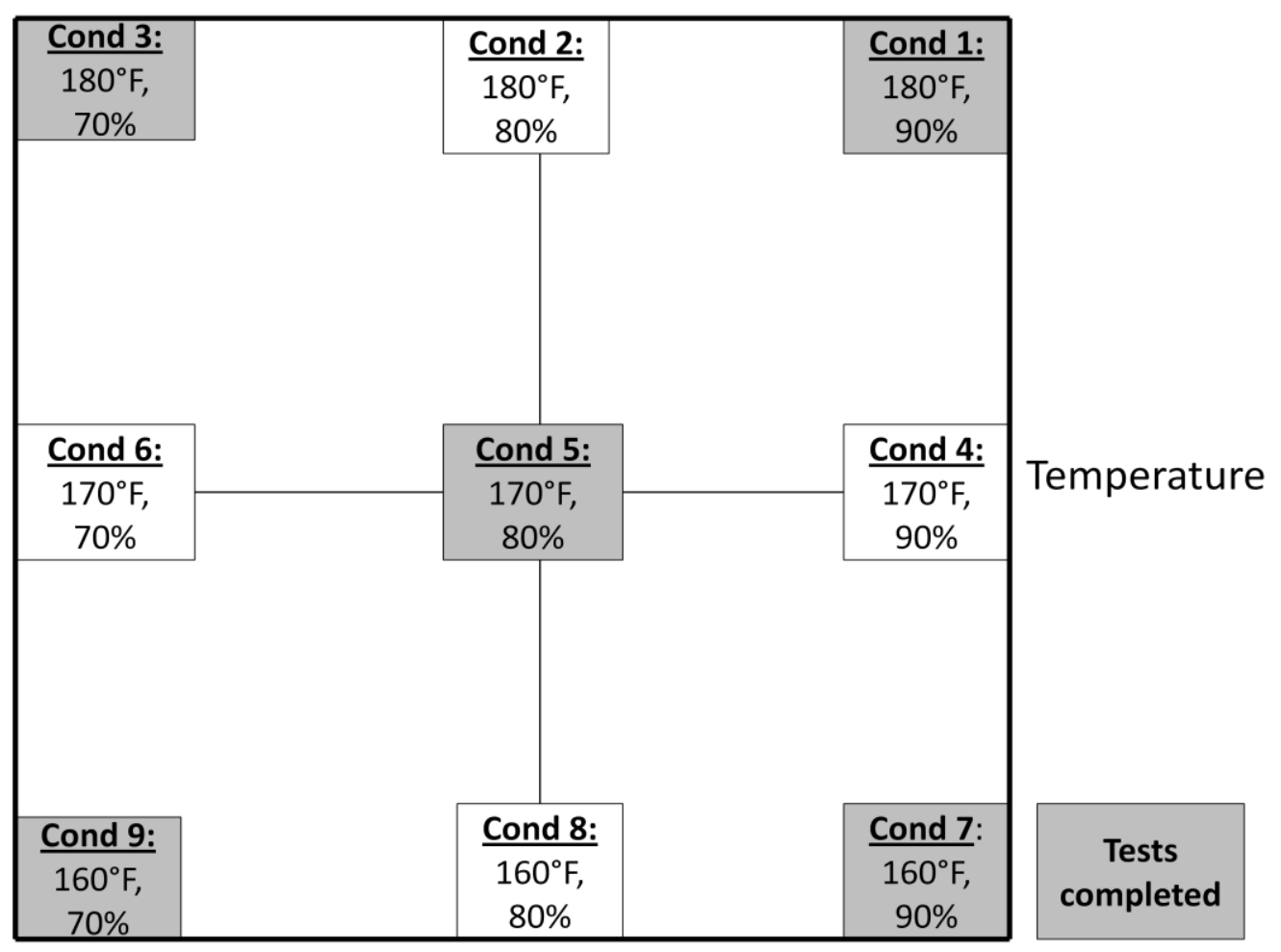

\section{Relative Humidity}

Figure 3-3-Decontamination test condition matrix 
Each testing condition had separate samples completed at times $0,24,48,72$, and 96 hours for high and low direct inoculation and aerosol deposition. Samples were completed at 120 hours for test conditions 5,7 and 9 because it was anticipated that the spores would not be inactivated at 96 hours for this combination of temperature and humidity. A total of 5 samples was collected at each time period for each test condition.

\section{Data Management and Statistics}

Inactivation rates were plotted using Microsoft Office Excel®. These plots included the average number of spores for each data point and the standard error for these points. A stepwise regression was completed on the inactivation data using Minitab®, v16 (State College, PA). This stepwise regression was completed on each different inoculation type (direct high, direct low, and aerosol deposition). The response variable in each model was the log value of the spores +1 . Explanatory variables were added or removed from the model during the stepwise regression using $\alpha=0.05$. Temperature, humidity, and treatment time were mandatory variables in the model. Once the additional predictors were included, a final regression model was completed with those variables selected during the step wise regression. Heat and humidity levels in the heat and humidity chamber were logged using Watview ${ }^{\circledR}$ Runtime Version 2.6.4 software, Watlow Electric Manufacturing Co (St Louis, MO).

\section{RESULTS}

\section{Spore removal efficiencies}

Testing for spore removals showed that the most effective method was using sonication followed by vortexing. Table 3-1 below shows the different recovery efficiencies for these tests. 
These efficiencies were determined by calculating the number of spores removed from the coupons divided by the theoretical number of spores that were inoculated on the coupon. The theoretical number of spores was based on the values provided by the spore supplier (Yakibou, Inc., Apex, NC). The standard error for each test is presented as well.

Table 3 - 1 - Spore recovery percentages from inoculated coupons

\begin{tabular}{|l|c|}
\hline \multicolumn{1}{|c|}{ Test method } & Percent recovery \\
\hline Sonicate 30 min, vortex 2 min & $142 \%( \pm 46.9 \%)$ \\
\hline Sonicate 30 min, vortex 30 sec & $165 \%( \pm 43.2 \%)$ \\
\hline Sonicate 30 min, vortex 10 sec & $180 \%( \pm 31.6 \%)$ \\
\hline Shake 60 min, vortex 2 min & $24.8 \%( \pm 18.0 \%)$ \\
\hline Vortex 2 min & $42.3 \%( \pm 30.3 \%)$ \\
\hline
\end{tabular}

Table 3-2 shows the spore recovery percentages from spiked tubes. These tubes did not have coupons, rather the spores were placed directly into the tubes. These were completed for comparison to the samples that did include the coupons.

Table 3 - 2 - Spore recovery percentages from spiked tubes

\begin{tabular}{|l|l|}
\hline \multicolumn{1}{|c|}{ Test method } & Percent recovery \\
\hline Vortex 30 seconds & $247 \%( \pm 15.29 \%)$ \\
\hline Vortex 5 seconds & $255 \%( \pm 11.7 \%)$ \\
\hline
\end{tabular}

As mentioned previously, all subsequent coupon analysis was completed using sonication for 30 minutes followed by vortexing for two minutes. This was completed for all samples and controls analyzed.

\section{Aerosol deposition removals}

The spore recovery efficiencies were evaluated by depositing the spores in goals of $10,000,100,000$, and 1,000,000 spores per sample, with each sample consisting of two one-inch square aluminum coupons. The deposition goals and average recoveries are presented in Table 3-3. The table includes the average recovery and the standard error for those recovery percentages. Each of these aerosolization and removal tests was performed once, with 18 
different coupon sets analyzed with each test run. Note that the test numbers are not sequential because all tests conducted were numbered as they were completed. After this, the assumption was made that $10 \%$ of the spores could be recovered through the aerosol deposition, which was then used to model the remaining experiments.

Table 3 - 3 - Spore recovery from aerosol deposition, aluminum coupons

\begin{tabular}{|l|c|c|c|c|}
\hline & $\begin{array}{c}\text { Test 77, } \\
\text { Aluminum }\end{array}$ & $\begin{array}{c}\text { Test 78, } \\
\text { Aluminum }\end{array}$ & $\begin{array}{c}\text { Test 79, } \\
\text { Aluminum }\end{array}$ & $\begin{array}{c}\text { Test 82, } \\
\text { Aluminum }\end{array}$ \\
\hline $\begin{array}{l}\text { Deposition } \\
\text { Goal }\end{array}$ & 10,000 & 10,000 & 100,000 & $1,000,000$ \\
\hline $\begin{array}{l}\text { Average } \\
\text { Recovery }\end{array}$ & $\begin{array}{c}28.8 \% \\
( \pm 13.8 \%)\end{array}$ & $\begin{array}{c}9.0 \% \\
( \pm 7.1 \%)\end{array}$ & $\begin{array}{c}8.3 \% \\
( \pm 7.2 \%)\end{array}$ & $\begin{array}{c}16.9 \% \\
( \pm 6.7 \%)\end{array}$ \\
\hline
\end{tabular}

$\mathrm{n}=18$ for each sample

\section{Initial decontamination tests}

The results of the initial decontamination tests, performed to determine sample size requirements, are presented in Figure 3-4. These tests were completed on two different sets of aluminum coupons and corresponding controls. These were direct inoculations with sample size of $5(\mathrm{n}=5)$. The top level in the Figure is time $=0$ for all samples. The sample size was 5 for each point $(n=5)$. 


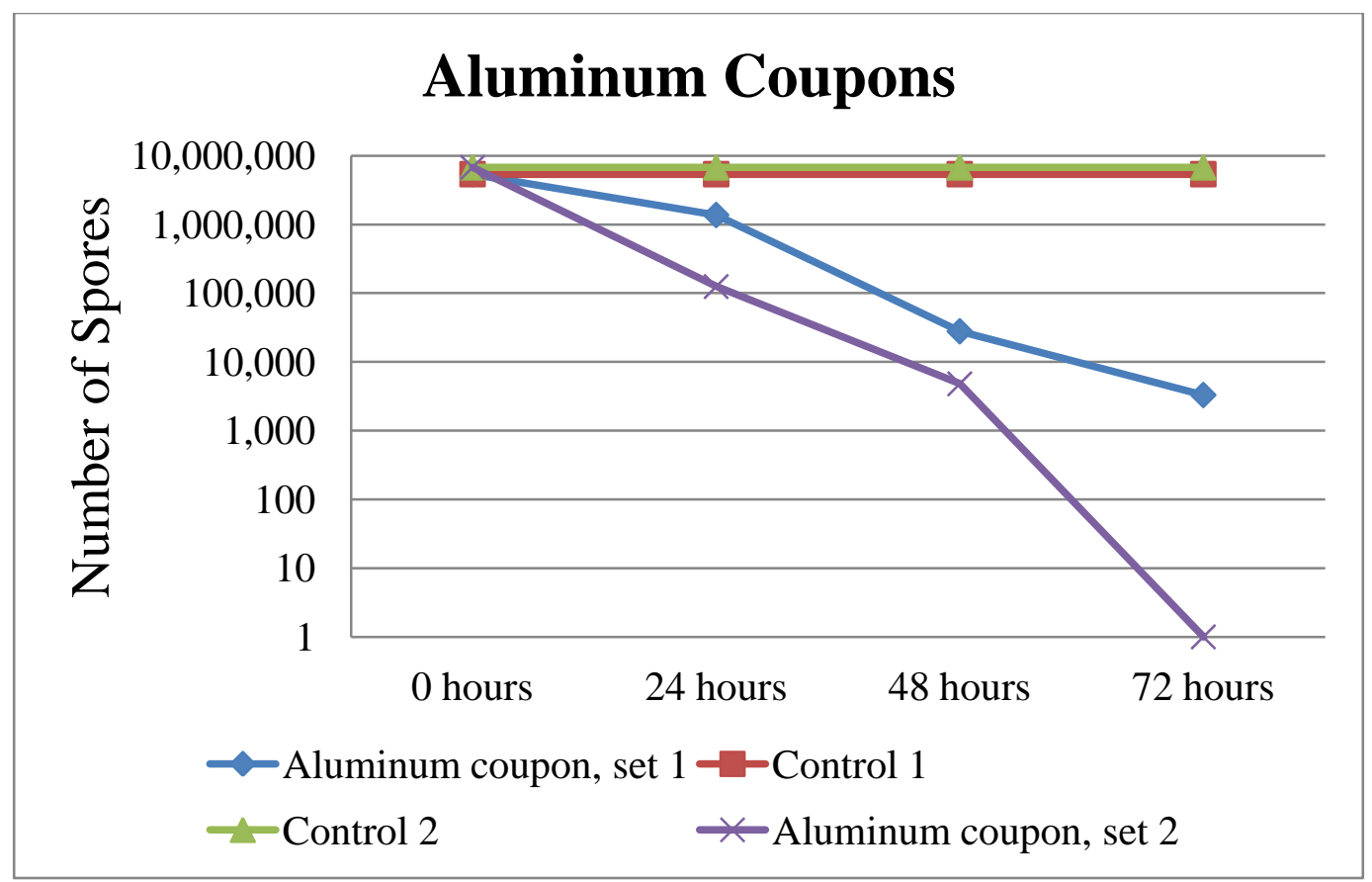

Figure 3- 4 - Initial spore decontamination tests

These data were analyzed with Minitab®, v16, using a general linear model to determine the number of samples required. The response variable was the log number of spores and the explanatory variables the coupon type (aluminum or plastic) and the treatment time $(0,24,48$, or 72 hours). The adjusted mean square error for the model was 1.072; therefore, the standard deviation for analysis was 1.035 . Assuming $\alpha=0.05$ and power $=0.8$, the 2 -sample t-test (one tail) testing the difference in means based on log reduction of spores from 14 to 2 would required from 1 to 7 samples, respectively as seen by Table 3-4 below. Following this analysis, all tests consisted of a sample size of $5(n=5)$. 
Table 3 - 4 - Sample size required based on initial decontamination tests

\begin{tabular}{|c|c|}
\hline $\begin{array}{c}\text { Difference of Means } \\
\text { (log of spores } \\
\text { inactivated) }\end{array}$ & $\begin{array}{c}\text { Sample size } \\
\text { (control and } \\
\text { treated) }\end{array}$ \\
\hline 1 & 14 \\
\hline 2 & 4 \\
\hline 3 & 4 \\
\hline 4 & 2 \\
\hline 5 & 2 \\
\hline 6 & 2 \\
\hline 7 & 2 \\
\hline
\end{tabular}

Note: The difference of means refers to the difference seen in the log of spores on the coupon for the treatment compared to the initial concentration of spores.

\section{Decontamination tests}

The five test conditions were completed and analyzed as depicted in Figure 3-3 above. These were completed for high and low direct inoculations and also aerosol depositions. There were two tests that had errors in that the data did not meet what was expected biologically. This was because the graphs, or inactivation rates, did not show a decrease uniformly over time, but rather there was one time point where all samples were zero and this was followed by the next period with samples of positive numbers. This occurred for the aerosol deposition for test condition 5, which had zero growth at 24 hours, but the spores did grow at 48 hours. Additionally, the samples for high direct inoculation for test condition 5 were rerun because there was a large variability in the data with several different time readings having zero spore growth at times that were not expected to have spore inactivation. Neither of these results could be explained; therefore, they were re-done and the subsequent data used.

\section{High direct inoculation inactivation}

The data for the spore inactivation are summarized graphically in Figure 3-5 and 3-6, which includes the high and low direct inoculations respectively. Aerosol deposition results are 
summarized graphically in Figures 3-7 through 3-11, which show total spores recovered on each sample. For high inoculations, test condition 1 had a rapid decrease with spore inactivation occurring by 48 hours. The only other test condition that had a decrease was test condition 5 . As seen in the graph below, the spore numbers increased for test condition 5 after the 96 hour point, which cannot be explained. All other test conditions did not exhibit a decrease in the spore numbers for the sampling times allotted. Each error bar presented in the figures is the standard error of the mean, that is, the standard deviation divided by the square root of the sample size (5 for all sample points).

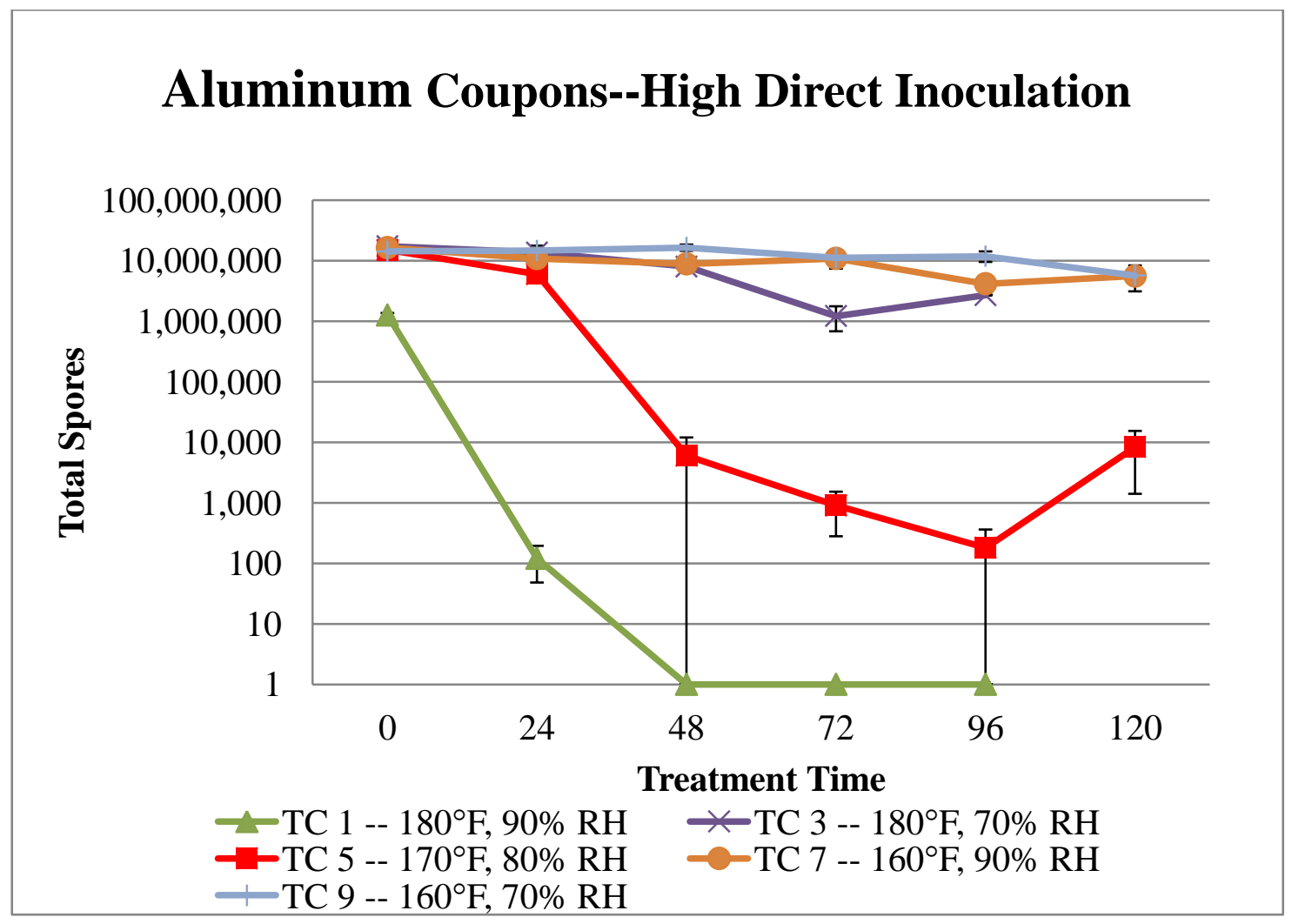

Figure 3-5 - Decontamination tests, high direct inoculation

\section{Low direct inoculation inactivation}

The low direct inoculation showed a similar response as the high concentrations. The spores from test condition 1 were all inactivated by 48 hours and the spores from test condition 5 
were reduced by 96 hours. Error bars are the same as in Figure 3-5. The other test conditions did not show a large degree of inactivation before the end of the tests.

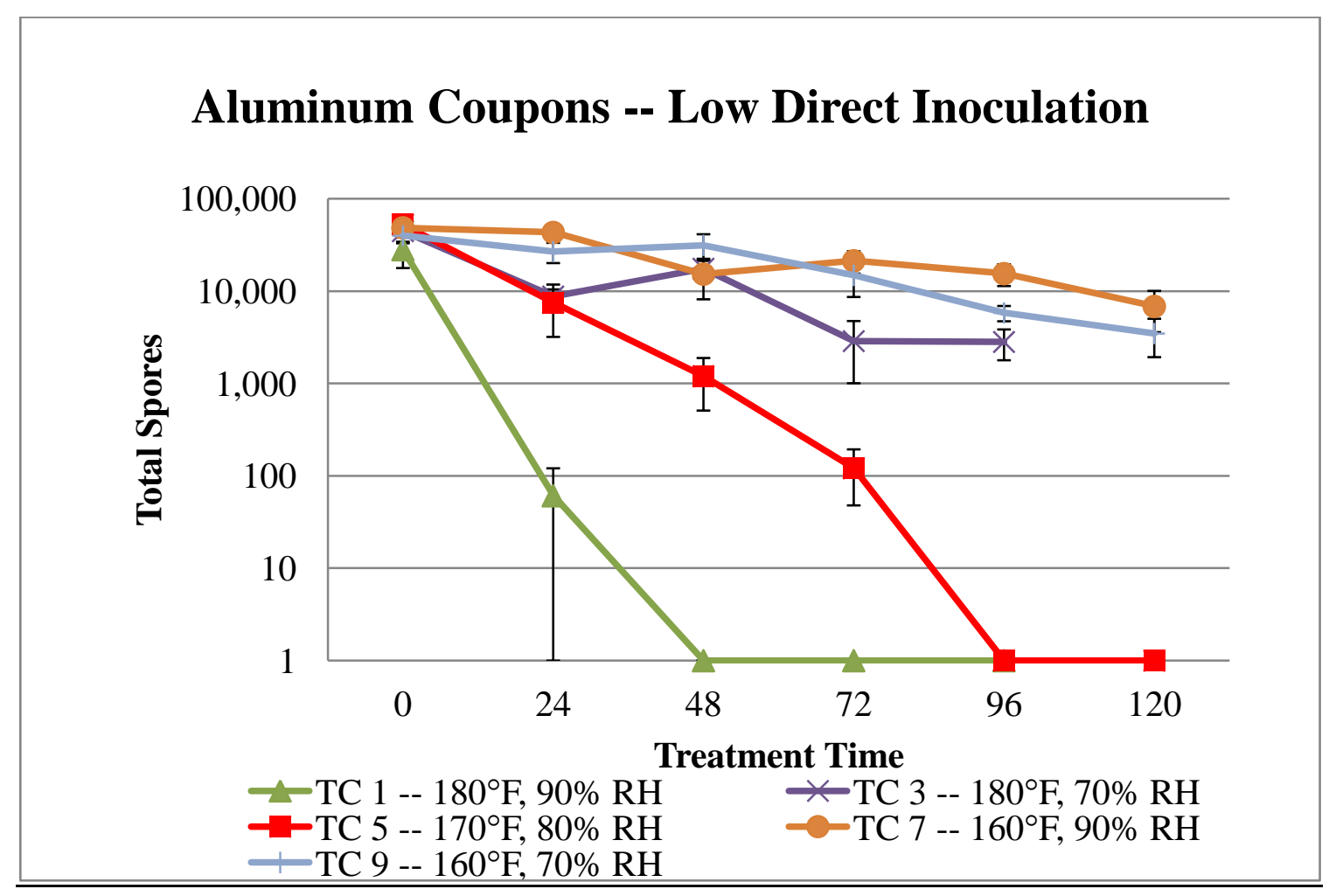

Figure 3- 6 - Decontamination tests, low direct inoculation

Aerosol deposition inoculation inactivation

The data for each aerosol test are included in separate figures because each corresponding sample point had a control. Each point in Figures 3-7 through 3-11include 5 data points $(n=5)$ for all aerosol deposition inactivation.

Figure 3-7, decontamination tests for aerosol deposition test condition 1 shows that the spores were inactivated within 24 hours. After this time point, all the samples were zero and all the controls remained positive, demonstrating successful inactivation. 


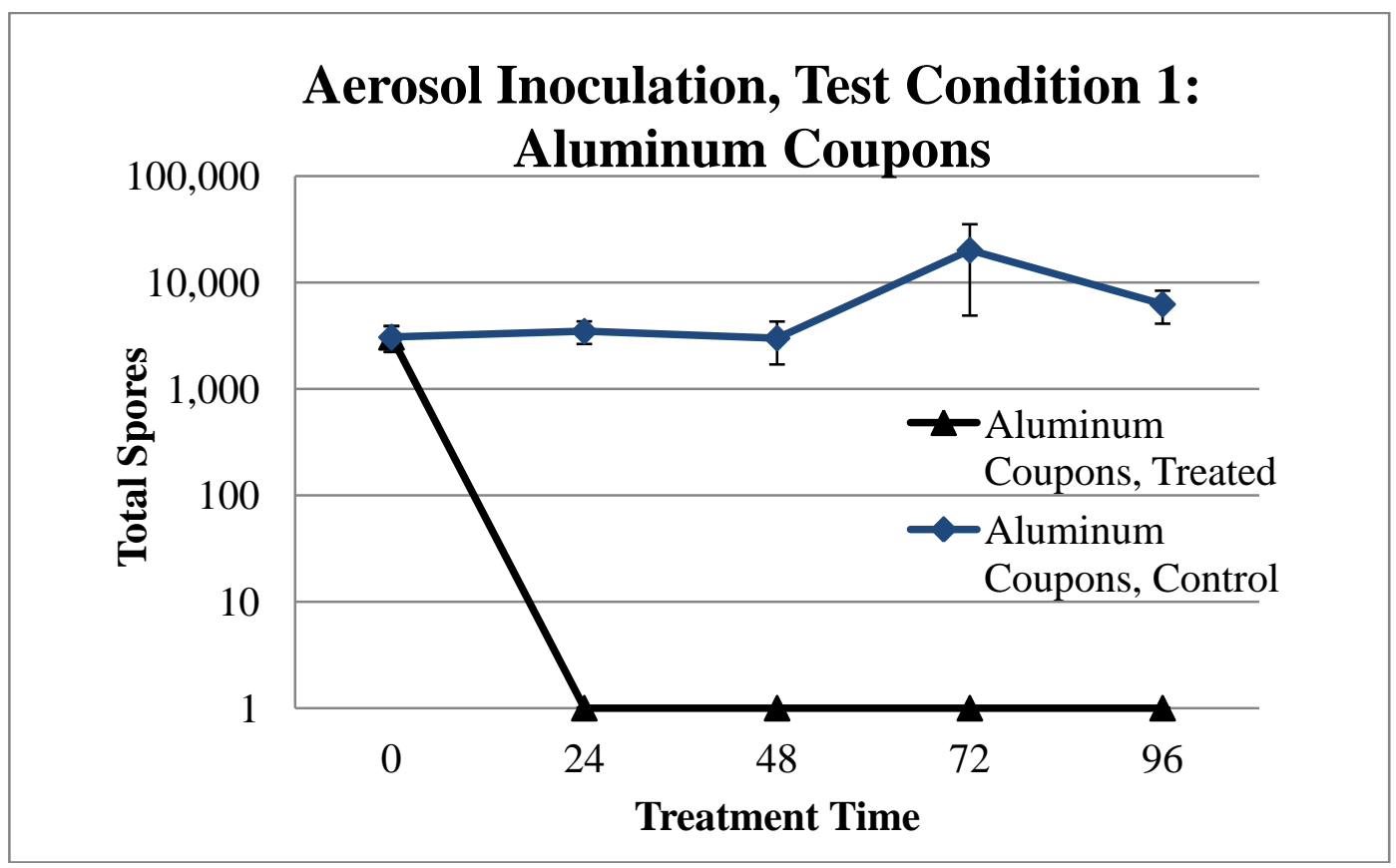

Figure 3- 7 - Decontamination tests, aerosol deposition, test condition 1

Figure 3-8, decontamination tests for aerosol deposition test condition 3, again shows successful decontamination; however, this was not seen until the 96 hour treatment point. The samples reached the zero mark at 96 hours and all the control samples remained positive, again demonstrating successful inactivation. 


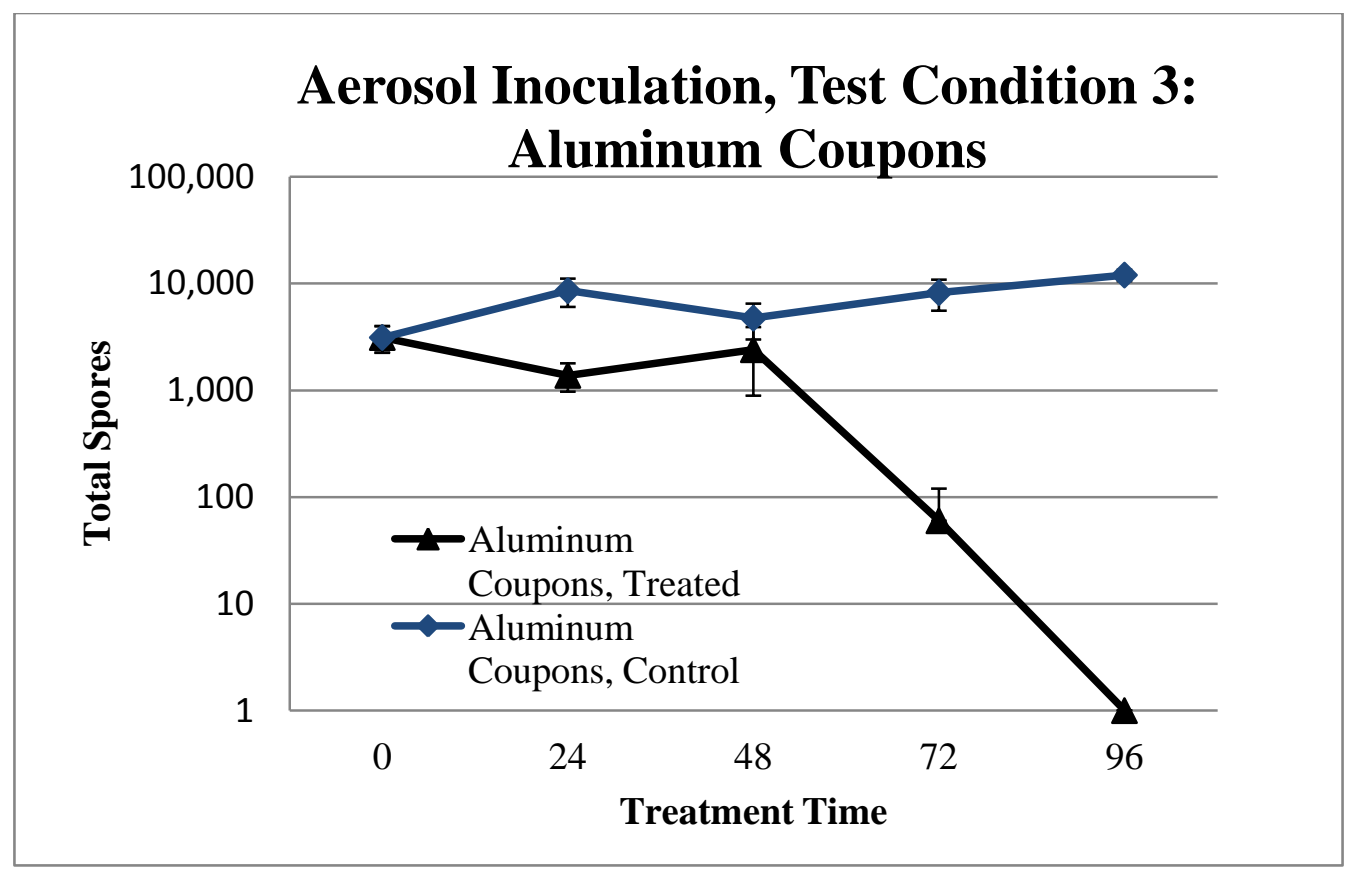

Figure 3- 8 - Decontamination tests, aerosol deposition, test condition 1

Figure 3-9, decontamination tests for aerosol deposition test condition 5, shows successful decontamination at 72 hours. Again, the controls remained positive during the samples.

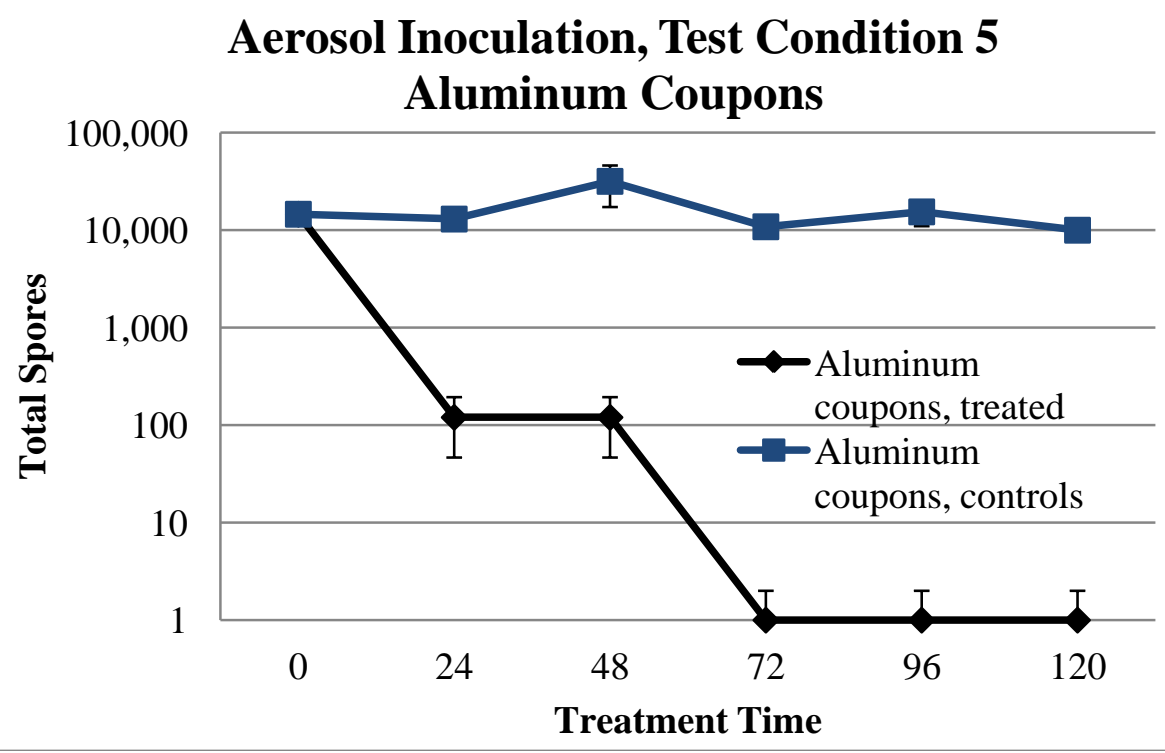

Figure 3- 9 - Decontamination tests, serosol deposition, test condition 5 
Figure 3-10, decontamination tests for aerosol deposition test condition 7, shows a longer time required for inactivation, with the samples reaching zero at 120 hours and the controls remaining positive.

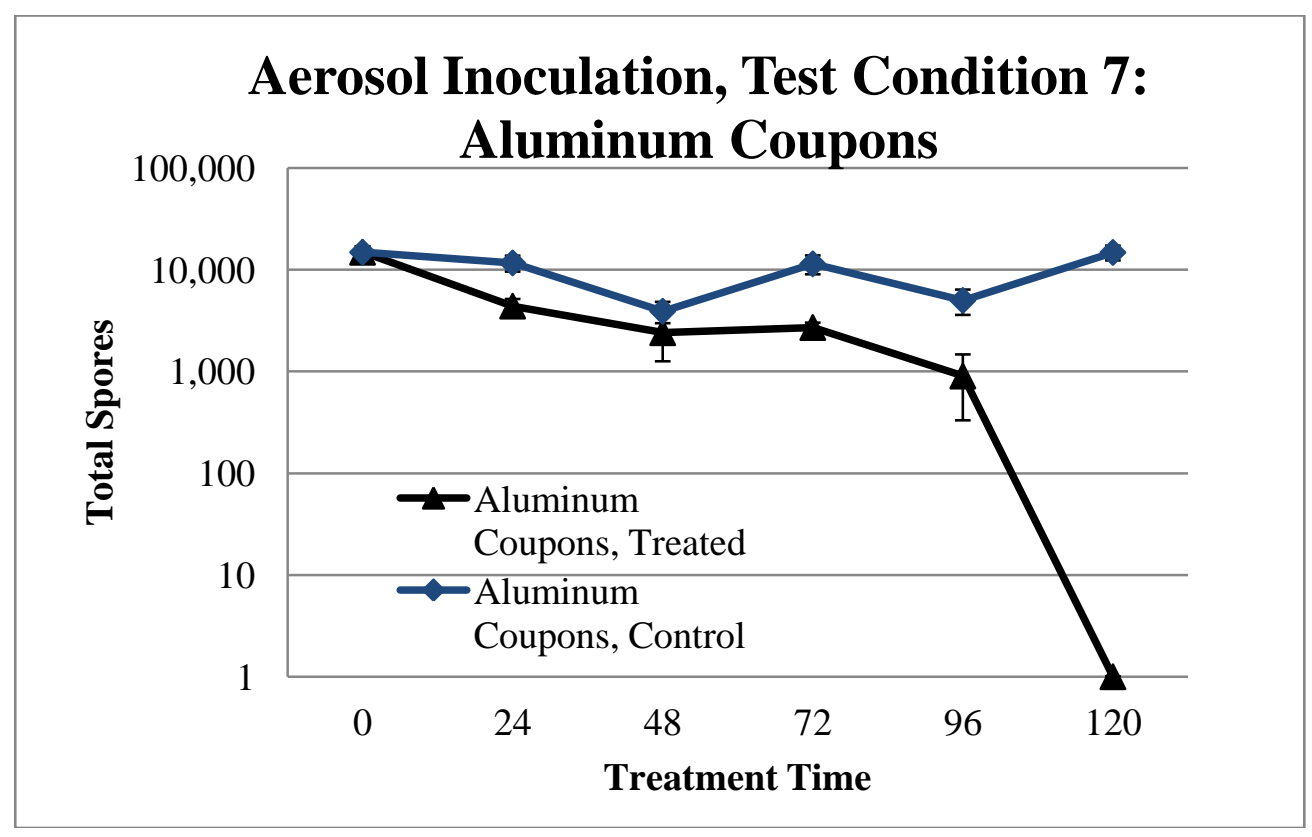

Figure 3- 10 - Decontamination tests, aerosol deposition, test condition 7

Figure 3-11, decontamination tests for aerosol deposition test condition 9, shows a similar pattern to test condition 7, with an abrupt decrease at 120 hours. Again, all controls were positive. 


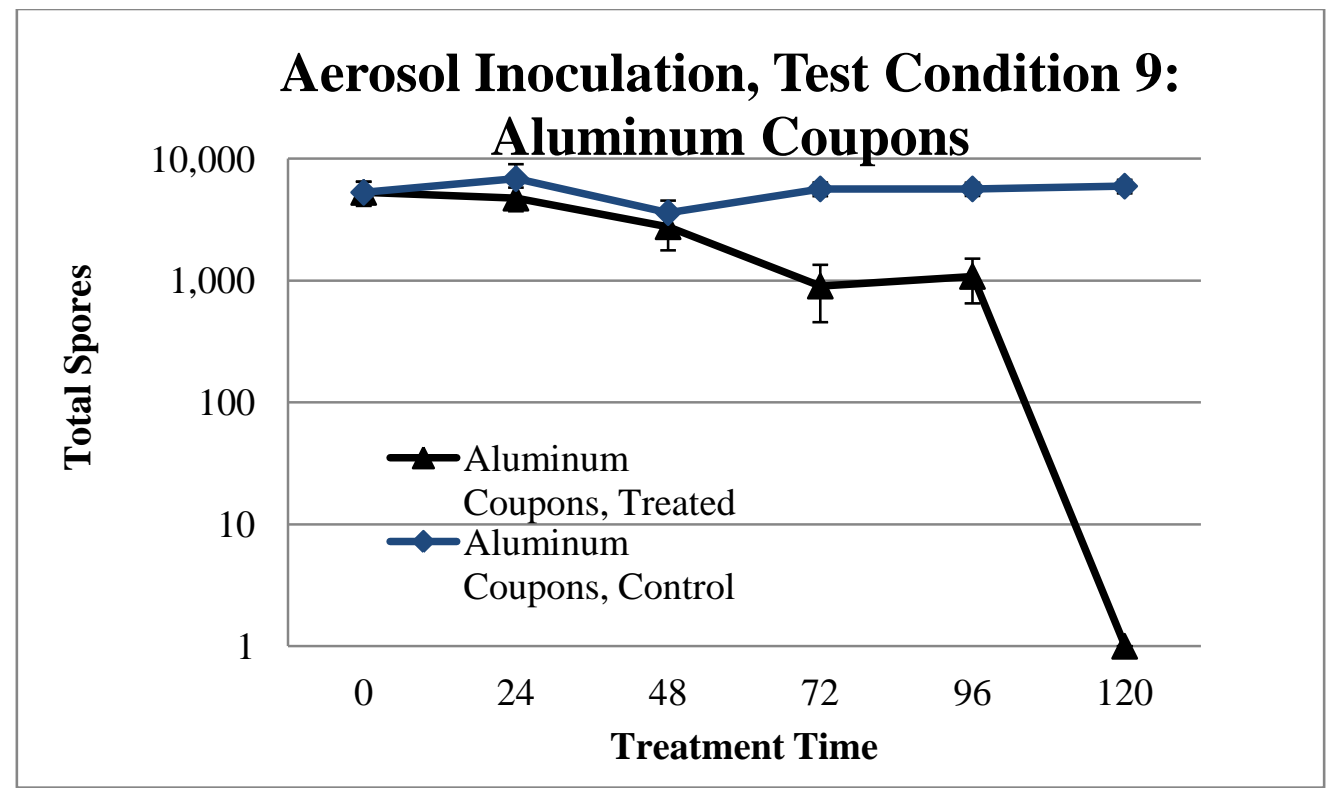

Figure 3- 11 - Decontamination tests, aerosol deposition, test condition 9

\section{Statistical analysis}

A stepwise regression was used to include the variables that would provide the best model for log value of the spores +1 . Temperature, humidity, and treatment time were mandatory variables in the model. Additional variables to be considered for the model were interactions of the main variables (temperature*humidity, temperature*time, and humidity* time). Squared terms of the main variables were also considered (temperature ${ }^{2}$, humidity ${ }^{2}$, and time $^{2}$ ). Because of the limits of the degrees of freedom on the temperature and humidity, these were limited to just the squared terms. The interactions of these terms with time ${ }^{2}$ were also included. Because there were more degrees of freedom for time, time ${ }^{3}$ was also considered. The stepwise regression was then completed using those terms with $\alpha=0.05$, that is, terms were added or removed from the model based on meeting this criteria. The variables selected in the stepwise regression for each deposition mechanism are included in Table 3-5. 
Table 3 - 5-Stepwise regression terms retained in models

\begin{tabular}{|c|c|c|c|}
\hline & $\begin{array}{l}\text { High direct } \\
\text { inoculation }\end{array}$ & $\begin{array}{l}\text { Low direct } \\
\text { inoculation }\end{array}$ & $\begin{array}{c}\text { Aerosol } \\
\text { deposition }\end{array}$ \\
\hline $\begin{array}{l}\text { Variables required in } \\
\text { every model }\end{array}$ & $\begin{array}{l}\text { Temperature } \\
\text { Humidity } \\
\text { Time }\end{array}$ & $\begin{array}{l}\text { Temperature } \\
\text { Humidity } \\
\text { Time }\end{array}$ & $\begin{array}{l}\text { Temperature } \\
\text { Humidity } \\
\text { Time }\end{array}$ \\
\hline $\begin{array}{l}\text { Additional } \\
\text { explanatory variables } \\
\text { retained in each } \\
\text { model }\end{array}$ & $\begin{array}{l}\text { Temp * Humidity } \\
\text { Time * Humidity } \\
\text { Temp * Time } \\
\text { Humidity } \\
\text { Temp }^{2} * \text { Time }^{2} \\
\text { Time }^{2} \\
\text { Humidity }^{*} \text { Time }^{2}\end{array}$ & $\begin{array}{l}\text { Temp * Humidity } \\
\text { Temp * Time } \\
\text { Humidity } \\
\text { Temp }^{2} * \text { Time }^{2} \\
\text { Time }^{3}\end{array}$ & $\begin{array}{l}\text { Temp }^{2} * \text { Time }^{2} \\
\text { Temp }^{2}\end{array}$ \\
\hline
\end{tabular}

Following the stepwise regression, a final regression model was completed using only

those terms retained in the model. These terms were entered into the model as presented below.

All of these terms were retained by the stepwise regression; there, they were all significant

predictors for the model. The results from these regressions models are included in Table 3-6

through 3-8, with the corresponding $\mathrm{R}^{2}$ presented.

Table 3 - 6 - High direct inoculation, regression model

\begin{tabular}{|c|c|c|c|}
\hline Term & Model Coeff & SE Coeff & $\mathrm{R}^{2}$ \\
\hline Temperature & 1.6023 & 0.2139 & \multirow[t]{11}{*}{$76.4 \%$} \\
\hline Humidity & -1.5149 & 0.5339 & \\
\hline Time & 0.23034 & 0.09977 & \\
\hline Temp * Humidity & -0.022625 & 0.002634 & \\
\hline Humidity $^{2}$ & 0.019994 & 0.003088 & \\
\hline Temp * Time & -0.022625 & 0.002634 & \\
\hline Temp $^{2} *$ Time $^{2}$ & -0.00000012 & 0.00000004 & \\
\hline Treatment time ${ }^{2}$ & -0.0010936 & 0.0008814 & \\
\hline Time*Humidity & -0.003629 & 0.001239 & \\
\hline Humidity * time ${ }^{2}$ & 0.00002589 & 0.00001063 & \\
\hline Constant & 14.47 & 25.58 & \\
\hline
\end{tabular}


Table 3 - 7 - Low direct inoculation, regression model

\begin{tabular}{|c|c|c|c|}
\hline Term & Model Coeff & SE Coeff & $\mathrm{R}^{2}$ \\
\hline Temperature & 0.9530 & 0.1502 & \multirow[t]{9}{*}{$71.5 \%$} \\
\hline Humidity & -1.0740 & 0.3787 & \\
\hline Time & 0.25914 & 0.06457 & \\
\hline Temp * Humidity & -0.012496 & 0.001828 & \\
\hline Humidity $^{2}$ & 0.012284 & 0.002201 & \\
\hline Temp * Time & -0.0043356 & 0.0009749 & \\
\hline Temp $^{2} *$ Time $^{2}$ & 0.00000015 & 0.00000005 & \\
\hline Time $^{3}$ & -0.00000423 & 0.00000153 & \\
\hline Constant & 14.44 & 18.10 & \\
\hline
\end{tabular}

Table 3 - 8 - Aerosol deposition, regression model

\begin{tabular}{|l|l|l|l|}
\hline \multicolumn{1}{|c|}{ Term } & Model Coeff & SE Coeff & $\mathrm{R}^{2}$ \\
\hline Temperature & -4.307 & 1.356 & \multirow{3}{*}{$38.5 \%$} \\
\cline { 1 - 3 } Humidity & -0.01139 & 0.01215 & \\
\cline { 1 - 3 } Time & 0.046437 & 0.009668 & \\
\cline { 1 - 3 } Temp $^{2}$ & 0.027749 & 0.008873 & \\
\cline { 1 - 3 } Temp $^{2} *$ time $^{2}$ & -0.00000009 & 0.00000001 & \\
\cline { 1 - 2 } Constant & 168.30 & 51.66 & \\
\hline
\end{tabular}

\section{DISCUSSION}

The goal of this research was to evaluate whether a Bacillus anthracis spore simulant could be inactivated over several different temperature and humidity levels and also three different inoculation methods. Initial parts of the project required substantiation of the methods to be used. Equations were derived and tested to verify the deposition of the spores within the bioaerosol deposition chamber. Spore removal from coupons, a critical component for measuring inactivation, was performed in several different methods. The method with the highest recovery was sonication and vortexing, recovering up to $180 \%$ of the estimated inoculated spores. The method of shaking was found to only remove $37.5 \%$ of the estimated spores. To validate these ranges, spores were also directly inoculated into a control tube without using a coupon. These controls showed recovery efficiencies up to $255 \%$. These efficiencies were based on the theoretical number of spores on the coupons. These results were consistent to 
other research (Brown et al., 2007b), which have shown it is not uncommon for recovery efficiencies to be over $100 \%$. A possible explanation for the high recovery could be that the sonication and vortexing breaking up clumps of spores, that is, the action of reducing agglomerations could have increased the colonies counted. These data were then used to select the method to remove aerosolized spores. Using the equations derived and the spore removal methods, it was determined that $10 \%$ of the theoretical number of spores could be recovered from a coupon. Again, this value is consistent with the most similar research and the number of spores removed from similar materials (Brown et al., 2007a; Lewandoski et al., 2010). Initial inactivation tests were performed, demonstrating effective spore kill. These tests showed that sample sizes of 5 tests for each temperature provided sufficient power for research goals

Clumping of the spores could lead to agglomerated particles containing more than one spore. The clumping was alleviated as much as possible with the use of Collison nebulizer, which generates aerosols from 1 to $3 \mu \mathrm{m}$ in diameter. Even with the small size of particles generated, spores could still clump after generation. This could impact the spore decontamination because a spore clump may be more difficult to inactivate when compared to a single spore. Additionally, the data for the sonication showed that these clumps were very likely broken into single spores during the sonication phase, as explained above.

The first tests performed were direct high inoculation with a goal of $10^{6}$ spores per coupon. The most effective temperature and humidity combinations for this inoculation were test condition $1\left(180^{\circ} \mathrm{F}\right.$ and $90 \%$ relative humidity) and test condition $5\left(170^{\circ} \mathrm{F}\right.$ and $80 \%$ relative humidity). Tests with low direct inoculations were then completed with an inoculation goal of $10^{4}$ spores per coupon. Again, the most effective combinations were test condition $1\left(180^{\circ} \mathrm{F}\right.$ and $90 \%$ relative humidity) and test condition $5\left(170^{\circ} \mathrm{F}\right.$ and $80 \%$ relative humidity), both with 
complete kills at 48 and 96 hours, respectively. The data for the aerosolized spores showed that all the spores were inactivated within 120 hours for all test conditions used. The other test conditions appear to be trending down at the 96 hour point; however, there was not a complete kill. One limitation of the methods used is that only culture-based methods were used. These methods only account for the spores that will germinate into a vegetative bacterial cell and does not include spores that may be active but not able to germinate.

The data for direct inoculation show that test condition $1\left(180^{\circ}\right.$ and $\left.90 \%\right)$ is the most effective method to inactivate the spores that are deposited directly onto the aluminum coupons. This is consistent with what was expected — the higher the temperature and humidity levels, the less time is required for inactivation. The spores for test condition $5\left(170^{\circ}\right.$ and $\left.80 \%\right)$ reached zero at 96 hours for the low inoculation and were trending lower for the high inoculation. This shows that these two levels are the most effective combinations to decontaminate aluminum coupons. Additionally, this is consistent with the statistical analysis that showed the highest $\mathrm{R}^{2}$ values occurred when temperature, humidity, treatment time, and temperature-humidity interactions were modeled. This was the case for both high and low direct inoculations.

A stepwise regression model was completed to determine the terms that would add significantly to a regression model. The stepwise regression included mandatory variables (or variables that had to be selected by the regression). These variables were time, temperature, and humidity. The data for the stepwise regression retained more variables for high direct inoculation (10 predictors) than low (8 predictors) or aerosol deposition (5 predictors). The only variable retained by all three models, besides the mandatory variables, was $\mathrm{Temp}^{2} * \mathrm{Time}^{2}$. For both of the direct inoculation methods, several of the same variables were retained, which included Temp*Humidity, Temp* Time, Humidity ${ }^{2}$, and Temp ${ }^{2} *$ Time $^{2}$. More of the predictor 
variables for high inoculation included an interaction with time when compared to the predictors for low inoculation. This was expected because it was assumed it would take more time to inactivate the high inoculation spores. It is also interesting to note time ${ }^{2}$ was retained for high inoculation and time ${ }^{3}$ was retained for low inoculation. It appears that temperature is a more critical variable than humidity for aerosol deposition because both retained terms included temperature (Temp ${ }^{2}$ and then the interaction between $\mathrm{Temp}^{2}$ and Time ${ }^{2}$ )— this shows humidity is not as critical of a variable for this deposition. The final regression models demonstrated reasonable $\mathrm{R}^{2}$ values for high and low inoculation methods, $76.4 \%$ and $71.5 \%$, respectively. The $\mathrm{R}^{2}$ for the aerosol deposition model was not as strong, being only $38.5 \%$, showing that a much smaller portion of the variability is captured by the model. The reason for this is likely because of the variability in the spore deposition onto the coupons. These regression models could be used to help determine when inactivation will occur, given the type of inoculation and also the treatment parameters.

The ideal humidity and temperature range is clearly the highest levels that can be delivered, reasonably maintained, and within proper engineering specifications. If $90 \%$ humidity cannot be easily generated or maintained throughout the body of an aircraft, the results show that $80 \%$ at the proper temperature $\left(170^{\circ} \mathrm{F}\right.$ or higher $)$ can be effective as well. Additionally, the delivery method impacts how long it will take to inactivate the spores, with aerosol delivered spores inactivated more quickly. A critical component that needs to be considered is time required to decontaminate the item. If the item being decontaminated can remain out of service for a longer period of time, lower temperatures and humidity levels could be used. This could be the case when power or even a water source to generate the humidity is not adequate to support these higher levels of humidity. 
Future research could focus on extending the treatment times for the test conditions that did not have full inactivation at the 120 hour time point. This would include test conditions 3,7 , and 9 for both the high and low direction inoculations. These data would be useful when the higher temperature and humidity levels are more difficult to maintain and a longer time period is allowed for decontamination efforts. The aerosol data showed that inactivation occurred within the 120 hour time periods; however, to obtain a more robust statistical analysis, smaller time increments could be analyzed. For instance, all spores were inactivated at the 24 hour time period for test condition 1; therefore, future tests could include a time period of 12 hours. Even though steps were taken to reduce the spore clumping, the phenomenon still could have occurred. Future studies could include microscopy analysis throughout each stage of the analysis to validate the extent that this occurs.

\section{Conclusion}

The goal of this research was to determine if a Bacillus anthracis simulant could be decontaminated from an aluminum coupon using high heat and humidity levels, while expanding on past research (AFRL, 2008). The results show there is a difference in the time required to inactivate the spores when delivered by the aerosol deposition method, which is a more realistic contamination method. These results show future research should focus more on these types of delivery mechanisms. These results do, however, confirm that when higher spore levels are inactivated, the lower levels of spores, delivered by direct inoculation or aerosol deposition, will also be inactivated. Overall, this research showed the spores can be effectively inactivated using high heat and humidity at specific combinations of these variables coupled with time. The results show promise for future efforts to inactivate biological agents safely, effectively, and also within aircraft engineering specifications. 


\section{ACKNOWLEDGEMENTS}

This study was funded in part by a grant from the Mountain and Plains Research Center (MAP ERC) through Dr. Reynolds, Deputy Director. Additional funds were provided through a grant from AFMSA/SGRS. Funds were committed with the assistance of William Kilpatrick (711 HPQ/RHXS), Gregory Sudberry (711 HPW/RHPC), SSgt Robert Arrington (711 HPW/RHPCB), SrA Ashley Grant (ASC/PKOA), and Tim Provens (711 HPW/RHPC). Aluminum coupons were provided by William Culhane (AFRL/RXSSO), USAF Coating Technology Integration Office. Spores were provided by Joe Dalmasso, Yakibou, Inc. 


\section{REFERENCES}

Air Force Research Laboratory (AFRL): Bio-thermal decontamination tests for large frame aircraft application laboratory and field test report, AFRL Document HEPC OSMSWP8Z, 5 Dec 2008.

Aizenberg, V., S.A. Grinshpun, K. Willeke, J. Smith, and P.A. Baron: Performance characteristics of the button personal inhalable aerosol sampler. American Industrial Hygiene Association Journal 61: 398-404 (2000a).

Andersen, B.M., M. Rasch, K. Hochlin, F.H. Jensen, P. Wismar, and J.E. Fredriksen: Decontamination of rooms, medical equipment and ambulances using an aerosol of hydrogen peroxide disinfectant. Journal of Hospital Infection Hosp Infection 62: 149-155 (2006).

Baron, P.A., C.F. Estill, J.K. Beard, M.J. Hein, and L. Larsen: Bacterial endospore inactivation caused by outgassing of vapourous hydrogen peroxide from polymethyl methacrylate (Plexiglas $\left.{ }^{\circledR}\right)$. Letters in Applied Microbiology 45: 485-490 (2007).

Baron, P.A., C.F. Estill, G.J. Deye, M.J. Heim, J.K. Beard, L.D. Larsen, and G.E. Dahlstrom: Development of an aerosol system for uniformly depositing Bacillus anthracis spore particles on surface. Aerosol Science and Technology 42: 159-172 (2008).

Barth, E., R. Rupert, F. Stroud, E. Rice, and B. Potoka: Environmental response to intentional dissemination of Bacillus anthracis spores in the United States 2001. Risk Management Research 13: 99-111 (2003).

BGI, Inc: Collison Nebulizer Droplet Number Output (Online) Available at http://bgiusa.com/agc/droplet_number_output.htm (accessed March 2011).

Brachman, P.S.: Bioterrorism: an update with a focus on anthrax. American Journal of Epidemiology 155: 981-987 (2002).

Brickhouse, M.: Innovative and Emerging Decontamination Technologies. In Report on Workshop on Decontamination, Cleanup and Associated Issues for Sites Contaminated with Chemical, Biological, or Radiological Materials, S. Dun, and J. Wood, US EPA: October 2005.

Brown, G.S.; R.G. Betty, J.E. Brockman, D.A. Lucero, C.A. Souza, K.S. Walsh, R.M. Boucher, M.S. Tezak, and M.C. Wilson: Evaluation of vacuum filter sock surface sample collection method for Bacillus spores from porous and non-porous surfaces. Journal of Environmental Monitoring 9(7): 666-671 (2007a).

Brown, G.S., R.G. Betty, J.E. Brockman, D.A. Lucero, C.A. Souza, K.S. Walsh, R.M. Boucher, M. Tezak, M.C. Wilson, and T. Rudolph: Evaluation of a wipe surface sample method for collection of Bacillus spores from nonporous surfaces. Applied and Environmental Microbiology 73(3): 706-710 (2007b). 
Brown, G.S., R.G. Betty, J.E. Brockmann, D.A. Lucero, C.A. Souza, K.S. Walsh, R.M. Boucher, M.S. Tezak, M.C. Wilson, T. Rudolph, H.D. Lindquist, and K.F. Martinez: Evaluation of rayon swab surface sample collection method for Bacillus spores from nonporous surfaces. Journal of Applied Microbiology 103(4): 1074-1080 (2007c).

Burgess, W.A., M.J. Ellenbecker, and R.D. Treitman: Ventilation for Control of the Work Environment. Hoboken, NJ: John Wiley \& Sons, 2004.

Burke, S.A., J.D. Wright, M.K. Robinson, B.V. Bronk, and R.L. Warren: Detection of molecular diversity in Bacillus atrophaeus by amplified fragment length polymorphism analysis. Applied and Environmental Microbiology 79(5): 2786-2790 (2004).

Burton, N.C., A. Adhikari, S.A. Grinshpun, R. Hornung, and T. Reponen: The effect of filter material on bioaerosol collection of Bacillus subtilis spores used as a Bacillus anthracis stimulant. Journal of Environmental Monitoring 7: 475-480 (2005).

Buttner, M. P., P. Cruz, L.D. Stetzenbach, A.K. Klima-Comba, V.L. Stevens, and P.A. Emanuel: Evaluation of the biological sampling kit (BiSKit) for large-area surface sampling. Applied Environmental Microbiology 70: 7040-7045 (2004).

Byrne, MA; A.J.H. Goddard, C. Lange, and J. Roed: Stable tracer aerosol deposition measurements in a test chamber. Journal of Aerosol Science 26(4): 645-653 (1995).

Canter, D.A., D. Gunning, P. Rodgers, L. O'Connor, C. Traunero, and C.J. Kempter: Remediation of Bacillus anthracis contamination in the U.S. Department of Justice mail facility. Biosecurity Bioterror 3(2): 119-127 (2005).

Centers for Disease Control: Investigation of bioterrorism-related anthrax - Connecticut. Morb Mortal Wkly Rep, 50: 1077-1079 (2001).

Chen, B.T., M.D. Hoover, G.J. Newton, S.J. Montano, and D.S. Gregory: Performance evaluation of the sampling head and annular kinetic impactor in the Savannah River site alpha continuous air monitor. Aerosol Science and Technology 31: 24-38 (1999).

Cordesman, A.H.: The Challenge of Biological Terrorism. Washington, DC: The CSIS Press (Center for Strategic and International Studies), 2005.

Chosewood, L.C., and D.E. Wilson, eds.: Biosafety in Microbiological and Biomedical Laboratories, $5^{\text {th }}$ Ed., Pub. No. 21-1112. Atlanta, GA: US Department of Health and Human Services, Centers for Disease Control, NIH, 1992.

Department of Defense: The Chemical, Biological, Radiological, and Nuclear (CBRN) Survivability Policy, Pub No. DoDI 3150.09 (August 17, 2009) 
Edmonds, J.M., P.J. Collett, E.R. Valdes, E.W. Skowronski, G.J. Pellar, and P.A. Emanuel: Surface sampling of spores in dry-deposition aerosols. Applied and Environmental Microbiology 75(1): 39-44 (2009).

Estill, C.F., P.A. Baron, J.K. Beard, M.J. Hein, L.D. Larsen, L. Rose, F.W. Schaefer III, J. Noble-Wang, L. Hodges, H.D.A. Lindquist, G.J. Deye, and M.J. Arduino: Recovery efficiency and limit of detection of aerosolized Bacillus anthracis sterne from environmental surface samples. Applied and Environmental Microbiology 75(13): 4297-4306 (2009).

Estill, C.F., and G.J. Deye: Personal communication, January 7, 2010.

Farnsworth, J.E., S.M. Goyal, S.W. Kim, T.H. Kuehn, P.C. Raynor, M.A. Ramakrishnan, S. Anantharaman, and W. Tang: Development of method for bacteria and virus recovery from heating, ventilation, and air conditioning (HVAC) filters. Journal of Environmental Monitoring 8: 1006-1013 (2006).

Feather, G.A., and B.T. Chen: Design and use of a settling chamber for sampler evaluation under calm-air conditions. Aerosol Science and Technology 37: 261-270 (2003).

Foarde, K.K., J.T. Janley, D.S. Ensor, and P. Roessler: Development of method for measuring single-pass bioaerosol removal efficiencies of a room air cleaner, Aerosol Science Technology 30: 223-234 (1999).

Gale, W.F., S. Hyacinth, and J. Watson: Field Evaluation of Whole Airliner Decontamination Technologies - Wide Body Aircraft with Dual-Use Application for Railcars, Federal Aviation Administration, Pub No. DOT/FAA/AM-08/4, (2008).

Gale, W.F., N.I. Sofyan, H.S. Gale, S.-F. Chou, J.W. Fergus, and C.G. Shannon: Effect of vapour phase hydrogen peroxide, as a decontaminant for civil aviation applications, on microstructure, tensile properties and corrosion resistance of 2024 and 7075 age hardenable aluminum alloys and 304 austenitic stainless steel. Materials Science and Technology 25(1): 7684 (2009).

Herzog, A.B., S.D. McLennan, A.K. Pandey, C.P. Gerba, C.N. Hass, J.B. Rose, and S.A. Hashsham: Implications of limits of detection of various methods for Bacillus anthracis in computing risks to human health. Applied and Environmental Microbiology 75(19): 6331-6339 (2009).

Hill S.C., R.G. Pinnick, S. Niles, Y.L. Pan, S. Holler, R.K. Chang, J. Bottiger, B.T. Chen, C.S. Orr, G. Feather, and A.P. Snyder: Real-time measurement of fluorescence spectra from single airborne biological particles. Field Analysis and Chemical Technology 3: 221-239 (1999).

Jensen, P.A., W.F. Todd, G.N. Davis, and P.V. Scarpino: Evaluation of eight bioaerosol samplers challenged with aerosols of free bacteria. American Industrial Hygiene Association Journal 53: 660-667 (1992). 
Jernigan, D.B., P.L. Raghunathan, B.P. Bell et al.: Investigation of bioterrorism-related anthrax, United States, 2001: epidemiologic findings. Emerging Infectious Diseases8: 10191028 (2002).

Kempter, J.: Crisis Exemptions for Products Intended to Inactivate Bacillus anthracis, In Report on Workshop on Decontamination, Cleanup and Associated Issues for Sites Contaminated with Chemical, Biological, or Radiological Materials, S. Dun, and J. Wood, US EPA: October 2005.

Kenny, L.C., R.J. Aitken, P.E.J. Baldwin, G.C. Beaumont, and A.D. Maynard: The sampling efficiency of personal inhalable aerosol samplers in low air movement environments. Journal of Aerosol Science 30(5): 627-638 (1999).

Kesavan, J., J.R. Bottiger, and A.R. McFarland: Bioaerosol concentrator performance: comparative tests with viable and with solid and liquid nonviable particles. Journal of Applied Microbiology 104: 285-295 (2008).

King, B., J. Kesavan, and J.-L. Sagripanti: Germicidal UV sensitivity of bacteria in aerosols and on contaminated surfaces. Aerosol Science and Technology 45(5): 645-653 (2011).

Koch, W., W. Dunkhort, and H. Lodding: Design and performance of a new personal aerosol monitor. Aerosol Science (1999).

Kournikakis, B., M. Walker, J. Ho et al.: Statistical analysis of bacterial spore aerosols created by opening a spore containing anthrax letter in an office. Journal of Aerosol Science 40: 514-522 (2009).

Lai, A.C.K.; M.A. Byrne, A.J.H. Goddard: Experimental studies of the effect of rough surfaces and air speed on aerosol deposition in a test chamber. Aerosol Science and Technology 36: 973-982 (2002).

Lewandowski, R., K. Kozłowska, M. Szpakowska, M. Stepin'ska, and E.A. Trafny: Use of a foam spatula for sampling surfaces after bioaerosol deposition. Applied and Environmental Microbiology 76(3): 688-694 (2010).

Li, C.S., M.L. Hao, W.H. Lin, C.W. Chang, and C.S. Wang: Evaluation of microbial samplers for bacterial microorganisms. Aerosol Science Technology 30: 100-108 (1999).

Mainelis, G., A. Adhikari, K. Willeke, S. Lee, T. Reponen, and S.A. Grinshpun: Collection of airborne microorganisms by a new electrostatic precipitator. Aerosol Science 33: 1417-1432 (2002).

Marple, V.A., and K.L. Rubow: An aerosol chamber for instrument evaluation and calibration. American Industrial Hygiene Association Journal 44: 361-367 (1983). 
Martin, B.: Opening Remarks, In Report on Workshop on Decontamination, Cleanup and Associated Issues for Sites Contaminated with Chemical, Biological, or Radiological Materials, S. Dun, and J. Wood, US EPA: October 2005.

Maus, R., A. Goppelsroder, and H. Umhauer: Atmospheric Environment 35: 105-113 (2001).

McVey, I.: STERIS Chem-Bio Decontamination, In Report on Workshop on Decontamination, Cleanup and Associated Issues for Sites Contaminated with Chemical, Biological, or Radiological Materials, S. Dun, and J. Wood, US EPA: October 2005.

Nicholson, W.L., P. Fajardo-Cavazos, R. Rebeil, T.A. Slieman, P.J. Riesenman, J.F. Law, and Y. Xue: Bacterial endospores and their significance in stress resistance. Antonie Leeuwenhoek 81: 27-32 (2002).

Oh, S.W., P.M. Gray, R.H. Dougherty, and D.H. Kang: Aerosolization as novel sanitizer delivery system to reduce food-borne pathogens. Lett Appl Microbiol 41: 56-60 (2005).

Orlusky, R.: Restoration From Decontamination: USPS Experience. In Report on Workshop on Decontamination, Cleanup and Associated Issues for Sites Contaminated with Chemical, Biological, or Radiological Materials, S. Dun, and J. Wood, US EPA: October 2005.

Park, J.M., J.C. Rock, L. Wang, Y.C. Seo, A. Bhatnagar, and S. Kim: Performance evaluation of six different aerosol samplers in a particulate matter generation chamber. Atmospheric Environment 43: 280-289 (2009).

Peccia J., H.M. Werth, S. Miller, \& M. Hernandez: Effects of relative humidity on the ultraviolet induced inactivation of airborne bacteria. Aerosol Science Technology 35: 728-740 (2001).

Perez, J., S. Springthorpe, and S.A. Sattar: Activity of selected oxidizing microbicides against the spores of Clostridium difficile: relevance to environmental control. American Journal of Infection Control 33: 320-325 (2005).

Perkins, J.J.: Principles and Methods of Sterilization in Health Sciences,. $2^{\text {nd }}$ ed.. Springfield, IL, Charles C. Thomas Publishers, 1983.

Peters, C.J., and D.M. Hartley: Anthrax inhalation and lethal human infection. Lancet. 359: 710-711 (2002).

Probst, A., R. Facius, R. Wirth, and C. Moissl-Eichinger: Validation of a nylon-flocked-swab protocol for efficient recovery of bacterial spores from smooth and rough surfaces. Applied and Environmental Microbiology 76(15): 5148-5158 (2010).

Rastogi, V.K., L. Wallace, L.S. Smith, S.P. Ryan, and M. Blair: Quantitative method to determine sporicidal decontamination of building surfaces by gaseous fumigants, and issues 
related to laboratory-scale studies. Applied and Environmental Microbiology 75(11): 3688-3694 (2009).

Rose, L.J., B. Jensen, A. Petersen, S.N. Banerjee, and M.J. Arduino,: Swab materials and Bacillus anthracis spore recovery from nonporous surfaces, Emerging Infectious Disease 10(6): 1023-1029 (2004).

Ryan, J.R., and J.F. Glarum: Biosecurity \& Bioterrorism: Containing and Preventing Biological Threats. Elsevier: New York, 2008.

Sagripanti, J.L., M. Carrera, J. Insalaco, M. Ziemski, J. Rogers, \& R. Zandomeni: Virulent spores of Bacillus anthracis and other bacillus species deposited on solid surfaces have similar sensitivity to chemical decontaminants. Journal of Applied Microbiology 102: 11-21 (2007).

Scheffrahn, R.: Whole-Structure Decontamination of Bacterial Spores by Methyl Bromide Fumigation. In Report on Workshop on Decontamination, Cleanup and Associated Issues for Sites Contaminated with Chemical, Biological, or Radiological Materials, S. Dun, and J. Wood, US EPA: October 2005.

Thatcher, T.L., and W.W. Nazaroff: Effects of small-scale obstructions and surface textures on particle deposition from natural convection flow. Aerosol Science Technology 27: 709-725 (1997).

Thomas, R.J., D. Webber, W. Sellors, A. Collinge, A. Frost, A.J. Stagg, S.C. Bailey, P.N. Jayasekera, R.R. Taylor, S. Eley, R.W. Titball: Characterization and deposition of respirable large- and small-particle bioaerosols. Applied and Environmental Microbiology 74(20): 64376443 (2008).

Uhm, H.S., H.Y. Lee, Y.C. Hong, D.H. Shin, Y.H. Park, Y.F. Hong: A decontamination study of simulated chemical and biological agents. Journal of Applied Physics 102 (2007).

Verce, M.F., B. Jayaraman, T.D. Ford, S.E. Fisher, A.J. Gadgil, and T.M. Carlsen: Minimizing decomposition of vaporized hydrogen peroxide for biological decontamination of galvanized steel ducting. Environ Sci Technol 42: 5765-5771 (2008).

Wagner, A., C.F. Green, V. Pedregon, E. Barth, S.G. Gibbs, and P.V. Scarpino: Inactivation of Bacillus subtilis on gypsum board using aerosolized chemical agents. Journal of Environmental Engineering Science 7: 159-164 (2008).

Wang, Z., T. Reponen, S.A. Grinshpun, R.L. Gorny, and K. Willeke: Effect of sampling time and air humidity on the bioefficiency of filter samplers for bioaerosol collection. Journal of Aerosol Science 32(5): 661-674 (2001).

Watson, A., and D. Keir: Information on which to base assessments of risk from environments contaminated with anthrax spores. Epidemiology and Infection 113: 479-90 (1994). 
Yah, M., and G. Mainelis: Use of portable microbial samples for estimating inhalation exposure to viable biological agents. Journal of Exposure Science and Environmental Epidemiology 17: 31-38 (2007). 


\section{APPENDIX 1: High direct inoculation test plots}

Data from the high direct inoculation tests were plotted using Minitab to show the inactivation rates against temperature and humidity. This included both surface plots and contour plots. Response plots for all of these included the $\log$ of the spores +1 .

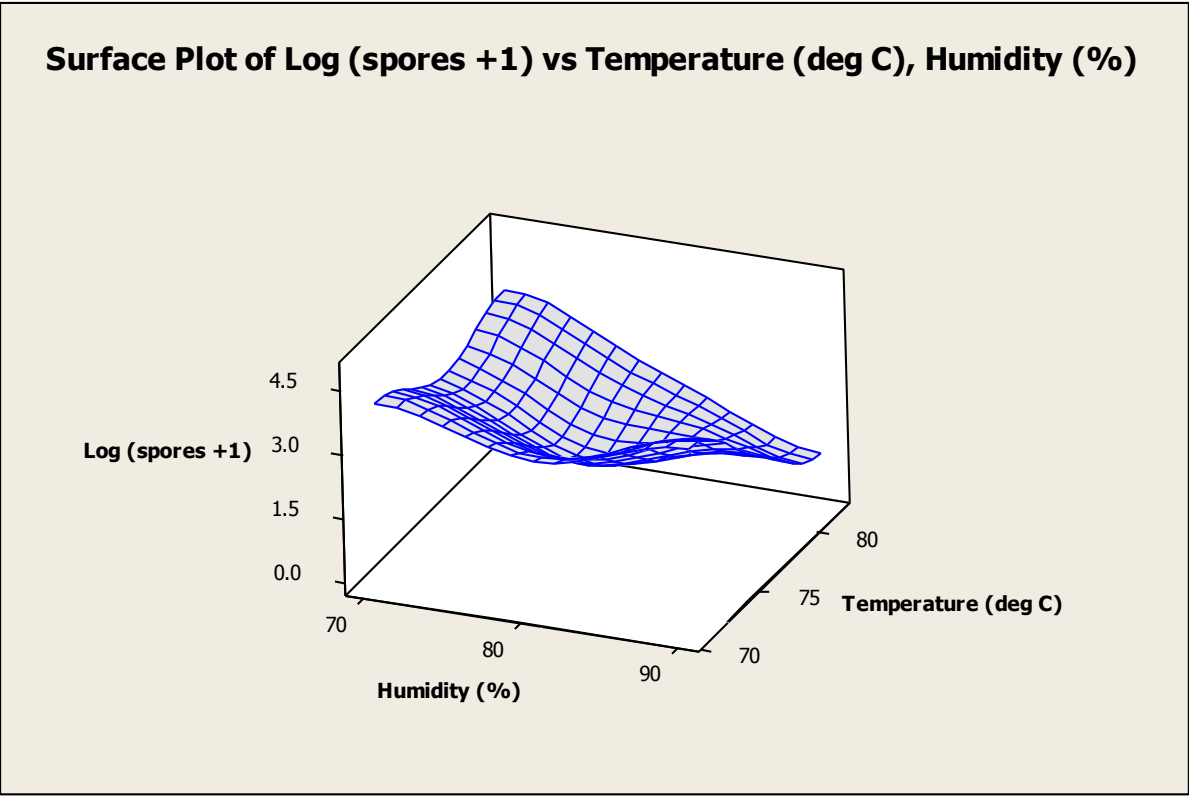

Figure 3- 12 -- High direct inoculation spore surface plot—spore log versus temperature and humidity

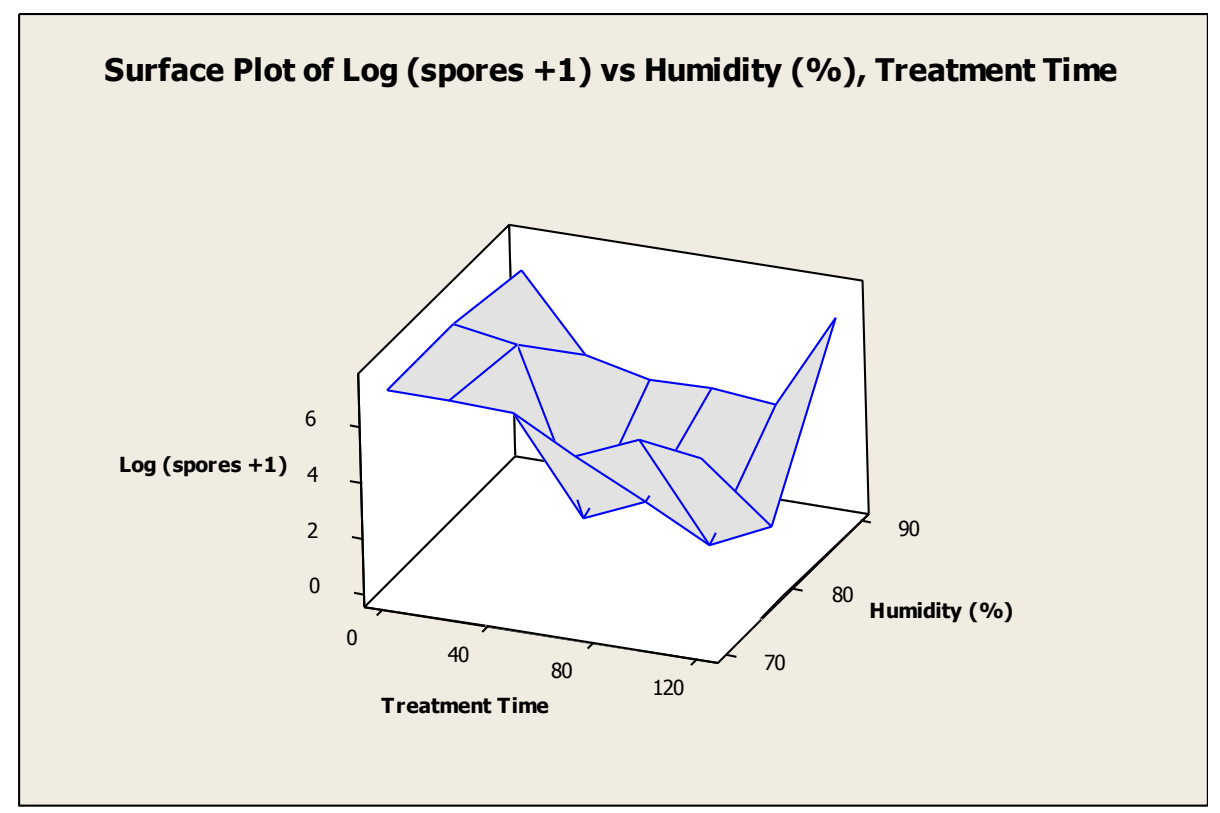

Figure 3-13 - High direct inoculation spore surface plot—spore log versus humidity and treatment time 


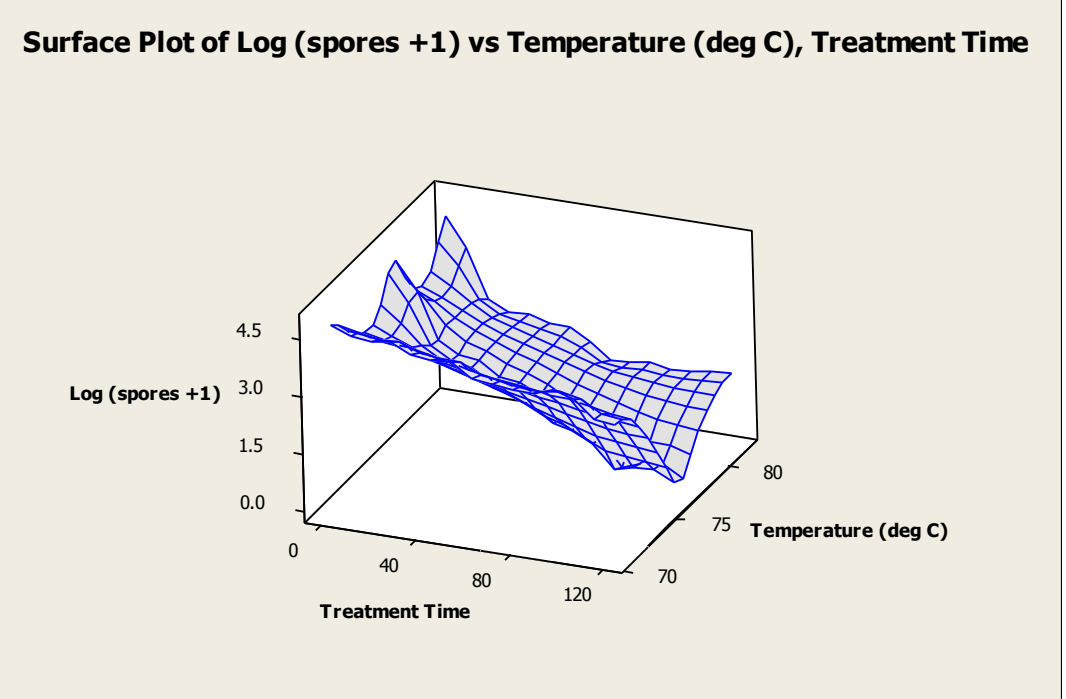

Figure 3-14 - High direct inoculation spore surface plot-spore log versus temperature and treatment time

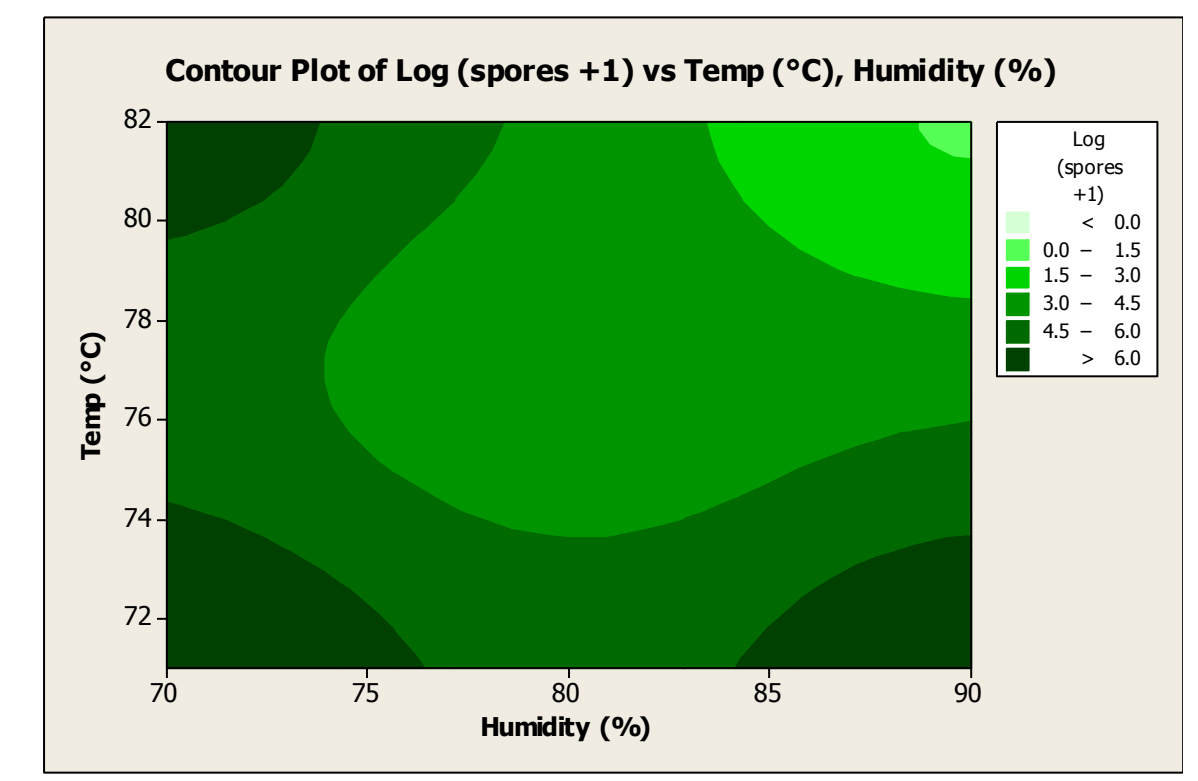

Figure 3-15 - High direct inoculation contour plot - spore log versus temperature and humidity 


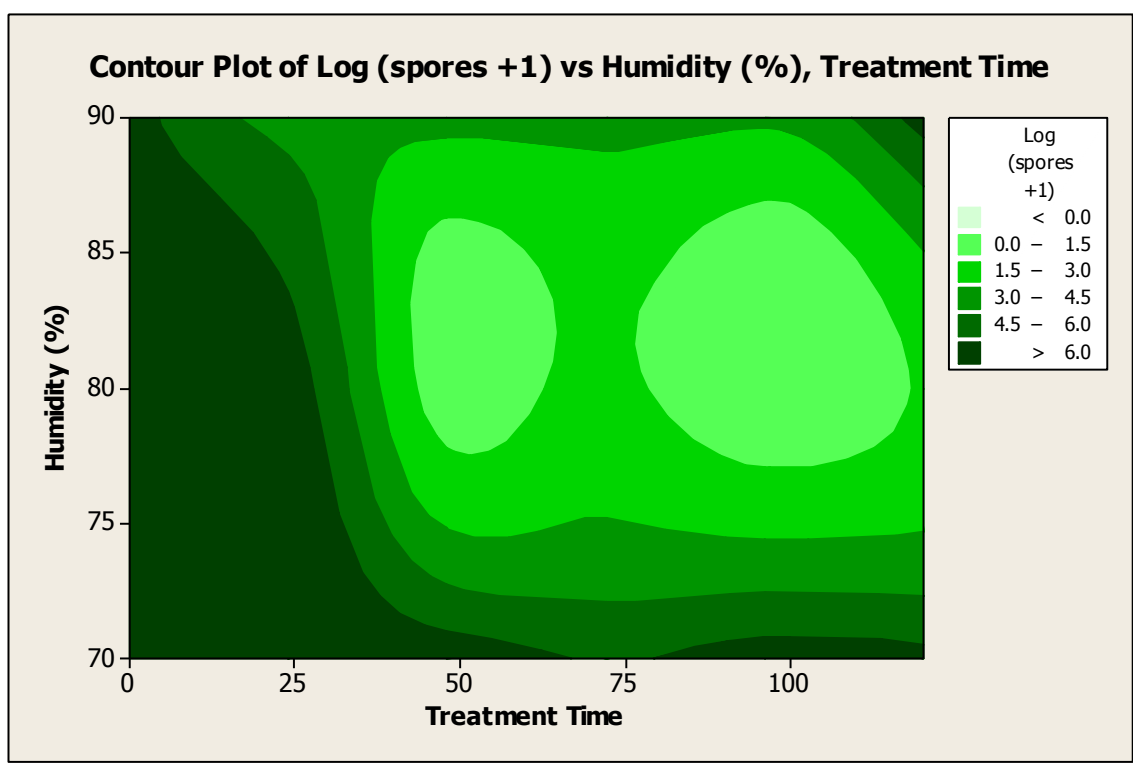

Figure 3-16 - High direct inoculation contour plot—spore log versus humidity and treatment time

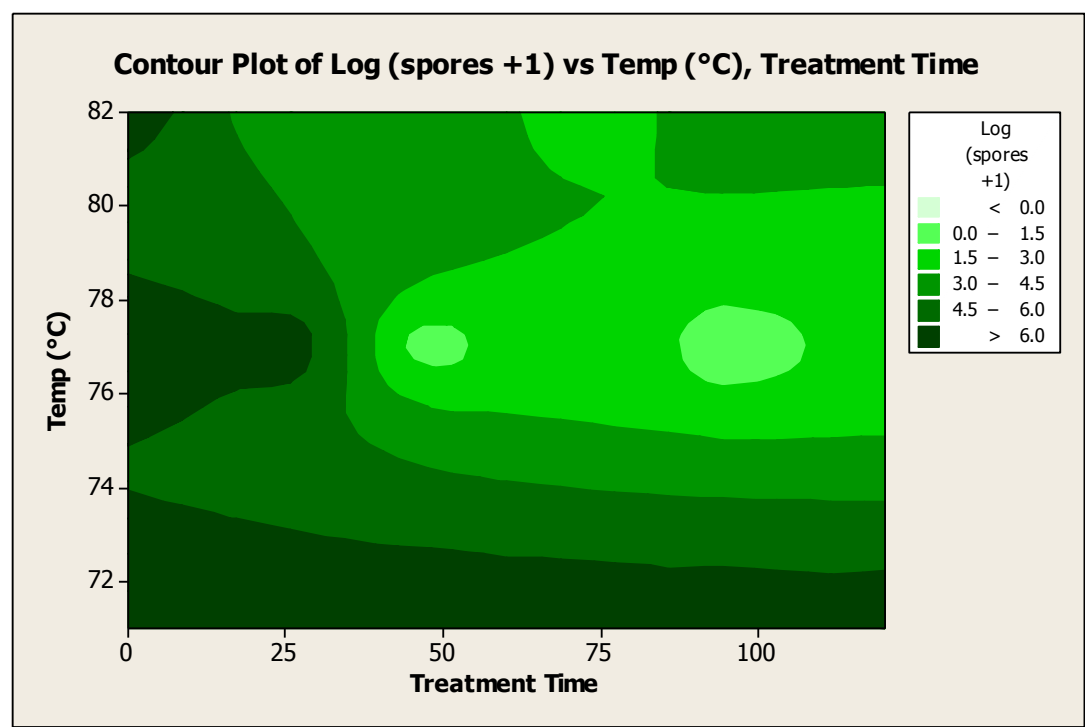

Figure 3-17 - High direct inoculation contour plot-spore log versus temperature and treatment time 


\section{APPENDIX 2: Low direct inoculation test plots}

Data from the low direct inoculation tests was plotted using Minitab to show the inactivation rates against temperature and humidity. This included both surface plots and contour plots. Response plots for all of these included the log of the spores +1 .

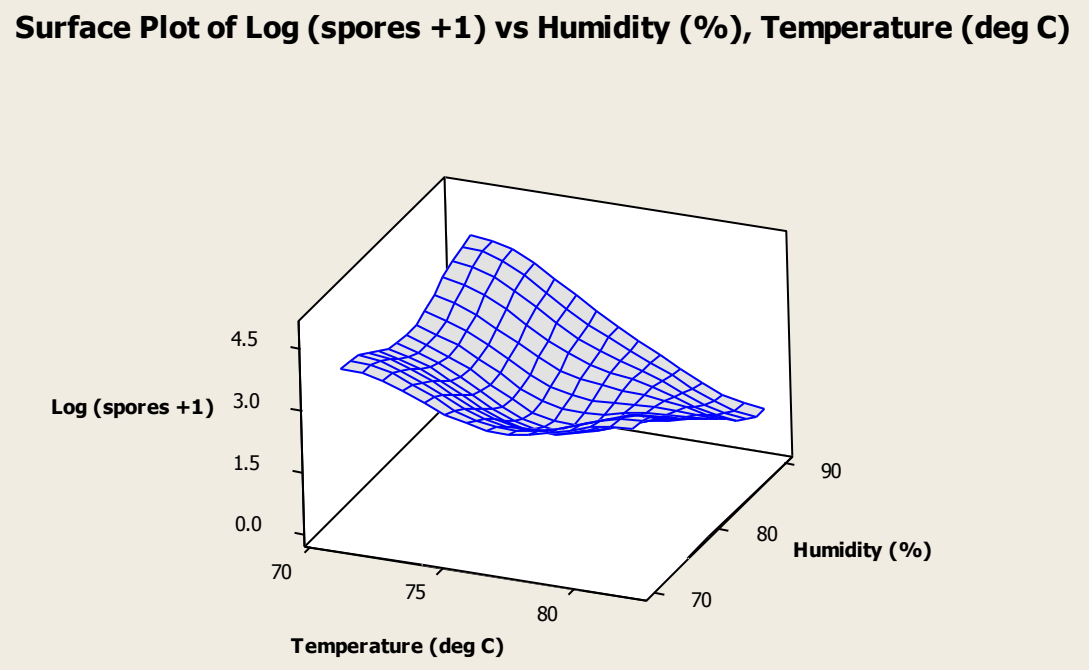

Figure 3-18 - Low direct inoculation spore surface plot-spore log versus humidity and temperature

Surface Plot of Log (spores +1) vs Humidity (\%), Treatment Time

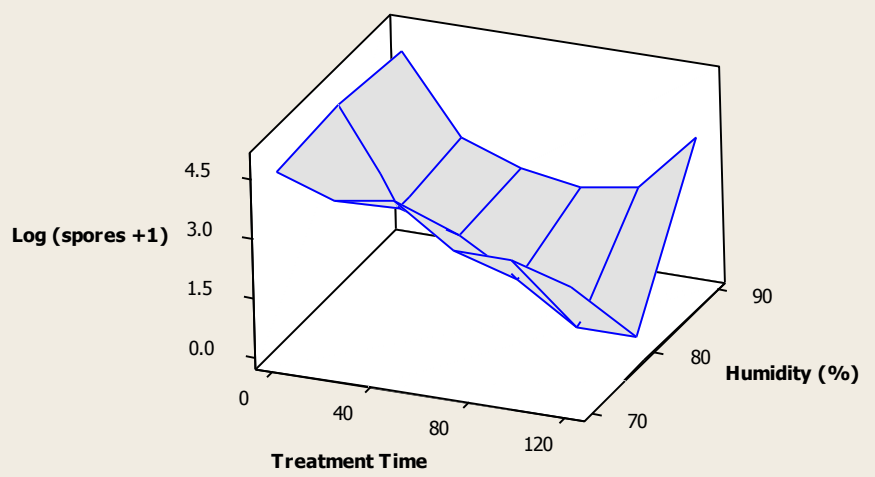

Figure 3-19 - Low direct inoculation spore surface plot-spore log versus humidity and treatment time 
Surface Plot of Log (spores +1) vs Temperature (deg C), Treatment Time

Log (spores +1)

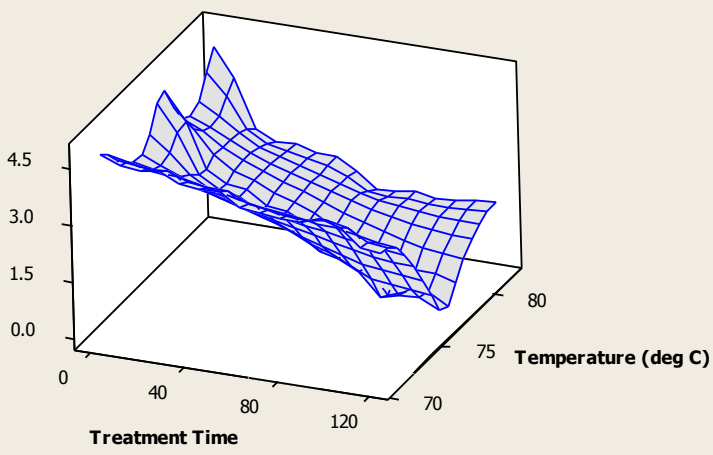

Figure 3-20 - Low direct inoculation spore surface plot-spore log versus temperature and treatment time

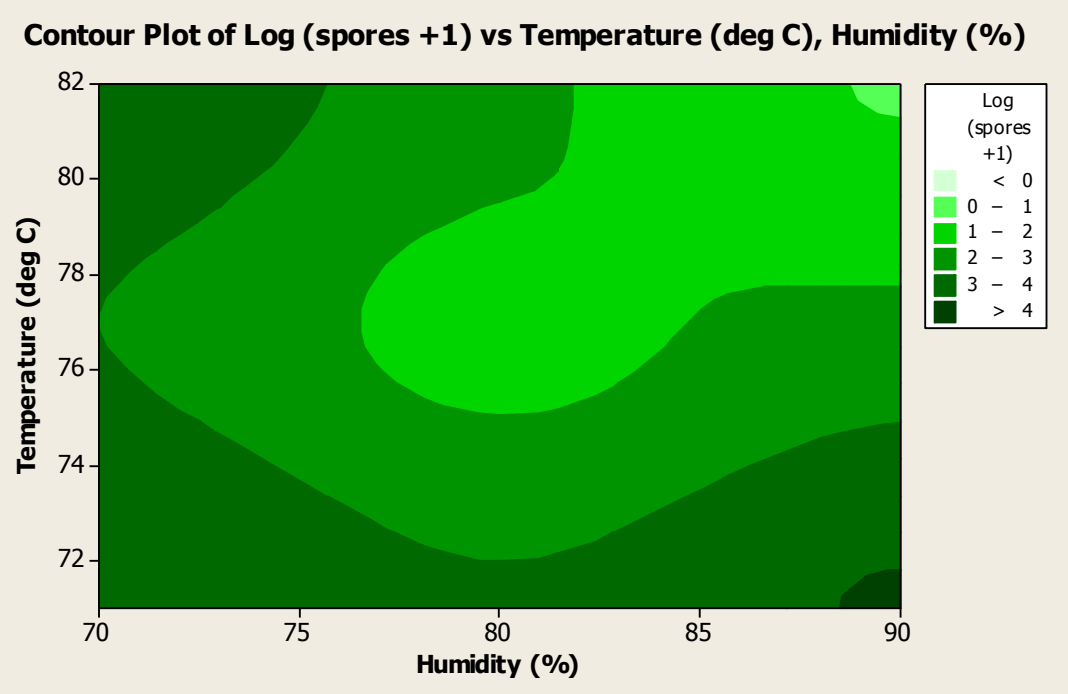

Figure 3- 21 - Low direct inoculation contour plot—spore log versus temperature and humidity 


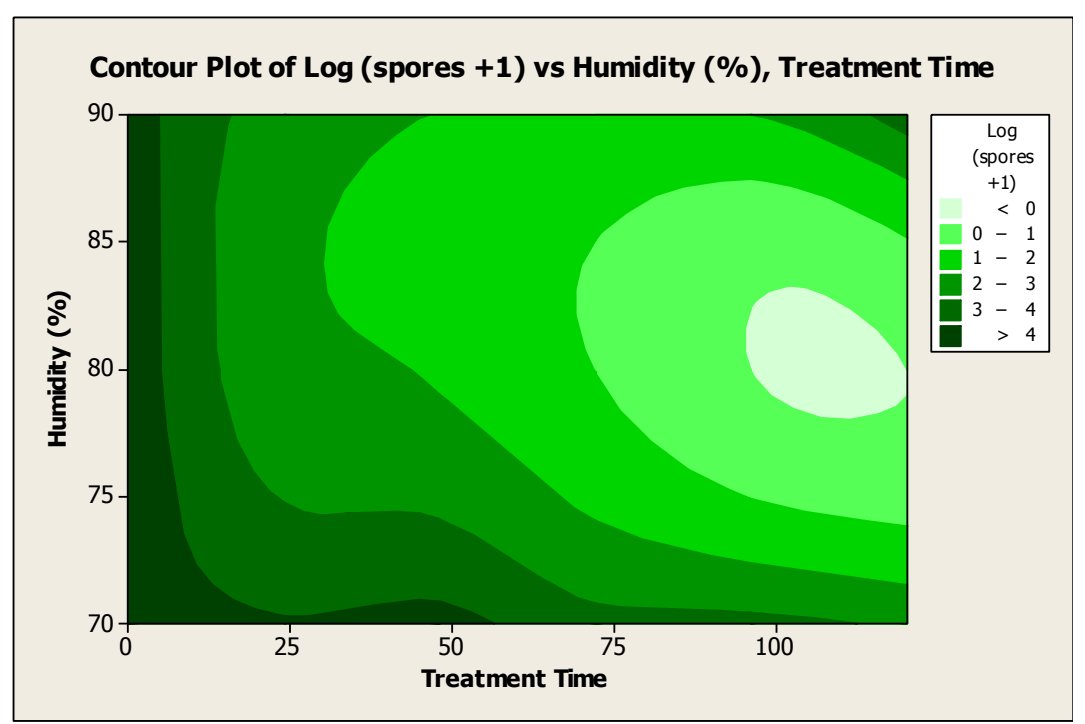

Figure 3- 22 - Low direct inoculation contour plot—spore log versus humidity and treatment time

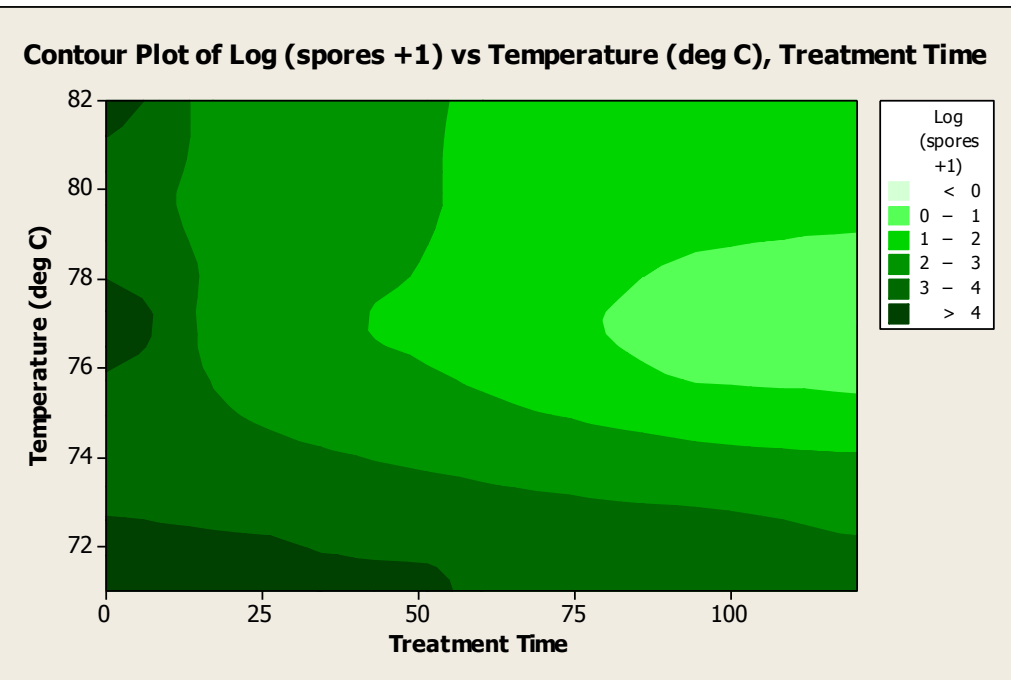

Figure 3- 23 - Low direct inoculation contour plot-spore log versus temperature and treatment time 


\section{APPENDIX 3: Aerosol deposition test plots}

Data from the low direct inoculation tests was plotted using Minitab to show the inactivation rates against temperature and humidity. This included both surface plots and contour plots. Because the all aerosol tests had corresponding controls, the response variables for these plots were the log of the control spores $(+1)$ - the log of the sample spores $(+1)$.

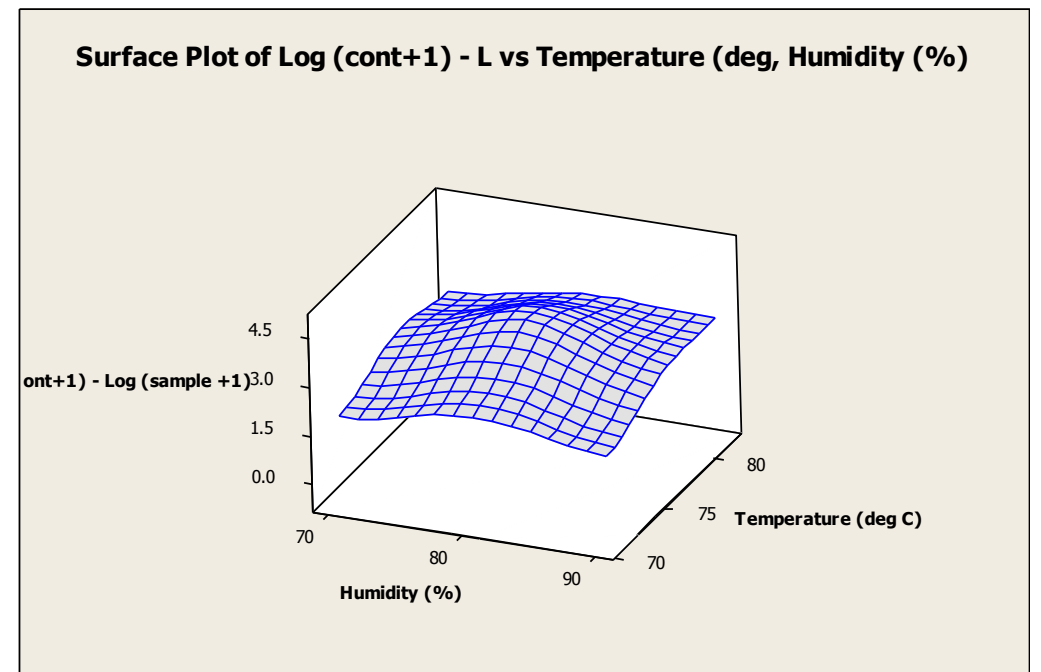

Figure 3-24 - Aerosol deposition surface plot—spore log versus humidity and temperature

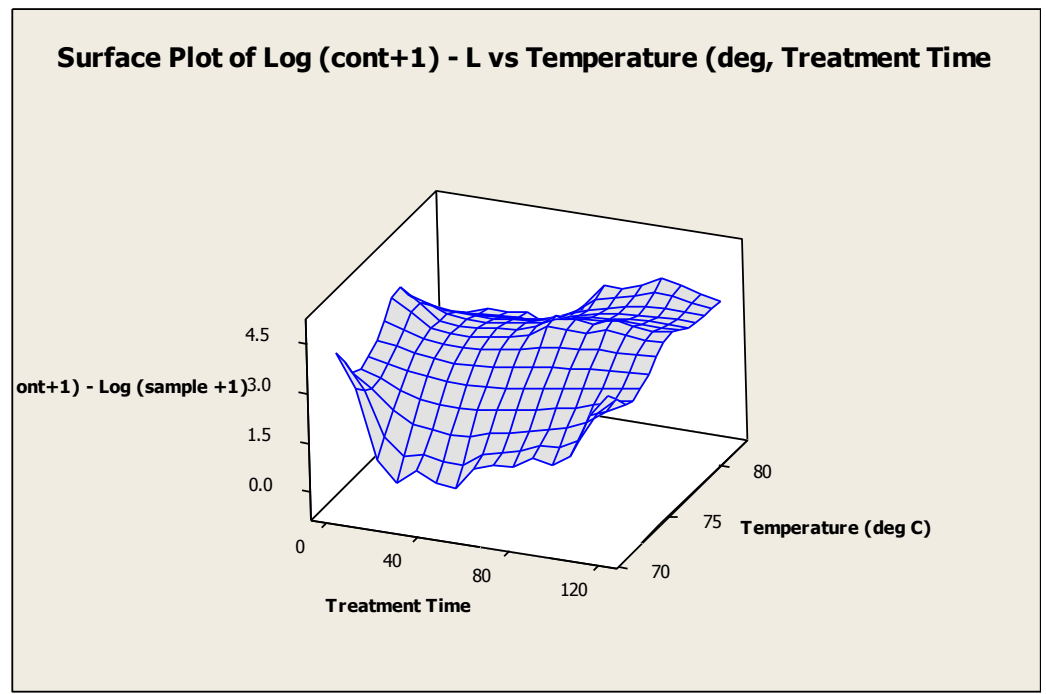

Figure 3-25 - Aerosol deposition surface plot—spore log versus humidity and treatment time 


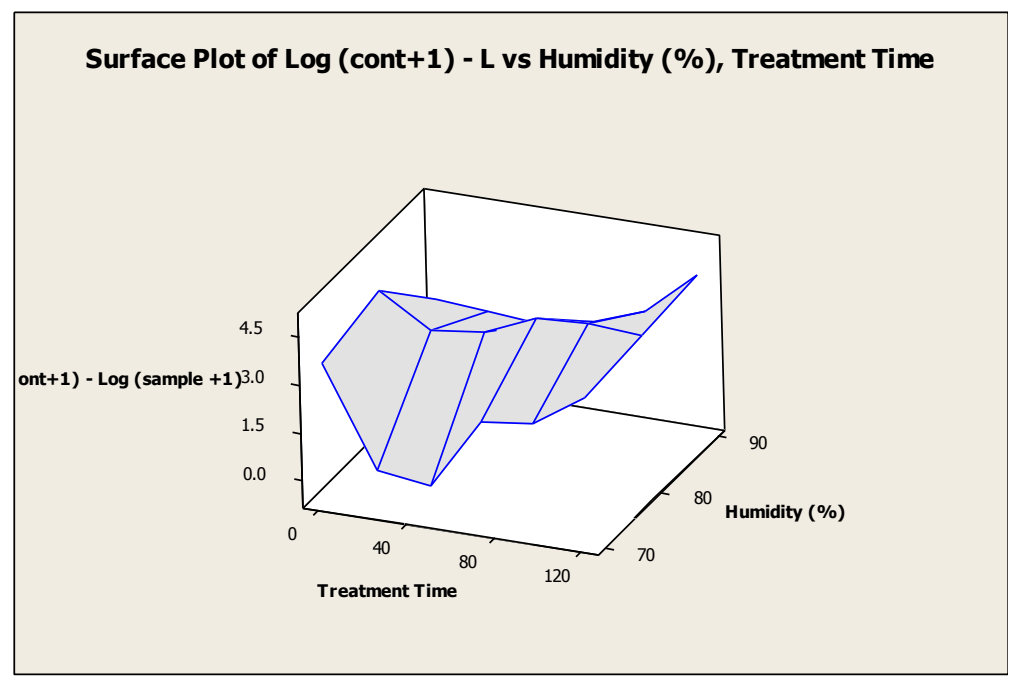

Figure 3- 26 - Aerosol deposition surface plot—-spore log versus temperature and treatment time

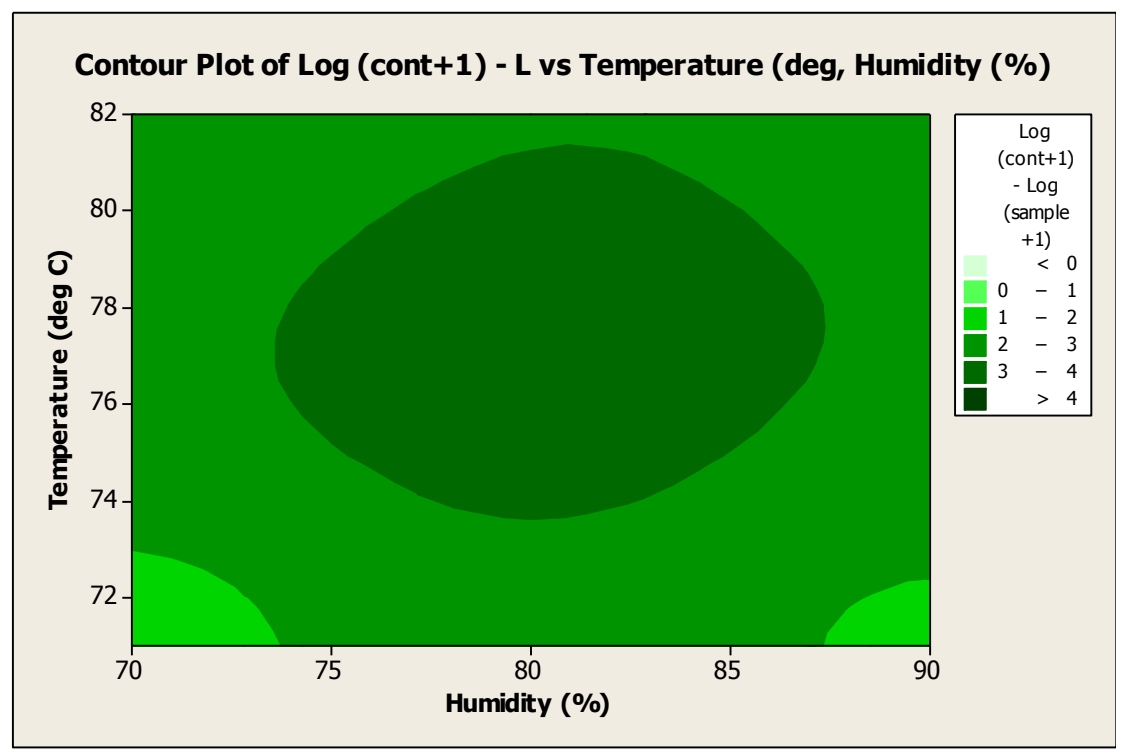

Figure 3- 27 - Aerosol deposition contour plot—-spore log versus temperature and humidity 


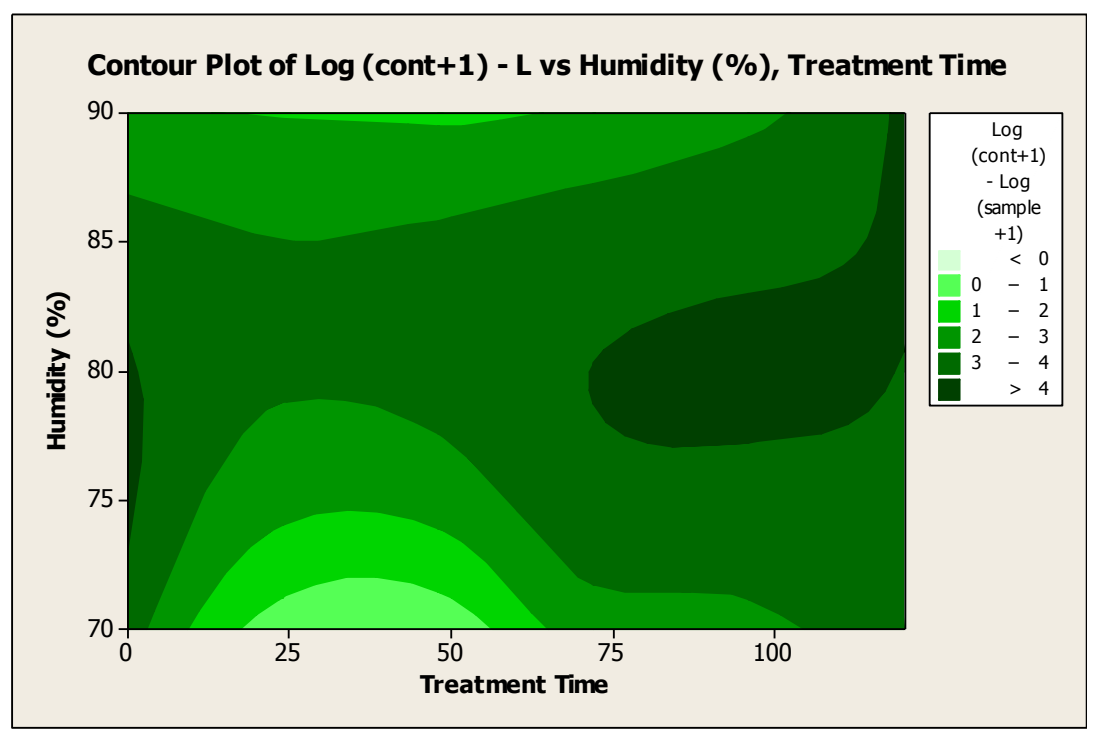

Figure 3- 28 - Aerosol deposition contour plot-spore log verusus humidity and treatment time

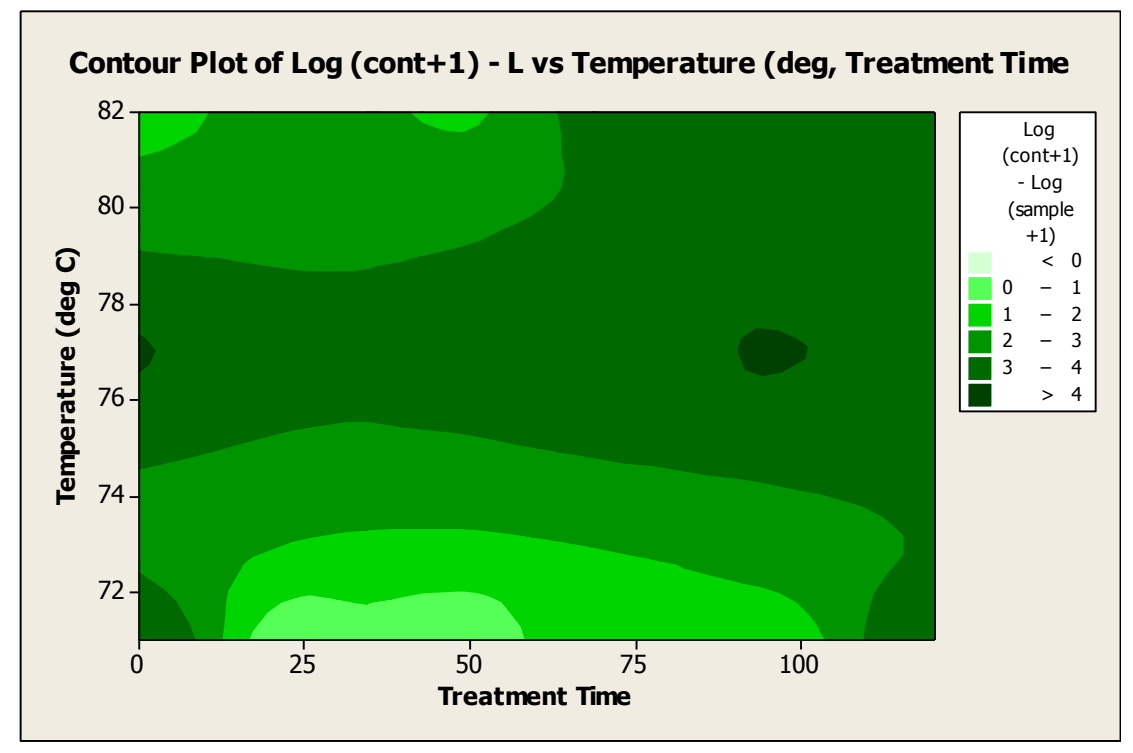

Figure 3-29 - Aerosol deposition contour plot—spore log versus temperature and treatment time 
APPENDIX 4: High direct inoculation data

Table A3 - 1-Test condition 1: 180 deg F, 90\% RH

\begin{tabular}{|c|c|c|c|c|c|c|c|c|c|}
\hline $\begin{array}{l}\text { Sample } \\
\text { Number }\end{array}$ & Time & $\begin{array}{l}\text { Spores } \\
\text { counted }\end{array}$ & $\begin{array}{c}\text { Serial } \\
\text { Dilution } \\
\text { Correction }\end{array}$ & $\begin{array}{c}\text { Dilution } \\
\text { Correction }^{2}\end{array}$ & $\begin{array}{c}\text { Agar } \\
\text { Volume }^{3}\end{array}$ & $\begin{array}{c}\text { Viable } \\
\text { spores }^{4}\end{array}$ & $\begin{array}{l}\text { Mean of } \\
\text { viable } \\
\text { spores }\end{array}$ & $\begin{array}{l}\text { SD of } \\
\text { viable } \\
\text { spores }\end{array}$ & $\begin{array}{l}\text { SE of } \\
\text { viable } \\
\text { spores }\end{array}$ \\
\hline $101 \mathrm{~A}$ & 0 & 228 & 20 & 30 & 0.1 & $1,368,000$ & \multirow{5}{*}{$1,269,600$} & \multirow{5}{*}{221,619} & \multirow{5}{*}{99,114} \\
\hline 101B & 0 & 189 & 20 & 30 & 0.1 & $1,134,000$ & & & \\
\hline $101 \mathrm{C}$ & 0 & 180 & 20 & 30 & 0.1 & $1,080,000$ & & & \\
\hline 101D & 0 & 269 & 20 & 30 & 0.1 & $1,614,000$ & & & \\
\hline $101 \mathrm{E}$ & 0 & 192 & 20 & 30 & 0.1 & $1,152,000$ & & & \\
\hline $103 \mathrm{~A}$ & 24 & 0 & 1 & 30 & 0.1 & 0 & \multirow{5}{*}{120} & \multirow{5}{*}{164} & \multirow{5}{*}{73} \\
\hline 103B & 24 & 0 & 1 & 30 & 0.1 & 0 & & & \\
\hline $103 \mathrm{C}$ & 24 & 0 & 1 & 30 & 0.1 & 0 & & & \\
\hline 103D & 24 & 1 & 1 & 30 & 0.1 & 300 & & & \\
\hline $103 \mathrm{E}$ & 24 & 1 & 1 & 30 & 0.1 & 300 & & & \\
\hline $105 \mathrm{~A}$ & 48 & 0 & 1 & 30 & 0.1 & 0 & \multirow{5}{*}{0} & \multirow{5}{*}{0} & \multirow{5}{*}{0} \\
\hline $105 \mathrm{~B}$ & 48 & 0 & 1 & 30 & 0.1 & 0 & & & \\
\hline $105 \mathrm{C}$ & 48 & 0 & 1 & 30 & 0.1 & 0 & & & \\
\hline $105 \mathrm{D}$ & 48 & 0 & 1 & 30 & 0.1 & 0 & & & \\
\hline $105 \mathrm{E}$ & 48 & 0 & 1 & 30 & 0.1 & 0 & & & \\
\hline $107 \mathrm{~A}$ & 72 & 0 & 1 & 30 & 0.1 & 0 & \multirow{5}{*}{0} & \multirow{5}{*}{0} & \multirow{5}{*}{0} \\
\hline 107B & 72 & 0 & 1 & 30 & 0.1 & 0 & & & \\
\hline $107 \mathrm{C}$ & 72 & 0 & 1 & 30 & 0.1 & 0 & & & \\
\hline 107D & 72 & 0 & 1 & 30 & 0.1 & 0 & & & \\
\hline $107 \mathrm{E}$ & 72 & 0 & 1 & 30 & 0.1 & 0 & & & \\
\hline 109A & 96 & 0 & 1 & 30 & 0.1 & 0 & \multirow{5}{*}{0} & \multirow{5}{*}{0} & \multirow{5}{*}{0} \\
\hline 109B & 96 & 0 & 1 & 30 & 0.1 & 0 & & & \\
\hline $109 \mathrm{C}$ & 96 & 0 & 1 & 30 & 0.1 & 0 & & & \\
\hline 109D & 96 & 0 & 1 & 30 & 0.1 & 0 & & & \\
\hline $109 \mathrm{E}$ & 96 & 0 & 1 & 30 & 0.1 & 0 & & & \\
\hline
\end{tabular}

1. The serial dilution correction is the value used to correct the spores based on the serial dilutions performed.

2. The dilution correction was a value used to correct for the volume of PBS in which the coupons were placed

3. The agar volume was a value used to correct for the volume of the PBS solution plated onto the petri dish

4. Viable spores was the number of spores after corrected for the dilution and agar volume corrections 
Table A3 - 2 - Test condition 3: $180 \mathrm{deg}$ F, 70\% RH

\begin{tabular}{|c|c|c|c|c|c|c|c|c|c|}
\hline $\begin{array}{l}\text { Sample } \\
\text { Number }\end{array}$ & Time & $\begin{array}{l}\text { Spores } \\
\text { counted }\end{array}$ & $\begin{array}{c}\text { Serial } \\
\text { Dilution } \\
\text { Correction }^{1}\end{array}$ & $\begin{array}{c}\text { Dilution } \\
\text { Correction }^{2}\end{array}$ & $\begin{array}{c}\text { Agar } \\
\text { Volume }^{3}\end{array}$ & $\begin{array}{l}\text { Viable } \\
\text { spores }\end{array}$ & $\begin{array}{l}\text { Mean of } \\
\text { viable } \\
\text { spores }\end{array}$ & $\begin{array}{l}\text { SD of } \\
\text { viable } \\
\text { spores }\end{array}$ & $\begin{array}{c}\text { SE of } \\
\text { viable } \\
\text { spores }\end{array}$ \\
\hline $150 \mathrm{~A}$ & 0 & 169 & 400 & 30 & 0.1 & $20,280,000$ & \multirow{5}{*}{$17,376,000$} & \multirow{5}{*}{$8,350,310$} & \multirow{5}{*}{$3,734,486$} \\
\hline $150 \mathrm{~B}$ & 0 & 65 & 400 & 30 & 0.1 & $7,800,000$ & & & \\
\hline $150 \mathrm{C}$ & 0 & 80 & 400 & 30 & 0.1 & $9,600,000$ & & & \\
\hline $150 \mathrm{D}$ & 0 & 183 & 400 & 30 & 0.1 & $21,960,000$ & & & \\
\hline $150 \mathrm{E}$ & 0 & 227 & 400 & 30 & 0.1 & $27,240,000$ & & & \\
\hline $152 \mathrm{~A}$ & 24 & 121 & 400 & 30 & 0.1 & $14,520,000$ & \multirow{5}{*}{$13,704,000$} & \multirow{5}{*}{$2,593,777$} & \multirow{5}{*}{$1,160,008$} \\
\hline $152 \mathrm{~B}$ & 24 & 136 & 400 & 30 & 0.1 & $16,320,000$ & & & \\
\hline $152 \mathrm{C}$ & 24 & 120 & 400 & 30 & 0.1 & $14,400,000$ & & & \\
\hline 152D & 24 & 116 & 400 & 30 & 0.1 & $13,920,000$ & & & \\
\hline $152 \mathrm{E}$ & 24 & 78 & 400 & 30 & 0.1 & $9,360,000$ & & & \\
\hline $154 \mathrm{~A}$ & 48 & 40 & 400 & 30 & 0.1 & $4,800,000$ & \multirow{5}{*}{$8,016,000$} & \multirow{5}{*}{$2,431,888$} & \multirow{5}{*}{$1,087,607$} \\
\hline 154B & 48 & 79 & 400 & 30 & 0.1 & $9,480,000$ & & & \\
\hline $154 \mathrm{C}$ & 48 & 68 & 400 & 30 & 0.1 & $8,160,000$ & & & \\
\hline 154D & 48 & 55 & 400 & 30 & 0.1 & $6,600,000$ & & & \\
\hline $154 \mathrm{E}$ & 48 & 92 & 400 & 30 & 0.1 & $11,040,000$ & & & \\
\hline $156 \mathrm{~A}$ & 72 & 20 & 400 & 30 & 0.1 & $2,400,000$ & \multirow{5}{*}{$1,224,000$} & \multirow{5}{*}{$1,213,128$} & \multirow{5}{*}{542,544} \\
\hline $156 \mathrm{~B}$ & 72 & 22 & 400 & 30 & 0.1 & $2,640,000$ & & & \\
\hline $156 \mathrm{C}$ & 72 & 3 & 400 & 30 & 0.1 & 360,000 & & & \\
\hline $156 \mathrm{D}$ & 72 & 6 & 400 & 30 & 0.1 & 720,000 & & & \\
\hline $156 \mathrm{E}$ & 72 & 0 & 400 & 30 & 0.1 & 0 & & & \\
\hline $158 \mathrm{~A}$ & 96 & 450 & 20 & 30 & 0.1 & $2,700,000$ & \multirow{5}{*}{$2,700,000$} & \multirow{5}{*}{0} & \multirow{5}{*}{0} \\
\hline 158B & 96 & 450 & 20 & 30 & 0.1 & $2,700,000$ & & & \\
\hline $158 \mathrm{C}$ & 96 & 450 & 20 & 30 & 0.1 & $2,700,000$ & & & \\
\hline $158 \mathrm{D}$ & 96 & 450 & 20 & 30 & 0.1 & $2,700,000$ & & & \\
\hline $158 \mathrm{E}$ & 96 & 450 & 20 & 30 & 0.1 & $2,700,000$ & & & \\
\hline $158 \mathrm{~A}$ & 96 & 23 & 400 & 30 & 0.1 & $2,760,000$ & \multirow{5}{*}{$4,416,000$} & \multirow{5}{*}{$1,494,952$} & \multirow{5}{*}{668,583} \\
\hline $158 \mathrm{~B}$ & 96 & 37 & 400 & 30 & 0.1 & $4,440,000$ & & & \\
\hline $158 \mathrm{C}$ & 96 & 33 & 400 & 30 & 0.1 & $3,960,000$ & & & \\
\hline $158 \mathrm{D}$ & 96 & 57 & 400 & 30 & 0.1 & $6,840,000$ & & & \\
\hline $159 \mathrm{E}$ & 96 & 34 & 400 & 30 & 0.1 & $4,080,000$ & & & \\
\hline
\end{tabular}

1. The serial dilution correction is the value used to correct the spores based on the serial dilutions performed.

2. The dilution correction was a value used to correct for the volume of PBS in which the coupons were placed

3. The agar volume was a value used to correct for the volume of the PBS solution plated onto the petri dish

4. Viable spores was the number of spores after corrected for the dilution and agar volume corrections 
Table A3 - 3- Test condition 5: 170 deg F, $80 \%$ RH

\begin{tabular}{|c|c|c|c|c|c|c|c|c|c|}
\hline $\begin{array}{l}\text { Sample } \\
\text { Number }\end{array}$ & Time & $\begin{array}{l}\text { Spores } \\
\text { counted }\end{array}$ & $\begin{array}{c}\text { Serial } \\
\text { Dilution } \\
\text { Correction }^{1}\end{array}$ & $\begin{array}{c}\text { Dilution } \\
\text { Correction }^{2}\end{array}$ & $\begin{array}{c}\text { Agar } \\
\text { Volume }^{3}\end{array}$ & $\begin{array}{c}\text { Viable } \\
\text { spores }^{4}\end{array}$ & $\begin{array}{c}\text { Mean of } \\
\text { viable } \\
\text { spores } \\
\end{array}$ & $\begin{array}{l}\text { SD of } \\
\text { viable } \\
\text { spores }\end{array}$ & $\begin{array}{c}\text { SE of } \\
\text { viable } \\
\text { spores }\end{array}$ \\
\hline $600 \mathrm{~A}$ & 0 & 156 & 400 & 30 & 0.1 & $18,720,000$ & \multirow{5}{*}{$15,072,000$} & \multirow{5}{*}{$6,071,929$} & \multirow{5}{*}{$2,715,532$} \\
\hline $600 \mathrm{~B}$ & 0 & 55 & 400 & 30 & 0.1 & $6,600,000$ & & & \\
\hline $600 \mathrm{C}$ & 0 & 186 & 400 & 30 & 0.1 & $22,320,000$ & & & \\
\hline $600 \mathrm{D}$ & 0 & 131 & 400 & 30 & 0.1 & $15,720,000$ & & & \\
\hline $600 \mathrm{E}$ & 0 & 100 & 400 & 30 & 0.1 & $12,000,000$ & & & \\
\hline $602 \mathrm{~A}$ & 24 & 74 & 400 & 30 & 0.1 & $8,880,000$ & \multirow{5}{*}{$6,024,000$} & \multirow{5}{*}{$3,058,706$} & \multirow{5}{*}{$1,367,936$} \\
\hline $602 B$ & 24 & 12 & 400 & 30 & 0.1 & $1,440,000$ & & & \\
\hline $602 \mathrm{C}$ & 24 & 61 & 400 & 30 & 0.1 & $7,320,000$ & & & \\
\hline 602D & 24 & 67 & 400 & 30 & 0.1 & $8,040,000$ & & & \\
\hline $602 \mathrm{E}$ & 24 & 37 & 400 & 30 & 0.1 & $4,440,000$ & & & \\
\hline $604 \mathrm{~A}$ & 48 & 0 & 20 & 30 & 0.1 & 0 & \multirow{5}{*}{6,000} & \multirow{5}{*}{13,416} & \multirow{5}{*}{6,000} \\
\hline $604 \mathrm{~B}$ & 48 & 0 & 20 & 30 & 0.1 & 0 & & & \\
\hline $602 C$ & 48 & 0 & 20 & 30 & 0.1 & 0 & & & \\
\hline $602 \mathrm{D}$ & 48 & 5 & 20 & 30 & 0.1 & 30,000 & & & \\
\hline $602 \mathrm{E}$ & 48 & 0 & 20 & 30 & 0.1 & 0 & & & \\
\hline $606 \mathrm{~A}$ & 72 & 1 & 1 & 30 & 0.1 & 300 & \multirow{5}{*}{900} & \multirow{5}{*}{1,391} & \multirow{5}{*}{622} \\
\hline $606 \mathrm{~B}$ & 72 & 0 & 1 & 30 & 0.1 & 0 & & & \\
\hline $606 \mathrm{C}$ & 72 & 0 & 1 & 30 & 0.1 & 0 & & & \\
\hline $606 \mathrm{D}$ & 72 & 11 & 1 & 30 & 0.1 & 3,300 & & & \\
\hline $606 \mathrm{E}$ & 72 & 3 & 1 & 30 & 0.1 & 900 & & & \\
\hline $608 \mathrm{~A}$ & 96 & 0 & 1 & 30 & 0.1 & 0 & \multirow{5}{*}{180} & \multirow{5}{*}{402} & \multirow{5}{*}{180} \\
\hline $608 \mathrm{~B}$ & 96 & 0 & 1 & 30 & 0.1 & 0 & & & \\
\hline $608 \mathrm{C}$ & 96 & 0 & 1 & 30 & 0.1 & 0 & & & \\
\hline $608 \mathrm{D}$ & 96 & 0 & 1 & 30 & 0.1 & 0 & & & \\
\hline $608 \mathrm{E}$ & 96 & 3 & 1 & 30 & 0.1 & 900 & & & \\
\hline $610 \mathrm{~A}$ & 120 & 0 & 20 & 30 & 0.1 & 0 & \multirow{5}{*}{8,400} & \multirow{5}{*}{15,646} & \multirow{5}{*}{6,997} \\
\hline $610 \mathrm{~B}$ & 120 & 0 & 20 & 30 & 0.1 & 0 & & & \\
\hline $610 \mathrm{C}$ & 120 & 0 & 20 & 30 & 0.1 & 0 & & & \\
\hline 610D & 120 & 6 & 20 & 30 & 0.1 & 36,000 & & & \\
\hline $610 \mathrm{E}$ & 120 & 1 & 20 & 30 & 0.1 & 6,000 & & & \\
\hline
\end{tabular}

1. The serial dilution correction is the value used to correct the spores based on the serial dilutions performed.

2. The dilution correction was a value used to correct for the volume of PBS in which the coupons were placed

3. The agar volume was a value used to correct for the volume of the PBS solution plated onto the petri dish

4. Viable spores was the number of spores after corrected for the dilution and agar volume corrections 
Table A3 - 4 - Test condition 7: 160 deg F, 90\% RH

\begin{tabular}{|c|c|c|c|c|c|c|c|c|c|}
\hline $\begin{array}{l}\text { Sample } \\
\text { Number }\end{array}$ & Time & $\begin{array}{l}\text { Spores } \\
\text { counted }\end{array}$ & $\begin{array}{c}\text { Serial } \\
\text { Dilution } \\
\text { Correction }^{1}\end{array}$ & $\begin{array}{c}\text { Dilution } \\
\text { Correction }^{2}\end{array}$ & $\begin{array}{c}\text { Agar } \\
\text { Volume }^{3}\end{array}$ & $\begin{array}{c}\text { Viable } \\
\text { spores }^{4}\end{array}$ & $\begin{array}{c}\text { Mean of } \\
\text { viable } \\
\text { spores } \\
\end{array}$ & $\begin{array}{c}\text { SD of } \\
\text { viable } \\
\text { spores }\end{array}$ & $\begin{array}{l}\text { SE of } \\
\text { viable } \\
\text { spores }\end{array}$ \\
\hline $200 \mathrm{~A}$ & 0 & 149 & 400 & 30 & 0.1 & $17,880,000$ & \multirow{5}{*}{$16,464,000$} & \multirow{5}{*}{$3,959,455$} & \multirow{5}{*}{$1,770,776$} \\
\hline 200B & 0 & 158 & 400 & 30 & 0.1 & $18,960,000$ & & & \\
\hline $200 \mathrm{C}$ & 0 & 133 & 400 & 30 & 0.1 & $15,960,000$ & & & \\
\hline 200D & 0 & 164 & 400 & 30 & 0.1 & $19,680,000$ & & & \\
\hline $200 \mathrm{E}$ & 0 & 82 & 400 & 30 & 0.1 & $9,840,000$ & & & \\
\hline $202 \mathrm{~A}$ & 24 & 158 & 400 & 30 & 0.1 & $18,960,000$ & \multirow{5}{*}{$10,848,000$} & \multirow{5}{*}{$5,632,080$} & \multirow{5}{*}{$2,518,819$} \\
\hline 202B & 24 & 112 & 400 & 30 & 0.1 & $13,440,000$ & & & \\
\hline $202 C$ & 24 & 88 & 400 & 30 & 0.1 & $10,560,000$ & & & \\
\hline 202D & 24 & 48 & 400 & 30 & 0.1 & $5,760,000$ & & & \\
\hline $202 \mathrm{E}$ & 24 & 46 & 400 & 30 & 0.1 & $5,520,000$ & & & \\
\hline $204 \mathrm{~A}$ & 48 & 50 & 400 & 30 & 0.1 & $6,000,000$ & \multirow{5}{*}{$8,880,000$} & \multirow{5}{*}{$4,071,167$} & \multirow{5}{*}{$1,820,737$} \\
\hline 204B & 48 & 45 & 400 & 30 & 0.1 & $5,400,000$ & & & \\
\hline $204 C$ & 48 & 113 & 400 & 30 & 0.1 & $13,560,000$ & & & \\
\hline 204D & 48 & 109 & 400 & 30 & 0.1 & $13,080,000$ & & & \\
\hline $204 \mathrm{E}$ & 48 & 53 & 400 & 30 & 0.1 & $6,360,000$ & & & \\
\hline $206 \mathrm{~A}$ & 72 & 85 & 400 & 30 & 0.1 & $10,200,000$ & \multirow{5}{*}{$10,968,000$} & \multirow{5}{*}{991,726} & \multirow{5}{*}{443,527} \\
\hline 206B & 72 & 82 & 400 & 30 & 0.1 & $9,840,000$ & & & \\
\hline $206 \mathrm{C}$ & 72 & 94 & 400 & 30 & 0.1 & $11,280,000$ & & & \\
\hline $206 \mathrm{D}$ & 72 & 93 & 400 & 30 & 0.1 & $11,160,000$ & & & \\
\hline $206 \mathrm{E}$ & 72 & 103 & 400 & 30 & 0.1 & $12,360,000$ & & & \\
\hline $208 \mathrm{~A}$ & 96 & 75 & 400 & 30 & 0.1 & $9,000,000$ & \multirow{5}{*}{$4,176,000$} & \multirow{5}{*}{$3,153,741$} & \multirow{5}{*}{$1,410,439$} \\
\hline $208 B$ & 96 & 7 & 400 & 30 & 0.1 & 840,000 & & & \\
\hline $208 \mathrm{C}$ & 96 & 38 & 400 & 30 & 0.1 & $4,560,000$ & & & \\
\hline 208D & 96 & 38 & 400 & 30 & 0.1 & $4,560,000$ & & & \\
\hline $208 \mathrm{E}$ & 96 & 16 & 400 & 30 & 0.1 & $1,920,000$ & & & \\
\hline $210 \mathrm{~A}$ & 120 & 64 & 400 & 30 & 0.1 & $7,680,000$ & \multirow{5}{*}{$5,592,000$} & \multirow{5}{*}{$1,768,932$} & \multirow{5}{*}{791,114} \\
\hline 210B & 120 & 57 & 400 & 30 & 0.1 & $6,840,000$ & & & \\
\hline $210 \mathrm{C}$ & 120 & 26 & 400 & 30 & 0.1 & $3,120,000$ & & & \\
\hline $210 \mathrm{D}$ & 120 & 45 & 400 & 30 & 0.1 & $5,400,000$ & & & \\
\hline $210 \mathrm{E}$ & 120 & 41 & 400 & 30 & 0.1 & $4,920,000$ & & & \\
\hline
\end{tabular}

1. The serial dilution correction is the value used to correct the spores based on the serial dilutions performed.

2. The dilution correction was a value used to correct for the volume of PBS in which the coupons were placed

3. The agar volume was a value used to correct for the volume of the PBS solution plated onto the petri dish

4. Viable spores was the number of spores after corrected for the dilution and agar volume corrections 
Table A3 - 5 - Test condition 9: $160 \mathrm{deg}$ F, 70\% RH

\begin{tabular}{|c|c|c|c|c|c|c|c|c|c|}
\hline $\begin{array}{l}\text { Sample } \\
\text { Number }\end{array}$ & Time & $\begin{array}{l}\text { Spores } \\
\text { counted }\end{array}$ & $\begin{array}{c}\text { Serial } \\
\text { Dilution } \\
\text { Correction }^{1}\end{array}$ & $\begin{array}{c}\text { Dilution } \\
\text { Correction }^{2}\end{array}$ & $\begin{array}{c}\text { Agar } \\
\text { Volume }^{3}\end{array}$ & $\begin{array}{c}\text { Viable } \\
\text { spores }^{4}\end{array}$ & $\begin{array}{c}\text { Mean of } \\
\text { viable } \\
\text { spores }\end{array}$ & $\begin{array}{c}\text { SD of } \\
\text { viable } \\
\text { spores }\end{array}$ & $\begin{array}{l}\text { SE of } \\
\text { viable } \\
\text { spores }\end{array}$ \\
\hline $400 \mathrm{~A}$ & 0 & 114 & 400 & 30 & 0.1 & $13,680,000$ & \multirow{5}{*}{$14,280,000$} & \multirow{5}{*}{$8,019,825$} & \multirow{5}{*}{$3,586,684$} \\
\hline $400 \mathrm{~B}$ & 0 & 61 & 400 & 30 & 0.1 & $7,320,000$ & & & \\
\hline $400 \mathrm{C}$ & 0 & 47 & 400 & 30 & 0.1 & $5,640,000$ & & & \\
\hline $400 \mathrm{D}$ & 0 & 196 & 400 & 30 & 0.1 & $23,520,000$ & & & \\
\hline $400 \mathrm{E}$ & 0 & 177 & 400 & 30 & 0.1 & $21,240,000$ & & & \\
\hline $402 \mathrm{~A}$ & 24 & 170 & 400 & 30 & 0.1 & $20,400,000$ & \multirow{5}{*}{$14,784,000$} & \multirow{5}{*}{$6,621,456$} & \multirow{5}{*}{$2,961,295$} \\
\hline $402 \mathrm{~B}$ & 24 & 35 & 400 & 30 & 0.1 & $4,200,000$ & & & \\
\hline $402 \mathrm{C}$ & 24 & 119 & 400 & 30 & 0.1 & $14,280,000$ & & & \\
\hline $402 \mathrm{D}$ & 24 & 170 & 400 & 30 & 0.1 & $20,400,000$ & & & \\
\hline $402 \mathrm{E}$ & 24 & 122 & 400 & 30 & 0.1 & $14,640,000$ & & & \\
\hline $404 \mathrm{~A}$ & 48 & 146 & 400 & 30 & 0.1 & $17,520,000$ & \multirow{5}{*}{$16,536,000$} & \multirow{5}{*}{$4,401,236$} & \multirow{5}{*}{$1,968,352$} \\
\hline 404B & 48 & 94 & 400 & 30 & 0.1 & $11,280,000$ & & & \\
\hline $404 \mathrm{C}$ & 48 & 107 & 400 & 30 & 0.1 & $12,840,000$ & & & \\
\hline 404D & 48 & 182 & 400 & 30 & 0.1 & $21,840,000$ & & & \\
\hline $404 \mathrm{E}$ & 48 & 160 & 400 & 30 & 0.1 & $19,200,000$ & & & \\
\hline $406 \mathrm{~A}$ & 72 & 181 & 400 & 30 & 0.1 & $21,720,000$ & \multirow{5}{*}{$11,160,000$} & \multirow{5}{*}{$8,423,111$} & \multirow{5}{*}{$3,767,044$} \\
\hline $406 \mathrm{~B}$ & 72 & 4 & 400 & 30 & 0.1 & 480,000 & & & \\
\hline $406 \mathrm{C}$ & 72 & 132 & 400 & 30 & 0.1 & $15,840,000$ & & & \\
\hline $406 \mathrm{D}$ & 72 & 104 & 400 & 30 & 0.1 & $12,480,000$ & & & \\
\hline $406 \mathrm{E}$ & 72 & 44 & 400 & 30 & 0.1 & $5,280,000$ & & & \\
\hline $408 \mathrm{~A}$ & 96 & 54 & 400 & 30 & 0.1 & $6,480,000$ & \multirow{5}{*}{$11,904,000$} & \multirow{5}{*}{$5,240,580$} & \multirow{5}{*}{$2,343,730$} \\
\hline 408B & 96 & 130 & 400 & 30 & 0.1 & $15,600,000$ & & & \\
\hline $408 \mathrm{C}$ & 96 & 94 & 400 & 30 & 0.1 & $11,280,000$ & & & \\
\hline 408D & 96 & 62 & 400 & 30 & 0.1 & $7,440,000$ & & & \\
\hline $408 \mathrm{E}$ & 96 & 156 & 400 & 30 & 0.1 & $18,720,000$ & & & \\
\hline $410 \mathrm{~A}$ & 120 & 18 & 400 & 30 & 0.1 & $2,160,000$ & \multirow{5}{*}{$5,736,000$} & \multirow{5}{*}{$5,855,705$} & \multirow{5}{*}{$2,618,830$} \\
\hline 410B & 120 & 98 & 400 & 30 & 0.1 & $11,760,000$ & & & \\
\hline $410 \mathrm{C}$ & 120 & 13 & 400 & 30 & 0.1 & $1,560,000$ & & & \\
\hline $410 \mathrm{D}$ & 120 & 6 & 400 & 30 & 0.1 & 720,000 & & & \\
\hline $410 \mathrm{E}$ & 120 & 104 & 400 & 30 & 0.1 & $12,480,000$ & & & \\
\hline
\end{tabular}

1. The serial dilution correction is the value used to correct the spores based on the serial dilutions performed.

2. The dilution correction was a value used to correct for the volume of PBS in which the coupons were placed

3. The agar volume was a value used to correct for the volume of the PBS solution plated onto the petri dish

4. Viable spores was the number of spores after corrected for the dilution and agar volume corrections 


\section{APPENDIX 5: Low direct inoculation data}

Table A3 - 6 - Test condition 1: 180 deg F, 90\% RH

\begin{tabular}{|c|c|c|c|c|c|c|c|c|}
\hline $\begin{array}{l}\text { Sample } \\
\text { Number }\end{array}$ & Time & $\begin{array}{l}\text { Spores } \\
\text { counted }\end{array}$ & $\begin{array}{c}\text { Dilution } \\
\text { Correction }^{1}\end{array}$ & $\begin{array}{c}\text { Agar } \\
\text { Volume }^{2}\end{array}$ & $\begin{array}{c}\text { Viable } \\
\text { spores }^{3}\end{array}$ & $\begin{array}{c}\text { Mean of } \\
\text { viable } \\
\text { spores } \\
\end{array}$ & $\begin{array}{l}\text { SD of } \\
\text { viable } \\
\text { spores }\end{array}$ & $\begin{array}{l}\text { SE of } \\
\text { viable } \\
\text { spores } \\
\end{array}$ \\
\hline $102 \mathrm{~A}$ & 0 & 23 & 30 & 0.1 & 6,900 & \multirow{5}{*}{28,140} & \multirow{5}{*}{23,116} & \multirow{5}{*}{10,338} \\
\hline 102B & 0 & 47 & 30 & 0.1 & 14,100 & & & \\
\hline $102 \mathrm{C}$ & 0 & 49 & 30 & 0.1 & 14,700 & & & \\
\hline 102D & 0 & 149 & 30 & 0.1 & 44,700 & & & \\
\hline $102 \mathrm{E}$ & 0 & 201 & 30 & 0.1 & 60,300 & & & \\
\hline $104 \mathrm{~A}$ & 24 & 1 & 30 & 0.1 & 300 & \multirow{5}{*}{60} & \multirow{5}{*}{134} & \multirow{5}{*}{60} \\
\hline 104B & 24 & 0 & 30 & 0.1 & 0 & & & \\
\hline $104 \mathrm{C}$ & 24 & 0 & 30 & 0.1 & 0 & & & \\
\hline 104D & 24 & 0 & 30 & 0.1 & 0 & & & \\
\hline $104 \mathrm{E}$ & 24 & 0 & 30 & 0.1 & 0 & & & \\
\hline $106 \mathrm{~A}$ & 48 & 0 & 30 & 0.1 & 0 & \multirow{5}{*}{0} & \multirow{5}{*}{0} & \multirow{5}{*}{0} \\
\hline 106B & 48 & 0 & 30 & 0.1 & 0 & & & \\
\hline $106 \mathrm{C}$ & 48 & 0 & 30 & 0.1 & 0 & & & \\
\hline $106 \mathrm{D}$ & 48 & 0 & 30 & 0.1 & 0 & & & \\
\hline $106 \mathrm{E}$ & 48 & 0 & 30 & 0.1 & 0 & & & \\
\hline $108 \mathrm{~A}$ & 72 & 0 & 30 & 0.1 & 0 & \multirow{5}{*}{0} & \multirow{5}{*}{0} & \multirow{5}{*}{0} \\
\hline 108B & 72 & 0 & 30 & 0.1 & 0 & & & \\
\hline $108 \mathrm{C}$ & 72 & 0 & 30 & 0.1 & 0 & & & \\
\hline 108D & 72 & 0 & 30 & 0.1 & 0 & & & \\
\hline $108 \mathrm{E}$ & 72 & 0 & 30 & 0.1 & 0 & & & \\
\hline $110 \mathrm{~A}$ & 96 & 0 & 30 & 0.1 & 0 & \multirow{5}{*}{0} & \multirow{5}{*}{0} & \multirow{5}{*}{0} \\
\hline 110B & 96 & 0 & 30 & 0.1 & 0 & & & \\
\hline $110 \mathrm{C}$ & 96 & 0 & 30 & 0.1 & 0 & & & \\
\hline 110D & 96 & 0 & 30 & 0.1 & 0 & & & \\
\hline $110 \mathrm{E}$ & 96 & 0 & 30 & 0.1 & 0 & & & \\
\hline
\end{tabular}

1. The dilution correction was a value used to correct for the volume of PBS in which the coupons were placed

2. The agar volume was a value used to correct for the volume of the PBS solution plated onto the petri dish

3. Viable spores was the number of spores after corrected for the dilution and agar volume corrections 
Table A3 - 7 - Test condition 3: 180 deg F, 70\% RH

\begin{tabular}{|c|c|c|c|c|c|c|c|c|}
\hline $\begin{array}{l}\text { Sample } \\
\text { Number }\end{array}$ & Time & $\begin{array}{l}\text { Spores } \\
\text { counted }\end{array}$ & $\begin{array}{c}\text { Dilution } \\
\text { Correction }^{1}\end{array}$ & $\begin{array}{c}\text { Agar } \\
\text { Volume }^{2}\end{array}$ & $\begin{array}{c}\text { Viable } \\
\text { spores }^{3}\end{array}$ & $\begin{array}{c}\text { Mean of } \\
\text { viable } \\
\text { spores } \\
\end{array}$ & $\begin{array}{l}\text { SD of } \\
\text { viable } \\
\text { spores } \\
\end{array}$ & $\begin{array}{l}\text { SE of } \\
\text { viable } \\
\text { spores } \\
\end{array}$ \\
\hline $151 \mathrm{~A}$ & 0 & 134 & 30 & 0.1 & 40,200 & \multirow{5}{*}{44,640} & \multirow{5}{*}{23,025} & \multirow{5}{*}{10,298} \\
\hline 151B & 0 & 75 & 30 & 0.1 & 22,500 & & & \\
\hline $151 \mathrm{C}$ & 0 & 78 & 30 & 0.1 & 23,400 & & & \\
\hline $151 \mathrm{D}$ & 0 & 219 & 30 & 0.1 & 65,700 & & & \\
\hline $151 \mathrm{E}$ & 0 & 238 & 30 & 0.1 & 71,400 & & & \\
\hline $153 \mathrm{~A}$ & 24 & 30 & 30 & 0.1 & 9,000 & \multirow{5}{*}{8,760} & \multirow{5}{*}{3,791} & \multirow{5}{*}{1,696} \\
\hline 153B & 24 & 9 & 30 & 0.1 & 2,700 & & & \\
\hline $153 \mathrm{C}$ & 24 & 31 & 30 & 0.1 & 9,300 & & & \\
\hline $153 \mathrm{D}$ & 24 & 32 & 30 & 0.1 & 9,600 & & & \\
\hline $153 \mathrm{E}$ & 24 & 44 & 30 & 0.1 & 13,200 & & & \\
\hline $155 \mathrm{~A}$ & 48 & 34 & 30 & 0.1 & 10,200 & \multirow{5}{*}{17,520} & \multirow{5}{*}{8,650} & \multirow{5}{*}{3,868} \\
\hline 155B & 48 & 22 & 30 & 0.1 & 6,600 & & & \\
\hline $155 \mathrm{C}$ & 48 & 68 & 30 & 0.1 & 20,400 & & & \\
\hline $155 \mathrm{D}$ & 48 & 83 & 30 & 0.1 & 24,900 & & & \\
\hline $155 \mathrm{E}$ & 48 & 85 & 30 & 0.1 & 25,500 & & & \\
\hline $157 \mathrm{~A}$ & 72 & 8 & 30 & 0.1 & 2,400 & \multirow{5}{*}{2,880} & \multirow{5}{*}{4,187} & \multirow{5}{*}{1,873} \\
\hline $157 \mathrm{~B}$ & 72 & 4 & 30 & 0.1 & 1,200 & & & \\
\hline $157 \mathrm{C}$ & 72 & 34 & 30 & 0.1 & 10,200 & & & \\
\hline 157D & 72 & 2 & 30 & 0.1 & 600 & & & \\
\hline $157 \mathrm{E}$ & 72 & 0 & 30 & 0.1 & 0 & & & \\
\hline $159 \mathrm{~A}$ & 96 & 0 & 30 & 0.1 & 0 & \multirow{5}{*}{2,820} & \multirow{5}{*}{2,310} & \multirow{5}{*}{1,033} \\
\hline 159B & 96 & 6 & 30 & 0.1 & 1,800 & & & \\
\hline $159 \mathrm{C}$ & 96 & 11 & 30 & 0.1 & 3,300 & & & \\
\hline 159D & 96 & 21 & 30 & 0.1 & 6,300 & & & \\
\hline $159 \mathrm{E}$ & 96 & 9 & 30 & 0.1 & 2,700 & & & \\
\hline
\end{tabular}

1. The dilution correction was a value used to correct for the volume of PBS in which the coupons were placed

2. The agar volume was a value used to correct for the volume of the PBS solution plated onto the petri dish

3. Viable spores was the number of spores after corrected for the dilution and agar volume corrections 
Table A3 - 8- Test condition 5: 170 deg F, 80\% RH

\begin{tabular}{|c|c|c|c|c|c|c|c|c|}
\hline $\begin{array}{l}\text { Sample } \\
\text { Number }\end{array}$ & Time & $\begin{array}{l}\text { Spores } \\
\text { counted }\end{array}$ & $\begin{array}{c}\text { Dilution } \\
\text { Correction }^{1}\end{array}$ & $\begin{array}{c}\text { Agar } \\
\text { Volume }^{2}\end{array}$ & $\begin{array}{c}\text { Viable } \\
\text { spores }^{3}\end{array}$ & $\begin{array}{c}\text { Mean of } \\
\text { viable } \\
\text { spores } \\
\end{array}$ & $\begin{array}{c}\text { SD of } \\
\text { viable } \\
\text { spores }\end{array}$ & $\begin{array}{c}\text { SE of } \\
\text { viable } \\
\text { spores }\end{array}$ \\
\hline $301 \mathrm{~A}$ & 0 & 194 & 30 & 0.1 & 58,200 & \multirow{5}{*}{52,560} & \multirow{5}{*}{16,624} & \multirow{5}{*}{7,435} \\
\hline 301B & 0 & 220 & 30 & 0.1 & 66,000 & & & \\
\hline $301 \mathrm{C}$ & 0 & 141 & 30 & 0.1 & 42,300 & & & \\
\hline $301 \mathrm{D}$ & 0 & 96 & 30 & 0.1 & 28,800 & & & \\
\hline $301 \mathrm{E}$ & 0 & 225 & 30 & 0.1 & 67,500 & & & \\
\hline $303 \mathrm{~A}$ & 24 & 0 & 30 & 0.1 & 0 & \multirow{5}{*}{7,500} & \multirow{5}{*}{9,614} & \multirow{5}{*}{4,300} \\
\hline $303 \mathrm{~B}$ & 24 & 0 & 30 & 0.1 & 0 & & & \\
\hline $303 \mathrm{C}$ & 24 & 58 & 30 & 0.1 & 17,400 & & & \\
\hline $303 \mathrm{D}$ & 24 & 62 & 30 & 0.1 & 18,600 & & & \\
\hline $303 \mathrm{E}$ & 24 & 5 & 30 & 0.1 & 1,500 & & & \\
\hline $305 \mathrm{~A}$ & 48 & 1 & 30 & 0.1 & 300 & \multirow{5}{*}{1,200} & \multirow{5}{*}{1,544} & \multirow{5}{*}{691} \\
\hline $305 \mathrm{~B}$ & 48 & 0 & 30 & 0.1 & 0 & & & \\
\hline $305 \mathrm{C}$ & 48 & 0 & 30 & 0.1 & 0 & & & \\
\hline $305 \mathrm{D}$ & 48 & 8 & 30 & 0.1 & 2,400 & & & \\
\hline $305 \mathrm{E}$ & 48 & 11 & 30 & 0.1 & 3,300 & & & \\
\hline $307 \mathrm{~A}$ & 72 & 1 & 30 & 0.1 & 300 & \multirow{5}{*}{120} & \multirow{5}{*}{164} & \multirow{5}{*}{73} \\
\hline 307B & 72 & 0 & 30 & 0.1 & 0 & & & \\
\hline $307 \mathrm{C}$ & 72 & 1 & 30 & 0.1 & 300 & & & \\
\hline $307 \mathrm{D}$ & 72 & 0 & 30 & 0.1 & 0 & & & \\
\hline $307 \mathrm{E}$ & 72 & 0 & 30 & 0.1 & 0 & & & \\
\hline $309 \mathrm{~A}$ & 96 & 0 & 30 & 0.1 & 0 & \multirow{5}{*}{0} & \multirow{5}{*}{0} & \multirow{5}{*}{0} \\
\hline 309B & 96 & 0 & 30 & 0.1 & 0 & & & \\
\hline $309 \mathrm{C}$ & 96 & 0 & 30 & 0.1 & 0 & & & \\
\hline $309 \mathrm{D}$ & 96 & 0 & 30 & 0.1 & 0 & & & \\
\hline $309 \mathrm{E}$ & 96 & 0 & 30 & 0.1 & 0 & & & \\
\hline $311 \mathrm{~A}$ & 120 & 0 & 30 & 0.1 & 0 & \multirow{5}{*}{0} & \multirow{5}{*}{0} & \multirow{5}{*}{0} \\
\hline $311 \mathrm{~B}$ & 120 & 0 & 30 & 0.1 & 0 & & & \\
\hline $311 \mathrm{C}$ & 120 & 0 & 30 & 0.1 & 0 & & & \\
\hline $311 \mathrm{D}$ & 120 & 0 & 30 & 0.1 & 0 & & & \\
\hline $311 \mathrm{E}$ & 120 & 0 & 30 & 0.1 & 0 & & & \\
\hline
\end{tabular}

1. The dilution correction was a value used to correct for the volume of PBS in which the coupons were placed

2. The agar volume was a value used to correct for the volume of the PBS solution plated onto the petri dish

3. Viable spores was the number of spores after corrected for the dilution and agar volume corrections 
Table A3 - 9 - Test condition 7: 160 deg F, 90\% RH

\begin{tabular}{|c|c|c|c|c|c|c|c|c|}
\hline $\begin{array}{l}\text { Sample } \\
\text { Number }\end{array}$ & Time & $\begin{array}{l}\text { Spores } \\
\text { counted }\end{array}$ & $\begin{array}{c}\text { Dilution } \\
\text { Correction }^{1}\end{array}$ & $\begin{array}{c}\text { Agar } \\
\text { Volume }^{2}\end{array}$ & $\begin{array}{c}\text { Viable } \\
\text { spores }^{3}\end{array}$ & $\begin{array}{c}\text { Mean of } \\
\text { viable } \\
\text { spores } \\
\end{array}$ & $\begin{array}{c}\text { SD of } \\
\text { viable } \\
\text { spores }\end{array}$ & $\begin{array}{l}\text { SE of } \\
\text { viable } \\
\text { spores }\end{array}$ \\
\hline $201 \mathrm{~A}$ & 0 & 166 & 30 & 0.1 & 49,800 & \multirow{5}{*}{48,540} & \multirow{5}{*}{12,509} & \multirow{5}{*}{5,595} \\
\hline 201B & 0 & 187 & 30 & 0.1 & 56,100 & & & \\
\hline $201 \mathrm{C}$ & 0 & 159 & 30 & 0.1 & 47,700 & & & \\
\hline $201 \mathrm{D}$ & 0 & 203 & 30 & 0.1 & 60,900 & & & \\
\hline $201 E$ & 0 & 94 & 30 & 0.1 & 28,200 & & & \\
\hline $203 \mathrm{~A}$ & 24 & 159 & 30 & 0.1 & 47,700 & \multirow{5}{*}{43,200} & \multirow{5}{*}{8,882} & \multirow{5}{*}{3,972} \\
\hline 203B & 24 & 105 & 30 & 0.1 & 31,500 & & & \\
\hline $203 C$ & 24 & 140 & 30 & 0.1 & 42,000 & & & \\
\hline 203D & 24 & 132 & 30 & 0.1 & 39,600 & & & \\
\hline $203 E$ & 24 & 184 & 30 & 0.1 & 55,200 & & & \\
\hline $205 \mathrm{~A}$ & 48 & 130 & 30 & 0.1 & 39,000 & \multirow{5}{*}{15,300} & \multirow{5}{*}{15,945} & \multirow{5}{*}{7,131} \\
\hline $205 B$ & 48 & 82 & 30 & 0.1 & 24,600 & & & \\
\hline $205 C$ & 48 & 19 & 30 & 0.1 & 5,700 & & & \\
\hline $205 \mathrm{D}$ & 48 & 16 & 30 & 0.1 & 4,800 & & & \\
\hline $205 \mathrm{E}$ & 48 & 8 & 30 & 0.1 & 2,400 & & & \\
\hline $207 \mathrm{~A}$ & 72 & 66 & 30 & 0.1 & 19,800 & \multirow{5}{*}{21,300} & \multirow{5}{*}{12,956} & \multirow{5}{*}{5,794} \\
\hline 207B & 72 & 0 & 30 & 0.1 & 0 & & & \\
\hline $207 C$ & 72 & 114 & 30 & 0.1 & 34,200 & & & \\
\hline $207 D$ & 72 & 87 & 30 & 0.1 & 26,100 & & & \\
\hline $207 E$ & 72 & 88 & 30 & 0.1 & 26,400 & & & \\
\hline $209 \mathrm{~A}$ & 96 & 76 & 30 & 0.1 & 22,800 & \multirow{5}{*}{15,480} & \multirow{5}{*}{9,180} & \multirow{5}{*}{4,105} \\
\hline 209B & 96 & 67 & 30 & 0.1 & 20,100 & & & \\
\hline $209 C$ & 96 & 3 & 30 & 0.1 & 900 & & & \\
\hline 209D & 96 & 40 & 30 & 0.1 & 12,000 & & & \\
\hline $209 \mathrm{E}$ & 96 & 72 & 30 & 0.1 & 21,600 & & & \\
\hline $211 \mathrm{~A}$ & 120 & 25 & 30 & 0.1 & 7,500 & \multirow{5}{*}{6,840} & \multirow{5}{*}{7,245} & \multirow{5}{*}{3,240} \\
\hline 211B & 120 & 1 & 30 & 0.1 & 300 & & & \\
\hline $211 \mathrm{C}$ & 120 & 24 & 30 & 0.1 & 7,200 & & & \\
\hline 211D & 120 & 3 & 30 & 0.1 & 900 & & & \\
\hline $211 \mathrm{E}$ & 120 & 61 & 30 & 0.1 & 18,300 & & & \\
\hline
\end{tabular}

1. The dilution correction was a value used to correct for the volume of PBS in which the coupons were placed

2. The agar volume was a value used to correct for the volume of the PBS solution plated onto the petri dish

3. Viable spores was the number of spores after corrected for the dilution and agar volume corrections 
Table A3 - 10 - Test condition 9: $160 \operatorname{deg}$ F, 70\% RH

\begin{tabular}{|c|c|c|c|c|c|c|c|c|}
\hline $\begin{array}{l}\text { Sample } \\
\text { Number }\end{array}$ & Time & $\begin{array}{l}\text { Spores } \\
\text { counted }\end{array}$ & $\begin{array}{c}\text { Dilution } \\
\text { Correction }^{1}\end{array}$ & $\begin{array}{c}\text { Agar } \\
\text { Volume }^{2}\end{array}$ & $\begin{array}{c}\text { Viable } \\
\text { spores }^{3}\end{array}$ & $\begin{array}{c}\text { Mean of } \\
\text { viable } \\
\text { spores }\end{array}$ & $\begin{array}{l}\text { SD of } \\
\text { viable } \\
\text { spores }\end{array}$ & $\begin{array}{l}\text { SE of } \\
\text { viable } \\
\text { spores }\end{array}$ \\
\hline $401 \mathrm{~A}$ & 0 & 100 & 30 & 0.1 & 30,000 & \multirow{5}{*}{39,780} & \multirow{5}{*}{15,149} & \multirow{5}{*}{6,775} \\
\hline $401 \mathrm{~B}$ & 0 & 98 & 30 & 0.1 & 29,400 & & & \\
\hline $401 \mathrm{C}$ & 0 & 108 & 30 & 0.1 & 32,400 & & & \\
\hline 401D & 0 & 139 & 30 & 0.1 & 41,700 & & & \\
\hline $401 \mathrm{E}$ & 0 & 218 & 30 & 0.1 & 65,400 & & & \\
\hline $403 \mathrm{~A}$ & 24 & 80 & 30 & 0.1 & 24,000 & \multirow{5}{*}{26,760} & \multirow{5}{*}{14,782} & \multirow{5}{*}{6,611} \\
\hline 403B & 24 & 7 & 30 & 0.1 & 2,100 & & & \\
\hline $403 \mathrm{C}$ & 24 & 113 & 30 & 0.1 & 33,900 & & & \\
\hline $403 \mathrm{D}$ & 24 & 120 & 30 & 0.1 & 36,000 & & & \\
\hline $403 \mathrm{E}$ & 24 & 126 & 30 & 0.1 & 37,800 & & & \\
\hline $405 \mathrm{~A}$ & 48 & 59 & 30 & 0.1 & 17,700 & \multirow{5}{*}{31,380} & \multirow{5}{*}{22,272} & \multirow{5}{*}{9,961} \\
\hline $405 \mathrm{~B}$ & 48 & 56 & 30 & 0.1 & 16,800 & & & \\
\hline $405 \mathrm{C}$ & 48 & 76 & 30 & 0.1 & 22,800 & & & \\
\hline $405 \mathrm{D}$ & 48 & 98 & 30 & 0.1 & 29,400 & & & \\
\hline $405 \mathrm{E}$ & 48 & 234 & 30 & 0.1 & 70,200 & & & \\
\hline $407 \mathrm{~A}$ & 72 & 31 & 30 & 0.1 & 9,300 & \multirow{5}{*}{14,820} & \multirow{5}{*}{13,781} & \multirow{5}{*}{6,163} \\
\hline 407B & 72 & 46 & 30 & 0.1 & 13,800 & & & \\
\hline $407 \mathrm{C}$ & 72 & 41 & 30 & 0.1 & 12,300 & & & \\
\hline 407D & 72 & 3 & 30 & 0.1 & 900 & & & \\
\hline $407 \mathrm{E}$ & 72 & 126 & 30 & 0.1 & 37,800 & & & \\
\hline $409 \mathrm{~A}$ & 96 & 6 & 30 & 0.1 & 1,800 & \multirow{5}{*}{5,820} & \multirow{5}{*}{2,452} & \multirow{5}{*}{1,097} \\
\hline $409 \mathrm{~B}$ & 96 & 22 & 30 & 0.1 & 6,600 & & & \\
\hline $409 \mathrm{C}$ & 96 & 27 & 30 & 0.1 & 8,100 & & & \\
\hline 409D & 96 & 24 & 30 & 0.1 & 7,200 & & & \\
\hline $409 \mathrm{E}$ & 96 & 18 & 30 & 0.1 & 5,400 & & & \\
\hline $411 \mathrm{~A}$ & 120 & 13 & 30 & 0.1 & 3,900 & \multirow{5}{*}{3,480} & \multirow{5}{*}{3,464} & \multirow{5}{*}{1,549} \\
\hline 411B & 120 & 11 & 30 & 0.1 & 3,300 & & & \\
\hline $411 \mathrm{C}$ & 120 & 0 & 30 & 0.1 & 0 & & & \\
\hline 411D & 120 & 30 & 30 & 0.1 & 9,000 & & & \\
\hline $411 \mathrm{E}$ & 120 & 4 & 30 & 0.1 & 1,200 & & & \\
\hline
\end{tabular}

1. The dilution correction was a value used to correct for the volume of PBS in which the coupons were placed

2. The agar volume was a value used to correct for the volume of the PBS solution plated onto the petri dish

3. Viable spores was the number of spores after corrected for the dilution and agar volume corrections 
Table A3 - 11 - Test condition 1: 180 deg F, 90\% RH, samples

\begin{tabular}{|c|c|c|c|c|c|c|c|c|c|}
\hline $\begin{array}{l}\text { Sample } \\
\text { Number }\end{array}$ & Time & Type & $\begin{array}{l}\text { Spores } \\
\text { counted }\end{array}$ & $\begin{array}{c}\text { Dilution } \\
\text { Correction }^{1}\end{array}$ & $\begin{array}{c}\text { Agar } \\
\text { Volume }^{2}\end{array}$ & $\begin{array}{c}\text { Viable } \\
\text { spores }^{3}\end{array}$ & $\begin{array}{c}\text { Mean } \\
\text { of } \\
\text { viable } \\
\text { spores }\end{array}$ & $\begin{array}{c}\text { SD of } \\
\text { viable } \\
\text { spores }\end{array}$ & $\begin{array}{l}\text { SE of } \\
\text { viable } \\
\text { spores }\end{array}$ \\
\hline $111 \mathrm{~A}$ & 0 & Sample & 2 & 30 & 0.1 & 600 & \multirow{5}{*}{3,060} & \multirow{5}{*}{1,866} & \multirow{5}{*}{835} \\
\hline 111B & 0 & Sample & 11 & 30 & 0.1 & 3,300 & & & \\
\hline $111 \mathrm{C}$ & 0 & Sample & 6 & 30 & 0.1 & 1,800 & & & \\
\hline 111D & 0 & Sample & 17 & 30 & 0.1 & 5,100 & & & \\
\hline $111 \mathrm{E}$ & 0 & Sample & 15 & 30 & 0.1 & 4,500 & & & \\
\hline $113 \mathrm{~A}$ & 24 & Sample & 0 & 30 & 0.1 & 0 & \multirow{5}{*}{0} & \multirow{5}{*}{0} & \multirow{5}{*}{0} \\
\hline 113B & 24 & Sample & 0 & 30 & 0.1 & 0 & & & \\
\hline $113 \mathrm{C}$ & 24 & Sample & 0 & 30 & 0.1 & 0 & & & \\
\hline 113D & 24 & Sample & 0 & 30 & 0.1 & 0 & & & \\
\hline $113 \mathrm{E}$ & 24 & Sample & 0 & 30 & 0.1 & 0 & & & \\
\hline $116 \mathrm{~A}$ & 48 & Sample & 0 & 30 & 0.1 & 0 & \multirow{5}{*}{0} & \multirow{5}{*}{0} & \multirow{5}{*}{0} \\
\hline 116B & 48 & Sample & 0 & 30 & 0.1 & 0 & & & \\
\hline $116 \mathrm{C}$ & 48 & Sample & 0 & 30 & 0.1 & 0 & & & \\
\hline $116 \mathrm{D}$ & 48 & Sample & 0 & 30 & 0.1 & 0 & & & \\
\hline $116 \mathrm{E}$ & 48 & Sample & 0 & 30 & 0.1 & 0 & & & \\
\hline $118 \mathrm{~A}$ & 72 & Sample & 0 & 30 & 0.1 & 0 & \multirow{5}{*}{0} & \multirow{5}{*}{0} & \multirow{5}{*}{0} \\
\hline 118B & 72 & Sample & 0 & 30 & 0.1 & 0 & & & \\
\hline $118 \mathrm{C}$ & 72 & Sample & 0 & 30 & 0.1 & 0 & & & \\
\hline $118 \mathrm{D}$ & 72 & Sample & 0 & 30 & 0.1 & 0 & & & \\
\hline $118 \mathrm{E}$ & 72 & Sample & 0 & 30 & 0.1 & 0 & & & \\
\hline $120 \mathrm{~A}$ & 96 & Sample & 0 & 30 & 0.1 & 0 & \multirow{5}{*}{0} & \multirow{5}{*}{0} & \multirow{5}{*}{0} \\
\hline $120 \mathrm{~B}$ & 96 & Sample & 0 & 30 & 0.1 & 0 & & & \\
\hline $120 \mathrm{C}$ & 96 & Sample & 0 & 30 & 0.1 & 0 & & & \\
\hline $120 \mathrm{D}$ & 96 & Sample & 0 & 30 & 0.1 & 0 & & & \\
\hline $120 \mathrm{E}$ & 96 & Sample & 0 & 30 & 0.1 & 0 & & & \\
\hline
\end{tabular}

1. The dilution correction was a value used to correct for the volume of PBS in which the coupons were placed

2. The agar volume was a value used to correct for the volume of the PBS solution plated onto the petri dish

3. Viable spores was the number of spores after corrected for the dilution and agar volume corrections 
Table A3 - 12 - Test condition 1: $180 \mathrm{deg}$ F, 90\% RH, controls

\begin{tabular}{|c|c|c|c|c|c|c|c|c|c|}
\hline $\begin{array}{l}\text { Sample } \\
\text { Number }\end{array}$ & Time & Type & $\begin{array}{l}\text { Spores } \\
\text { counted }\end{array}$ & $\begin{array}{c}\text { Dilution } \\
\text { Correction }^{1}\end{array}$ & $\begin{array}{c}\text { Agar } \\
\text { Volume }^{2}\end{array}$ & $\begin{array}{c}\text { Viable } \\
\text { spores }^{3}\end{array}$ & $\begin{array}{c}\text { Mean } \\
\text { of } \\
\text { viable } \\
\text { spores }\end{array}$ & $\begin{array}{c}\text { SD of } \\
\text { viable } \\
\text { spores }\end{array}$ & $\begin{array}{c}\text { SE of } \\
\text { viable } \\
\text { spores }\end{array}$ \\
\hline $113 \mathrm{~A}$ & 24 & Control & 15 & 30 & 0.1 & 4,500 & \multirow{5}{*}{3,480} & \multirow{5}{*}{1,869} & \multirow{5}{*}{836} \\
\hline 113B & 24 & Control & 8 & 30 & 0.1 & 2,400 & & & \\
\hline $113 \mathrm{C}$ & 24 & Control & 19 & 30 & 0.1 & 5,700 & & & \\
\hline $113 \mathrm{D}$ & 24 & Control & 3 & 30 & 0.1 & 900 & & & \\
\hline $113 \mathrm{E}$ & 24 & Control & 13 & 30 & 0.1 & 3,900 & & & \\
\hline $115 \mathrm{~A}$ & 48 & Control & 20 & 30 & 0.1 & 6,000 & \multirow{5}{*}{3,000} & \multirow{5}{*}{2,909} & \multirow{5}{*}{1,301} \\
\hline 115B & 48 & Control & 1 & 30 & 0.1 & 300 & & & \\
\hline $115 \mathrm{C}$ & 48 & Control & 21 & 30 & 0.1 & 6,300 & & & \\
\hline $115 \mathrm{D}$ & 48 & Control & 5 & 30 & 0.1 & 1,500 & & & \\
\hline $115 \mathrm{E}$ & 48 & Control & 3 & 30 & 0.1 & 900 & & & \\
\hline $117 \mathrm{~A}$ & 72 & Control & 268 & 30 & 0.1 & 80,400 & \multirow{5}{*}{20,100} & \multirow{5}{*}{34,007} & \multirow{5}{*}{15,209} \\
\hline 117B & 72 & Control & 1 & 30 & 0.1 & 300 & & & \\
\hline $117 \mathrm{C}$ & 72 & Control & 9 & 30 & 0.1 & 2,700 & & & \\
\hline 117D & 72 & Control & 41 & 30 & 0.1 & 12,300 & & & \\
\hline $117 \mathrm{E}$ & 72 & Control & 16 & 30 & 0.1 & 4,800 & & & \\
\hline $119 \mathrm{~A}$ & 96 & Control & 48 & 30 & 0.1 & 14,400 & \multirow{5}{*}{6,240} & \multirow{5}{*}{4,778} & \multirow{5}{*}{2,137} \\
\hline 119B & 96 & Control & 21 & 30 & 0.1 & 6,300 & & & \\
\hline $119 \mathrm{C}$ & 96 & Control & 12 & 30 & 0.1 & 3,600 & & & \\
\hline 119D & 96 & Control & 15 & 30 & 0.1 & 4,500 & & & \\
\hline $119 \mathrm{E}$ & 96 & Control & 8 & 30 & 0.1 & 2,400 & & & \\
\hline
\end{tabular}

1. The dilution correction was a value used to correct for the volume of PBS in which the coupons were placed

2. The agar volume was a value used to correct for the volume of the PBS solution plated onto the petri dish

3. Viable spores was the number of spores after corrected for the dilution and agar volume corrections 
Table A3 - 13 - Test condition 3: $180 \mathrm{deg}$ F, 70\% RH, samples

\begin{tabular}{|c|c|c|c|c|c|c|c|c|c|}
\hline $\begin{array}{l}\text { Sample } \\
\text { Number }\end{array}$ & Time & Type & $\begin{array}{l}\text { Spores } \\
\text { counted }\end{array}$ & $\begin{array}{c}\text { Dilution } \\
\text { Correction }^{1}\end{array}$ & $\begin{array}{c}\text { Agar } \\
\text { Volume }^{2}\end{array}$ & $\begin{array}{l}\text { Viable } \\
\text { spores }^{3}\end{array}$ & $\begin{array}{c}\text { Mean } \\
\text { of } \\
\text { viable } \\
\text { spores }\end{array}$ & $\begin{array}{l}\text { SD of } \\
\text { viable } \\
\text { spores }\end{array}$ & $\begin{array}{l}\text { SE of } \\
\text { viable } \\
\text { spores }\end{array}$ \\
\hline $161 \mathrm{~A}$ & 0 & Sample & 2 & 30 & 0.1 & 600 & \multirow{5}{*}{3,120} & \multirow{5}{*}{1,951} & \multirow{5}{*}{873} \\
\hline $161 \mathrm{~B}$ & 0 & Sample & 7 & 30 & 0.1 & 2,100 & & & \\
\hline $161 \mathrm{C}$ & 0 & Sample & 19 & 30 & 0.1 & 5,700 & & & \\
\hline 161D & 0 & Sample & 14 & 30 & 0.1 & 4,200 & & & \\
\hline $161 \mathrm{E}$ & 0 & Sample & 10 & 30 & 0.1 & 3,000 & & & \\
\hline $164 \mathrm{~A}$ & 24 & Sample & 1 & 30 & 0.1 & 300 & \multirow{5}{*}{1,380} & \multirow{5}{*}{915} & \multirow{5}{*}{409} \\
\hline 164B & 24 & Sample & 5 & 30 & 0.1 & 1,500 & & & \\
\hline $164 \mathrm{C}$ & 24 & Sample & 8 & 30 & 0.1 & 2,400 & & & \\
\hline 164D & 24 & Sample & 2 & 30 & 0.1 & 600 & & & \\
\hline $164 \mathrm{E}$ & 24 & Sample & 7 & 30 & 0.1 & 2,100 & & & \\
\hline $166 \mathrm{~A}$ & 48 & Sample & 1 & 30 & 0.1 & 300 & \multirow{5}{*}{2,400} & \multirow{5}{*}{3,374} & \multirow{5}{*}{1,509} \\
\hline 166B & 48 & Sample & 4 & 30 & 0.1 & 1,200 & & & \\
\hline $166 \mathrm{C}$ & 48 & Sample & 4 & 30 & 0.1 & 1,200 & & & \\
\hline 166D & 48 & Sample & 28 & 30 & 0.1 & 8,400 & & & \\
\hline $166 \mathrm{E}$ & 48 & Sample & 3 & 30 & 0.1 & 900 & & & \\
\hline $168 \mathrm{~A}$ & 72 & Sample & 0 & 30 & 0.1 & 0 & \multirow{5}{*}{60} & \multirow{5}{*}{134} & \multirow{5}{*}{60} \\
\hline 168B & 72 & Sample & 0 & 30 & 0.1 & 0 & & & \\
\hline $168 \mathrm{C}$ & 72 & Sample & 0 & 30 & 0.1 & 0 & & & \\
\hline 168D & 72 & Sample & 0 & 30 & 0.1 & 0 & & & \\
\hline $168 \mathrm{E}$ & 72 & Sample & 1 & 30 & 0.1 & 300 & & & \\
\hline $170 \mathrm{~A}$ & 96 & Sample & 0 & 30 & 0.1 & 0 & \multirow{5}{*}{0} & \multirow{5}{*}{0} & \multirow{5}{*}{0} \\
\hline 170B & 96 & Sample & 0 & 30 & 0.1 & 0 & & & \\
\hline $170 \mathrm{C}$ & 96 & Sample & 0 & 30 & 0.1 & 0 & & & \\
\hline 170D & 96 & Sample & 0 & 30 & 0.1 & 0 & & & \\
\hline $170 \mathrm{E}$ & 96 & Sample & 0 & 30 & 0.1 & 0 & & & \\
\hline
\end{tabular}

1. The dilution correction was a value used to correct for the volume of PBS in which the coupons were placed

2. The agar volume was a value used to correct for the volume of the PBS solution plated onto the petri dish

3. Viable spores was the number of spores after corrected for the dilution and agar volume corrections 
Table A3 - 14 - Test condition 3: $180 \mathrm{deg}$ F, 70\% RH, controls

\begin{tabular}{|c|c|c|c|c|c|c|c|c|c|}
\hline $\begin{array}{l}\text { Sample } \\
\text { Number }\end{array}$ & Time & Type & $\begin{array}{l}\text { Spores } \\
\text { counted }\end{array}$ & $\begin{array}{c}\text { Dilution } \\
\text { Correction }^{1}\end{array}$ & $\begin{array}{c}\text { Agar } \\
\text { Volume }^{2}\end{array}$ & $\begin{array}{c}\text { Viable } \\
\text { spores }^{3}\end{array}$ & $\begin{array}{c}\text { Mean } \\
\text { of } \\
\text { viable } \\
\text { spores }\end{array}$ & $\begin{array}{l}\text { SD of } \\
\text { viable } \\
\text { spores }\end{array}$ & $\begin{array}{c}\text { SE of } \\
\text { viable } \\
\text { spores }\end{array}$ \\
\hline $163 \mathrm{~A}$ & 24 & Control & 47 & 30 & 0.1 & 14,100 & \multirow{5}{*}{8,580} & \multirow{5}{*}{5,679} & \multirow{5}{*}{2,540} \\
\hline 163B & 24 & Control & 18 & 30 & 0.1 & 5,400 & & & \\
\hline $163 \mathrm{C}$ & 24 & Control & 50 & 30 & 0.1 & 15,000 & & & \\
\hline 163D & 24 & Control & 7 & 30 & 0.1 & 2,100 & & & \\
\hline $163 \mathrm{E}$ & 24 & Control & 21 & 30 & 0.1 & 6,300 & & & \\
\hline $165 \mathrm{~A}$ & 48 & Control & 1 & 30 & 0.1 & 300 & \multirow{5}{*}{4,740} & \multirow{5}{*}{3,908} & \multirow{5}{*}{1,748} \\
\hline $165 \mathrm{~B}$ & 48 & Control & 21 & 30 & 0.1 & 6,300 & & & \\
\hline $165 \mathrm{C}$ & 48 & Control & 35 & 30 & 0.1 & 10,500 & & & \\
\hline $165 \mathrm{D}$ & 48 & Control & 14 & 30 & 0.1 & 4,200 & & & \\
\hline $165 \mathrm{E}$ & 48 & Control & 8 & 30 & 0.1 & 2,400 & & & \\
\hline $167 \mathrm{~A}$ & 72 & Control & 52 & 30 & 0.1 & 15,600 & \multirow{5}{*}{8,220} & \multirow{5}{*}{5,931} & \multirow{5}{*}{2,652} \\
\hline 167B & 72 & Control & 44 & 30 & 0.1 & 13,200 & & & \\
\hline $167 \mathrm{C}$ & 72 & Control & 12 & 30 & 0.1 & 3,600 & & & \\
\hline $167 \mathrm{D}$ & 72 & Control & 7 & 30 & 0.1 & 2,100 & & & \\
\hline $167 \mathrm{E}$ & 72 & Control & 22 & 30 & 0.1 & 6,600 & & & \\
\hline $169 \mathrm{~A}$ & 96 & Control & 27 & 30 & 0.1 & 8,100 & \multirow{5}{*}{12,000} & \multirow{5}{*}{3,300} & \multirow{5}{*}{1,476} \\
\hline 169B & 96 & Control & 32 & 30 & 0.1 & 9,600 & & & \\
\hline $169 \mathrm{C}$ & 96 & Control & 41 & 30 & 0.1 & 12,300 & & & \\
\hline 169D & 96 & Control & 45 & 30 & 0.1 & 13,500 & & & \\
\hline $169 \mathrm{E}$ & 96 & Control & 55 & 30 & 0.1 & 16,500 & & & \\
\hline
\end{tabular}

1. The dilution correction was a value used to correct for the volume of PBS in which the coupons were placed

2. The agar volume was a value used to correct for the volume of the PBS solution plated onto the petri dish

3. Viable spores was the number of spores after corrected for the dilution and agar volume corrections 
Table A3 - 15 - Test condition 5: $170 \mathrm{deg}$ F, 80\% RH, samples

\begin{tabular}{|c|c|c|c|c|c|c|c|c|c|}
\hline $\begin{array}{l}\text { Sample } \\
\text { Number }\end{array}$ & Time & Type & $\begin{array}{l}\text { Spores } \\
\text { counted }\end{array}$ & $\begin{array}{c}\text { Dilution } \\
\text { Correction }^{1}\end{array}$ & $\begin{array}{c}\text { Agar } \\
\text { Volume }^{2}\end{array}$ & $\begin{array}{c}\text { Viable } \\
\text { spores }^{3}\end{array}$ & $\begin{array}{c}\text { Mean } \\
\text { of } \\
\text { viable } \\
\text { spores }\end{array}$ & $\begin{array}{l}\text { SD of } \\
\text { viable } \\
\text { spores }\end{array}$ & $\begin{array}{c}\text { SE of } \\
\text { viable } \\
\text { spores }\end{array}$ \\
\hline $621 \mathrm{~A}$ & 0 & Sample & 42 & 30 & 0.1 & 12,600 & \multirow{5}{*}{14,520} & \multirow{5}{*}{3,892} & \multirow{5}{*}{1,741} \\
\hline $621 B$ & 0 & Sample & 40 & 30 & 0.1 & 12,000 & & & \\
\hline $621 \mathrm{C}$ & 0 & Sample & 51 & 30 & 0.1 & 15,300 & & & \\
\hline $621 \mathrm{D}$ & 0 & Sample & 70 & 30 & 0.1 & 21,000 & & & \\
\hline $621 \mathrm{E}$ & 0 & Sample & 39 & 30 & 0.1 & 11,700 & & & \\
\hline $622 \mathrm{~A}$ & 24 & Sample & 0 & 30 & 0.1 & 0 & \multirow{5}{*}{120} & \multirow{5}{*}{164} & \multirow{5}{*}{73} \\
\hline $622 B$ & 24 & Sample & 0 & 30 & 0.1 & 0 & & & \\
\hline $622 C$ & 24 & Sample & 1 & 30 & 0.1 & 300 & & & \\
\hline $622 \mathrm{D}$ & 24 & Sample & 1 & 30 & 0.1 & 300 & & & \\
\hline $622 \mathrm{E}$ & 24 & Sample & 0 & 30 & 0.1 & 0 & & & \\
\hline $624 \mathrm{~A}$ & 48 & Sample & 0 & 30 & 0.1 & 0 & \multirow{5}{*}{120} & \multirow{5}{*}{164} & \multirow{5}{*}{73} \\
\hline $624 B$ & 48 & Sample & 0 & 30 & 0.1 & 0 & & & \\
\hline $624 C$ & 48 & Sample & 1 & 30 & 0.1 & 300 & & & \\
\hline $624 \mathrm{D}$ & 48 & Sample & 0 & 30 & 0.1 & 0 & & & \\
\hline $624 \mathrm{E}$ & 48 & Sample & 1 & 30 & 0.1 & 300 & & & \\
\hline $626 \mathrm{~A}$ & 72 & Sample & 0 & 30 & 0.1 & 0 & \multirow{5}{*}{0} & \multirow{5}{*}{0} & \multirow{5}{*}{0} \\
\hline $626 \mathrm{~B}$ & 72 & Sample & 0 & 30 & 0.1 & 0 & & & \\
\hline $626 \mathrm{C}$ & 72 & Sample & 0 & 30 & 0.1 & 0 & & & \\
\hline $626 \mathrm{D}$ & 72 & Sample & 0 & 30 & 0.1 & 0 & & & \\
\hline $626 \mathrm{E}$ & 72 & Sample & 0 & 30 & 0.1 & 0 & & & \\
\hline $628 \mathrm{~A}$ & 96 & Sample & 0 & 30 & 0.1 & 0 & \multirow{5}{*}{0} & \multirow{5}{*}{0} & \multirow{5}{*}{0} \\
\hline $628 B$ & 96 & Sample & 0 & 30 & 0.1 & 0 & & & \\
\hline $628 \mathrm{C}$ & 96 & Sample & 0 & 30 & 0.1 & 0 & & & \\
\hline $628 \mathrm{D}$ & 96 & Sample & 0 & 30 & 0.1 & 0 & & & \\
\hline $628 \mathrm{E}$ & 96 & Sample & 0 & 30 & 0.1 & 0 & & & \\
\hline $630 \mathrm{~A}$ & 120 & Sample & 0 & 30 & 0.1 & 0 & \multirow{5}{*}{0} & \multirow{5}{*}{0} & \multirow{5}{*}{0} \\
\hline $630 B$ & 120 & Sample & 0 & 30 & 0.1 & 0 & & & \\
\hline $630 \mathrm{C}$ & 120 & Sample & 0 & 30 & 0.1 & 0 & & & \\
\hline $630 \mathrm{D}$ & 120 & Sample & 0 & 30 & 0.1 & 0 & & & \\
\hline $630 \mathrm{E}$ & 120 & Sample & 0 & 30 & 0.1 & 0 & & & \\
\hline
\end{tabular}

1. The dilution correction was a value used to correct for the volume of PBS in which the coupons were placed

2. The agar volume was a value used to correct for the volume of the PBS solution plated onto the petri dish

3. Viable spores was the number of spores after corrected for the dilution and agar volume corrections 
Table A3 - 16 - Test condition 5: 170 deg F, 80\% RH, controls

\begin{tabular}{|c|c|c|c|c|c|c|c|c|c|}
\hline $\begin{array}{l}\text { Sample } \\
\text { Number }\end{array}$ & Time & Type & $\begin{array}{l}\text { Spores } \\
\text { counted }\end{array}$ & $\begin{array}{c}\text { Dilution } \\
\text { Correction }^{1}\end{array}$ & $\begin{array}{c}\text { Agar } \\
\text { Volume }^{2}\end{array}$ & $\begin{array}{c}\text { Viable } \\
\text { spores }^{3}\end{array}$ & $\begin{array}{c}\text { Mean } \\
\text { of } \\
\text { viable } \\
\text { spores }\end{array}$ & $\begin{array}{l}\text { SD of } \\
\text { viable } \\
\text { spores }\end{array}$ & $\begin{array}{l}\text { SE of } \\
\text { viable } \\
\text { spores }\end{array}$ \\
\hline $623 \mathrm{~A}$ & 24 & Control & 53 & 30 & 0.1 & 15,900 & \multirow{5}{*}{12,960} & \multirow{5}{*}{1,960} & \multirow{5}{*}{877} \\
\hline $623 \mathrm{~B}$ & 24 & Control & 38 & 30 & 0.1 & 11,400 & & & \\
\hline $623 C$ & 24 & Control & 37 & 30 & 0.1 & 11,100 & & & \\
\hline $623 \mathrm{D}$ & 24 & Control & 42 & 30 & 0.1 & 12,600 & & & \\
\hline $623 \mathrm{E}$ & 24 & Control & 46 & 30 & 0.1 & 13,800 & & & \\
\hline $625 \mathrm{~A}$ & 48 & Control & 30 & 30 & 0.1 & 9,000 & \multirow{5}{*}{31,560} & \multirow{5}{*}{32,078} & \multirow{5}{*}{14,346} \\
\hline $625 B$ & 48 & Control & 26 & 30 & 0.1 & 7,800 & & & \\
\hline $625 \mathrm{C}$ & 48 & Control & 56 & 30 & 0.1 & 16,800 & & & \\
\hline $625 \mathrm{D}$ & 48 & Control & 134 & 30 & 0.1 & 40,200 & & & \\
\hline $625 \mathrm{E}$ & 48 & Control & 280 & 30 & 0.1 & 84,000 & & & \\
\hline $627 \mathrm{~A}$ & 72 & Control & 45 & 30 & 0.1 & 13,500 & \multirow{5}{*}{10,800} & \multirow{5}{*}{3,594} & \multirow{5}{*}{1,607} \\
\hline $627 \mathrm{~B}$ & 72 & Control & 41 & 30 & 0.1 & 12,300 & & & \\
\hline $627 \mathrm{C}$ & 72 & Control & 16 & 30 & 0.1 & 4,800 & & & \\
\hline $627 \mathrm{D}$ & 72 & Control & 34 & 30 & 0.1 & 10,200 & & & \\
\hline $627 \mathrm{E}$ & 72 & Control & 44 & 30 & 0.1 & 13,200 & & & \\
\hline $629 \mathrm{~A}$ & 96 & Control & 25 & 30 & 0.1 & 7,500 & \multirow{5}{*}{15,360} & \multirow{5}{*}{9,844} & \multirow{5}{*}{4,402} \\
\hline 629B & 96 & Control & 22 & 30 & 0.1 & 6,600 & & & \\
\hline $629 \mathrm{C}$ & 96 & Control & 100 & 30 & 0.1 & 30,000 & & & \\
\hline $629 \mathrm{D}$ & 96 & Control & 68 & 30 & 0.1 & 20,400 & & & \\
\hline $629 \mathrm{E}$ & 96 & Control & 41 & 30 & 0.1 & 12,300 & & & \\
\hline $631 \mathrm{~A}$ & 120 & Control & 26 & 30 & 0.1 & 7,800 & \multirow{5}{*}{9,960} & \multirow{5}{*}{2,849} & \multirow{5}{*}{1,274} \\
\hline $631 \mathrm{~B}$ & 120 & Control & 29 & 30 & 0.1 & 8,700 & & & \\
\hline $631 \mathrm{C}$ & 120 & Control & 35 & 30 & 0.1 & 10,500 & & & \\
\hline 631D & 120 & Control & 27 & 30 & 0.1 & 8,100 & & & \\
\hline $631 \mathrm{E}$ & 120 & Control & 49 & 30 & 0.1 & 14,700 & & & \\
\hline
\end{tabular}

1. The dilution correction was a value used to correct for the volume of PBS in which the coupons were placed

2. The agar volume was a value used to correct for the volume of the PBS solution plated onto the petri dish

3. Viable spores was the number of spores after corrected for the dilution and agar volume corrections 
Table A3 - 17 - Test condition 7: 160 deg F, 90\%, samples

\begin{tabular}{|c|c|c|c|c|c|c|c|c|c|}
\hline $\begin{array}{l}\text { Sample } \\
\text { Number }\end{array}$ & Time & Type & $\begin{array}{l}\text { Spores } \\
\text { counted }\end{array}$ & $\begin{array}{c}\text { Dilution } \\
\text { Correction }^{1}\end{array}$ & $\begin{array}{c}\text { Agar } \\
\text { Volume }^{2}\end{array}$ & $\begin{array}{c}\text { Viable } \\
\text { spores }^{3}\end{array}$ & $\begin{array}{c}\text { Mean } \\
\text { of } \\
\text { viable } \\
\text { spores }\end{array}$ & $\begin{array}{l}\text { SD of } \\
\text { viable } \\
\text { spores }\end{array}$ & $\begin{array}{c}\text { SE of } \\
\text { viable } \\
\text { spores }\end{array}$ \\
\hline $221 \mathrm{~A}$ & 0 & Sample & 62 & 30 & 0.1 & 18,600 & \multirow{5}{*}{14,820} & \multirow{5}{*}{4,770} & \multirow{5}{*}{2,133} \\
\hline 221B & 0 & Sample & 33 & 30 & 0.1 & 9,900 & & & \\
\hline $221 C$ & 0 & Sample & 38 & 30 & 0.1 & 11,400 & & & \\
\hline 221D & 0 & Sample & 70 & 30 & 0.1 & 21,000 & & & \\
\hline $221 \mathrm{E}$ & 0 & Sample & 44 & 30 & 0.1 & 13,200 & & & \\
\hline $222 \mathrm{~A}$ & 24 & Sample & 15 & 30 & 0.1 & 4,500 & \multirow{5}{*}{4,380} & \multirow{5}{*}{1,718} & \multirow{5}{*}{768} \\
\hline 222B & 24 & Sample & 10 & 30 & 0.1 & 3,000 & & & \\
\hline $222 C$ & 24 & Sample & 14 & 30 & 0.1 & 4,200 & & & \\
\hline 222D & 24 & Sample & 10 & 30 & 0.1 & 3,000 & & & \\
\hline $222 \mathrm{E}$ & 24 & Sample & 24 & 30 & 0.1 & 7,200 & & & \\
\hline $224 \mathrm{~A}$ & 48 & Sample & 10 & 30 & 0.1 & 3,000 & \multirow{5}{*}{2,400} & \multirow{5}{*}{2,554} & \multirow{5}{*}{1,142} \\
\hline 224B & 48 & Sample & 3 & 30 & 0.1 & 900 & & & \\
\hline $224 C$ & 48 & Sample & 22 & 30 & 0.1 & 6,600 & & & \\
\hline $224 \mathrm{D}$ & 48 & Sample & 4 & 30 & 0.1 & 1,200 & & & \\
\hline $224 \mathrm{E}$ & 48 & Sample & 1 & 30 & 0.1 & 300 & & & \\
\hline $226 \mathrm{~A}$ & 72 & Sample & 6 & 30 & 0.1 & 1,800 & \multirow{5}{*}{2,580} & \multirow{5}{*}{622} & \multirow{5}{*}{278} \\
\hline $226 B$ & 72 & Sample & 11 & 30 & 0.1 & 3,300 & & & \\
\hline $226 \mathrm{C}$ & 72 & Sample & 9 & 30 & 0.1 & 2,700 & & & \\
\hline $226 \mathrm{D}$ & 72 & Sample & 7 & 30 & 0.1 & 2,100 & & & \\
\hline $226 \mathrm{E}$ & 72 & Sample & 10 & 30 & 0.1 & 3,000 & & & \\
\hline $228 \mathrm{~A}$ & 96 & Sample & 0 & 30 & 0.1 & 0 & \multirow{5}{*}{900} & \multirow{5}{*}{1,273} & \multirow{5}{*}{569} \\
\hline 229B & 96 & Sample & 4 & 30 & 0.1 & 1,200 & & & \\
\hline $228 \mathrm{C}$ & 96 & Sample & 10 & 30 & 0.1 & 3,000 & & & \\
\hline $228 \mathrm{D}$ & 96 & Sample & 1 & 30 & 0.1 & 300 & & & \\
\hline $228 \mathrm{E}$ & 96 & Sample & 0 & 30 & 0.1 & 0 & & & \\
\hline $230 \mathrm{~A}$ & 120 & Sample & 0 & 30 & 0.1 & 0 & \multirow{5}{*}{0} & \multirow{5}{*}{0} & \multirow{5}{*}{0} \\
\hline $230 \mathrm{~B}$ & 120 & Sample & 0 & 30 & 0.1 & 0 & & & \\
\hline $230 \mathrm{C}$ & 120 & Sample & 0 & 30 & 0.1 & 0 & & & \\
\hline $230 \mathrm{D}$ & 120 & Sample & 0 & 30 & 0.1 & 0 & & & \\
\hline $230 \mathrm{E}$ & 120 & Sample & 0 & 30 & 0.1 & 0 & & & \\
\hline
\end{tabular}

1. The dilution correction was a value used to correct for the volume of PBS in which the coupons were placed

2. The agar volume was a value used to correct for the volume of the PBS solution plated onto the petri dish

3. Viable spores was the number of spores after corrected for the dilution and agar volume corrections 
Table A3 - 18 - Test condition 7: $160 \mathrm{deg}$ F, 90\% RH, controls

\begin{tabular}{|c|c|c|c|c|c|c|c|c|c|}
\hline $\begin{array}{l}\text { Sample } \\
\text { Number }\end{array}$ & Time & Type & $\begin{array}{l}\text { Spores } \\
\text { counted }\end{array}$ & $\begin{array}{c}\text { Dilution } \\
\text { Correction }^{1}\end{array}$ & $\begin{array}{c}\text { Agar } \\
\text { Volume }^{2}\end{array}$ & $\begin{array}{c}\text { Viable } \\
\text { spores }^{3}\end{array}$ & $\begin{array}{c}\text { Mean } \\
\text { of } \\
\text { viable } \\
\text { spores }\end{array}$ & $\begin{array}{l}\text { SD of } \\
\text { viable } \\
\text { spores }\end{array}$ & $\begin{array}{l}\text { SE of } \\
\text { viable } \\
\text { spores }\end{array}$ \\
\hline $223 \mathrm{~A}$ & 24 & Control & 54 & 30 & 0.1 & 16,200 & \multirow{5}{*}{11,640} & \multirow{5}{*}{4,654} & \multirow{5}{*}{2,082} \\
\hline 223B & 24 & Control & 35 & 30 & 0.1 & 10,500 & & & \\
\hline $223 \mathrm{C}$ & 24 & Control & 43 & 30 & 0.1 & 12,900 & & & \\
\hline 223D & 24 & Control & 48 & 30 & 0.1 & 14,400 & & & \\
\hline $223 E$ & 24 & Control & 14 & 30 & 0.1 & 4,200 & & & \\
\hline $225 \mathrm{~A}$ & 48 & Control & 8 & 30 & 0.1 & 2,400 & \multirow{5}{*}{3,900} & \multirow{5}{*}{2,068} & \multirow{5}{*}{925} \\
\hline $225 B$ & 48 & Control & 11 & 30 & 0.1 & 3,300 & & & \\
\hline $225 \mathrm{C}$ & 48 & Control & 9 & 30 & 0.1 & 2,700 & & & \\
\hline $225 \mathrm{D}$ & 48 & Control & 25 & 30 & 0.1 & 7,500 & & & \\
\hline $225 \mathrm{E}$ & 48 & Control & 12 & 30 & 0.1 & 3,600 & & & \\
\hline $227 \mathrm{~A}$ & 72 & Control & 36 & 30 & 0.1 & 10,800 & \multirow{5}{*}{11,400} & \multirow{5}{*}{5,354} & \multirow{5}{*}{2,394} \\
\hline 227B & 72 & Control & 25 & 30 & 0.1 & 7,500 & & & \\
\hline $227 C$ & 72 & Control & 22 & 30 & 0.1 & 6,600 & & & \\
\hline 227D & 72 & Control & 67 & 30 & 0.1 & 20,100 & & & \\
\hline $227 E$ & 72 & Control & 40 & 30 & 0.1 & 12,000 & & & \\
\hline $229 \mathrm{~A}$ & 96 & Control & 29 & 30 & 0.1 & 8,700 & \multirow{5}{*}{4,980} & \multirow{5}{*}{3,108} & \multirow{5}{*}{1,390} \\
\hline 229B & 96 & Control & 10 & 30 & 0.1 & 3,000 & & & \\
\hline $229 \mathrm{C}$ & 96 & Control & 4 & 30 & 0.1 & 1,200 & & & \\
\hline 229D & 96 & Control & 25 & 30 & 0.1 & 7,500 & & & \\
\hline $229 E$ & 96 & Control & 15 & 30 & 0.1 & 4,500 & & & \\
\hline $231 \mathrm{~A}$ & 120 & Control & 29 & 30 & 0.1 & 8,700 & \multirow{5}{*}{14,760} & \multirow{5}{*}{5,464} & \multirow{5}{*}{2,444} \\
\hline 231B & 120 & Control & 48 & 30 & 0.1 & 14,400 & & & \\
\hline $231 \mathrm{C}$ & 120 & Control & 36 & 30 & 0.1 & 10,800 & & & \\
\hline $231 \mathrm{D}$ & 120 & Control & 58 & 30 & 0.1 & 17,400 & & & \\
\hline $231 \mathrm{E}$ & 120 & Control & 75 & 30 & 0.1 & 22,500 & & & \\
\hline
\end{tabular}

1. The dilution correction was a value used to correct for the volume of PBS in which the coupons were placed

2. The agar volume was a value used to correct for the volume of the PBS solution plated onto the petri dish

3. Viable spores was the number of spores after corrected for the dilution and agar volume corrections 
Table A3 - 19 - Test condition 9: $160 \mathrm{deg}$ F, 70\% RH, samples

\begin{tabular}{|c|c|c|c|c|c|c|c|c|c|}
\hline $\begin{array}{l}\text { Sample } \\
\text { Number }\end{array}$ & Time & Type & $\begin{array}{l}\text { Spores } \\
\text { counted }\end{array}$ & $\begin{array}{c}\text { Dilution } \\
\text { Correction }^{1}\end{array}$ & $\begin{array}{c}\text { Agar } \\
\text { Volume }^{2}\end{array}$ & $\begin{array}{l}\text { Viable } \\
\text { spores }^{3}\end{array}$ & $\begin{array}{c}\text { Mean } \\
\text { of } \\
\text { viable } \\
\text { spores }\end{array}$ & $\begin{array}{l}\text { SD of } \\
\text { viable } \\
\text { spores }\end{array}$ & $\begin{array}{l}\text { SE of } \\
\text { viable } \\
\text { spores }\end{array}$ \\
\hline $421 \mathrm{~A}$ & 0 & Sample & 11 & 30 & 0.1 & 3,300 & \multirow{5}{*}{5,280} & \multirow{5}{*}{2,638} & \multirow{5}{*}{1,180} \\
\hline 421B & 0 & Sample & 32 & 30 & 0.1 & 9,600 & & & \\
\hline $421 \mathrm{C}$ & 0 & Sample & 20 & 30 & 0.1 & 6,000 & & & \\
\hline $421 \mathrm{D}$ & 0 & Sample & 12 & 30 & 0.1 & 3,600 & & & \\
\hline $421 \mathrm{E}$ & 0 & Sample & 13 & 30 & 0.1 & 3,900 & & & \\
\hline $422 \mathrm{~A}$ & 24 & Sample & 8 & 30 & 0.1 & 2,400 & \multirow{5}{*}{4,740} & \multirow{5}{*}{2,328} & \multirow{5}{*}{1,041} \\
\hline 422B & 24 & Sample & 8 & 30 & 0.1 & 2,400 & & & \\
\hline $422 C$ & 24 & Sample & 24 & 30 & 0.1 & 7,200 & & & \\
\hline 422D & 24 & Sample & 23 & 30 & 0.1 & 6,900 & & & \\
\hline $422 \mathrm{E}$ & 24 & Sample & 16 & 30 & 0.1 & 4,800 & & & \\
\hline $424 \mathrm{~A}$ & 48 & Sample & 22 & 30 & 0.1 & 6,600 & \multirow{5}{*}{2,760} & \multirow{5}{*}{2,219} & \multirow{5}{*}{992} \\
\hline 424B & 48 & Sample & 8 & 30 & 0.1 & 2,400 & & & \\
\hline $424 \mathrm{C}$ & 48 & Sample & 7 & 30 & 0.1 & 2,100 & & & \\
\hline $424 \mathrm{D}$ & 48 & Sample & 6 & 30 & 0.1 & 1,800 & & & \\
\hline $424 \mathrm{E}$ & 48 & Sample & 3 & 30 & 0.1 & 900 & & & \\
\hline $426 \mathrm{~A}$ & 72 & Sample & 0 & 30 & 0.1 & 0 & \multirow{5}{*}{900} & \multirow{5}{*}{995} & \multirow{5}{*}{445} \\
\hline 426B & 72 & Sample & 6 & 30 & 0.1 & 1,800 & & & \\
\hline $426 \mathrm{C}$ & 72 & Sample & 0 & 30 & 0.1 & 0 & & & \\
\hline $426 \mathrm{D}$ & 72 & Sample & 2 & 30 & 0.1 & 600 & & & \\
\hline $426 \mathrm{E}$ & 72 & Sample & 7 & 30 & 0.1 & 2,100 & & & \\
\hline $428 \mathrm{~A}$ & 96 & Sample & 0 & 30 & 0.1 & 0 & \multirow{5}{*}{1,080} & \multirow{5}{*}{963} & \multirow{5}{*}{431} \\
\hline 428B & 96 & Sample & 1 & 30 & 0.1 & 300 & & & \\
\hline $428 \mathrm{C}$ & 96 & Sample & 8 & 30 & 0.1 & 2,400 & & & \\
\hline $428 \mathrm{D}$ & 96 & Sample & 4 & 30 & 0.1 & 1,200 & & & \\
\hline $428 \mathrm{E}$ & 96 & Sample & 5 & 30 & 0.1 & 1,500 & & & \\
\hline $430 \mathrm{~A}$ & 120 & Sample & 0 & 30 & 0.1 & 0 & \multirow{5}{*}{0} & \multirow{5}{*}{0} & \multirow{5}{*}{0} \\
\hline $430 \mathrm{~B}$ & 120 & Sample & 0 & 30 & 0.1 & 0 & & & \\
\hline $430 \mathrm{C}$ & 120 & Sample & 0 & 30 & 0.1 & 0 & & & \\
\hline $430 \mathrm{D}$ & 120 & Sample & 0 & 30 & 0.1 & 0 & & & \\
\hline $430 \mathrm{E}$ & 120 & Sample & 0 & 30 & 0.1 & 0 & & & \\
\hline
\end{tabular}

1. The dilution correction was a value used to correct for the volume of PBS in which the coupons were placed

2. The agar volume was a value used to correct for the volume of the PBS solution plated onto the petri dish

3. Viable spores was the number of spores after corrected for the dilution and agar volume corrections 
Table A3 - 20 - Test condition 9: 160 deg F, 70\% RH, controls

\begin{tabular}{|c|c|c|c|c|c|c|c|c|c|}
\hline $\begin{array}{l}\text { Sample } \\
\text { Number }\end{array}$ & Time & Type & $\begin{array}{l}\text { Spores } \\
\text { counted }\end{array}$ & $\begin{array}{c}\text { Dilution } \\
\text { Correction }^{1}\end{array}$ & $\begin{array}{c}\text { Agar } \\
\text { Volume }^{2}\end{array}$ & $\begin{array}{c}\text { Viable } \\
\text { spores }^{3}\end{array}$ & $\begin{array}{c}\text { Mean } \\
\text { of } \\
\text { viable } \\
\text { spores }\end{array}$ & $\begin{array}{l}\text { SD of } \\
\text { viable } \\
\text { spores }\end{array}$ & $\begin{array}{l}\text { SE of } \\
\text { viable } \\
\text { spores }\end{array}$ \\
\hline $423 \mathrm{~A}$ & 24 & Control & 48 & 30 & 0.1 & 14,400 & \multirow{5}{*}{6,840} & \multirow{5}{*}{4,811} & \multirow{5}{*}{2,152} \\
\hline 423B & 24 & Control & 11 & 30 & 0.1 & 3,300 & & & \\
\hline $423 \mathrm{C}$ & 24 & Control & 23 & 30 & 0.1 & 6,900 & & & \\
\hline $423 \mathrm{D}$ & 24 & Control & 7 & 30 & 0.1 & 2,100 & & & \\
\hline $423 \mathrm{E}$ & 24 & Control & 25 & 30 & 0.1 & 7,500 & & & \\
\hline $425 \mathrm{~A}$ & 48 & Control & 10 & 30 & 0.1 & 3,000 & \multirow{5}{*}{3,600} & \multirow{5}{*}{2,068} & \multirow{5}{*}{925} \\
\hline $425 B$ & 48 & Control & 7 & 30 & 0.1 & 2,100 & & & \\
\hline $425 \mathrm{C}$ & 48 & Control & 8 & 30 & 0.1 & 2,400 & & & \\
\hline $425 \mathrm{D}$ & 48 & Control & 11 & 30 & 0.1 & 3,300 & & & \\
\hline $425 \mathrm{E}$ & 48 & Control & 24 & 30 & 0.1 & 7,200 & & & \\
\hline $427 \mathrm{~A}$ & 72 & Control & 24 & 30 & 0.1 & 7,200 & \multirow{5}{*}{5,640} & \multirow{5}{*}{1,621} & \multirow{5}{*}{725} \\
\hline $427 \mathrm{~B}$ & 72 & Control & 18 & 30 & 0.1 & 5,400 & & & \\
\hline $427 \mathrm{C}$ & 72 & Control & 10 & 30 & 0.1 & 3,000 & & & \\
\hline 427D & 72 & Control & 22 & 30 & 0.1 & 6,600 & & & \\
\hline $427 \mathrm{E}$ & 72 & Control & 20 & 30 & 0.1 & 6,000 & & & \\
\hline $429 \mathrm{~A}$ & 96 & Control & 17 & 30 & 0.1 & 5,100 & \multirow{5}{*}{5,640} & \multirow{5}{*}{1,565} & \multirow{5}{*}{700} \\
\hline 429B & 96 & Control & 20 & 30 & 0.1 & 6,000 & & & \\
\hline $429 \mathrm{C}$ & 96 & Control & 25 & 30 & 0.1 & 7,500 & & & \\
\hline 429D & 96 & Control & 11 & 30 & 0.1 & 3,300 & & & \\
\hline $429 \mathrm{E}$ & 96 & Control & 21 & 30 & 0.1 & 6,300 & & & \\
\hline $431 \mathrm{~A}$ & 120 & Control & 16 & 30 & 0.1 & 4,800 & \multirow{5}{*}{5,940} & \multirow{5}{*}{1,689} & \multirow{5}{*}{755} \\
\hline 431B & 120 & Control & 19 & 30 & 0.1 & 5,700 & & & \\
\hline $431 \mathrm{C}$ & 120 & Control & 13 & 30 & 0.1 & 3,900 & & & \\
\hline $431 \mathrm{D}$ & 120 & Control & 25 & 30 & 0.1 & 7,500 & & & \\
\hline $431 \mathrm{E}$ & 120 & Control & 26 & 30 & 0.1 & 7,800 & & & \\
\hline
\end{tabular}

1. The dilution correction was a value used to correct for the volume of PBS in which the coupons were placed

2. The agar volume was a value used to correct for the volume of the PBS solution plated onto the petri dish

3. Viable spores was the number of spores after corrected for the dilution and agar volume corrections 


\section{CHAPTER 4 -- DECONTAMINATION OF A BACILLUS ANTHRACIS SPORE SIMULANT ON AIRCRAFT PLASTIC COUPONS USING HIGH HEAT AND HUMIDITY WITHIN AIRCRAFT ENGINEERING TOLERANCES}

\section{SUMMARY}

The goal of this research was to determine if plastic coupons, indicative of aircraft materials, could be effectively decontaminated from a Bacillus anthracis simulant (Bacillus atrophaeus subsp globigii $[\mathrm{BG}])$ using high heat and humidity within the engineering specifications of aircraft. These spores were deposited using a high direct inoculation $\left(10^{6}\right.$ spores per coupon), low direction inoculation ( $10^{4}$ spores per coupon), and an innovative aerosol deposition method using a bioaerosol test chamber $\left(10^{4}\right.$ spores per coupon). Previous studies have evaluated only direct inoculations in the range of $10^{6}$ spores and only on aluminum coupons. Five different test conditions of temperature and humidity (ranging from an upper limit combination of $180^{\circ} \mathrm{F}$ and $90 \%$ relative humidity $[\mathrm{RH}]$ to a lower limit of $160^{\circ} \mathrm{F}$ and $70 \% \mathrm{RH}$ ) were evaluated over 24 hour increments with an upper time limit of 120 hours. Decontamination tests showed that the high concentrations of spores were inactivated within 48 hours at $180^{\circ} \mathrm{F}$ and $90 \% \mathrm{RH}$. No other treatment temperatures or humidity ranges inactivated all spores within the time allotted of 120 hours. Tests using low direct inoculations showed complete kills at 48 hours with a treatment of $180^{\circ} \mathrm{F}$ with $90 \% \mathrm{RH}$ and $170^{\circ} \mathrm{F}$ with $80 \% \mathrm{RH}$. Additionally, all spores were inactivated at 120 hours $160{ }^{\circ} \mathrm{F}$ with $90 \% \mathrm{RH}$. Aerosol deposited spores were inactivated within 48 hours for all five test conditions, except for treatment with $160^{\circ} \mathrm{F}$ with $70 \% \mathrm{RH}$, which still had active spores at the 120 hour point. A stepwise regression was performed to determine which variables are significant to predict the inactivation rates $(\alpha=0.05$ was used to keep or discard terms). For this regression, there were three variables required to be in each modeltime, temperature, and humidity. The stepwise regression resulted in approximately the same 
number of terms being retained in the models with high, low, and aerosol deposition have 7, 6, and 8 terms, respectively. Besides the mandatory variables (time, temperature, and humidity), there were no variables retained in all three models. The statistical analysis does indicate humidity is a critical factor, as nearly all variables retained in these models contain humidityeach model only has one variable that does not contain humidity. The $\mathrm{R}^{2}$ values are reasonable for these models, with the values being $76.6 \%, 68.8 \%$, and $77.8 \%$, for high and low direct inoculation and aerosol deposition, respectively. Thus most of the variability for the spore inactivation is explained by the models.

Data from a Chapter 3 completed on aluminum coupons were used to determine if inactivation rates were significantly different for plastic coupons. The slopes for inactivation lines were compared for plastic and aluminum coupons for each test condition. For high direction inoculation, there was a significant difference for test condition $5\left(170{ }^{\circ} \mathrm{F}\right.$ with $80 \%$ $\mathrm{RH})$ and test condition $7\left(160^{\circ} \mathrm{F}\right.$ with $\left.90 \% \mathrm{RH}\right)$, with inactivation being faster for plastic coupons. For low direct inoculation there was only one test condition that was significantly different for the testing conditions and this was test condition $7\left(160^{\circ} \mathrm{F}\right.$ with $\left.90 \% \mathrm{RH}\right)$, again with plastic being faster. A tobit analysis showed the plastic coupon inactivation rates were significantly faster for test condition $3\left(180^{\circ} \mathrm{F}\right.$ with $\left.70 \% \mathrm{RH}\right), 5\left(170^{\circ} \mathrm{F}\right.$ with $\left.80 \% \mathrm{RH}\right)$, and 7 (160 ${ }^{\circ} \mathrm{F}$ with $\left.90 \% \mathrm{RH}\right)$.

The research demonstrated that the optimal heat and humidity ranges are those that can be maintained at the highest levels within engineering tolerances. Additionally, the best combination for decontamination was test condition $1\left(180^{\circ} \mathrm{F}\right.$ and $90 \%$ relative humidity) which inactivated all the spores in all test conditions within 48 hours. The next best combination was test condition $5\left(170{ }^{\circ} \mathrm{F}\right.$ and $80 \%$ relative humidity). The data also showed that only 6 of the 15 
test conditions had significantly different inactivation rates for the plastic versus aluminum coupons. These results suggest that the material is an important consideration, but that at the proper temperature and humidity levels, the spores will be inactivated in the required time. Overall, this research demonstrated that these spores can be inactivated safely, effectively, and also within aircraft engineering specifications using high heat and humidity.

\section{INTRODUCTION}

Bioterrorism is defined as a use or threatened use of biological agents against individuals to obtain advantage for a specific purpose such as intimidation, ideological principles, or disruption of everyday activities (Brachman, 2002). Any act of biological terrorism or warfare can be very difficult to diagnose the actual agent in a short time (Estill et al., 2009), which may hamper decontamination efforts. To minimize illnesses, decontamination to an acceptable level in a very short time is critical (Uhm et al., 2007).

DoDI 3150.09 “The Chemical, Biological, Radiological, and Nuclear (CBRN) Survivability Policy" requires all DoD assets to be able to continue operations even in the presence of biological agents, including the capability to be decontaminated properly (DoD, 2009). More detailed definitions can be found in Chapter 3. It is important to note, however, that decontamination is generally referred to as a "process making material safe by absorbing, destroying, neutralizing, rendering harmless, or removing chemical or biological agents and radiological contamination" (DoD, 2009) and that most field tests have targeted a 6-log reduction in the contaminant (Gale et al., 2009). Others have stated that any detectable Bacillus anthracis spore would constitute an unacceptable risk (Herzog et al., 2009); however, this does not take into normal environmental risks in that these spores are indigenous in certain areas (Chosewood and Wilson, 2009). 
Regardless of the level of decontamination required, decontamination tests are generally completed on spores. The U.S. Army Edgewood Chemical and Biological Center (ECBC) requires the decontamination methods be effective against spores, and more specifically, Bacillus anthracis spores. These spores are the target because it they are considered the most difficult biological warfare agent to decontaminate. The endospores are metabolically inactive and are highly resistant to many physical stresses such as wet and dry heat, chemical agents, UV and gamma radiation, oxidizing agents, vacuums and ultra-high hydrostatic pressures (Nicholson et al., 2002). The spores are stable for up to 60 years in soil and water and can resist sunlight for varying periods (Chosewood and Wilson, 2009; Perkins, 1983). Because of the lethality of Bacillus anthracis spores, simulants, including Bacillus subtilis var niger (also known as Bacillus globigii (BG) or Bacillus subtilis have been used extensively in studies (Aizenberg et al., 2000; Burton et al., 2005; Carrera et al., 2005; Farnsworth et al., 2006; Foarde et al., 1999; Jensen, 1992; Mainelis et al., 2002; Hill et al., 1999; Maus et al., 2001; Li and Lin, 2001; Sagripanti et al., 2007; Wagner et al., 2008; Yah and Mainelis, 2007;). Additionally, Bacillus atrophaeus, is also used in the past because it is virtually indistinguishable from Bacillus subtilis. Some of the Bacillus subtilis lines used in the past were identified as a new strain, Bacillus atrophaeus susp globigii (Burke et al., 2004). Bacillus atropheaus spores have been used in several studies as well (Lewandowski et al., 2010; Brown et al., 2008a; Carrera et al., 2005; Kesavan, 2008; Thomas et al., 2008; Martin and Moore, 2001).

Several test chambers have been designed to aerosolize and then deposit biological simulants onto some type of coupon for further testing (Baron et al., 2007; Baron et al., 2008; Brown et al., 2007a, 2007b, 2007c; Buttner et al., 2004; Chen et al., 1999; Edmonds et al. 2009; Estill et al., 2009; Kesavan, 2008; King et al., 2011; Farnsworth et al., 2006;; Feather and Chen, 
2003; Byrne et al., 1995; Lai et al., 2002; Kenny et al., 1999; Koch et al., 1999; Lewandowski et al., 2010; Marple and Rubow, 1983; Park et al., 2009; Thatcher and Nazaroff, 1997). With few exceptions (King, 2010), these chambers were constructed to evaluate deposition or swipe sampling and have not been used to evaluate the effectiveness of decontamination methods.

The actual decontamination can be completed in several different methods. During the 2001 anthrax attacks, chlorine dioxide, vaporized hydrogen peroxide, paraformaldehyde, methyl bromide, and ethylene oxide were approved for use (Kempter, 2005). Hydrogen peroxide in the vapor phase was used with a high degree of success during the 2001 attacks (McVey, 2005) and tests in both the laboratory (Andersen et al., 2006; Oh et al., 2005) and field studies on grounded aircraft (Gale et al., 2008) have shown its efficacy. This method cannot be used on airworthy aircraft because it has detrimental material impacts (Gale et al., 2009; Verce et al., 2008). Chlorine dioxide was also used during the 2001 attacks (Barth et al., 2003; Canter et al., 2005; Rastogi et al., 2009) and has shown to effective in laboratory studies (Perez et al., 2005; Wagner et al., 2008). Despite its effectiveness, chlorine dioxide has shown to be damaging to materials as well (Orlusky, 2005). Because all of these decontamination methods are at least somewhat hazardous to aircraft materials, they cannot be used on Air Force aircraft (AFRL, 2008). All aircraft must meet strict engineering specifications, including withstanding high temperature storage greater than $185^{\circ} \mathrm{F}$ at $100 \%$ relative humidity $(\mathrm{RH})$ for prolonged periods, which gives a possibility of decontamination using these levels (AFRL, 2008). For these reasons, the Air Force has evaluated using high heat and humidity for decontaminating aircraft. Several studies have been conducted on grounded aircraft to determine if the technology is feasible. These tests, completed at $180^{\circ} \mathrm{F}$ and $\mathrm{RH}$ ranges from 75 to $90 \%$, showed a 5 to $6 \log$ reduction in Bacillus 
thuringiensis var kurstaki (BtK or Bt) spores. The studies have shown that these ranges are capable of inactivating the spores within the engineering specifications (AFRL, 2008).

Previous studies have been completed analyzing high heat and humidity inactivation rates on Bacillus anthracis spore simulants at high concentrations directly deposited onto aluminum coupons. These studies have only focused on the upper limits of the heat and humidity engineering limits of the aircraft. A better understanding of the inactivation rates of these spores on other aircraft materials, lower heat and humidity ranges, and also lower inoculation rates is critical for understanding the best method for safely decontaminating aircraft. The research tested the inactivation rates of a Bacillus anthracis spore simulant using five combinations of high heat and humidity levels, all within the engineering specifications of aircraft. The spores were delivered to plastic coupons in three different methods - high direct inoculation, low direct inoculation, and an aerosol deposition method using a previously described test chamber. The inactivation rates for the plastic coupons were then compared to the aluminum coupons tested previously. Data on inactivation rates on different aircraft materials are critical to verify decontamination can be completed on the entire aircraft.

\section{MATERIALS AND METHODS}

\section{Test chamber}

A bioaerosol test chamber was designed and built to deposit a Bacillus anthracis spore simulant onto plastic coupons to test inactivation rates when exposed to high heat and humidity. These chamber design and testing methods are described in more detail in Chapter 2. Equations were derived to model spore deposition in the chamber using a 6-jet Collison nebulizer (BGI, Waltham, MA). These equations, based on the general ventilation dilution equations (Burgess, 
Ellenbecker, and Treitman, 2004), are explained more thoroughly in Chapter 2 . The final equation for surface spore deposition is defined in equation 1 below.

(1) $\mathrm{S}_{\mathrm{c}}=\left(\left(\frac{\mathrm{G}}{\mathrm{Q}_{\text {in }}}\right) * \mathrm{H}\right) * \mathrm{SA}_{\mathrm{C}}$

$$
\begin{aligned}
& \mathrm{G}=\text { Generation rate for spores }\left(\frac{C F U}{\text { minute }}\right) \\
& \text { Where: } \mathrm{Q}_{\text {in }}=\text { Air generation rate into chamber }\left(\frac{m^{3}}{\text { minute }}\right) \\
& \text { (Controllable throughout experiment) } \\
& \mathrm{H}=\text { Chamber height }(1.22 \mathrm{~m}) \\
& \mathrm{SA}_{\mathrm{c}}=\text { Coupon surface area }\left(1 \mathrm{in}^{2}=6.45 \times 10^{-4} \mathrm{~m}^{2}\right)
\end{aligned}
$$

Aerosol generation was completed in the same manner as described in Chapter 3. This included particle free air to generate spores using a Collison nebulizer (BGI, Waltham, MA), with neutralization completed with a TSI Kr-85 neutralizer.

\section{Test Coupons}

Plastic sheets were provided by Dr. Ken Heater and Daniel E. Badowski, METSS Corporation, per the recommended material of the Air Force Research Laboratory. The plastic was 1/8" uncoated Makrolon ${ }^{\circledR}$ Polycarbonate plastic, Sheffield Plastics, Inc. (Sheffield, MA). The material was provided in sheets and cut to one-inch squares by Fort Collins Plastics Inc, Fort Collins, CO. Before each test, the coupons were rinsed with tap water and then deionized water to remove all biological material and chlorine ion residuals. The coupons were then autoclaved at $121^{\circ} \mathrm{C}$ for 30 minutes. Inoculations were performed using aseptic techniques explained later. 


\section{Biological methods}

Spores

Tests were completed using Bacillus atrophaeus subsp globigii (BG), obtained from Yakibou, Inc (Apex, NC). The spores were provided in two concentrations-3.1 $10^{8}$ spores $/ \mathrm{mL}$ and $2.2 \times 10^{9}$ spores $/ \mathrm{mL}$. For nebulization, the spores were diluted in Phosphate Buffered Saline (PBS) with 0.05\% Tween 20 (Fisher Scientific). The PBS, delivered as a dry powder, was mixed with laboratory grade water from a Barnstead NANOpure Diamond ${ }^{\mathrm{TM}}$ purification system.

Spore inoculation methods

Direct

Spore inoculations were performed in the same manner as described in Chapter 3. The inoculation levels were high ( $10^{6}$ spores per coupon) and low $\left(10^{4}\right.$ spores per coupons). Aerosol spore deposition

The spores were aerosolized within the test chamber based on the deposition goal of $10^{4}$ spores per coupon. This deposition number was used to correspond to the low direct inoculation level. Methods to deposit these spores were the same methods as described in Chapter 3.

\section{Sample processing}

Removal of the spores from the coupons was completed in the method as developed and described in Chapter 3. This method was 30 minutes of sonication followed by 2 minutes of vortexing. Plating was also completed as described in Chapter 3. This included completing serial dilutions with a goal of 300 spores or less per petri dish. 


\section{Inactivation tests}

The environmental test chamber used was a reconditioned Blue M, HR-381C

Temperature/Humidity chamber purchased from Technical Equipment Sales, Inc, (Severance, $\mathrm{CO})$, capable of maintaining a temperature up to $150^{\circ} \mathrm{C}\left(300^{\circ} \mathrm{F}\right)$ and relative humidity levels from $10 \%$ to $95 \%$. The chamber was updated with an electronic controller operated by Watlow Electric Manufacturing Co, Watview ${ }^{\circledR}$ Runtime Version 2.6.4 software (St. Louis, MO).

Initial inactivation tests showed that test sizes needed to be 5 samples. Again, this is described more in-depth in Chapter 3. Based on this number, a decontamination testing matrix was developed, shown in Figure 4-1.

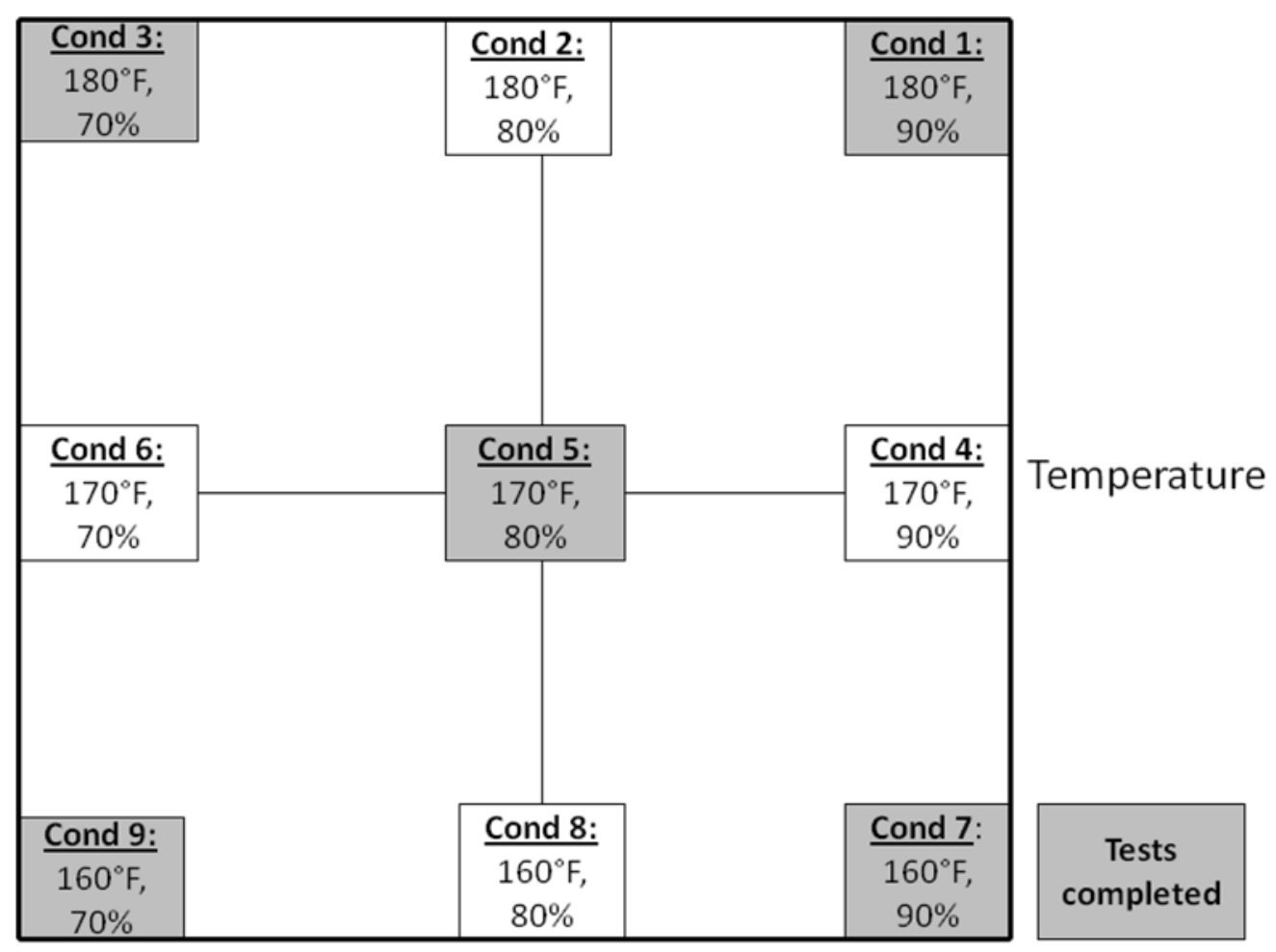

Relative Humidity

Figure 4- 1 - Decontamination test matrix 
Each testing condition had separate samples completed at times $0,24,48,72$, and 96 hours for high and low direct inoculation and also aerosol deposition. Samples were completed at 120 hours for test conditions 5, 7 and 9 because it was anticipated the spores would not be inactivated at 96 hours for this combination of temperature and humidity. A total of 5 samples were collected at each time period for each test condition.

\section{Data Management and Statistics}

Inactivation rates were plotted using Microsoft Office Excel®. These plots included the average number of spores for each data point and the standard error for these points. A stepwise regression was completed on the inactivation data using Minitab®, v16 (State College, PA). This stepwise regression was completed on each different inoculation type (direct high, direct low, and aerosol deposition). The response variable in each model was the log value of the spores +1 . Explanatory variables were added or removed from the model during the stepwise regression using $\alpha=0.05$. Temperature, humidity, and treatment time were mandatory variables in the model. Once the additional predictors were included, a final regression model was completed with those variables selected during the step wise regression. Heat and humidity levels in the heat and humidity chamber were logged using Watview ${ }^{\circledR}$ Runtime Version 2.6.4 software, Watlow Electric Manufacturing Co (St Louis, MO). 


\section{RESULTS}

\section{Spore removal efficiencies}

Testing, discussed in Chapter 3, showed for both aluminum and plastic coupons the optimal method for removing spores was 30 minutes of sonication followed by 2 minutes of vortexing. Based on this result, all spore removals were completed using these methods.

\section{Aerosol deposition removals}

The spore recovery efficiencies were evaluated by depositing the spores in goals of 10,$000 ; 100,000 ;$ and 1,000,000 spores per sample, with each sample consisting of two one-inch square plastic coupons. The deposition goals and average recoveries are presented in Table 4-1. Each of these aerosolization and removal tests was performed once, with 18 different coupon sets analyzed. Note the test numbers are not sequential because all tests conducted were numbered as they were completed. The data for spore recovery efficiencies ranged from $28.8 \%$ to $4.8 \%$, for both aluminum and plastic coupons. These values are consistent with the closest comparisons available in the literature (Brown et al., 2007a; Lewandoski et al., 2010). After this analysis, the tests were completed assuming $10 \%$ of the modeled spores generated would actually be deposited and removed from the coupons. This assumption was made in order to keep all modeling parameters constant throughout the remaining experiments.

Table 4 - 1 - Spore recovery from aerosol deposition, plastic and aluminum coupons

\begin{tabular}{|l|c|c|c|c|c|c|c|}
\hline & $\begin{array}{c}\text { Test 77, } \\
\text { Alum }\end{array}$ & $\begin{array}{c}\text { Test 78, } \\
\text { Alum }\end{array}$ & $\begin{array}{c}\text { Test 79, } \\
\text { Alum }\end{array}$ & $\begin{array}{c}\text { Test 82, } \\
\text { Alum }\end{array}$ & $\begin{array}{c}\text { Test 78, } \\
\text { Plastic }\end{array}$ & $\begin{array}{c}\text { Test 79, } \\
\text { Plastic }\end{array}$ & $\begin{array}{c}\text { Test 82, } \\
\text { Plastic }\end{array}$ \\
\hline $\begin{array}{l}\text { Deposition } \\
\text { Goal }\end{array}$ & 10,000 & 10,000 & 100,000 & $1,000,000$ & 10,000 & 100,000 & $1,000,000$ \\
\hline $\begin{array}{l}\text { Average } \\
\text { Recovery }\end{array}$ & $\begin{array}{c}28.8 \% \\
( \pm 13.8 \%)\end{array}$ & $\begin{array}{c}9.0 \% \\
( \pm 7.1 \%)\end{array}$ & $\begin{array}{c}8.3 \% \\
( \pm 7.2 \%)\end{array}$ & $\begin{array}{c}16.9 \% \\
( \pm 6.7 \%)\end{array}$ & $\begin{array}{c}5.3 \% \\
( \pm 6.7 \%)\end{array}$ & $\begin{array}{c}4.8 \% \\
( \pm 3.2 \%)\end{array}$ & $\begin{array}{c}13.5 \% \\
( \pm 4.6 \%)\end{array}$ \\
\hline
\end{tabular}

$\mathrm{n}=18$ for each sample 


\section{Initial decontamination tests}

Initial decontamination tests were completed to determine the sample sizes required. This was done by initial decontamination tests on plastic and aluminum coupons, followed by a general linear model in Minitab®, v16, to determine the sample size required. It was found that a sample size of $5(n=5)$ would be required, assuming $\alpha=0.05$ and power $=0.8$. This analysis is described more thoroughly in Chapter 3.

\section{Decontamination tests}

The five test conditions were completed and analyzed as depicted in Figure 4-1 above. These were completed for high and low direct inoculations and aerosol depositions. There were two tests that had errors, that is, the data did not meet what was expected biologically. This was because the graphs, or inactivation rates, did not show a decrease uniformly, but rather there was one time point where all samples were zero and this was followed by the next period with samples of positive numbers. It was not known why this occurred. These samples were aerosol deposition for test conditions 3 and 9. These test conditions were re-accomplished because the data points at treatment time of 24 hours were all zero (all five samples had spore readings of zero); however, the readings at treatment time 48 and 72 had positive spore growth.

\section{High direct inoculation inactivation}

Figure 4-2 includes the data for high direct inoculations. Each data point in the graphs included 5 samples $(n=5)$. Error bars are standard error, or the standard deviation divided by the square root of the sample size. Test condition 1 had full inactivation of the spores occurring at the 48 hour time period. None of the other test conditions showed a full inactivation within the 
time constraints; however, test conditions 5 and 7 showed decreases within the time limits. Test condition 9 showed little to no impact on the spores.

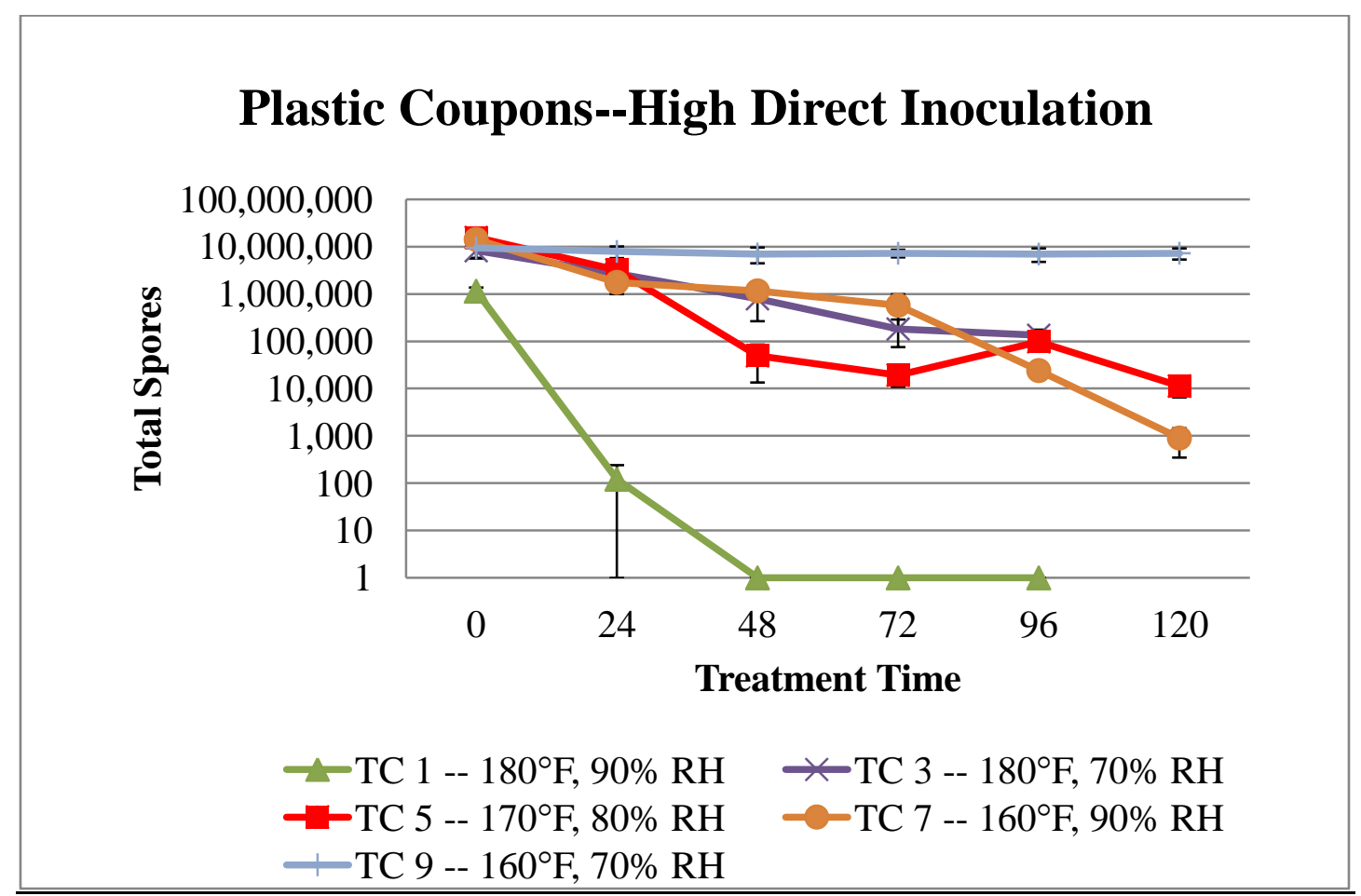

Figure 4- 2 - Decontamination tests, high direct inoculation

\section{Low direct inoculation inactivation}

Data for low direct inoculations are in Figure 4-3. Each data point in the graphs included 5 samples $(n=5)$. Error bars are the same as in Figure 4-2. These data show full inactivation occurred for the spores for test conditions 1 and 5 within 48 hours and test condition 7 at 120 hours. Test condition 3 showed a trend that was decreasing at the upper time limit. The data for test condition 9 showed inactivation at 72 hours but the spore numbers increased until the end point. The reason for this is unknown; however, the figure shows that test condition 9 is not as effective in inactivating the spores. 


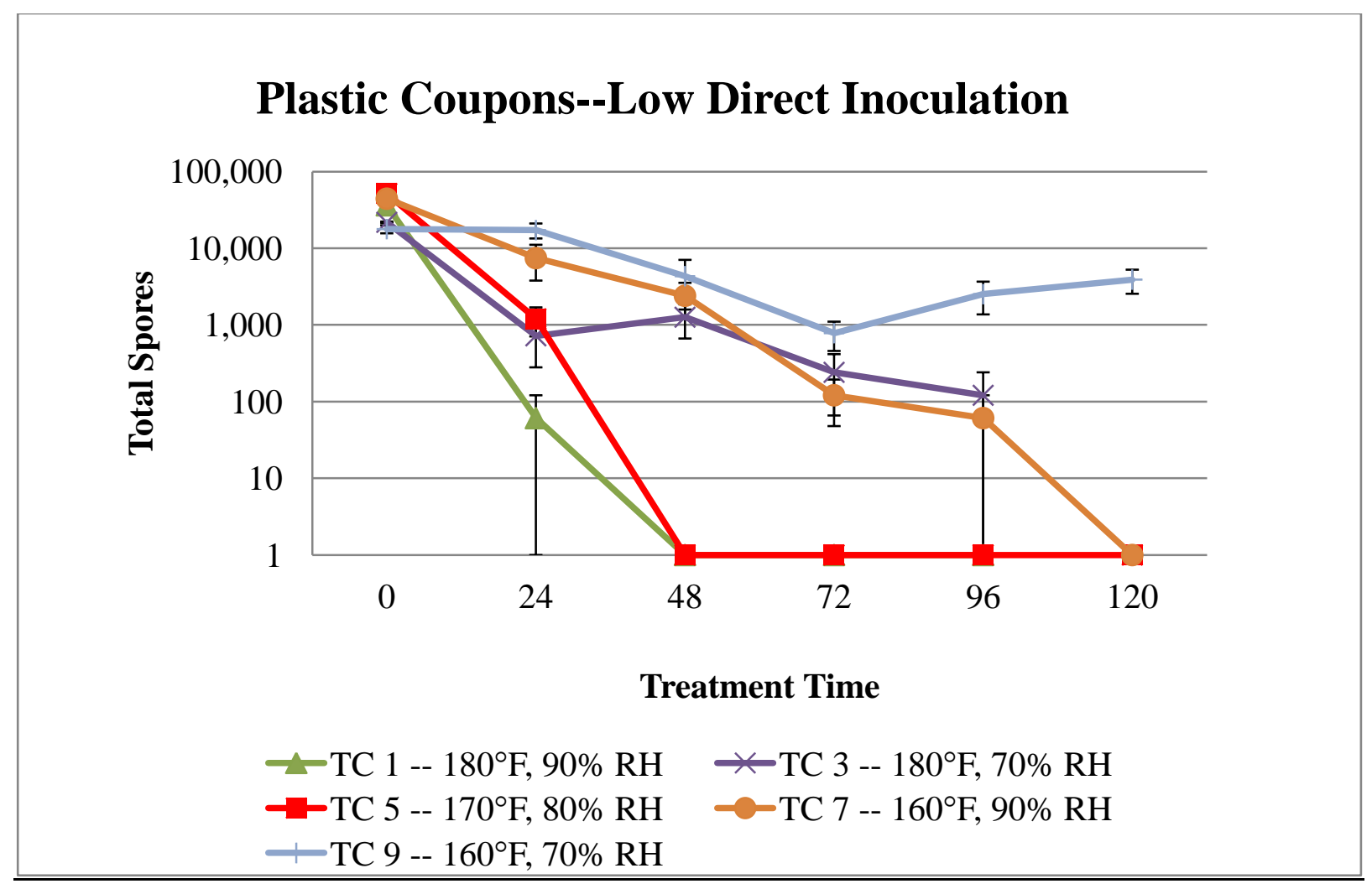

Figure 4- 3 - Decontamination tests, low direct inoculation

Aerosol deposition inoculation inactivation

The aerosol deposition data are in Figures 4-4 through 4-8. The data for each aerosol test is included in separate figures because each sample point had a corresponding control, with each data point in the graphs including 5 samples $(n=5)$.

Figure 4-4a, decontamination tests for aerosol deposition test condition 1, shows that the spores were inactivated within 24 hours. After this time point, all the samples were zero and all the controls remained positive, demonstrating successful inactivation. 


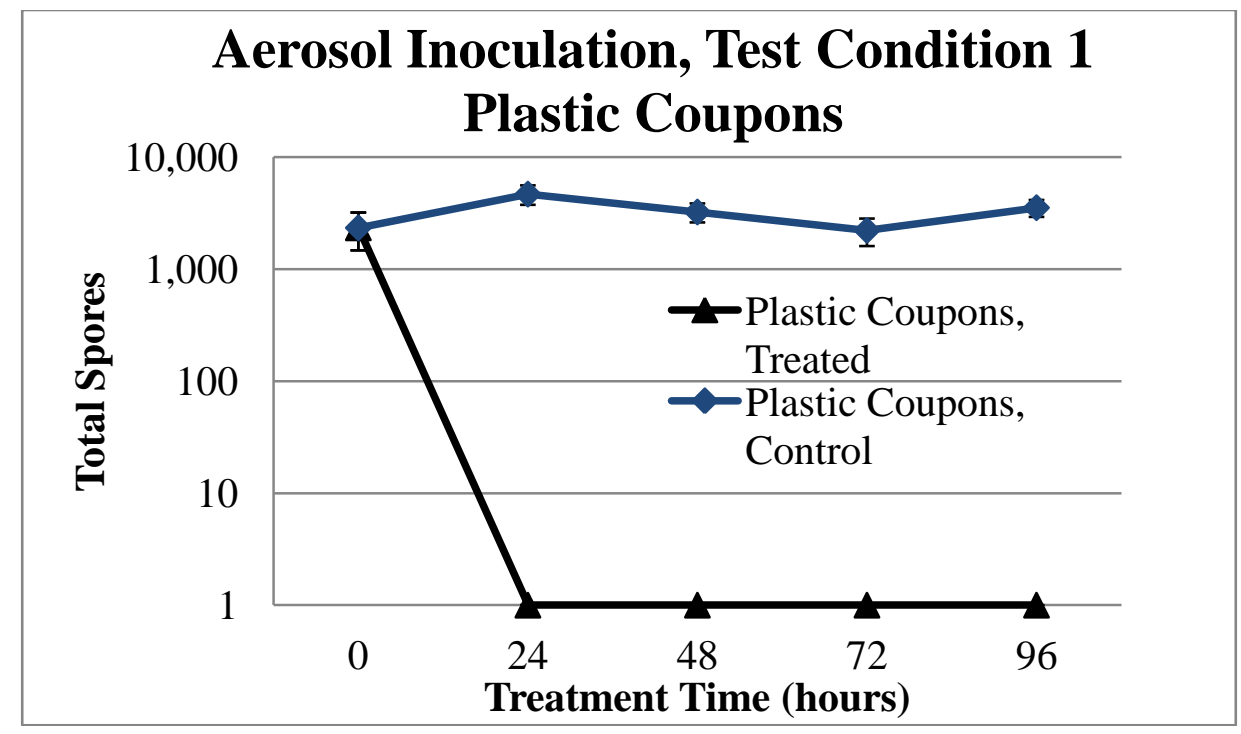

Figure 4- 4-Decontamination tests, aerosol deposition, test condition 1

Figure 4-5, decontamination tests for aerosol deposition test condition 3 again shows successful decontamination; however, this was not seen until the 48 hour treatment point. The samples reached the zero mark at this point and all control samples remained positive, again demonstrating successful spore inactivation. 


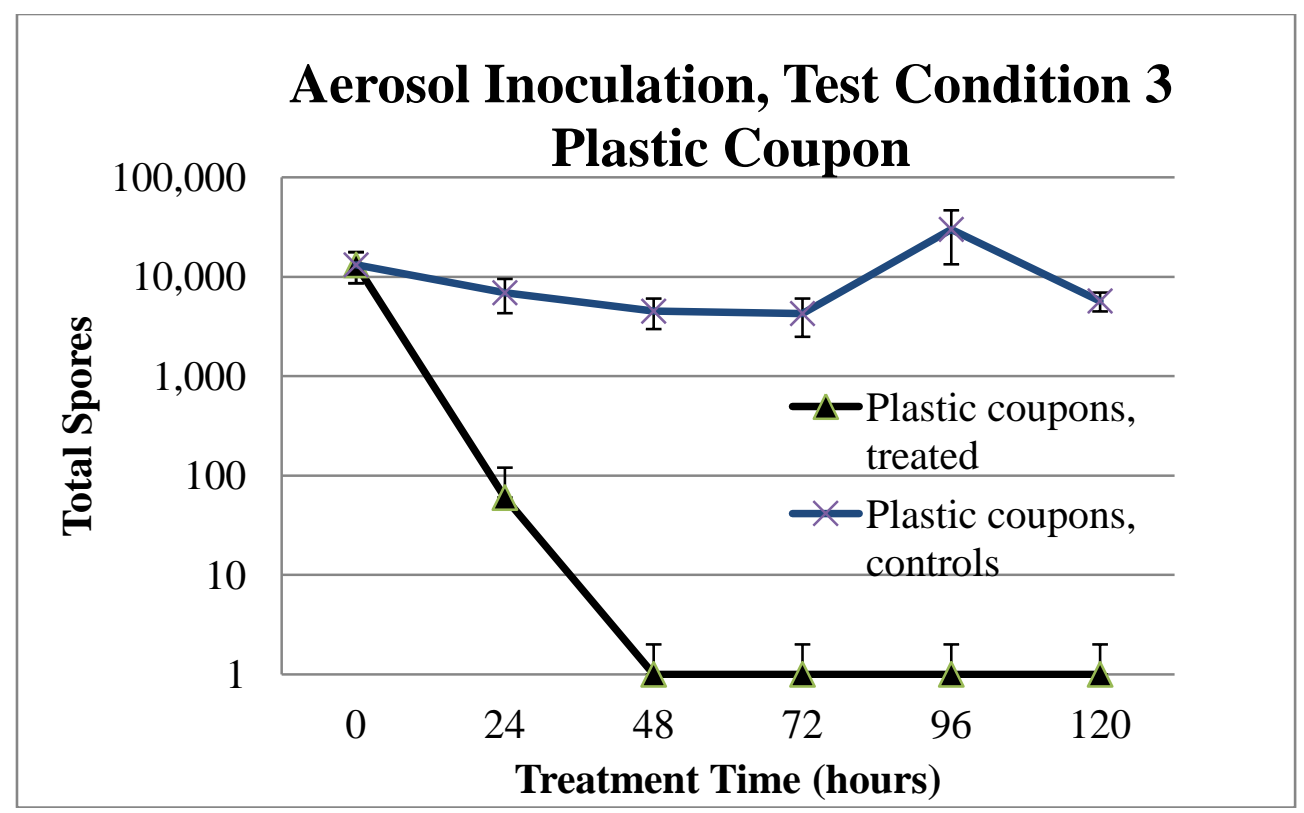

Figure 4- 5 - Decontamination tests, aerosol deposition, test condition 3

Figure 4-6, decontamination tests for aerosol deposition test condition 5 shows successful decontamination at 24 hours. Again, the controls remained positive during the samples.

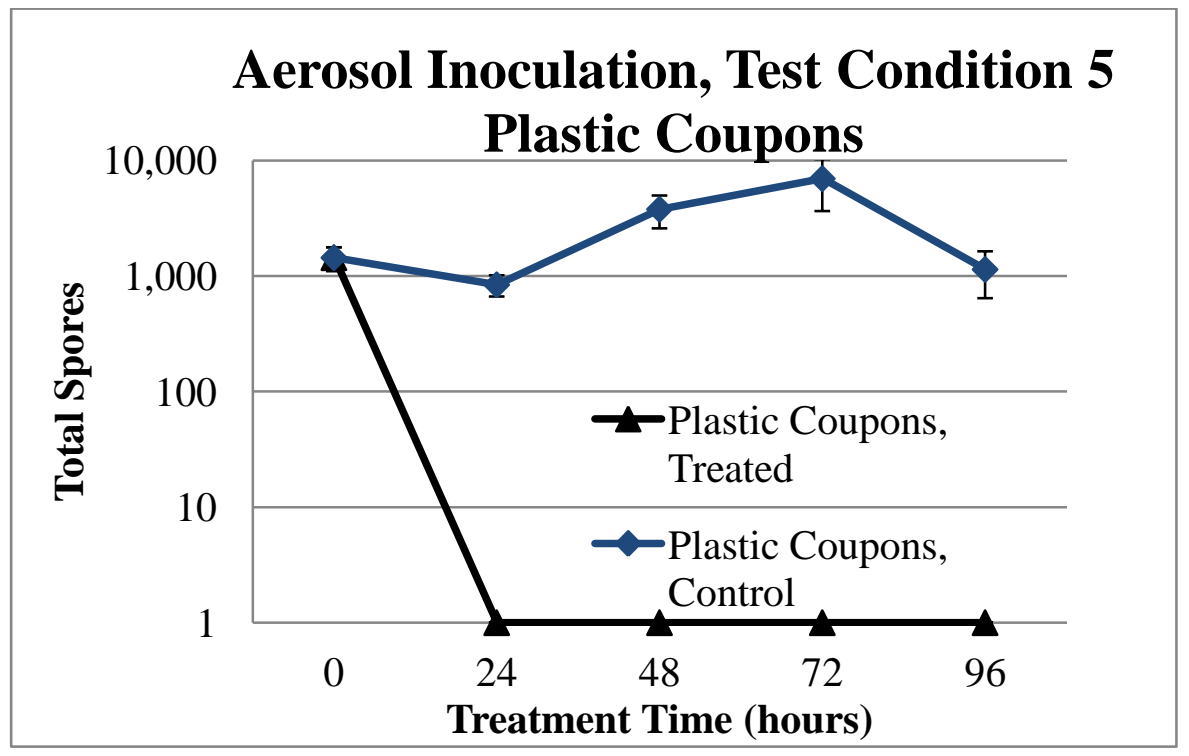

Figure 4- 6-Decontamination tests, aerosol deposition, test condition 5 
Figure 4-7, decontamination tests for aerosol deposition test condition 7 , shows the spores were inactivated at 24 hours, again all control samples remained positive.

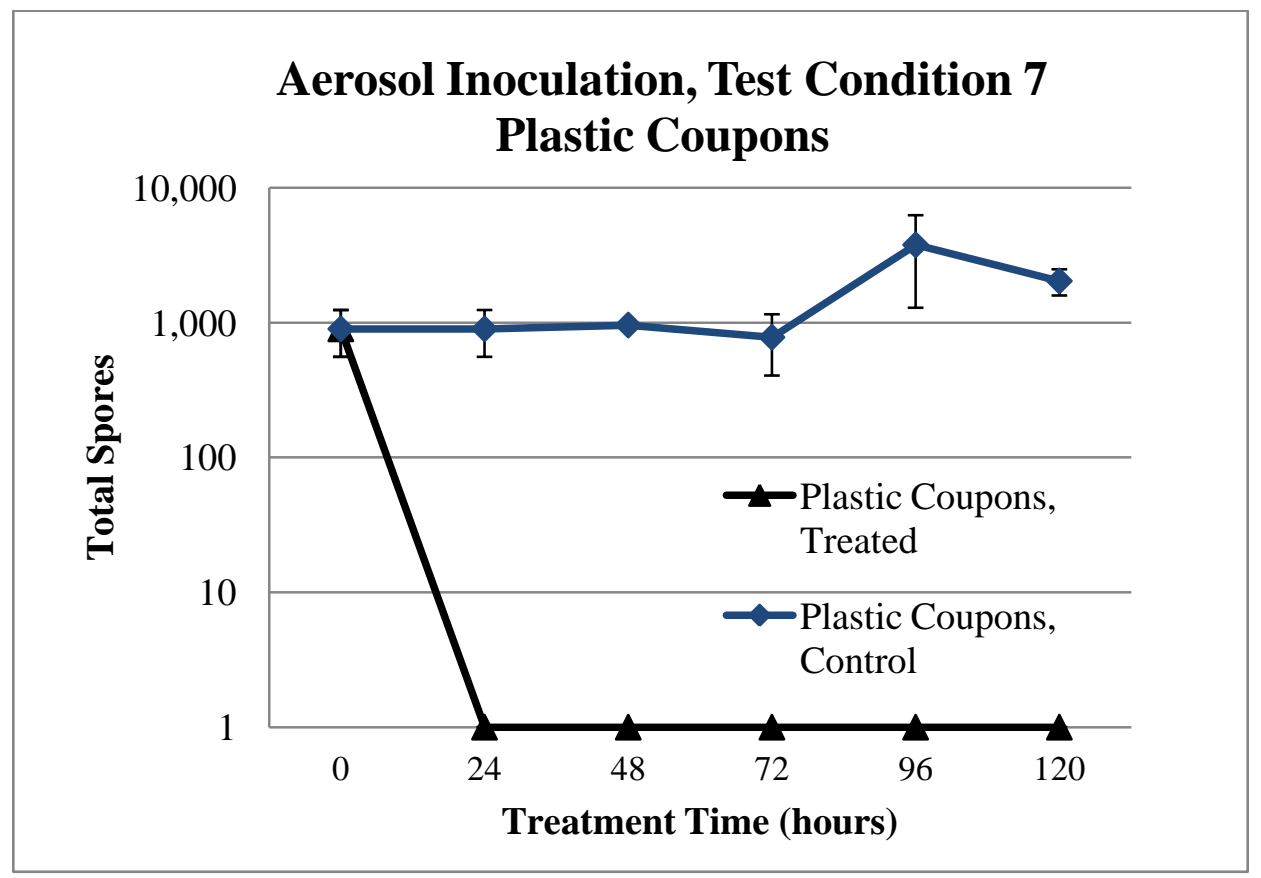

Figure 4- 7 - Decontamination tests, aerosol deposition, test condition 7

Figure 4-8, decontamination tests for aerosol deposition test condition 9, shows that inactivation did not occur within 120 hours of treatment. There was a trend for the spore numbers to decrease; however, they did not reach zero. 


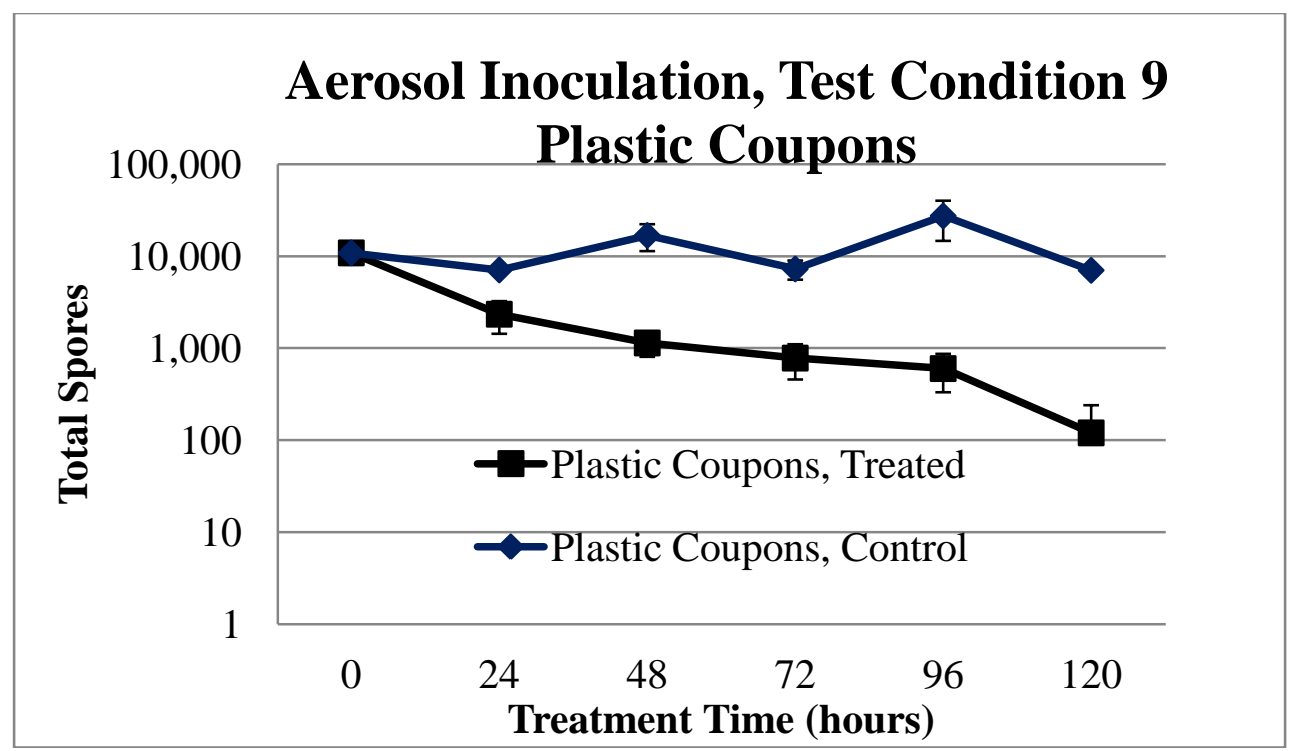

Figure 4- 8 - Decontamination tests, aerosol deposition, test condition 9

\section{Statistical analysis}

A stepwise regression was used to include the variables that would provide the best model for log value of the spores +1 . Temperature, humidity, and treatment time were mandatory variables in the model. Additional variables to be considered for the model were interactions of the main variables (temperature*humidity, temperature*time, and humidity* time). Squared terms of the main variables were also considered (temperature ${ }^{2}$, humidity ${ }^{2}$, and time $^{2}$ ). Because of the limits of the degrees of freedom on the temperature and humidity, these were limited to just the squared terms. The interactions of these terms with time ${ }^{2}$ were included. Because there were more degrees of freedom for time, time ${ }^{3}$ was also considered. The stepwise regression was then completed using those terms with $\alpha=0.05$, that is, terms were added or removed from the model based on meeting this criteria. The variables selected in the stepwise regression for each deposition mechanism are included in Table 4-2. 
Table 4 - 2 - Stepwise regression terms retained in models

\begin{tabular}{|c|c|c|c|}
\hline & High direct inoculation & Low direct inoculation & Aerosol deposition \\
\hline $\begin{array}{l}\text { Mandatory } \\
\text { model variables }\end{array}$ & $\begin{array}{l}\text { Temperature } \\
\text { Humidity } \\
\text { Time }\end{array}$ & $\begin{array}{l}\text { Temperature } \\
\text { Humidity } \\
\text { Time }\end{array}$ & $\begin{array}{l}\text { Temperature } \\
\text { Humidity } \\
\text { Time }\end{array}$ \\
\hline $\begin{array}{l}\text { Additional } \\
\text { explanatory } \\
\text { variables } \\
\text { retained in each } \\
\text { model }\end{array}$ & $\begin{array}{l}\text { Time } * \text { Humidity } \\
\text { Temp } * \text { Humidity } \\
\text { Temp*Humidity } * \text { Time }^{2} \\
\text { Temp }^{2}\end{array}$ & $\begin{array}{l}\text { Time }^{2} \\
\text { Time*Humidity } * \text { Time }^{2} \\
\text { Humidity }^{2}\end{array}$ & $\begin{array}{l}\text { Time * Humidity } \\
\text { Temp * Humidity } \\
\text { Time*Humidity * } \text { time }^{2} \\
\text { Humid*Time } \\
\text { Temp }^{2}\end{array}$ \\
\hline
\end{tabular}

Following the stepwise regression, a final regression model was completed using only

those terms retained in the model. These terms were entered into the model as presented below.

All of these terms were retained by the stepwise regression; there, they were all significant

predictors for the model. The results from these regressions models are included in Tables 4-3

through 4-5, with the corresponding $\mathrm{R}^{2}$ presented.

Table 4 - 3 - High direct inoculation, regression model

\begin{tabular}{|c|c|c|c|}
\hline Term & Model Coeff & SE Coeff & $\mathrm{R}^{2}$ \\
\hline Temperature & 3.859 & 1.315 & \multirow[t]{8}{*}{$76.6 \%$} \\
\hline Humidity & 0.9168 & 0.1683 & \\
\hline Time & 0.13213 & 0.02483 & \\
\hline Time*Humidity & -0.0023182 & 0.0003271 & \\
\hline Temp * Humidity & -0.012488 & 0.002164 & \\
\hline Temp*RH $*$ time $^{2}$ & 0.00000004 & 0.00000001 & \\
\hline Temp $^{2}$ & -0.020340 & 0.008530 & \\
\hline Constant & -166.04 & 51.38 & \\
\hline
\end{tabular}

Table 4 - 4- Low direct inoculation, regression model

\begin{tabular}{|c|c|c|c|}
\hline Term & Model Coeff & SE Coeff & $\mathrm{R}^{2}$ \\
\hline Temperature & -0.12882 & 0.01953 & \multirow[t]{7}{*}{$68.8 \%$} \\
\hline Humidity & -1.1378 & 0.3652 & \\
\hline Time & -0.11719 & 0.01252 & \\
\hline Time $^{2}$ & 0.0013334 & 0.000232 & \\
\hline Time*humidity $*$ time $^{2}$ & -0.00000007 & 0.00000002 & \\
\hline Humidity $^{2}$ & 0.006973 & 0.002280 & \\
\hline Constant & 60.21 & 14.38 & \\
\hline
\end{tabular}


Table 4 - 5 - Aerosol deposition, regression model

\begin{tabular}{|c|c|c|c|}
\hline Term & Model Coeff & SE Coeff & $\mathrm{R}^{2}$ \\
\hline Temperature & -3.3606 & 0.7919 & \multirow[t]{9}{*}{$77.8 \%$} \\
\hline Humidity & -0.68092 & 0.09925 & \\
\hline Time & -0.06095 & 0.01417 & \\
\hline Humid $^{*}$ time $^{2}$ & 0.00002269 & 0.00000309 & \\
\hline Temp * Humidity & 0.007893 & 0.001281 & \\
\hline Time*Humidity $*$ Time $^{2}$ & -0.00000010 & 0.00000002 & \\
\hline Time $*$ Humidity & -0.0008490 & 0.0002333 & \\
\hline Temp $^{2}$ & 0.017406 & 0.005115 & \\
\hline Constant & 164.24 & 31.06 & \\
\hline
\end{tabular}

\section{Total inactivation comparisons}

Inactivation values from all tests, high direct, low direct, and aerosol deposition are included in Tables 4-6 through 4-8 below. These tables also include the aluminum values from Chapter 3. These tables show the log inactivation numbers of the spores which was obtained by taking the number of spores at treatment time 0 hours and subtracting the number of spores left at the end of each test. The log of this number is presented in the tables below. The tables also show the time (if applicable) when all spores were inactivated.

Table 4 - 6- High direct inoculation test summaries, plastic and aluminum coupons

\begin{tabular}{|c|c|c|c|c|c|c|}
\hline \multirow[b]{2}{*}{$\begin{array}{c}\text { Test } \\
\text { Condition }\end{array}$} & \multirow[b]{2}{*}{ Temp } & \multirow[b]{2}{*}{$\begin{array}{l}\text { Relative } \\
\text { Humidity }\end{array}$} & \multicolumn{2}{|c|}{ Plastic coupons } & \multicolumn{2}{|c|}{ Aluminum coupons } \\
\hline & & & $\begin{array}{c}\log \\
\text { inactivated }\end{array}$ & $\begin{array}{l}\text { Time at full } \\
\text { inactivation }\end{array}$ & $\begin{array}{c}\log \\
\text { inactivated }\end{array}$ & $\begin{array}{l}\text { Time at full } \\
\text { inactivation }\end{array}$ \\
\hline 1 & $180^{\circ} \mathrm{F}$ & $90 \%$ & $6.06^{*}$ & $48 \mathrm{hrs}$ & $6.10 *$ & $48 \mathrm{hrs}$ \\
\hline 3 & $180^{\circ} \mathrm{F}$ & $70 \%$ & 1.79 & NA & 0.81 & NA \\
\hline 5 & $170^{\circ} \mathrm{F}$ & $80 \%$ & 3.14 & NA & 3.26 & NA \\
\hline 7 & $160^{\circ} \mathrm{F}$ & $90 \%$ & 4.20 & NA & 0.47 & NA \\
\hline 9 & $160^{\circ} \mathrm{F}$ & $70 \%$ & 0.11 & NA & 0.40 & NA \\
\hline
\end{tabular}

*Full inactivation 
Table 4 - 7 - Low direct inoculation test summaries, plastic and aluminum coupons

\begin{tabular}{|c|c|c|c|c|c|c|}
\hline \multirow[b]{2}{*}{$\begin{array}{c}\text { Test } \\
\text { Condition }\end{array}$} & \multirow[b]{2}{*}{ Temp } & \multirow[b]{2}{*}{$\begin{array}{l}\text { Relative } \\
\text { Humidity }\end{array}$} & \multicolumn{2}{|c|}{ Plastic coupons } & \multicolumn{2}{|c|}{ Aluminum coupons } \\
\hline & & & $\begin{array}{c}\text { Log } \\
\text { inactivated }\end{array}$ & $\begin{array}{l}\text { Time at full } \\
\text { inactivation }\end{array}$ & $\begin{array}{c}\text { Log } \\
\text { inactivated }\end{array}$ & $\begin{array}{l}\text { Time at full } \\
\text { inactivation }\end{array}$ \\
\hline 1 & $180^{\circ} \mathrm{F}$ & $90 \%$ & $4.56 *$ & $48 \mathrm{hrs}$ & $4.45 *$ & $48 \mathrm{hrs}$ \\
\hline 3 & $180^{\circ} \mathrm{F}$ & $70 \%$ & 2.26 & NA & 1.20 & NA \\
\hline 5 & $170^{\circ} \mathrm{F}$ & $80 \%$ & $4.71 *$ & $48 \mathrm{hrs}$ & $4.72 *$ & $96 \mathrm{hrs}$ \\
\hline 7 & $160^{\circ} \mathrm{F}$ & $90 \%$ & $4.65 *$ & $120 \mathrm{hrs}$ & 0.85 & NA \\
\hline 9 & $160^{\circ} \mathrm{F}$ & $70 \%$ & 0.66 & NA & 1.06 & NA \\
\hline
\end{tabular}

*Full inactivation

Table 4 - 8-Aerosol deposistion test summaries, plastic and aluminum coupons

\begin{tabular}{|c|c|c|c|c|c|c|}
\hline \multirow{2}{*}{$\begin{array}{c}\text { Test } \\
\text { Condition }\end{array}$} & \multirow{2}{*}{ Temp } & \multirow{2}{*}{$\begin{array}{c}\text { Relative } \\
\text { Humidity }\end{array}$} & $\begin{array}{c}\text { Plastic coupons } \\
\text { inactivated }\end{array}$ & $\begin{array}{c}\text { Time at full } \\
\text { inactivation }\end{array}$ & $\begin{array}{c}\text { Log } \\
\text { inactivated }\end{array}$ & $\begin{array}{c}\text { Time at full } \\
\text { inactivation }\end{array}$ \\
\hline 1 & $180^{\circ} \mathrm{F}$ & $90 \%$ & $3.37^{*}$ & $24 \mathrm{hrs}$ & $3.48^{*}$ & $24 \mathrm{hrs}$ \\
\hline 3 & $180^{\circ} \mathrm{F}$ & $70 \%$ & $4.12^{*}$ & $48 \mathrm{hrs}$ & $3.49^{*}$ & $96 \mathrm{hrs}$ \\
\hline 5 & $170^{\circ} \mathrm{F}$ & $80 \%$ & $3.12^{*}$ & $24 \mathrm{hrs}$ & $4.16^{*}$ & $72 \mathrm{hrs}$ \\
\hline 7 & $160^{\circ} \mathrm{F}$ & $90 \%$ & $2.95^{*}$ & $24 \mathrm{hrs}$ & $4.17^{*}$ & $120 \mathrm{hrs}$ \\
\hline 9 & $160^{\circ} \mathrm{F}$ & $70 \%$ & 1.95 & NA & $3.72^{*}$ & $120 \mathrm{hrs}$ \\
\hline
\end{tabular}

*Full inactivation

\section{Direct inoculation inactivation rate comparisons}

Analysis was performed to determine if the inactivation rates were significantly different for aluminum and plastic for the direct inoculation methods. This was completed by using linear regression analyses in Minitab with the response variable being the log of the spores +1 . The explanatory variables were a dummy variable ( 0 for aluminum; 1 for plastic), treatment time, and then the dummy variable * time. Each separate test condition was analyzed in this manner to compare the slope of the line and thus the inactivation rates. Tables 4-9 and 4-10 show the pvalues for each of these analyses and whether the slopes of the lines are significantly different $(\mathrm{p}<0.05)$ 
Table 4 - 9 - Slope comparison, high direct inoculations

\begin{tabular}{|c|c|c|c|c|}
\hline $\begin{array}{c}\text { Test } \\
\text { Condition }\end{array}$ & Temperature & $\begin{array}{c}\text { Relative } \\
\text { Humidity }\end{array}$ & p-value & $\begin{array}{c}\text { Significantly } \\
\text { different? }\end{array}$ \\
\hline 1 & $180^{\circ} \mathrm{F}$ & $90 \%$ & 0.858 & No \\
\hline 3 & $180^{\circ} \mathrm{F}$ & $70 \%$ & 0.793 & No \\
\hline 5 & $170^{\circ} \mathrm{F}$ & $80 \%$ & 0.018 & Yes \\
\hline 7 & $160^{\circ} \mathrm{F}$ & $90 \%$ & 0.000 & Yes \\
\hline 9 & $160^{\circ} \mathrm{F}$ & $70 \%$ & 0.124 & No \\
\hline
\end{tabular}

Table 4 - 10 - Slope comparison, low direct inoculations

\begin{tabular}{|c|c|c|c|c|}
\hline $\begin{array}{c}\text { Test } \\
\text { Condition }\end{array}$ & Temperature & $\begin{array}{c}\text { Relative } \\
\text { Humidity }\end{array}$ & p-value & $\begin{array}{c}\text { Significantly } \\
\text { different? }\end{array}$ \\
\hline 1 & $180^{\circ} \mathrm{F}$ & $90 \%$ & 0.880 & No \\
\hline 3 & $180^{\circ} \mathrm{F}$ & $70 \%$ & 0.253 & No \\
\hline 5 & $170^{\circ} \mathrm{F}$ & $80 \%$ & 0.882 & No \\
\hline 7 & $160^{\circ} \mathrm{F}$ & $90 \%$ & 0.000 & Yes \\
\hline 9 & $160^{\circ} \mathrm{F}$ & $70 \%$ & 0.811 & No \\
\hline
\end{tabular}

\section{Aerosol deposition inactivation rate comparisons}

Analysis was performed to determine the D-value, which is the time when $90 \%$ of the microorganisms are inactivated (Prescott, et al, 2002). The spore values were graphed using Microsoft Office Excel ${ }^{\circledR} 2007$, with a trend line plotted. These trend lines were chosen based on the best fit, with a goal of $r^{2}$ being 0.95 or higher. The equations for the trend lines were used to calculate when $90 \%$ of the spores were inactivated. These values were analyzed using SAS 9.2 using a tobit model, which is a censored regression model designed to estimate linear relationships when one of the variables to be compared is censored. For this case, the values were censored if the spores were not inactivated fully within the time limits. The only censored values were for test condition 9 for plastic coupons. The D-values and corresponding p-values are in Table 4-11. 
Table 4 - 11 - D-value analysis, plastic versus aluminum coupons

\begin{tabular}{|c|c|c|c|c|c|c|}
\hline \multirow{2}{*}{$\begin{array}{c}\text { Test } \\
\text { Condition }\end{array}$} & \multirow{2}{*}{ Temperature } & \multirow{2}{*}{$\begin{array}{l}\text { Relative } \\
\text { Humidity }\end{array}$} & \multicolumn{2}{|c|}{ D-value* } & \multirow{2}{*}{ p-value } & \multirow{2}{*}{$\begin{array}{c}\text { Significantly } \\
\text { different? }\end{array}$} \\
\hline & & & Aluminum & Plastic & & \\
\hline 1 & $180^{\circ} \mathrm{F}$ & $90 \%$ & 21.6 & 21.6 & 1 & No \\
\hline 3 & $180^{\circ} \mathrm{F}$ & $70 \%$ & 66.03 & 22.18 & $<0.0001$ & Yes \\
\hline 5 & $170^{\circ} \mathrm{F}$ & $80 \%$ & 43.64 & 21.60 & 0.007 & Yes \\
\hline 7 & $160^{\circ} \mathrm{F}$ & $90 \%$ & 91.3 & 21.6 & $<0.0001$ & Yes \\
\hline 9 & $160^{\circ} \mathrm{F}$ & $70 \%$ & 88.72 & 102.1 & 0.2659 & $\mathrm{No}$ \\
\hline
\end{tabular}

$* \mathrm{D}$-value is defined as the time (in hours) to inactivate $90 \%$ of the spores.

\section{DISCUSSION}

The goal of this research project was to determine if a Bacillus anthracis simulant could be effectively decontaminated from a plastic coupon using high heat and humidity. Plastic decontamination tests showed full spore inactivation for the high inoculation $\left(10^{6}\right.$ spores per coupon) after 48 hours with $180{ }^{\circ} \mathrm{F}$ and $90 \% \mathrm{RH}$ (test condition 1) and partially inactivated at $170^{\circ} \mathrm{F}$ and $80 \% \mathrm{RH}$ (test condition 5 ), $180^{\circ} \mathrm{F}$ and $70 \% \mathrm{RH}$ (test condition 3 ), and $160^{\circ} \mathrm{F}$ and 90\% RH (test condition 7). Test condition 9 had minimal to no inactivation on the spores during the time limit of 120 hours. Tests with low direct inoculation $\left(10^{4}\right.$ spores per coupon $)$ showed complete spore kills at 48 hours when treatment was $180{ }^{\circ} \mathrm{F}$ with $90 \% \mathrm{RH}$ (test condition 1) and $170{ }^{\circ} \mathrm{F}$ with $80 \% \mathrm{RH}$ (test condition 5). Additionally, all spores were inactivated at 120 hours $160^{\circ} \mathrm{F}$ with $90 \%$ (test condition 7). Finally, all spores deposited by aerosols were inactivated within 48 hours, except for test condition 9 (160 ${ }^{\circ} \mathrm{F}$ with $\left.70 \% \mathrm{RH}\right)$, which still had active spores at the 120 hour point.

The stepwise regression resulted in approximately the same number of terms being retained in the models with high, low, and aerosol deposition have 7, 6, and 8 terms, respectively. Besides the mandatory variables (time, temperature, and humidity), there were no variables retained in all three models. This analysis does indicate humidity is a critical factor, as nearly all variables retained in these models contain humidity-each model only has one variable 
that does not contain humidity. The $\mathrm{R}^{2}$ values are reasonable for these models, with the values being $76.6 \%, 68.8 \%$, and $77.8 \%$, for high and low direct inoculation and aerosol deposition, respectively. Thus most of the variability for the spore inactivation is explained by the models.

The ideal humidity and temperature range for plastic coupon decontamination is clearly the highest levels that can delivered and maintained through the time being decontaminated. If $90 \%$ humidity cannot be easily generated or maintained throughout the body of an aircraft, the results show that $80 \%$ at the proper temperature $\left(170^{\circ} \mathrm{F}\right.$ or higher $)$ can be effective as well. For the plastic coupons, all spores were inactivated within 48 hours using the highest temperature and humidity levels. No other levels completely inactivated the spores at the high inoculations. Low inoculations were inactive within 48 and 120 hours using test conditions 5 and 7, respectfully. The aerosol deposited spores were all inactivated within 48 hours, except for test condition 9. This data shows that the higher the temperature and humidity levels, the more effective the decontamination will be.

A final evaluation was completed to determine if there was a significant difference in the inactivation rates of the plastic or aluminum materials. Analysis was completed on both direct inoculations by comparing the line slopes for the different coupons at each test condition. For high direction inoculation, there was a significant difference for test condition $5\left(170^{\circ} \mathrm{F}\right.$ with $80 \% \mathrm{RH})$ and test condition $7\left(160^{\circ} \mathrm{F}\right.$ with $\left.90 \% \mathrm{RH}\right)$, with inactivation being faster on the plastic coupons. It should be noted however that test condition 1 may not have been significantly different because all spores were inactivated at the 48 hour time point for both plastic and aluminum. For low direct inoculation, only test condition $7\left(160^{\circ} \mathrm{F}\right.$ with $\left.90 \% \mathrm{RH}\right)$ was significantly different, with the inactivation occurring faster for plastic coupons again. Also, all spores were inactivated at the 48 hour mark for test condition 1 , which may be why they were 
not significantly different at that level. A tobit analysis was completed to compare plastic and aluminum inactivation after aerosol deposition, showing the inactivation rates were significantly different for test conditions $3\left(180^{\circ} \mathrm{F}\right.$ with $\left.70 \% \mathrm{RH}\right), 5\left(170^{\circ} \mathrm{F}\right.$ with $\left.80 \% \mathrm{RH}\right)$, and $7\left(160^{\circ} \mathrm{F}\right.$ with $90 \% \mathrm{RH})$ with the corresponding rates being faster for plastic coupons $(\mathrm{p}<0.05)$. Test condition 1 was not significantly different; however, all the spores were inactivated for both materials within 24 hours at that temperature and humidity. It is worth noting that for test condition 9, the mean value for spore inactivation was lower for aluminum coupons; however, this was not statistically significant. All other test conditions showed the plastic coupons were inactivated at a faster rate than then aluminum coupons.

Analyzing the results from the aluminum coupon data from Chapter 3, it is evident that the optimal temperature and humidity ranges for both coupons are the highest levels that can be effectively maintained within engineering specifications. Test condition $1\left(180^{\circ} \mathrm{F}\right.$ with $\left.90 \% \mathrm{RH}\right)$ inactivated all spores on all coupons with all the inoculation methods. This was the only test condition that met this goal. Test condition $5\left(170^{\circ} \mathrm{F}\right.$ with $\left.80 \% \mathrm{RH}\right)$ inactivated all spores for all tests except for the high direct inoculation on plastic coupons. It is important to note, however, that the spores for this test condition were trending down at the upper time limit for test condition 5.

Another key result of these studies shows that the decontamination method of high heat and humidity will inactivate plastics and aluminum at approximately the same times. The studies showed that only 6 of 15 tests were significantly different for the plastics versus aluminum coupons, with plastic coupons being inactivated at a faster rate. That shows that when the spores are inactivated on aluminum, they will be inactivated on plastic. 
Future research could focus on extending the treatment times for the test conditions that did not have full inactivation at the 120 hour time point. This would include test conditions 3,5 , 7, and 9 for the high inoculations; test conditions 3 and 9 for the low inoculations; and test condition 9 for the aerosol deposition. This data would be useful when the higher temperature and humidity levels are more difficult to maintain and a longer time period is allowed for decontamination efforts. Shorter time evaluations would be beneficial for the aerosol deposited data as well - this is because the spores were all inactivated at several test conditions within 24 hours. Future tests could include a time period of 12 hours. Additionally, more aircraft materials should be tested. This research showed that spores on plastics are inactivated more quickly than aluminum, a critical piece of information; however, other materials need to be tested to verify this. Such materials could include other metals, plastics, or aircraft fabrics (canvas). One limitation of the study is that the analysis only included those spores that could germinate, that is, those spores that could culture. There could still be spores that are active but could not be cultured. Additionally, clumping of spores could have resulted which could lead to agglomerated particles containing more than one spore. This was alleviated as much as possible with the use of Collison nebulizer, which generates aerosols from 1 to $3 \mu \mathrm{m}$ in diameter. Even with this small size, spores could still clump after generation and until deposition. This could impact the spore decontamination because a spore clump may be more difficult to inactivate (compared to a single spore). Future studies should evaluate this more closely through the use of electron microscopy throughout all stages of the experiments. 


\section{Conclusion}

The goal of this research was to determine if a Bacillus anthracis simulant could be decontaminated from plastic coupons using high heat and humidity. The results show that spores can be inactivated if the proper temperature and humidity levels are applied. There is a difference in the method of spore application, with those deposited via aerosol deposition having a more effective inactivation within the allotted time. These results also confirm that when higher spore levels are inactivated, the lower levels of spores, delivered by direct inoculation or aerosol deposition, will also be inactivated. Additionally, comparisons of plastic versus aluminum coupons showed that plastic coupons were decontaminated quicker for 6 of 15 tests. Again, this shows that if the aluminum coupons are effectively decontaminated, the plastic materials will be as well. Overall, this research showed the spores can be effectively inactivated using high heat and humidity at specific combinations of these variables coupled with time. This shows promise for future efforts to inactivate biological agents safely, effectively, and also within aircraft engineering specifications.

\section{ACKNOWLEDGEMENTS}

This study was funded in part by a grant from the Mountain and Plains Research Center (MAP ERC) through Dr. Reynolds, Deputy Director. Additional funds were provided through a grant from AFMSA/SGRS. Funds were committed with the assistance of William Kilpatrick

(711 HPQ/RHXS), Gregory Sudberry (711 HPW/RHPC), SSgt Robert Arrington (711 HPW/RHPCB), SrA Ashley Grant (ASC/PKOA), and Tim Provens (711 HPW/RHPC). Plastic coupons were provided by Dr. Ken Heater and Daniel E. Badowski, Research Scientist, METSS Corporation. Spores were provided by Joe Dalmasso, Yakibou, Inc. 


\section{REFERENCES}

Air Force Research Laboratory (AFRL): Bio-thermal decontamination tests for large frame aircraft application laboratory and field test report, AFRL Document HEPC OSMSWP8Z, 5 Dec 2008.

Aizenberg, V., S.A. Grinshpun, K. Willeke, J. Smith, and P.A. Baron: Performance characteristics of the button personal inhalable aerosol sampler. American Industrial Hygiene Association Journal 61: 398-404 (2000a).

Andersen, B.M., M. Rasch, K. Hochlin, F.H. Jensen, P. Wismar, and J.E. Fredriksen: Decontamination of rooms, medical equipment and ambulances using an aerosol of hydrogen peroxide disinfectant. Journal of Hospital Infection Hosp Infection 62: 149-155 (2006).

Baron, P.A., C.F. Estill, J.K. Beard, M.J. Hein, and L. Larsen: Bacterial endospore inactivation caused by outgassing of vapourous hydrogen peroxide from polymethyl methacrylate (Plexiglas $\left.{ }^{\circledR}\right)$. Letters in Applied Microbiology 45: 485-490 (2007).

Baron, P.A., C.F. Estill, G.J. Deye, M.J. Heim, J.K. Beard, L.D. Larsen, and G.E. Dahlstrom: Development of an aerosol system for uniformly depositing Bacillus anthracis spore particles on surface. Aerosol Science and Technology 42: 159-172 (2008).

Barth, E., R. Rupert, F. Stroud, E. Rice, and B. Potoka: Environmental response to intentional dissemination of Bacillus anthracis spores in the United States 2001. Risk Management Research 13: 99-111 (2003).

BGI, Inc: Collison Nebulizer Droplet Number Output (Online) Available at http://bgiusa.com/agc/droplet_number_output.htm (accessed March 2011).

Brickhouse, M.: Innovative and Emerging Decontamination Technologies. In Report on Workshop on Decontamination, Cleanup and Associated Issues for Sites Contaminated with Chemical, Biological, or Radiological Materials, S. Dun, and J. Wood, US EPA: October 2005.

Brown, G.S.; R.G. Betty, J.E. Brockman, D.A. Lucero, C.A. Souza, K.S. Walsh, R.M. Boucher, M.S. Tezak, and M.C. Wilson: Evaluation of vacuum filter sock surface sample collection method for Bacillus spores from porous and non-porous surfaces. Journal of Environmental Monitoring 9(7): 666-671 (2007a).

Brown, G.S., R.G. Betty, J.E. Brockman, D.A. Lucero, C.A. Souza, K.S. Walsh, R.M. Boucher, M. Tezak, M.C. Wilson, and T. Rudolph: Evaluation of a wipe surface sample method for collection of Bacillus spores from nonporous surfaces. Applied and Environmental Microbiology 73(3): 706-710 (2007b).

Brown, G.S., R.G. Betty, J.E. Brockmann, D.A. Lucero, C.A. Souza, K.S. Walsh, R.M. Boucher, M.S. Tezak, M.C. Wilson, T. Rudolph, H.D. Lindquist, and K.F. Martinez: 
Evaluation of rayon swab surface sample collection method for Bacillus spores from nonporous surfaces. Journal of Applied Microbiology 103(4): 1074-1080 (2007c).

Burgess, W.A., M.J. Ellenbecker, and R.D. Treitman: Ventilation for Control of the Work Environment. Hoboken, NJ: John Wiley \& Sons, 2004.

Burke, S.A., J.D. Wright, M.K. Robinson, B.V. Bronk, and R.L. Warren: Detection of molecular diversity in Bacillus atrophaeus by amplified fragment length polymorphism analysis. Applied and Environmental Microbiology 79(5): 2786-2790 (2004).

Burton, N.C., A. Adhikari, S.A. Grinshpun, R. Hornung, and T. Reponen: The effect of filter material on bioaerosol collection of Bacillus subtilis spores used as a Bacillus anthracis stimulant. Journal of Environmental Monitoring 7: 475-480 (2005).

Buttner, M. P., P. Cruz, L.D. Stetzenbach, A.K. Klima-Comba, V.L. Stevens, and P.A. Emanuel: Evaluation of the biological sampling kit (BiSKit) for large-area surface sampling. Applied Environmental Microbiology 70: 7040-7045 (2004).

Byrne, MA; A.J.H. Goddard, C. Lange, and J. Roed: Stable tracer aerosol deposition measurements in a test chamber. Journal of Aerosol Science 26(4): 645-653 (1995).

Canter, D.A., D. Gunning, P. Rodgers, L. O'Connor, C. Traunero, and C.J. Kempter: Remediation of Bacillus anthracis contamination in the U.S. Department of Justice mail facility. Biosecurity Bioterror 3(2): 119-127 (2005).

Centers for Disease Control: Investigation of bioterrorism-related anthrax - Connecticut. Morb Mortal Wkly Rep, 50: 1077-1079 (2001).

Chen, B.T., M.D. Hoover, G.J. Newton, S.J. Montano, and D.S. Gregory: Performance evaluation of the sampling head and annular kinetic impactor in the Savannah River site alpha continuous air monitor. Aerosol Science and Technology 31: 24-38 (1999).

Cordesman, A.H.: The Challenge of Biological Terrorism. Washington, DC: The CSIS Press (Center for Strategic and International Studies), 2005.

Chosewood, L.C., and D.E. Wilson, eds.: Biosafety in Microbiological and Biomedical Laboratories, $5^{\text {th }}$ Ed., Pub. No. 21-1112. Atlanta, GA: US Department of Health and Human Services, Centers for Disease Control, NIH, 1992.

Department of Defense: The Chemical, Biological, Radiological, and Nuclear (CBRN) Survivability Policy, Pub No. DoDI 3150.09 (August 17, 2009)

Edmonds, J.M., P.J. Collett, E.R. Valdes, E.W. Skowronski, G.J. Pellar, and P.A. Emanuel: Surface sampling of spores in dry-deposition aerosols. Applied and Environmental Microbiology 75(1): 39-44 (2009). 
Estill, C.F., P.A. Baron, J.K. Beard, M.J. Hein, L.D. Larsen, L. Rose, F.W. Schaefer III, J. Noble-Wang, L. Hodges, H.D.A. Lindquist, G.J. Deye, and M.J. Arduino: Recovery efficiency and limit of detection of aerosolized Bacillus anthracis sterne from environmental surface samples. Applied and Environmental Microbiology 75(13): 4297-4306 (2009).

Estill, C.F., and G.J. Deye: Personal communication, January 7, 2010.

Farnsworth, J.E., S.M. Goyal, S.W. Kim, T.H. Kuehn, P.C. Raynor, M.A. Ramakrishnan, S. Anantharaman, and W. Tang: Development of method for bacteria and virus recovery from heating, ventilation, and air conditioning (HVAC) filters. Journal of Environmental Monitoring 8: 1006-1013 (2006).

Feather, G.A., and B.T. Chen: Design and use of a settling chamber for sampler evaluation under calm-air conditions. Aerosol Science and Technology 37: 261-270 (2003).

Foarde, K.K., J.T. Janley, D.S. Ensor, and P. Roessler: Development of method for measuring single-pass bioaerosol removal efficiencies of a room air cleaner, Aerosol Science Technology 30: 223-234 (1999).

Gale, W.F., S. Hyacinth, and J. Watson: Field Evaluation of Whole Airliner Decontamination Technologies - Wide Body Aircraft with Dual-Use Application for Railcars, Federal Aviation Administration, Pub No. DOT/FAA/AM-08/4, (2008).

Gale, W.F., N.I. Sofyan, H.S. Gale, S.-F. Chou, J.W. Fergus, and C.G. Shannon: Effect of vapour phase hydrogen peroxide, as a decontaminant for civil aviation applications, on microstructure, tensile properties and corrosion resistance of 2024 and 7075 age hardenable aluminum alloys and 304 austenitic stainless steel. Materials Science and Technology 25(1): 7684 (2009).

Herzog, A.B., S.D. McLennan, A.K. Pandey, C.P. Gerba, C.N. Hass, J.B. Rose, and S.A. Hashsham: Implications of limits of detection of various methods for Bacillus anthracis in computing risks to human health. Applied and Environmental Microbiology 75(19): 6331-6339 (2009).

Hill S.C., R.G. Pinnick, S. Niles, Y.L. Pan, S. Holler, R.K. Chang, J. Bottiger, B.T. Chen, C.S. Orr, G. Feather, and A.P. Snyder: Real-time measurement of fluorescence spectra from single airborne biological particles. Field Analysis and Chemical Technology 3: 221-239 (1999).

Jensen, P.A., W.F. Todd, G.N. Davis, and P.V. Scarpino: Evaluation of eight bioaerosol samplers challenged with aerosols of free bacteria. American Industrial Hygiene Association Journal 53: 660-667 (1992).

Jernigan, J.A., D.S. Stephens, D.A. Ashford, C. Omenaca, M.S. Topeil, M. Galbraigh, M. Tapper, T.L. Fish, S. Saki, T. Popovic, R.F. Meyer, C.P. Quinn, S.A. Harper, S.K. Fridkin, J.J. Sejvar, C.W. Shepard, M. McConnell, J. Guarner, W. Sheih, J.M. Malecki, J.L. Gerberding, J.M. Hughes, B.A. Perkins, and members of the Anthrax Bioterrorism 
Investigation Team: Bioterrorism-related inhalational anthrax: the first 10 cases reported in the United States. Emerging Infectious Diseases 7: 933-944 (2001).

Kempter, J.: Crisis Exemptions for Products Intended to Inactivate Bacillus anthracis, In Report on Workshop on Decontamination, Cleanup and Associated Issues for Sites Contaminated with Chemical, Biological, or Radiological Materials, S. Dun, and J. Wood, US EPA: October 2005.

Kenny, L.C., R.J. Aitken, P.E.J. Baldwin, G.C. Beaumont, and A.D. Maynard: The sampling efficiency of personal inhalable aerosol samplers in low air movement environments. Journal of Aerosol Science 30(5): 627-638 (1999).

Kesavan, J., J.R. Bottiger, and A.R. McFarland: Bioaerosol concentrator performance: comparative tests with viable and with solid and liquid nonviable particles. Journal of Applied Microbiology 104: 285-295 (2008).

King, B., J. Kesavan, and J.-L. Sagripanti: Germicidal UV sensitivity of bacteria in aerosols and on contaminated surfaces. Aerosol Science and Technology 45(5): 645-653 (2011).

Koch, W., W. Dunkhort, and H. Lodding: Design and performance of a new personal aerosol monitor. Aerosol Science (1999).

Kournikakis, B., M. Walker, J. Ho et al.: Statistical analysis of bacterial spore aerosols created by opening a spore containing anthrax letter in an office. Journal of Aerosol Science 40: 514-522 (2009).

Lai, A.C.K.; M.A. Byrne, A.J.H. Goddard: Experimental studies of the effect of rough surfaces and air speed on aerosol deposition in a test chamber. Aerosol Science and Technology 36: 973-982 (2002).

Lewandowski, R., K. Kozłowska, M. Szpakowska, M. Stepin'ska, and E.A. Trafny: Use of a foam spatula for sampling surfaces after bioaerosol deposition. Applied and Environmental Microbiology 76(3): 688-694 (2010).

Li, C.S., and Y.C. Lin: Storage effects on bacterial concentration: determination of impinge and filter samples. Science of the Total Environment 278: 231-237 (2001).

Mainelis, G., A. Adhikari, K. Willeke, S. Lee, T. Reponen, and S.A. Grinshpun: Collection of airborne microorganisms by a new electrostatic precipitator. Aerosol Science 33: 1417-1432 (2002).

Marple, V.A., and K.L. Rubow: An aerosol chamber for instrument evaluation and calibration. American Industrial Hygiene Association Journal 44: 361-367 (1983). 
Martin, D. D., and L. M. Moore: Technical report for the comparison of swab-sampling techniques for recovery of bacterial spores from three surfaces. West Desert Test Center document no. WDTC-TR-00-018, U.S. Army Dugway Proving Ground, Utah, (2001).

Maus, R., A. Goppelsroder, and H. Umhauer: Atmospheric Environment 35: 105-113 (2001).

McVey, I.: STERIS Chem-Bio Decontamination, In Report on Workshop on Decontamination, Cleanup and Associated Issues for Sites Contaminated with Chemical, Biological, or Radiological Materials, S. Dun, and J. Wood, US EPA: October 2005.

Nicholson, W.L., P. Fajardo-Cavazos, R. Rebeil, T.A. Slieman, P.J. Riesenman, J.F. Law, and Y. Xue: Bacterial endospores and their significance in stress resistance. Antonie Leeuwenhoek 81: 27-32 (2002).

Oh, S.W., P.M. Gray, R.H. Dougherty, and D.H. Kang: Aerosolization as novel sanitizer delivery system to reduce food-borne pathogens. Lett Appl Microbiol 41: 56-60 (2005).

Orlusky, R.: Restoration From Decontamination: USPS Experience. In Report on Workshop on Decontamination, Cleanup and Associated Issues for Sites Contaminated with Chemical, Biological, or Radiological Materials, S. Dun, and J. Wood, US EPA: October 2005.

Park, J.M., J.C. Rock, L. Wang, Y.C. Seo, A. Bhatnagar, and S. Kim: Performance evaluation of six different aerosol samplers in a particulate matter generation chamber. Atmospheric Environment 43: 280-289 (2009).

Peccia J., H.M. Werth, S. Miller, \& M. Hernandez: Effects of relative humidity on the ultraviolet induced inactivation of airborne bacteria. Aerosol Science Technology 35: 728-740 (2001).

Perez, J., S. Springthorpe, and S.A. Sattar: Activity of selected oxidizing microbicides against the spores of Clostridium difficile: relevance to environmental control. American Journal of Infection Control 33: 320-325 (2005).

Perkins, J.J.: Principles and Methods of Sterilization in Health Sciences,. $2^{\text {nd }}$ ed.. Springfield, IL, Charles C. Thomas Publishers, 1983.

Prescott, L.M., J.P. Harley, and D.A. Klein: Microbiology, $5^{\text {th }}$ ed. New York: McGraw-Hill Higher Education, 2002.

Probst, A., R. Facius, R. Wirth, and C. Moissl-Eichinger: Validation of a nylon-flocked-swab protocol for efficient recovery of bacterial spores from smooth and rough surfaces. Applied and Environmental Microbiology 76(15): 5148-5158 (2010).

Rastogi, V.K., L. Wallace, L.S. Smith, S.P. Ryan, and M. Blair: Quantitative method to determine sporicidal decontamination of building surfaces by gaseous fumigants, and issues 
related to laboratory-scale studies. Applied and Environmental Microbiology 75(11): 3688-3694 (2009).

Rose, L.J., B. Jensen, A. Petersen, S.N. Banerjee, and M.J. Arduino,: Swab materials and Bacillus anthracis spore recovery from nonporous surfaces, Emerging Infectious Disease 10(6): 1023-1029 (2004).

Ryan, J.R., and J.F. Glarum: Biosecurity \& Bioterrorism: Containing and Preventing Biological Threats. Elsevier: New York, 2008.

Sagripanti, J.L., M. Carrera, J. Insalaco, M. Ziemski, J. Rogers, \& R. Zandomeni: Virulent spores of Bacillus anthracis and other bacillus species deposited on solid surfaces have similar sensitivity to chemical decontaminants. Journal of Applied Microbiology 102: 11-21 (2007).

Scheffrahn, R.: Whole-Structure Decontamination of Bacterial Spores by Methyl Bromide Fumigation. In Report on Workshop on Decontamination, Cleanup and Associated Issues for Sites Contaminated with Chemical, Biological, or Radiological Materials, S. Dun, and J. Wood, US EPA: October 2005.

Thatcher, T.L., and W.W. Nazaroff: Effects of small-scale obstructions and surface textures on particle deposition from natural convection flow. Aerosol Science Technology 27: 709-725 (1997).

Thomas, R.J., D. Webber, W. Sellors, A. Collinge, A. Frost, A.J. Stagg, S.C. Bailey, P.N. Jayasekera, R.R. Taylor, S. Eley, R.W. Titball: Characterization and deposition of respirable large- and small-particle bioaerosols. Applied and Environmental Microbiology 74(20): 64376443 (2008).

Uhm, H.S., H.Y. Lee, Y.C. Hong, D.H. Shin, Y.H. Park, Y.F. Hong: A decontamination study of simulated chemical and biological agents. Journal of Applied Physics 102 (2007).

Verce, M.F., B. Jayaraman, T.D. Ford, S.E. Fisher, A.J. Gadgil, and T.M. Carlsen: Minimizing decomposition of vaporized hydrogen peroxide for biological decontamination of galvanized steel ducting. Environ Sci Technol 42: 5765-5771 (2008).

Wagner, A., C.F. Green, V. Pedregon, E. Barth, S.G. Gibbs, and P.V. Scarpino: Inactivation of Bacillus subtilis on gypsum board using aerosolized chemical agents. Journal of Environmental Engineering Science 7: 159-164 (2008).

Wang, Z., T. Reponen, S.A. Grinshpun, R.L. Gorny, and K. Willeke: Effect of sampling time and air humidity on the bioefficiency of filter samplers for bioaerosol collection. Journal of Aerosol Science 32(5): 661-674 (2001).

Watson, A., and D. Keir: Information on which to base assessments of risk from environments contaminated with anthrax spores. Epidemiology and Infection 113: 479-90 (1994). 
Yah, M., and G. Mainelis: Use of portable microbial samples for estimating inhalation exposure to viable biological agents. Journal of Exposure Science and Environmental Epidemiology 17: 31-38 (2007). 


\section{APPENDIX 1: High direct inoculation test plots}

Data from the high direct inoculation tests was plotted using Minitab to show the inactivation rates against temperature and humidity. This included both surface plots and contour plots. Response plots for all of these included the log of the spores +1 .

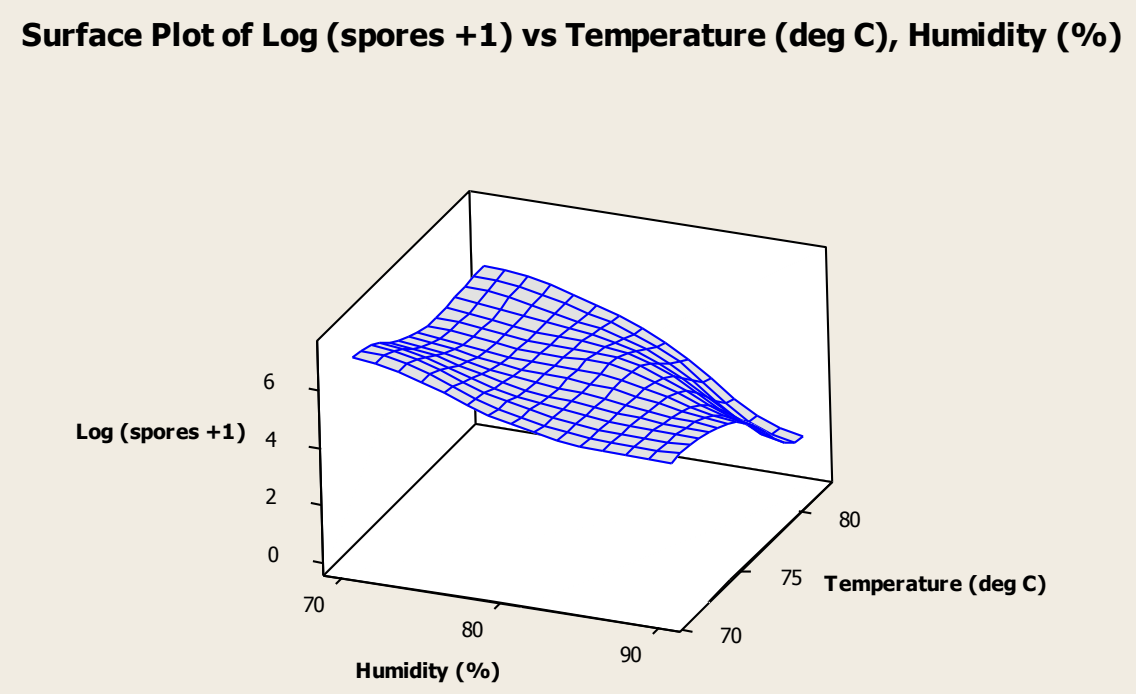

Figure 4- 9- High direct inoculation spore surface plot—-spore log versus temperature and humidity

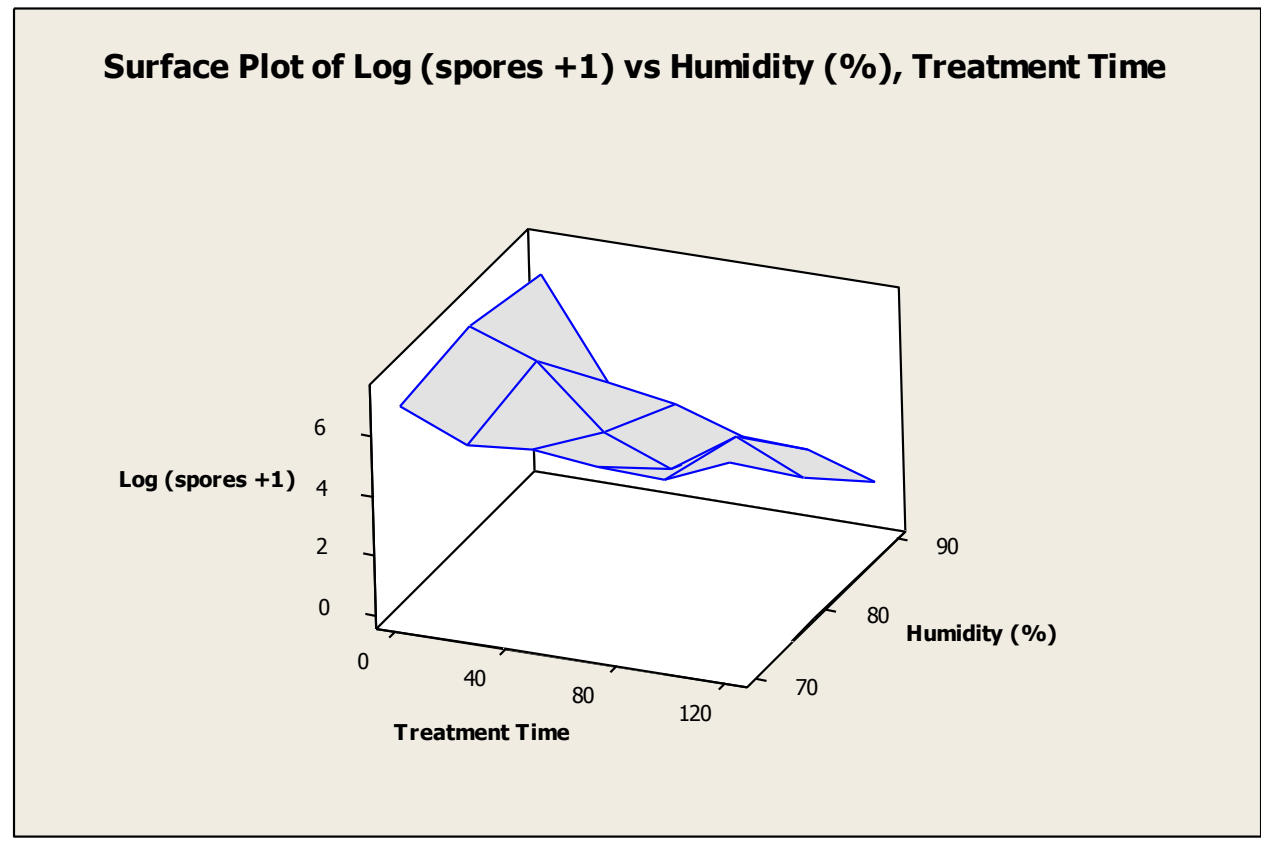

Figure 4- 10 - High direct inoculation spore surface plot—spore log versus humidity and treatment time 


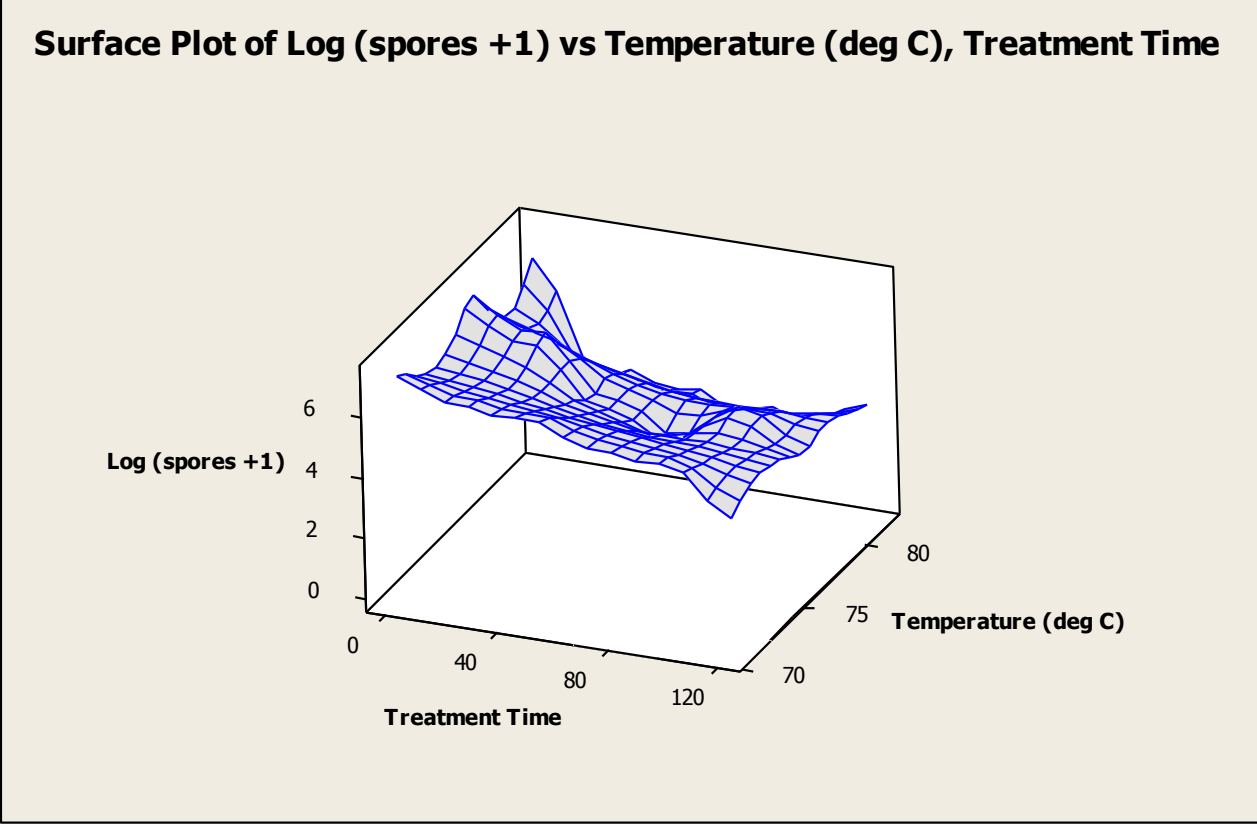

Figure 4- 11 - High direct inoculation spore surface plot—spore log versus temperature and treatment time

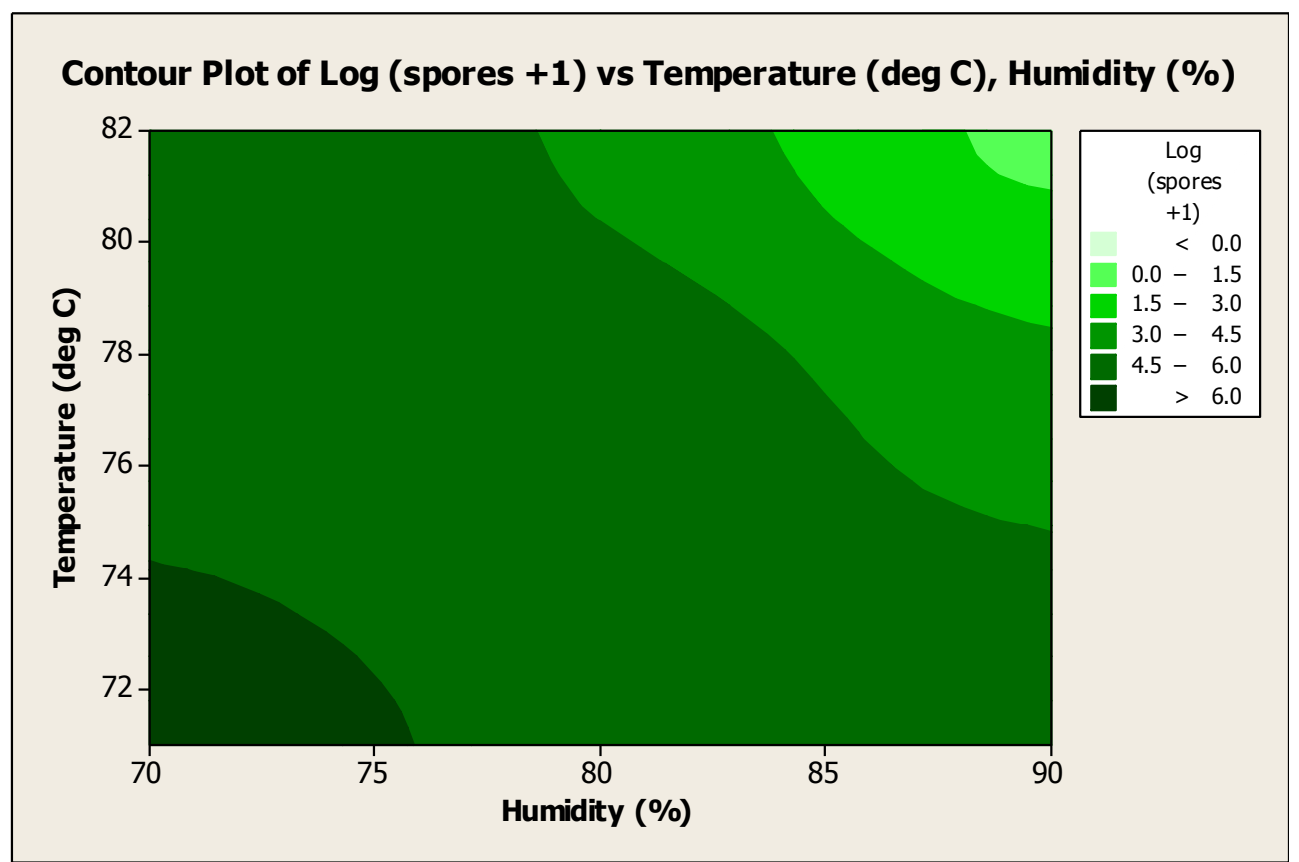

Figure 4- 12 - High direct inoculation contour plot—spore log versus temperature and humidity 


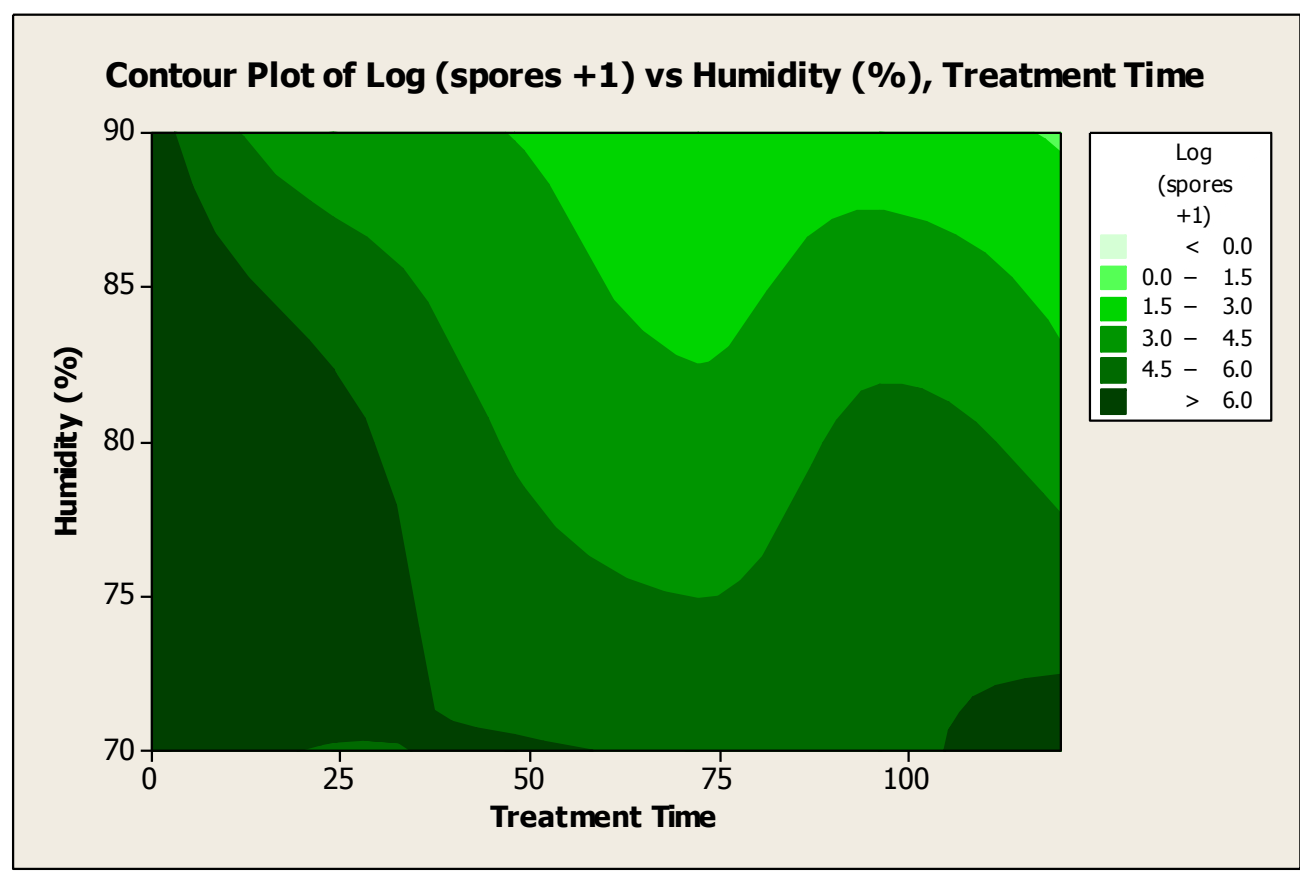

Figure 4- 13 - High direct inoculation contour plot—spore log versus humidity and treatment time

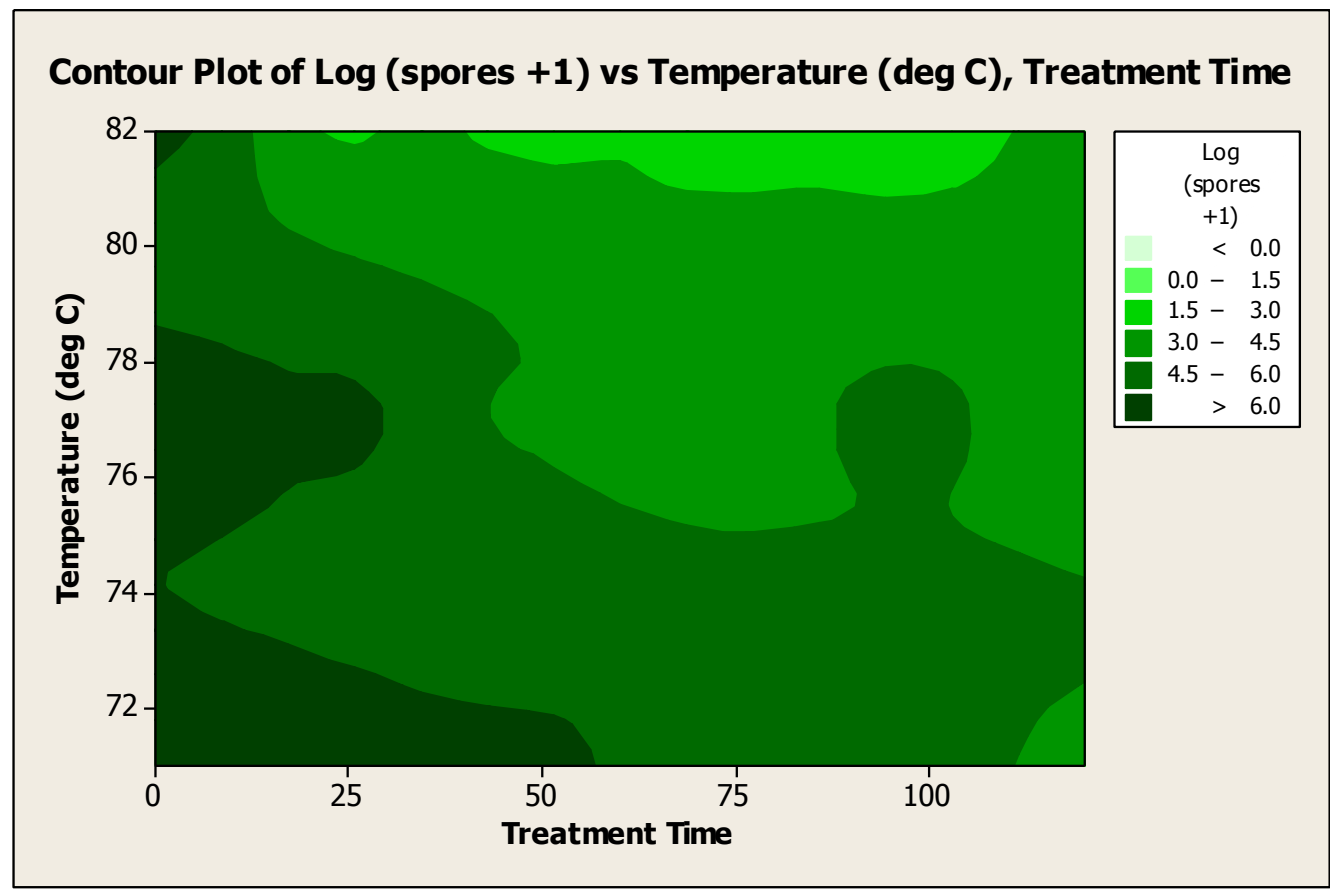

Figure 4- 14 - High direct inoculation contour plot—spore log versus temperature and treatment time 


\section{APPENDIX 2: Low direct inoculation test plots}

Data from the low direct inoculation tests was plotted using Minitab to show the inactivation rates against temperature and humidity. This included both surface plots and contour plots. Response plots for all of these included the log of the spores +1 .

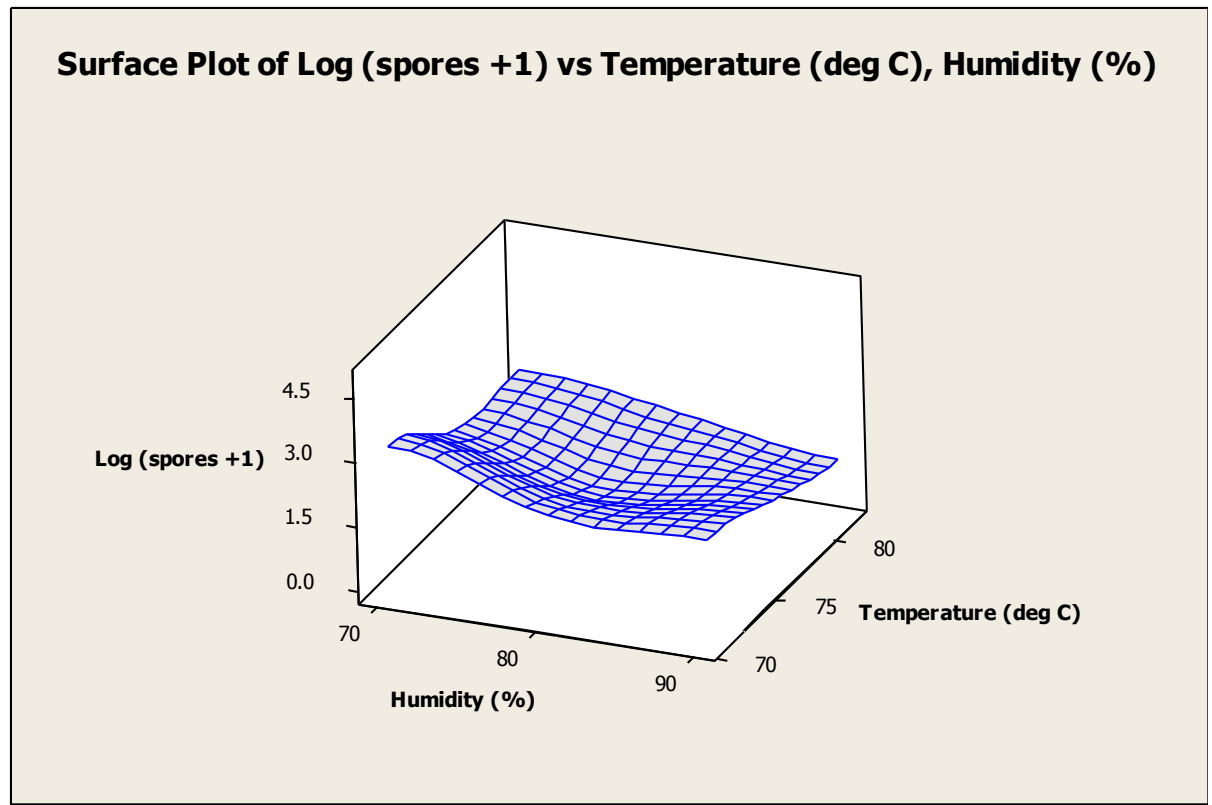

Figure 4- 15 - Low direct inoculation spore surface plot-spore log versus humidity and temperature

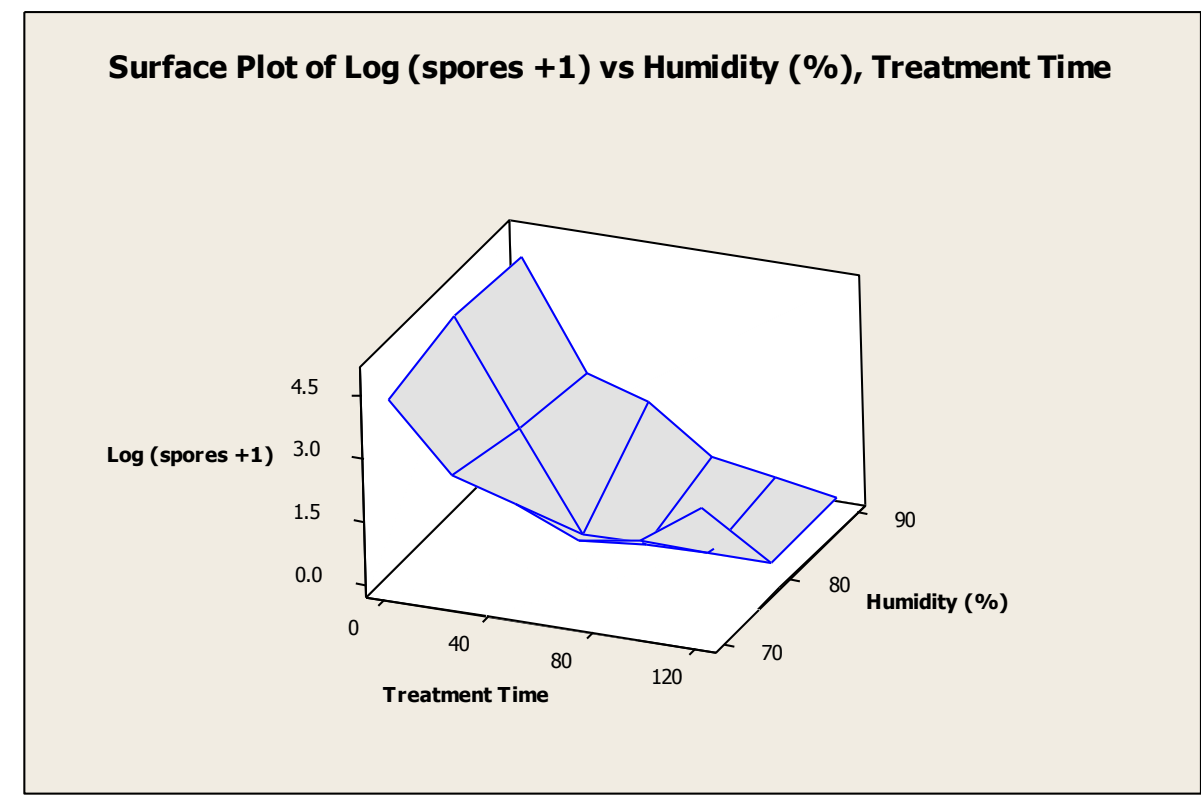

Figure 4- 16 - Low direct inoculation spore surface plot-spore log versus humidity and treatment time 


\section{Surface Plot of Log (spores +1) vs Temperature (deg C), Treatment Time}

Figure 4- 17 - Low direct inoculation spore surface plot-spore log versus temperature and treatment time

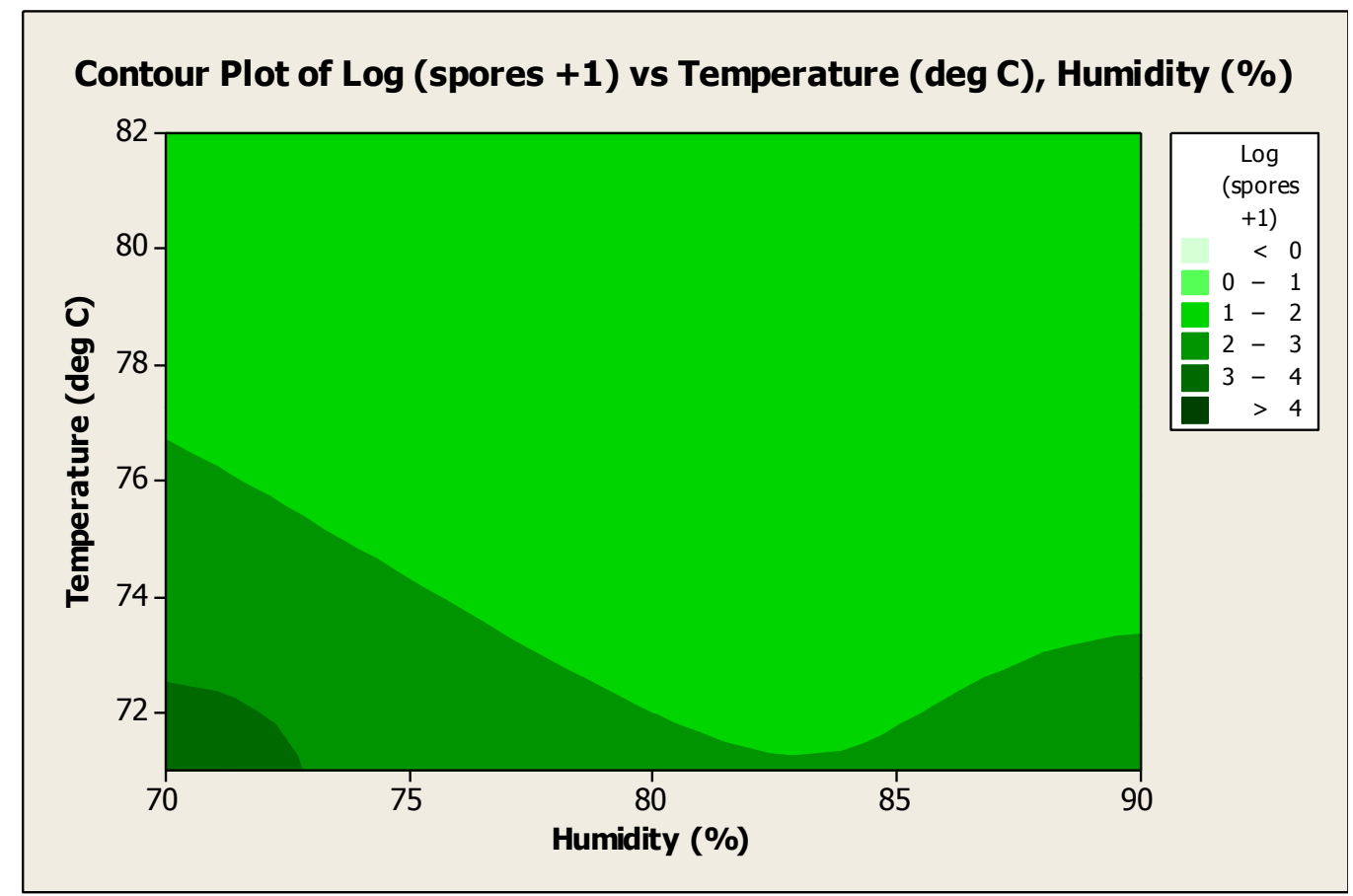

Figure 4- 18 - Low direct inoculation contour plot-spore log versus temperature and humidity 


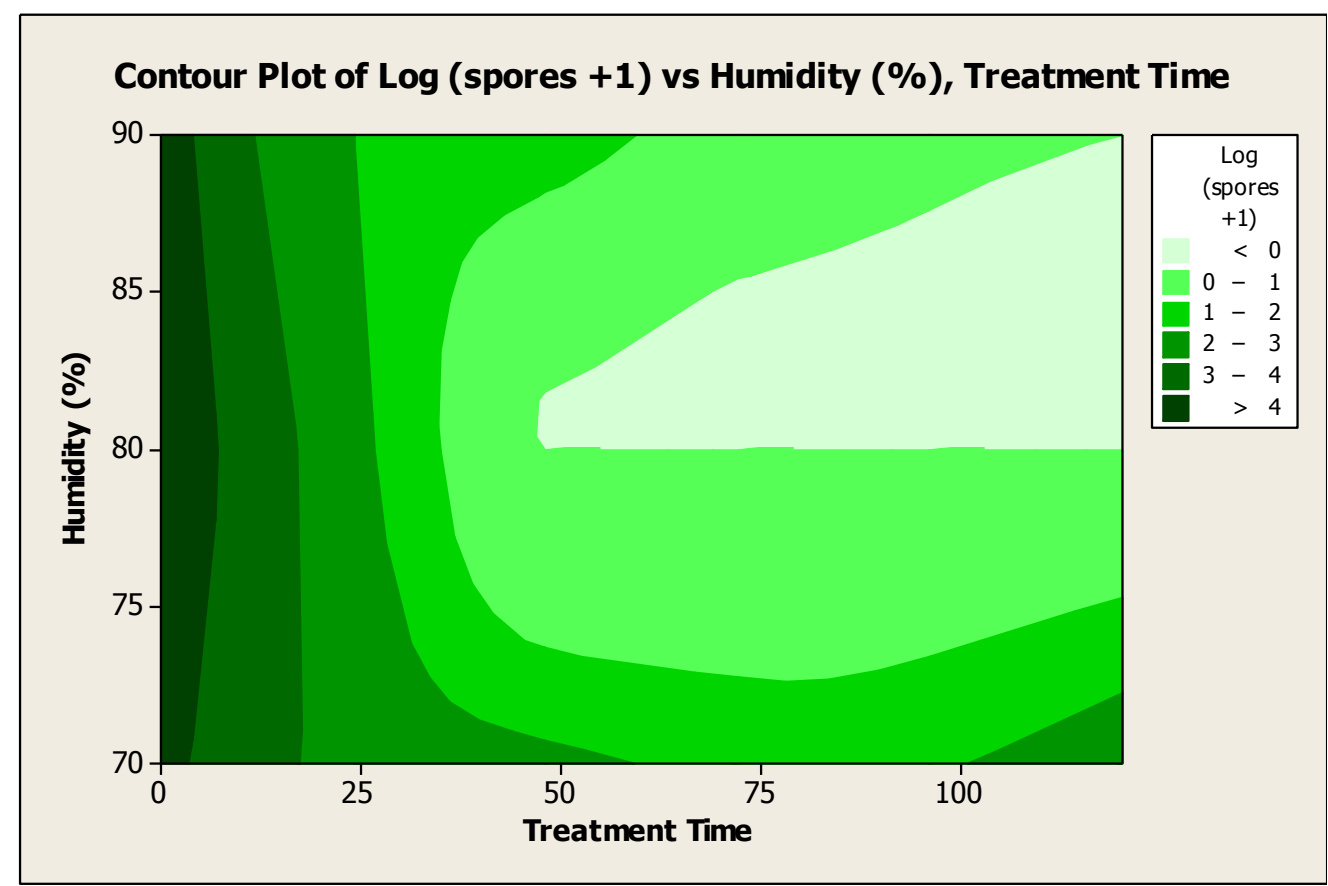

Figure 4- 19- Low direct inoculation contour plot—spore log versus humidity and treatment time

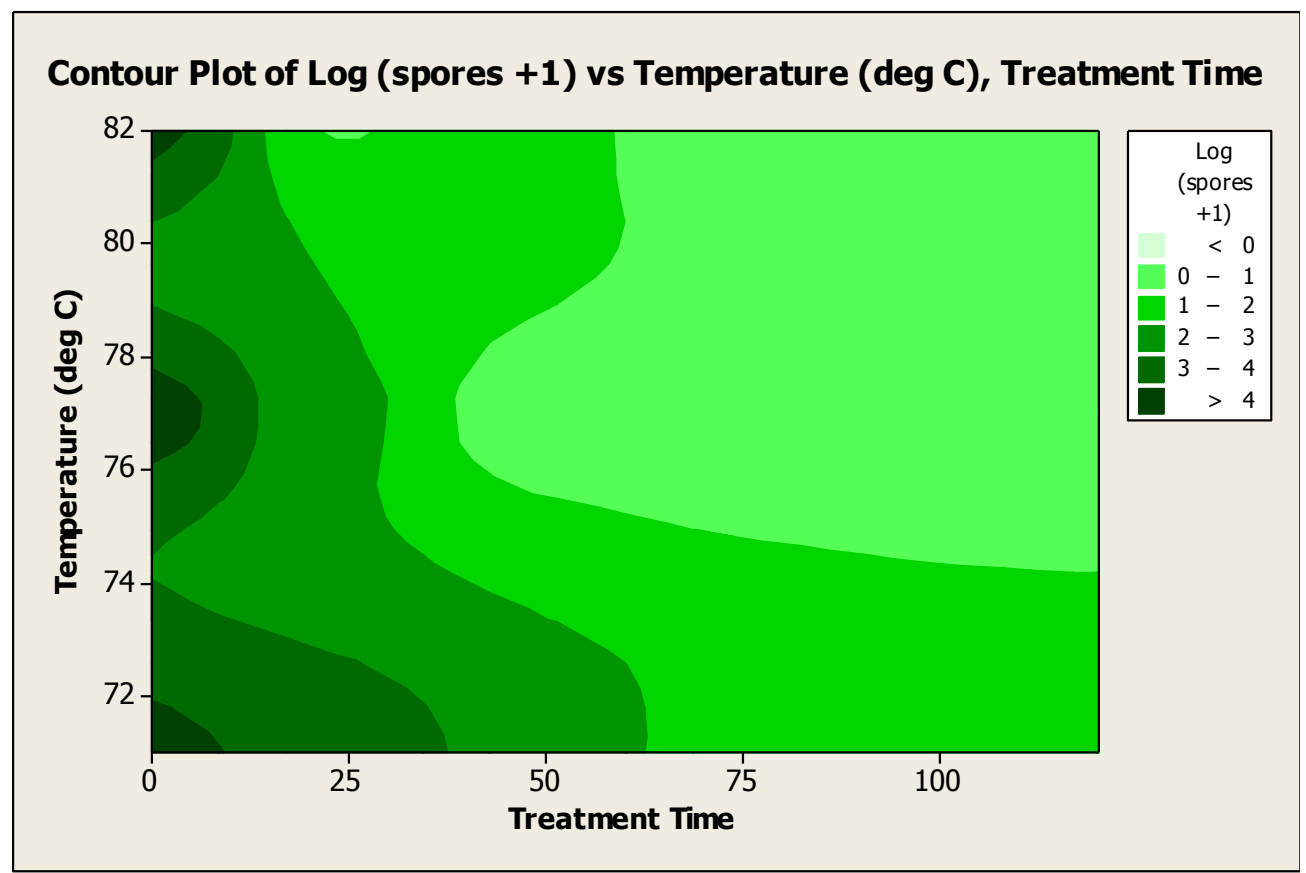

Figure 4- 20 - Low direct inoculation contour plot-spore log versus temperature and treatment time 


\section{APPENDIX 3: Aerosol deposition test plots}

Data from the low direct inoculation tests was plotted using Minitab to show the inactivation rates against temperature and humidity. This included both surface plots and contour plots. Because the all aerosol tests had corresponding controls, the response variables for these plots were the log of the control spores $(+1)$ - the log of the sample spores $(+1)$.

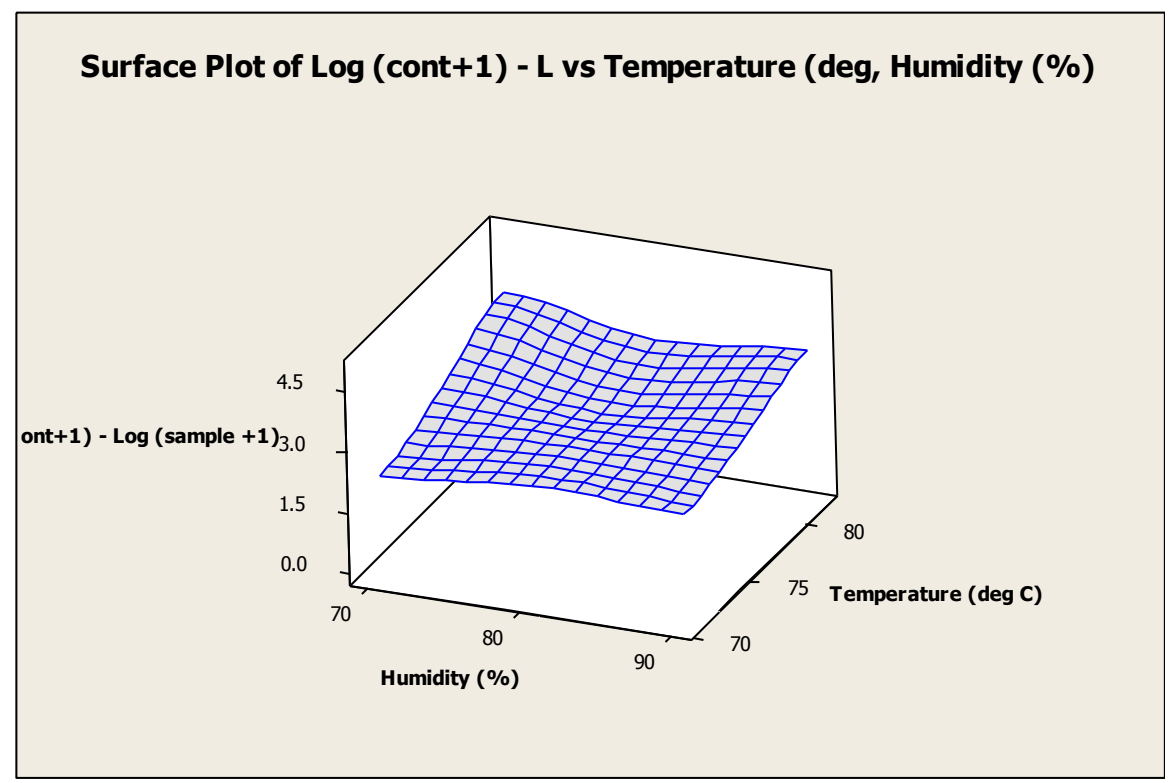

Figure 4- 21 - Aerosol deposition spore surface plot—spore log versus humidity and temperature

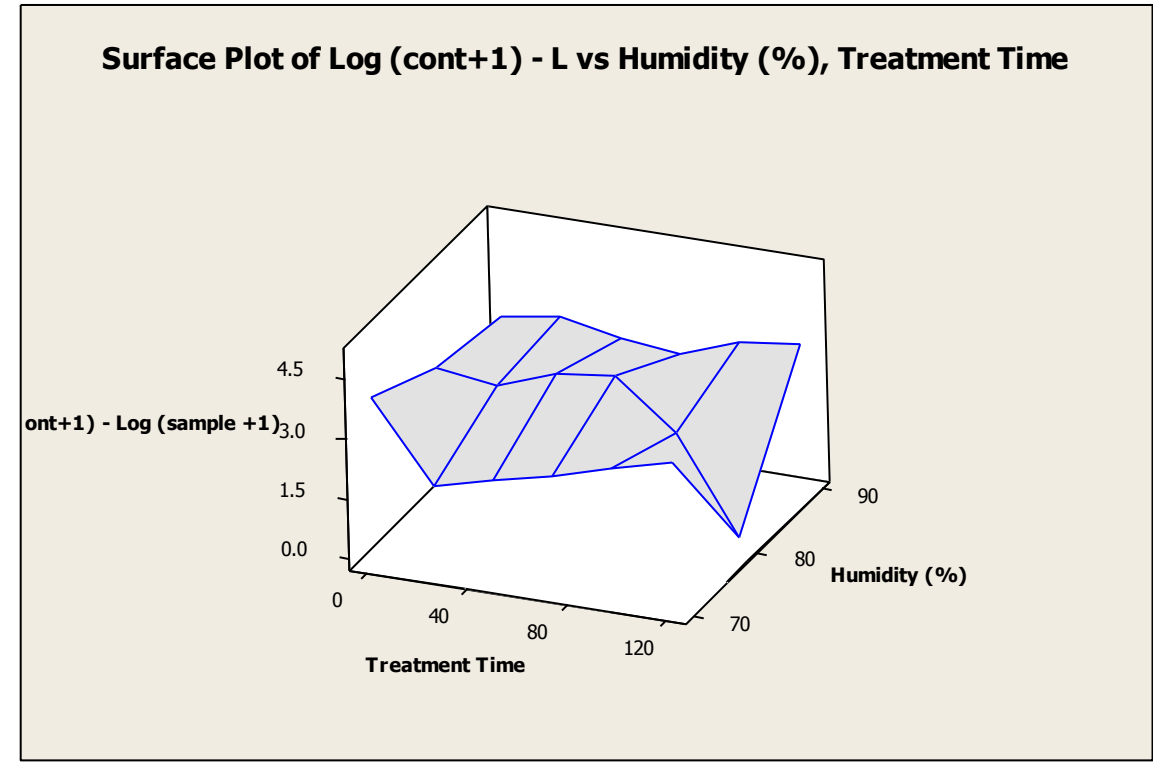

Figure 4- 22 - Aerosol deposition surface plot—spore log versus humidity and treatment time 


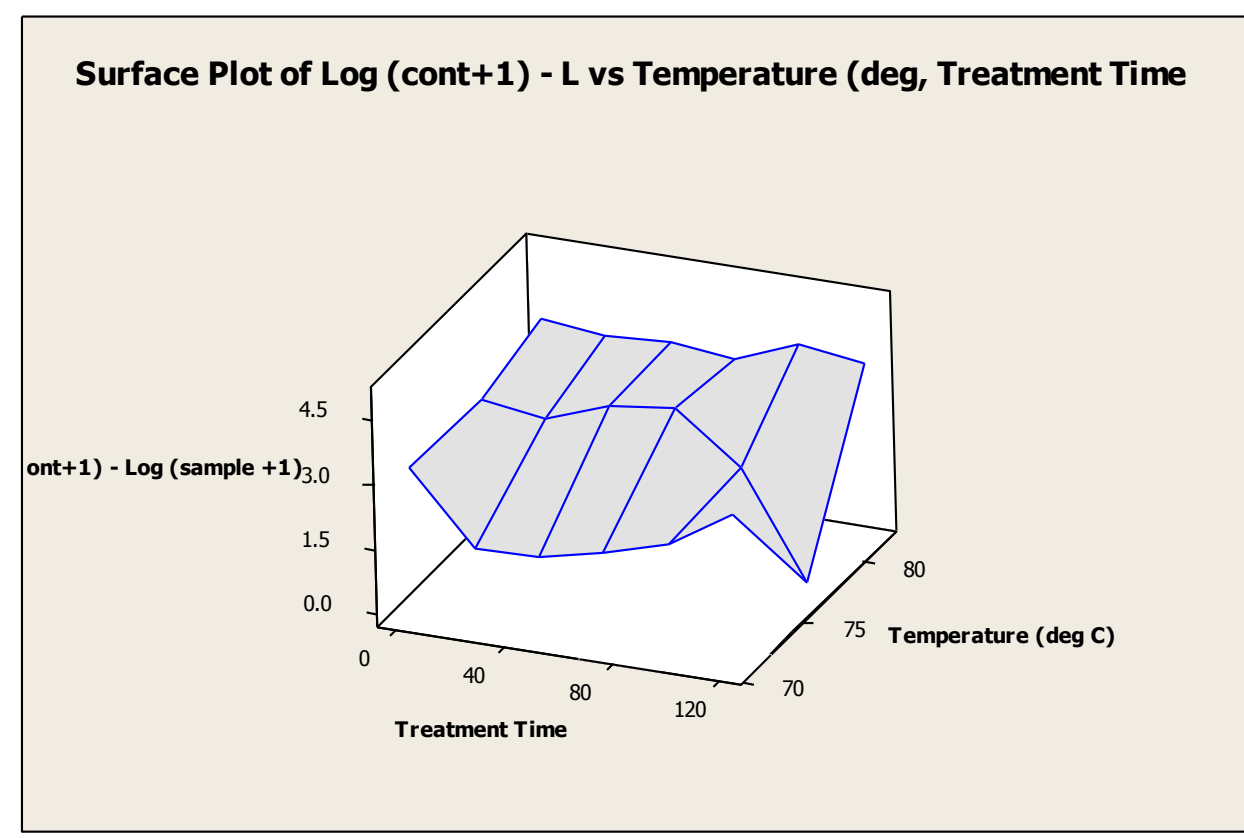

Figure 4- 23 - Aerosol deposition surface plot-spore log versus temperature and treatment time

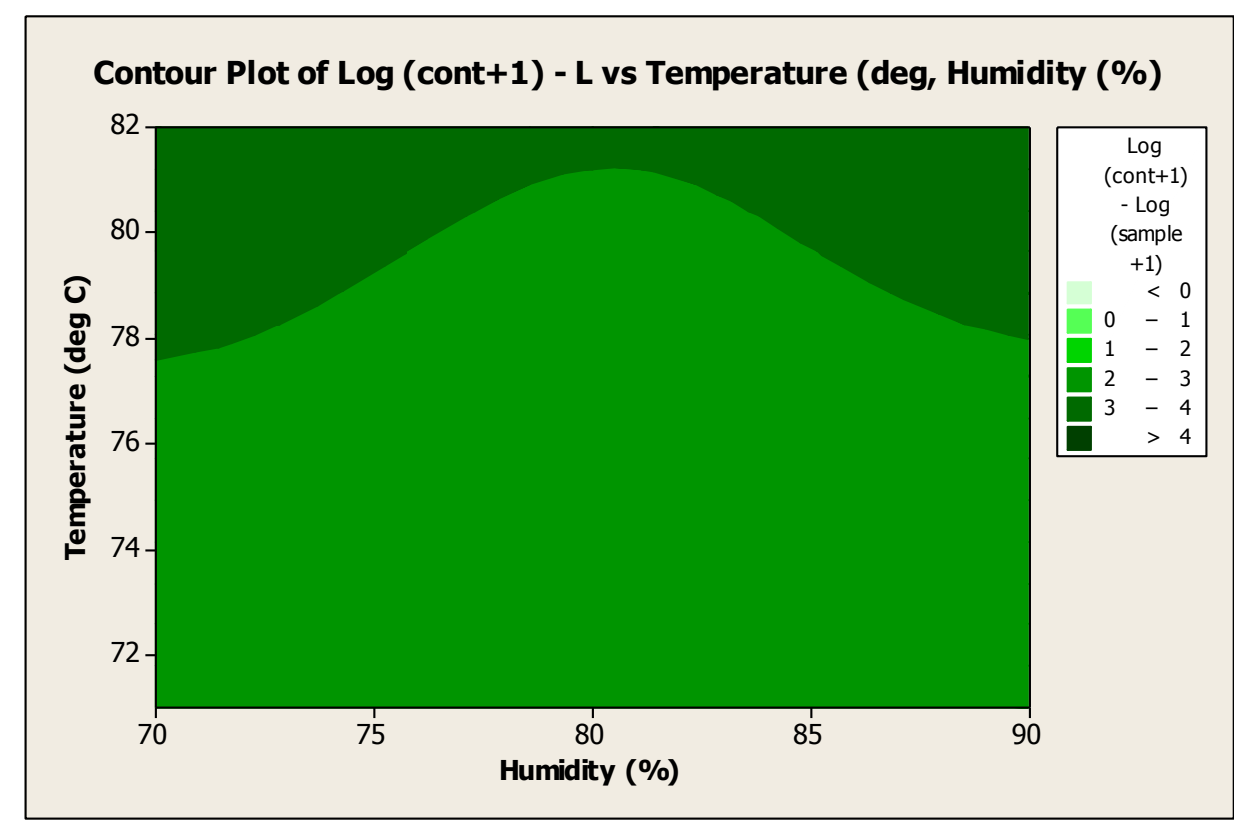

Figure 4- 24 - Aerosol deposition contour plot-spore log versus temperature and humidity 


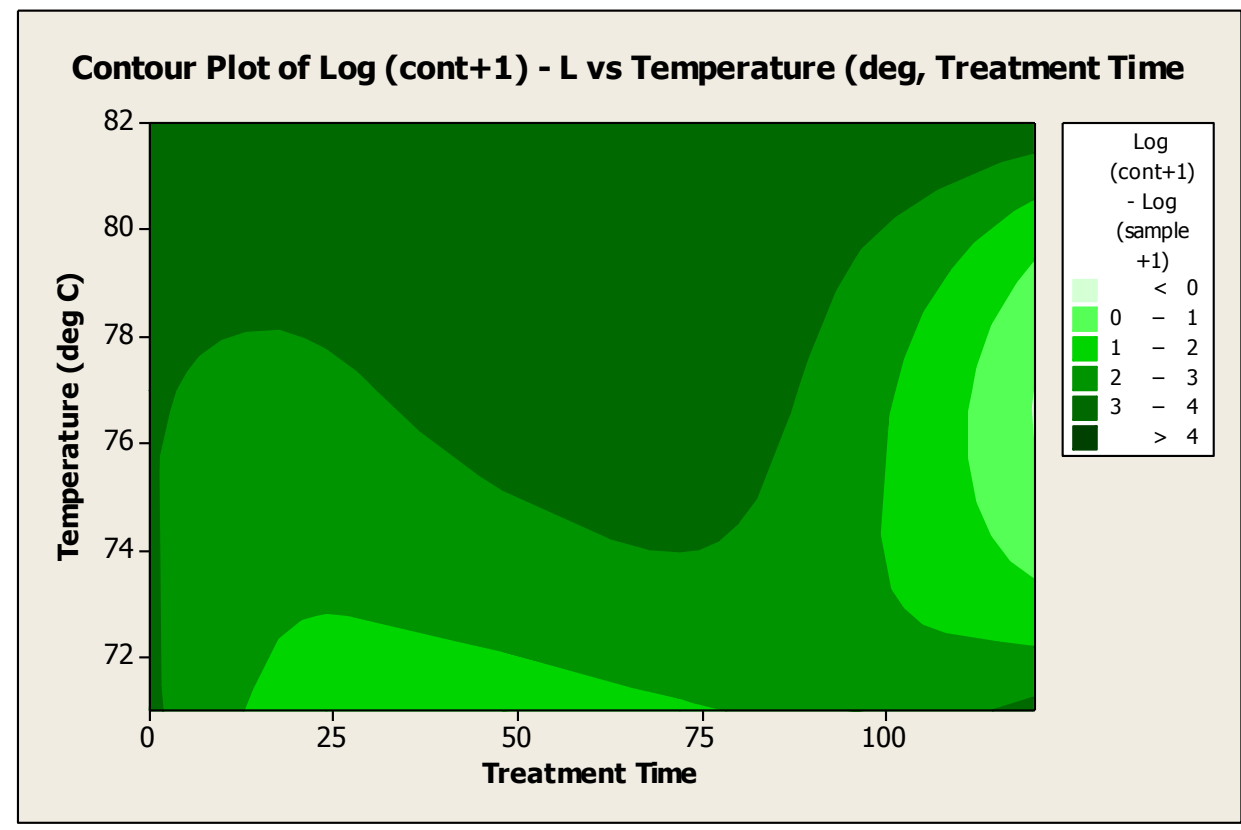

Figure 4- 25 - Aerosol deposition contour plot—spore log versus humidity and treatment time

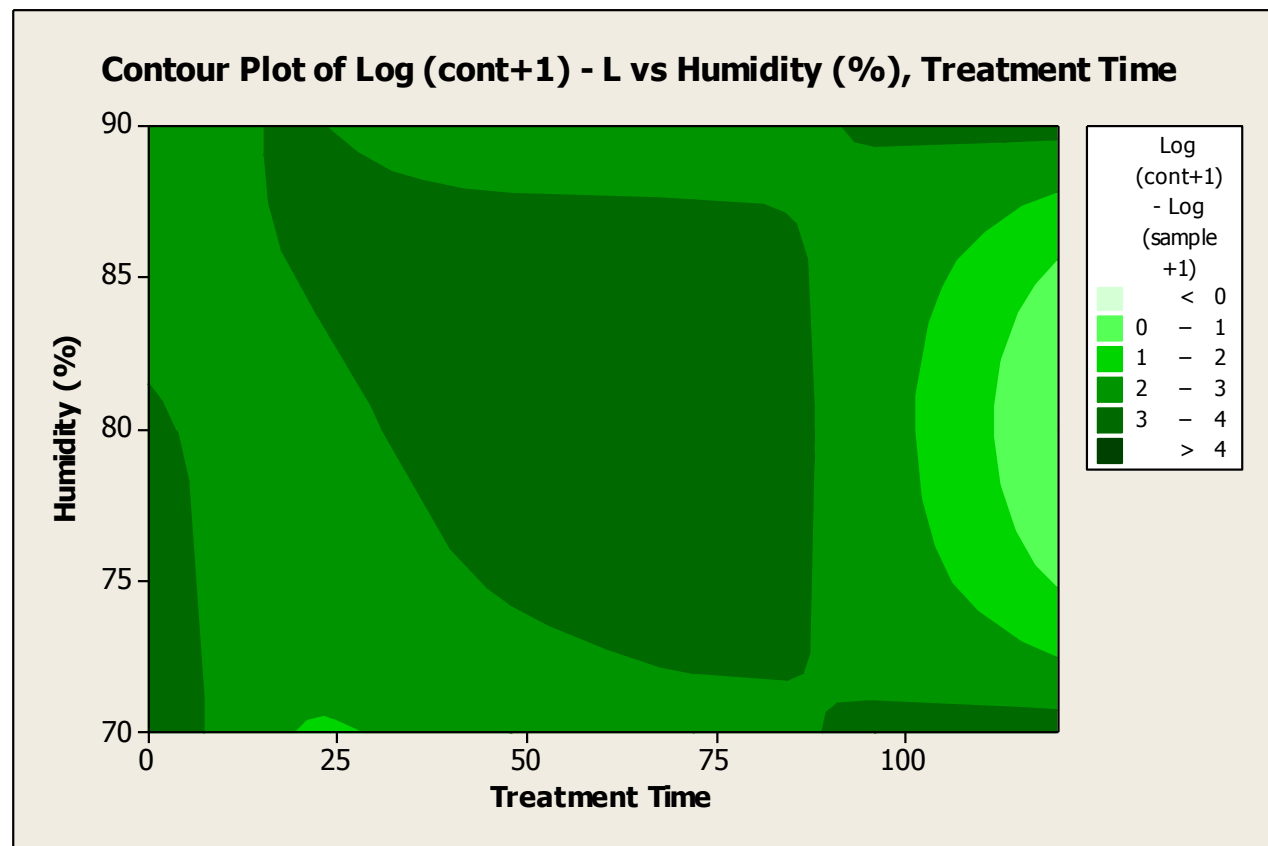

Figure 4- 26-Aerosol deposition contour plot-spore log versus temperature and treatment time 


\section{APPENDIX 4: High direct inoculation data}

Table A4 - 1 - Test condition 1: 180 deg F, $80 \%$ RH

\begin{tabular}{|c|c|c|c|c|c|c|c|c|c|}
\hline $\begin{array}{l}\text { Sample } \\
\text { Number }\end{array}$ & Time & $\begin{array}{l}\text { Spores } \\
\text { counted }\end{array}$ & $\begin{array}{c}\text { Serial } \\
\text { Dilution } \\
\text { Correction }^{1}\end{array}$ & $\begin{array}{c}\text { Dilution } \\
\text { Correction }^{2}\end{array}$ & $\begin{array}{c}\text { Agar } \\
\text { Volume }^{3}\end{array}$ & $\begin{array}{c}\text { Viable } \\
\text { spores }\end{array}$ & $\begin{array}{l}\text { Mean of } \\
\text { viable } \\
\text { spores }\end{array}$ & $\begin{array}{l}\text { SD of } \\
\text { viable } \\
\text { spores }\end{array}$ & $\begin{array}{c}\text { SE of } \\
\text { viable } \\
\text { spores }\end{array}$ \\
\hline $101 \mathrm{~A}$ & 0 & 81 & 20 & 30 & 0.1 & 486,000 & \multirow{5}{*}{$1,136,400$} & \multirow{5}{*}{500,445} & \multirow{5}{*}{223,812} \\
\hline 101B & 0 & 161 & 20 & 30 & 0.1 & 966,000 & & & \\
\hline $101 \mathrm{C}$ & 0 & 229 & 20 & 30 & 0.1 & $1,374,000$ & & & \\
\hline 101D & 0 & 171 & 20 & 30 & 0.1 & $1,026,000$ & & & \\
\hline $101 \mathrm{E}$ & 0 & 305 & 20 & 30 & 0.1 & $1,830,000$ & & & \\
\hline $103 \mathrm{~A}$ & 24 & 0 & 1 & 30 & 0.1 & 0 & \multirow{5}{*}{120} & \multirow{5}{*}{268} & \multirow{5}{*}{120.0} \\
\hline 103B & 24 & 0 & 1 & 30 & 0.1 & 0 & & & \\
\hline $103 \mathrm{C}$ & 24 & 2 & 1 & 30 & 0.1 & 600 & & & \\
\hline $103 \mathrm{D}$ & 24 & 0 & 1 & 30 & 0.1 & 0 & & & \\
\hline $103 E$ & 24 & 0 & 1 & 30 & 0.1 & 0 & & & \\
\hline $105 \mathrm{~A}$ & 48 & 0 & 1 & 30 & 0.1 & 0 & \multirow{5}{*}{0} & \multirow{5}{*}{0} & \multirow{5}{*}{0} \\
\hline $105 \mathrm{~B}$ & 48 & 0 & 1 & 30 & 0.1 & 0 & & & \\
\hline $105 \mathrm{C}$ & 48 & 0 & 1 & 30 & 0.1 & 0 & & & \\
\hline $105 \mathrm{D}$ & 48 & 0 & 1 & 30 & 0.1 & 0 & & & \\
\hline $105 \mathrm{E}$ & 48 & 0 & 1 & 30 & 0.1 & 0 & & & \\
\hline $107 \mathrm{~A}$ & 72 & 0 & 1 & 30 & 0.1 & 0 & \multirow{5}{*}{0} & \multirow{5}{*}{0} & \multirow{5}{*}{0} \\
\hline 107B & 72 & 0 & 1 & 30 & 0.1 & 0 & & & \\
\hline $107 \mathrm{C}$ & 72 & 0 & 1 & 30 & 0.1 & 0 & & & \\
\hline 107D & 72 & 0 & 1 & 30 & 0.1 & 0 & & & \\
\hline $108 \mathrm{E}$ & 72 & 0 & 1 & 30 & 0.1 & 0 & & & \\
\hline 109A & 96 & 0 & 1 & 30 & 0.1 & 0 & \multirow{5}{*}{0} & \multirow{5}{*}{0} & \multirow{5}{*}{0} \\
\hline 109B & 96 & 0 & 1 & 30 & 0.1 & 0 & & & \\
\hline $109 \mathrm{C}$ & 96 & 0 & 1 & 30 & 0.1 & 0 & & & \\
\hline 109D & 96 & 0 & 1 & 30 & 0.1 & 0 & & & \\
\hline $109 \mathrm{E}$ & 96 & 0 & 1 & 30 & 0.1 & 0 & & & \\
\hline
\end{tabular}

1. The serial dilution correction is the value used to correct the spores based on the serial dilutions performed.

2. The dilution correction was a value used to correct for the volume of PBS in which the coupons were placed

3. The agar volume was a value used to correct for the volume of the PBS solution plated onto the petri dish

4. Viable spores was the number of spores after corrected for the dilution and agar volume corrections 
Table A4 - 2- Test condition 3: $180 \mathrm{deg}$ F, 70\% RH

\begin{tabular}{|c|c|c|c|c|c|c|c|c|c|}
\hline $\begin{array}{l}\text { Sample } \\
\text { Number }\end{array}$ & Time & $\begin{array}{l}\text { Spores } \\
\text { counted }\end{array}$ & $\begin{array}{c}\text { Serial } \\
\text { Dilution } \\
\text { Correction }^{1}\end{array}$ & $\begin{array}{c}\text { Dilution } \\
\text { Correction }^{2}\end{array}$ & $\begin{array}{c}\text { Agar } \\
\text { Volume }^{3}\end{array}$ & $\begin{array}{c}\text { Viable } \\
\text { spores }\end{array}$ & $\begin{array}{c}\text { Mean of } \\
\text { viable } \\
\text { spores } \\
\end{array}$ & $\begin{array}{c}\text { SD of } \\
\text { viable } \\
\text { spores }\end{array}$ & $\begin{array}{c}\text { SE of } \\
\text { viable } \\
\text { spores }\end{array}$ \\
\hline $150 \mathrm{~A}$ & 0 & 147 & 400 & 30 & 0.1 & $17,640,000$ & \multirow{5}{*}{$8,184,000$} & \multirow{5}{*}{$5,794,522$} & \multirow{5}{*}{$2,591,468$} \\
\hline $150 \mathrm{~B}$ & 0 & 33 & 400 & 30 & 0.1 & $3,960,000$ & & & \\
\hline $150 \mathrm{C}$ & 0 & 35 & 400 & 30 & 0.1 & $4,200,000$ & & & \\
\hline $150 \mathrm{D}$ & 0 & 82 & 400 & 30 & 0.1 & $9,840,000$ & & & \\
\hline $150 \mathrm{E}$ & 0 & 44 & 400 & 30 & 0.1 & $5,280,000$ & & & \\
\hline $152 \mathrm{~A}$ & 24 & 11 & 400 & 30 & 0.1 & $1,320,000$ & \multirow{5}{*}{$2,688,000$} & \multirow{5}{*}{$2,751,640$} & \multirow{5}{*}{$1,230,608$} \\
\hline 152B & 24 & 59 & 400 & 30 & 0.1 & $7,080,000$ & & & \\
\hline $152 \mathrm{C}$ & 24 & 29 & 400 & 30 & 0.1 & $3,480,000$ & & & \\
\hline $152 \mathrm{D}$ & 24 & 13 & 400 & 30 & 0.1 & $1,560,000$ & & & \\
\hline $152 \mathrm{E}$ & 24 & 0 & 400 & 30 & 0.1 & 0 & & & \\
\hline $154 \mathrm{~A}$ & 48 & 84 & 20 & 30 & 0.1 & 504,000 & \multirow{5}{*}{788,400} & \multirow{5}{*}{$1,170,774$} & \multirow{5}{*}{523,602} \\
\hline $154 \mathrm{~B}$ & 48 & 11 & 20 & 30 & 0.1 & 66,000 & & & \\
\hline $154 \mathrm{C}$ & 48 & 30 & 20 & 30 & 0.1 & 180,000 & & & \\
\hline 154D & 48 & 55 & 20 & 30 & 0.1 & 330,000 & & & \\
\hline $154 \mathrm{E}$ & 48 & 477 & 20 & 30 & 0.1 & $2,862,000$ & & & \\
\hline $156 \mathrm{~A}$ & 72 & 2 & 20 & 30 & 0.1 & 12,000 & \multirow{5}{*}{181,200} & \multirow{5}{*}{237,338} & \multirow{5}{*}{106,144} \\
\hline $156 \mathrm{~B}$ & 72 & 3 & 20 & 30 & 0.1 & 18,000 & & & \\
\hline $156 \mathrm{C}$ & 72 & 98 & 20 & 30 & 0.1 & 588,000 & & & \\
\hline $156 \mathrm{D}$ & 72 & 19 & 20 & 30 & 0.1 & 114,000 & & & \\
\hline $156 \mathrm{E}$ & 72 & 29 & 20 & 30 & 0.1 & 174,000 & & & \\
\hline $158 \mathrm{~A}$ & 96 & 1 & 20 & 30 & 0.1 & 6,000 & \multirow{5}{*}{133,200} & \multirow{5}{*}{93,857} & \multirow{5}{*}{41,976} \\
\hline 158B & 96 & 30 & 20 & 30 & 0.1 & 180,000 & & & \\
\hline $158 \mathrm{C}$ & 96 & 11 & 20 & 30 & 0.1 & 66,000 & & & \\
\hline $158 \mathrm{D}$ & 96 & 30 & 20 & 30 & 0.1 & 180,000 & & & \\
\hline $158 \mathrm{E}$ & 96 & 39 & 20 & 30 & 0.1 & 234,000 & & & \\
\hline
\end{tabular}

1. The serial dilution correction is the value used to correct the spores based on the serial dilutions performed.

2. The dilution correction was a value used to correct for the volume of PBS in which the coupons were placed

3. The agar volume was a value used to correct for the volume of the PBS solution plated onto the petri dish

4. Viable spores was the number of spores after corrected for the dilution and agar volume corrections 
Table A4 - 3 - Test condition 5: $170 \mathrm{deg}$ F, 80\% RH

\begin{tabular}{|c|c|c|c|c|c|c|c|c|c|}
\hline $\begin{array}{l}\text { Sample } \\
\text { Number }\end{array}$ & Time & $\begin{array}{l}\text { Spores } \\
\text { counted }\end{array}$ & $\begin{array}{c}\text { Serial } \\
\text { Dilution } \\
\text { Correction }^{1}\end{array}$ & $\begin{array}{c}\text { Dilution } \\
\text { Correction }^{2}\end{array}$ & $\begin{array}{c}\text { Agar } \\
\text { Volume }^{3}\end{array}$ & $\begin{array}{l}\text { Viable } \\
\text { spores }^{4}\end{array}$ & $\begin{array}{c}\text { Mean of } \\
\text { viable } \\
\text { spores } \\
\end{array}$ & $\begin{array}{l}\text { SD of } \\
\text { viable } \\
\text { spores }\end{array}$ & $\begin{array}{l}\text { SE of } \\
\text { viable } \\
\text { spores }\end{array}$ \\
\hline $601 \mathrm{~A}$ & 0 & 161 & 400 & 30 & 0.1 & $19,320,000$ & \multirow{5}{*}{$15,504,000$} & \multirow{5}{*}{$2,748,760$} & \multirow{5}{*}{$1,229,320$} \\
\hline $601 \mathrm{~B}$ & 0 & 105 & 400 & 30 & 0.1 & $12,600,000$ & & & \\
\hline $601 \mathrm{C}$ & 0 & 114 & 400 & 30 & 0.1 & $13,680,000$ & & & \\
\hline 601D & 0 & 144 & 400 & 30 & 0.1 & $17,280,000$ & & & \\
\hline $601 \mathrm{E}$ & 0 & 122 & 400 & 30 & 0.1 & $14,640,000$ & & & \\
\hline $603 \mathrm{~A}$ & 24 & 56 & 400 & 30 & 0.1 & $6,720,000$ & \multirow{5}{*}{$3,216,000$} & \multirow{5}{*}{$2,221,459$} & \multirow{5}{*}{993,497} \\
\hline $603 \mathrm{~B}$ & 24 & 21 & 400 & 30 & 0.1 & $2,520,000$ & & & \\
\hline $603 C$ & 24 & 28 & 400 & 30 & 0.1 & $3,360,000$ & & & \\
\hline 603D & 24 & 5 & 400 & 30 & 0.1 & 600,000 & & & \\
\hline $603 \mathrm{E}$ & 24 & 24 & 400 & 30 & 0.1 & $2,880,000$ & & & \\
\hline $605 \mathrm{~A}$ & 48 & 2 & 20 & 30 & 0.1 & 12,000 & \multirow{5}{*}{49,200} & \multirow{5}{*}{79,982} & \multirow{5}{*}{35,770} \\
\hline $605 \mathrm{~B}$ & 48 & 32 & 20 & 30 & 0.1 & 192,000 & & & \\
\hline $605 \mathrm{C}$ & 48 & 3 & 20 & 30 & 0.1 & 18,000 & & & \\
\hline $605 \mathrm{D}$ & 48 & 1 & 20 & 30 & 0.1 & 6,000 & & & \\
\hline $605 \mathrm{E}$ & 48 & 3 & 20 & 30 & 0.1 & 18,000 & & & \\
\hline $607 \mathrm{~A}$ & 72 & 1 & 20 & 30 & 0.1 & 6,000 & \multirow{5}{*}{19,200} & \multirow{5}{*}{18,687} & \multirow{5}{*}{8,357} \\
\hline $607 \mathrm{~B}$ & 72 & 4 & 20 & 30 & 0.1 & 24,000 & & & \\
\hline $607 \mathrm{C}$ & 72 & 8 & 20 & 30 & 0.1 & 48,000 & & & \\
\hline $607 \mathrm{D}$ & 72 & 3 & 20 & 30 & 0.1 & 18,000 & & & \\
\hline $607 \mathrm{E}$ & 72 & 0 & 20 & 30 & 0.1 & 0 & & & \\
\hline $609 \mathrm{~A}$ & 96 & 208 & 1 & 30 & 0.1 & 62,400 & \multirow{5}{*}{97,800} & \multirow{5}{*}{78,161} & \multirow{5}{*}{34,956} \\
\hline 609B & 96 & 456 & 1 & 30 & 0.1 & 136,800 & & & \\
\hline $609 \mathrm{C}$ & 96 & 162 & 1 & 30 & 0.1 & 48,600 & & & \\
\hline 609D & 96 & 84 & 1 & 30 & 0.1 & 25,200 & & & \\
\hline $609 \mathrm{E}$ & 96 & 720 & 1 & 30 & 0.1 & 216,000 & & & \\
\hline $611 \mathrm{~A}$ & 120 & 89 & 1 & 30 & 0.1 & 26,700 & \multirow{5}{*}{11,280} & \multirow{5}{*}{10,718} & \multirow{5}{*}{4,793} \\
\hline $611 B$ & 120 & 60 & 1 & 30 & 0.1 & 18,000 & & & \\
\hline $611 \mathrm{C}$ & 120 & 5 & 1 & 30 & 0.1 & 1,500 & & & \\
\hline $611 \mathrm{D}$ & 120 & 22 & 1 & 30 & 0.1 & 6,600 & & & \\
\hline $611 \mathrm{E}$ & 120 & 12 & 1 & 30 & 0.1 & 3,600 & & & \\
\hline
\end{tabular}

1. The serial dilution correction is the value used to correct the spores based on the serial dilutions performed.

2. The dilution correction was a value used to correct for the volume of PBS in which the coupons were placed

3. The agar volume was a value used to correct for the volume of the PBS solution plated onto the petri dish

4. Viable spores was the number of spores after corrected for the dilution and agar volume corrections 
Table A4 - 4-Test condition 7: $160 \mathrm{deg}$ F, 90\% RH

\begin{tabular}{|c|c|c|c|c|c|c|c|c|c|}
\hline $\begin{array}{l}\text { Sample } \\
\text { Number }\end{array}$ & Time & $\begin{array}{l}\text { Spores } \\
\text { counted }\end{array}$ & $\begin{array}{c}\text { Serial } \\
\text { Dilution } \\
\text { Correction }^{1}\end{array}$ & $\begin{array}{c}\text { Dilution } \\
\text { Correction }^{2}\end{array}$ & $\begin{array}{c}\text { Agar } \\
\text { Volume }^{3}\end{array}$ & $\begin{array}{l}\text { Viable } \\
\text { spores }^{4}\end{array}$ & $\begin{array}{c}\text { Mean of } \\
\text { viable } \\
\text { spores } \\
\end{array}$ & $\begin{array}{l}\text { SD of } \\
\text { viable } \\
\text { spores }\end{array}$ & $\begin{array}{l}\text { SE of } \\
\text { viable } \\
\text { spores }\end{array}$ \\
\hline $200 \mathrm{~A}$ & 0 & 153 & 400 & 30 & 0.1 & $18,360,000$ & \multirow{5}{*}{$14,232,000$} & \multirow{5}{*}{$3,982,301$} & \multirow{5}{*}{$1,780,993$} \\
\hline $200 \mathrm{~B}$ & 0 & 156 & 400 & 30 & 0.1 & $18,720,000$ & & & \\
\hline $200 \mathrm{C}$ & 0 & 103 & 400 & 30 & 0.1 & $12,360,000$ & & & \\
\hline 200D & 0 & 91 & 400 & 30 & 0.1 & $10,920,000$ & & & \\
\hline $200 \mathrm{E}$ & 0 & 90 & 400 & 30 & 0.1 & $10,800,000$ & & & \\
\hline $202 \mathrm{~A}$ & 24 & 13 & 400 & 30 & 0.1 & $1,560,000$ & \multirow{5}{*}{$1,752,000$} & \multirow{5}{*}{$1,706,786$} & \multirow{5}{*}{763,321} \\
\hline 202B & 24 & 2 & 400 & 30 & 0.1 & 240,000 & & & \\
\hline $202 C$ & 24 & 39 & 400 & 30 & 0.1 & $4,680,000$ & & & \\
\hline 202D & 24 & 10 & 400 & 30 & 0.1 & $1,200,000$ & & & \\
\hline $202 \mathrm{E}$ & 24 & 9 & 400 & 30 & 0.1 & $1,080,000$ & & & \\
\hline $204 \mathrm{~A}$ & 48 & 387 & 20 & 30 & 0.1 & $2,322,000$ & \multirow{5}{*}{$1,156,800$} & \multirow{5}{*}{843,383} & \multirow{5}{*}{377,184} \\
\hline 204B & 48 & 77 & 20 & 30 & 0.1 & 462,000 & & & \\
\hline $204 \mathrm{C}$ & 48 & 61 & 20 & 30 & 0.1 & 366,000 & & & \\
\hline $204 \mathrm{D}$ & 48 & 287 & 20 & 30 & 0.1 & $1,722,000$ & & & \\
\hline $204 \mathrm{E}$ & 48 & 152 & 20 & 30 & 0.1 & 912,000 & & & \\
\hline $206 \mathrm{~A}$ & 72 & 4 & 400 & 30 & 0.1 & 480,000 & \multirow{5}{*}{576,000} & \multirow{5}{*}{903,593} & \multirow{5}{*}{404,111} \\
\hline $206 B$ & 72 & 0 & 400 & 30 & 0.1 & 0 & & & \\
\hline $206 C$ & 72 & 1 & 400 & 30 & 0.1 & 120,000 & & & \\
\hline $206 \mathrm{D}$ & 72 & 1 & 400 & 30 & 0.1 & 120,000 & & & \\
\hline $206 \mathrm{E}$ & 72 & 18 & 400 & 30 & 0.1 & $2,160,000$ & & & \\
\hline $208 \mathrm{~A}$ & 96 & 1 & 400 & 30 & 0.1 & 120,000 & \multirow{5}{*}{48,000} & \multirow{5}{*}{65,727} & \multirow{5}{*}{29,395} \\
\hline 208B & 96 & 0 & 400 & 30 & 0.1 & 0 & & & \\
\hline $208 \mathrm{C}$ & 96 & 0 & 400 & 30 & 0.1 & 0 & & & \\
\hline 208D & 96 & 1 & 400 & 30 & 0.1 & 120,000 & & & \\
\hline $208 \mathrm{E}$ & 96 & 0 & 400 & 30 & 0.1 & 0 & & & \\
\hline $210 \mathrm{~A}$ & 120 & 8 & 1 & 30 & 0.1 & 2,400 & \multirow{5}{*}{900} & \multirow{5}{*}{1,237} & \multirow{5}{*}{553} \\
\hline $210 \mathrm{~B}$ & 120 & 0 & 1 & 30 & 0.1 & 0 & & & \\
\hline $210 \mathrm{C}$ & 120 & 7 & 1 & 30 & 0.1 & 2,100 & & & \\
\hline $210 \mathrm{D}$ & 120 & 0 & 1 & 30 & 0.1 & 0 & & & \\
\hline $210 \mathrm{E}$ & 120 & 0 & 1 & 30 & 0.1 & 0 & & & \\
\hline
\end{tabular}

1. The serial dilution correction is the value used to correct the spores based on the serial dilutions performed.

2. The dilution correction was a value used to correct for the volume of PBS in which the coupons were placed

3. The agar volume was a value used to correct for the volume of the PBS solution plated onto the petri dish

4. Viable spores was the number of spores after corrected for the dilution and agar volume corrections 
Table A4 - 5 - Test condition 9: $160 \mathrm{deg}$ F, 70\% RH

\begin{tabular}{|c|c|c|c|c|c|c|c|c|c|}
\hline $\begin{array}{l}\text { Sample } \\
\text { Number }\end{array}$ & Time & $\begin{array}{l}\text { Spores } \\
\text { counted }\end{array}$ & $\begin{array}{c}\text { Serial } \\
\text { Dilution } \\
\text { Correction }\end{array}$ & $\begin{array}{c}\text { Dilution } \\
\text { Correction }^{2}\end{array}$ & $\begin{array}{c}\text { Agar } \\
\text { Volume }^{3}\end{array}$ & $\begin{array}{c}\text { Viable } \\
\text { spores }\end{array}$ & $\begin{array}{l}\text { Mean of } \\
\text { viable } \\
\text { spores }\end{array}$ & $\begin{array}{l}\text { SD of } \\
\text { viable } \\
\text { spores }\end{array}$ & $\begin{array}{c}\text { SE of } \\
\text { viable } \\
\text { spores }\end{array}$ \\
\hline $400 \mathrm{~A}$ & 0 & 34 & 400 & 30 & 0.1 & $4,080,000$ & \multirow{5}{*}{$9,336,000$} & \multirow{5}{*}{$7,991,676$} & \multirow{5}{*}{$3,574,095$} \\
\hline $400 \mathrm{~B}$ & 0 & 16 & 400 & 30 & 0.1 & $1,920,000$ & & & \\
\hline $400 \mathrm{C}$ & 0 & 39 & 400 & 30 & 0.1 & $4,680,000$ & & & \\
\hline $400 \mathrm{D}$ & 0 & 156 & 400 & 30 & 0.1 & $18,720,000$ & & & \\
\hline $400 \mathrm{E}$ & 0 & 144 & 400 & 30 & 0.1 & $17,280,000$ & & & \\
\hline $402 \mathrm{~A}$ & 24 & 5 & 400 & 30 & 0.1 & 600,000 & \multirow{5}{*}{$7,872,000$} & \multirow{5}{*}{$4,861,555$} & \multirow{5}{*}{$2,174,220$} \\
\hline 402B & 24 & 112 & 400 & 30 & 0.1 & $13,440,000$ & & & \\
\hline $402 \mathrm{C}$ & 24 & 64 & 400 & 30 & 0.1 & $7,680,000$ & & & \\
\hline $402 \mathrm{D}$ & 24 & 91 & 400 & 30 & 0.1 & $10,920,000$ & & & \\
\hline $402 \mathrm{E}$ & 24 & 56 & 400 & 30 & 0.1 & $6,720,000$ & & & \\
\hline $404 \mathrm{~A}$ & 48 & 78 & 400 & 30 & 0.1 & $9,360,000$ & \multirow{5}{*}{$6,984,000$} & \multirow{5}{*}{$5,708,142$} & \multirow{5}{*}{$2,552,836$} \\
\hline 404B & 48 & 121 & 400 & 30 & 0.1 & $14,520,000$ & & & \\
\hline $404 \mathrm{C}$ & 48 & 11 & 400 & 30 & 0.1 & $1,320,000$ & & & \\
\hline 404D & 48 & 71 & 400 & 30 & 0.1 & $8,520,000$ & & & \\
\hline $404 \mathrm{E}$ & 48 & 10 & 400 & 30 & 0.1 & $1,200,000$ & & & \\
\hline $406 \mathrm{~A}$ & 72 & 84 & 400 & 30 & 0.1 & $10,080,000$ & \multirow{5}{*}{$7,224,000$} & \multirow{5}{*}{$2,953,317$} & \multirow{5}{*}{$1,320,804$} \\
\hline 406B & 72 & 38 & 400 & 30 & 0.1 & $4,560,000$ & & & \\
\hline $406 \mathrm{C}$ & 72 & 51 & 400 & 30 & 0.1 & $6,120,000$ & & & \\
\hline $406 \mathrm{D}$ & 72 & 89 & 400 & 30 & 0.1 & $10,680,000$ & & & \\
\hline $406 \mathrm{E}$ & 72 & 39 & 400 & 30 & 0.1 & $4,680,000$ & & & \\
\hline $408 \mathrm{~A}$ & 96 & 15 & 400 & 30 & 0.1 & $1,800,000$ & \multirow{5}{*}{$6,936,000$} & \multirow{5}{*}{$4,922,487$} & \multirow{5}{*}{$2,201,470$} \\
\hline 408B & 96 & 122 & 400 & 30 & 0.1 & $14,640,000$ & & & \\
\hline $408 \mathrm{C}$ & 96 & 71 & 400 & 30 & 0.1 & $8,520,000$ & & & \\
\hline 408D & 96 & 42 & 400 & 30 & 0.1 & $5,040,000$ & & & \\
\hline $408 \mathrm{E}$ & 96 & 39 & 400 & 30 & 0.1 & $4,680,000$ & & & \\
\hline $410 \mathrm{~A}$ & 120 & 40 & 400 & 30 & 0.1 & $4,800,000$ & \multirow{5}{*}{$7,224,000$} & \multirow{5}{*}{$4,261,605$} & \multirow{5}{*}{$1,905,906$} \\
\hline $410 \mathrm{~B}$ & 120 & 74 & 400 & 30 & 0.1 & $8,880,000$ & & & \\
\hline $410 \mathrm{C}$ & 120 & 91 & 400 & 30 & 0.1 & $10,920,000$ & & & \\
\hline $410 \mathrm{D}$ & 120 & 88 & 400 & 30 & 0.1 & $10,560,000$ & & & \\
\hline $410 \mathrm{E}$ & 120 & 8 & 400 & 30 & 0.1 & 960,000 & & & \\
\hline
\end{tabular}

1. The serial dilution correction is the value used to correct the spores based on the serial dilutions performed.

2. The dilution correction was a value used to correct for the volume of PBS in which the coupons were placed

3. The agar volume was a value used to correct for the volume of the PBS solution plated onto the petri dish

4. Viable spores was the number of spores after corrected for the dilution and agar volume corrections 


\section{APPENDIX 5: Low direct inoculation data}

Table A4 - 6- Test condition 1: $180 \mathrm{deg}$ F, 90\% RH

\begin{tabular}{|c|c|c|c|c|c|c|c|c|}
\hline $\begin{array}{l}\text { Sample } \\
\text { Number }\end{array}$ & Time & $\begin{array}{l}\text { Spores } \\
\text { counted }\end{array}$ & $\begin{array}{c}\text { Dilution } \\
\text { Correction }^{1}\end{array}$ & $\begin{array}{c}\text { Agar } \\
\text { Volume }^{2}\end{array}$ & $\begin{array}{c}\text { Viable } \\
\text { spores }^{3}\end{array}$ & $\begin{array}{c}\text { Mean } \\
\text { of } \\
\text { viable } \\
\text { spores }\end{array}$ & $\begin{array}{c}\text { SD of } \\
\text { viable } \\
\text { spores }\end{array}$ & $\begin{array}{c}\text { SE of } \\
\text { viable } \\
\text { spores }\end{array}$ \\
\hline $102 \mathrm{~A}$ & 0 & 68 & 30 & 0.1 & 20,400 & \multirow{5}{*}{36,300} & \multirow{5}{*}{19,079} & \multirow{5}{*}{8,533} \\
\hline 102B & 0 & 59 & 30 & 0.1 & 17,700 & & & \\
\hline $102 \mathrm{C}$ & 0 & 101 & 30 & 0.1 & 30,300 & & & \\
\hline 102D & 0 & 186 & 30 & 0.1 & 55,800 & & & \\
\hline $102 \mathrm{E}$ & 0 & 191 & 30 & 0.1 & 57,300 & & & \\
\hline $104 \mathrm{~A}$ & 24 & 1 & 30 & 0.1 & 300 & \multirow{5}{*}{60} & \multirow{5}{*}{134} & \multirow{5}{*}{60} \\
\hline 104B & 24 & 0 & 30 & 0.1 & 0 & & & \\
\hline $104 C$ & 24 & 0 & 30 & 0.1 & 0 & & & \\
\hline 104D & 24 & 0 & 30 & 0.1 & 0 & & & \\
\hline $104 \mathrm{E}$ & 24 & 0 & 30 & 0.1 & 0 & & & \\
\hline $106 \mathrm{~A}$ & 48 & 0 & 30 & 0.1 & 0 & \multirow{5}{*}{0} & \multirow{5}{*}{0} & \multirow{5}{*}{0} \\
\hline 106B & 48 & 0 & 30 & 0.1 & 0 & & & \\
\hline $106 \mathrm{C}$ & 48 & 0 & 30 & 0.1 & 0 & & & \\
\hline $106 \mathrm{D}$ & 48 & 0 & 30 & 0.1 & 0 & & & \\
\hline $106 \mathrm{E}$ & 48 & 0 & 30 & 0.1 & 0 & & & \\
\hline $108 \mathrm{~A}$ & 72 & 0 & 30 & 0.1 & 0 & \multirow{5}{*}{0} & \multirow{5}{*}{0} & \multirow{5}{*}{0} \\
\hline 108B & 72 & 0 & 30 & 0.1 & 0 & & & \\
\hline $108 \mathrm{C}$ & 72 & 0 & 30 & 0.1 & 0 & & & \\
\hline 108D & 72 & 0 & 30 & 0.1 & 0 & & & \\
\hline $108 \mathrm{E}$ & 72 & 0 & 30 & 0.1 & 0 & & & \\
\hline $110 \mathrm{~A}$ & 96 & 0 & 30 & 0.1 & 0 & \multirow{5}{*}{0} & \multirow{5}{*}{0} & \multirow{5}{*}{0} \\
\hline 110B & 96 & 0 & 30 & 0.1 & 0 & & & \\
\hline $110 \mathrm{C}$ & 96 & 0 & 30 & 0.1 & 0 & & & \\
\hline 110D & 96 & 0 & 30 & 0.1 & 0 & & & \\
\hline $110 \mathrm{E}$ & 96 & 0 & 30 & 0.1 & 0 & & & \\
\hline
\end{tabular}

1. The dilution correction was a value used to correct for the volume of PBS in which the coupons were placed

2. The agar volume was a value used to correct for the volume of the PBS solution plated onto the petri dish

3. Viable spores was the number of spores after corrected for the dilution and agar volume corrections 
Table A4 - 7 - Test condition 3: $180 \mathrm{deg}$ F, $70 \%$ RH

\begin{tabular}{|c|c|c|c|c|c|c|c|c|}
\hline $\begin{array}{l}\text { Sample } \\
\text { Number }\end{array}$ & Time & $\begin{array}{l}\text { Spores } \\
\text { counted }\end{array}$ & $\begin{array}{c}\text { Dilution } \\
\text { Correction }^{1}\end{array}$ & $\begin{array}{c}\text { Agar } \\
\text { Volume }^{2}\end{array}$ & $\begin{array}{c}\text { Viable } \\
\text { spores }^{3}\end{array}$ & $\begin{array}{c}\text { Mean } \\
\text { of } \\
\text { viable } \\
\text { spores }\end{array}$ & $\begin{array}{l}\text { SD of } \\
\text { viable } \\
\text { spores }\end{array}$ & $\begin{array}{c}\text { SE of } \\
\text { viable } \\
\text { spores }\end{array}$ \\
\hline $151 \mathrm{~A}$ & 0 & 71 & 30 & 0.1 & 21,300 & \multirow{5}{*}{21,780} & \multirow{5}{*}{1,094} & \multirow{5}{*}{489} \\
\hline 151B & 0 & 79 & 30 & 0.1 & 23,700 & & & \\
\hline $151 \mathrm{C}$ & 0 & 72 & 30 & 0.1 & 21,600 & & & \\
\hline 151D & 0 & 70 & 30 & 0.1 & 21,000 & & & \\
\hline $151 \mathrm{E}$ & 0 & 71 & 30 & 0.1 & 21,300 & & & \\
\hline $153 \mathrm{~A}$ & 24 & 0 & 30 & 0.1 & 0 & \multirow{5}{*}{720} & \multirow{5}{*}{986} & \multirow{5}{*}{441} \\
\hline 153B & 24 & 0 & 30 & 0.1 & 0 & & & \\
\hline $153 \mathrm{C}$ & 24 & 6 & 30 & 0.1 & 1,800 & & & \\
\hline 153D & 24 & 6 & 30 & 0.1 & 1,800 & & & \\
\hline $153 \mathrm{E}$ & 24 & 0 & 30 & 0.1 & 0 & & & \\
\hline $155 \mathrm{~A}$ & 48 & 1 & 30 & 0.1 & 300 & \multirow{5}{*}{1,260} & \multirow{5}{*}{1,332} & \multirow{5}{*}{596} \\
\hline $155 \mathrm{~B}$ & 48 & 0 & 30 & 0.1 & 0 & & & \\
\hline $155 \mathrm{C}$ & 48 & 2 & 30 & 0.1 & 600 & & & \\
\hline $155 \mathrm{D}$ & 48 & 9 & 30 & 0.1 & 2,700 & & & \\
\hline $155 \mathrm{E}$ & 48 & 9 & 30 & 0.1 & 2,700 & & & \\
\hline $157 \mathrm{~A}$ & 72 & 1 & 30 & 0.1 & 300 & \multirow{5}{*}{240} & \multirow{5}{*}{391} & \multirow{5}{*}{175} \\
\hline 157B & 72 & 0 & 30 & 0.1 & 0 & & & \\
\hline $157 \mathrm{C}$ & 72 & 0 & 30 & 0.1 & 0 & & & \\
\hline 157D & 72 & 3 & 30 & 0.1 & 900 & & & \\
\hline $157 \mathrm{E}$ & 72 & 0 & 30 & 0.1 & 0 & & & \\
\hline $159 \mathrm{~A}$ & 96 & 0 & 30 & 0.1 & 0 & \multirow{5}{*}{120} & \multirow{5}{*}{268} & \multirow{5}{*}{120} \\
\hline 159B & 96 & 2 & 30 & 0.1 & 600 & & & \\
\hline $159 \mathrm{C}$ & 96 & 0 & 30 & 0.1 & 0 & & & \\
\hline 159D & 96 & 0 & 30 & 0.1 & 0 & & & \\
\hline $159 \mathrm{E}$ & 96 & 0 & 30 & 0.1 & 0 & & & \\
\hline
\end{tabular}

1. The dilution correction was a value used to correct for the volume of PBS in which the coupons were placed

2. The agar volume was a value used to correct for the volume of the PBS solution plated onto the petri dish

3. Viable spores was the number of spores after corrected for the dilution and agar volume corrections 
Table A4 - 8- Test condition 5: 170 deg F, $80 \%$ RH

\begin{tabular}{|c|c|c|c|c|c|c|c|c|}
\hline $\begin{array}{l}\text { Sample } \\
\text { Number }\end{array}$ & Time & $\begin{array}{l}\text { Spores } \\
\text { counted }\end{array}$ & $\begin{array}{c}\text { Dilution } \\
\text { Correction }^{1}\end{array}$ & $\begin{array}{c}\text { Agar } \\
\text { Volume }^{2}\end{array}$ & $\begin{array}{c}\text { Viable } \\
\text { spores }^{3}\end{array}$ & $\begin{array}{c}\begin{array}{c}\text { Mean } \\
\text { of } \\
\text { viable } \\
\text { spores }\end{array} \\
\end{array}$ & $\begin{array}{c}\text { SD of } \\
\text { viable } \\
\text { spores }\end{array}$ & $\begin{array}{c}\text { SE of } \\
\text { viable } \\
\text { spores }\end{array}$ \\
\hline $301 \mathrm{~A}$ & 0 & 132 & 30 & 0.1 & 39,600 & \multirow{5}{*}{51,600} & \multirow{5}{*}{12,291} & \multirow{5}{*}{5,497} \\
\hline $301 \mathrm{~B}$ & 0 & 150 & 30 & 0.1 & 45,000 & & & \\
\hline $301 \mathrm{C}$ & 0 & 226 & 30 & 0.1 & 67,800 & & & \\
\hline $301 \mathrm{D}$ & 0 & 205 & 30 & 0.1 & 61,500 & & & \\
\hline $301 \mathrm{E}$ & 0 & 147 & 30 & 0.1 & 44,100 & & & \\
\hline $303 \mathrm{~A}$ & 24 & 4 & 30 & 0.1 & 1,200 & \multirow{5}{*}{1,200} & \multirow{5}{*}{1,102} & \multirow{5}{*}{493} \\
\hline 303B & 24 & 4 & 30 & 0.1 & 1,200 & & & \\
\hline $303 \mathrm{C}$ & 24 & 1 & 30 & 0.1 & 300 & & & \\
\hline $303 \mathrm{D}$ & 24 & 10 & 30 & 0.1 & 3,000 & & & \\
\hline $303 \mathrm{E}$ & 24 & 1 & 30 & 0.1 & 300 & & & \\
\hline $305 \mathrm{~A}$ & 48 & 0 & 30 & 0.1 & 0 & \multirow{5}{*}{0} & \multirow{5}{*}{0} & \multirow{5}{*}{0} \\
\hline $305 \mathrm{~B}$ & 48 & 0 & 30 & 0.1 & 0 & & & \\
\hline $305 \mathrm{C}$ & 48 & 0 & 30 & 0.1 & 0 & & & \\
\hline $305 \mathrm{D}$ & 48 & 0 & 30 & 0.1 & 0 & & & \\
\hline $305 \mathrm{E}$ & 48 & 0 & 30 & 0.1 & 0 & & & \\
\hline $307 \mathrm{~A}$ & 72 & 0 & 30 & 0.1 & 0 & \multirow{5}{*}{0} & \multirow{5}{*}{0} & \multirow{5}{*}{0} \\
\hline $307 \mathrm{~B}$ & 72 & 0 & 30 & 0.1 & 0 & & & \\
\hline $307 \mathrm{C}$ & 72 & 0 & 30 & 0.1 & 0 & & & \\
\hline $307 \mathrm{D}$ & 72 & 0 & 30 & 0.1 & 0 & & & \\
\hline $307 \mathrm{E}$ & 72 & 0 & 30 & 0.1 & 0 & & & \\
\hline $309 \mathrm{~A}$ & 96 & 0 & 30 & 0.1 & 0 & \multirow{5}{*}{0} & \multirow{5}{*}{0} & \multirow{5}{*}{0} \\
\hline 309B & 96 & 0 & 30 & 0.1 & 0 & & & \\
\hline $309 \mathrm{C}$ & 96 & 0 & 30 & 0.1 & 0 & & & \\
\hline 309D & 96 & 0 & 30 & 0.1 & 0 & & & \\
\hline $309 E$ & 96 & 0 & 30 & 0.1 & 0 & & & \\
\hline $311 \mathrm{~A}$ & 120 & 0 & 30 & 0.1 & 0 & \multirow{5}{*}{0} & \multirow{5}{*}{0} & \multirow{5}{*}{0} \\
\hline 311B & 120 & 0 & 30 & 0.1 & 0 & & & \\
\hline $311 \mathrm{C}$ & 120 & 0 & 30 & 0.1 & 0 & & & \\
\hline $311 \mathrm{D}$ & 120 & 0 & 30 & 0.1 & 0 & & & \\
\hline $311 \mathrm{E}$ & 120 & 0 & 30 & 0.1 & 0 & & & \\
\hline
\end{tabular}

1. The dilution correction was a value used to correct for the volume of PBS in which the coupons were placed

2. The agar volume was a value used to correct for the volume of the PBS solution plated onto the petri dish

3. Viable spores was the number of spores after corrected for the dilution and agar volume corrections 
Table A4 - 9- Test condition 7: 160 deg F, 90\% RH

\begin{tabular}{|c|c|c|c|c|c|c|c|c|}
\hline $\begin{array}{l}\text { Sample } \\
\text { Number }\end{array}$ & Time & $\begin{array}{l}\text { Spores } \\
\text { counted }\end{array}$ & $\begin{array}{c}\text { Dilution } \\
\text { Correction }^{1}\end{array}$ & $\begin{array}{c}\text { Agar } \\
\text { Volume }^{2}\end{array}$ & $\begin{array}{c}\text { Viable } \\
\text { spores }^{3}\end{array}$ & $\begin{array}{c}\text { Mean } \\
\text { of } \\
\text { viable } \\
\text { spores }\end{array}$ & $\begin{array}{c}\text { SD of } \\
\text { viable } \\
\text { spores }\end{array}$ & $\begin{array}{c}\text { SE of } \\
\text { viable } \\
\text { spores }\end{array}$ \\
\hline $201 \mathrm{~A}$ & 0 & 156 & 30 & 0.1 & 46,800 & \multirow{5}{*}{44,280} & \multirow{5}{*}{18,953} & \multirow{5}{*}{8,476} \\
\hline $201 B$ & 0 & 109 & 30 & 0.1 & 32,700 & & & \\
\hline $201 C$ & 0 & 254 & 30 & 0.1 & 76,200 & & & \\
\hline 201D & 0 & 100 & 30 & 0.1 & 30,000 & & & \\
\hline $201 E$ & 0 & 119 & 30 & 0.1 & 35,700 & & & \\
\hline $203 \mathrm{~A}$ & 24 & 1 & 30 & 0.1 & 300 & \multirow{5}{*}{7,440} & \multirow{5}{*}{8,173} & \multirow{5}{*}{3,655} \\
\hline 203B & 24 & 56 & 30 & 0.1 & 16,800 & & & \\
\hline $203 C$ & 24 & 7 & 30 & 0.1 & 2,100 & & & \\
\hline 203D & 24 & 53 & 30 & 0.1 & 15,900 & & & \\
\hline $203 E$ & 24 & 7 & 30 & 0.1 & 2,100 & & & \\
\hline $205 \mathrm{~A}$ & 48 & 10 & 30 & 0.1 & 3,000 & \multirow{5}{*}{2,400} & \multirow{5}{*}{2,554} & \multirow{5}{*}{1,142} \\
\hline 205B & 48 & 3 & 30 & 0.1 & 900 & & & \\
\hline $205 \mathrm{C}$ & 48 & 22 & 30 & 0.1 & 6,600 & & & \\
\hline $205 \mathrm{D}$ & 48 & 4 & 30 & 0.1 & 1,200 & & & \\
\hline $205 \mathrm{E}$ & 48 & 1 & 30 & 0.1 & 300 & & & \\
\hline $207 \mathrm{~A}$ & 72 & 0 & 30 & 0.1 & 0 & \multirow{5}{*}{120} & \multirow{5}{*}{164} & \multirow{5}{*}{73} \\
\hline $207 \mathrm{~B}$ & 72 & 0 & 30 & 0.1 & 0 & & & \\
\hline $207 C$ & 72 & 1 & 30 & 0.1 & 300 & & & \\
\hline 207D & 72 & 0 & 30 & 0.1 & 0 & & & \\
\hline $207 \mathrm{E}$ & 72 & 1 & 30 & 0.1 & 300 & & & \\
\hline $209 \mathrm{~A}$ & 96 & 1 & 30 & 0.1 & 300 & \multirow{5}{*}{60} & \multirow{5}{*}{134} & \multirow{5}{*}{60} \\
\hline 209B & 96 & 0 & 30 & 0.1 & 0 & & & \\
\hline $209 \mathrm{C}$ & 96 & 0 & 30 & 0.1 & 0 & & & \\
\hline 209D & 96 & 0 & 30 & 0.1 & 0 & & & \\
\hline $209 E$ & 96 & 0 & 30 & 0.1 & 0 & & & \\
\hline $211 \mathrm{~A}$ & 120 & 0 & 30 & 0.1 & 0 & \multirow{5}{*}{0} & \multirow{5}{*}{0} & \multirow{5}{*}{0} \\
\hline 211B & 120 & 0 & 30 & 0.1 & 0 & & & \\
\hline $211 \mathrm{C}$ & 120 & 0 & 30 & 0.1 & 0 & & & \\
\hline 211D & 120 & 0 & 30 & 0.1 & 0 & & & \\
\hline $211 \mathrm{E}$ & 120 & 0 & 30 & 0.1 & 0 & & & \\
\hline
\end{tabular}

1. The dilution correction was a value used to correct for the volume of PBS in which the coupons were placed

2. The agar volume was a value used to correct for the volume of the PBS solution plated onto the petri dish

3. Viable spores was the number of spores after corrected for the dilution and agar volume corrections 
Table A4 - 10 - Test condition 9: $160 \mathrm{deg}$ F, 70\% RH

\begin{tabular}{|c|c|c|c|c|c|c|c|c|}
\hline $\begin{array}{l}\text { Sample } \\
\text { Number }\end{array}$ & Time & $\begin{array}{l}\text { Spores } \\
\text { counted }\end{array}$ & $\begin{array}{c}\text { Dilution } \\
\text { Correction }^{1}\end{array}$ & $\begin{array}{c}\text { Agar } \\
\text { Volume }^{2}\end{array}$ & $\begin{array}{l}\text { Viable } \\
\text { spores }^{3}\end{array}$ & $\begin{array}{c}\text { Mean } \\
\text { of } \\
\text { viable } \\
\text { spores }\end{array}$ & $\begin{array}{l}\text { SD of } \\
\text { viable } \\
\text { spores }\end{array}$ & $\begin{array}{l}\text { SE of } \\
\text { viable } \\
\text { spores }\end{array}$ \\
\hline $401 \mathrm{~A}$ & 0 & 58 & 30 & 0.1 & 17,400 & \multirow{5}{*}{17,760} & \multirow{5}{*}{4,693} & \multirow{5}{*}{2,099} \\
\hline $401 \mathrm{~B}$ & 0 & 71 & 30 & 0.1 & 21,300 & & & \\
\hline $401 \mathrm{C}$ & 0 & 36 & 30 & 0.1 & 10,800 & & & \\
\hline $401 \mathrm{D}$ & 0 & 76 & 30 & 0.1 & 22,800 & & & \\
\hline $401 \mathrm{E}$ & 0 & 55 & 30 & 0.1 & 16,500 & & & \\
\hline $403 \mathrm{~A}$ & 24 & 81 & 30 & 0.1 & 24,300 & \multirow{5}{*}{17,220} & \multirow{5}{*}{8,524} & \multirow{5}{*}{3,812} \\
\hline 403B & 24 & 55 & 30 & 0.1 & 16,500 & & & \\
\hline $403 C$ & 24 & 82 & 30 & 0.1 & 24,600 & & & \\
\hline 403D & 24 & 12 & 30 & 0.1 & 3,600 & & & \\
\hline $403 \mathrm{E}$ & 24 & 57 & 30 & 0.1 & 17,100 & & & \\
\hline $405 \mathrm{~A}$ & 48 & 46 & 30 & 0.1 & 13,800 & \multirow{5}{*}{4,320} & \multirow{5}{*}{6,110} & \multirow{5}{*}{2,733} \\
\hline 405B & 48 & 2 & 30 & 0.1 & 600 & & & \\
\hline $405 \mathrm{C}$ & 48 & 0 & 30 & 0.1 & 0 & & & \\
\hline $405 \mathrm{D}$ & 48 & 24 & 30 & 0.1 & 7,200 & & & \\
\hline $405 \mathrm{E}$ & 48 & 0 & 30 & 0.1 & 0 & & & \\
\hline $407 \mathrm{~A}$ & 72 & 1 & 30 & 0.1 & 300 & \multirow{5}{*}{780} & \multirow{5}{*}{722} & \multirow{5}{*}{323} \\
\hline $407 \mathrm{~B}$ & 72 & 6 & 30 & 0.1 & 1,800 & & & \\
\hline $407 \mathrm{C}$ & 72 & 2 & 30 & 0.1 & 600 & & & \\
\hline 407D & 72 & 0 & 30 & 0.1 & 0 & & & \\
\hline $407 \mathrm{E}$ & 72 & 4 & 30 & 0.1 & 1,200 & & & \\
\hline 409A & 96 & 22 & 30 & 0.1 & 6,600 & \multirow{5}{*}{2,520} & \multirow{5}{*}{2,568} & \multirow{5}{*}{1,149} \\
\hline 409B & 96 & 11 & 30 & 0.1 & 3,300 & & & \\
\hline $409 \mathrm{C}$ & 96 & 6 & 30 & 0.1 & 1,800 & & & \\
\hline 409D & 96 & 2 & 30 & 0.1 & 600 & & & \\
\hline $409 \mathrm{E}$ & 96 & 1 & 30 & 0.1 & 300 & & & \\
\hline $411 \mathrm{~A}$ & 120 & 0 & 30 & 0.1 & 0 & \multirow{5}{*}{3,900} & \multirow{5}{*}{3,022} & \multirow{5}{*}{1,352} \\
\hline 411B & 120 & 17 & 30 & 0.1 & 5,100 & & & \\
\hline $411 \mathrm{C}$ & 120 & 19 & 30 & 0.1 & 5,700 & & & \\
\hline $411 \mathrm{D}$ & 120 & 24 & 30 & 0.1 & 7,200 & & & \\
\hline $411 \mathrm{E}$ & 120 & 5 & 30 & 0.1 & 1,500 & & & \\
\hline
\end{tabular}

1. The dilution correction was a value used to correct for the volume of PBS in which the coupons were placed

2. The agar volume was a value used to correct for the volume of the PBS solution plated onto the petri dish

3. Viable spores was the number of spores after corrected for the dilution and agar volume corrections 
Table A4 - 11 - Test condition 1: 180 deg F, 90\% RH, samples

\begin{tabular}{|c|c|c|c|c|c|c|c|c|c|}
\hline $\begin{array}{l}\text { Sample } \\
\text { Number }\end{array}$ & Time & Type & $\begin{array}{l}\text { Spores } \\
\text { counted }\end{array}$ & $\begin{array}{c}\text { Dilution } \\
\text { Correction }^{1}\end{array}$ & $\begin{array}{c}\text { Agar } \\
\text { Volume }^{2}\end{array}$ & $\begin{array}{c}\text { Viable } \\
\text { spores }^{3}\end{array}$ & $\begin{array}{c}\text { Mean } \\
\text { of } \\
\text { viable } \\
\text { spores }\end{array}$ & $\begin{array}{c}\text { SD of } \\
\text { viable } \\
\text { spores }\end{array}$ & $\begin{array}{l}\text { SE of } \\
\text { viable } \\
\text { spores }\end{array}$ \\
\hline $111 \mathrm{~A}$ & 0 & Sample & 4 & 30 & 0.1 & 1,200 & \multirow{5}{*}{2,340} & \multirow{5}{*}{1,937} & \multirow{5}{*}{866} \\
\hline 111B & 0 & Sample & 18 & 30 & 0.1 & 5,400 & & & \\
\hline $111 \mathrm{C}$ & 0 & Sample & 1 & 30 & 0.1 & 300 & & & \\
\hline 111D & 0 & Sample & 7 & 30 & 0.1 & 2,100 & & & \\
\hline $111 \mathrm{E}$ & 0 & Sample & 9 & 30 & 0.1 & 2,700 & & & \\
\hline $114 \mathrm{~A}$ & 24 & Sample & 0 & 30 & 0.1 & 0 & \multirow{5}{*}{0} & \multirow{5}{*}{0} & \multirow{5}{*}{0} \\
\hline 114B & 24 & Sample & 0 & 30 & 0.1 & 0 & & & \\
\hline $114 \mathrm{C}$ & 24 & Sample & 0 & 30 & 0.1 & 0 & & & \\
\hline 114D & 24 & Sample & 0 & 30 & 0.1 & 0 & & & \\
\hline $114 \mathrm{E}$ & 24 & Sample & 0 & 30 & 0.1 & 0 & & & \\
\hline $116 \mathrm{~A}$ & 48 & Sample & 0 & 30 & 0.1 & 0 & \multirow{5}{*}{0} & \multirow{5}{*}{0} & \multirow{5}{*}{0} \\
\hline 116B & 48 & Sample & 0 & 30 & 0.1 & 0 & & & \\
\hline $116 \mathrm{C}$ & 48 & Sample & 0 & 30 & 0.1 & 0 & & & \\
\hline $116 \mathrm{D}$ & 48 & Sample & 0 & 30 & 0.1 & 0 & & & \\
\hline $116 \mathrm{E}$ & 48 & Sample & 0 & 30 & 0.1 & 0 & & & \\
\hline $118 \mathrm{~A}$ & 72 & Sample & 0 & 30 & 0.1 & 0 & \multirow{5}{*}{0} & \multirow{5}{*}{0} & \multirow{5}{*}{0} \\
\hline 118B & 72 & Sample & 0 & 30 & 0.1 & 0 & & & \\
\hline $118 \mathrm{C}$ & 72 & Sample & 0 & 30 & 0.1 & 0 & & & \\
\hline $118 \mathrm{D}$ & 72 & Sample & 0 & 30 & 0.1 & 0 & & & \\
\hline $118 \mathrm{E}$ & 72 & Sample & 0 & 30 & 0.1 & 0 & & & \\
\hline $120 \mathrm{~A}$ & 96 & Sample & 0 & 30 & 0.1 & 0 & \multirow{5}{*}{0} & \multirow{5}{*}{0} & \multirow{5}{*}{0} \\
\hline 120B & 96 & Sample & 0 & 30 & 0.1 & 0 & & & \\
\hline $120 \mathrm{C}$ & 96 & Sample & 0 & 30 & 0.1 & 0 & & & \\
\hline 120D & 96 & Sample & 0 & 30 & 0.1 & 0 & & & \\
\hline $120 \mathrm{E}$ & 96 & Sample & 0 & 30 & 0.1 & 0 & & & \\
\hline
\end{tabular}

1. The dilution correction was a value used to correct for the volume of PBS in which the coupons were placed

2. The agar volume was a value used to correct for the volume of the PBS solution plated onto the petri dish

3. Viable spores was the number of spores after corrected for the dilution and agar volume corrections 
Table A4 - 12 - Test condition 1: $180 \mathrm{deg}$ F, 90\% RH, controls

\begin{tabular}{|c|c|c|c|c|c|c|c|c|c|}
\hline $\begin{array}{l}\text { Sample } \\
\text { Number }\end{array}$ & Time & Type & $\begin{array}{l}\text { Spores } \\
\text { counted }\end{array}$ & $\begin{array}{c}\text { Dilution } \\
\text { Correction }^{1}\end{array}$ & $\begin{array}{c}\text { Agar } \\
\text { Volume }^{2}\end{array}$ & $\begin{array}{c}\text { Viable } \\
\text { spores }^{3}\end{array}$ & $\begin{array}{c}\text { Mean } \\
\text { of } \\
\text { viable } \\
\text { spores }\end{array}$ & $\begin{array}{l}\text { SD of } \\
\text { viable } \\
\text { spores }\end{array}$ & $\begin{array}{l}\text { SE of } \\
\text { viable } \\
\text { spores }\end{array}$ \\
\hline $113 \mathrm{~A}$ & 24 & Control & 23 & 30 & 0.1 & 6,900 & \multirow{5}{*}{4,680} & \multirow{5}{*}{2,063} & \multirow{5}{*}{923} \\
\hline 113B & 24 & Control & 20 & 30 & 0.1 & 6,000 & & & \\
\hline $113 \mathrm{C}$ & 24 & Control & 16 & 30 & 0.1 & 4,800 & & & \\
\hline $113 \mathrm{D}$ & 24 & Control & 5 & 30 & 0.1 & 1,500 & & & \\
\hline $113 \mathrm{E}$ & 24 & Control & 14 & 30 & 0.1 & 4,200 & & & \\
\hline $115 \mathrm{~A}$ & 48 & Control & 4 & 30 & 0.1 & 1,200 & \multirow{5}{*}{3,240} & \multirow{5}{*}{1,397} & \multirow{5}{*}{625} \\
\hline $115 \mathrm{~B}$ & 48 & Control & 10 & 30 & 0.1 & 3,000 & & & \\
\hline $115 \mathrm{C}$ & 48 & Control & 12 & 30 & 0.1 & 3,600 & & & \\
\hline $115 \mathrm{D}$ & 48 & Control & 17 & 30 & 0.1 & 5,100 & & & \\
\hline $115 \mathrm{E}$ & 48 & Control & 11 & 30 & 0.1 & 3,300 & & & \\
\hline $117 \mathrm{~A}$ & 72 & Control & 14 & 30 & 0.1 & 4,200 & \multirow{5}{*}{2,220} & \multirow{5}{*}{1,368} & \multirow{5}{*}{612} \\
\hline 117B & 72 & Control & 3 & 30 & 0.1 & 900 & & & \\
\hline $117 \mathrm{C}$ & 72 & Control & 10 & 30 & 0.1 & 3,000 & & & \\
\hline 117D & 72 & Control & 6 & 30 & 0.1 & 1,800 & & & \\
\hline $117 \mathrm{E}$ & 72 & Control & 4 & 30 & 0.1 & 1,200 & & & \\
\hline 119A & 96 & Control & 13 & 30 & 0.1 & 3,900 & \multirow{5}{*}{3,540} & \multirow{5}{*}{1,365} & \multirow{5}{*}{610} \\
\hline 119B & 96 & Control & 9 & 30 & 0.1 & 2,700 & & & \\
\hline $119 \mathrm{C}$ & 96 & Control & 6 & 30 & 0.1 & 1,800 & & & \\
\hline 119D & 96 & Control & 13 & 30 & 0.1 & 3,900 & & & \\
\hline $119 \mathrm{E}$ & 96 & Control & 18 & 30 & 0.1 & 5,400 & & & \\
\hline
\end{tabular}

1. The dilution correction was a value used to correct for the volume of PBS in which the coupons were placed

2. The agar volume was a value used to correct for the volume of the PBS solution plated onto the petri dish

3. Viable spores was the number of spores after corrected for the dilution and agar volume corrections 
Table A4 - 13 - Test condition 3: $180 \mathrm{deg}$ F, 70\% RH, samples

\begin{tabular}{|c|c|c|c|c|c|c|c|c|c|}
\hline $\begin{array}{l}\text { Sample } \\
\text { Number }\end{array}$ & Time & Type & $\begin{array}{l}\text { Spores } \\
\text { counted }\end{array}$ & $\begin{array}{c}\text { Dilution } \\
\text { Correction }^{1}\end{array}$ & $\begin{array}{c}\text { Agar } \\
\text { Volume }^{2}\end{array}$ & $\begin{array}{c}\text { Viable } \\
\text { spores }^{3}\end{array}$ & $\begin{array}{c}\text { Mean } \\
\text { of } \\
\text { viable } \\
\text { spores }\end{array}$ & $\begin{array}{l}\text { SD of } \\
\text { viable } \\
\text { spores }\end{array}$ & $\begin{array}{l}\text { SE of } \\
\text { viable } \\
\text { spores }\end{array}$ \\
\hline $701 \mathrm{~A}$ & 0 & Sample & 63 & 30 & 0.1 & 18,900 & \multirow{5}{*}{13,140} & \multirow{5}{*}{10,148} & \multirow{5}{*}{4,538} \\
\hline $701 \mathrm{~B}$ & 0 & Sample & 10 & 30 & 0.1 & 3,000 & & & \\
\hline $701 \mathrm{C}$ & 0 & Sample & 4 & 30 & 0.1 & 1,200 & & & \\
\hline $701 \mathrm{D}$ & 0 & Sample & 70 & 30 & 0.1 & 21,000 & & & \\
\hline $701 \mathrm{E}$ & 0 & Sample & 72 & 30 & 0.1 & 21,600 & & & \\
\hline $702 \mathrm{~A}$ & 24 & Sample & 0 & 30 & 0.1 & 0 & \multirow{5}{*}{60} & \multirow{5}{*}{134} & \multirow{5}{*}{60} \\
\hline $702 B$ & 24 & Sample & 0 & 30 & 0.1 & 0 & & & \\
\hline $702 \mathrm{C}$ & 24 & Sample & 1 & 30 & 0.1 & 300 & & & \\
\hline $702 \mathrm{D}$ & 24 & Sample & 0 & 30 & 0.1 & 0 & & & \\
\hline $702 \mathrm{E}$ & 24 & Sample & 0 & 30 & 0.1 & 0 & & & \\
\hline $704 \mathrm{~A}$ & 48 & Sample & 0 & 30 & 0.1 & 0 & \multirow{5}{*}{0} & \multirow{5}{*}{0} & \multirow{5}{*}{0} \\
\hline 704B & 48 & Sample & 0 & 30 & 0.1 & 0 & & & \\
\hline $704 \mathrm{C}$ & 48 & Sample & 0 & 30 & 0.1 & 0 & & & \\
\hline $704 \mathrm{D}$ & 48 & Sample & 0 & 30 & 0.1 & 0 & & & \\
\hline $704 \mathrm{E}$ & 48 & Sample & 0 & 30 & 0.1 & 0 & & & \\
\hline $706 \mathrm{~A}$ & 72 & Sample & 0 & 30 & 0.1 & 0 & \multirow{5}{*}{0} & \multirow{5}{*}{0} & \multirow{5}{*}{0} \\
\hline $706 \mathrm{~B}$ & 72 & Sample & 0 & 30 & 0.1 & 0 & & & \\
\hline $706 \mathrm{C}$ & 72 & Sample & 0 & 30 & 0.1 & 0 & & & \\
\hline $706 \mathrm{D}$ & 72 & Sample & 0 & 30 & 0.1 & 0 & & & \\
\hline $706 \mathrm{E}$ & 72 & Sample & 0 & 30 & 0.1 & 0 & & & \\
\hline $708 \mathrm{~A}$ & 96 & Sample & 0 & 30 & 0.1 & 0 & \multirow{5}{*}{0} & \multirow{5}{*}{0} & \multirow{5}{*}{0} \\
\hline $708 \mathrm{~B}$ & 96 & Sample & 0 & 30 & 0.1 & 0 & & & \\
\hline $708 \mathrm{C}$ & 96 & Sample & 0 & 30 & 0.1 & 0 & & & \\
\hline $708 \mathrm{D}$ & 96 & Sample & 0 & 30 & 0.1 & 0 & & & \\
\hline $708 \mathrm{E}$ & 96 & Sample & 0 & 30 & 0.1 & 0 & & & \\
\hline $710 \mathrm{~A}$ & 120 & Sample & 0 & 30 & 0.1 & 0 & \multirow{5}{*}{0} & \multirow{5}{*}{0} & \multirow{5}{*}{0} \\
\hline $710 \mathrm{~B}$ & 120 & Sample & 0 & 30 & 0.1 & 0 & & & \\
\hline $710 \mathrm{C}$ & 120 & Sample & 0 & 30 & 0.1 & 0 & & & \\
\hline $710 \mathrm{D}$ & 120 & Sample & 0 & 30 & 0.1 & 0 & & & \\
\hline $710 \mathrm{E}$ & 120 & Sample & 0 & 30 & 0.1 & 0 & & & \\
\hline
\end{tabular}

1. The dilution correction was a value used to correct for the volume of PBS in which the coupons were placed

2. The agar volume was a value used to correct for the volume of the PBS solution plated onto the petri dish

3. Viable spores was the number of spores after corrected for the dilution and agar volume corrections 
Table A4 - 14 - Test condition 3: $180 \operatorname{deg}$ F, 70\% RH, controls

\begin{tabular}{|c|c|c|c|c|c|c|c|c|c|}
\hline $\begin{array}{l}\text { Sample } \\
\text { Number }\end{array}$ & Time & Type & $\begin{array}{l}\text { Spores } \\
\text { counted }\end{array}$ & $\begin{array}{c}\text { Dilution } \\
\text { Correction }^{1}\end{array}$ & $\begin{array}{c}\text { Agar } \\
\text { Volume }^{2}\end{array}$ & $\begin{array}{c}\text { Viable } \\
\text { spores }^{3}\end{array}$ & $\begin{array}{c}\text { Mean } \\
\text { of } \\
\text { viable } \\
\text { spores }\end{array}$ & $\begin{array}{l}\text { SD of } \\
\text { viable } \\
\text { spores }\end{array}$ & $\begin{array}{l}\text { SE of } \\
\text { viable } \\
\text { spores }\end{array}$ \\
\hline $703 \mathrm{~A}$ & 24 & Control & 54 & 30 & 0.1 & 16,200 & \multirow{5}{*}{6,900} & \multirow{5}{*}{5,821} & \multirow{5}{*}{2,603} \\
\hline $703 \mathrm{~B}$ & 24 & Control & 5 & 30 & 0.1 & 1,500 & & & \\
\hline $703 \mathrm{C}$ & 24 & Control & 12 & 30 & 0.1 & 3,600 & & & \\
\hline $703 \mathrm{D}$ & 24 & Control & 15 & 30 & 0.1 & 4,500 & & & \\
\hline $703 \mathrm{E}$ & 24 & Control & 29 & 30 & 0.1 & 8,700 & & & \\
\hline $705 \mathrm{~A}$ & 48 & Control & 13 & 30 & 0.1 & 3,900 & \multirow{5}{*}{4,500} & \multirow{5}{*}{3,414} & \multirow{5}{*}{1,527} \\
\hline $705 \mathrm{~B}$ & 48 & Control & 5 & 30 & 0.1 & 1,500 & & & \\
\hline $705 \mathrm{C}$ & 48 & Control & 5 & 30 & 0.1 & 1,500 & & & \\
\hline $705 \mathrm{D}$ & 48 & Control & 20 & 30 & 0.1 & 6,000 & & & \\
\hline $705 \mathrm{E}$ & 48 & Control & 32 & 30 & 0.1 & 9,600 & & & \\
\hline $707 \mathrm{~A}$ & 72 & Control & 3 & 30 & 0.1 & 900 & \multirow{5}{*}{4,260} & \multirow{5}{*}{3,965} & \multirow{5}{*}{1,773} \\
\hline 707B & 72 & Control & 11 & 30 & 0.1 & 3,300 & & & \\
\hline $707 \mathrm{C}$ & 72 & Control & 1 & 30 & 0.1 & 300 & & & \\
\hline $707 \mathrm{D}$ & 72 & Control & 30 & 30 & 0.1 & 9,000 & & & \\
\hline $707 \mathrm{E}$ & 72 & Control & 26 & 30 & 0.1 & 7,800 & & & \\
\hline $709 \mathrm{~A}$ & 96 & Control & 55 & 30 & 0.1 & 16,500 & \multirow{5}{*}{29,940} & \multirow{5}{*}{37,144} & \multirow{5}{*}{16,612} \\
\hline 709B & 96 & Control & 320 & 30 & 0.1 & 96,000 & & & \\
\hline $709 \mathrm{C}$ & 96 & Control & 53 & 30 & 0.1 & 15,900 & & & \\
\hline $709 \mathrm{D}$ & 96 & Control & 22 & 30 & 0.1 & 6,600 & & & \\
\hline $709 \mathrm{E}$ & 96 & Control & 49 & 30 & 0.1 & 14,700 & & & \\
\hline $711 \mathrm{~A}$ & 120 & Control & 15 & 30 & 0.1 & 4,500 & \multirow{5}{*}{5,700} & \multirow{5}{*}{2,741} & \multirow{5}{*}{1,226} \\
\hline $711 \mathrm{~B}$ & 120 & Control & 31 & 30 & 0.1 & 9,300 & & & \\
\hline $711 \mathrm{C}$ & 120 & Control & 9 & 30 & 0.1 & 2,700 & & & \\
\hline $711 \mathrm{D}$ & 120 & Control & 14 & 30 & 0.1 & 4,200 & & & \\
\hline $711 \mathrm{E}$ & 120 & Control & 26 & 30 & 0.1 & 7,800 & & & \\
\hline
\end{tabular}

1. The dilution correction was a value used to correct for the volume of PBS in which the coupons were placed

2. The agar volume was a value used to correct for the volume of the PBS solution plated onto the petri dish

3. Viable spores was the number of spores after corrected for the dilution and agar volume corrections 
Table A4 - 15 - Test condition 5: $170 \mathrm{deg}$ F, 80\% RH, samples

\begin{tabular}{|c|c|c|c|c|c|c|c|c|c|}
\hline $\begin{array}{l}\text { Sample } \\
\text { Number }\end{array}$ & Time & Type & $\begin{array}{l}\text { Spores } \\
\text { counted }\end{array}$ & $\begin{array}{c}\text { Dilution } \\
\text { Correction }^{1}\end{array}$ & $\begin{array}{c}\text { Agar } \\
\text { Volume }^{2}\end{array}$ & $\begin{array}{l}\text { Viable } \\
\text { spores }^{3}\end{array}$ & $\begin{array}{c}\text { Mean } \\
\text { of } \\
\text { viable } \\
\text { spores } \\
\end{array}$ & $\begin{array}{c}\text { SD of } \\
\text { viable } \\
\text { spores }\end{array}$ & $\begin{array}{l}\text { SE of } \\
\text { viable } \\
\text { spores }\end{array}$ \\
\hline $341 \mathrm{~A}$ & 0 & Sample & 5 & 30 & 0.1 & 1,500 & \multirow{5}{*}{1,440} & \multirow{5}{*}{747} & \multirow{5}{*}{334} \\
\hline 341B & 0 & Sample & 5 & 30 & 0.1 & 1,500 & & & \\
\hline $341 \mathrm{C}$ & 0 & Sample & 8 & 30 & 0.1 & 2,400 & & & \\
\hline $341 \mathrm{D}$ & 0 & Sample & 1 & 30 & 0.1 & 300 & & & \\
\hline $341 \mathrm{E}$ & 0 & Sample & 5 & 30 & 0.1 & 1,500 & & & \\
\hline $342 \mathrm{~A}$ & 24 & Sample & 0 & 30 & 0.1 & 0 & \multirow{5}{*}{0} & \multirow{5}{*}{0} & \multirow{5}{*}{0} \\
\hline 342B & 24 & Sample & 0 & 30 & 0.1 & 0 & & & \\
\hline $342 \mathrm{C}$ & 24 & Sample & 0 & 30 & 0.1 & 0 & & & \\
\hline 342D & 24 & Sample & 0 & 30 & 0.1 & 0 & & & \\
\hline $342 \mathrm{E}$ & 24 & Sample & 0 & 30 & 0.1 & 0 & & & \\
\hline $344 \mathrm{~A}$ & 48 & Sample & 0 & 30 & 0.1 & 0 & \multirow{5}{*}{0} & \multirow{5}{*}{0} & \multirow{5}{*}{0} \\
\hline 344B & 48 & Sample & 0 & 30 & 0.1 & 0 & & & \\
\hline $344 \mathrm{C}$ & 48 & Sample & 0 & 30 & 0.1 & 0 & & & \\
\hline $344 \mathrm{D}$ & 48 & Sample & 0 & 30 & 0.1 & 0 & & & \\
\hline $344 \mathrm{E}$ & 48 & Sample & 0 & 30 & 0.1 & 0 & & & \\
\hline $346 \mathrm{~A}$ & 72 & Sample & 0 & 30 & 0.1 & 0 & \multirow{5}{*}{0} & \multirow{5}{*}{0} & \multirow{5}{*}{0} \\
\hline 346B & 72 & Sample & 0 & 30 & 0.1 & 0 & & & \\
\hline $346 \mathrm{C}$ & 72 & Sample & 0 & 30 & 0.1 & 0 & & & \\
\hline $346 \mathrm{D}$ & 72 & Sample & 0 & 30 & 0.1 & 0 & & & \\
\hline $346 \mathrm{E}$ & 72 & Sample & 0 & 30 & 0.1 & 0 & & & \\
\hline $348 \mathrm{~A}$ & 96 & Sample & 0 & 30 & 0.1 & 0 & \multirow{5}{*}{0} & \multirow{5}{*}{0} & \multirow{5}{*}{0} \\
\hline 348B & 96 & Sample & 0 & 30 & 0.1 & 0 & & & \\
\hline $348 \mathrm{C}$ & 96 & Sample & 0 & 30 & 0.1 & 0 & & & \\
\hline $348 \mathrm{D}$ & 96 & Sample & 0 & 30 & 0.1 & 0 & & & \\
\hline $348 \mathrm{E}$ & 96 & Sample & 0 & 30 & 0.1 & 0 & & & \\
\hline $350 \mathrm{~A}$ & 120 & Sample & 0 & 30 & 0.1 & 0 & \multirow{5}{*}{0} & \multirow{5}{*}{0} & \multirow{5}{*}{0} \\
\hline $350 \mathrm{~B}$ & 120 & Sample & 0 & 30 & 0.1 & 0 & & & \\
\hline $350 \mathrm{C}$ & 120 & Sample & 0 & 30 & 0.1 & 0 & & & \\
\hline $350 \mathrm{D}$ & 120 & Sample & 0 & 30 & 0.1 & 0 & & & \\
\hline $350 \mathrm{E}$ & 120 & Sample & 0 & 30 & 0.1 & 0 & & & \\
\hline
\end{tabular}

1. The dilution correction was a value used to correct for the volume of PBS in which the coupons were placed

2. The agar volume was a value used to correct for the volume of the PBS solution plated onto the petri dish

3. Viable spores was the number of spores after corrected for the dilution and agar volume corrections 
Table A4 - 16 - Test condition 5: $170 \mathrm{deg}$ F, 80\% RH, controls

\begin{tabular}{|c|c|c|c|c|c|c|c|c|c|}
\hline $\begin{array}{l}\text { Sample } \\
\text { Number }\end{array}$ & Time & Type & $\begin{array}{l}\text { Spores } \\
\text { counted }\end{array}$ & $\begin{array}{c}\text { Dilution } \\
\text { Correction }^{1}\end{array}$ & $\begin{array}{c}\text { Agar } \\
\text { Volume }^{2}\end{array}$ & $\begin{array}{c}\text { Viable } \\
\text { spores }^{3}\end{array}$ & $\begin{array}{c}\text { Mean } \\
\text { of } \\
\text { viable } \\
\text { spores }\end{array}$ & $\begin{array}{l}\text { SD of } \\
\text { viable } \\
\text { spores }\end{array}$ & $\begin{array}{l}\text { SE of } \\
\text { viable } \\
\text { spores }\end{array}$ \\
\hline $343 \mathrm{~A}$ & 24 & Control & 1 & 30 & 0.1 & 300 & \multirow{5}{*}{840} & \multirow{5}{*}{391} & \multirow{5}{*}{175} \\
\hline 343B & 24 & Control & 4 & 30 & 0.1 & 1,200 & & & \\
\hline $343 C$ & 24 & Control & 2 & 30 & 0.1 & 600 & & & \\
\hline 343D & 24 & Control & 3 & 30 & 0.1 & 900 & & & \\
\hline $343 \mathrm{E}$ & 24 & Control & 4 & 30 & 0.1 & 1,200 & & & \\
\hline $345 \mathrm{~A}$ & 48 & Control & 17 & 30 & 0.1 & 5,100 & \multirow{5}{*}{3,780} & \multirow{5}{*}{2,672} & \multirow{5}{*}{1,195} \\
\hline $345 B$ & 48 & Control & 6 & 30 & 0.1 & 1,800 & & & \\
\hline $345 \mathrm{C}$ & 48 & Control & 23 & 30 & 0.1 & 6,900 & & & \\
\hline $345 \mathrm{D}$ & 48 & Control & 16 & 30 & 0.1 & 4,800 & & & \\
\hline $345 \mathrm{E}$ & 48 & Control & 1 & 30 & 0.1 & 300 & & & \\
\hline $347 \mathrm{~A}$ & 72 & Control & 30 & 30 & 0.1 & 9,000 & \multirow{5}{*}{6,960} & \multirow{5}{*}{7,402} & \multirow{5}{*}{3,310} \\
\hline 347B & 72 & Control & 63 & 30 & 0.1 & 18,900 & & & \\
\hline $347 \mathrm{C}$ & 72 & Control & 15 & 30 & 0.1 & 4,500 & & & \\
\hline 347D & 72 & Control & 4 & 30 & 0.1 & 1,200 & & & \\
\hline $347 E$ & 72 & Control & 4 & 30 & 0.1 & 1,200 & & & \\
\hline $349 \mathrm{~A}$ & 96 & Control & 9 & 30 & 0.1 & 2,700 & \multirow{5}{*}{1,140} & \multirow{5}{*}{1,110} & \multirow{5}{*}{497} \\
\hline 349B & 96 & Control & 0 & 30 & 0.1 & 0 & & & \\
\hline $349 \mathrm{C}$ & 96 & Control & 3 & 30 & 0.1 & 900 & & & \\
\hline 349D & 96 & Control & 6 & 30 & 0.1 & 1,800 & & & \\
\hline $349 E$ & 96 & Control & 1 & 30 & 0.1 & 300 & & & \\
\hline $351 \mathrm{~A}$ & 120 & Control & 0 & 30 & 0.1 & 0 & \multirow{5}{*}{300} & \multirow{5}{*}{367} & \multirow{5}{*}{164} \\
\hline $351 \mathrm{~B}$ & 120 & Control & 0 & 30 & 0.1 & 0 & & & \\
\hline $351 \mathrm{C}$ & 120 & Control & 1 & 30 & 0.1 & 300 & & & \\
\hline $351 \mathrm{D}$ & 120 & Control & 1 & 30 & 0.1 & 300 & & & \\
\hline $351 \mathrm{E}$ & 120 & Control & 3 & 30 & 0.1 & 900 & & & \\
\hline
\end{tabular}

1. The dilution correction was a value used to correct for the volume of PBS in which the coupons were placed

2. The agar volume was a value used to correct for the volume of the PBS solution plated onto the petri dish

3. Viable spores was the number of spores after corrected for the dilution and agar volume corrections 
Table A4 - 17 - Test condition 7: $160 \mathrm{deg}$ F, 90\% RH, controls

\begin{tabular}{|c|c|c|c|c|c|c|c|c|c|}
\hline $\begin{array}{l}\text { Sample } \\
\text { Number }\end{array}$ & Time & Type & $\begin{array}{l}\text { Spores } \\
\text { counted }\end{array}$ & $\begin{array}{c}\text { Dilution } \\
\text { Correction }^{1}\end{array}$ & $\begin{array}{c}\text { Agar } \\
\text { Volume }^{2}\end{array}$ & $\begin{array}{l}\text { Viable } \\
\text { spores }^{3}\end{array}$ & $\begin{array}{c}\begin{array}{c}\text { Mean } \\
\text { of } \\
\text { viable }\end{array} \\
\text { spores } \\
\end{array}$ & $\begin{array}{c}\text { SD of } \\
\text { viable } \\
\text { spores }\end{array}$ & $\begin{array}{l}\text { SE of } \\
\text { viable } \\
\text { spores }\end{array}$ \\
\hline $253 \mathrm{~A}$ & 0 & Sample & 5 & 30 & 0.1 & 1,500 & \multirow{5}{*}{900} & \multirow{5}{*}{765} & \multirow{5}{*}{342} \\
\hline $253 \mathrm{~B}$ & 0 & Sample & 6 & 30 & 0.1 & 1,800 & & & \\
\hline $253 \mathrm{C}$ & 0 & Sample & 0 & 30 & 0.1 & 0 & & & \\
\hline 253D & 0 & Sample & 1 & 30 & 0.1 & 300 & & & \\
\hline $253 \mathrm{E}$ & 0 & Sample & 3 & 30 & 0.1 & 900 & & & \\
\hline $252 \mathrm{~A}$ & 24 & Sample & 0 & 30 & 0.1 & 0 & \multirow{5}{*}{0} & \multirow{5}{*}{0} & \multirow{5}{*}{0} \\
\hline $252 \mathrm{~B}$ & 24 & Sample & 0 & 30 & 0.1 & 0 & & & \\
\hline $252 \mathrm{C}$ & 24 & Sample & 0 & 30 & 0.1 & 0 & & & \\
\hline 252D & 24 & Sample & 0 & 30 & 0.1 & 0 & & & \\
\hline $252 \mathrm{E}$ & 24 & Sample & 0 & 30 & 0.1 & 0 & & & \\
\hline $254 \mathrm{~A}$ & 48 & Sample & 0 & 30 & 0.1 & 0 & \multirow{5}{*}{0} & \multirow{5}{*}{0} & \multirow{5}{*}{0} \\
\hline 254B & 48 & Sample & 0 & 30 & 0.1 & 0 & & & \\
\hline $254 \mathrm{C}$ & 48 & Sample & 0 & 30 & 0.1 & 0 & & & \\
\hline $254 \mathrm{D}$ & 48 & Sample & 0 & 30 & 0.1 & 0 & & & \\
\hline $254 \mathrm{E}$ & 48 & Sample & 0 & 30 & 0.1 & 0 & & & \\
\hline $256 \mathrm{~A}$ & 72 & Sample & 0 & 30 & 0.1 & 0 & \multirow{5}{*}{0} & \multirow{5}{*}{0} & \multirow{5}{*}{0} \\
\hline $256 \mathrm{~B}$ & 72 & Sample & 0 & 30 & 0.1 & 0 & & & \\
\hline $256 \mathrm{C}$ & 72 & Sample & 0 & 30 & 0.1 & 0 & & & \\
\hline $256 \mathrm{D}$ & 72 & Sample & 0 & 30 & 0.1 & 0 & & & \\
\hline $256 \mathrm{E}$ & 72 & Sample & 0 & 30 & 0.1 & 0 & & & \\
\hline $258 \mathrm{~A}$ & 96 & Sample & 0 & 30 & 0.1 & 0 & \multirow{5}{*}{0} & \multirow{5}{*}{0} & \multirow{5}{*}{0} \\
\hline 258B & 96 & Sample & 0 & 30 & 0.1 & 0 & & & \\
\hline $258 \mathrm{C}$ & 96 & Sample & 0 & 30 & 0.1 & 0 & & & \\
\hline $258 \mathrm{D}$ & 96 & Sample & 0 & 30 & 0.1 & 0 & & & \\
\hline $258 \mathrm{E}$ & 96 & Sample & 0 & 30 & 0.1 & 0 & & & \\
\hline $260 \mathrm{~A}$ & 120 & Sample & 0 & 30 & 0.1 & 0 & \multirow{5}{*}{0} & \multirow{5}{*}{0} & \multirow{5}{*}{0} \\
\hline 260B & 120 & Sample & 0 & 30 & 0.1 & 0 & & & \\
\hline $260 \mathrm{C}$ & 120 & Sample & 0 & 30 & 0.1 & 0 & & & \\
\hline 260D & 120 & Sample & 0 & 30 & 0.1 & 0 & & & \\
\hline $260 \mathrm{E}$ & 120 & Sample & 0 & 30 & 0.1 & 0 & & & \\
\hline
\end{tabular}

1. The dilution correction was a value used to correct for the volume of PBS in which the coupons were placed

2. The agar volume was a value used to correct for the volume of the PBS solution plated onto the petri dish

3. Viable spores was the number of spores after corrected for the dilution and agar volume corrections 
Table A4 - 18 - Test condition 7: $160 \operatorname{deg}$ F, 90\% RH, controls

\begin{tabular}{|c|c|c|c|c|c|c|c|c|c|}
\hline $\begin{array}{l}\text { Sample } \\
\text { Number }\end{array}$ & Time & Type & $\begin{array}{l}\text { Spores } \\
\text { counted }\end{array}$ & $\begin{array}{c}\text { Dilution } \\
\text { Correction }^{1}\end{array}$ & $\begin{array}{c}\text { Agar } \\
\text { Volume }^{2}\end{array}$ & $\begin{array}{c}\text { Viable } \\
\text { spores }^{3}\end{array}$ & $\begin{array}{c}\begin{array}{c}\text { Mean } \\
\text { of } \\
\text { viable } \\
\text { spores }\end{array} \\
\end{array}$ & $\begin{array}{l}\text { SD of } \\
\text { viable } \\
\text { spores }\end{array}$ & $\begin{array}{l}\text { SE of } \\
\text { viable } \\
\text { spores }\end{array}$ \\
\hline $253 \mathrm{~A}$ & 24 & Control & 5 & 30 & 0.1 & 1,500 & \multirow{5}{*}{900} & \multirow{5}{*}{765} & \multirow{5}{*}{342} \\
\hline 253B & 24 & Control & 6 & 30 & 0.1 & 1,800 & & & \\
\hline $253 \mathrm{C}$ & 24 & Control & 0 & 30 & 0.1 & 0 & & & \\
\hline 253D & 24 & Control & 1 & 30 & 0.1 & 300 & & & \\
\hline $253 \mathrm{E}$ & 24 & Control & 3 & 30 & 0.1 & 900 & & & \\
\hline $255 \mathrm{~A}$ & 48 & Control & 0 & 30 & 0.1 & 0 & \multirow{5}{*}{960} & \multirow{5}{*}{934} & \multirow{5}{*}{418} \\
\hline $255 \mathrm{~B}$ & 48 & Control & 5 & 30 & 0.1 & 1,500 & & & \\
\hline $255 \mathrm{C}$ & 48 & Control & 0 & 30 & 0.1 & 0 & & & \\
\hline $255 \mathrm{D}$ & 48 & Control & 4 & 30 & 0.1 & 1,200 & & & \\
\hline $255 \mathrm{E}$ & 48 & Control & 7 & 30 & 0.1 & 2,100 & & & \\
\hline $257 \mathrm{~A}$ & 72 & Control & 0 & 30 & 0.1 & 0 & \multirow{5}{*}{780} & \multirow{5}{*}{838} & \multirow{5}{*}{375} \\
\hline 257B & 72 & Control & 2 & 30 & 0.1 & 600 & & & \\
\hline $257 \mathrm{C}$ & 72 & Control & 5 & 30 & 0.1 & 1,500 & & & \\
\hline 257D & 72 & Control & 6 & 30 & 0.1 & 1,800 & & & \\
\hline $257 \mathrm{E}$ & 72 & Control & 0 & 30 & 0.1 & 0 & & & \\
\hline $259 \mathrm{~A}$ & 96 & Control & 3 & 30 & 0.1 & 900 & \multirow{5}{*}{2,880} & \multirow{5}{*}{5,781} & \multirow{5}{*}{2,585} \\
\hline $259 \mathrm{~B}$ & 96 & Control & 44 & 30 & 0.1 & 13,200 & & & \\
\hline $259 \mathrm{C}$ & 96 & Control & 0 & 30 & 0.1 & 0 & & & \\
\hline 259D & 96 & Control & 0 & 30 & 0.1 & 0 & & & \\
\hline $259 \mathrm{E}$ & 96 & Control & 1 & 30 & 0.1 & 300 & & & \\
\hline $261 \mathrm{~A}$ & 120 & Control & 9 & 30 & 0.1 & 2,700 & \multirow{5}{*}{2,040} & \multirow{5}{*}{1,004} & \multirow{5}{*}{449} \\
\hline 261B & 120 & Control & 11 & 30 & 0.1 & 3,300 & & & \\
\hline $261 \mathrm{C}$ & 120 & Control & 4 & 30 & 0.1 & 1,200 & & & \\
\hline 261D & 120 & Control & 3 & 30 & 0.1 & 900 & & & \\
\hline $271 \mathrm{E}$ & 120 & Control & 7 & 30 & 0.1 & 2,100 & & & \\
\hline
\end{tabular}

1. The dilution correction was a value used to correct for the volume of PBS in which the coupons were placed

2. The agar volume was a value used to correct for the volume of the PBS solution plated onto the petri dish

3. Viable spores was the number of spores after corrected for the dilution and agar volume corrections 
Table A4 - 19 - Test condition 9: $160 \mathrm{deg}$ F, 70\% RH, samples

\begin{tabular}{|c|c|c|c|c|c|c|c|c|c|}
\hline $\begin{array}{l}\text { Sample } \\
\text { Number }\end{array}$ & Time & Type & $\begin{array}{l}\text { Spores } \\
\text { counted }\end{array}$ & $\begin{array}{c}\text { Dilution } \\
\text { Correction }^{1}\end{array}$ & $\begin{array}{c}\text { Agar } \\
\text { Volume }^{2}\end{array}$ & $\begin{array}{c}\text { Viable } \\
\text { spores }^{3}\end{array}$ & $\begin{array}{c}\text { Mean } \\
\text { of } \\
\text { viable } \\
\text { spores }\end{array}$ & $\begin{array}{l}\text { SD of } \\
\text { viable } \\
\text { spores }\end{array}$ & $\begin{array}{l}\text { SE of } \\
\text { viable } \\
\text { spores }\end{array}$ \\
\hline $501 \mathrm{~A}$ & 0 & Sample & 15 & 30 & 0.1 & 4,500 & \multirow{5}{*}{10,860} & \multirow{5}{*}{6,233} & \multirow{5}{*}{2,788} \\
\hline $501 \mathrm{~B}$ & 0 & Sample & 66 & 30 & 0.1 & 19,800 & & & \\
\hline $501 \mathrm{C}$ & 0 & Sample & 20 & 30 & 0.1 & 6,000 & & & \\
\hline 501D & 0 & Sample & 33 & 30 & 0.1 & 9,900 & & & \\
\hline $501 \mathrm{E}$ & 0 & Sample & 47 & 30 & 0.1 & 14,100 & & & \\
\hline $502 \mathrm{~A}$ & 24 & Sample & 4 & 30 & 0.1 & 1,200 & \multirow{5}{*}{2,340} & \multirow{5}{*}{2,028} & \multirow{5}{*}{907} \\
\hline 502B & 24 & Sample & 18 & 30 & 0.1 & 5,400 & & & \\
\hline $502 \mathrm{C}$ & 24 & Sample & 5 & 30 & 0.1 & 1,500 & & & \\
\hline 502D & 24 & Sample & 11 & 30 & 0.1 & 3,300 & & & \\
\hline $502 \mathrm{E}$ & 24 & Sample & 1 & 30 & 0.1 & 300 & & & \\
\hline $504 \mathrm{~A}$ & 48 & Sample & 2 & 30 & 0.1 & 600 & \multirow{5}{*}{1,140} & \multirow{5}{*}{747} & \multirow{5}{*}{334} \\
\hline 504B & 48 & Sample & 2 & 30 & 0.1 & 600 & & & \\
\hline $504 \mathrm{C}$ & 48 & Sample & 3 & 30 & 0.1 & 900 & & & \\
\hline 504D & 48 & Sample & 8 & 30 & 0.1 & 2,400 & & & \\
\hline $504 \mathrm{E}$ & 48 & Sample & 4 & 30 & 0.1 & 1,200 & & & \\
\hline $506 \mathrm{~A}$ & 72 & Sample & 4 & 30 & 0.1 & 1,200 & \multirow{5}{*}{780} & \multirow{5}{*}{722} & \multirow{5}{*}{323} \\
\hline $506 \mathrm{~B}$ & 72 & Sample & 5 & 30 & 0.1 & 1,500 & & & \\
\hline $506 \mathrm{C}$ & 72 & Sample & 0 & 30 & 0.1 & 0 & & & \\
\hline 506D & 72 & Sample & 0 & 30 & 0.1 & 0 & & & \\
\hline $506 \mathrm{E}$ & 72 & Sample & 4 & 30 & 0.1 & 1,200 & & & \\
\hline $508 \mathrm{~A}$ & 96 & Sample & 0 & 30 & 0.1 & 0 & \multirow{5}{*}{600} & \multirow{5}{*}{600} & \multirow{5}{*}{268} \\
\hline 508B & 96 & Sample & 1 & 30 & 0.1 & 300 & & & \\
\hline $508 \mathrm{C}$ & 96 & Sample & 1 & 30 & 0.1 & 300 & & & \\
\hline 508D & 96 & Sample & 5 & 30 & 0.1 & 1,500 & & & \\
\hline $508 \mathrm{E}$ & 96 & Sample & 3 & 30 & 0.1 & 900 & & & \\
\hline $510 \mathrm{~A}$ & 120 & Sample & 0 & 30 & 0.1 & 0 & \multirow{5}{*}{120} & \multirow{5}{*}{268} & \multirow{5}{*}{120} \\
\hline $510 \mathrm{~B}$ & 120 & Sample & 0 & 30 & 0.1 & 0 & & & \\
\hline $510 \mathrm{C}$ & 120 & Sample & 0 & 30 & 0.1 & 0 & & & \\
\hline 510D & 120 & Sample & 0 & 30 & 0.1 & 0 & & & \\
\hline $510 \mathrm{E}$ & 120 & Sample & 2 & 30 & 0.1 & 600 & & & \\
\hline
\end{tabular}

1. The dilution correction was a value used to correct for the volume of PBS in which the coupons were placed

2. The agar volume was a value used to correct for the volume of the PBS solution plated onto the petri dish

3. Viable spores was the number of spores after corrected for the dilution and agar volume corrections 
Table A4 - 20 - Test condition 9: 160 deg F, 70\% RH, controls

\begin{tabular}{|c|c|c|c|c|c|c|c|c|c|}
\hline $\begin{array}{l}\text { Sample } \\
\text { Number }\end{array}$ & Time & Type & $\begin{array}{l}\text { Spores } \\
\text { counted }\end{array}$ & $\begin{array}{c}\text { Dilution } \\
\text { Correction }^{1}\end{array}$ & $\begin{array}{c}\text { Agar } \\
\text { Volume }^{2}\end{array}$ & $\begin{array}{c}\text { Viable } \\
\text { spores }^{3}\end{array}$ & $\begin{array}{c}\text { Mean } \\
\text { of } \\
\text { viable } \\
\text { spores }\end{array}$ & $\begin{array}{c}\text { SD of } \\
\text { viable } \\
\text { spores }\end{array}$ & $\begin{array}{c}\text { SE of } \\
\text { viable } \\
\text { spores }\end{array}$ \\
\hline $503 \mathrm{~A}$ & 24 & Control & 25 & 30 & 0.1 & 7,500 & \multirow{5}{*}{7,080} & \multirow{5}{*}{915} & \multirow{5}{*}{409} \\
\hline 503D & 24 & Control & 20 & 30 & 0.1 & 6,000 & & & \\
\hline $503 C$ & 24 & Control & 22 & 30 & 0.1 & 6,600 & & & \\
\hline 503D & 24 & Control & 28 & 30 & 0.1 & 8,400 & & & \\
\hline $503 \mathrm{E}$ & 24 & Control & 23 & 30 & 0.1 & 6,900 & & & \\
\hline $505 \mathrm{~A}$ & 48 & Control & 27 & 30 & 0.1 & 8,100 & \multirow{5}{*}{16,800} & \multirow{5}{*}{12,182} & \multirow{5}{*}{5,448} \\
\hline 505B & 48 & Control & 32 & 30 & 0.1 & 9,600 & & & \\
\hline $505 \mathrm{C}$ & 48 & Control & 29 & 30 & 0.1 & 8,700 & & & \\
\hline $505 \mathrm{D}$ & 48 & Control & 71 & 30 & 0.1 & 21,300 & & & \\
\hline $505 \mathrm{E}$ & 48 & Control & 121 & 30 & 0.1 & 36,300 & & & \\
\hline $507 \mathrm{~A}$ & 72 & Control & 23 & 30 & 0.1 & 6,900 & \multirow{5}{*}{7,260} & \multirow{5}{*}{3,815} & \multirow{5}{*}{1,706} \\
\hline 507B & 72 & Control & 46 & 30 & 0.1 & 13,800 & & & \\
\hline $507 \mathrm{C}$ & 72 & Control & 20 & 30 & 0.1 & 6,000 & & & \\
\hline 507D & 72 & Control & 13 & 30 & 0.1 & 3,900 & & & \\
\hline $507 \mathrm{E}$ & 72 & Control & 19 & 30 & 0.1 & 5,700 & & & \\
\hline $509 \mathrm{~A}$ & 96 & Control & 26 & 30 & 0.1 & 7,800 & \multirow{5}{*}{27,360} & \multirow{5}{*}{28,398} & \multirow{5}{*}{12,700} \\
\hline 509B & 96 & Control & 158 & 30 & 0.1 & 47,400 & & & \\
\hline $509 \mathrm{C}$ & 96 & Control & 225 & 30 & 0.1 & 67,500 & & & \\
\hline 509D & 96 & Control & 18 & 30 & 0.1 & 5,400 & & & \\
\hline $509 \mathrm{E}$ & 96 & Control & 29 & 30 & 0.1 & 8,700 & & & \\
\hline $511 \mathrm{~A}$ & 120 & Control & 20 & 30 & 0.1 & 6,000 & \multirow{5}{*}{7,020} & \multirow{5}{*}{1,744} & \multirow{5}{*}{780} \\
\hline $511 \mathrm{~B}$ & 120 & Control & 22 & 30 & 0.1 & 6,600 & & & \\
\hline $511 \mathrm{C}$ & 120 & Control & 18 & 30 & 0.1 & 5,400 & & & \\
\hline $511 \mathrm{D}$ & 120 & Control & 24 & 30 & 0.1 & 7,200 & & & \\
\hline $511 \mathrm{E}$ & 120 & Control & 33 & 30 & 0.1 & 9,900 & & & \\
\hline
\end{tabular}

1. The dilution correction was a value used to correct for the volume of PBS in which the coupons were placed

2. The agar volume was a value used to correct for the volume of the PBS solution plated onto the petri dish

3. Viable spores was the number of spores after corrected for the dilution and agar volume corrections 


\section{CHAPTER 5 -- CONCLUSIONS}

\section{Introduction}

The overall goal of this dissertation research was to determine if aircraft materials, consisting of plastic and aluminum coupons, could be effectively decontaminated from a Bacillus anthracis simulant using high heat and humidity ranges within aircraft engineering specifications. Three different methods of inoculations were used-high direct, low direct, and an aerosol deposition. The first study (Chapter 2) evaluated the deposition characteristics of an innovative bioaerosol test chamber. This study included design, build, and evaluation of the deposition characteristics of the bioaerosol used. Aluminum coupon decontamination tests (Chapter 3) were completed at five different temperature and humidity ranges using three different deposition mechanisms, which included high direct, low direct, and deposition in the aerosol test chamber. The third study (Chapter 4$)$ evaluated the inactivation rates on plastic coupons using the same deposition methods. The differences in inactivation were compared between aluminum and plastic coupons to determine if they were significantly different.

\section{Summary and significance of each study}

A bioaerosol test chamber can be designed and built to model biological agent deposition on aircraft materials.

The bioaerosol test chamber was the first one to be designed, built, and tested to evaluate inactivation of a biological warfare agent simulant after deposition onto coupons. The study found that the coefficients of variation for the spore depositions were less than $25.5 \%$ for the

final four studies completed, which is an acceptable level. Contour plots completed on the $\log$ transformed data showed that the deposition for each test was even; however, there were no 
discernible patterns over all the four tests. Additionally, equations derived to model the deposition showed that spore recovery efficiencies were from $8.67 \%$ to $31.0 \%$ of the total spores modeled. These tests showed the recovery of the spores could be modeled appropriately with the equations derived. This study showed the test chamber could be used effectively to model spore depositions if control coupons were used.

Aluminum coupons, indicative of aircraft materials, can be effectively decontaminated using high heat and humidity.

The spores deposited on aluminum coupons were decontaminated effectively using high heat and humidity. These tests showed that the spores were all inactivated following 24 hours of treatment at $180^{\circ} \mathrm{F}$ with $90 \% \mathrm{RH}$ and partially inactivated at $170^{\circ} \mathrm{F}$ with $80 \% \mathrm{RH}$. A stepwise regression model was completed to determine the terms that would add significantly to a regression model. The stepwise regression included mandatory variables (or variables that had to be selected by the regression). These variables were time, temperature, and humidity. The data for the stepwise regression retained more variables for high direct inoculation (10 predictors) than low (8 predictors) or aerosol deposition (5). The only variable retained by all three models, besides the mandatory variables, was Temp ${ }^{2} * \mathrm{Time}^{2}$. For both of the direct inoculation methods, several of the same variables were retained, which included Temp*Humidity, Temp* Time, Humidity ${ }^{2}$, and Temp ${ }^{2} *$ Time $^{2}$. More of the predictor variables for high inoculation included an interaction with time when compared to the predictors for low inoculation. This was expected because it was assumed it would take more time to inactivate the high inoculation spores. It is also interesting to note Time $^{2}$ was retained for high inoculation and time $^{3}$ was retained for low inoculation. It appears that temperature is a more critical variable 
than humidity for aerosol deposition because both retained terms included temperature $\left(\right.$ Temp $^{2}$ and then the interaction between Temp ${ }^{2}$ and Time ${ }^{2}$ ) - this shows humidity is not as critical of a variable for this deposition. Overall this showed the spores deposited onto aluminum coupons can be effectively inactivated using high heat and humidity at specific combinations of these variables coupled with time. This shows promise for future efforts to inactivate biological agents safely, effectively, and also within aircraft engineering specifications.

Plastic coupons, similar to materials used on aircraft, can be effectively decontaminated using high heat and humidity.

Decontamination tests for the plastic coupons showed full spore inactivation for the high inoculation ( $10^{6}$ spores per coupon) after 48 hours with $180^{\circ} \mathrm{F}$ and $90 \% \mathrm{RH}$ (test condition 1 ) and partially inactivated at $170^{\circ} \mathrm{F}$ and $80 \% \mathrm{RH}$ (test condition 5), $180^{\circ} \mathrm{F}$ and $70 \% \mathrm{RH}$ (test condition 3), and $160^{\circ} \mathrm{F}$ and $90 \% \mathrm{RH}$ (test condition 7). Test condition 9 had minimal to no inactivation on the spores during the time limit of 120 hours. Tests with low direct inoculation $\left(10^{4}\right.$ spores per coupon) showed complete spore kills at 48 hours when treatment was $180{ }^{\circ} \mathrm{F}$ with $90 \% \mathrm{RH}$ (test condition 1 ) and $170{ }^{\circ} \mathrm{F}$ with $80 \% \mathrm{RH}$ (test condition 5). Additionally, all spores were inactivated at 120 hours $160^{\circ} \mathrm{F}$ with $90 \%$ (test condition 7 ). Finally, all spores deposited by aerosols were inactivated within 48 hours, except for test condition $9\left(160{ }^{\circ} \mathrm{F}\right.$ with $70 \% \mathrm{RH})$, which still had active spores at the 120 hour point. The stepwise regression resulted in approximately the same number of terms being retained in the models with high, low, and aerosol deposition have 7, 6, and 8 terms, respectively. Besides the mandatory variables (time, temperature, and humidity), there were no variables retained in all three models. This analysis does indicate humidity is a critical factor, as nearly all variables retained in these models contain 
humidity—each model only has one variable that does not contain humidity. The $\mathrm{R}^{2}$ values for these models were $76.6 \%, 68.8 \%$, and $77.8 \%$, for high and low direct inoculation and aerosol deposition, respectively. Thus most of the variability for the spore inactivation is explained by the models. Overall this showed the spores deposited onto plastic coupons can be effectively inactivated using high heat and humidity at specific combinations of these variables coupled with time. This shows promise for future efforts to inactivate biological agents safely, effectively, and also within aircraft engineering specifications.

There were six tests that showed a significant difference between the two different materials.

Finally, the data from the aluminum and plastic coupons were evaluated to determine if there was a difference in the coupon materials for spore inactivation. Of 15 total tests, only 6 showed a significant difference in inactivation rates, all demonstrating faster inactivation rates for plastic coupons. For high direction inoculation, there was a significant difference for test condition $5\left(170^{\circ} \mathrm{F}\right.$ with $\left.80 \% \mathrm{RH}\right)$ and test condition $7\left(160^{\circ} \mathrm{F}\right.$ with $\left.90 \% \mathrm{RH}\right)$. For low direct inoculation there was only one test condition that was significantly different for the testing conditions and this was test condition $7\left(160^{\circ} \mathrm{F}\right.$ with $\left.90 \% \mathrm{RH}\right)$. A tobit analysis showed there plastic coupon inactivation rates were significantly different (faster inactivation) plastic coupons for test condition $3\left(180^{\circ} \mathrm{F}\right.$ with $\left.70 \% \mathrm{RH}\right), 5\left(170^{\circ} \mathrm{F}\right.$ with $\left.80 \% \mathrm{RH}\right)$, and $7\left(160^{\circ} \mathrm{F}\right.$ with $\left.90 \% \mathrm{RH}\right)$. This data showed that if the spores are inactivated on aluminum coupons, they will be inactivated on the plastic coupons as well. The optimal heat and humidity levels for both plastic and aluminum coupons is the highest temperature and humidity that can be maintained effectively within engineering specifications. 


\section{Conclusion}

The goal of this research was to determine if a Bacillus anthracis simulant could be decontaminated from aluminum and plastic coupons using high heat and humidity levels and evaluating three different deposition mechanisms. The results show there is a difference in the time required to inactivate the spores when delivered by the aerosol deposition method, which is a more realistic contamination method. These results show future research should focus more on these types of delivery mechanisms. These results do, however, confirm that when higher spore levels are inactivated, the lower levels of spores, delivered by direct inoculation or aerosol deposition, will also be inactivated. Additionally, comparisons of plastic versus aluminum coupons showed that plastic coupons were decontaminated quicker for 6 of 15 tests. Again, if the aluminum coupons are effectively decontaminated, the plastic materials will be as well. Overall, this research showed the spores can be effectively inactivated using high heat and humidity at specific combinations of these variables coupled with time. This shows promise for future efforts to inactivate biological agents safely, effectively, and also within aircraft engineering specifications.

\section{Future Research}

Findings of this dissertation raised several questions for future investigation including:

1. Investigation of more condensed time intervals. These studies found that several temperature and humidity ranges inactivated the spores within 24 hours. Additional data on shorter time periods could be beneficial, including determination if the activation rates are significantly different for these lower levels of treatment. 
2. Investigation of longer time periods. The upper time limits of this study were placed at 120 hours. Several different heat and humidity levels showed the spore numbers were decreasing, yet not fully inactivated at 120 hours. Additional samples showed very little spore inactivation, even after the 120 hour treatment time. Additional tests extending these times up to 168 hours (7 days) or longer could provide useful data.

3. Investigate inactivation rates of spores fumed with silica. This research used BG spores diluted in phosphate buffered saline with tween, aerosolized using a Collison 6-jet nebulizer. Spores are available with fumed silica that can be delivered using a dry method. While the aerosol deposited spores in this study provided a unique analysis, generating spores with dry-fumed silica would be provide an even more realistic deposition method. This would also provide data on whether the fumed silica provides some level of protection to the spores.

4. Different aircraft materials. Previous studies researched only aircraft aluminum coupons - this is the first study to have analyzed a different material and the results were promising in that the inactivation rates were faster for plastic coupons. Additional data on aircraft materials could be beneficial. This could include non-coated aluminum, stainless steel, and aircraft cloth material such as canvas.

5. Investigation of active, but not culturable spores. This research focused on a culture method to evaluate the inactivation of spores. Some spores could be present that are active but are not culturable. Methods to distinguish the difference between these are very limited. If advances in technology allow this (for instance, a flow cytometry dye that could distinguish between an active and inactive spore), such methods would help 
delineate the differences in these types of spores and should be considered for future research. 


\section{LIST OF UNITS}

$\begin{array}{ll}{ }^{\circ} \mathrm{C} & \text { Degrees Celsius } \\ \mathrm{Cfm} & \text { Cubic feet per minute } \\ \mathrm{cfu} & \text { Colony forming unit } \\ \mathrm{cm} & \text { Centimeter } \\ \mathrm{cm}^{2} & \text { Square centimeter } \\ { }^{\circ} \mathrm{F} & \text { Degrees Fahrenheit } \\ \text { “ } & \text { Inch } \\ \mathrm{in} & \text { Inch } \\ \mathrm{kPa} & \text { Kilopascal } \\ \mathrm{L} / \mathrm{s} & \text { Liters per second } \\ \mathrm{lpm} & \text { Liters per minute } \\ \mathrm{m} & \text { Meter } \\ \mathrm{MBq} & \text { Megabecquerel } \\ \mathrm{mil} & \text { Length equal to 0.001 inch } \\ \mathrm{mL} & \text { Milliliter } \\ \mathrm{mm} & \text { Millimeter } \\ \mathrm{ppm} & \text { Parts per million } \\ \mathrm{psi} & \text { Pounds per square inch } \\ \mathrm{SCFH} \text { Air } & \text { Standard cubic feet per hour } \\ \mu \mathrm{m} & \text { Micrometer } \\ \mu \mathrm{L} & \text { Microliter }\end{array}$




\section{LIST OF ABBREVIATIONS AND SYMBOLS}

ACGIH

AF

AFI

AFRL

AMC

APS

$\mathrm{Ba}$

BBT

BG

BSL

BTK or BT

$\mathrm{C}_{2} \mathrm{H}_{4} \mathrm{O}_{3}$

CBRN

$\mathrm{Cc}$

$\mathrm{CDC}$

$\mathrm{CFU}$

$\mathrm{ClO}_{2}$

$\mathrm{C}_{\max }$

$\mathrm{C}_{\text {neb }}$

$\mathrm{CSU}$

CV

DMM

DoD

DoDI

$\mathrm{D}_{\mathrm{p}}$

ECS

EPA

EU

g

G

GS

$\mathrm{H}$

$\mathrm{H}_{2} \mathrm{O}_{2}$

$\mathrm{H}_{\mathrm{o}}$

HEPA

HPV

HVAC

$\mathrm{KCl}$

$\mathrm{Kr}-85$

$\mathrm{LD}_{50}$

LFA

LOD

LOQ
American Conference of Governmental Industrial Hygienists

Air Force

Air Force Instruction

Air Force Research Laboratory

Air Mobility Command

Aerodynamic Particle Sizer

Bacillus anthracis

Butterfield buffer, with tween

Bacillus atrophaeus subspecies globigii

Biosafety Level

Bacillus Thuringiensis var kurstaki

Peroxyacetic acid

Chemical, biological, radiological, and nuclear

Cunningham correction factor

Centers for Disease Control

Colony forming units

Chlorine dioxide

Total number concentration (CFU/meter ${ }^{3}$ )

Spore concentration in nebulizer (CFU/milliliter)

Colorado State University

coefficients of variations

Design Metal Manufacturing, Fort Collins, CO

Department of Defense

Department of Defense Instruction

Particle diameter (meter)

Environmental control system

Environmental Protection Agency

Endotoxin unit

Acceleration of gravity (meter/second ${ }^{2}$ )

Generation rate (CFU/minutes)

Galvanized steel

Chamber height

Hydrogen peroxide

Null hypothesis

High efficiency particle air

Hydrogen peroxide vapor

Heating, ventilation, and air conditioning

Potassium chloride

Krypton 85

Lethal dose 50 (median concentration of a toxicant that will kill $50 \%$ of the population within a designated period)

Large frame aircraft

Limit of detection

Limit of quantification 


\begin{tabular}{|c|c|}
\hline MCE & Mixed cellulose ester \\
\hline MIL-PRF & Military Performance \\
\hline MIL-STD & Military Standard \\
\hline MMAD & Mass median aerodynamic diameter \\
\hline $\mathrm{n}$ & Number of samples \\
\hline$Q_{\text {in }}$ & Air generation rate into chamber (meter ${ }^{3} /$ minute $)$ \\
\hline $\mathrm{Q}_{\text {liq }}$ & Liquid use rate for nebulizer (milliliter/minute) \\
\hline $\mathrm{NaOCl}$ & Sodium hypochlorite \\
\hline NIOSH & National Institute for Occupational Safety and Health \\
\hline PBS & Phosphate buffered solution \\
\hline PPE & Personal protective equipment \\
\hline PSL & Polystyrene latex spheres \\
\hline PTFE & Polytetrafluoroethylene \\
\hline $\mathrm{R}^{2}$ & Coefficient of determination \\
\hline RFU & Relative fluorescence units \\
\hline RH & Relative humidity \\
\hline $\mathrm{SA}_{\mathrm{c}}$ & Coupon surface area \\
\hline $\mathrm{S}_{\mathrm{c}}$ & Surface concentration per coupon (CFU) \\
\hline SGR & Surgeon General's Office, Modernization Branch \\
\hline$S_{\mathrm{V}}$ & Viable surface concentration (CFU/meter $\left.{ }^{2}\right)$ \\
\hline TC & Test Condition \\
\hline Time $_{\text {Set }}$ & Time to setting (seconds) \\
\hline TSA & Typticase soy agar \\
\hline USAF & United States Air Force \\
\hline USAMRIID & United States Army Medical Institute of Infectious Diseases \\
\hline UDRI & University of Dayton Research Institute \\
\hline UV & Ultraviolet \\
\hline VHP & Vaporized hydrogen peroxide \\
\hline $\mathrm{V}_{\mathrm{ts}}$ & Settling velocity (meter/second) \\
\hline$\rho_{\mathrm{p}}$ & Symbol for density of particle (kilogram/meter ${ }^{3}$ ) \\
\hline$\eta$ & $\begin{array}{l}\text { Symbol for viscosity of gas (air), (Pascals/second) or } \\
\text { (kilogram/meter* second) }\end{array}$ \\
\hline
\end{tabular}

